NIST NCSTAR 1-3C

Federal Building and Fire Safety Investigation of the World Trade Center Disaster

\title{
Damage and Failure Modes of Structural Steel Components
}

\section{(Appendices A-G)}

Stephen W. Banovic

Timothy Foecke 


\section{Appendix A \\ EXAmples of Perimeter PANel FloOR TRuss Connectors}

The floor trusses were attached to the perimeter panels at the interior intersection of the columns and spandrels by different types of connectors. The following appendix displays photographic examples, taken by National Institute of Standards and Technology (NIST), of these types of connectors as observed on the recovered panels. 


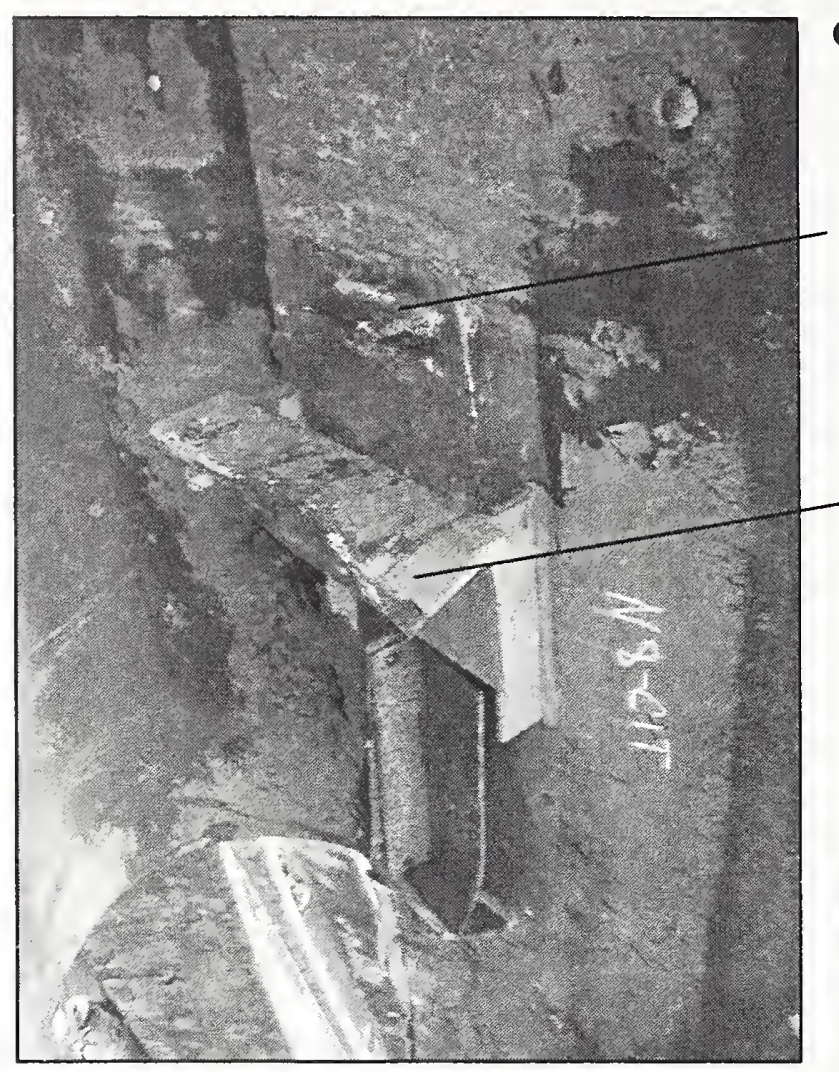

\section{Connection N8-CIT}

Portion of welded gusset plate with backing bar

Seat with 2 intact bolt holes for truss attachment

Figure Ala: Type A floor truss connector

Standoff plates used to attach seat to column interior

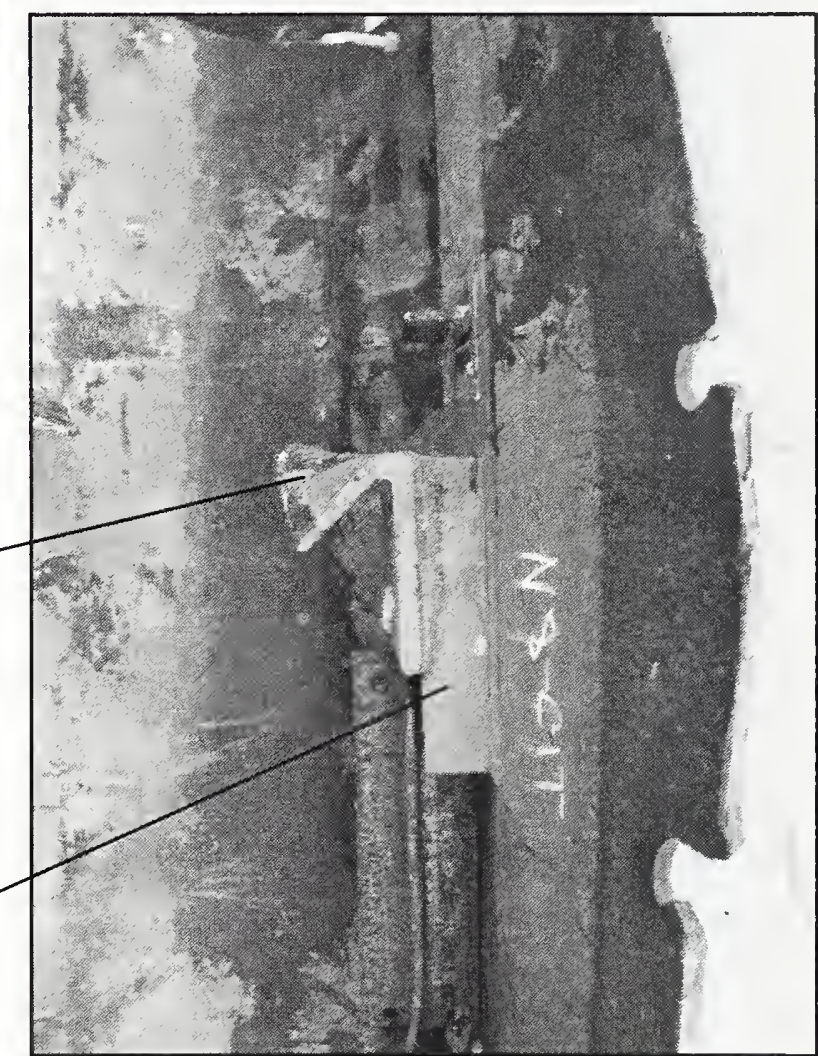

Figure A1b: Type A floor truss connector Figure A-1. Type A Floor Truss Connector (primary truss seat). 


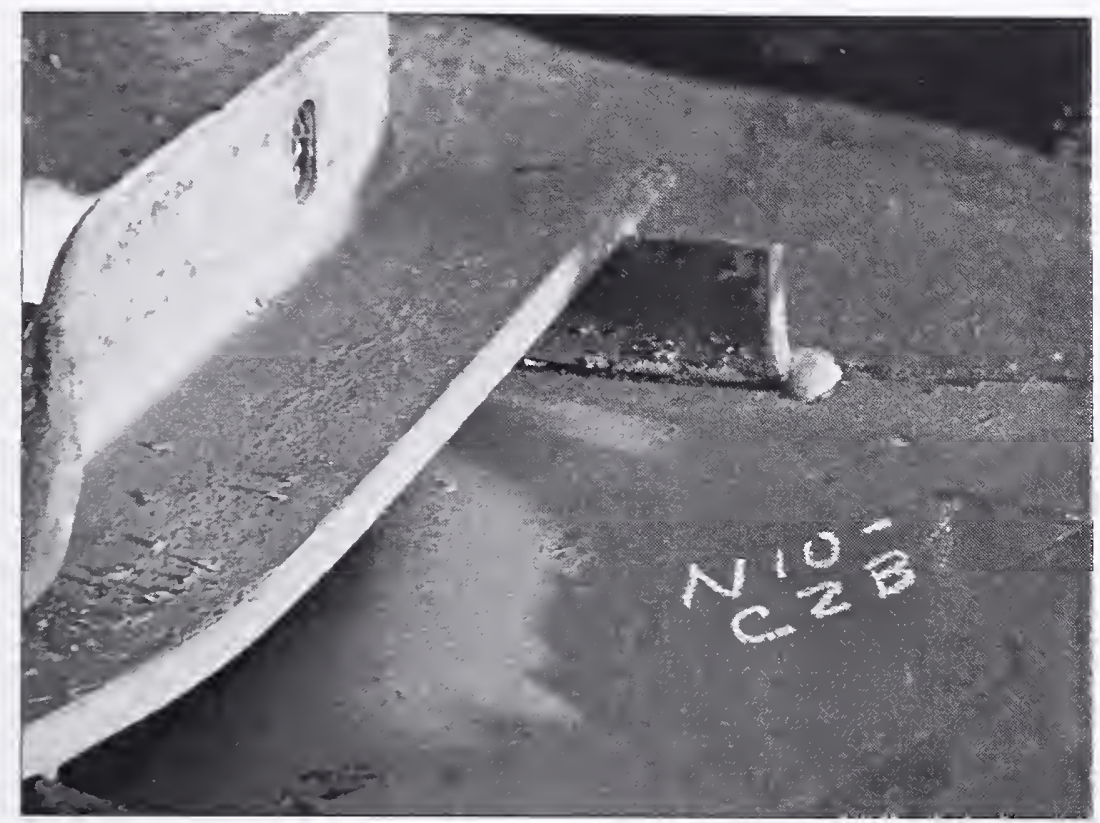

\section{Connection N10-C2B}

Standoff plate welded on only one side

Figure Alc: Type A floor truss connector

\section{Connection N8-CIT}

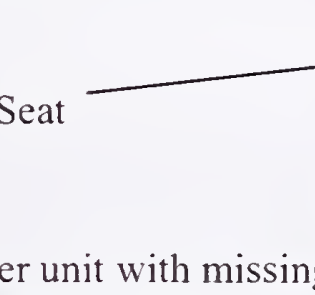

Damper unit with missi
viscoelastic material

Gusset plate for viscoelastic damper unit attachment to column interior

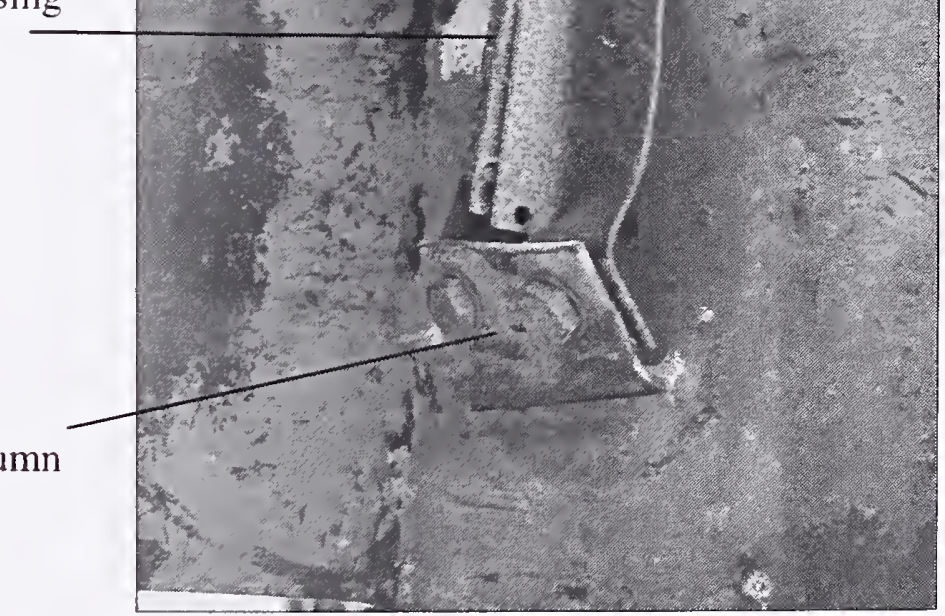

Figure Ald: Type A floor truss connector

Figure A-1. Type A Floor Truss Connector (primary truss seat) (cont.). 


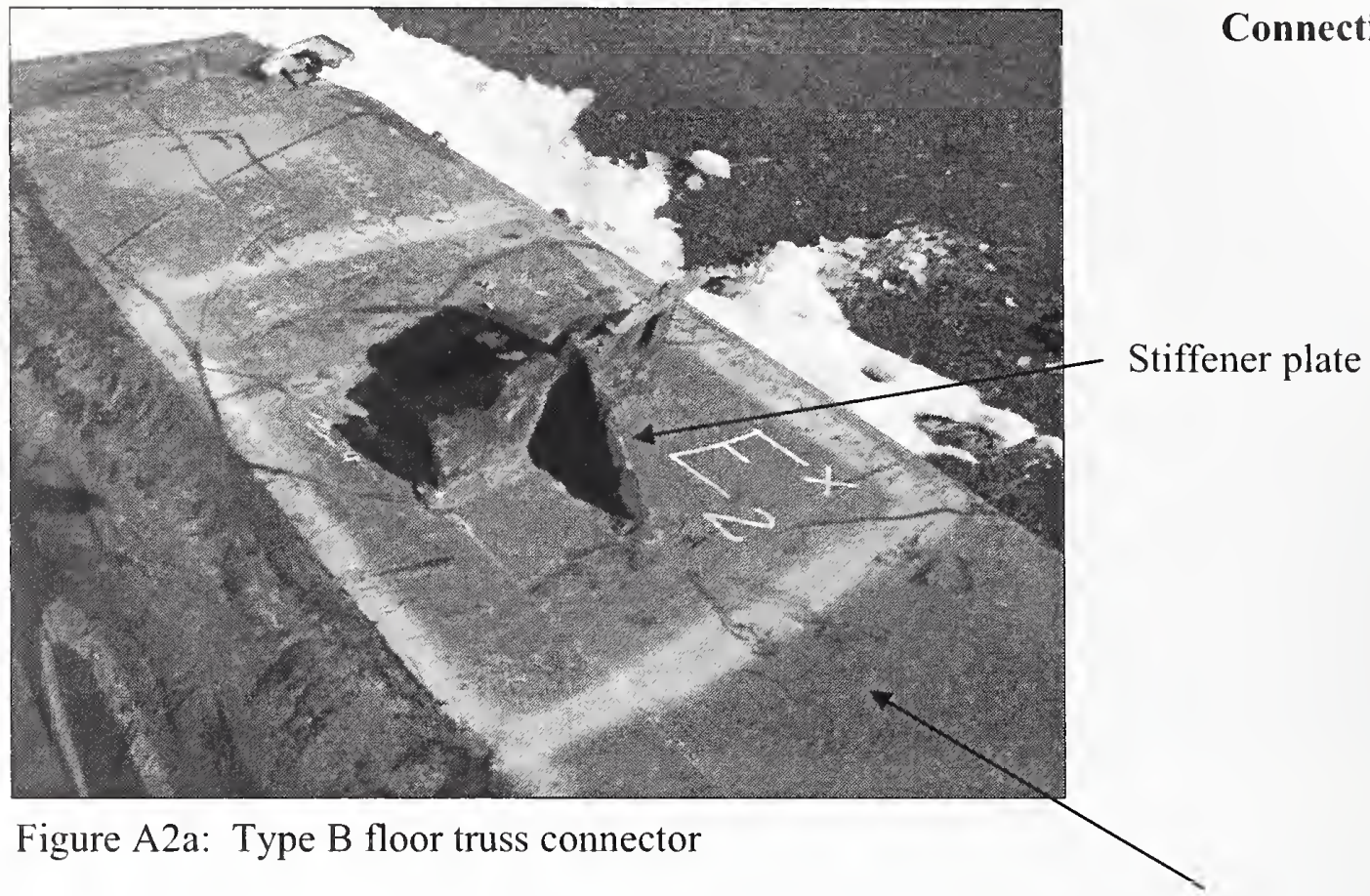

No gusset plate for damper unit

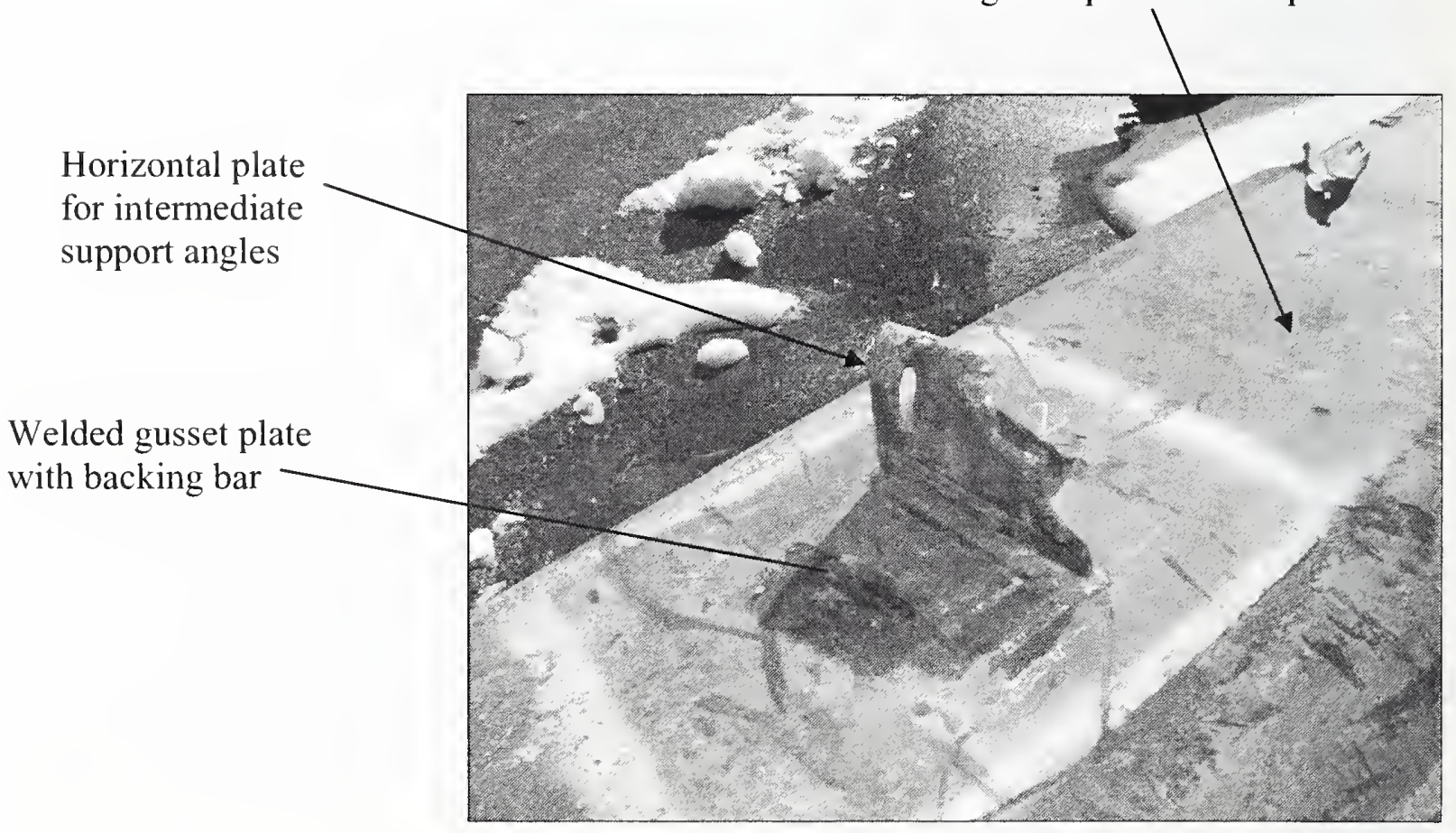

Figure A2b: Type B floor truss connector

Figure A-2. Type B Floor Truss Connector (intermediate deck support angles). 


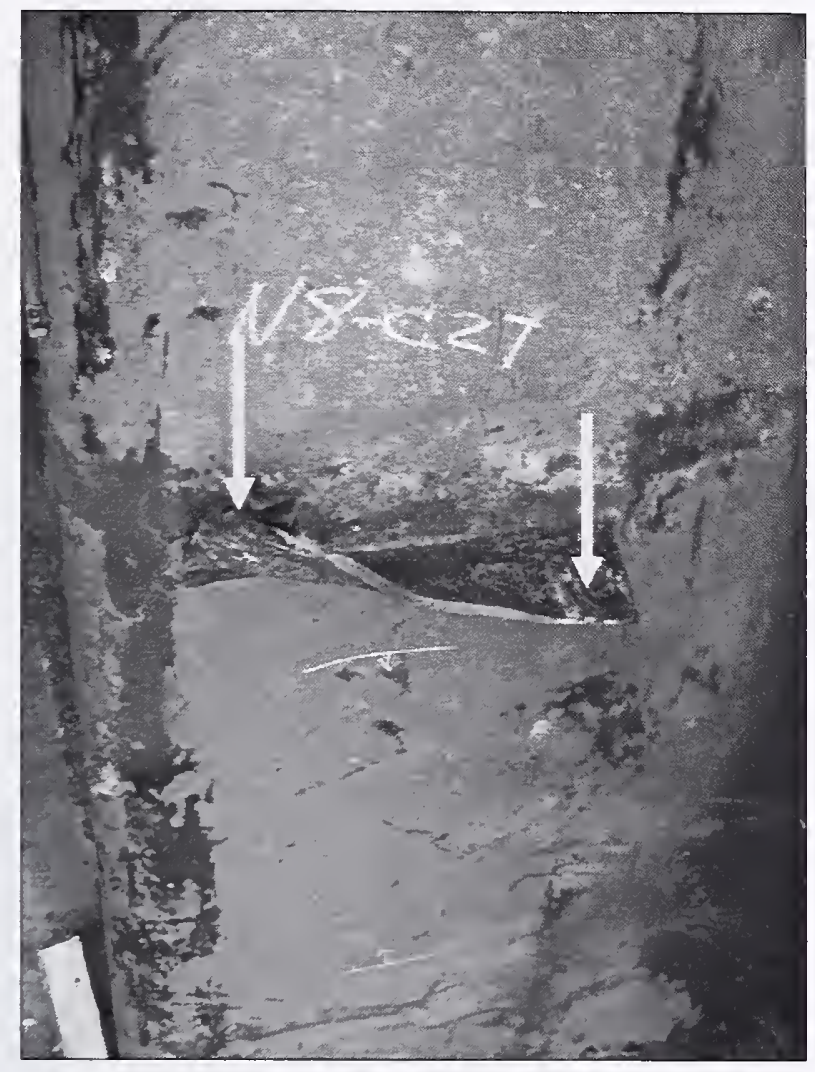

\section{Connection N8-C2T}

Diagonal bracing straps attached to gusset plate in locations indicated by arrows

\section{Various shapes for gusset plate}
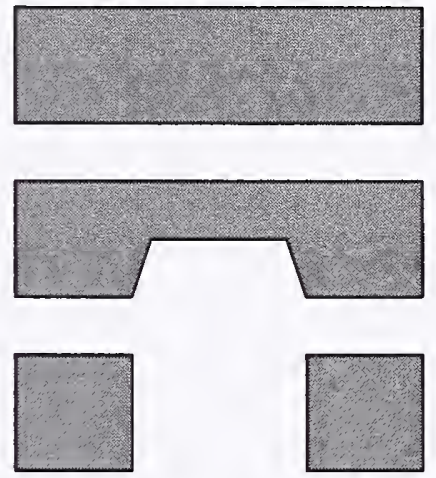

Figure A3a: Type $\mathrm{C}$ floor truss connector

\section{Sample M-29}

Portion of diagonal bracing strap

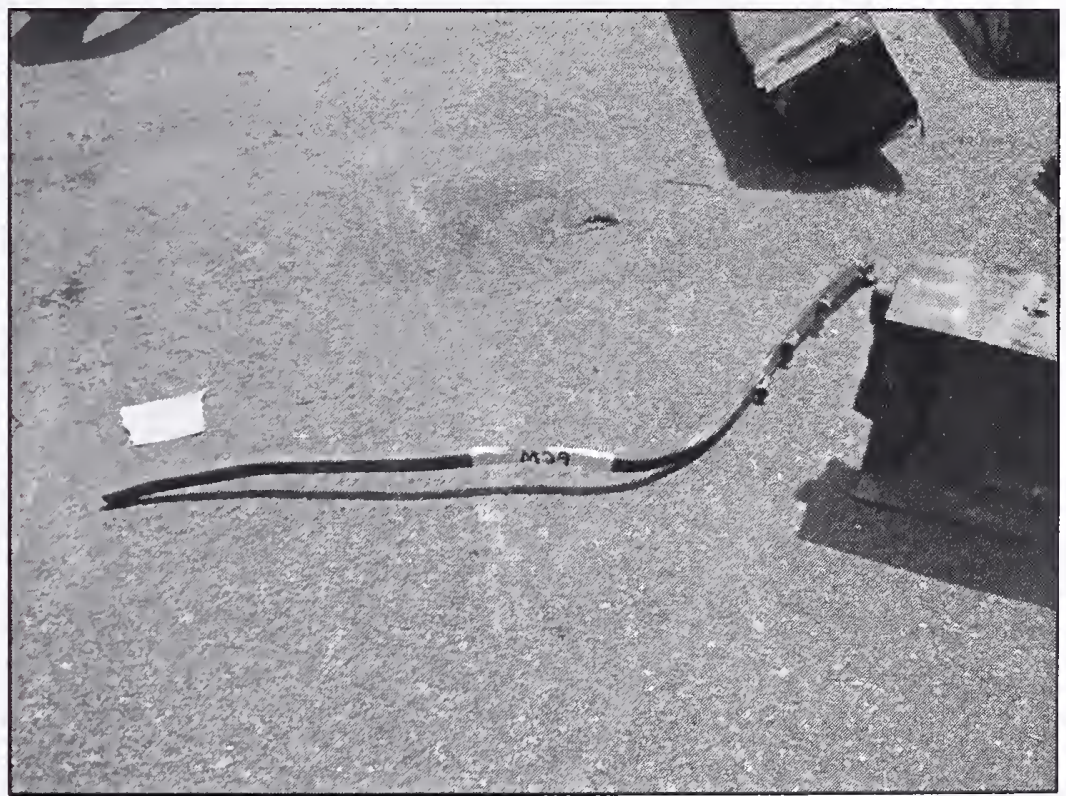

Figure A3b: Type $\mathrm{C}$ floor truss connector

Figure A-3. Type C Floor Truss connector (anchor for diagonal bracing strap). 


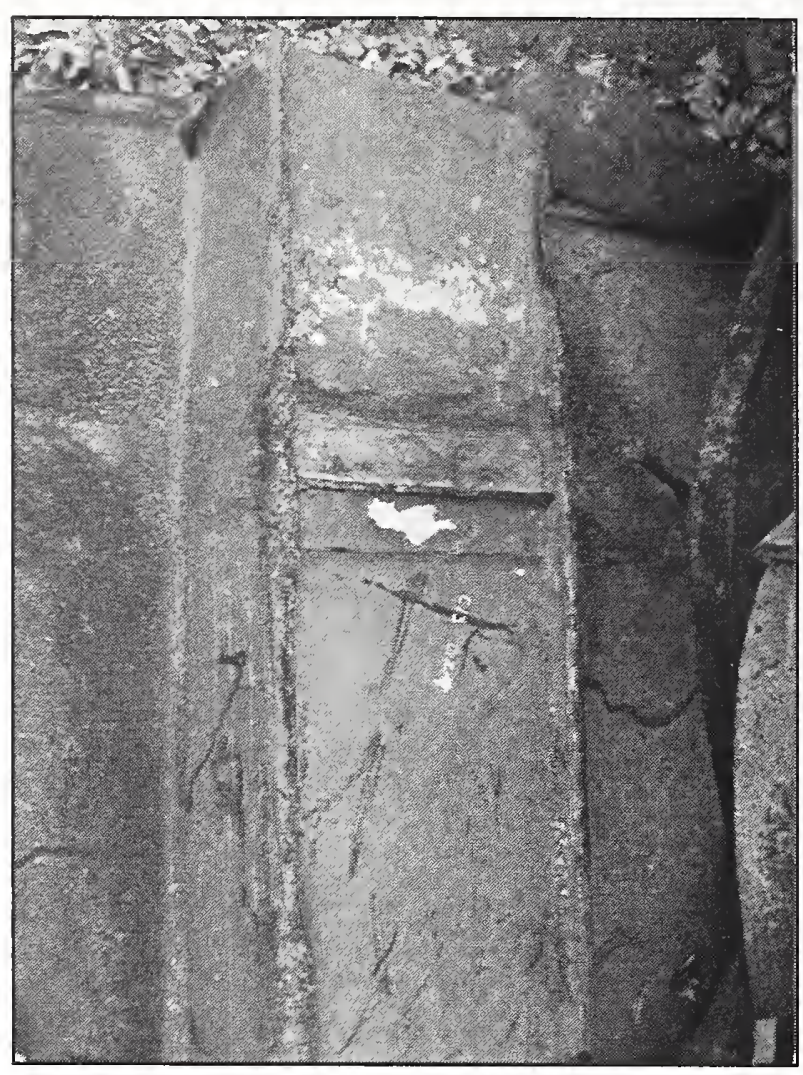

Figure A3c: Type C floor truss connector

$\underline{\text { Shape for gusset plate }}$

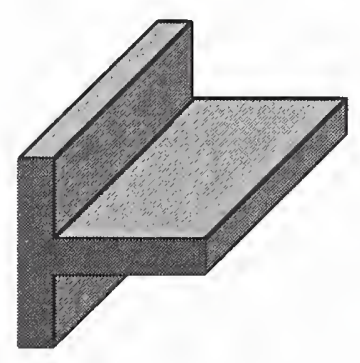

Connection C13-C2B

\section{Connection C13-C2T}

Diagonal bracing strap attached to gusset plate

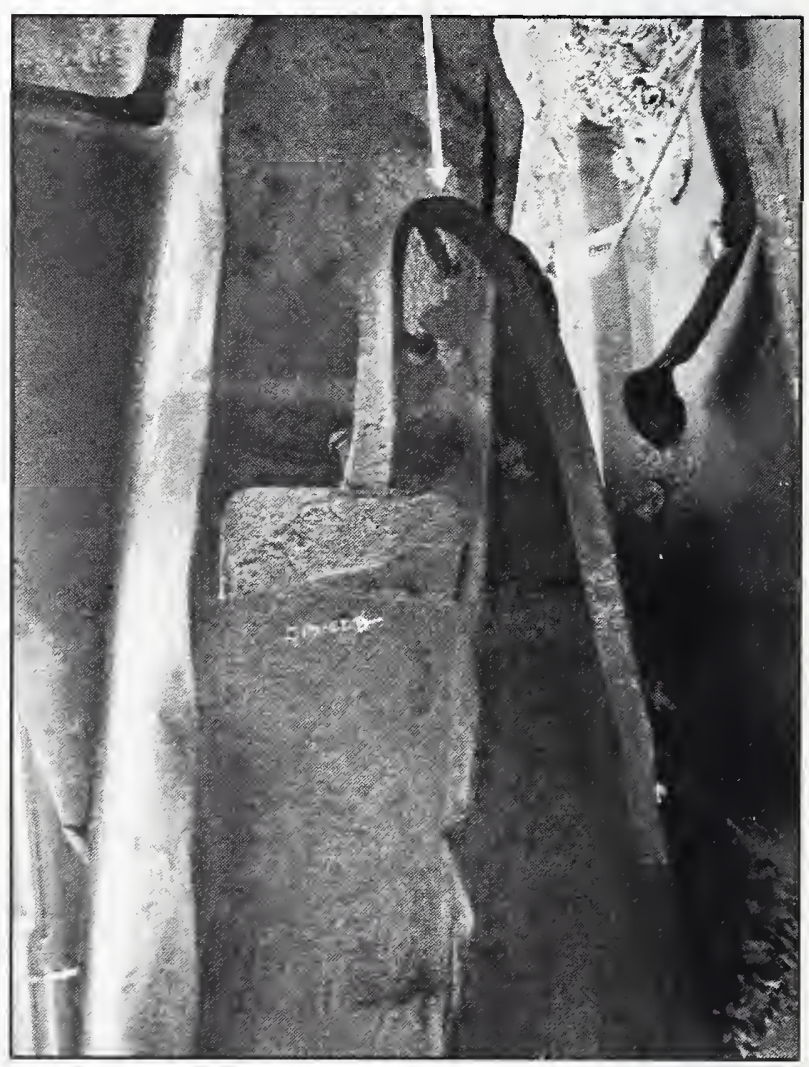

Figure A3d: Type C floor truss connector

Figure A-3. Type C Floor Truss Connector (anchor for diagonal bracing strap) (cont.). 


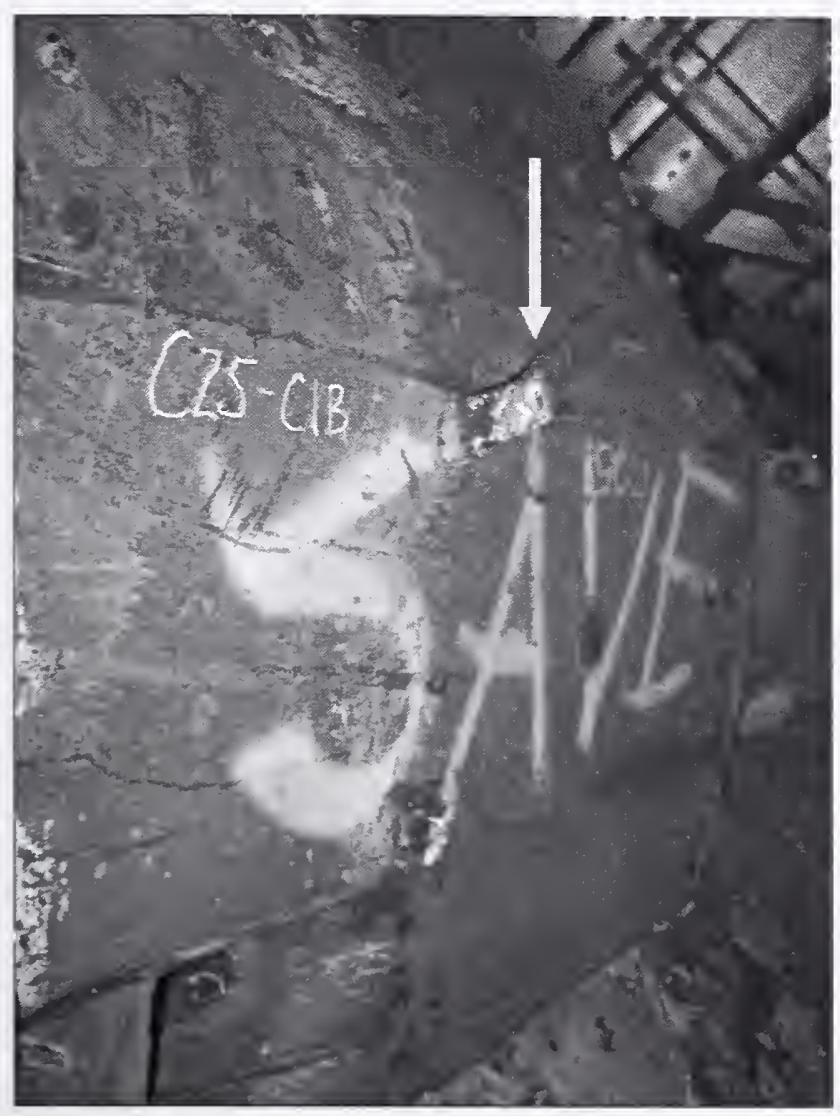

\section{Connection C25-C1B}

Portion of diagonal bracing straps

remain attached to spandrel;

welded directly to the spandrel.

Figure A4a: Type D floor truss connector

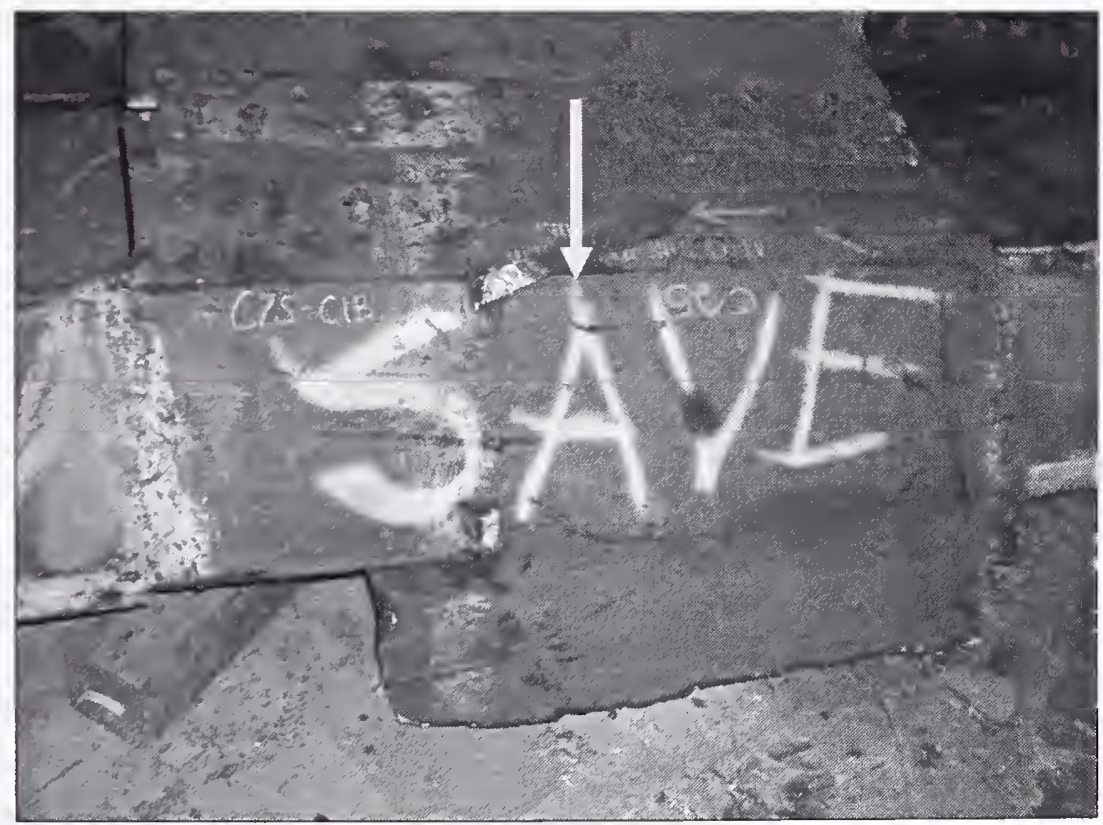

Figure A4b: Type D floor truss connector

Figure A-4. Type D Floor Truss Connector (diagonal bracing strap welded directly to column). 
This page intentionally left blank. 


\section{Appendix B \\ Recovered Perimeter Panel Floor Truss Connectors from IDENTIFIED PANELS}

The following appendix displays images, taken by National Institute of Standards and Technology, of the recovered floor truss connectors for unambiguously identified exterior panels. Panels B-1024, B-1043, and B-1044, located at John F. Kennedy International Airport, were not fully documented and are not present in this appendix. Connectors from sample C-117, a partially identified panel, were also shown. 
a)

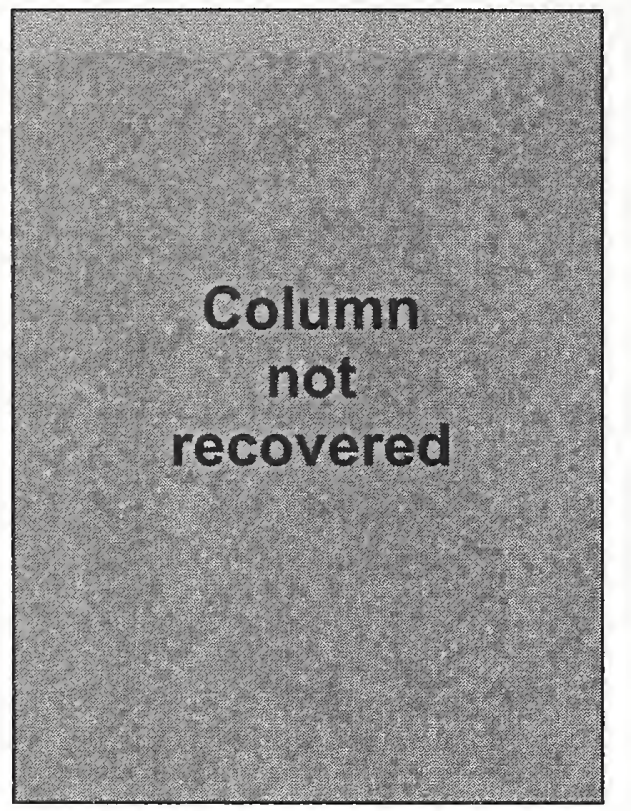

c)

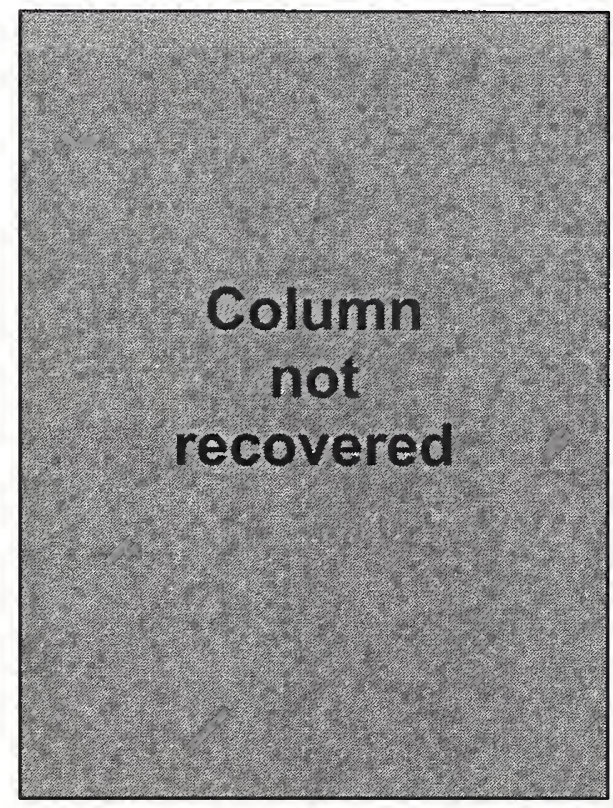

\section{Mechanical floor panel}

ASCE2-C1T (WTC 2, Col.331, Fl 43)

NA

b)

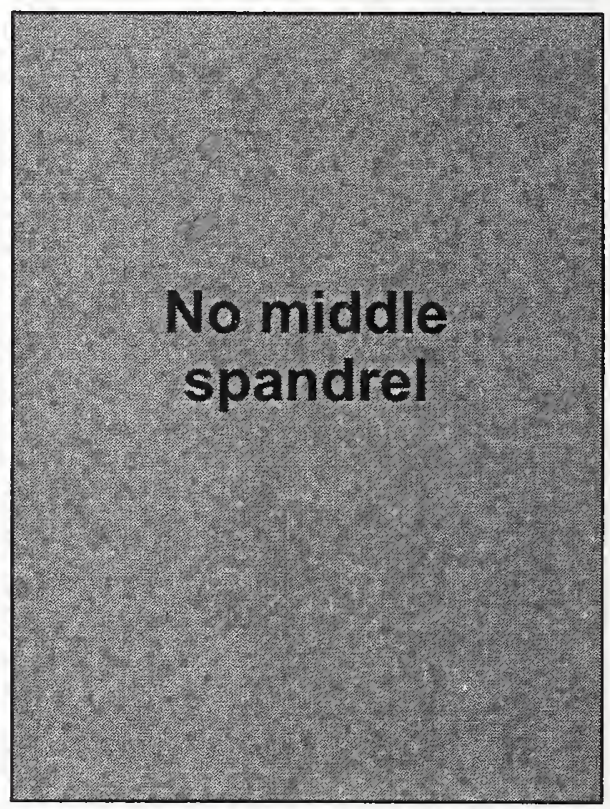

ASCE2-C1B (WTC 2, Col.331, Fl 41)

NA

Figure B-1. Floor truss connectors found on recovered panel ASCE-2 (B330: 40-43) from WTC 2. Column 331 was not recovered. 
d)

\section{Column not recovered}

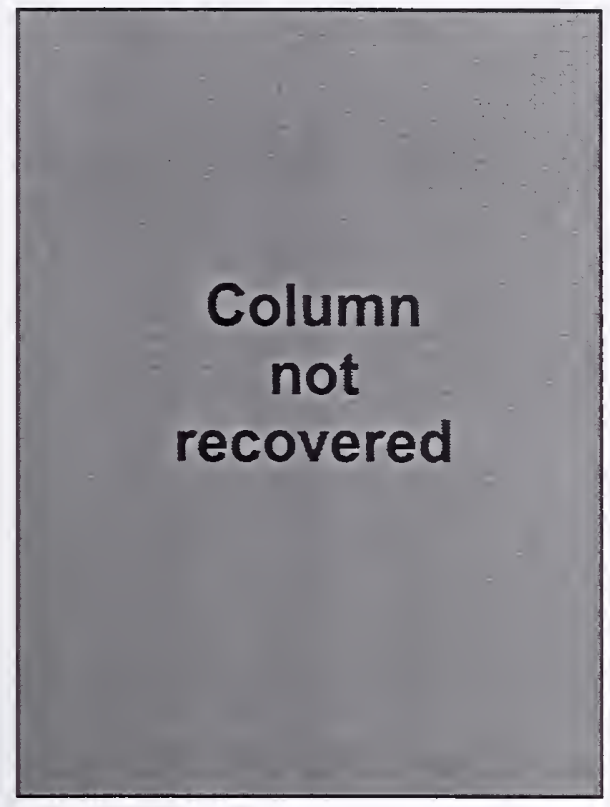

f)

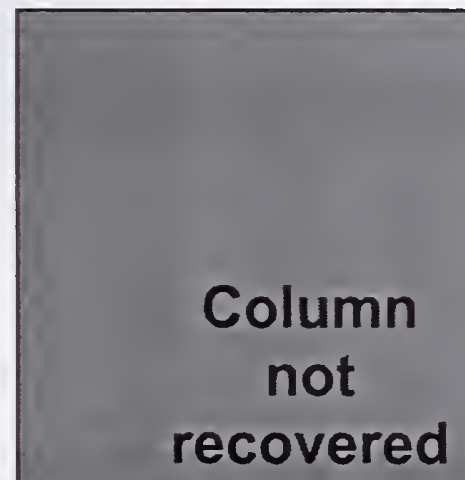

Figure B-1. Floor truss connectors found on recovered panel ASCE-2 (B330: 40-43) from WTC 2. Column 330 was not recovered (cont.).
ASCE2-C2T (WTC 2, Col.330, Fl 43)

NA

e)

No middle spandrel

ASCE2-C2B (WTC 2, Col.330, Fl 41)

NA 
g)

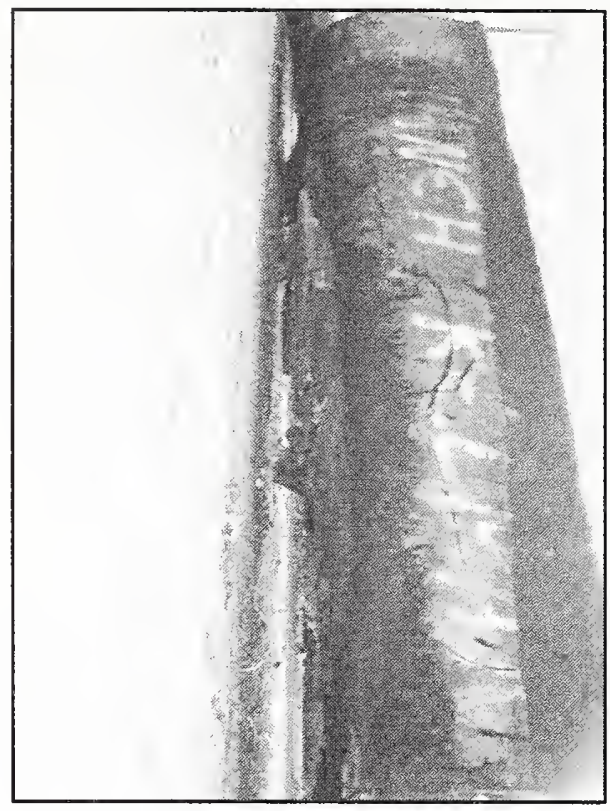

\section{Mechanical floor panel}

ASCE2-C3T (WTC 2, Col.329, Fl 43)

Type A

Seat Detail: 4424

Gusset plate ripped completely out of spandrel; Small seat intact, bent downwards, 1 bolt hole intact, other ripped out;

No evidence of gusset plate for damper unit.

h)

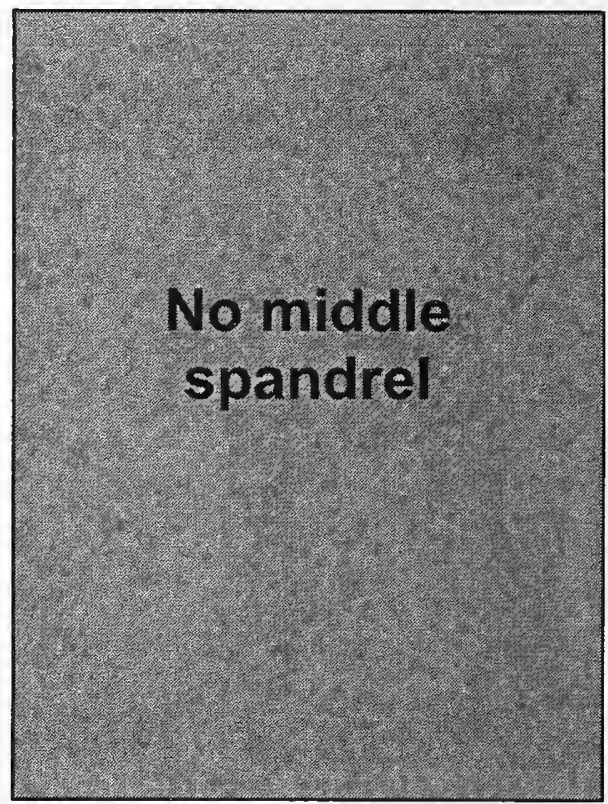

ASCE2-C3B (WTC 2, Col.329, Fl 41)

Type A

Seat Detail: 4424

Portion of gusset plate remains;

Small seat intact, bent downwards, 1 bolt hole intact;

No evidence of gusset plate for damper unit.

Figure B-1. Floor truss connectors found on recovered panel ASCE-2 (B330: 40-43) from WTC 2. Shown are connectors found on column 329 at different floor levels (cont.). 
a)

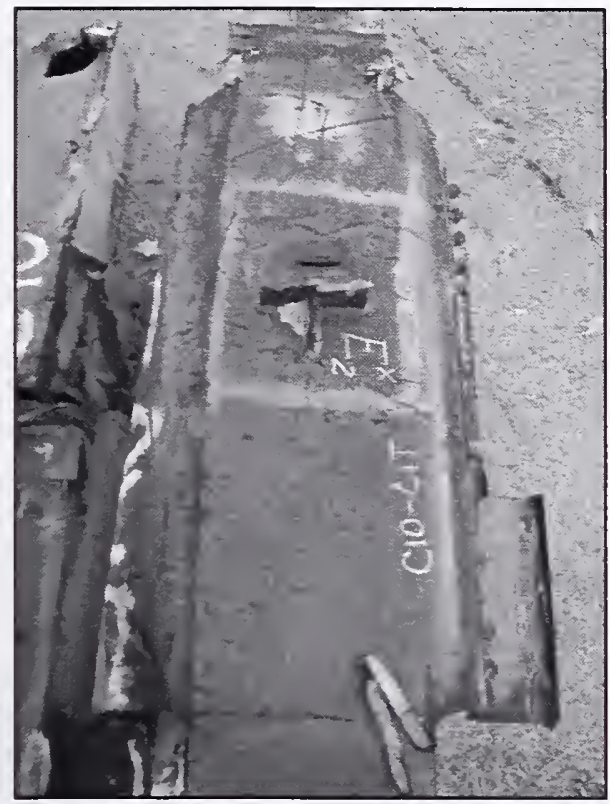

C10-C1M (WTC 1, Col.452, FI 87)

Type B

Seat Detail: 5010

Gusset plate ripped completely out of spandrel;

Small seat intact, corners bent downwards, 1 bolt hole intact other ripped out;

No evidence of gusset plate for damper unit.

c)

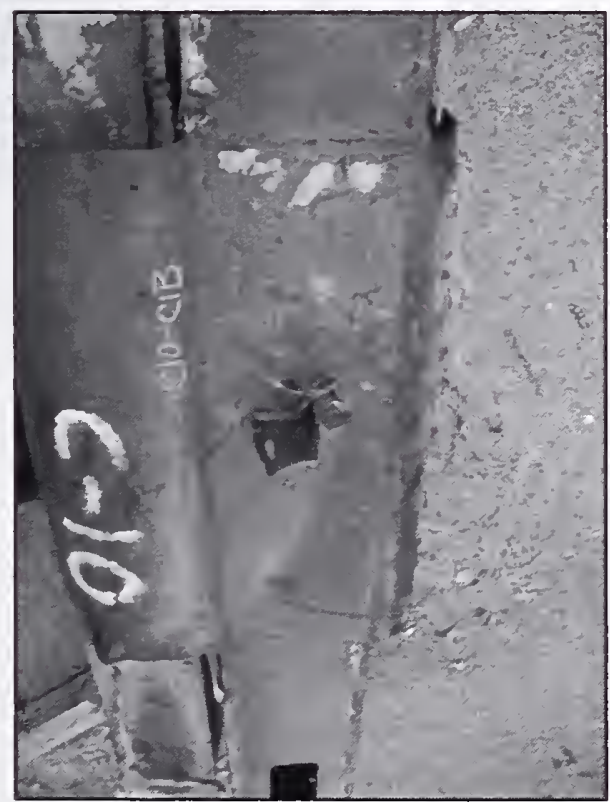

Type B

Seat Detail: 5010 intact;
C10-C1T (WTC 1, Col.452, Fl 88)

Type B

Seat Detail: 5010

Gusset plate ripped completely out of spandrel; Small seat intact, bent downwards, 1 bolt hole intact, other ripped out;

No evidence of gusset plate for damper unit. b)

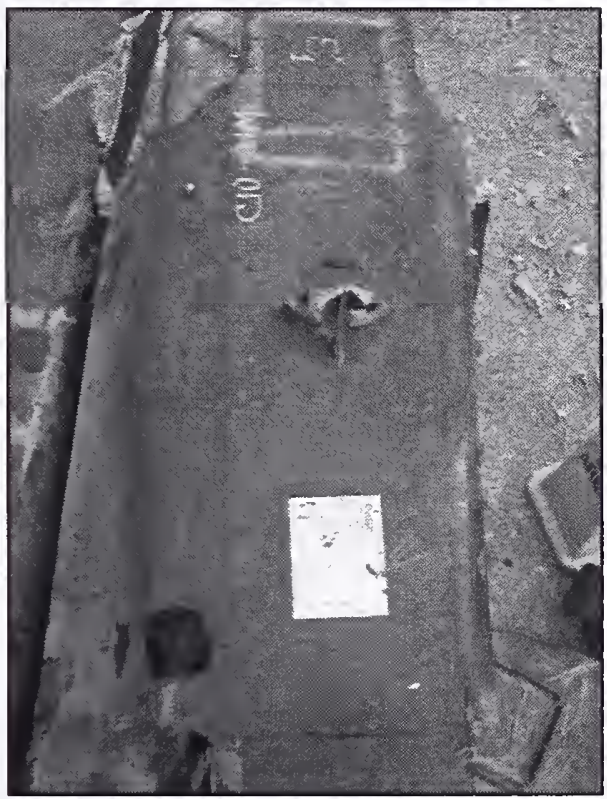

C10-C1B (WTC 1, Col.452, Fl 86)

Portion of gusset plate remains;

Small seat intact, bent downwards, 1 bolt hole

No evidence of gusset plate for damper unit.

Figure B-2. Floor truss connectors found on recovered panel C-10 (A451: 85-88) from WTC 1. Shown are connectors found on column 452 at different floor levels. 
d)

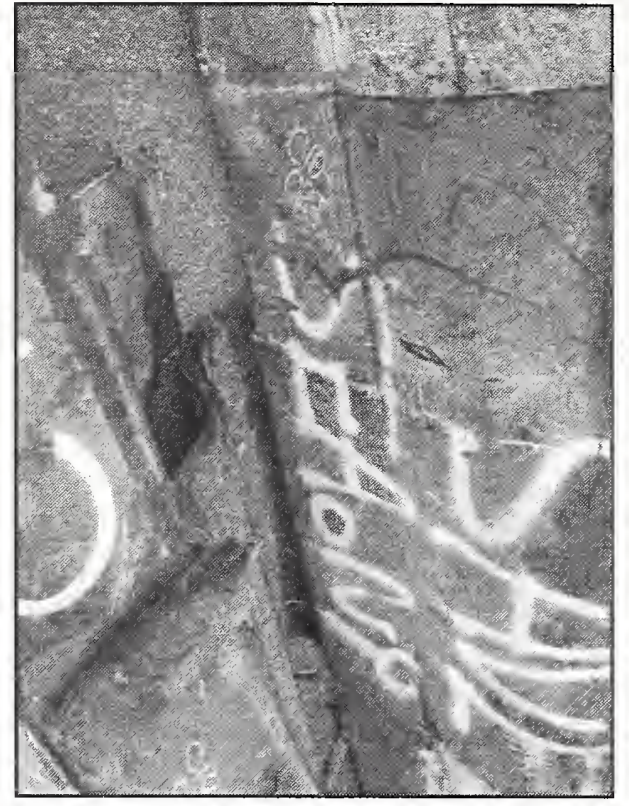

\section{C10-C2T (WTC 1, Col.451, Fl 88)}

Type D

Seat Detail: 5210

2 diagonal bracing straps ripped off at weld.

C10-C2M (WTC 1, Col.451, F1 87)

Type D

Seat Detail: 5210

2 1-inch portions of diagonal bracing straps remain.

f)

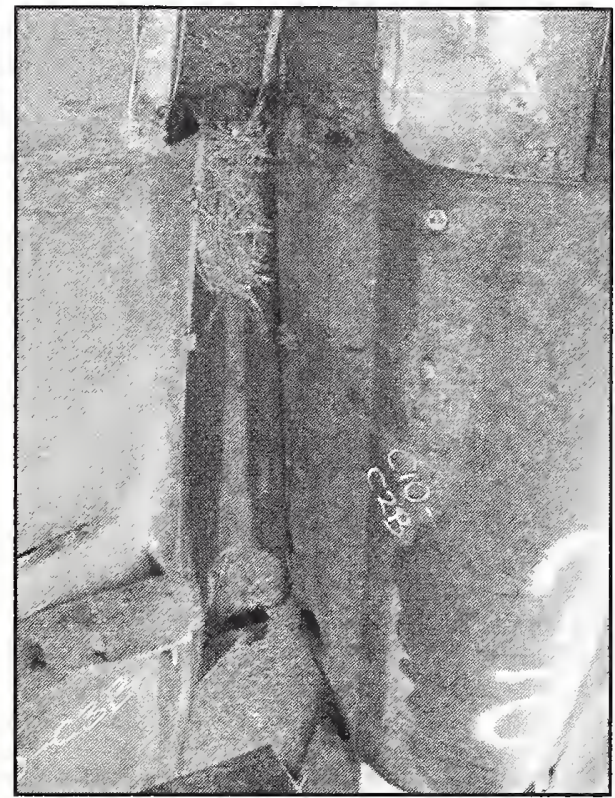

e)

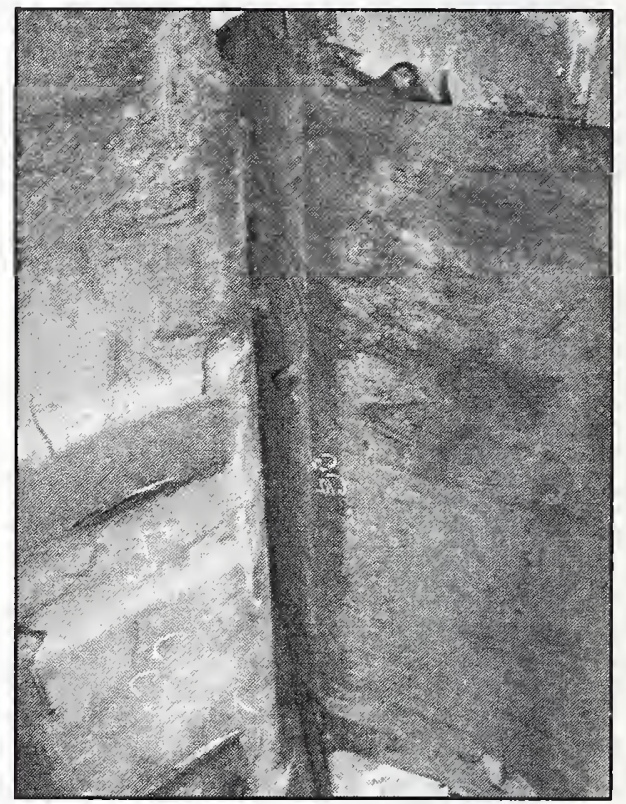

C10-C2B (WTC 1, Col.451, F1 86)

Type D

Seat Detail: 5210

2 diagonal bracing straps ripped off at weld.

Figure B-2. Floor truss connectors found on recovered panel C-10 (A451: 85-88) from WTC 1. Shown are connectors found on column 451 at different floor levels (cont.). 
g)

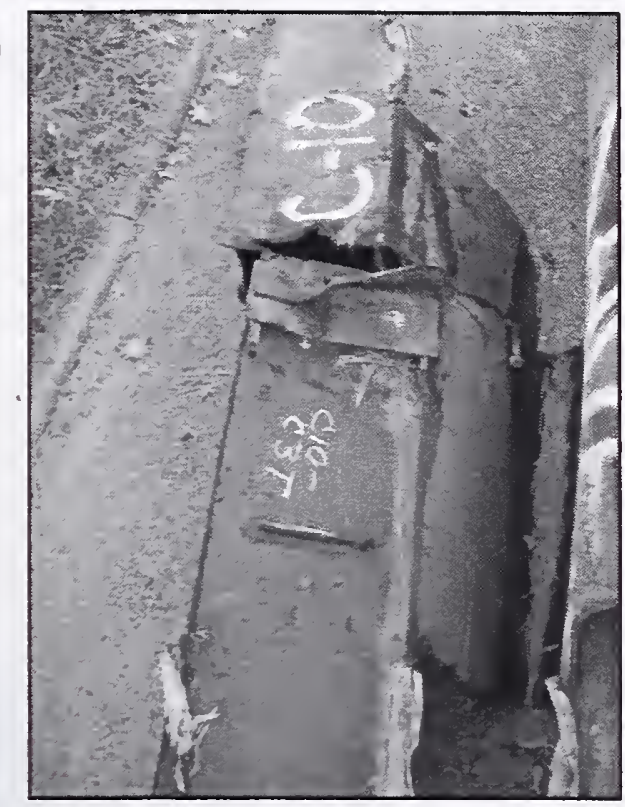

C10-C3M (WTC 1, Col.450, F1 87)

Type A

Seat Detail: 1212

Gusset plate ripped completely out of spandrel; Only 1 standoff plate remains, rest ripped off at welds;

Gusset plate with damper unit intact, bent downwards.

i)

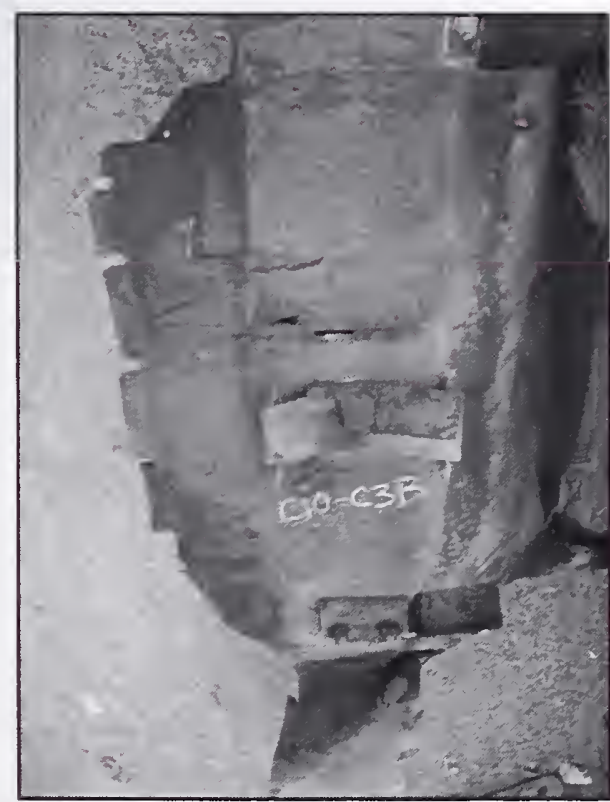

Type A

Seat Detail: 1212

\section{C10-C3T (WTC 1, Col.450, Fl 88)}

Type A

Seat Detail: 1212

Gusset plate ripped completely out of spandrel, large tear due to internal strengthening plate in the area; Seat intact, bent downwards, both bolt holes intact; Gusset plate for damper unit ripped off at weld. h)

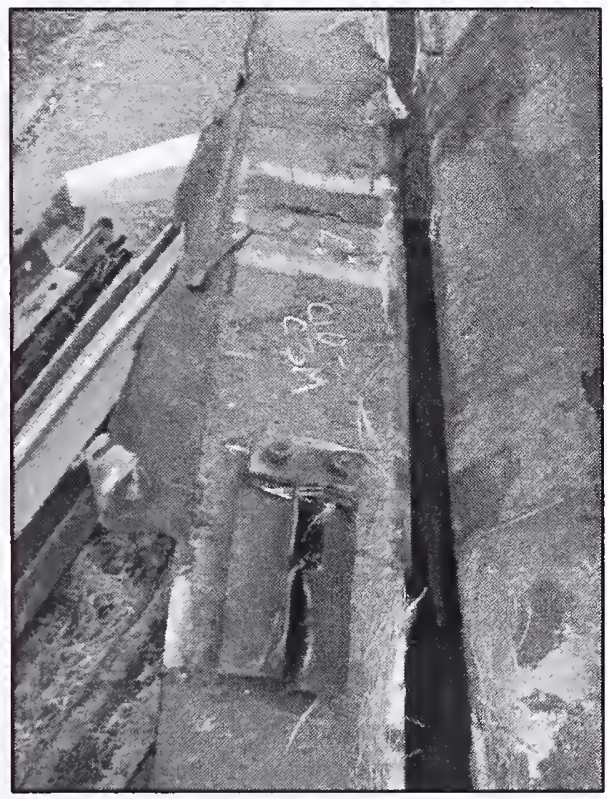

\section{C10-C3B (WTC 1, Col.450, Fl 86)}

Gusset plate ripped completely out of spandrel;

Seat intact, bent towards column and downwards, 1 bolt hole intact, other ripped through;

Gusset plate with damper unit intact, bent downwards.

Figure B-2. Floor truss connectors found on recovered panel C-10 (A451: 85-88) from WTC 1. Shown are connectors found on column 450 at different floor levels (cont.). 
a)

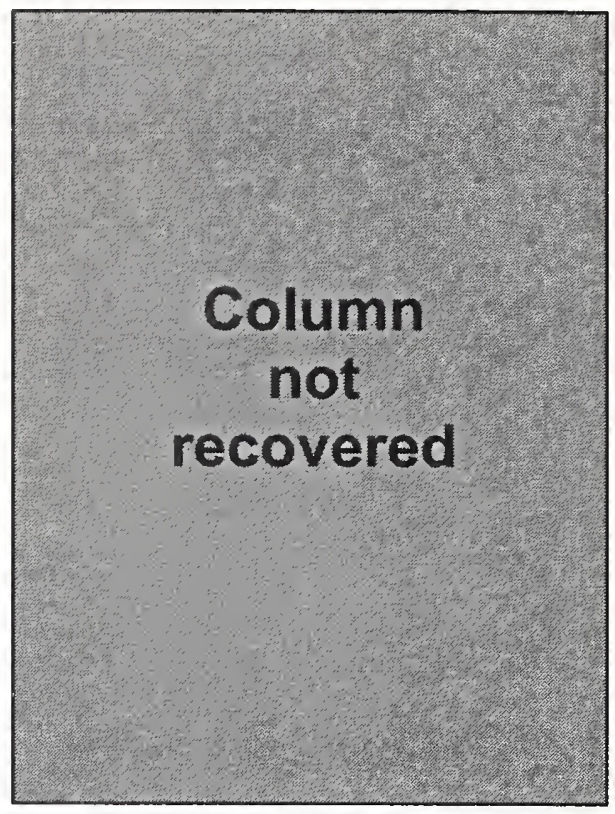

c)

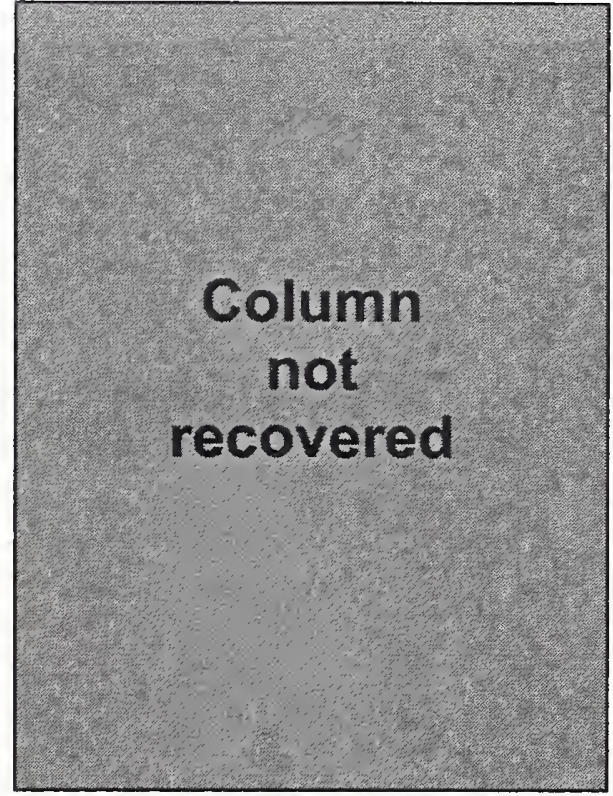

\section{Panel at chamfered corner}

C13-C1T

NA

b)

\section{No middle spandrel}

C13-C1B

NA

Figure B-3. Floor truss connectors found on recovered panel C-13 (B200: 90-92) from WTC 2. Column 201 was not recovered. 
d)

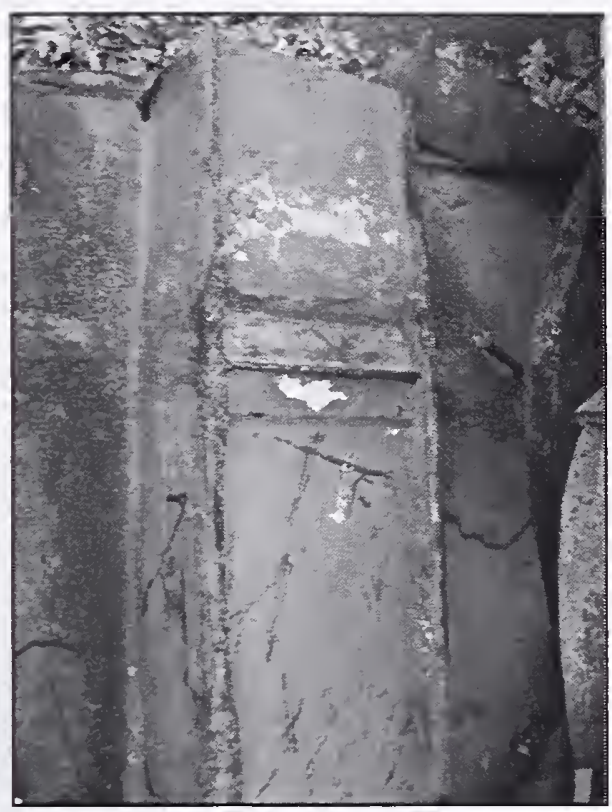

f)

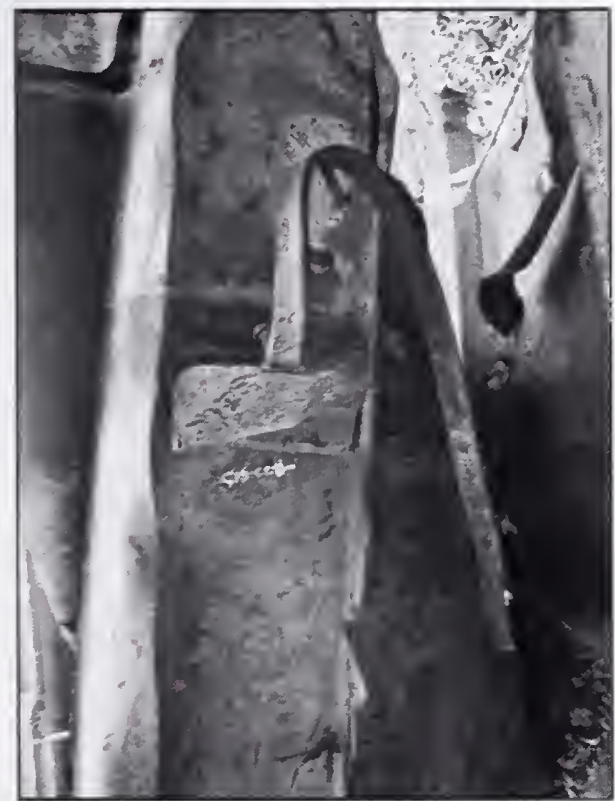

\section{$\underline{\text { Panel at chamfered corner }}$}

C13-C2T (WTC 2, Col.200, Fl 92)

Type C

Seat Detail: 5010

Gusset plate ripped off at weld.

e)

No middle spandrel

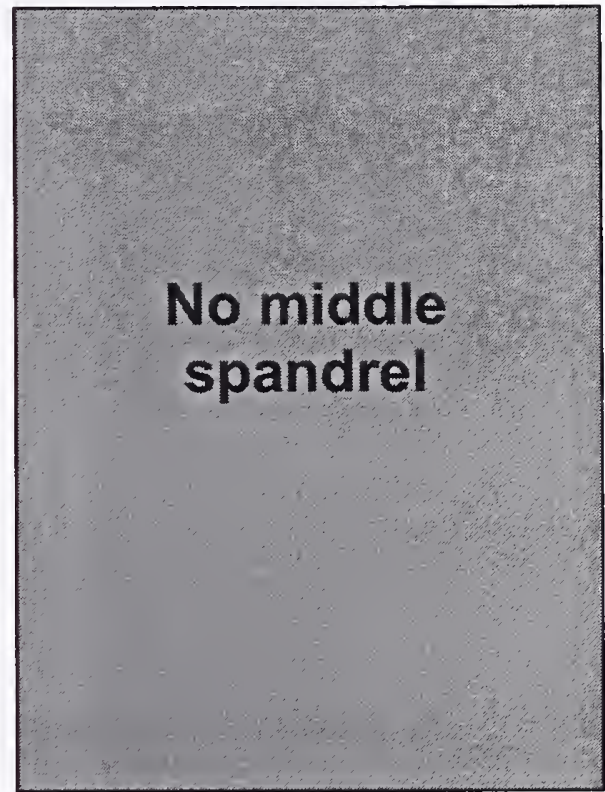

C13-C2B (WTC 2, Col.200, F1 91)

Type $\mathrm{C}$

Seat Detail: 5010

Gusset plate remains, bent upwards, one diagonal bracing strap attached, other ripped off at weld.

Figure B-3. Floor truss connectors found on recovered panel C-13 (B200: 90-92) from WTC 2. Shown are connectors found on column 200 at different floor levels (cont.). 
g)

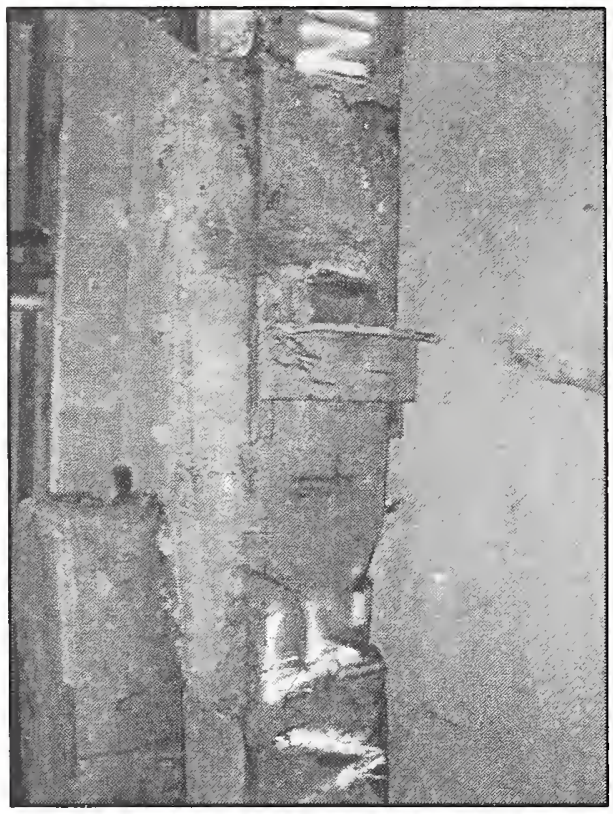

i)

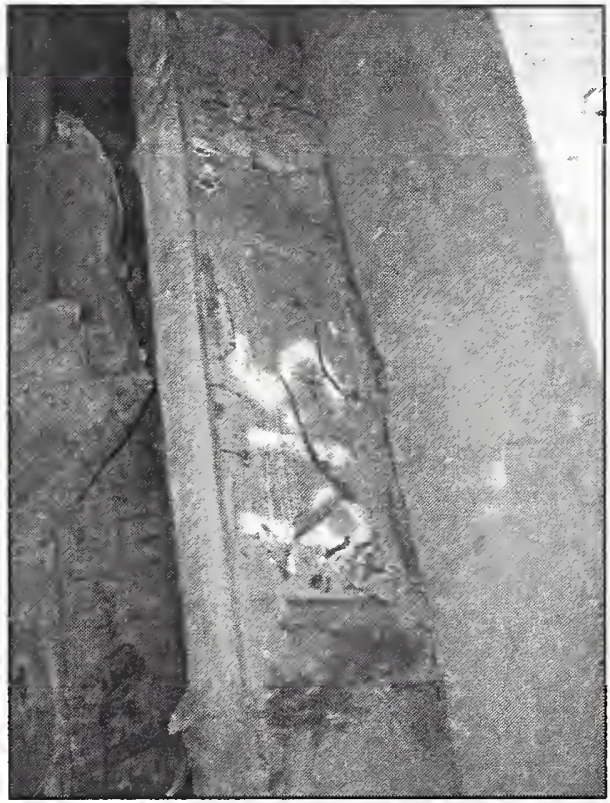

\section{$\underline{\text { Panel at chamfered corner }}$}

C13a-C3T (WTC 2, Col.159, Fl 92)

Type A

Seat Detail: 2310

Gusset plate remains;

Seat intact with both bolt holes intact;

Gusset plate for damper unit ripped off at weld.

h)

\section{No middle spandrel}

C13a-C3B (WTC 2, Col.159, Fl 91)

Type A

Seat Detail: 1313

Gusset plate ripped completely out of spandrel; Both standoff plates remain, rest ripped off at welds; Gusset plate for damper unit ripped off at weld.

Figure B-3. Floor truss connectors found on recovered panel C-13 (B200: 90-92) from WTC 2. Shown are connectors found on column 159 at different floor levels (cont.). 
a)

\section{Column not recovered}

\section{Panel at chamfered corner}

\section{C14-C1T}

NA

c)

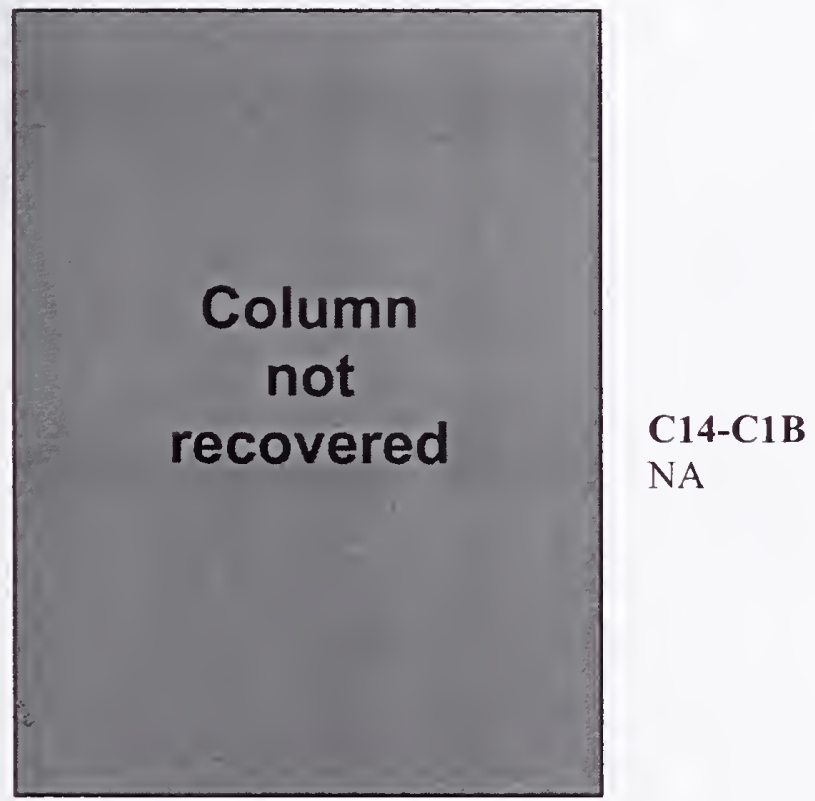

b)

\section{No middle spandrel}

Figure B-4. Floor truss connectors found on recovered panel C-14 (B300: 85-87) from WTC 2. Column 301 was not recovered. 
d)

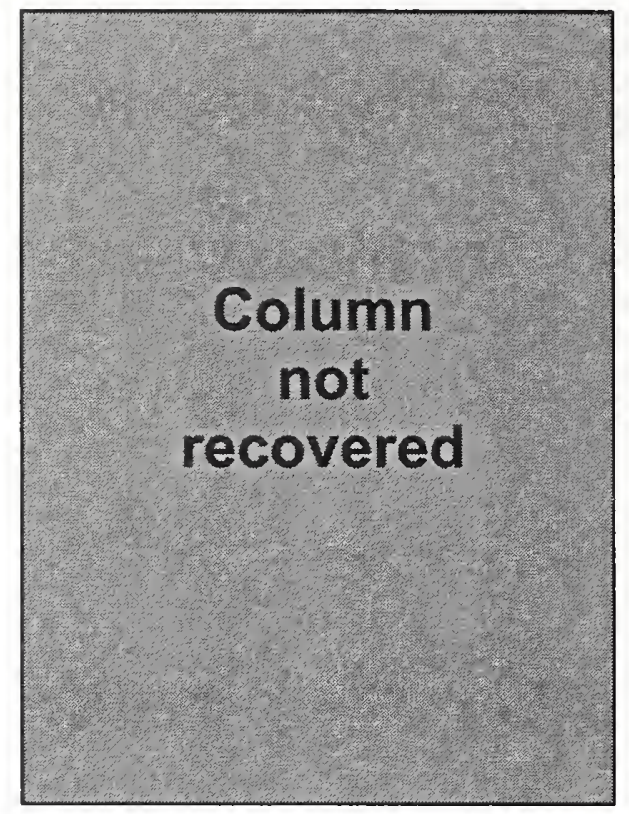

f)

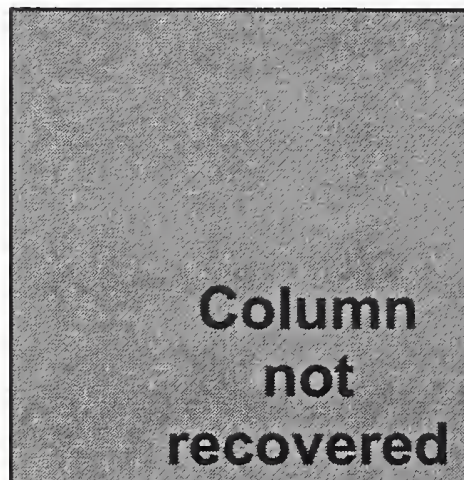

\section{Panel at chamfered corner}

C14-C2T

NA e)

\section{No middle spandrel}

C14-C2B

NA 
g)

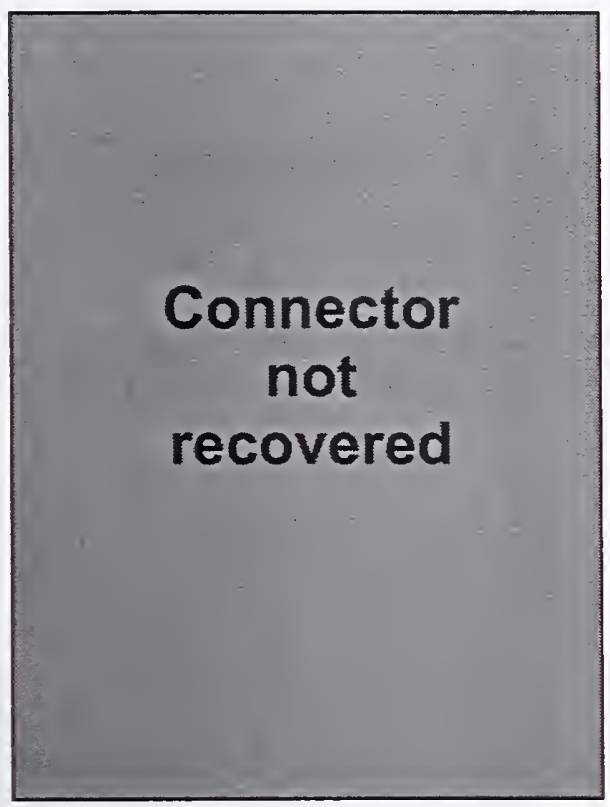

i)

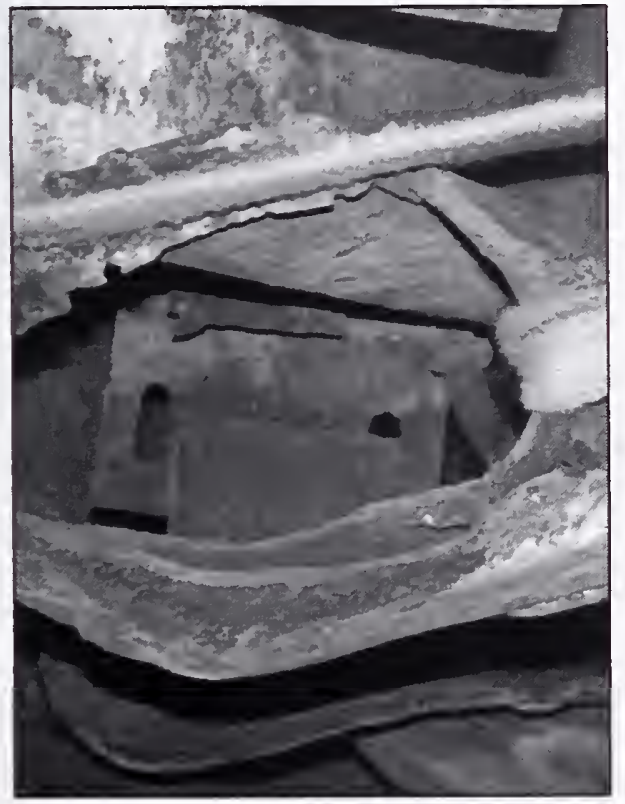

\section{Panel at chamfered corner}

\section{C14-C3T}

NA

h)

\section{No middle spandrel}

\section{C14-C3B (WTC 2, Col.259, Fl 86)}

Type A

Seat Detail: 1313

\section{Gusset plate intact;}

Small seat intact with both bolt holes intact;

Gusset plate for damper unit ripped off at weld.

Figure B-4. Floor truss connectors found on recovered panel C-14 (B300: 85-87) from WTC 2. Shown are connectors found on column 259 at different floor levels (cont.). 
a)

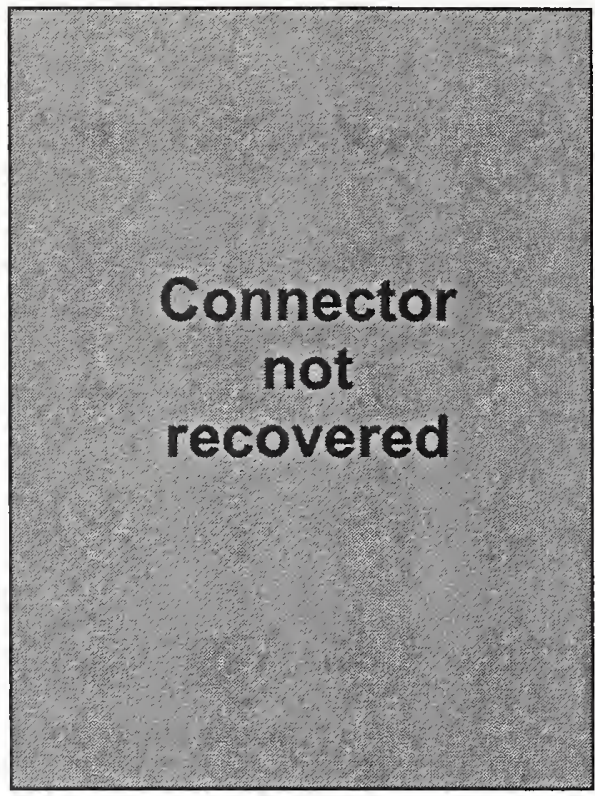

\section{C18-C1T}

NA

\section{C18-C1M (WTC 2, Col.231, Fl 95)}

Type A

Seat Detail: 1111

Gusset plate intact;

Seat intact, both bolt holes intact, slight damage to one corner, bent downwards;

Gusset plate with damper unit intact, bent upwards.

c)

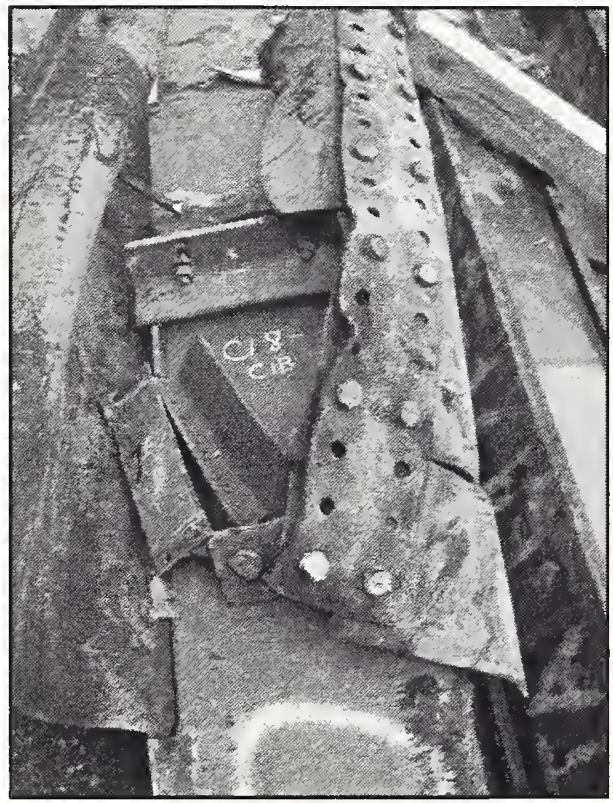

b)

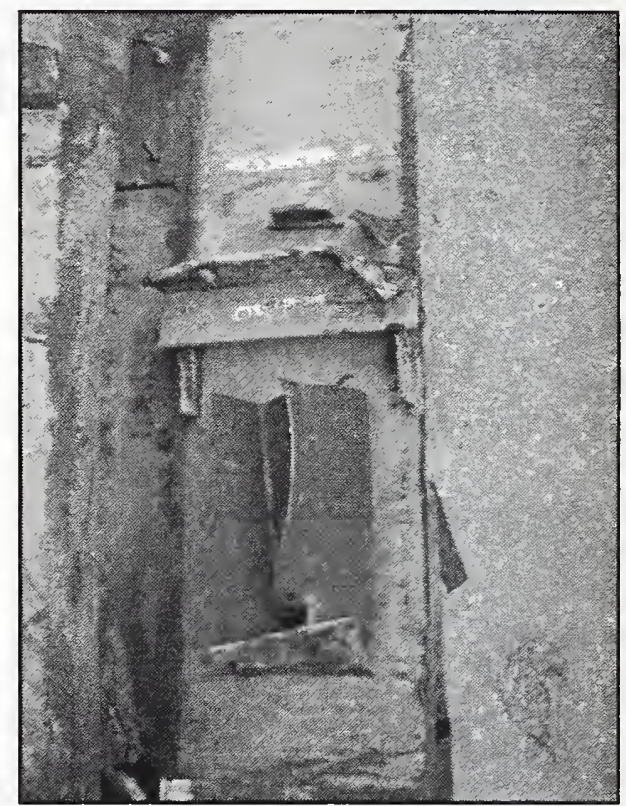

\section{C18-C1B (WTC 2, Col.231, Fl 94)}

Type A

Seat Detail: 1111

Gusset plate intact;

Seat intact, both bolt holes intact with bolts and nuts; Gusset plate with damper unit intact, bent upwards.

Figure B-5. Floor truss connectors found on recovered panel C-18 (B230: 93-96) from WTC 2. Shown are connectors found on column 231 at different floor levels. 
d)

\section{Connector not recovered}

\section{C18-C2T}

NA

C18-C2M (WTC 2, Col.230, FI 95)

Type C

Seat Detail: 5210

Very small portion of large gusset plate remains, bent upwards.

f)

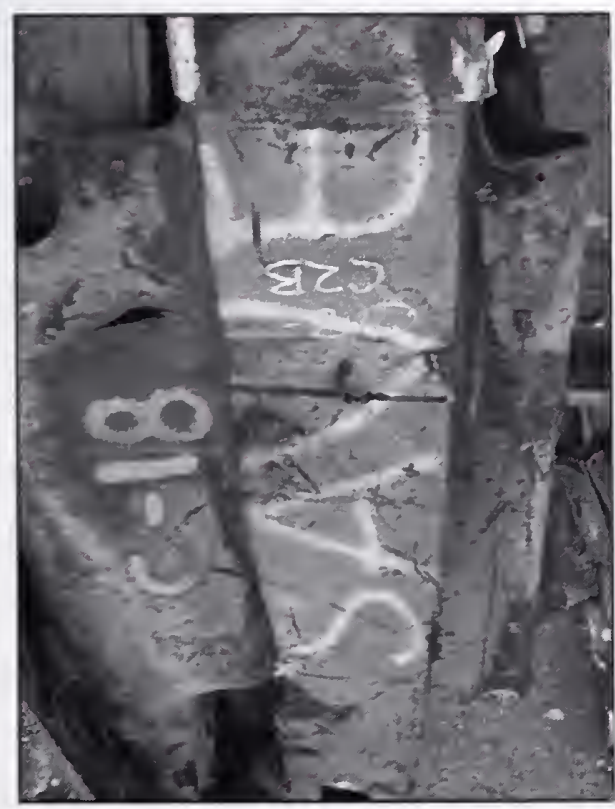

C18-C2B (WTC 2, Col.230, FI 94)

Type C

Seat Detail: 5210

Gusset plate ripped off at weld.

Figure B-5. Floor truss connectors found on recovered panel C-18 (B230: 93-96) from WTC 2. Shown are connectors found on column 230 at different floor levels (cont.). 
g)

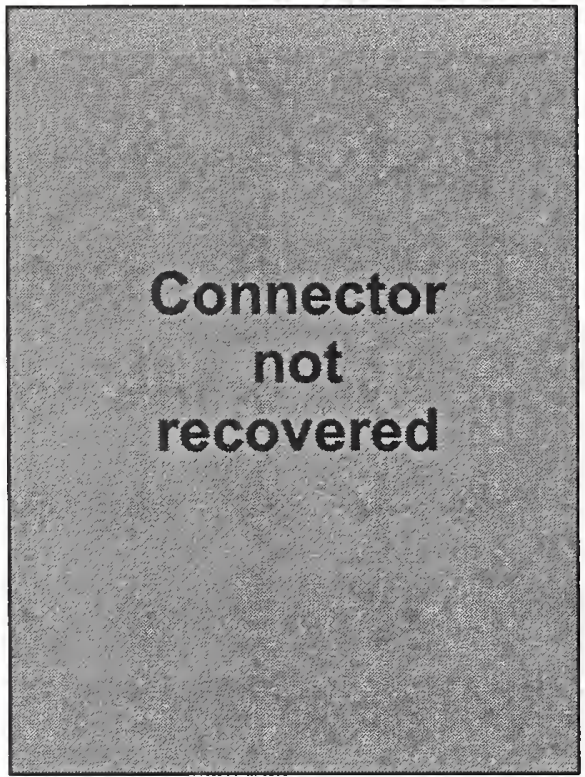

\section{C18-C3T}

NA

C18-C3M (WTC 2, Col.229, FI 95)

Type A

Seat Detail: 1111

Gusset plate intact, bent downwards;

Seat intact, bent down and in towards column, 1 bolt hole intact, other ripped out;

Gusset plate for damper unit ripped off at weld.

i)

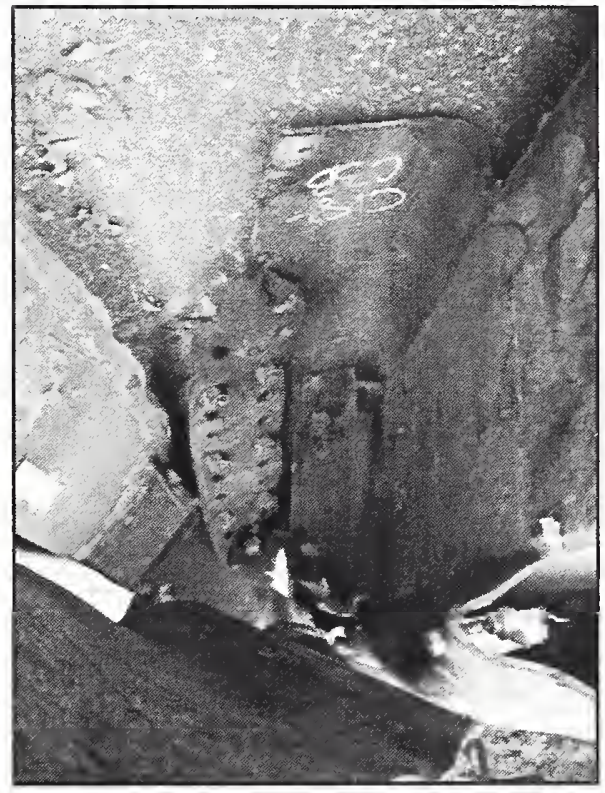

h)

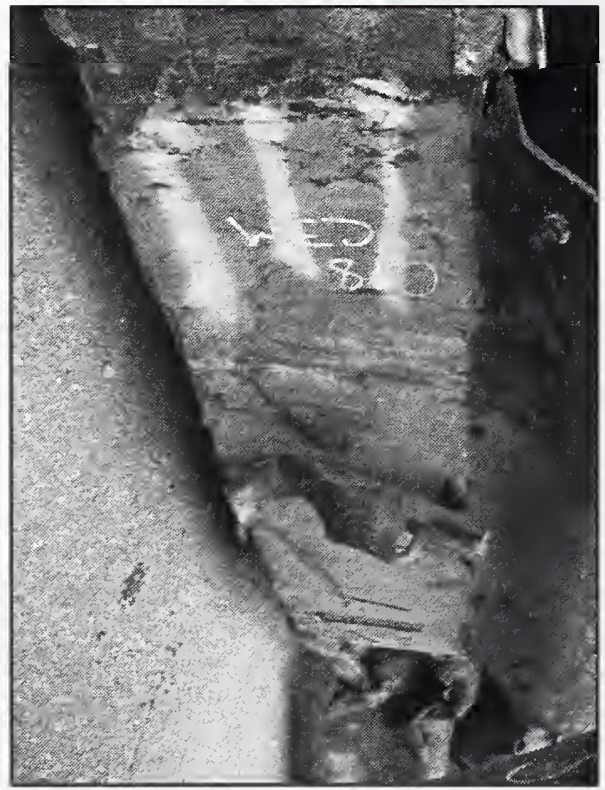

C18-C3B (WTC 2, Col.229, Fl 94)

Type A

Seat Detail: 1111

Gusset plate intact;

Seat intact, both bolt holes intact with 1 bolt and nut, some damage to corners;

Gusset plate with damper unit intact, bent downwards.

Figure B-5. Floor truss connectors found on recovered panel C-18 (B230: 93-96) from WTC 2. Shown are connectors found on column 229 at different floor levels (cont.). 
a)

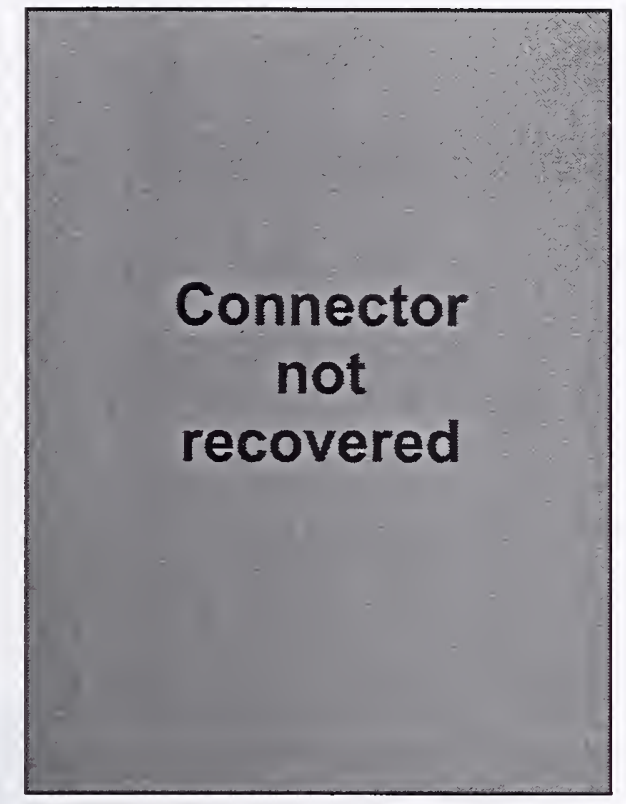

C22-C1M (IVTC 1, Col.158, Fl 95)

Type C

Seat Detail: 5210

Large gusset plate ripped off at weld.
C22-C1T

NA b)

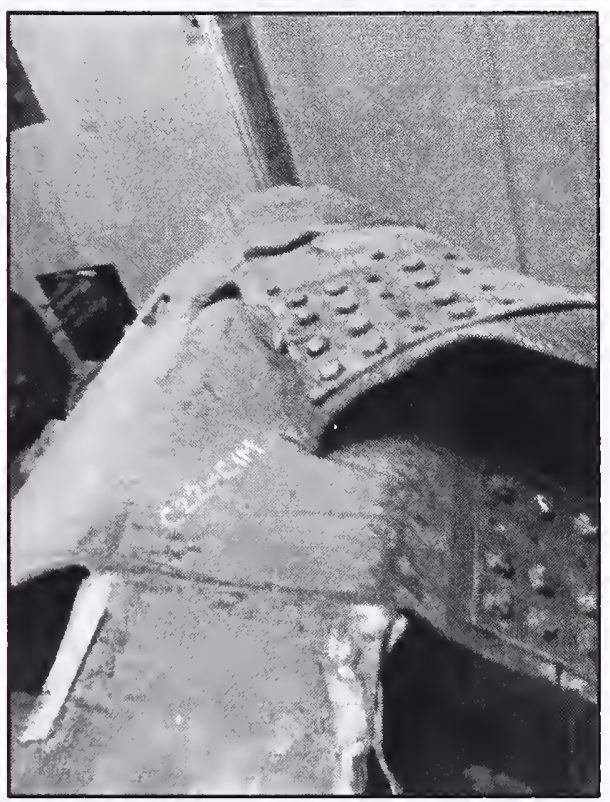

c)

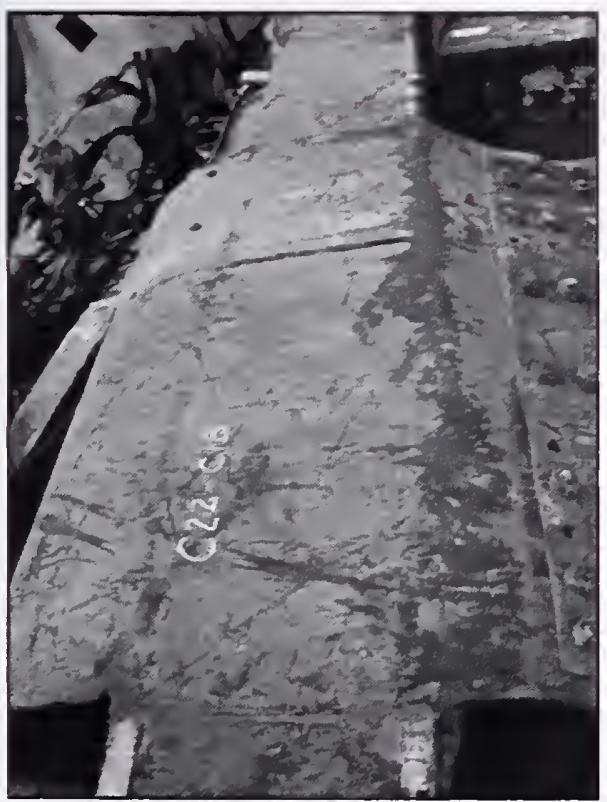

C22-C1B (WTC 1, Col.158, Fl 94)

Type $\mathrm{C}$

Seat Detail: 5210

Large gusset plate ripped off at weld.

Figure B-6. Floor truss connectors found on recovered panel C-22 (A157: 93-96) from WTC 1. Shown are connectors found on column 158 at different floor levels. 
d)

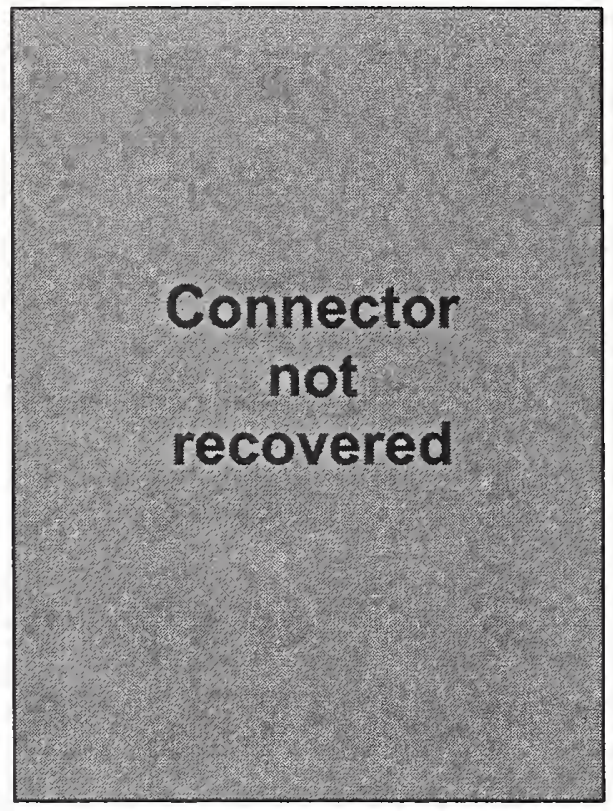

C22-C2M (WTC 1, Col.157, Fl 95)

Type A

Seat Detail: 1311

Gusset plate ripped completely out of spandrel;

Only standoff plates remain, seat ripped off at welds; Gusset for damper unit ripped off at weld.

f)

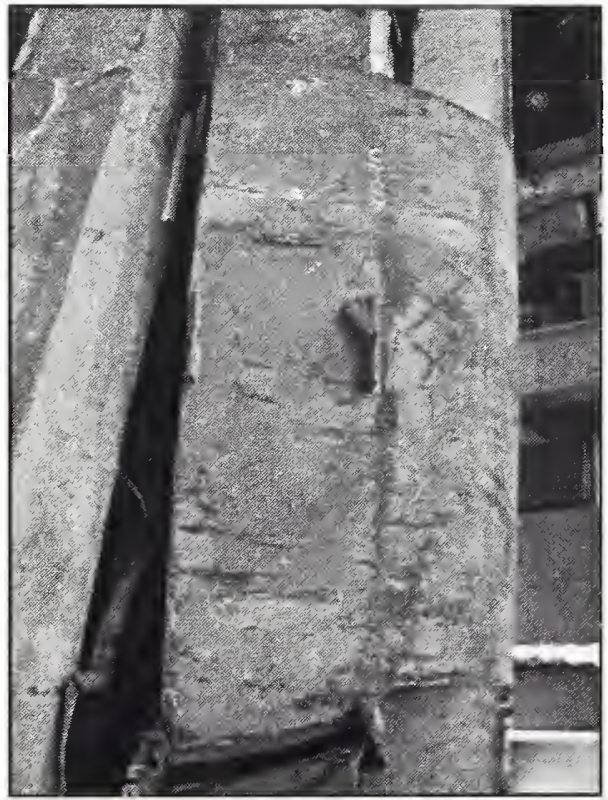

Type A

Seat Detail: 1311
C22-C2T

NA

e)

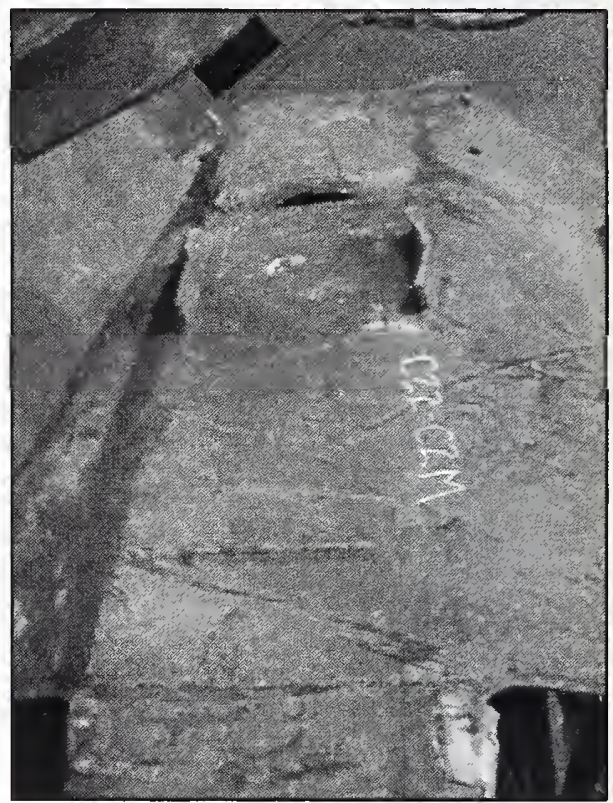

C22-C2B (WTC 1, Col.157, Fl 94)

Gusset plate ripped off at weld;

Only standoff plates remain, seat ripped off at welds;

Gusset for damper unit ripped off at weld.

Figure B-6. Floor truss connectors found on recovered panel C-22 (A157: 93-96) from WTC 1. Shown are connectors found on column 157 at different floor levels (cont.). 
g)

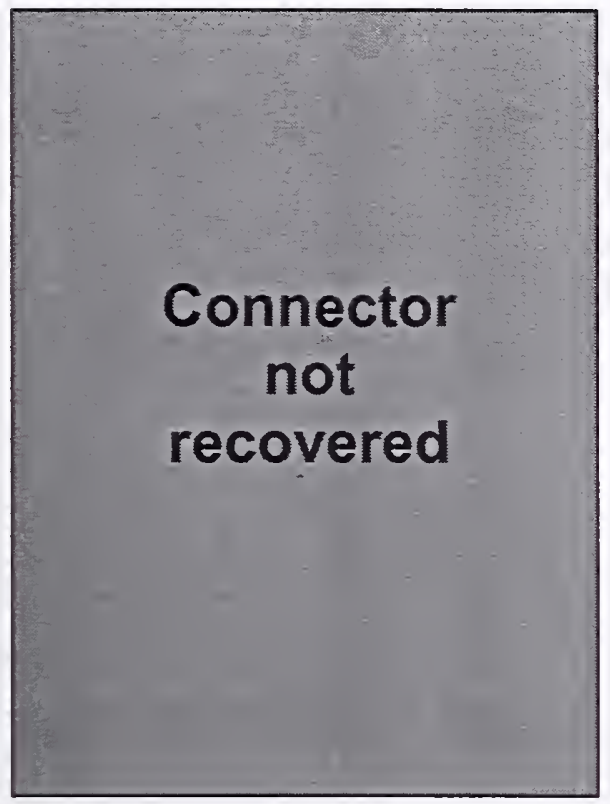

C22-C3M (WTC 1, Col.156, Fl 95)

Type C

Seat Detail: 5110

Large gusset plate ripped off at weld.
C22-C3T

NA h)

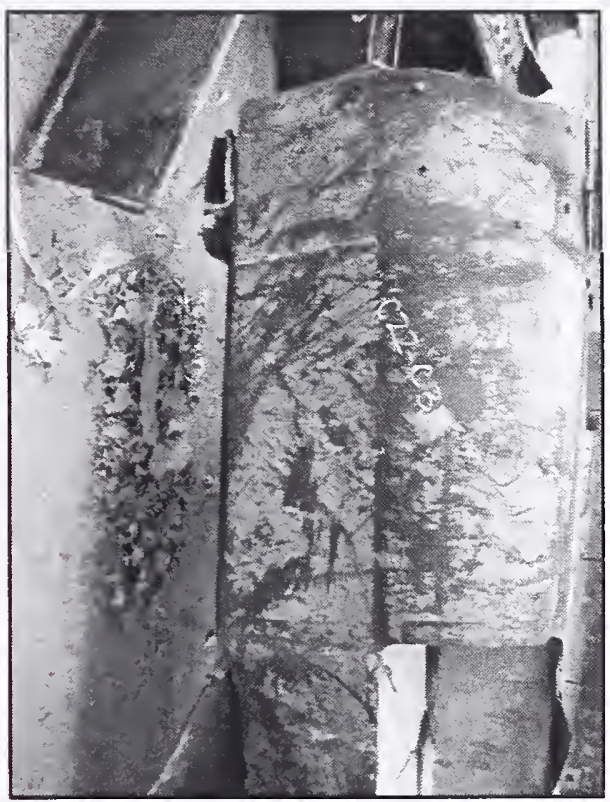

i)

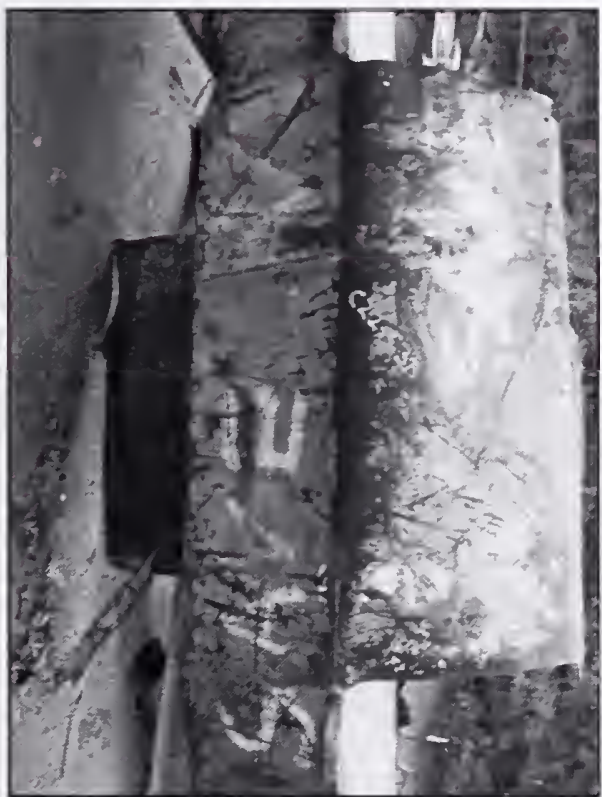

C22-C3B (WTC 1, Col.156, FI 94)

Type C

Seat Detail: 5110

Large gusset plate ripped off at weld.

Figure B-6. Floor truss connectors found on recovered panel C-22 (A157: 93-96) from WTC 1. Shown are connectors found on column 156 at different floor levels (cont.). 
a)

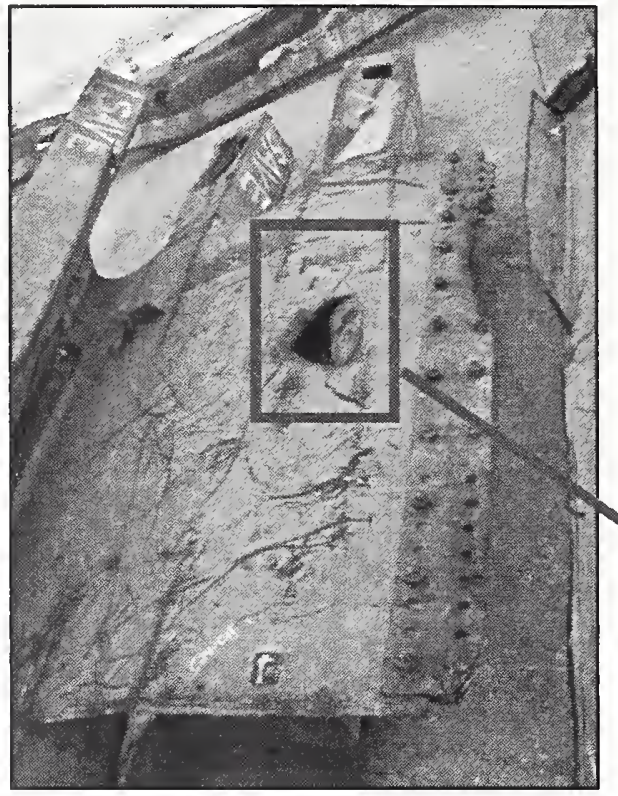

c)

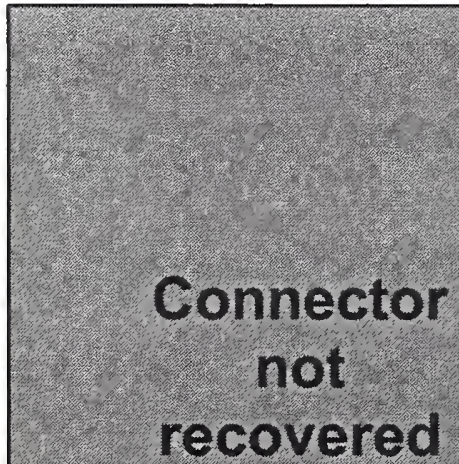

\section{Mechanical floor panel}

C24-C1T (WTC 2, Col. 204, Fl 77)

Type A

Seat Detail: 7010

No evidence of gusset plate;

Seat that was welded directly to column (no shear plates) ripped off at welds;

No evidence of gusset plate for damper unit.

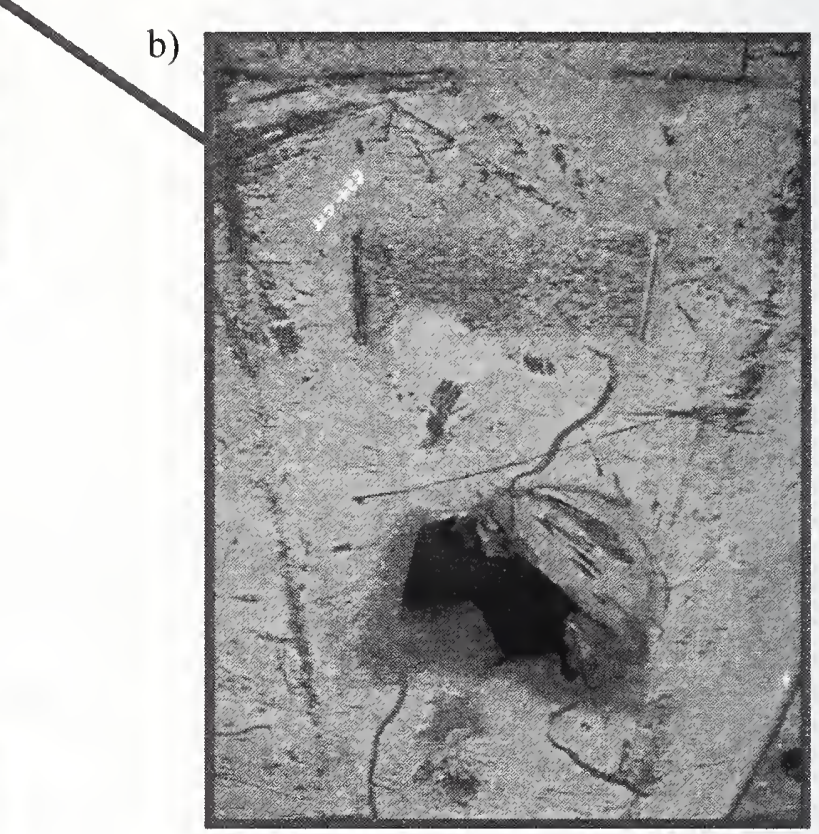

C24-C1B

NA

Figure B-7. Floor truss connectors found on recovered panel C-24 (B203: 74-77) from WTC 2. Shown are connectors found on column 204 at different floor levels. 
d)

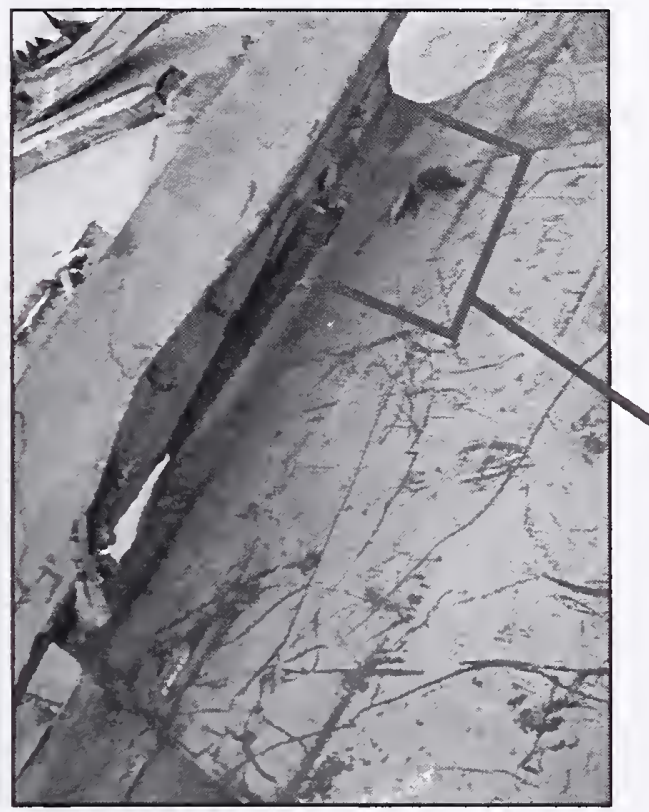

\section{Mechanical floor panel}

C24-C2T (WTC 2, Col.203, Fl 77)

Type C

Seat Detail: 6220

Gusset plate with vertical support, torn and bent down wards.

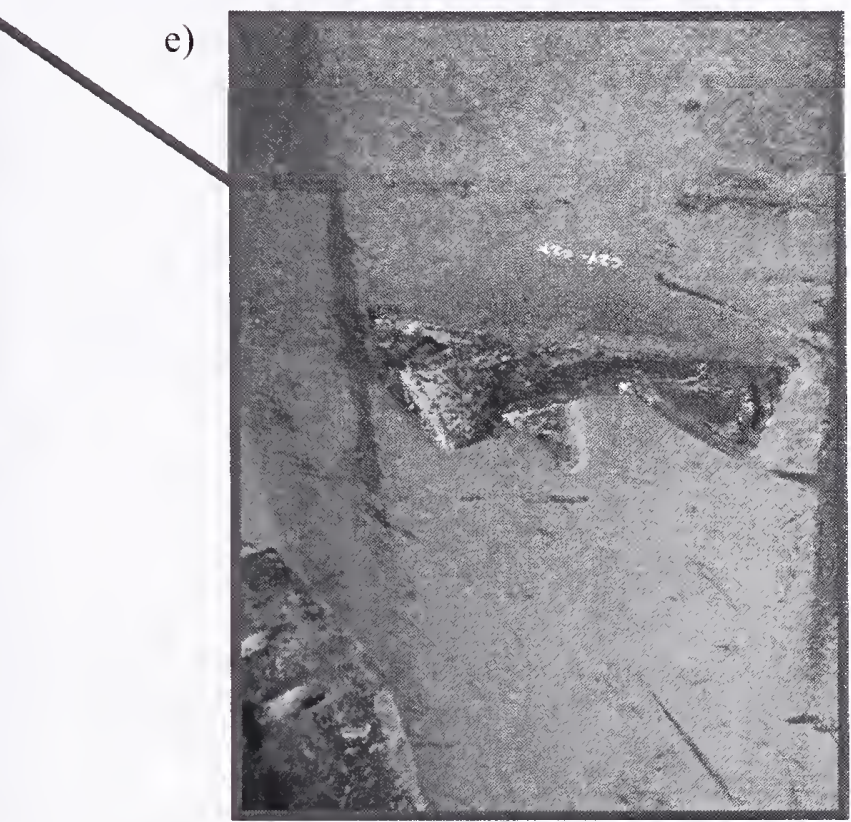

f)

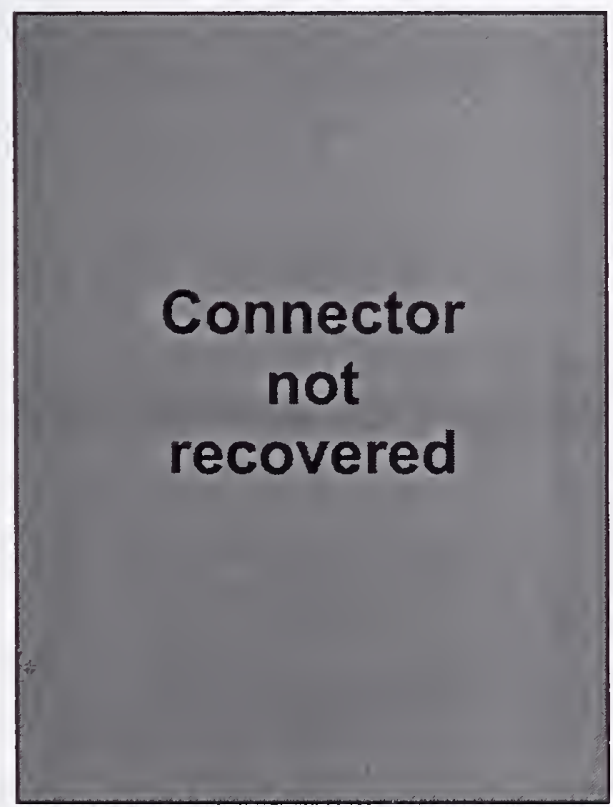

C24-C2B

NA

Figure B-7. Floor truss connectors found on recovered panel C-24 (B203: 74-77) from WTC 2. Shown are connectors found on column 203 at different floor levels (cont.). 
g)

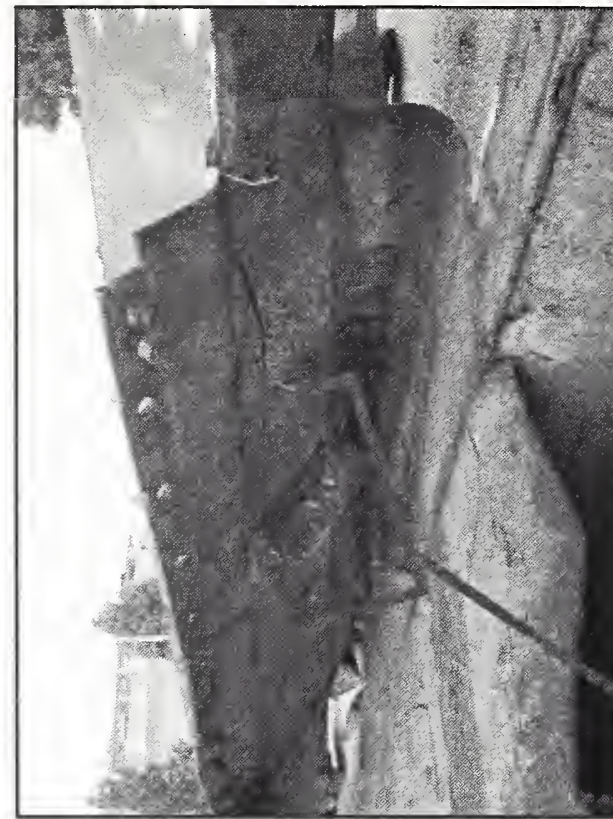

\section{Mechanical floor panel}

C24-C3T (WTC 2, Col.202, Fl 77)

Type A

Seat Detail: 7494

Gusset plate intact;

Seat intact, 1 side bent down, both bolt holes intact;

Gusset plate for dampers ripped off at welds.

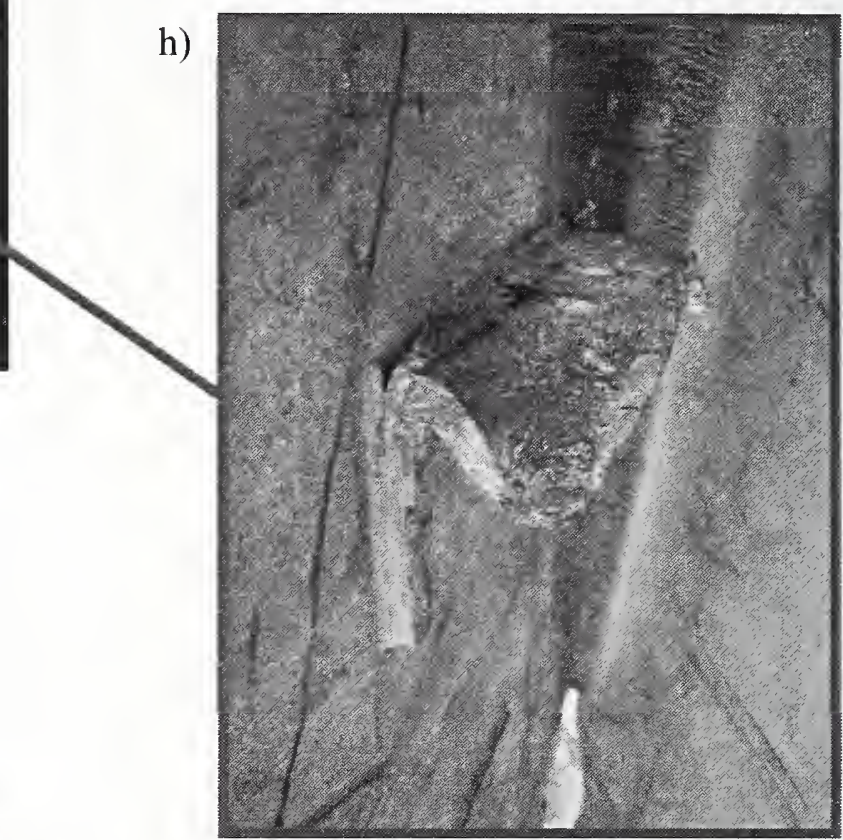

i)

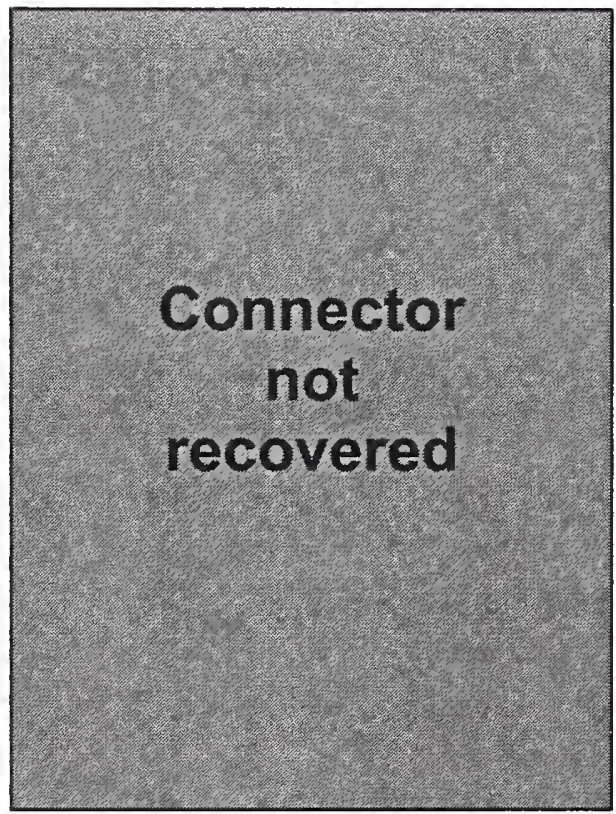

C24-C3B

NA

Figure B-7. Floor truss connectors found on recovered panel C-24 (B203: 74-77) from WTC 2. Shown are connectors found on column 202 at different floor levels (cont.). 
a)

Connector not recovered

\section{C25-C1T}

NA

C25-C1M (WTC 1, Col.207, Fl 91) Type D

Seat Detail: 5110

1-in portion of diagonal bracing strap remains, other is ripped off at weld.

c)

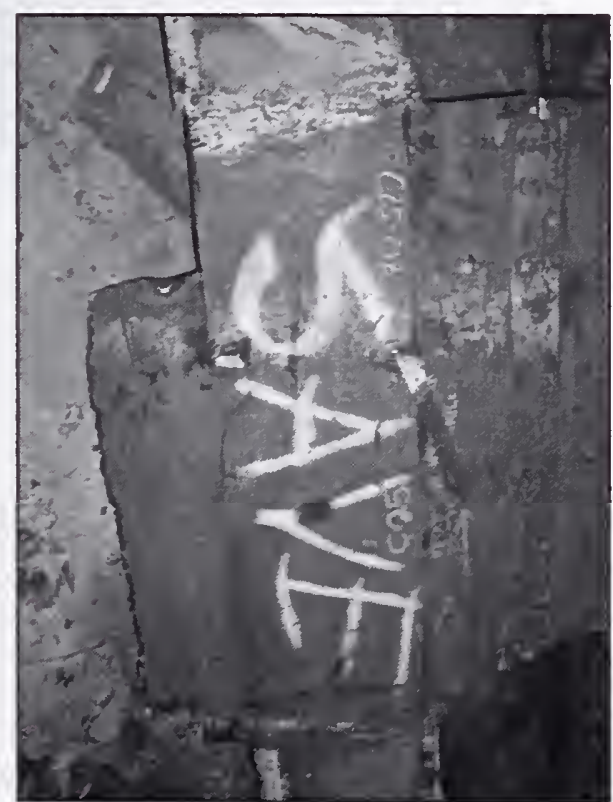

b)

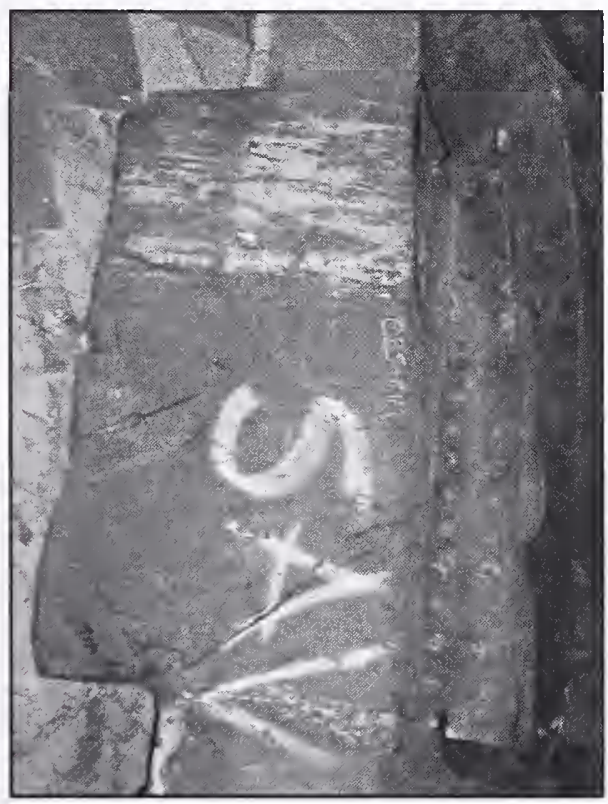

C25-C1B (WTC 1, Col.207, Fl 90)

Type D

Seat Detail: 5110

$1-\mathrm{ft}$ and 1 -in portions of diagonal bracing straps remain.

Figure B-8. Floor truss connectors found on recovered panel C-25 (A206: 89-92) from WTC 1. Shown are connectors found on column 207 at different floor levels. 
d)

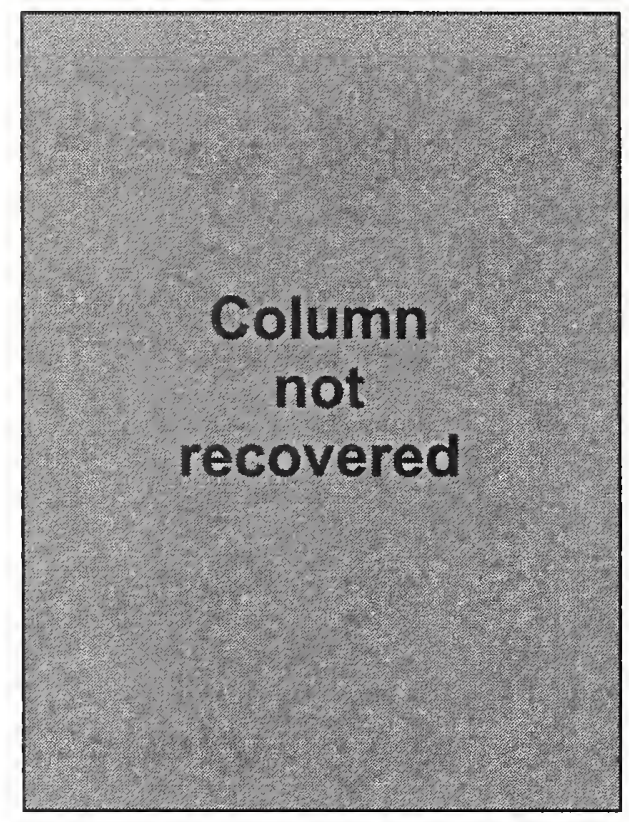

C25-C2M

NA

f)

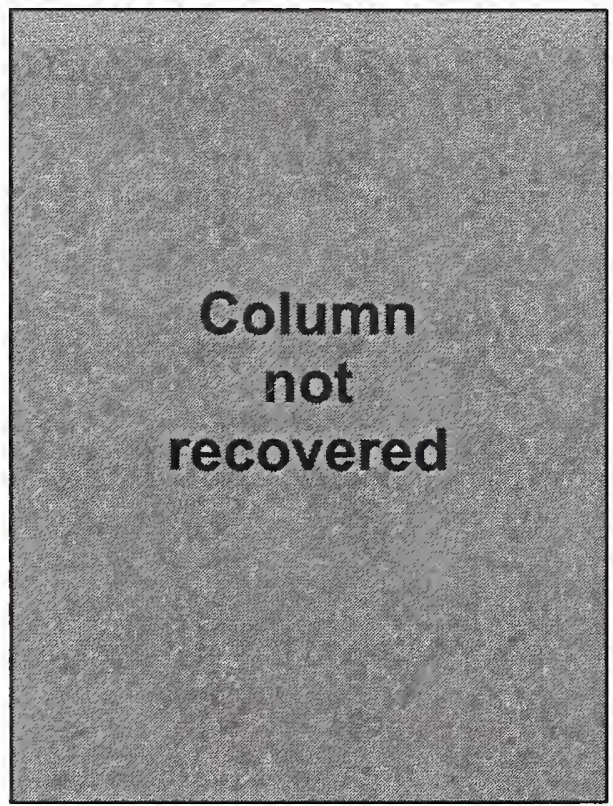

\section{C25-C2T}

NA e)

\section{Column not recovered}

C25-C2B

NA

Figure B-8. Floor truss connectors found on recovered panel C-25 (A206: 89-92) from WTC 1. Column 206 was not recovered (cont.). 
g)

\section{Column not recovered}

\section{C25-C3T}

NA

h)

C25-C3M

NA

i)

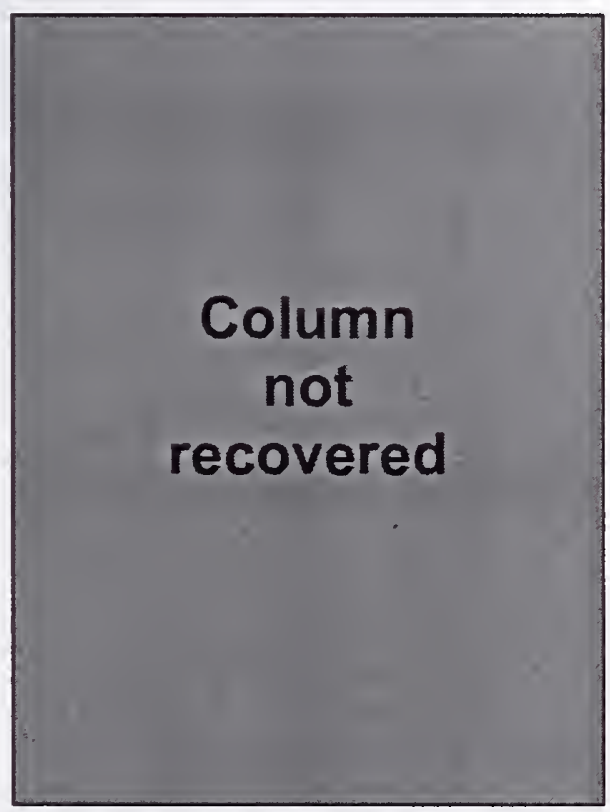

C25-C3B

NA

Figure B-8. Floor truss connectors found on recovered panel C-25 (A206: 89-92) from WTC 1. Column 205 was not recovered (cont.). 
a)

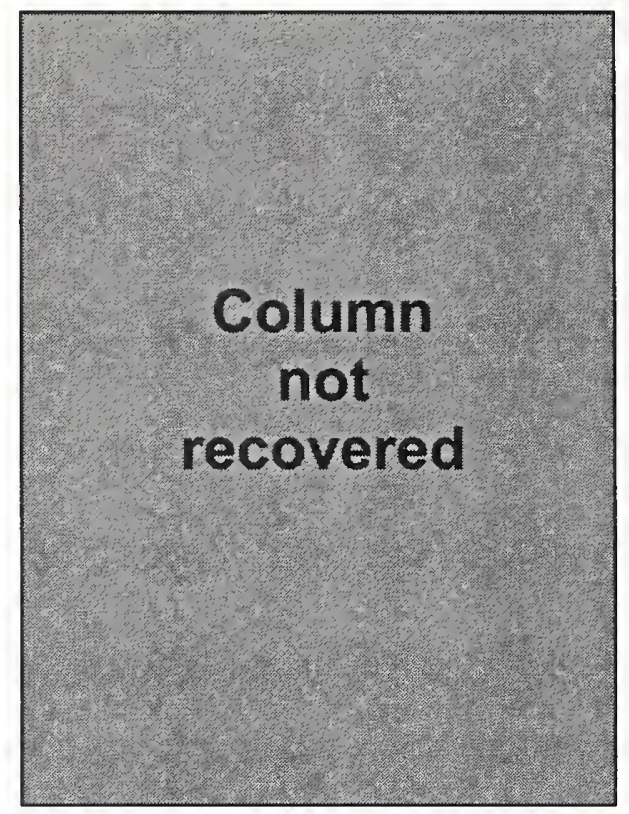

C40-C1M

NA
C40-C1T

NA b)

\section{Column not recovered}

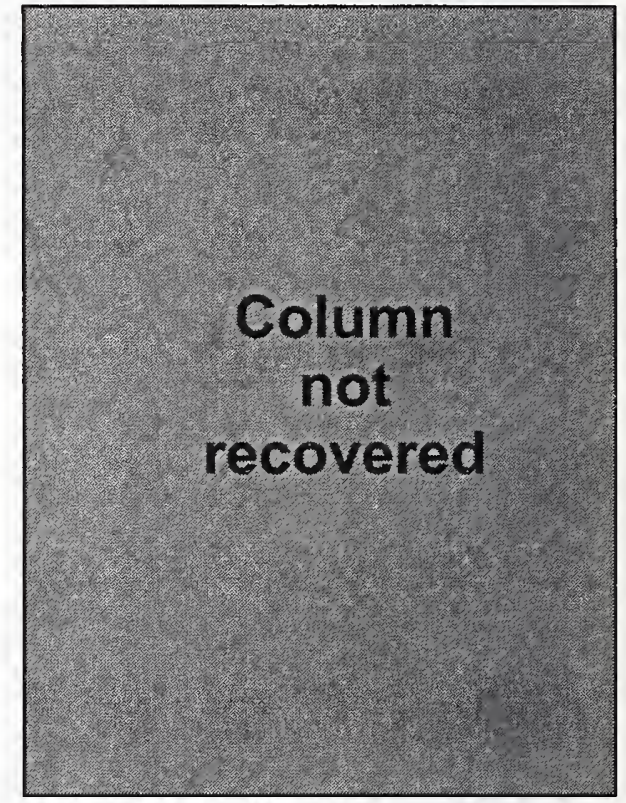

C40-C1B

NA

Figure B-9. Floor truss connectors found on recovered panel C-40 (A136: 98-101) from WTC 1. Column 137 was not recovered. 
d)

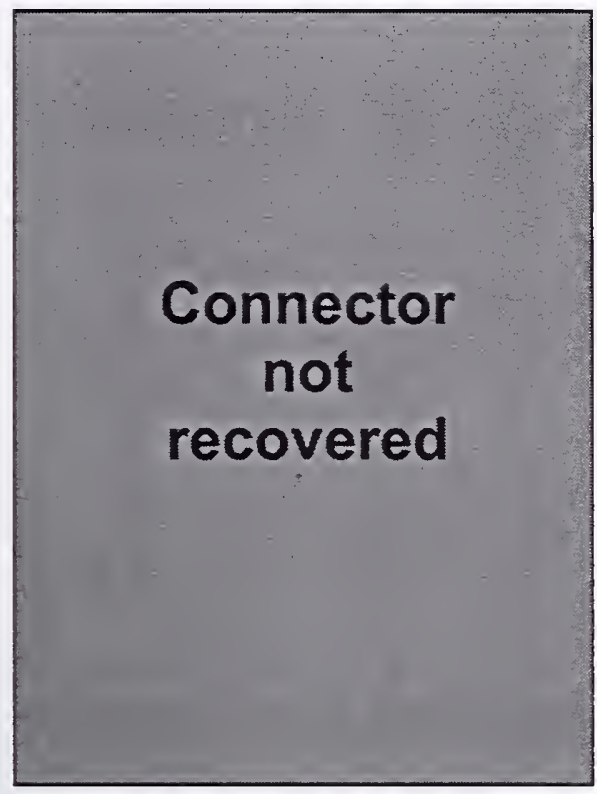

C40-C2M (WTC 1, Col.136, Fl 100)

Type C

Seat Detail: 5110

Large gusset plate intact with portions of diagonal bracing straps attached, partially bent downwards; Smaller gusset plate also found lower on the spandrel.

f)

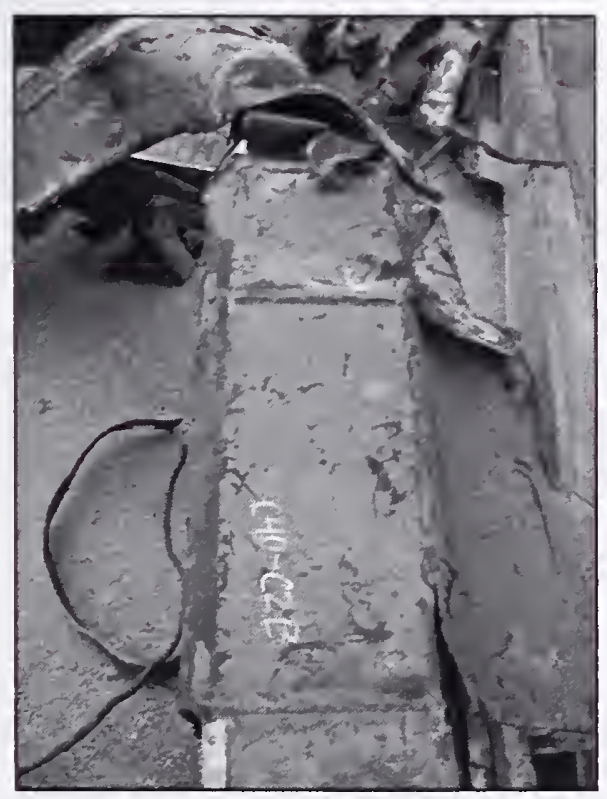

C40-C2T

NA
Type $\mathrm{C}$

Seat Detail: 5110 e)

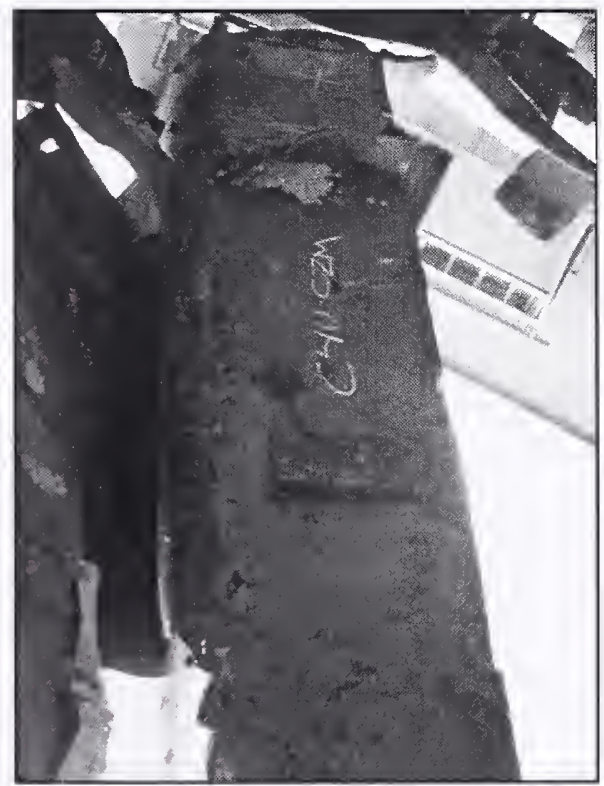

C40-C2B (WTC 1, Col.136, Fl 99)

Large gusset plate ripped off at weld.

Figure B-9. Floor truss connectors found on recovered panel C-40 (A136: 98-101) from WTC 1. Shown are connectors found on column 136 at different floor levels (cont.). 
g)

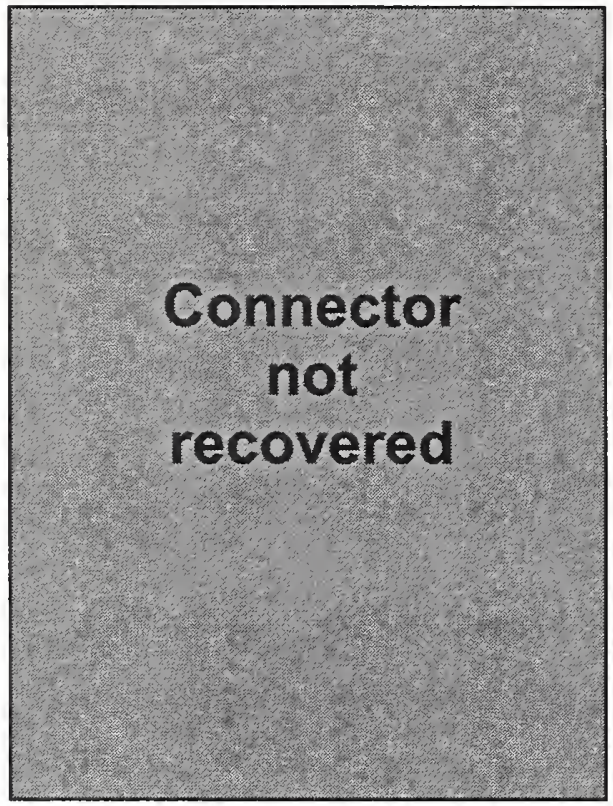

C40-C3M (WTC 1, Col.135, Fl 100)

Type A

Seat Detail: 1411

Portion of gusset plate intact;

Seat intact, both bolt holes intact with bolts and nuts remaining;

Gusset for damper unit ripped off at weld.

i)

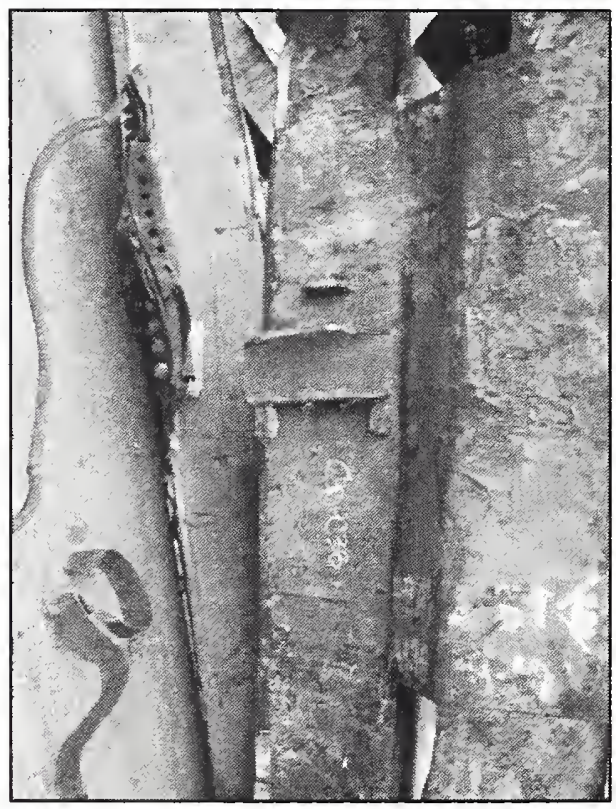

Type A

Seat Detail: 1411
C40-C3T

NA

h)

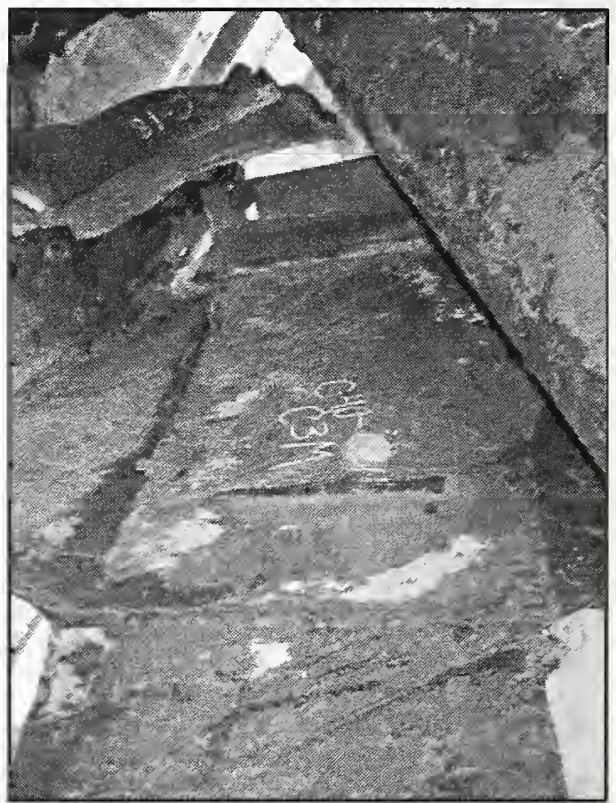

C40-C3B (WTC 1, Col.135, Fl 99)

Portion of gusset plate intact;

Seat intact, both bolt holes ripped out;

Gusset for damper unit ripped out at weld.

Figure B-9. Floor truss connectors found on recovered panel C-40 (A136: 98-101) from WTC 1. Shown are connectors found on column 135 at different floor levels (cont.). 
a)

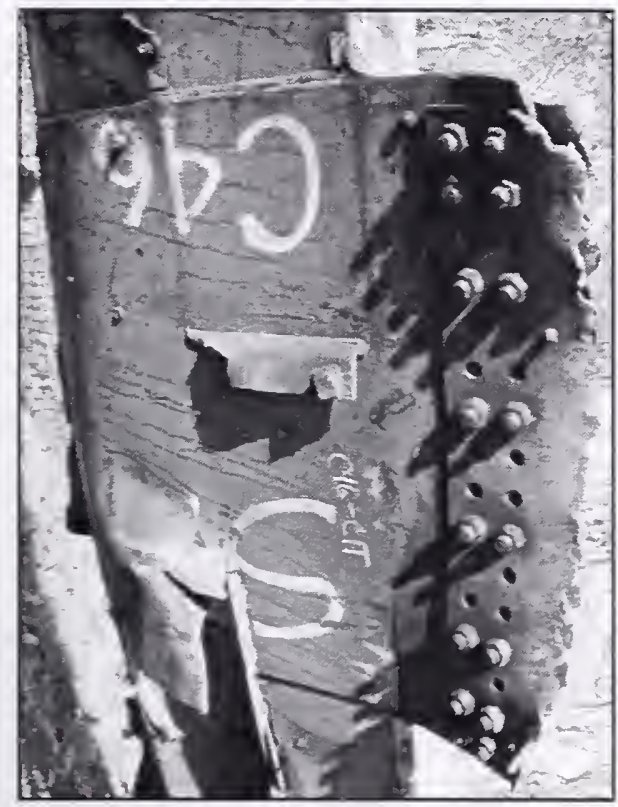

C46-C1M (WTC 2, Col.158, Fl 70)

Type C

Seat Detail: 5110

Portion of gusset plate remains, bent downward.

c)

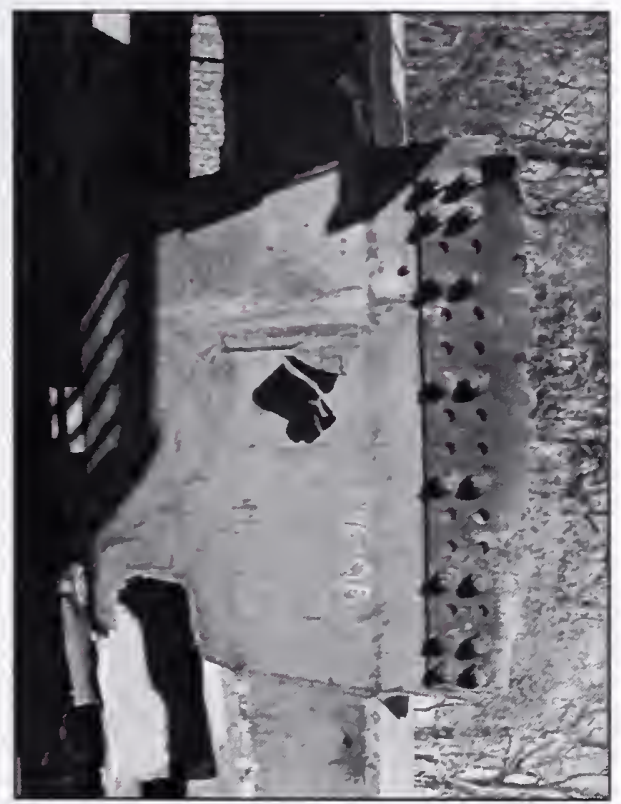

Type $\mathrm{C}$

Seat Detail: 5110
C46-C1T (WTC 2, Col.158, FI 71)

Type C

Seat Detail: 5110

Gusset plate remains, bent downward. b)

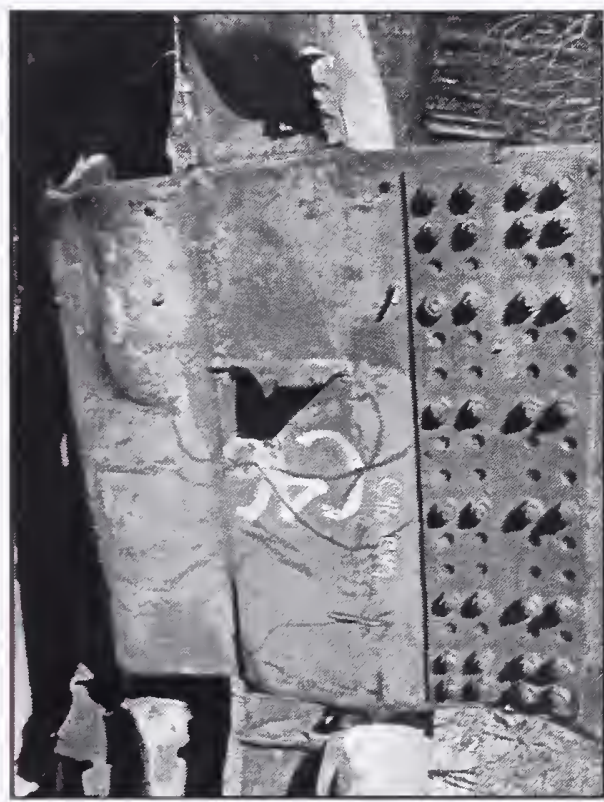

C46-C1B (WTC 2, Col.158, FI 69)

Gusset plate remains, but half is ripped away from spandrel at weld, bent downwards.

Figure B-10. Floor truss connectors found on recovered panel C-46 (B157: 68-72) from WTC 2. Shown are connectors found on column 158 at different floor levels. 
d)

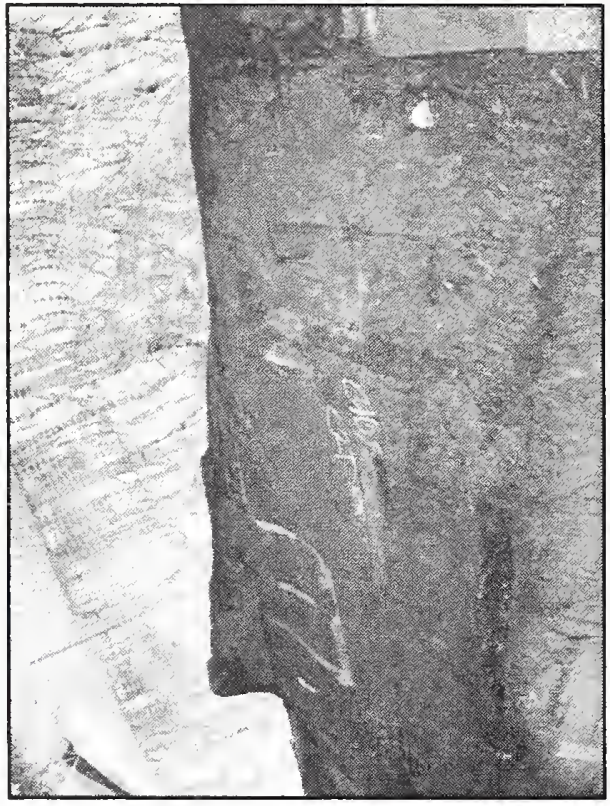

C46-C2M (WTC 2, Col.157, FI 70)

Type A

Seat Detail: 1311

Gusset plate intact;

Only portions of standoff plates remain;

Gusset plate for damper unit ripped off at weld.

\section{C46-C2T (WTC 2, Col.157, Fl 71)}

Type A

Seat Detail: 1311

Gusset plate completely ripped out of spandrel;

Standoff plates ripped of at weld;

Gusset plate for damper unit ripped off at weld. f)

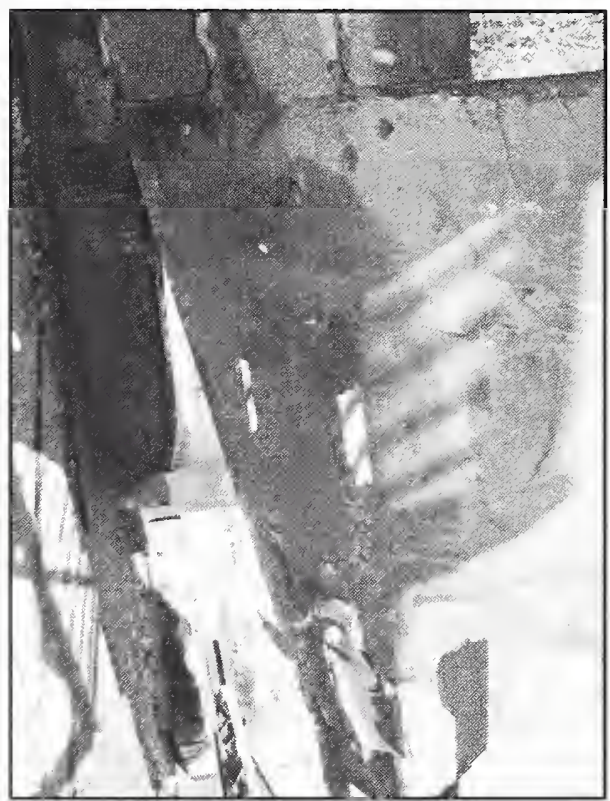

e)

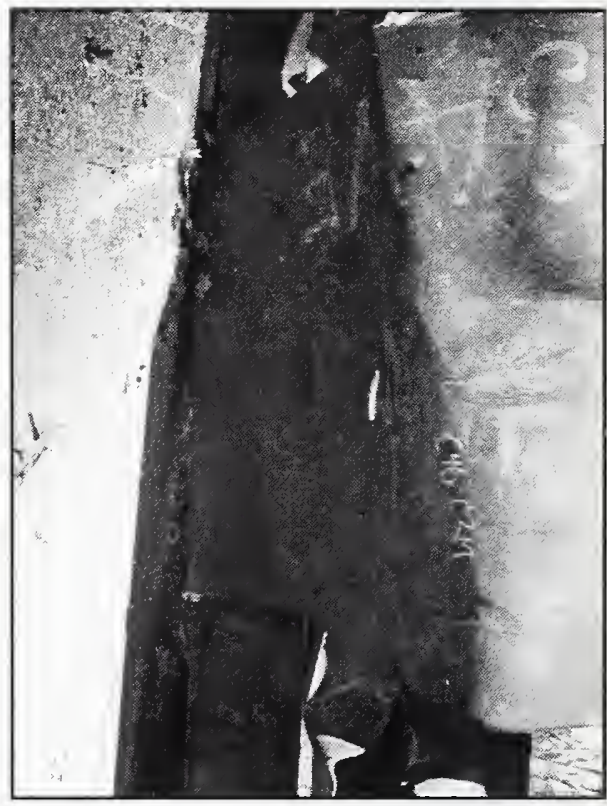

C46-C2B (WTC 2, Col.157, Fl 69)

Type A

Seat Detail: 1311

Gusset plate ripped off at weld;

Only standoff plates remain, rest ripped off at welds;

Gusset plate with damper unit intact, bent downwards.

Figure B-10. Floor truss connectors found on recovered panel C-46 (B157: 68-72) from WTC 2. Shown are connectors found on column 157 at different floor levels (cont.). 
g)

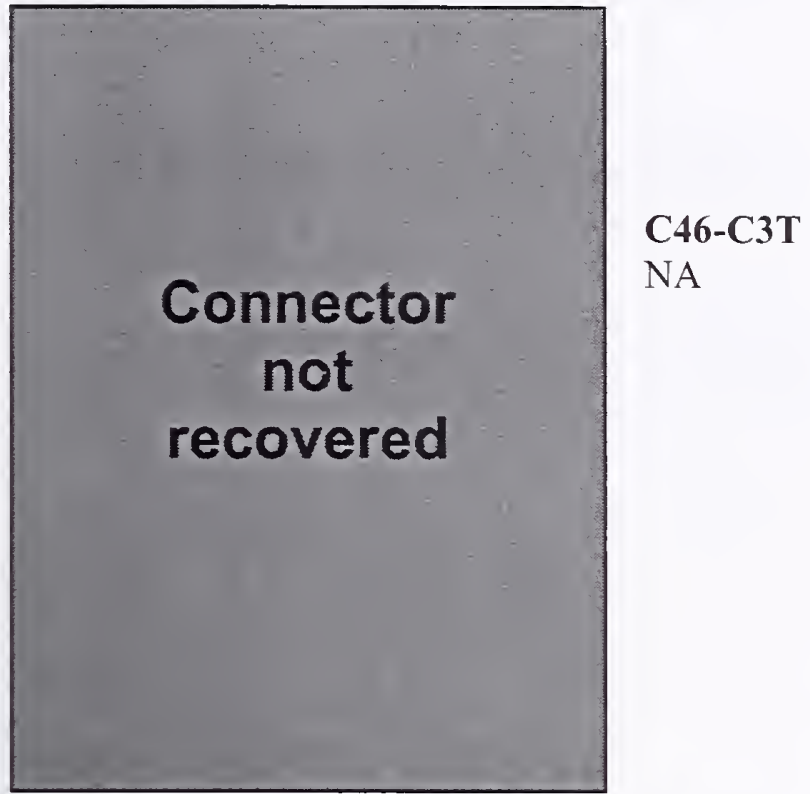

C46-C3M (WTC 2, Col.156, Fl 70)

Type C

Seat Detail: 5210

Large gusset plate intact, bent downwards, 2 long diagonal bracing straps remain as well.

h)

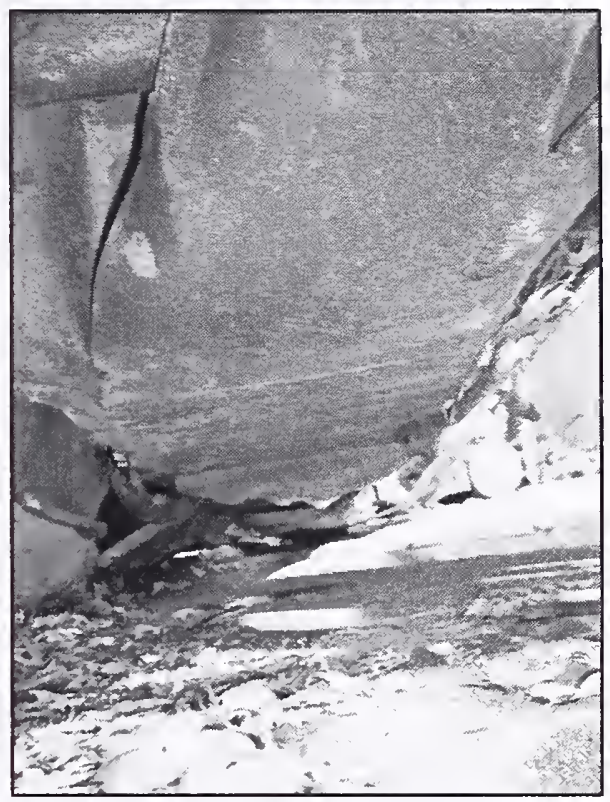

i)

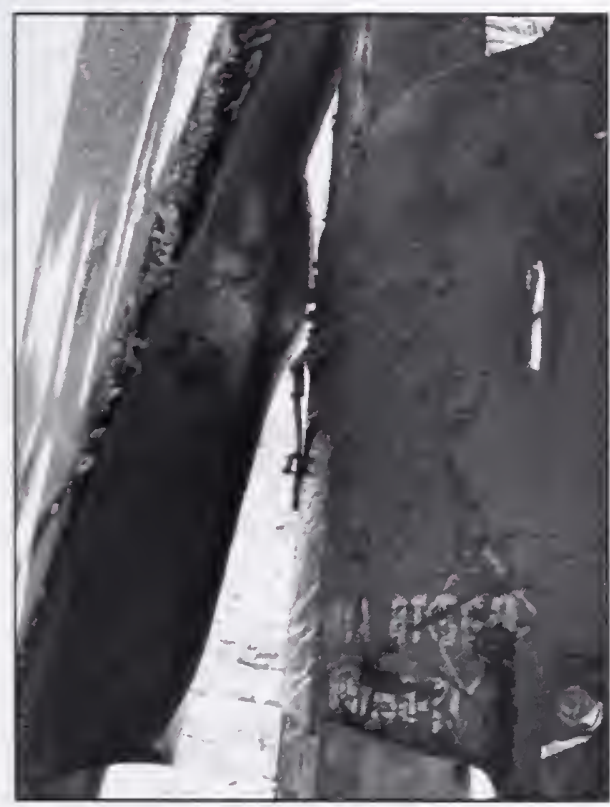

C46-C3B (WTC 2, Col.156, Fl 69)

Type C

Seat Detail: 5210

Large gusset plate intact, bent downwards, 2 long diagonal bracing straps remain as well.

Figure B-10. Floor truss connectors found on recovered panel C-46 (B157: 68-72) from WTC 2. Shown are connectors found on column 156 at different floor levels (cont.). 
a)

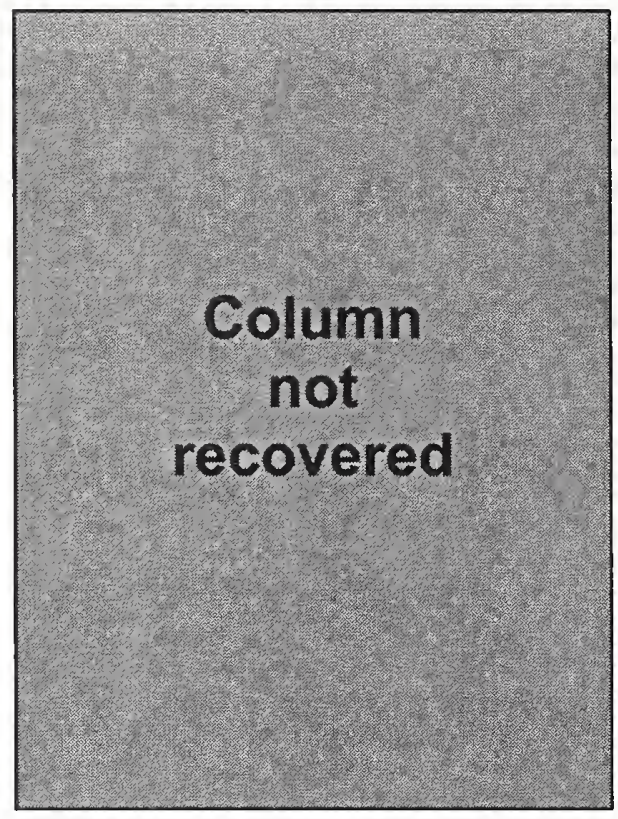

C48-C1T

NA

C48-C1M

NA

c)

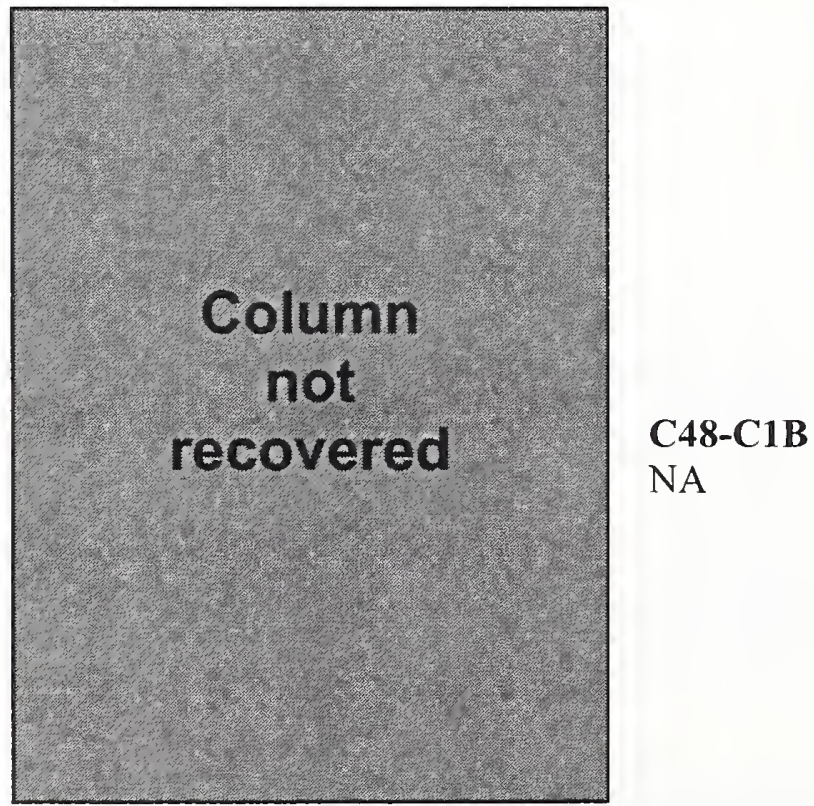

b)

Column

not

recovered

Figure B-11. Floor truss connectors found on recovered panel C-48 (B442: 91-94) from WTC 2. Column 443 was not recovered. 
d)

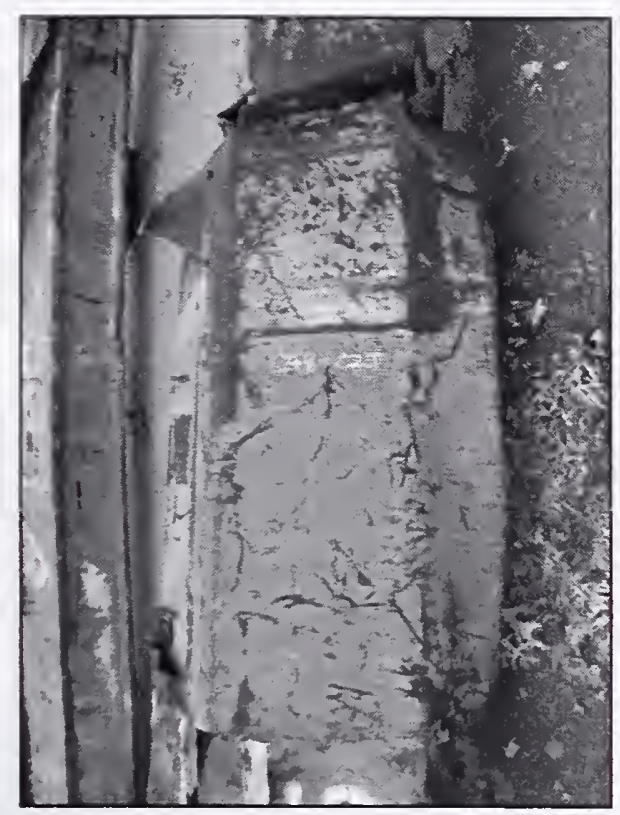

C48-C2M (IVTC 2, Col.442, Fl 93)

Type C

Seat Detail: 5210

Large gusset plate ripped off at weld.
C48-C2T (WTC 2, Col.442, FI 94)

Type $\mathrm{C}$

Seat Detail: 5210

Large gusset plate ripped off at weld. f)

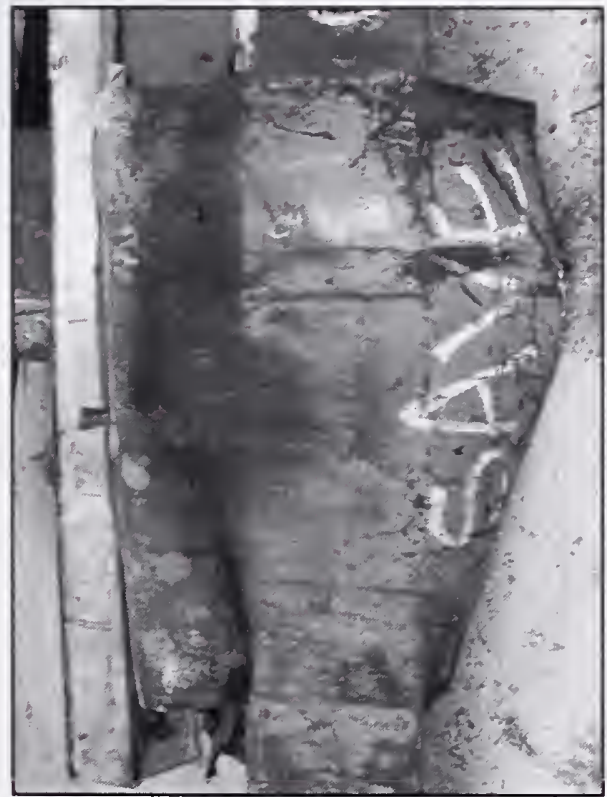

e)

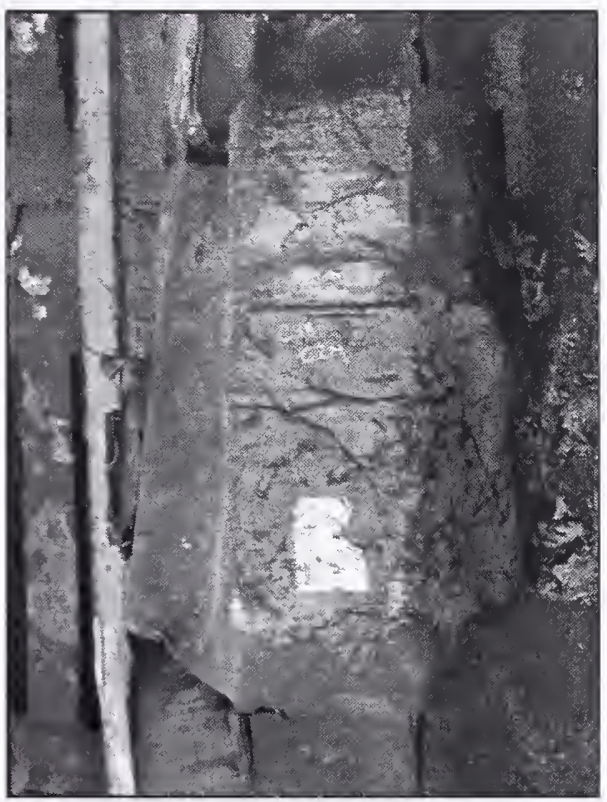

C-48-C2B (WTC 2, Col.442, Fl 92)

Type C

Seat Detail: 5210

Large gusset plate ripped off at weld.

Figure B-11. Floor truss connectors found on recovered panel C-48 (B442: 91-94) from WTC 2. Shown are connectors found on column 442 at different floor levels (cont.). 
g)

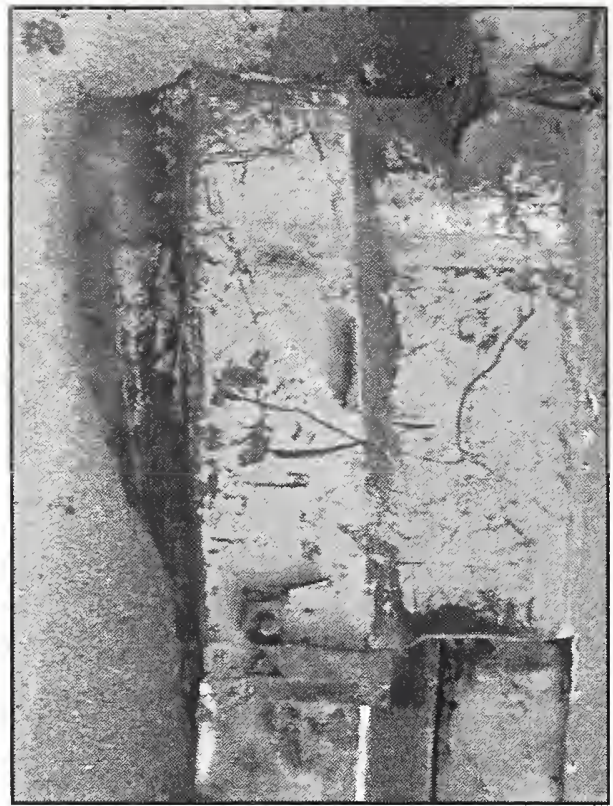

\section{C48-C3T (WTC 2, Col.441, FI 94)}

Type A

Seat Detail: 1111

Gusset plate intact;

1 standoff plate remains, rest ripped of at welds;

Portion of gusset plate with damper unit intact, bent downwards.

C48-C3M (WTC 2, Col.441, Fl 93)

Type A

Seat Detail: 1111

Gusset plate intact;

Seat intact, bent downwards, both bolt holes intact, 1 with bolt and nut;

Gusset plate and damper unit intact, bent upwards.

i)

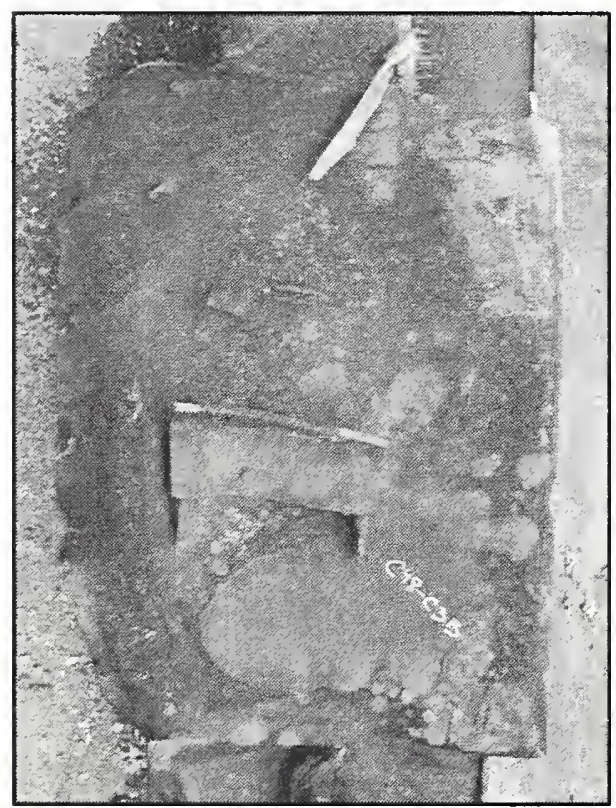

h)

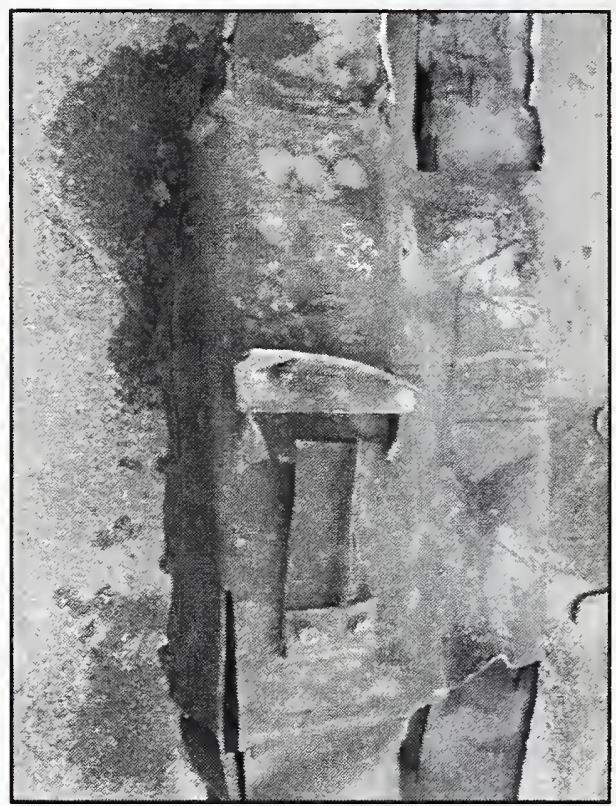

C48-C3B (WTC 2, Col.441, Fl 92)

Type A

Seat Detail: 1111

Gusset plate intact;

Seat intact, bent in towards column, both bolt holes intact;

No evidence of gusset plate for damper unit.

Figure B-11. Floor truss connectors found on recovered panel C-48 (B442: 91-94) from WTC 2. Shown are connectors found on column 441 at different floor levels (cont.). 
a)

\section{Column not recovered}

\section{C55-C1M}

NA

c)

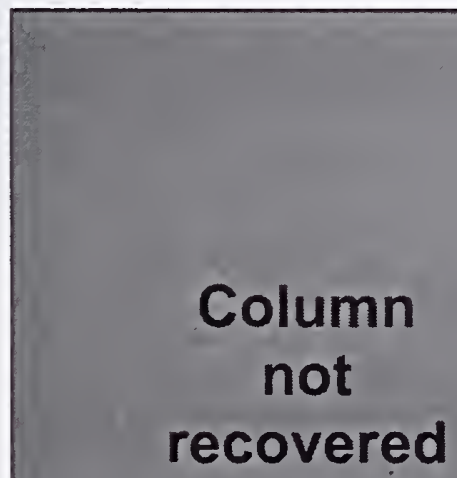

C55-C1T

NA

\section{Column not recovered}

b)

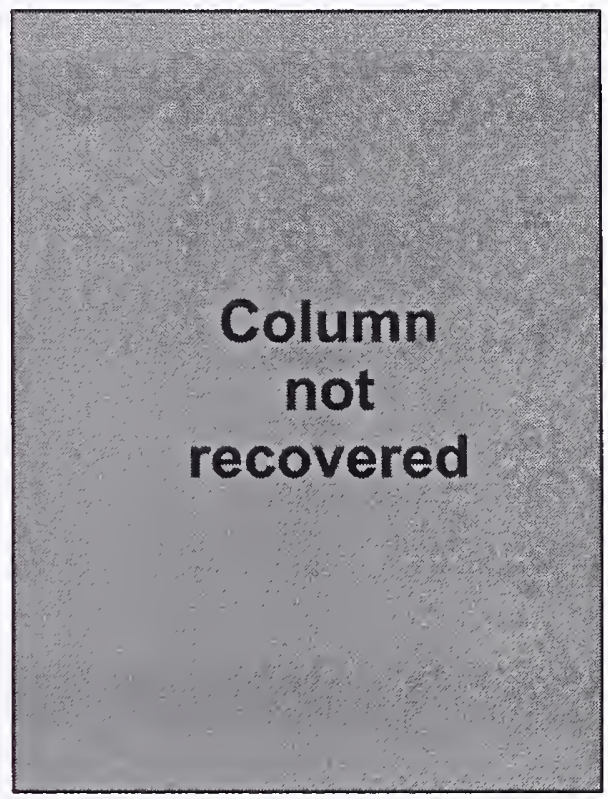

C55-C1B

NA

Figure B-12. Floor truss connectors found on recovered panel C-55 (A209: 94-97) from WTC 1. Column 210 was not recovered. 
d)

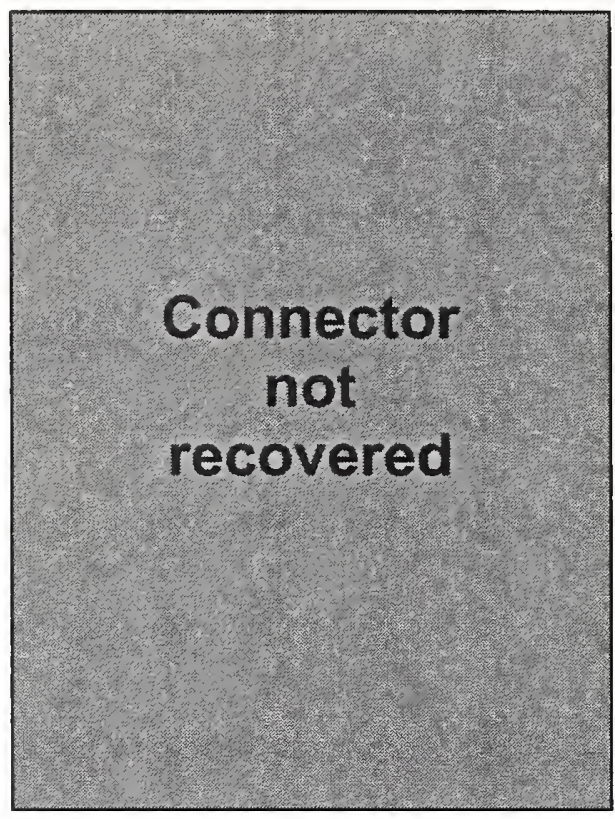

C55-C2M

NA

f)

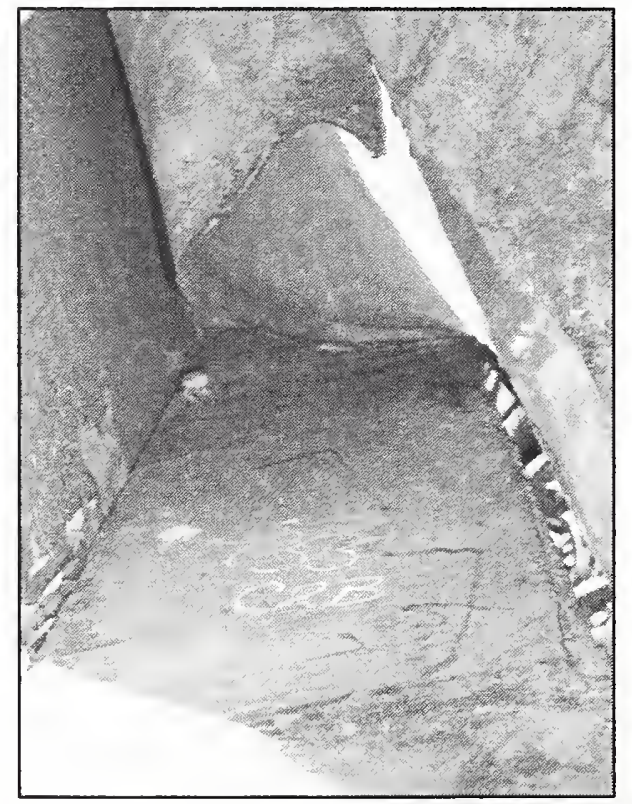

\section{C55-C2T}

NA e)

\section{Connector not} recovered

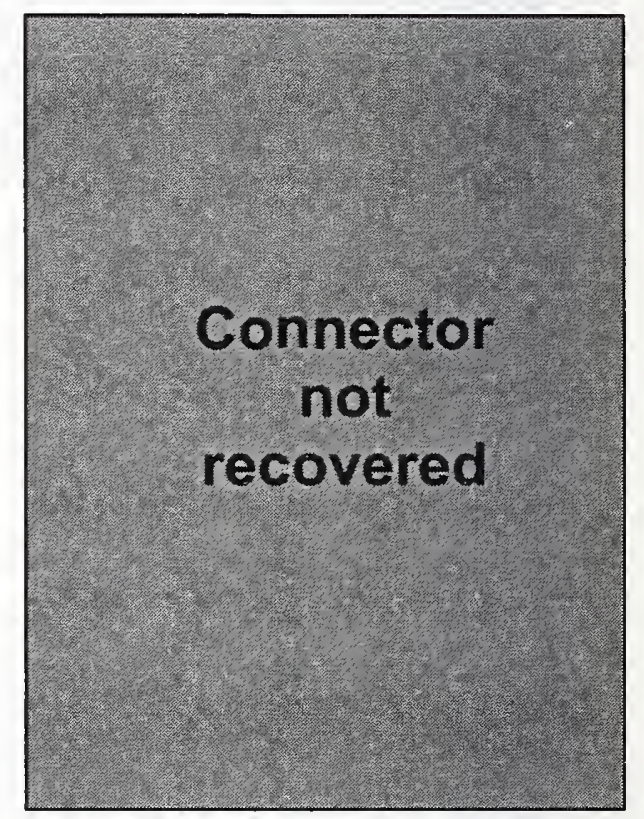

C55-C2B (WTC 1, Col.209, Fl 95)

Type D

Seat Detail: 5210

One diagonal bracing strap ripped off at weld, other Has about 1 in left.

Figure B-12. Floor truss connectors found on recovered panel C-55 (A209: 94-97) from WTC 1. Shown are connectors found on column 209 at different floor levels (cont.). 
g)

\section{Column not recovered}

C55-C3M

NA

i)

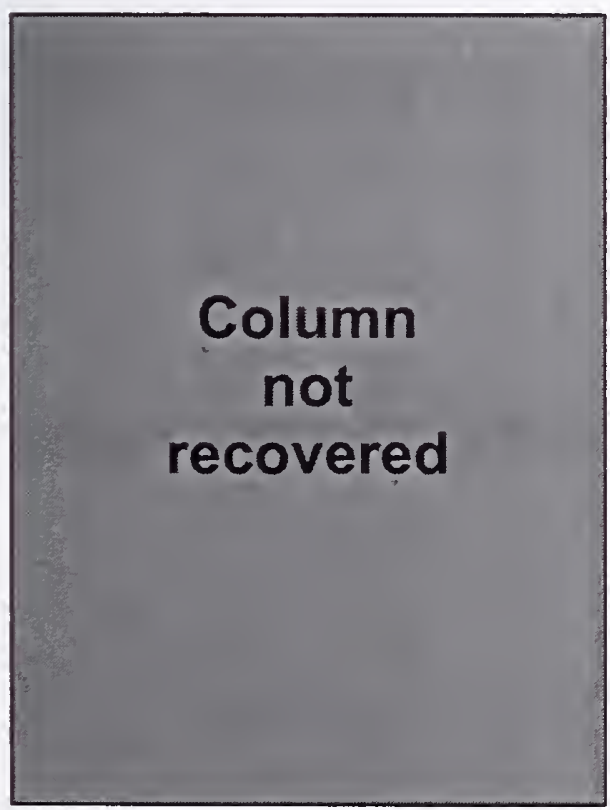

C55-C3T

NA

h)

Column not recovered

\section{C55-C3B}

NA

Figure B-12. Floor truss connectors found on recovered panel C-55 (A209: 94-97) from WTC 1. Column 208 was not recovered (cont.). 
a)

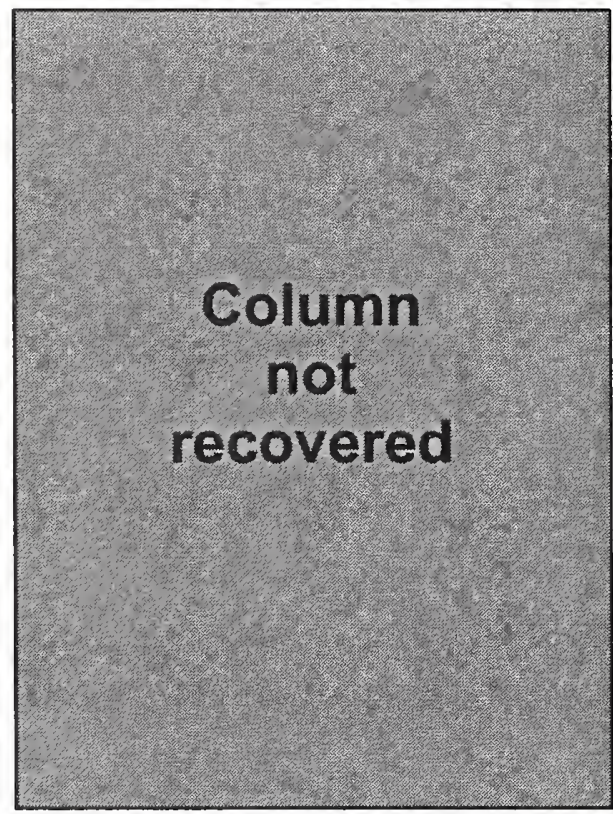

C89-C1M

NA

\section{C89-C1T}

NA

\section{Column not recovered}

b)

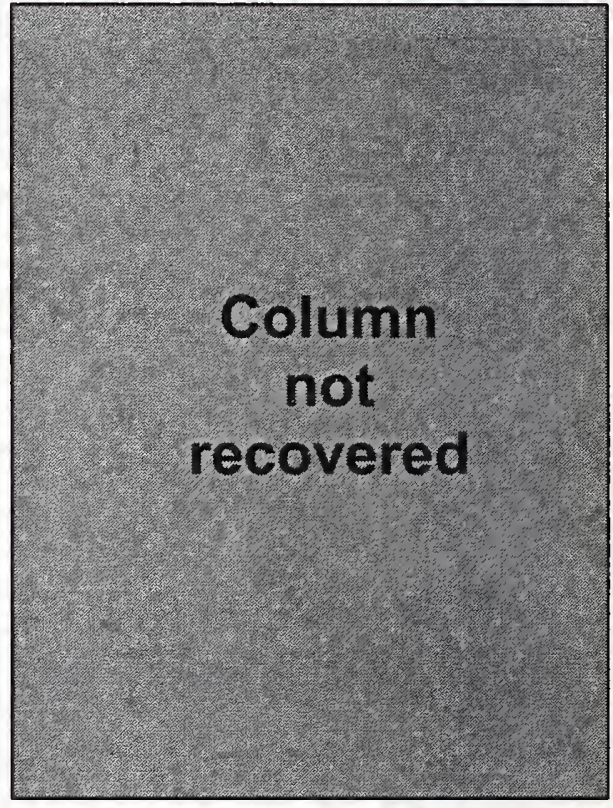

C89-C1B

NA

Figure B-13. Floor truss connectors found on recovered panel C-89 (B215: 12-15) from WTC 2. Column 216 was not recovered. 
d)

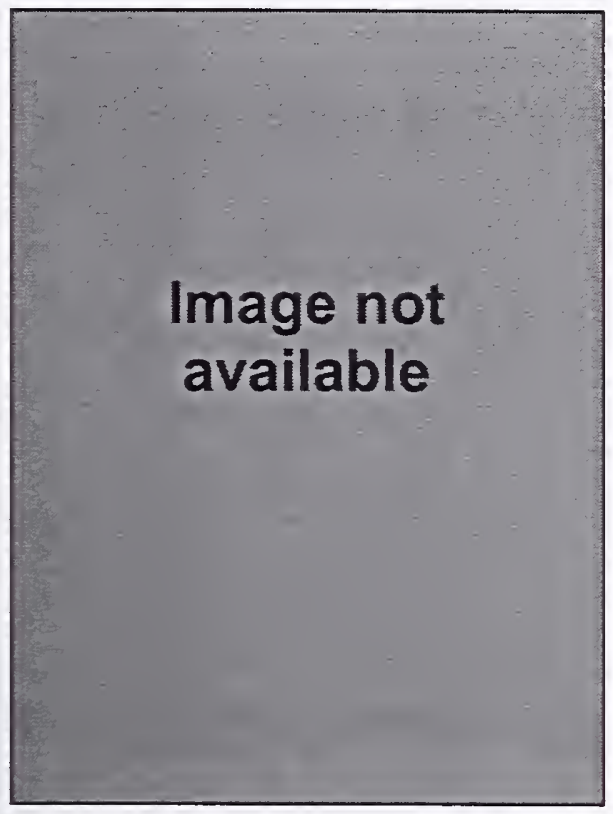

C89-C2M (WTC 2, Col.215, Fl 14)

Type B

Seat Detail: 5510

Gusset plate ripped off at weld;

Small seat ripped of at weld:

No evidence of gusset plate for damper unit.

f)

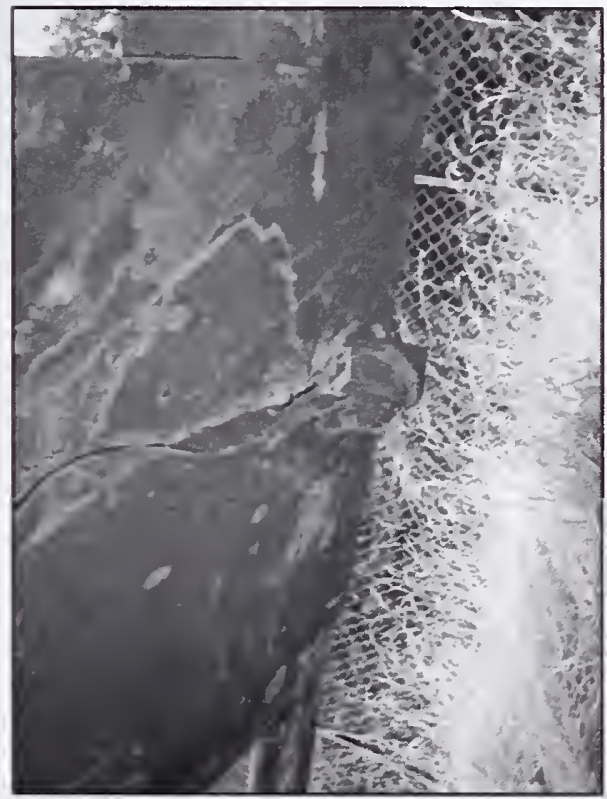

Type B

\section{C89-C2T (WTC 2, Col.215, Fl 15)}

Type B

Seat Detail: 5510

Gusset plate ripped off at weld;

Small seat intact, both bolt holes ripped through;

No evidence of gusset plate for damper unit. e)

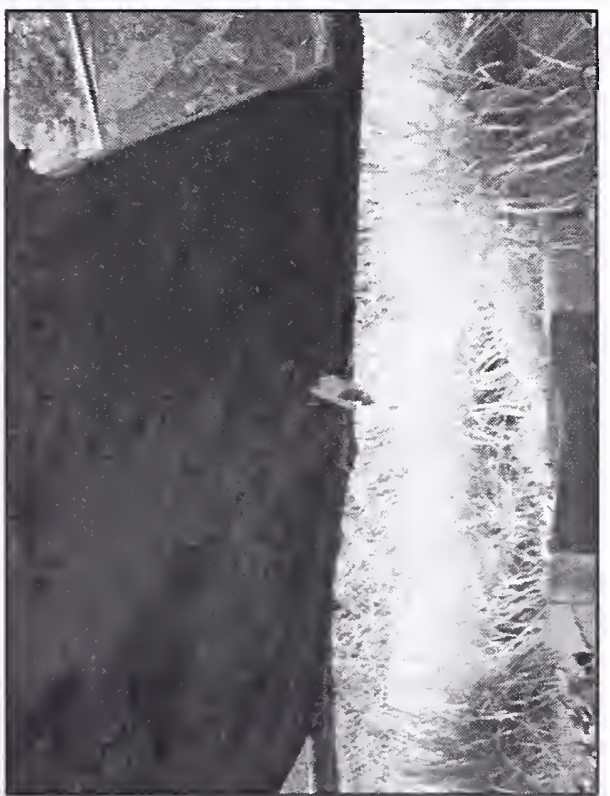

C89-C2B (WTC 2, Col.215, Fl 13)

Seat Detail: 5510

No gusset plate, appears to have partial of 1 diagonal bracing strap remaining;

Small seat intact, both bolt holes ok, small wide flange section remains welded to seat;

No evidence of gusset plate for damper unit.

Figure B-13. Floor truss connectors found on recovered panel C-89 (B215: 12-15) from WTC 2. Shown are connectors found on column 215 at different floor levels (cont.). 
g)

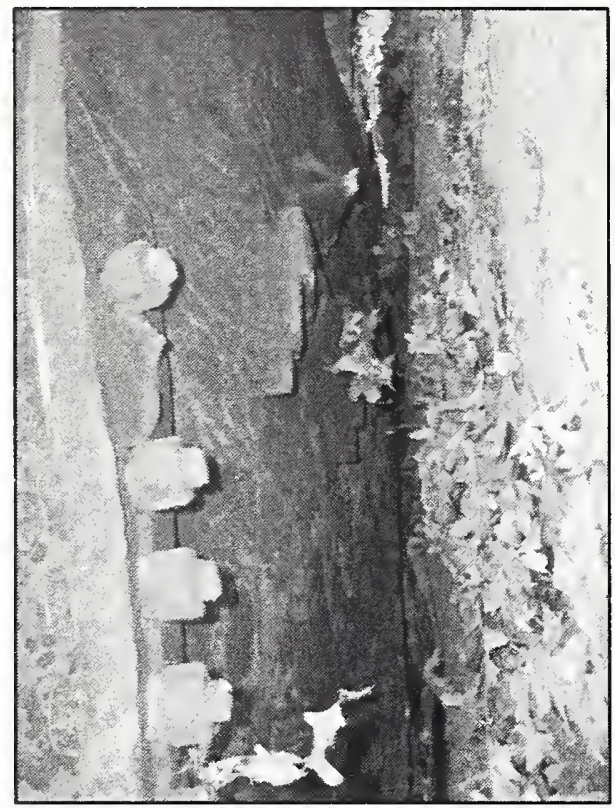

C89-C3M (WTC 2, Col.214, Fl 14)

Type A

Seat Detail: 1212

Gusset plate ripped off at weld;

Standoff plates ripped off at welds;

Portion of gusset plate for damper unit remains, damper units gone.

i)

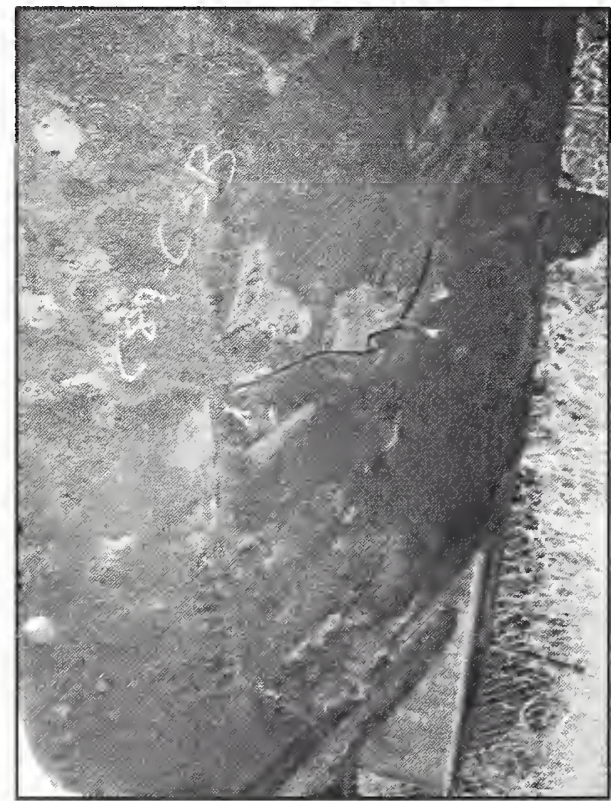

Type A

Seat Detail: 1113
C89-C3T (WTC 2, Col.214, FI 15)

Type A

Seat Detail: 1212

Gusset plate intact with shear bolt, bent up;

Seat intact, bent down, 1 bolt hole intact, other ripped through;

Gusset plate for damper unit ripped off at weld. h)

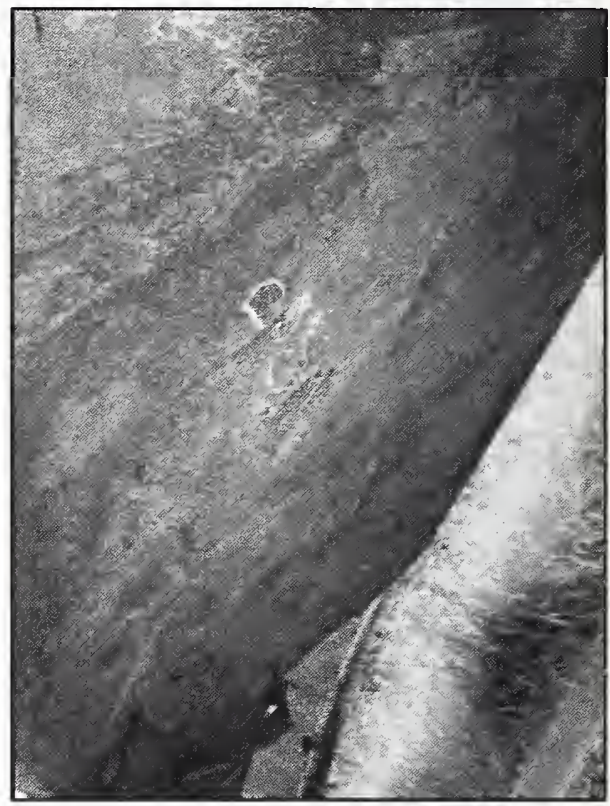

C89-C3B (WTC 2, Col.214, Fl 13)

Gusset plate intact, pushed inwards;

Seat intact, bent up, both bolt holes ripped through; Gusset plate with damper unit intact, bent sideways.

Figure B-13. Floor truss connectors found on recovered panel C-89 (B215: 12-15) from WTC 2. Shown are connectors found on column 214 at different floor levels (cont.). 
a)

Connector

C92-C1T

not

recovered

NA

C92-C1M (WTC 2, Col.131, Fl 95)

Type A

Seat Detail: 1411

Gusset plate intact;

Seat intact, $1 / 2$ bent down $1 / 2$ bent up, both bolt holes ripped through;

Gusset plate with damper unit intact, half bent up.

c)

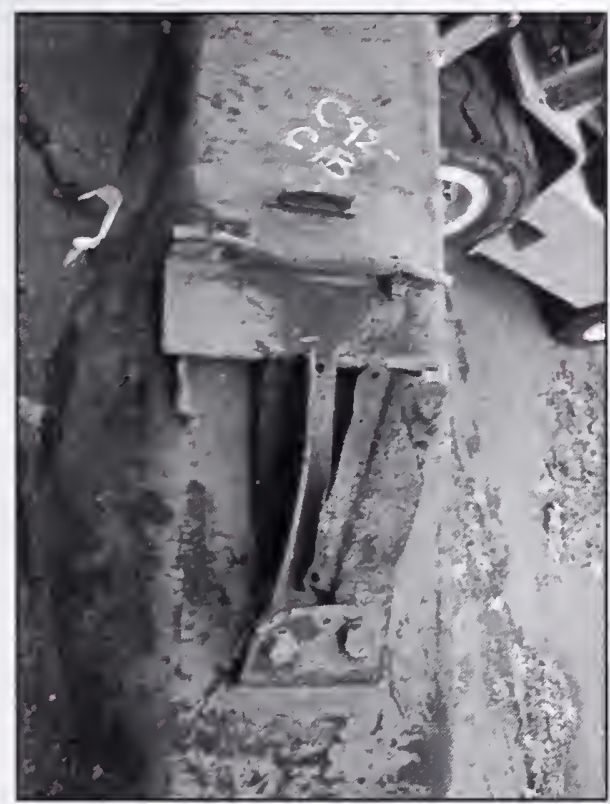

b)

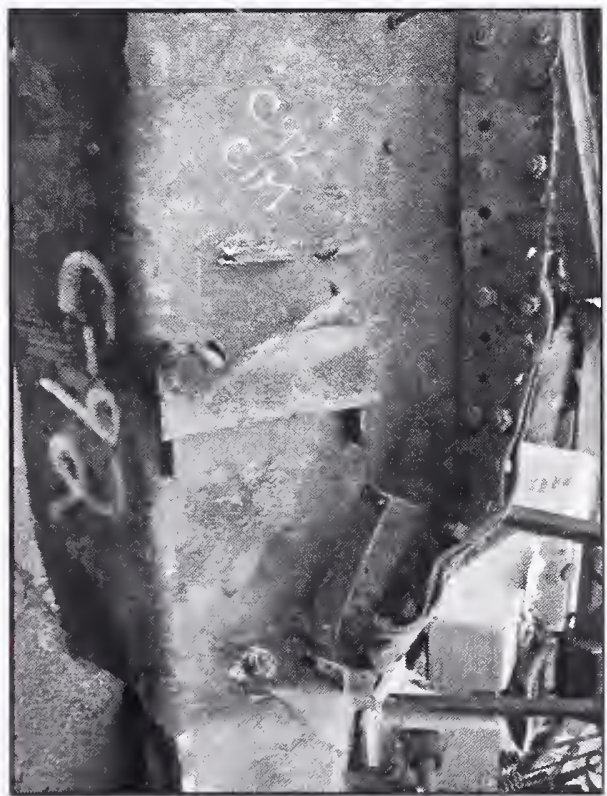

C92-C1B (WTC 2, Col.131, FI 94)

Type A

Seat Detail: 1411

Gusset plate intact;

Seat intact, bent down, both bolt holes ok, 1 with bolt;

Gusset plate with damper unit intact, bent up.

Figure B-14. Floor truss connectors found on recovered panel C-92 (B130: 93-96) from WTC 2. Shown are connectors found on column 131 at different floor levels. 
d)

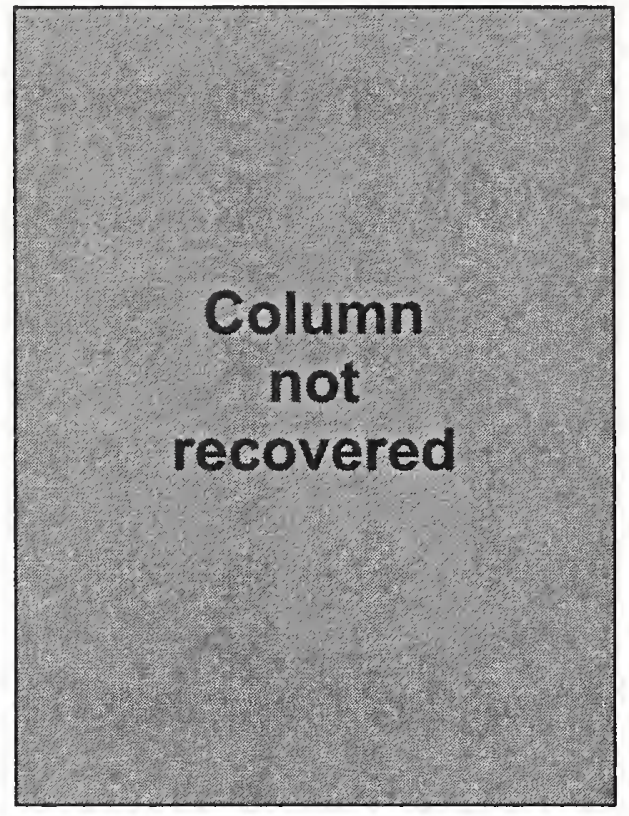

C92-C2M

NA

f)

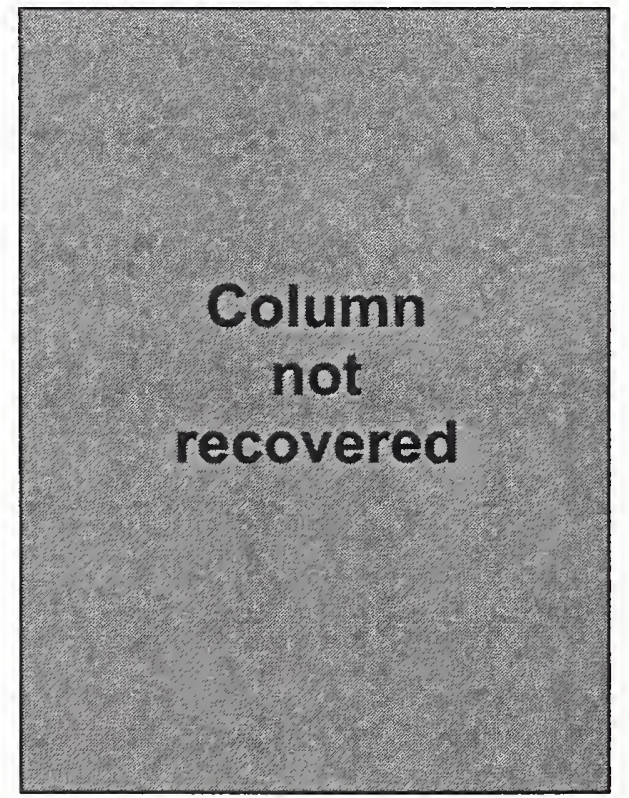

C92-C2T

NA

e)

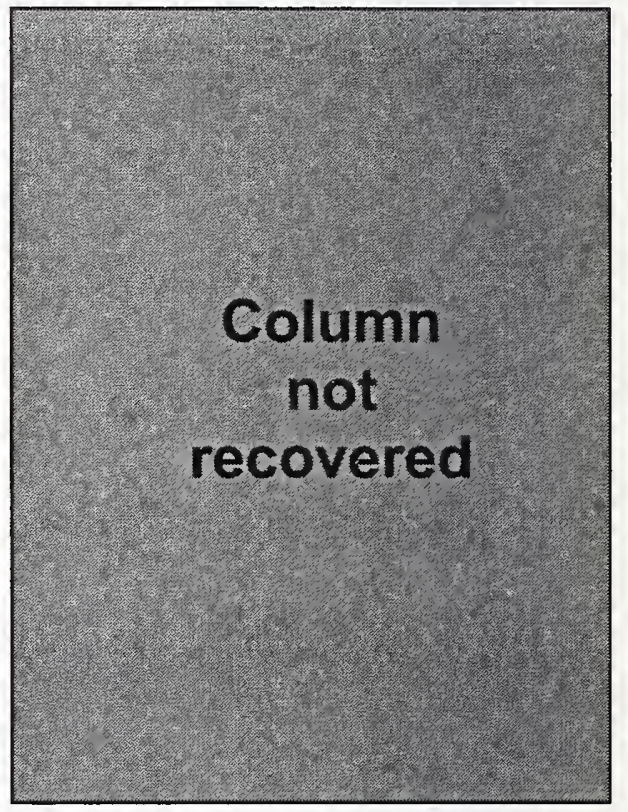

C92-C2B

NA

Figure B-14. Floor truss connectors found on recovered panel C-92 (B130: 93-96) from WTC 2. Column 130 was not recovered (cont.). 
g)

Column not recovered

\section{C92-C3T}

NA

h)

\section{Column not recovered}

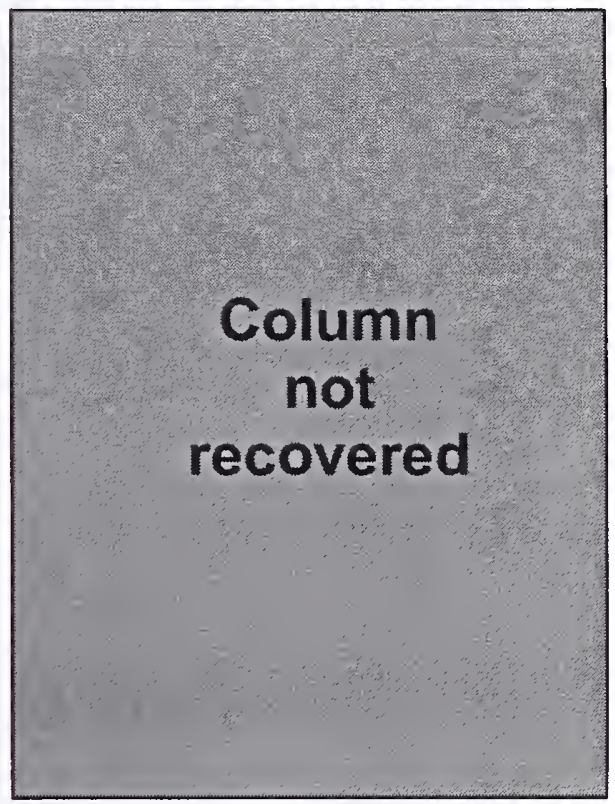

C92-C3B

NA

Figure B-14. Floor truss connectors found on recovered panel C-92 (B130: 93-96) from WTC 2. Column 129 was not recovered (cont.). 
a)

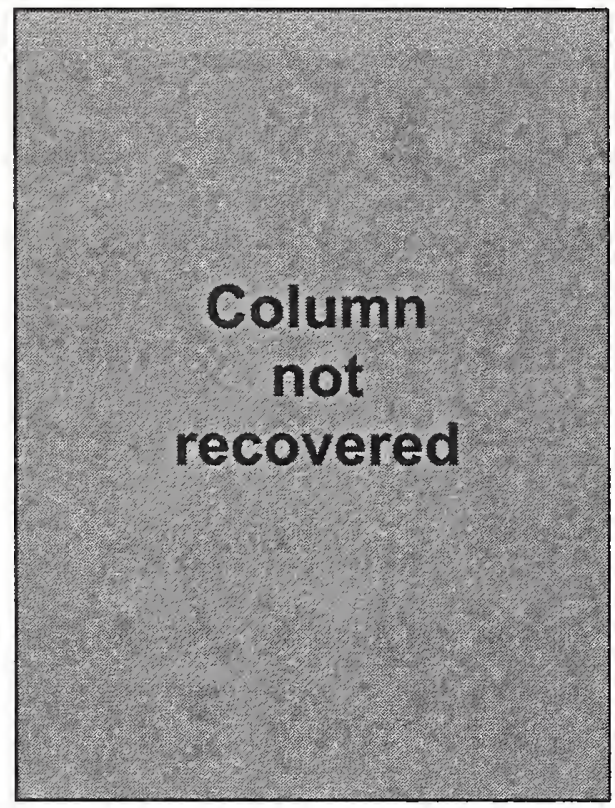

C93-C1M

NA c)

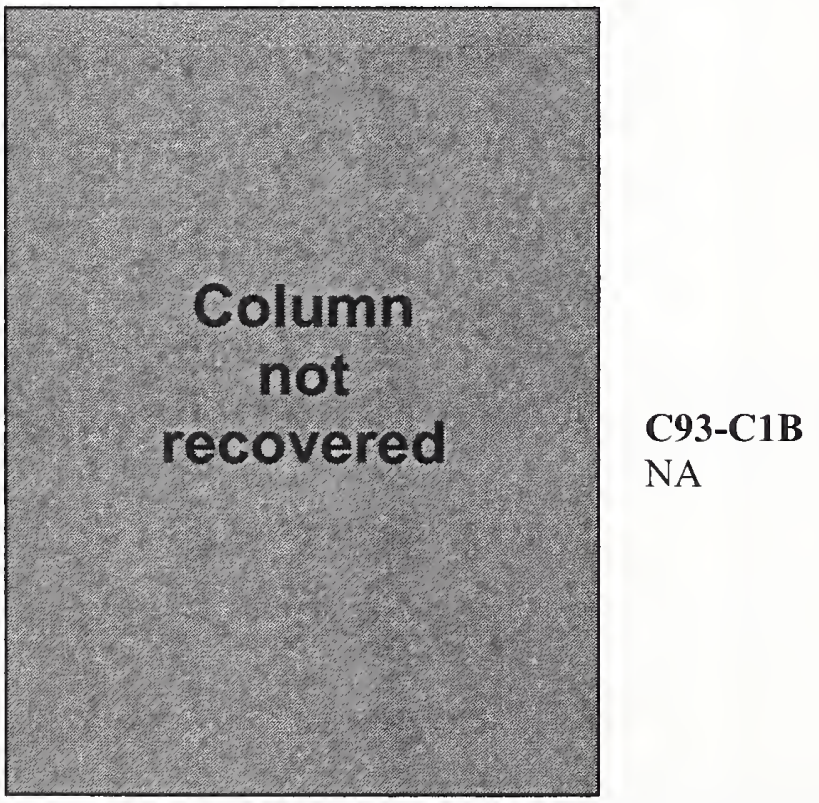

C93-C1T

NA b)

\section{Column not recovered}

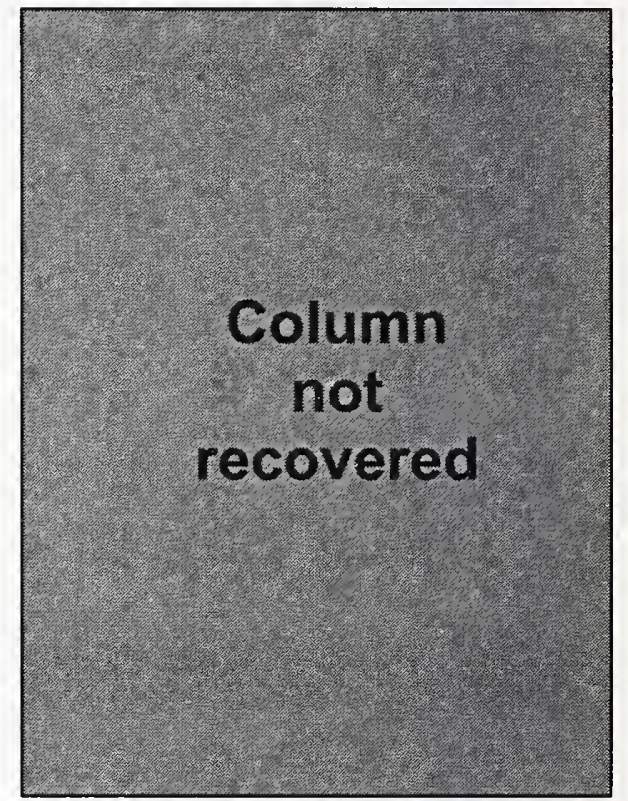

Figure B-15. Floor truss connectors found on recovered panel C-93 (A339: 99-102) from WTC 1. Column 340 was not recovered. 
d)

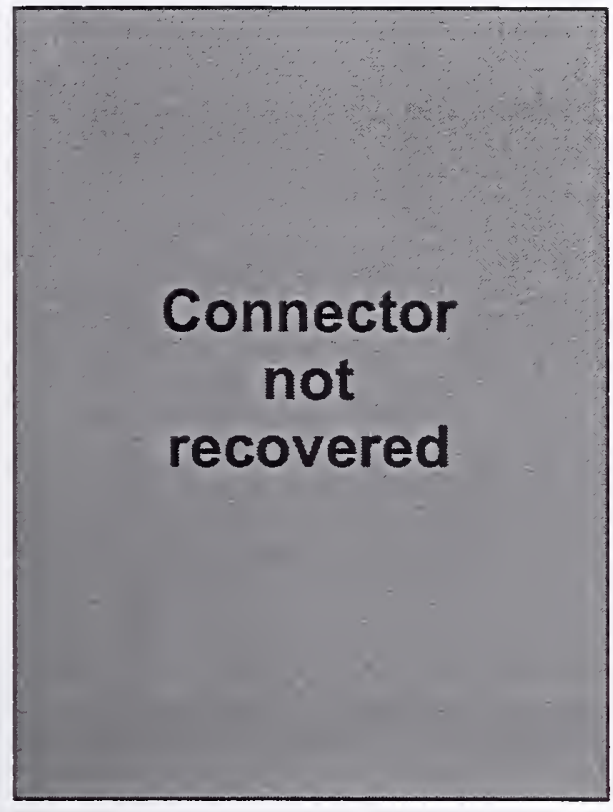

C93-C2M

NA

f)

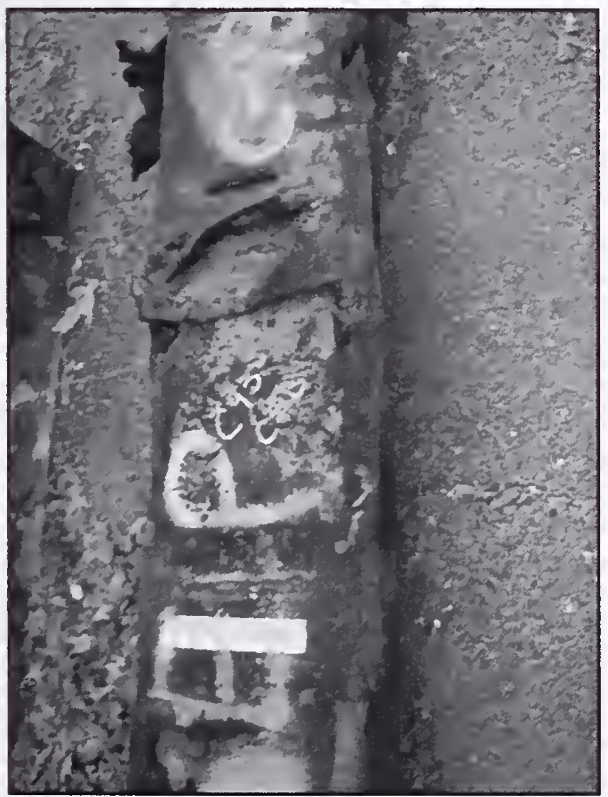

C93-C2T

NA

e)

Connector not recovered

\section{C93-C2B (WTC 1, Col.339, Fl 98)}

Type A

Seat Detail: 1411

Gusset plate ripped off at weld;

Seat intact, pushed in and bent down, both bolt holes ripped through;

Gusset plate for damper unit ripped off at weld.

Figure B-15. Floor truss connectors found on recovered panel C-93 (A339: 99-102) from WTC 1. Shown are connectors found on column 339 at different floor levels (cont.). 
g)

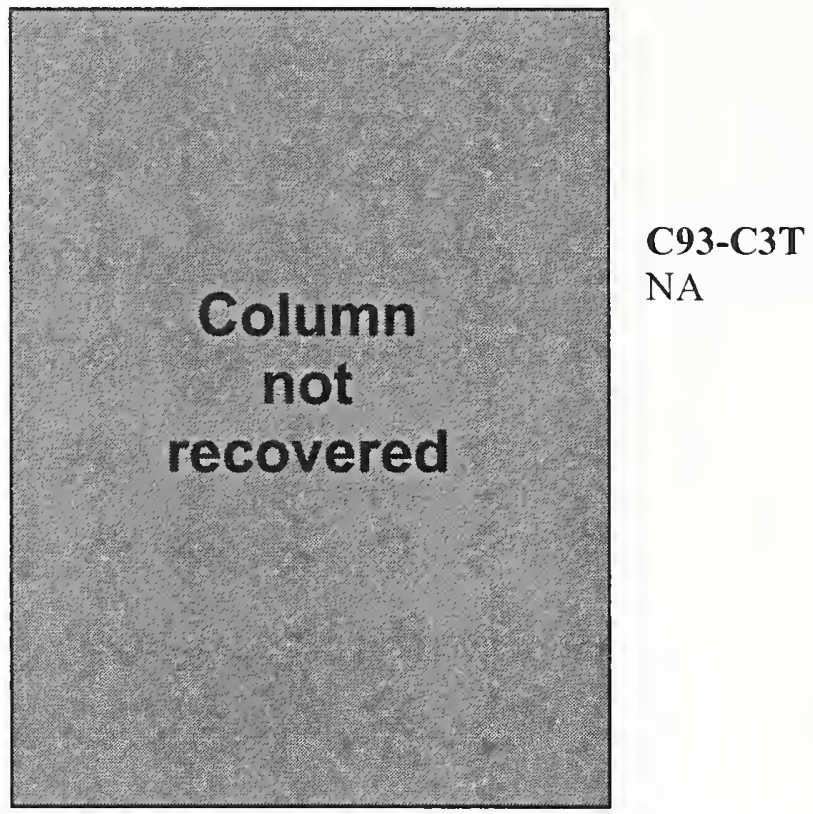

h)

C93-C3M

NA

i)

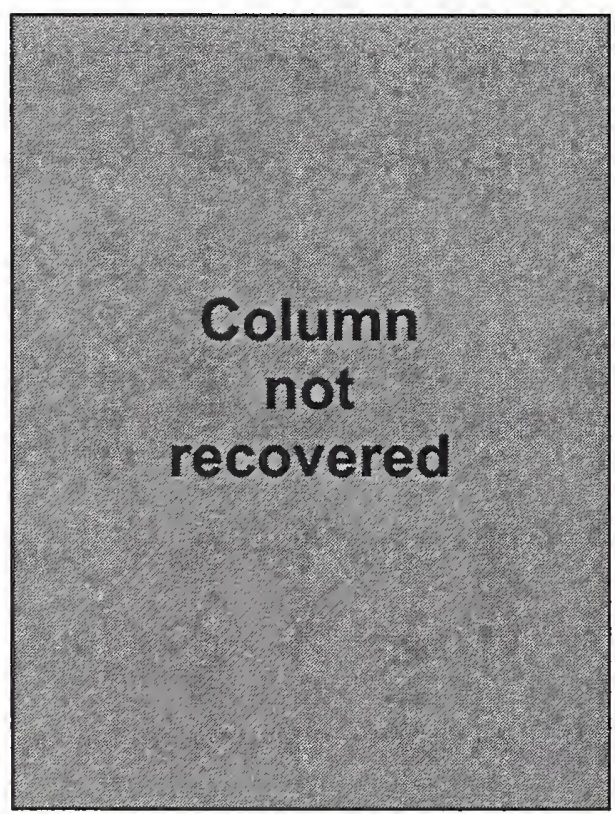

Column not recovered
C93-C3B

NA

Figure B-15. Floor truss connectors found on recovered panel C-93 (A339: 99-102) from WTC 1. Column 338 was not recovered (cont.). 
a)

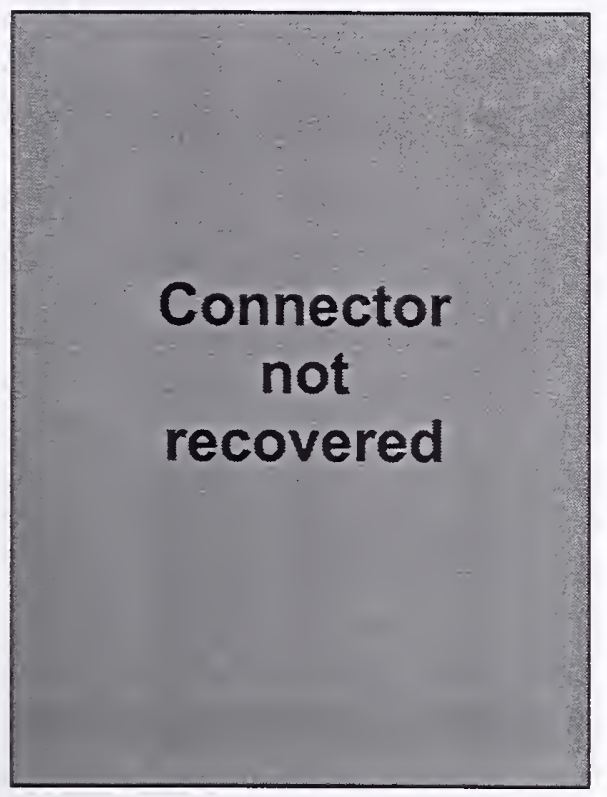

\section{C117-C1M}

NA

c)

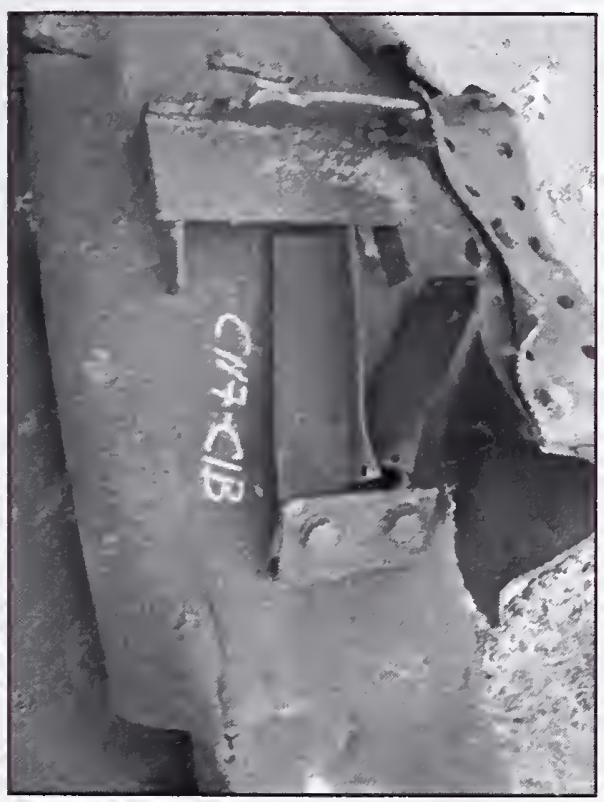

\section{C117-C1T}

NA

b)

Connector not

recovered

C117-C1B (Col.unknown, Fl 102)

Type A

Seat Detail:

Gusset plate ripped off at weld;

Seat intact, 1 bolt holes ripped out, other intact with bolt;

Gusset plate and damper unit attached, bent upwards.

Figure B-16. Floor truss connectors found on recovered panel $\mathrm{C}-117$ (building and column line unknown, floors 101-104). Connectors are shown on the unknown column at different floor levels. 
d)

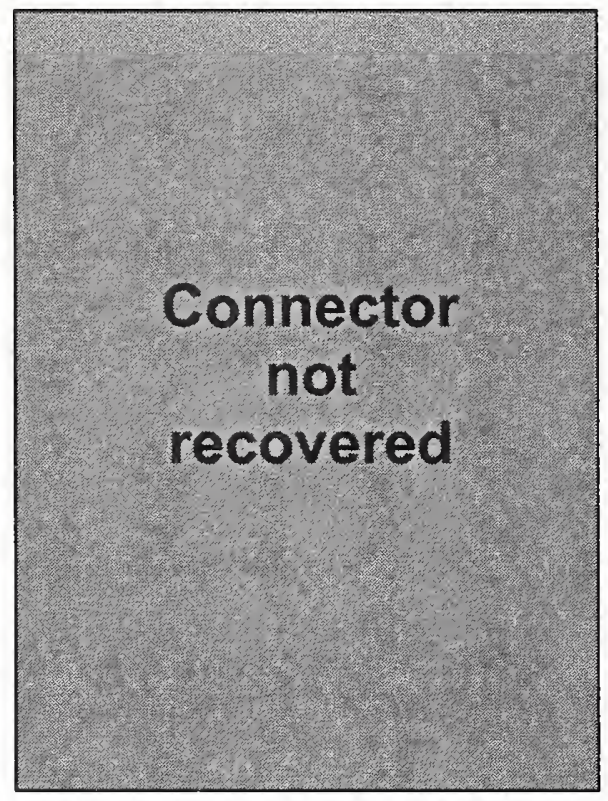

C117-C2M

NA

\section{C117-C2T}

NA

e)

Connector not recovered f)

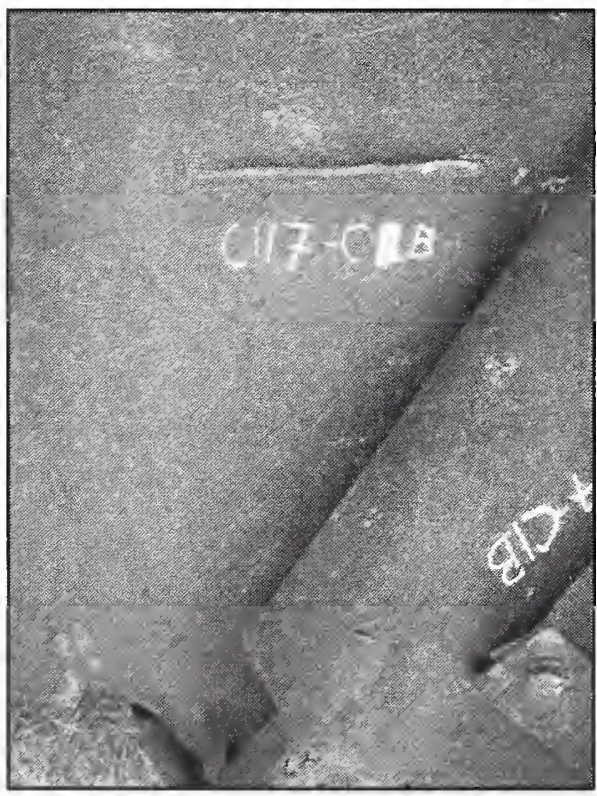

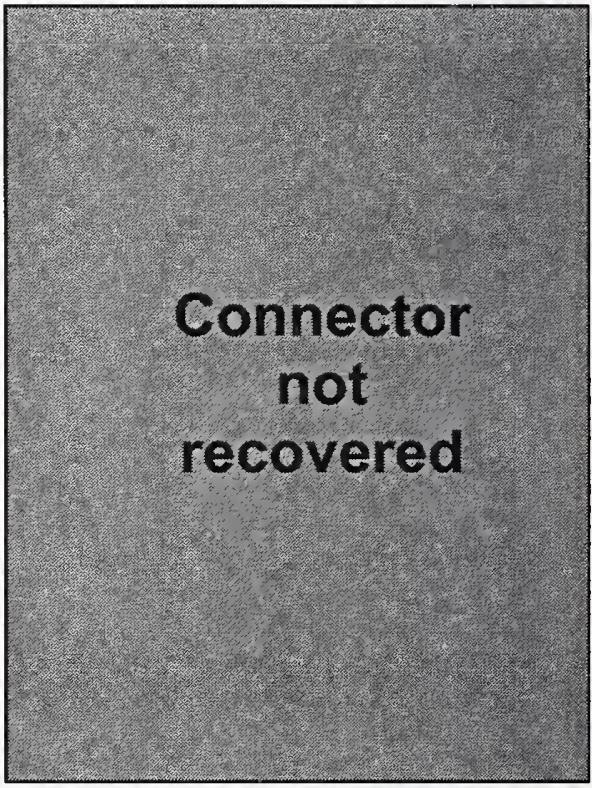

C117-C2B (Col.unknown, Fl 102)

Type C

Seat Detail:

Portion of gusset plate remains.

Figure B-16. Floor truss connectors found on recovered panel C-117 (building and column line unknown, floors 101-104). Connectors are shown on the unknown column at different floor levels (cont.). 
g)

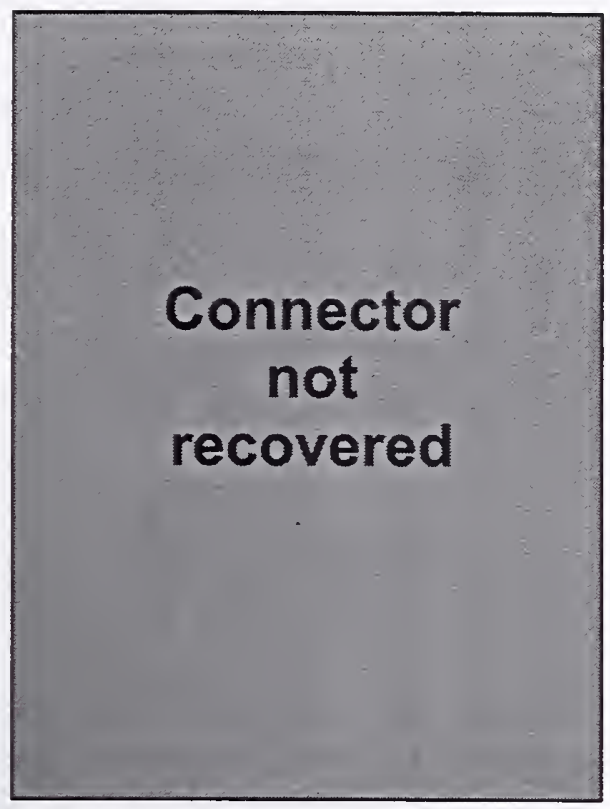

C117-C3M

NA

i)

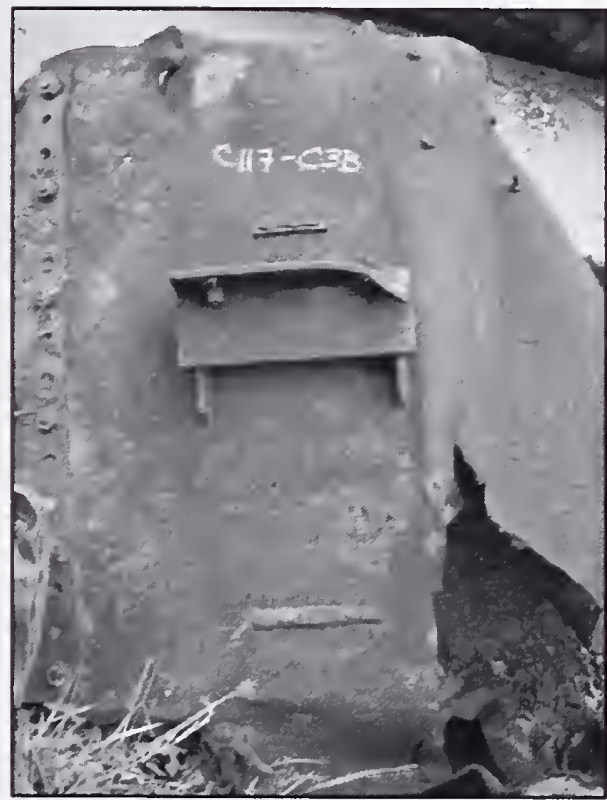

C117-C3T

NA h)

Connector not recovered

\section{C117-C3B (Col.unknown, Fl 102)}

Type A

Seat Detail:

Gusset plate ripped off at weld;

Seat intact, half bent down, both bolt holes intact with bolts;

Gusset plate for damper unit ripped off at weld.

Figure B-16. Floor truss connectors found on recovered panel C-117 (building and column line unknown, floors 101-104). Connectors are shown on the unknown column at different floor levels (cont.). 
a)

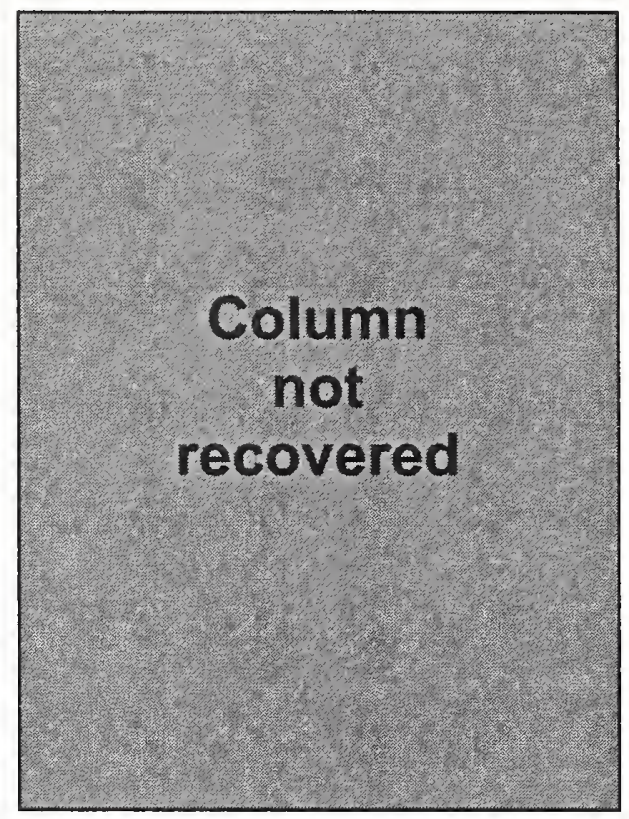

CC-C1M

NA

c)

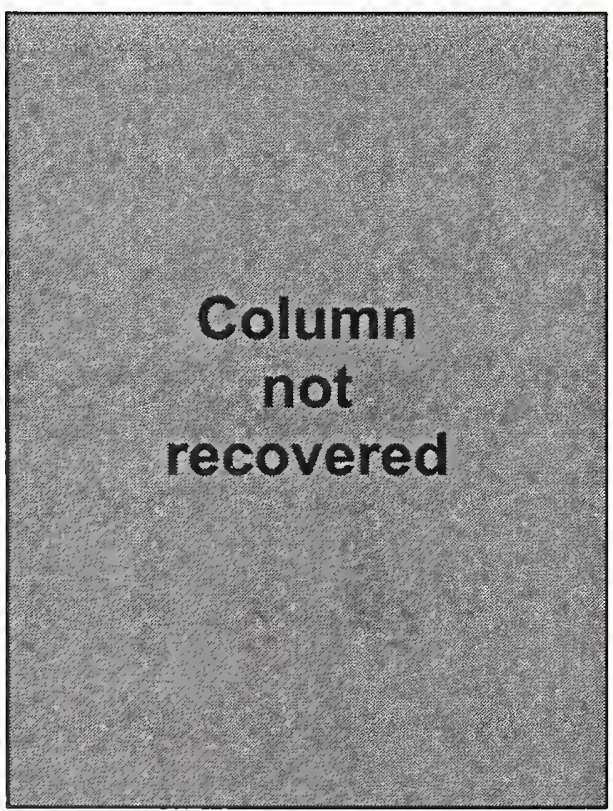

Figure B-17. Floor truss connectors found on recovered panel CC (A124: 70-73) from WTC 1. Column 125 was not recovered.
CC-C1T

NA

b)

\section{Column not recovered}

CC-C1B

NA 
d)

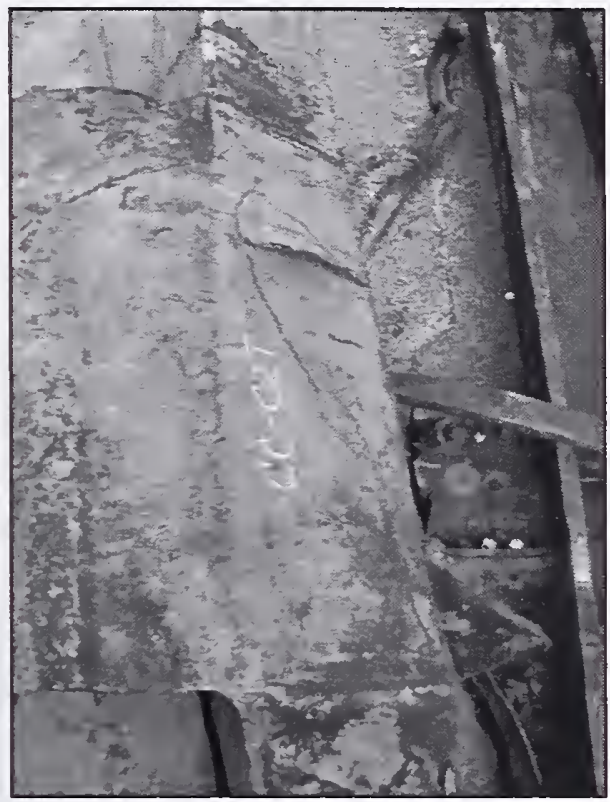

CC-C2T (WTC 1, Col.124, Fl 73)

Type C

Seat Detail: 5110

Portion of large gusset plate remains.

CC-C2M (WTC 1, Col.124, FI 72)

Type C

Seat Detail: 5110

Large gusset plate ripped off at weld.

e)

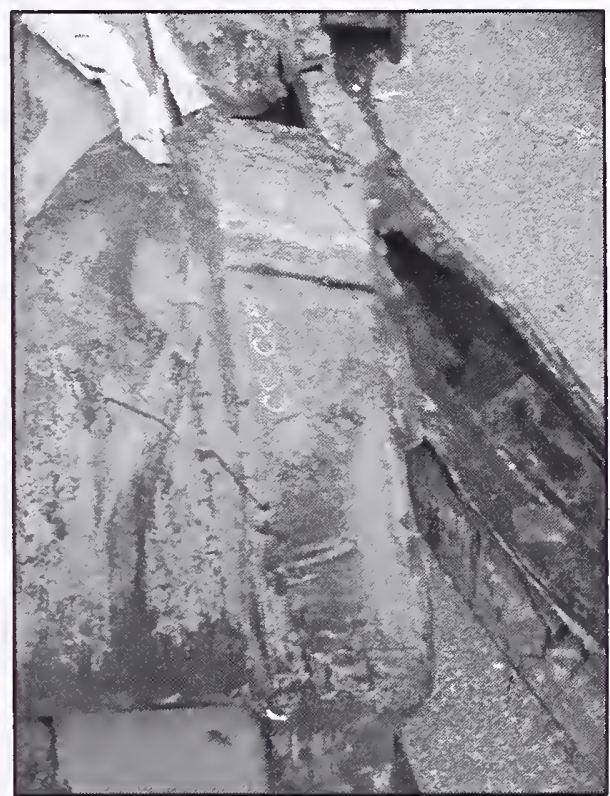

f)

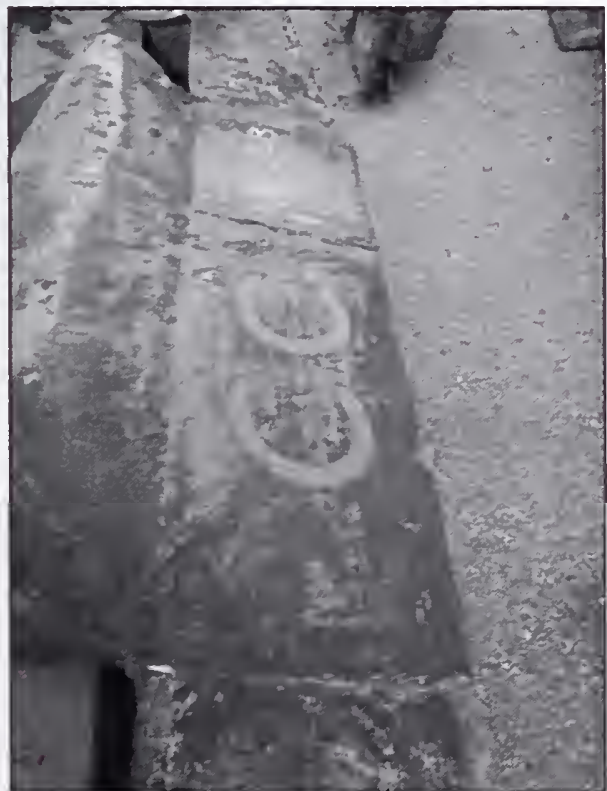

CC-C2B (WTC 1, Col.124, FI 71)

Type $\mathrm{C}$

Seat Detail: 5110

Large gusset plate ripped off at weld.

Figure B-17. Floor truss connectors found on recovered panel CC (A124: 70-73) from WTC 1. Shown are connectors found on column 124 at different floor levels (cont.). 
g)

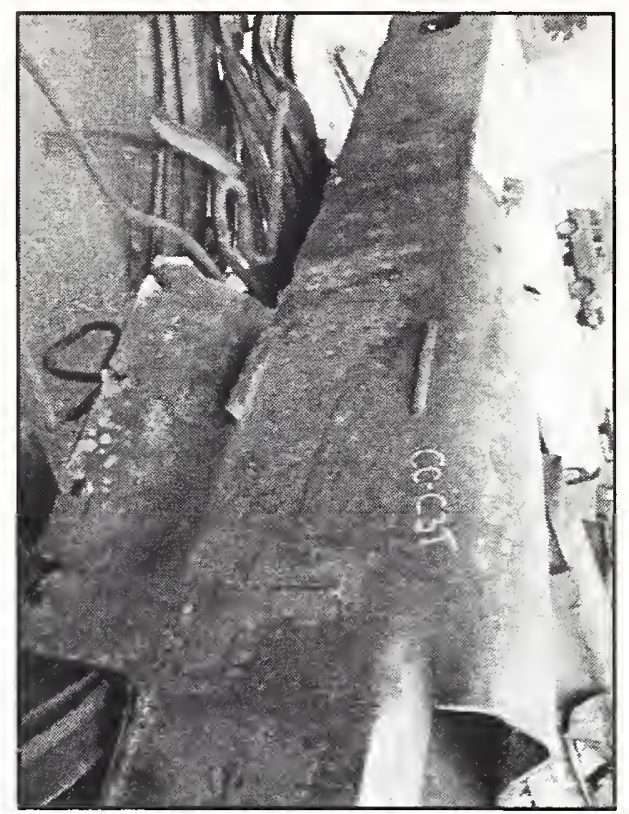

CC-C3M (WTC 1, Col.123, Fl 72)

Type A

Seat Detail: 1411

Gusset plate ripped off at weld;

Standoff plates ripped off at welds;

Gusset plate for damper unit ripped off at weld.

i)

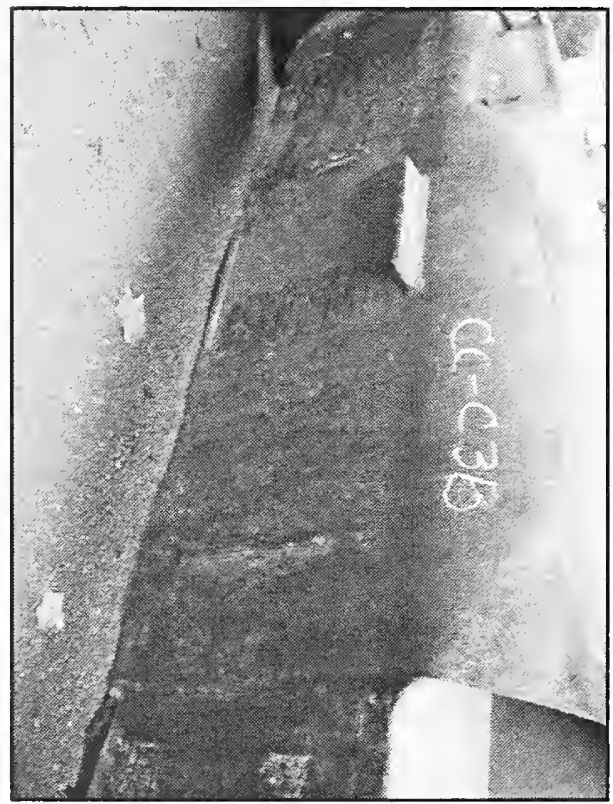

Type A

Seat Detail: 1411 welds;
CC-C3T (WTC 1, Col.123, Fl 73)

Type A

Seat Detail: 1411

Gusset plate ripped off at weld;

Only 1 standoff plate remains, rest ripped off at welds;

Gusset plate for damper unit ripped off at weld. h)

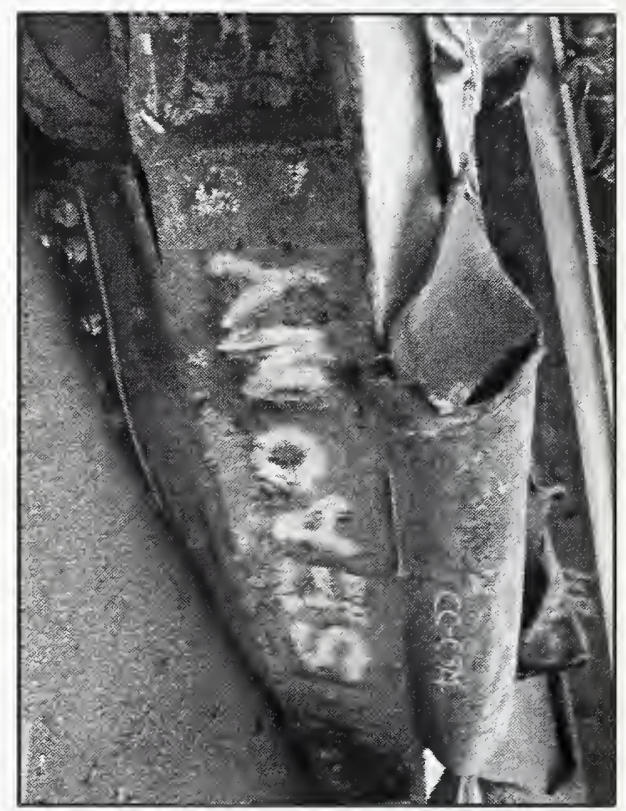

CC-C3B (WTC 1, Col.123, FI 71)

Gusset plate ripped off at weld;

Only 1 standoff plate remains, rest ripped off at

Gusset plate for damper unit ripped off at weld.

Figure B-17. Floor truss connectors found on recovered panel CC (A124: 70-73) from WTC 1. Shown are connectors found on column 123 at different floor levels (cont.). 
a)

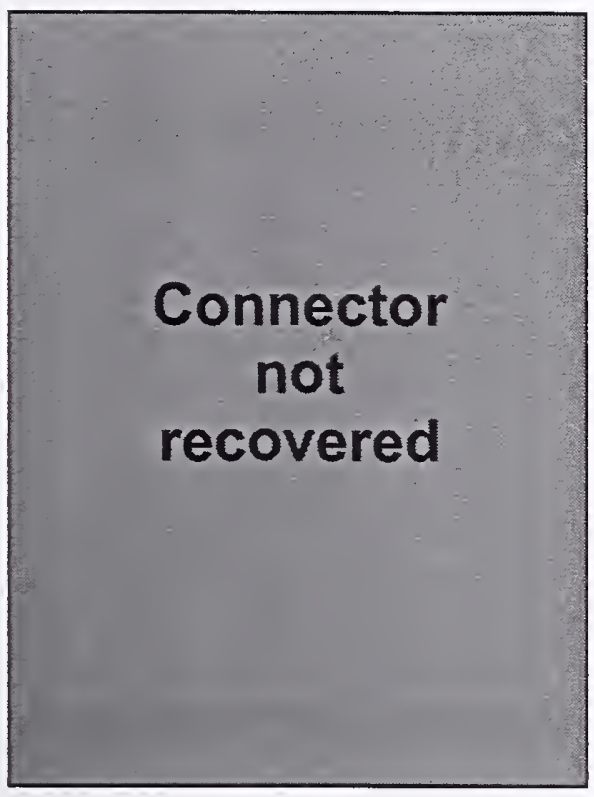

K1-C1M

NA
K1-C1T

NA

b) c)

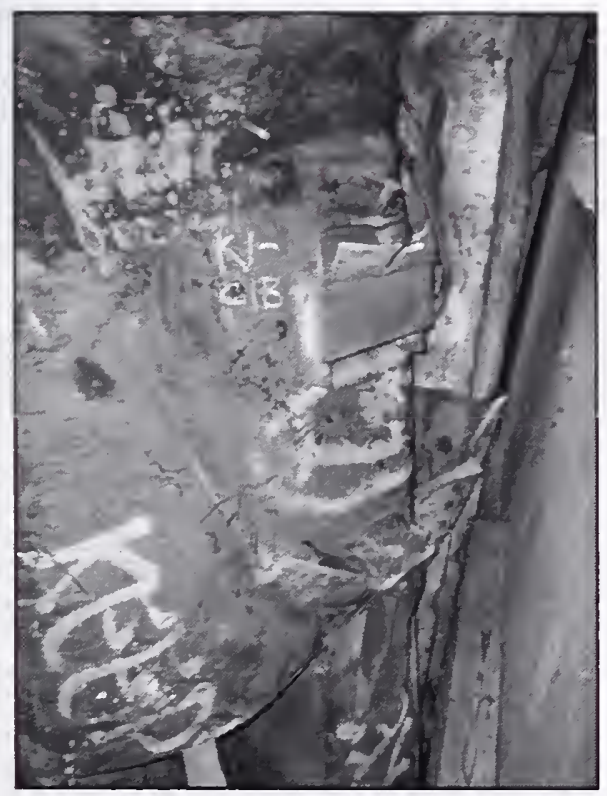

Connector not recovered

)

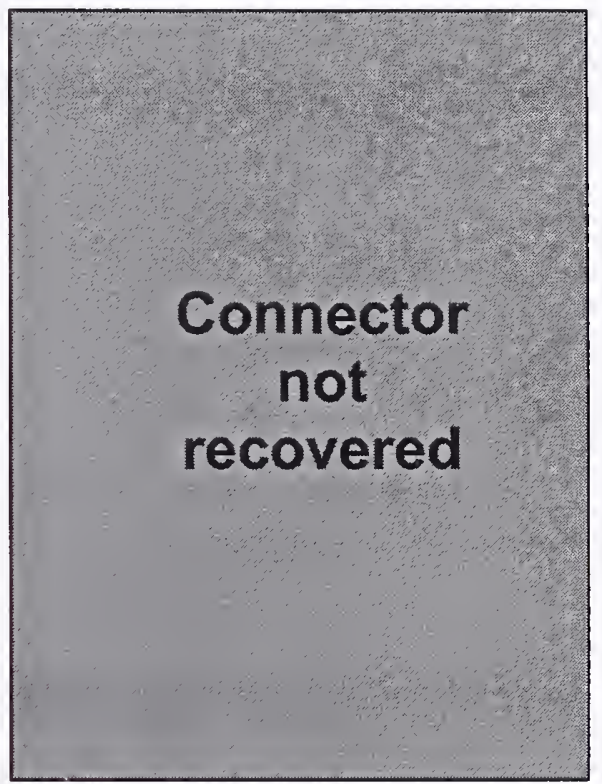

K1-C1B (WTC 1, Col.210. Fl 98)

Type A

Seat Detail: 1212

Gusset plate with shear bolt intact;

Seat intact, bent upwards, both bolt holes ripped through;

Gusset plate with damper unit intact, bent downwards.

Figure B-18. Floor truss connectors found on recovered panel K-1 (A209: 97-100) from WTC 1. Shown are connectors found on column 210 at different floor levels. 
d)

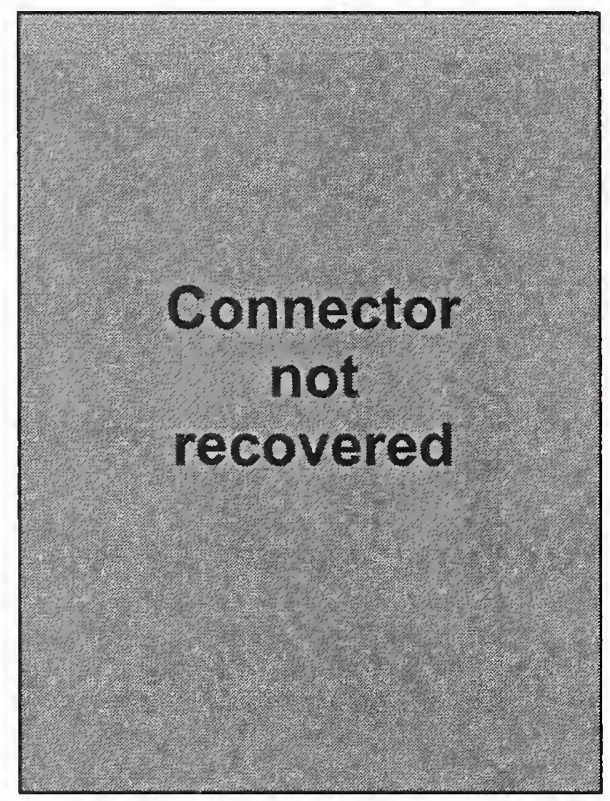

K1-C2M

NA
K1-C2T

NA e)

Connector not recovered

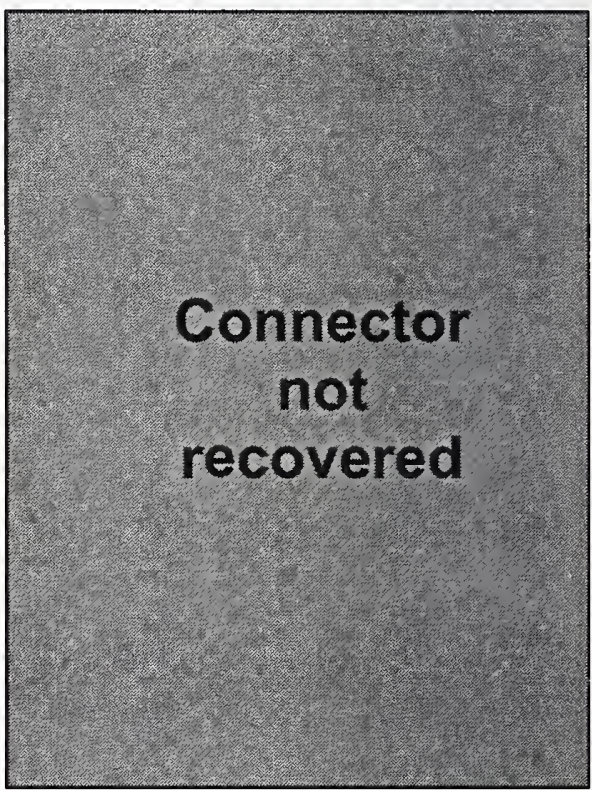

K1-C2B (WTC 1, Col.209, Fl 98)

Type D

Seat Detail: 5210

Both diagonal bracing straps ripped off at weld.

Figure B-18. Floor truss connectors found on recovered panel K-1 (A209: 97-100) from WTC 1. Shown are connectors found on column 209 at different floor levels (cont.). 
g)

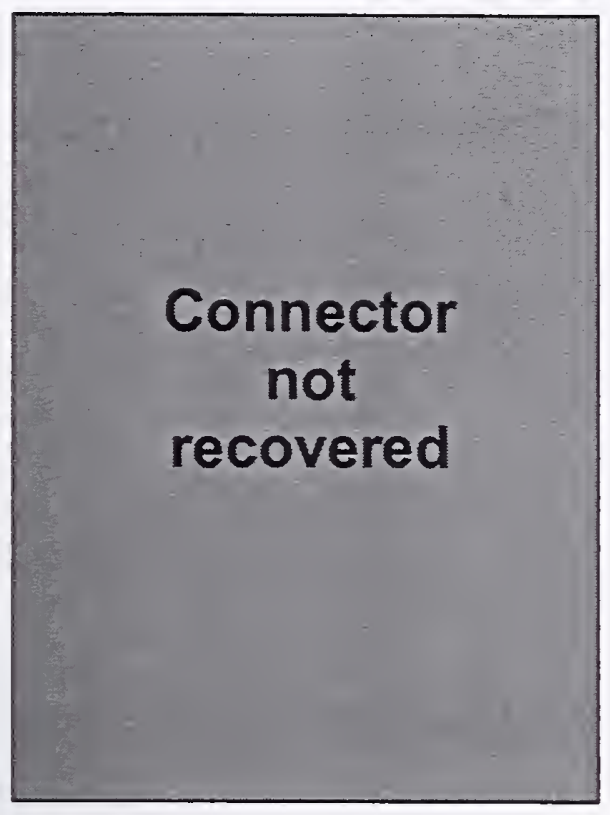

K1-C3M

NA

i)

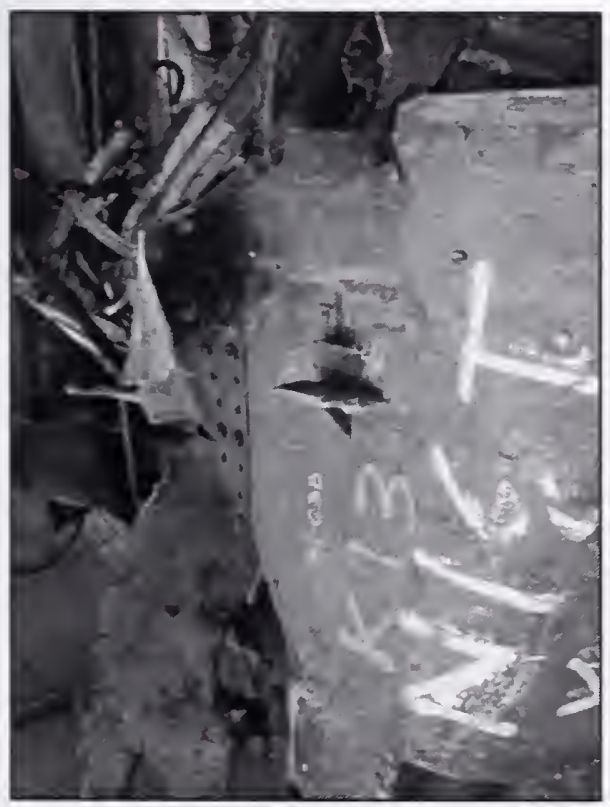

K1-C3T

NA h)

\section{Connector not recovered}

K1-C3B (WTC 1, Col.208, Fl 98)

Type B

Seat Detail: 5010

Gusset plate remains with shear bolt;

Small seat intact, 1 bolt hole intact, other is covered by a welded plate;

No evidence of gusset plate for damper unit.

Figure B-18. Floor truss connectors found on recovered panel K-1 (A209: 97-100) from WTC 1. Shown are connectors found on column 208 at different floor levels (cont.). 
a)

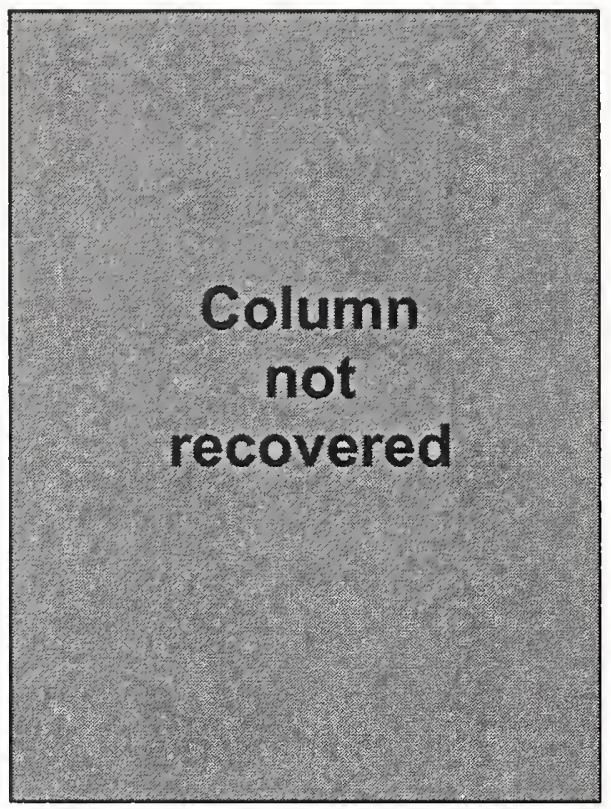

K2-C1T

NA

K2-C1M

NA

\section{Column not recovered.}

c)

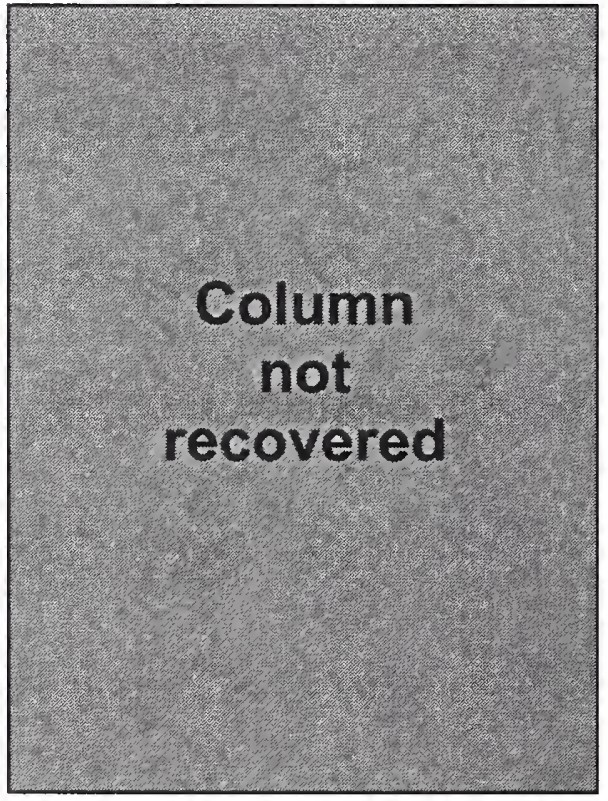

K2-C1B

NA

Figure B-19. Floor truss connectors found on recovered panel K-2 (A236: 92-95) from WTC 1. Column 237 was not recovered. 
d)

Connector
not
recovered

\section{K2-C2T}

NA

K2-C2M (WTC 1, Col.236, F1 94)

Type C

Seat Detail: 5110

Gusset plate ripped off at weld.

f)

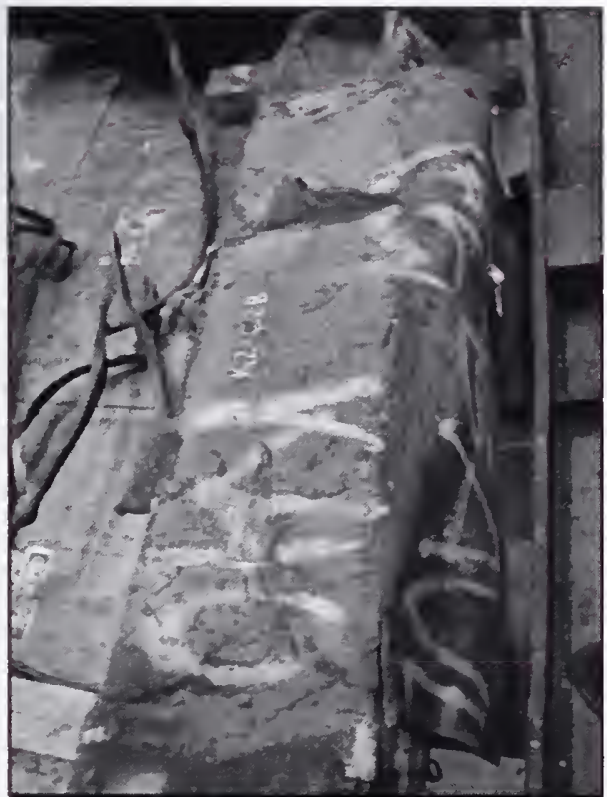

e)

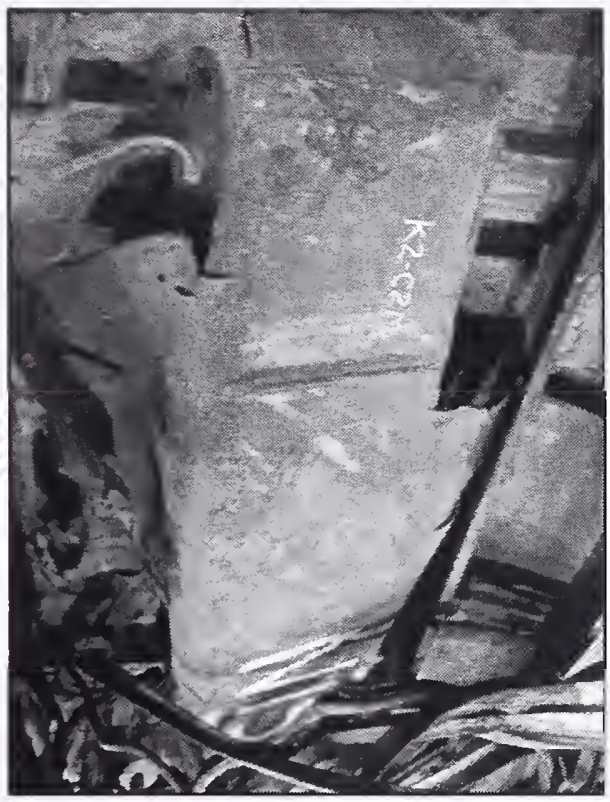

K2-C2B (WTC 1, Col.236, Fl 93)

Type C

Seat Detail: 5110

Portion of gusset plate remains.

Figure B-19. Floor truss connectors found on recovered panel K-2 (A236: 92-95) from WTC 1. Shown are connectors found on column 236 at different floor levels (cont.). 
g)

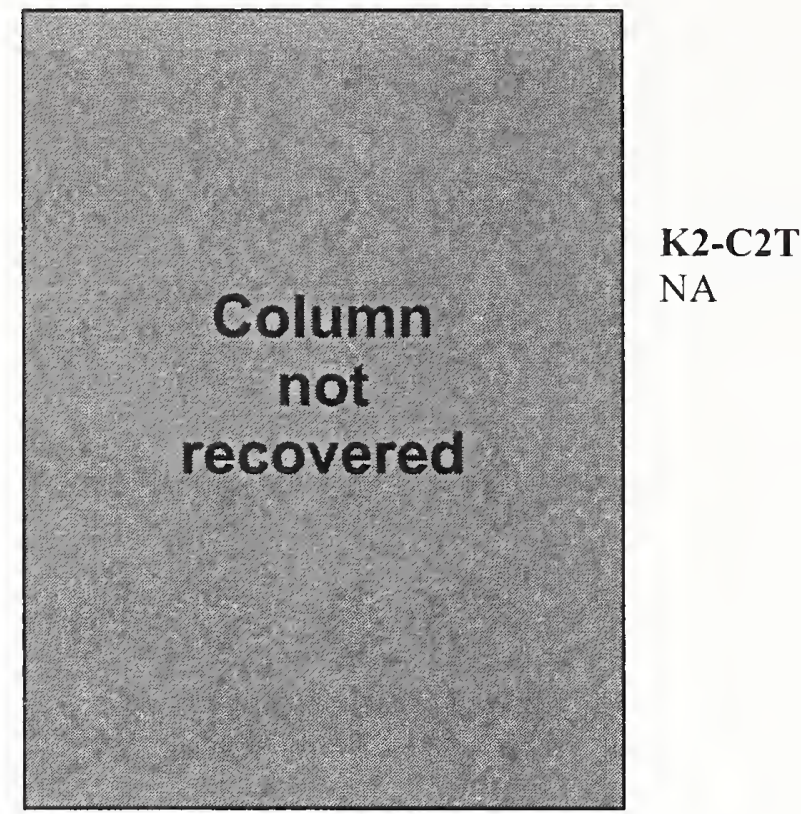

h)

K2-C3M

$\mathrm{NA}$

i)

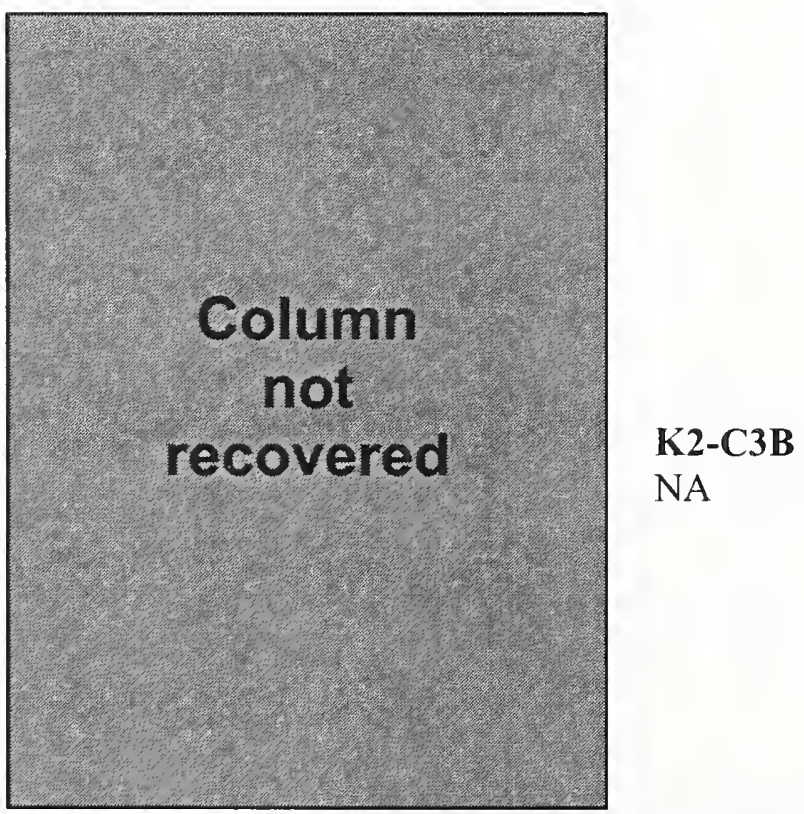

Figure B-19. Floor truss connectors found on recovered panel K-2 (A236: 92-95) from WTC 1. Column 235 was not recovered (cont.). 
a)

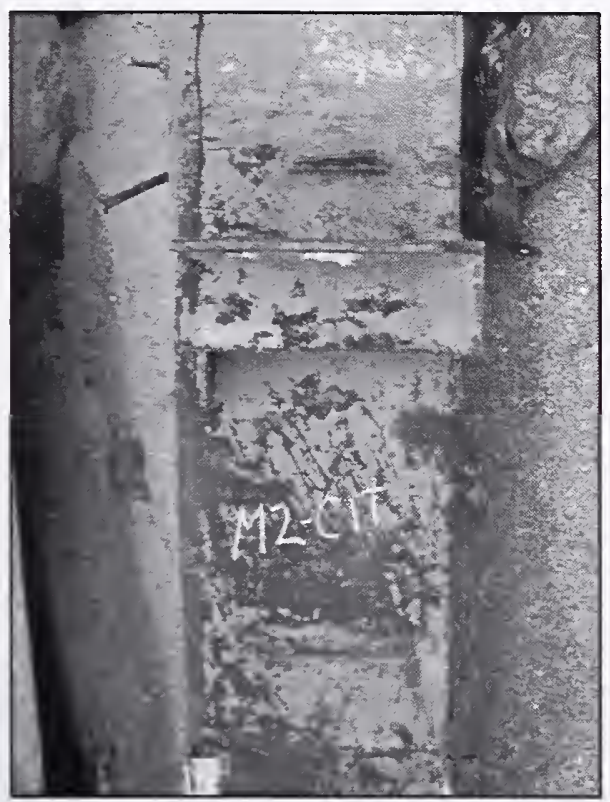

M2-C1M (WTC 1, Col.131, Fl 98)

Type A

Seat Detail: 1411

Gusset plate intact;

1 standoff plate remains, seat and other standoff plate ripped off at welds;

Gusset plate with damper unit intact, bent up.

c)

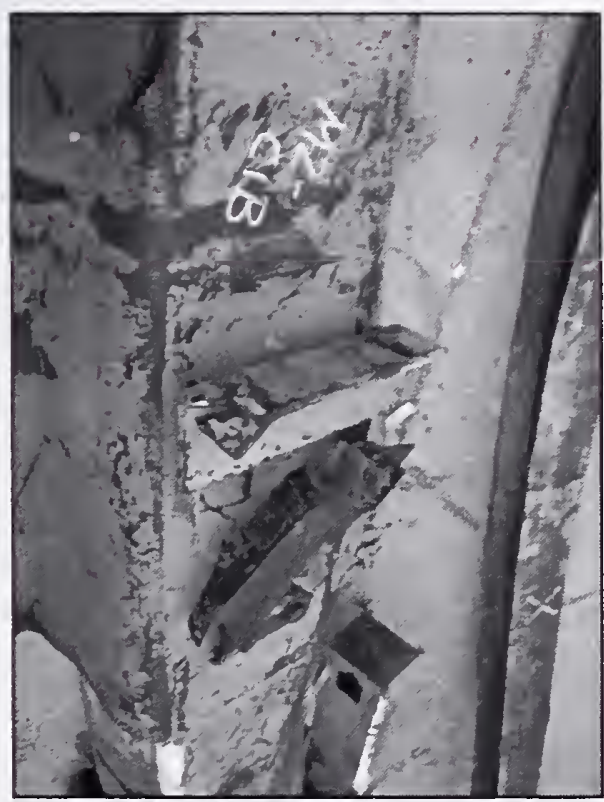

M2-C1'T (W'TC 1, Col.131, FI 99)

Type A

Seat Detail: 1411

Gusset plate remains;

Seat intact, 1 bolt hole remains intact, other ripped out;

Gusset plate for damper unit ripped off at weld. b)

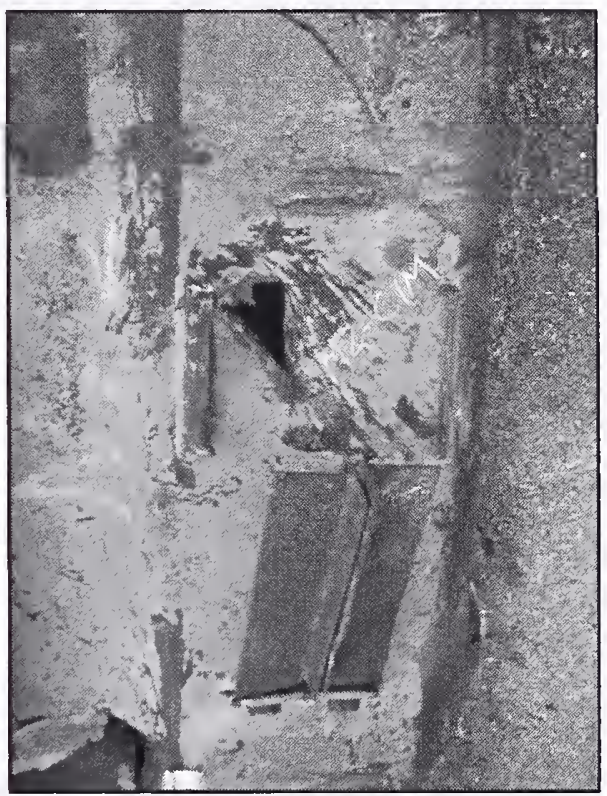

M2-C1B (WTC 1, Col.131, Fl 97)

Type A

Seat Detail: 1411

Gusset plate ripped off at weld

Seat intact, 1 corner bent downwards, 1 bolt hole intact with bolt, other ripped out;

Gusset plate with damper unit intact, bent up.

Figure B-20. Floor truss connectors found on recovered panel M-2 (A130: 96-99) from WTC 1. Shown are connectors found on column 131 at different floor leveis. 
d)

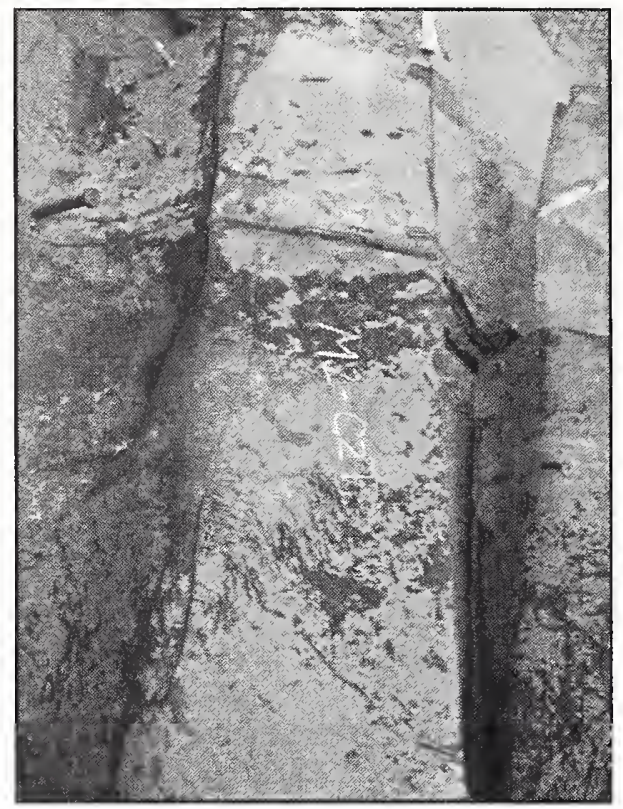

M2-C2M (WTC 1, Col.130, FI 98)

Type C

Seat Detail: 5210

Portion of gusset plate remains, bent up.

f)

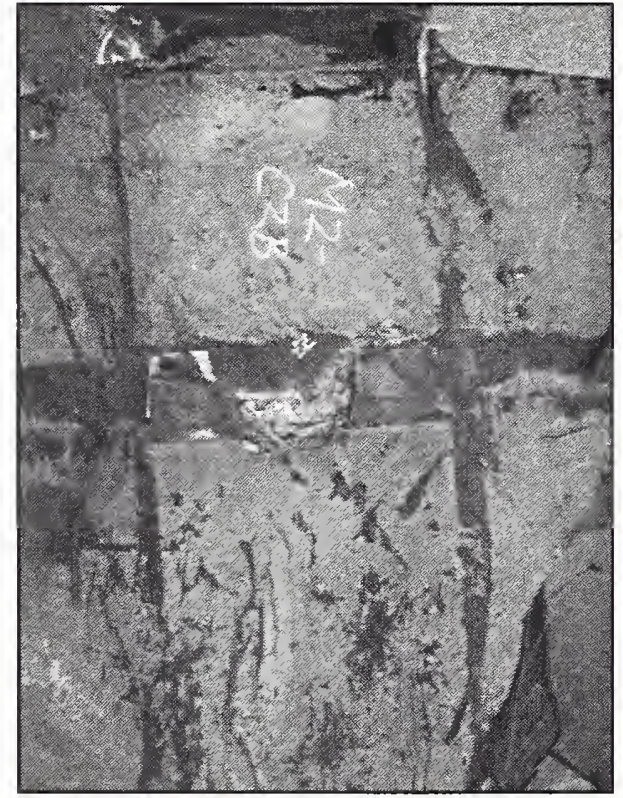

Type C
M2-C2T (WTC 1, Col.130, FI 99)

Type C

Seat Detail: 5210

Gusset plate ripped off at weld. e)

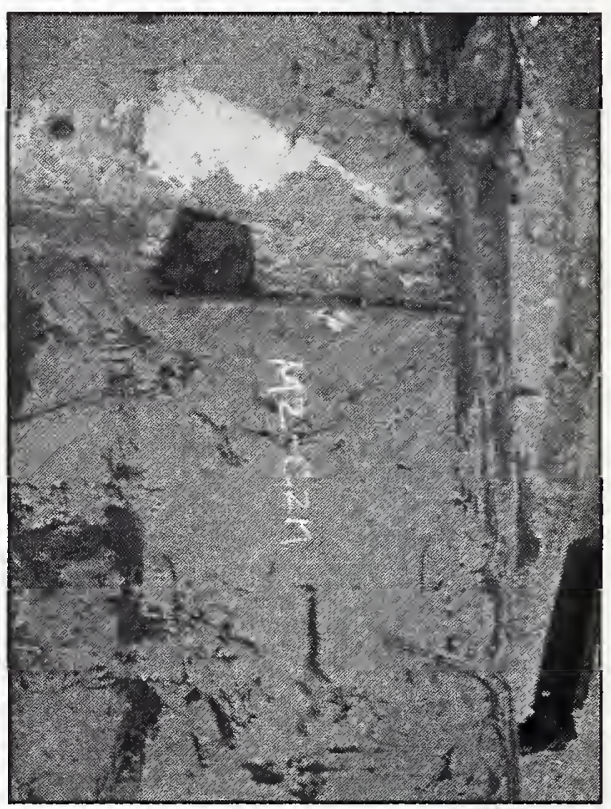

M2-C2B (WTC 1, Col.130, F1 97)

Seat Detail: 5210

Gusset plate remains, bent up.

Figure B-20. Floor truss connectors found on recovered panel M-2 (A130: 96-99) from WTC 1. Shown are connectors found on column 130 at different floor levels (cont.). 
g)

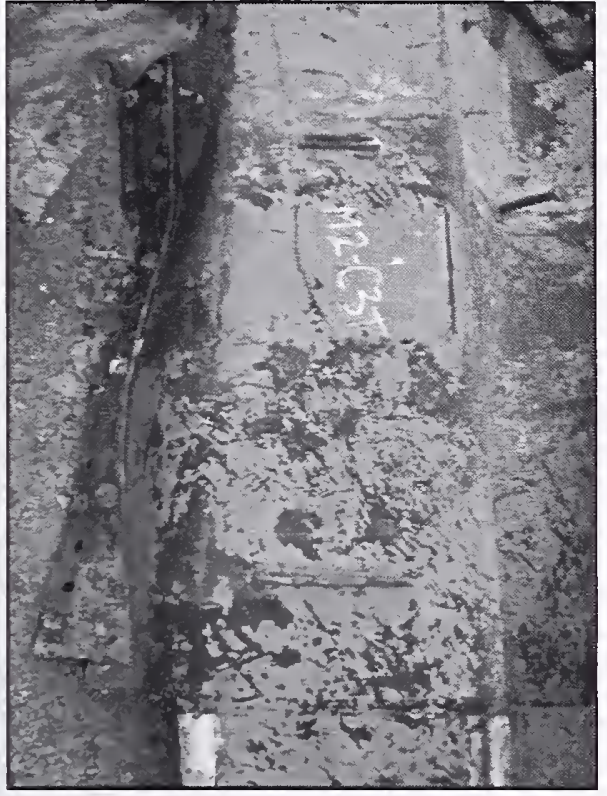

M2-C3M (WTC 1, Col.129, Fl 98)

Type A

Seat Detail: 1411

Gusset plate remains;

Seat intact, bent down, 1 bolt hole remains intact with bolt, other ripped out;

Gusset plate with damper unit intact, bent up.

i)

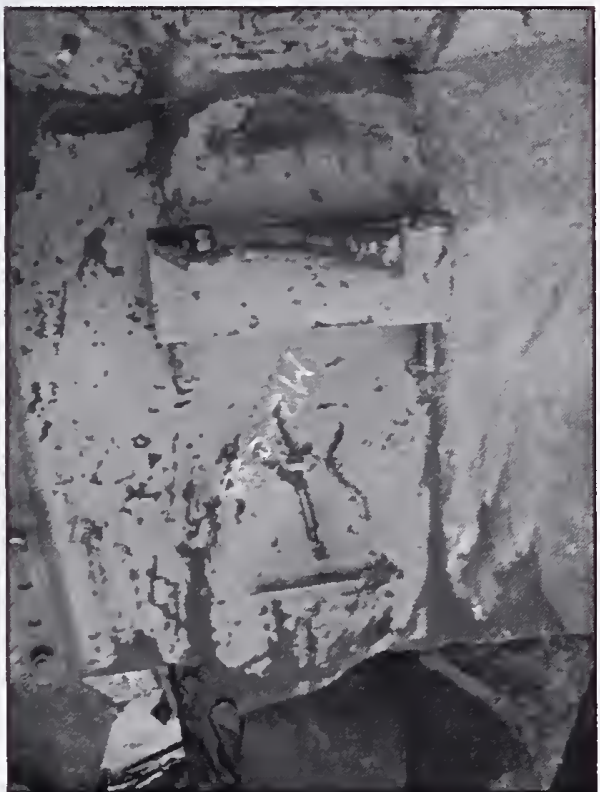

Type A

Seat Detail: 1411 other ripped out;
M2-C3T (WTC 1, Col.129, FI 99)

Type A

Seat Detail: 1411

Gusset plate ripped off at weld;

Standoff plates ripped off at welds;

Gusset plate for damper unit ripped off at weld. h)

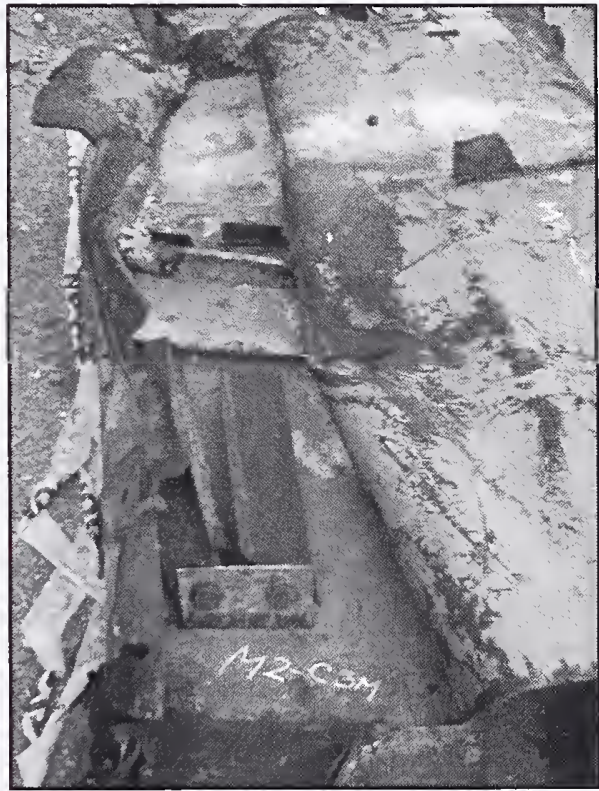

M2-C3B (WTC 1, Col.129, Fl 97)

Gusset plate ripped off at weld;

Seat intact, bent down, 1 bolt hole remains intact,

Gusset for damper unit ripped off at weld.

Figure B-20. Floor truss connectors found on recovered panel M-2 (A130: 96-99) from WTC 1. Shown are connectors found on column 129 at different floor levels (cont.). 
a)

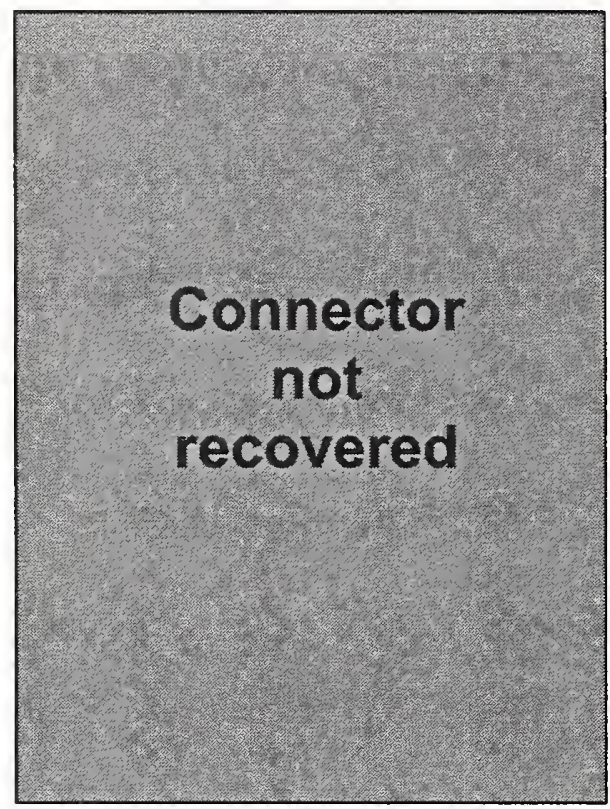

M10a-C1T

NA

M10a-C1M (WTC 2, Col.210, Fl 84)

Type A

Seat Detail: 1212

Gusset plate ripped completely out of spandrel; Only portions of standoff plates remain;

Gusset plate with damper unit intact, partially ripped at weld, bent downwards, portion of 1 damper remains.

c)

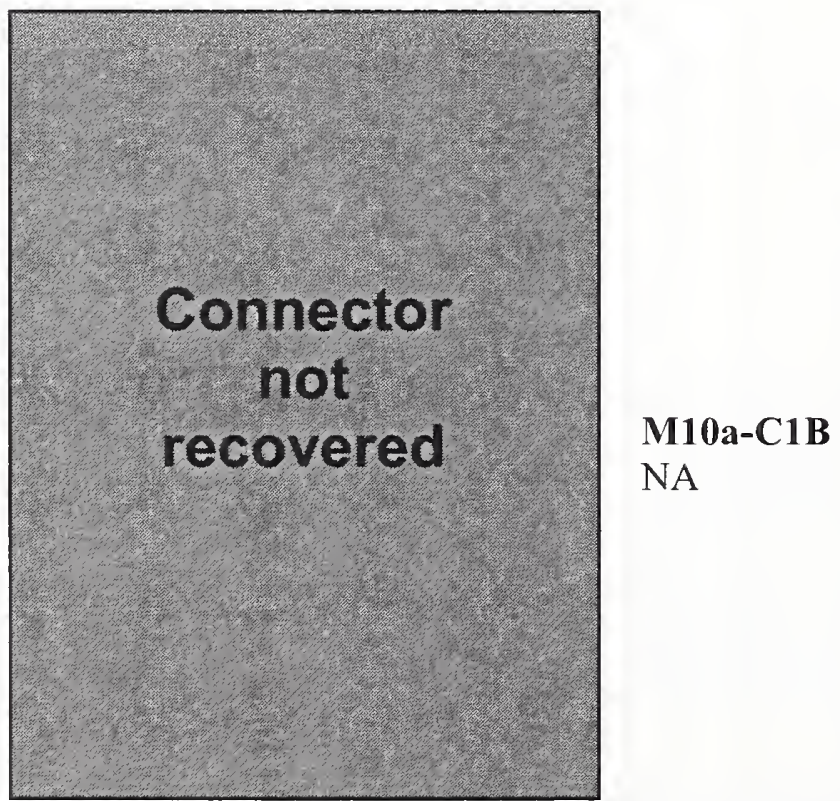

b)

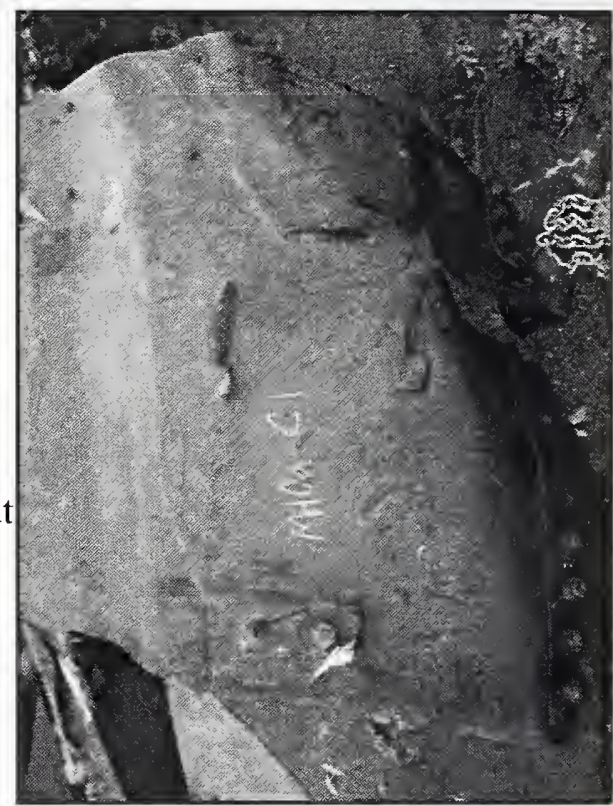

Figure B-21. Floor truss connectors found on recovered panel M-10a (B209: 82-85) from WTC 2. Shown are connectors found on column 210 at different floor levels. 
d)

Connector

M10a-C2T

not

recovered

NA

M10a-C2N (WTC 2, Col.209, Fl 84)

Type D

Seat Detail: 5210

Both diagonal bracing straps ripped off at weld.

e)

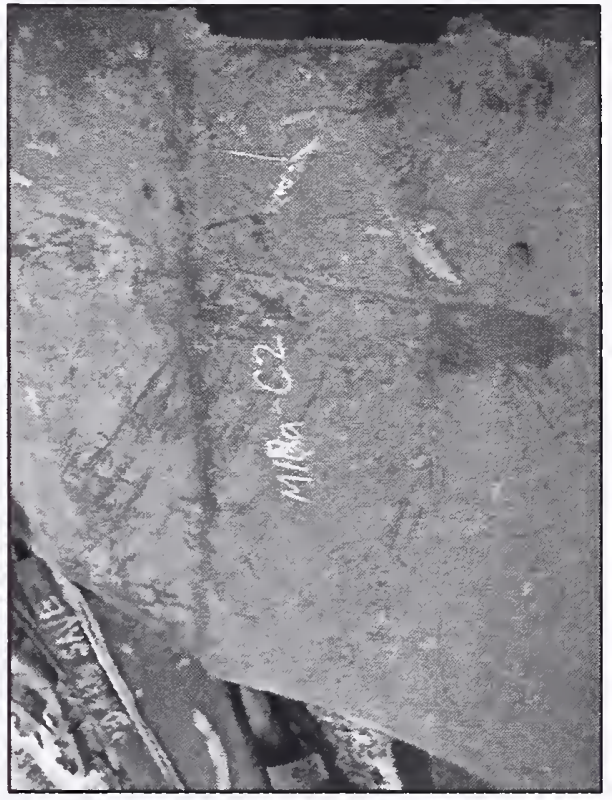

f)

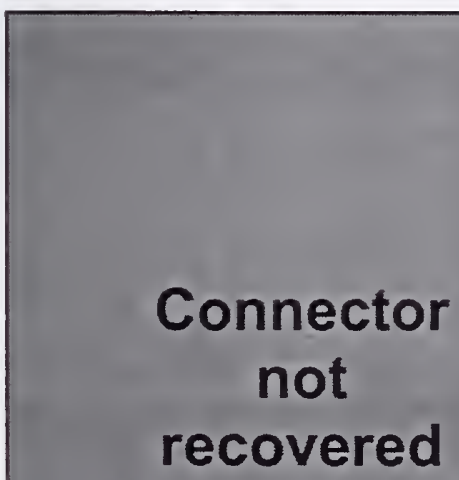

M10a-C2B

NA

Figure B-21. Floor truss connectors found on recovered panel M-10a (B209: 82-85) from WTC 2. Shown are connectors found on column 209 at different floor levels (cont.). 
g)

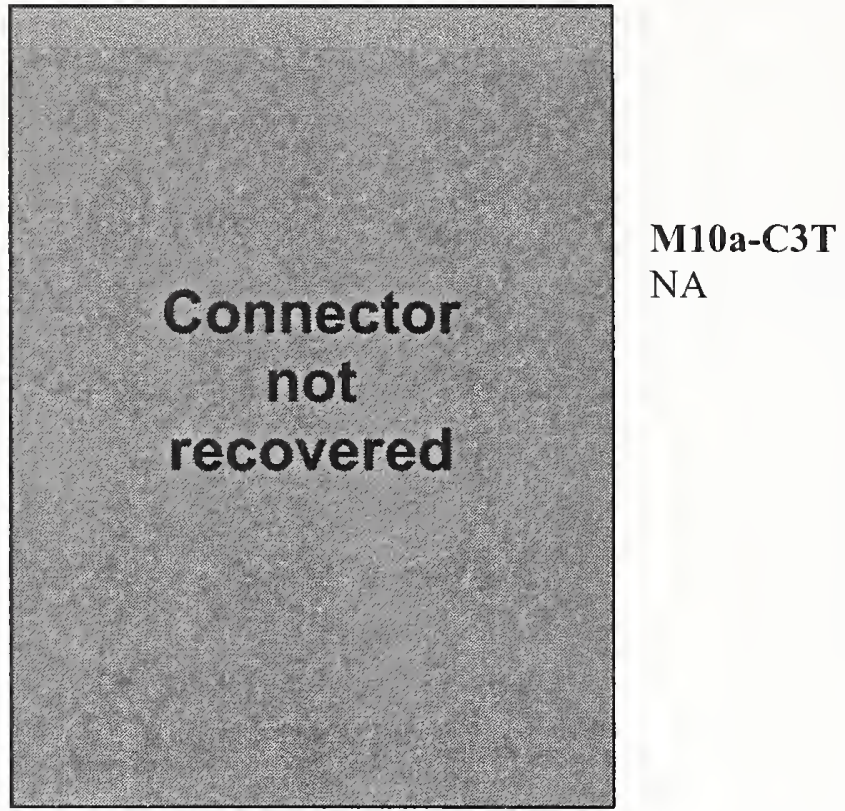

M10a-C3M (WTC 2, Col.208, Fl 84)

Type B

Seat Detail: 5010

Gusset plate ripped completely out of spandrel; Small seat remains, highly deformed, bent downwards, 1 bolt hole intact;

No evidence of gusset plate for damper unit.

i)

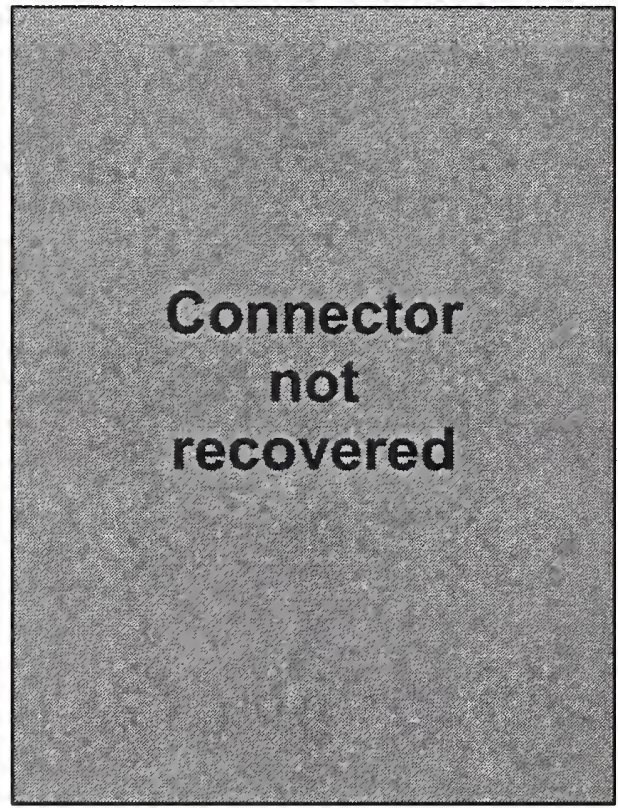

h)

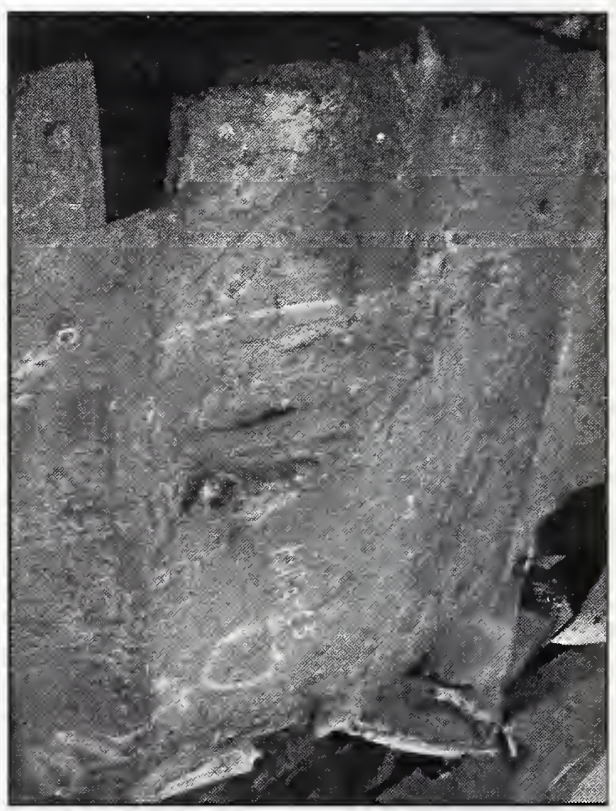

M10a-C3B

NA

Figure B-21. Floor truss connectors found on recovered panel M-10a (B209: 82-85) from WTC 2. Shown are connectors found on column 208 at different floor levels (cont.). 
a)

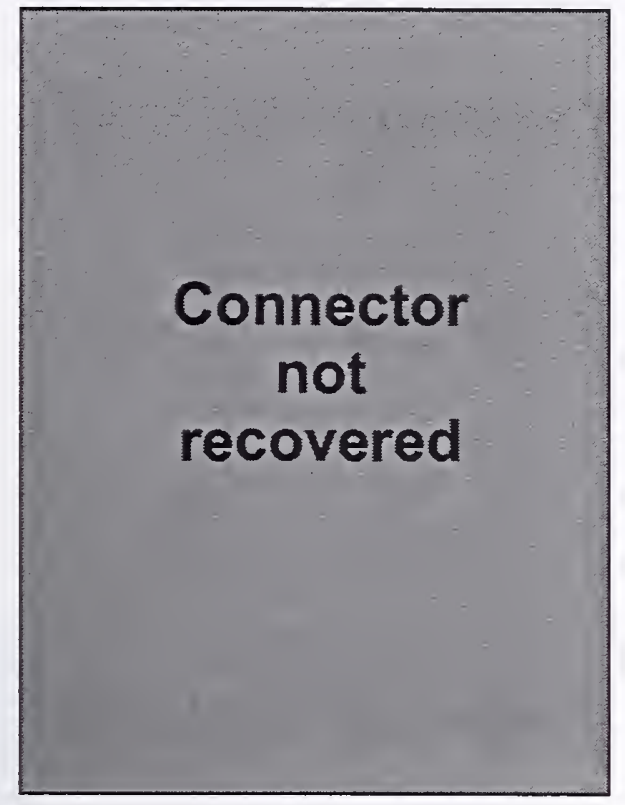

M10b-C1M

NA

c)

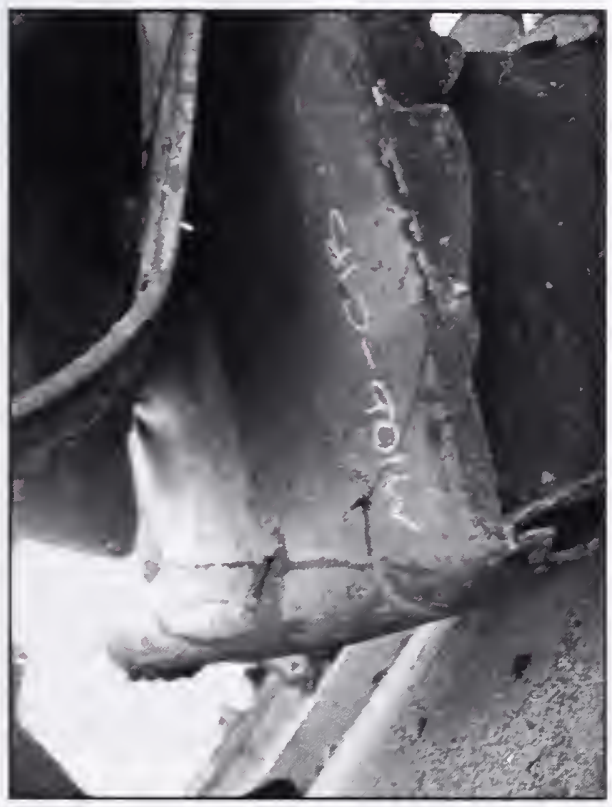

M10b-C1T

NA b)

Connector not recovered

M10b-C1B (WTC 2, Col.207, Fl 84)

Type D

Seat Detail: 5110

Both diagonal bracing straps ripped off at weld.

Figure B-22. Floor truss connectors found on recovered panel M-10b (B206: 83-86) from WTC 2. Shown are connectors found on column 207 at different floor levels. 
d)

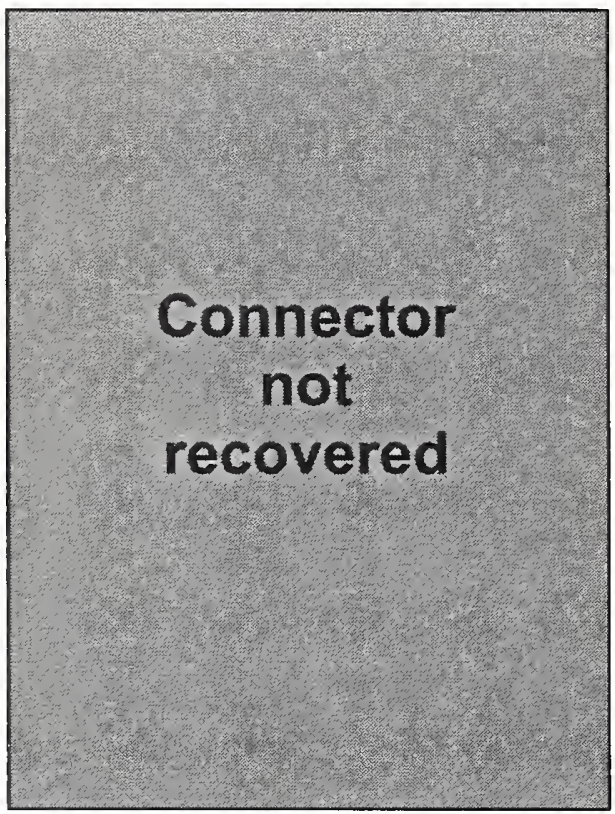

M10b-C2M

NA

f)

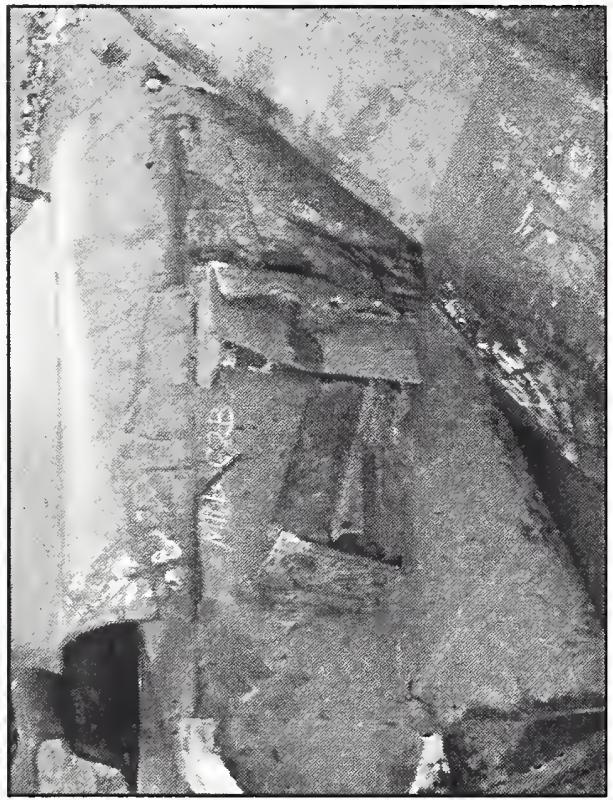

M10b-C2T

NA e)

\section{Connector} not recovered

\section{M10b-C2B (WTC 2, Col.206, Fl 84)}

Type A

Seat Detail: 1212

Gusset plate ripped completely out of spandrel;

Seat intact, bent in towards column and downwards, only 1 bolt hole intact, other ripped through; Gusset plate with damper unit intact, bent upwards.

Figure B-22. Floor truss connectors found on recovered panel M-10b (B206: 83-86) from WTC 2. Shown are connectors found on column 206 at different floor levels (cont.). 
g)

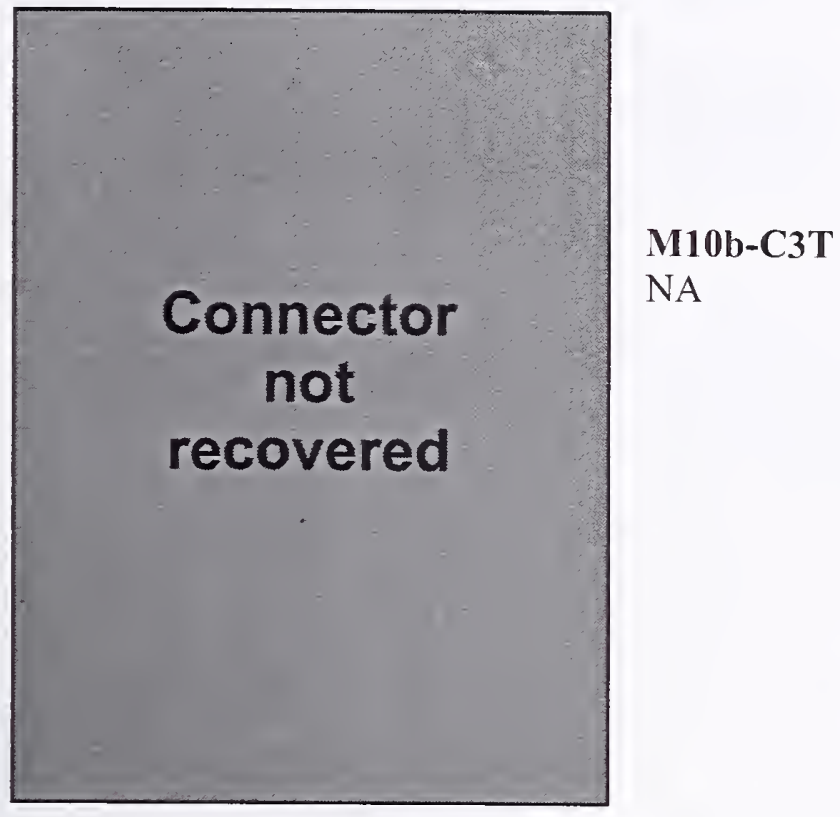

h)

M10b-C3M

NA

i)

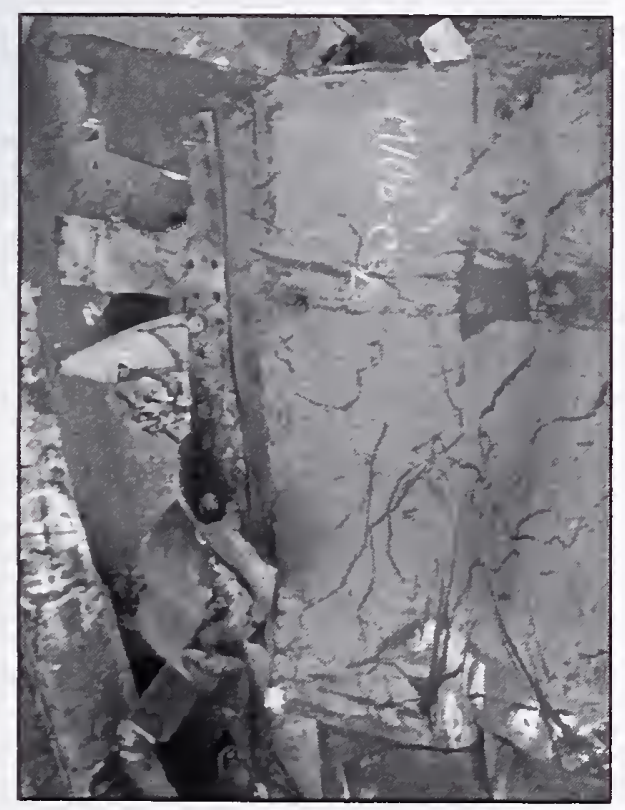

Connector not recovered

M10b-C3B (WTC 2, Col.205, FI 84)

Type D

Seat Detail: 5110

1 -in portion of diagonal bracing strap remaining, other ripped completely out of spandrel.

Figure B-22. Floor truss connectors found on recovered panel M-10b (B206: 83-86) from WTC 2. Shown are connectors found on column 205 at different floor levels (cont.). 
a)

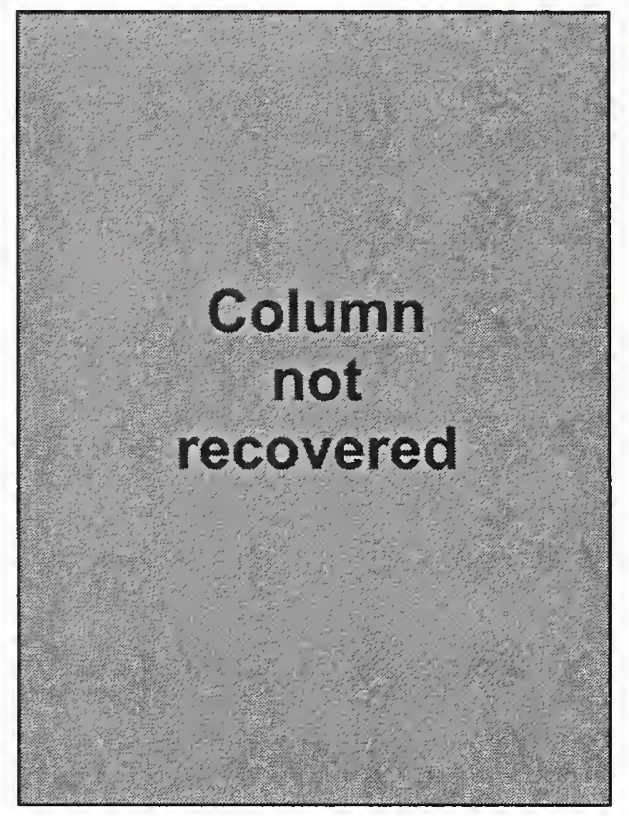

M20-C1M

NA
M20-C1T

NA c)

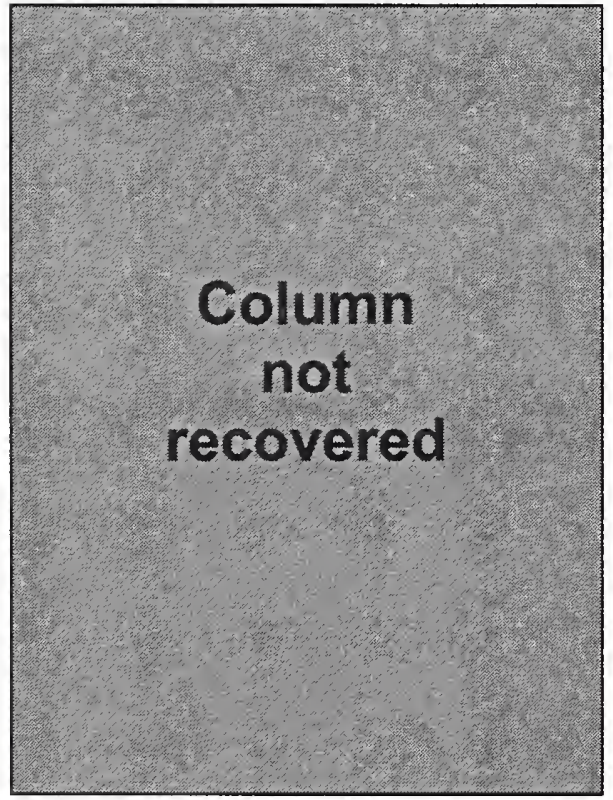

b)

\section{Column not} recovered

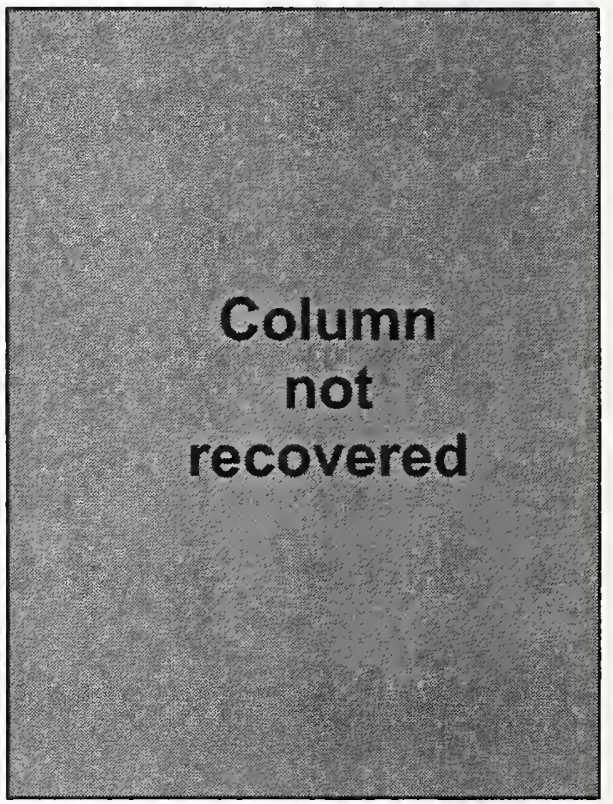

M20-C1B

NA

Figure B-23. Floor truss connectors found on recovered panel M-20 (A121: 99-102) from WTC 1. Shown are connectors found on column 122 at different floor levels. 
d)

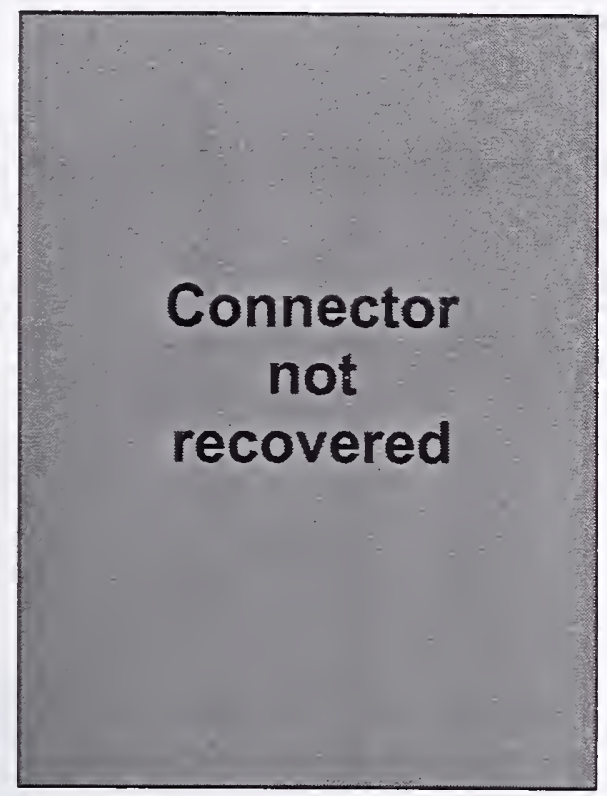

M20-C2M

NA

f)

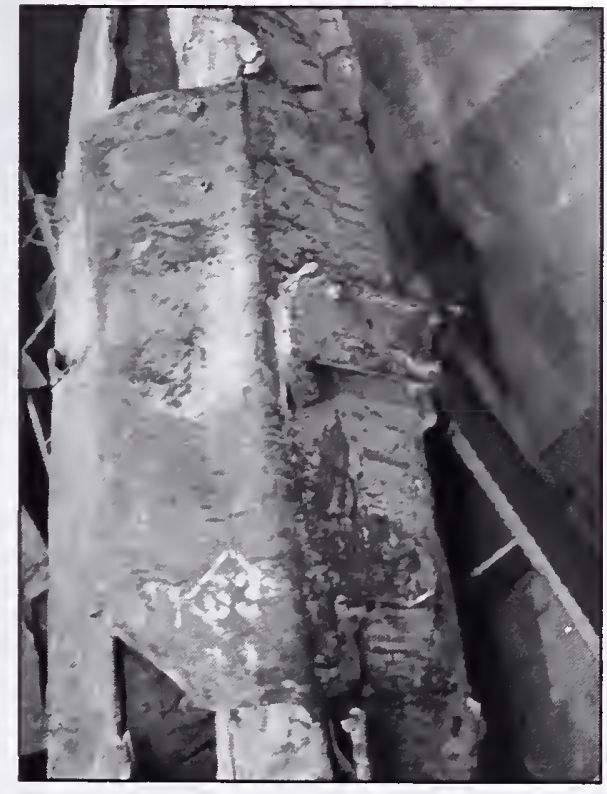

M20-C2T

NA e)

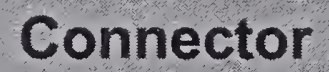
not recovered

M20-C2B (WTC 1, Col.121, FI 100)

Type A

Seat Detail: 1411

Gusset plate ripped completely out of spandrel;

Heavy seat intact, has additional stiffener, both bolt holes intact, 2 bolts remain;

Gusset for damper unit ripped off at weld.

Figure B-23. Floor truss connectors found on recovered panel $M-20$ (A121: 99-102) from WTC 1. Shown are connectors found on column 121 at different floor levels (cont.). 
g)

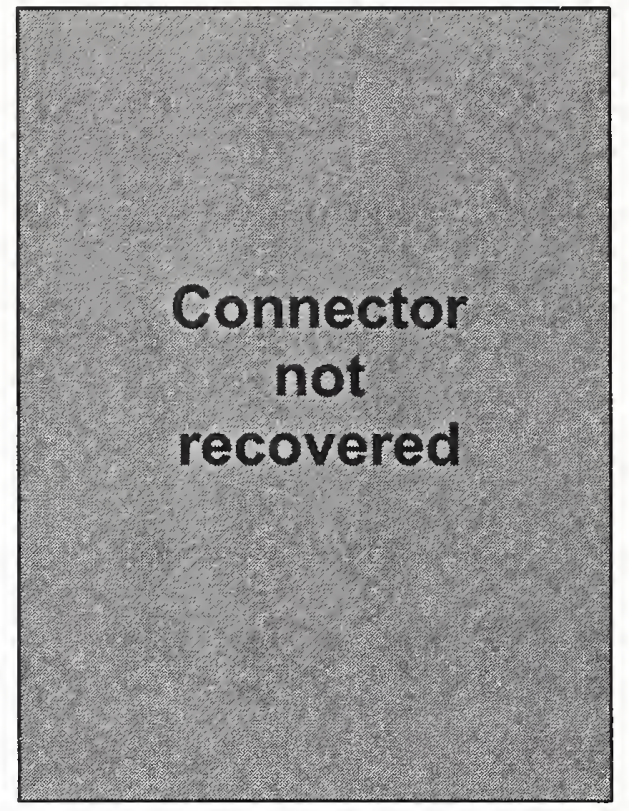

M20-C3M

NA
M20-C3T

NA i)

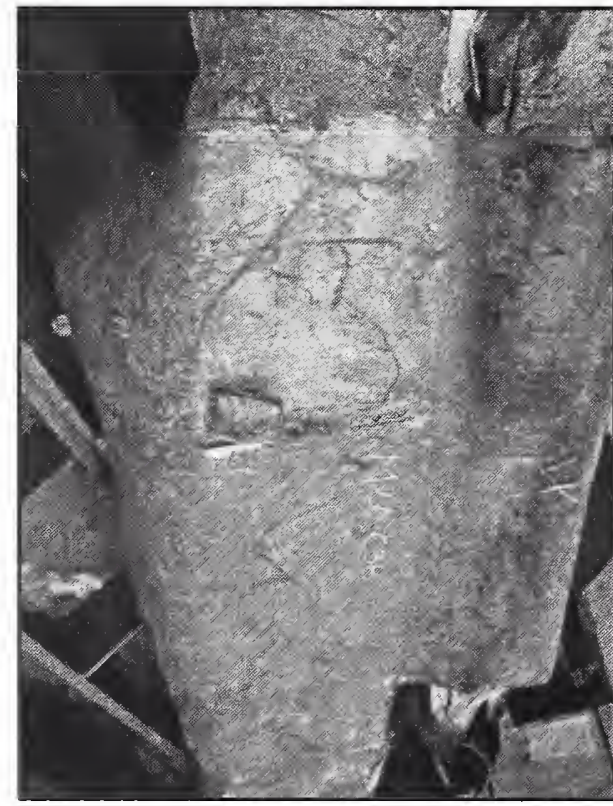

h)

\section{Connector} not recovered

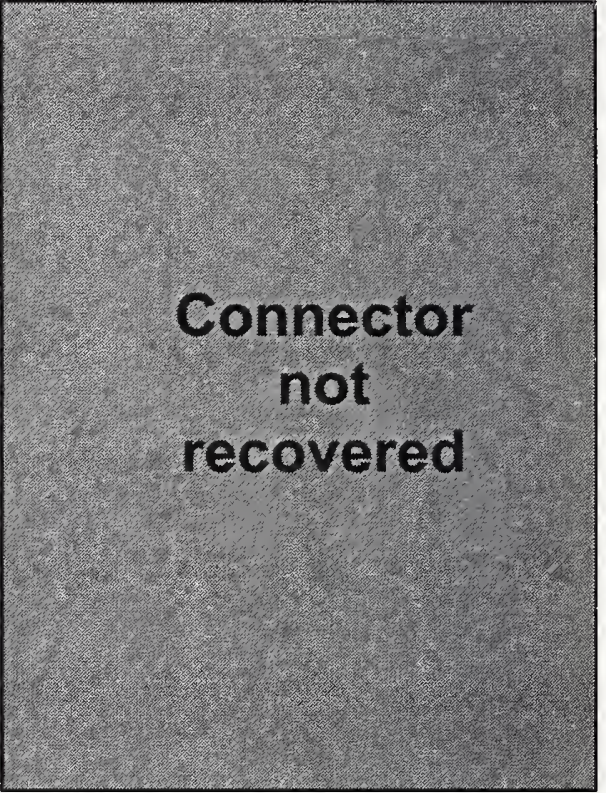

M20-C3B (WTC 1, Col.120, Fl 100)

Type $\mathrm{C}$

Seat Detail: 5210

Portion of gusset plate remains, bent upward, rest ripped off at weld.

Figure B-23. Floor truss connectors found on recovered panel M-20 (A121: 99-102) from WTC 1. Shown are connectors found on column 120 at different floor levels (cont.). 
a)

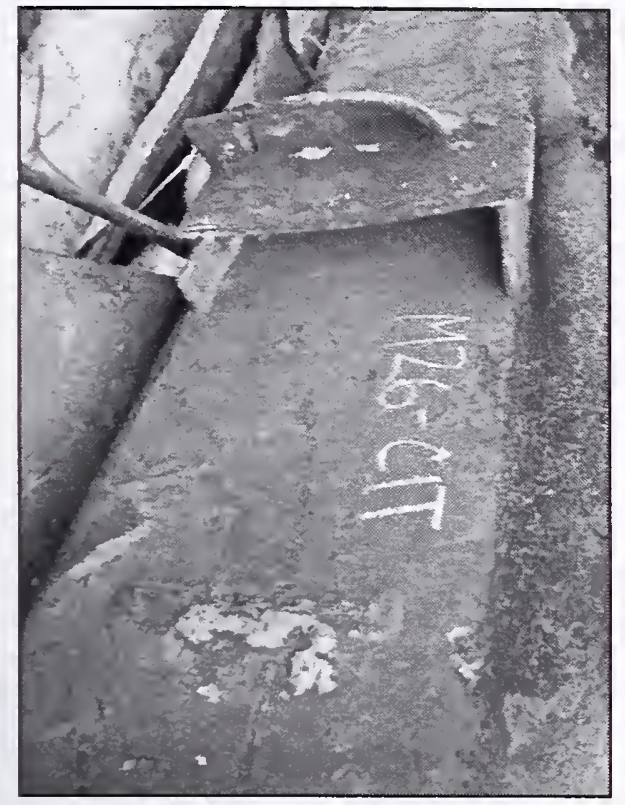

M26-C1M (WTC 1, Col.131, Fl 92)

Type A

Seat Detail: 2410

Gusset plate ripped off at weld;

Seat intact, bent downwards, 1 bolt hole remains intact. other ripped out;

No evidence of gusset for damper unit.

c)

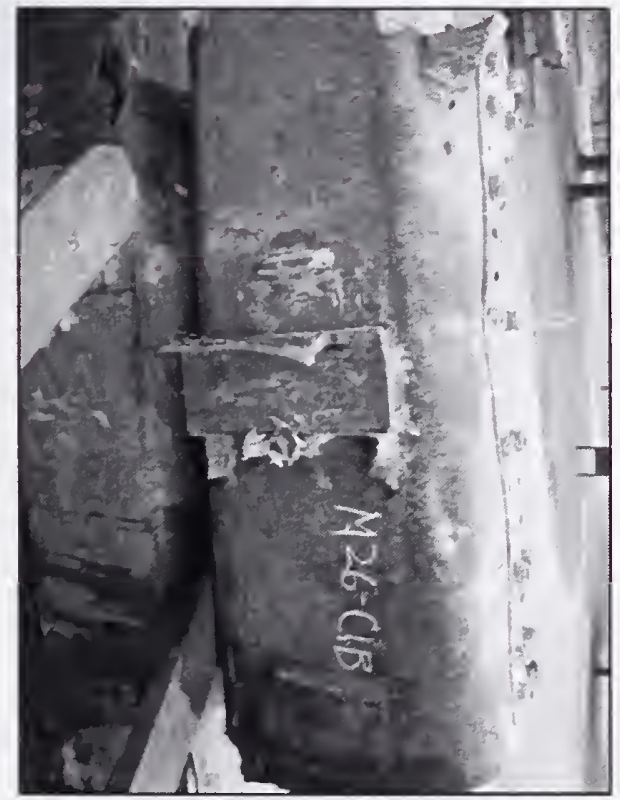

\section{M26-C1T (WTC 1, Col.131, Fl 93)}

Type A

Seat Detail: 1411

Gusset plate ripped off at weld;

Seat intact, bent downwards, 1 bolt hole remains intact, other ripped out;

Gusset for damper unit ripped off at weld b)

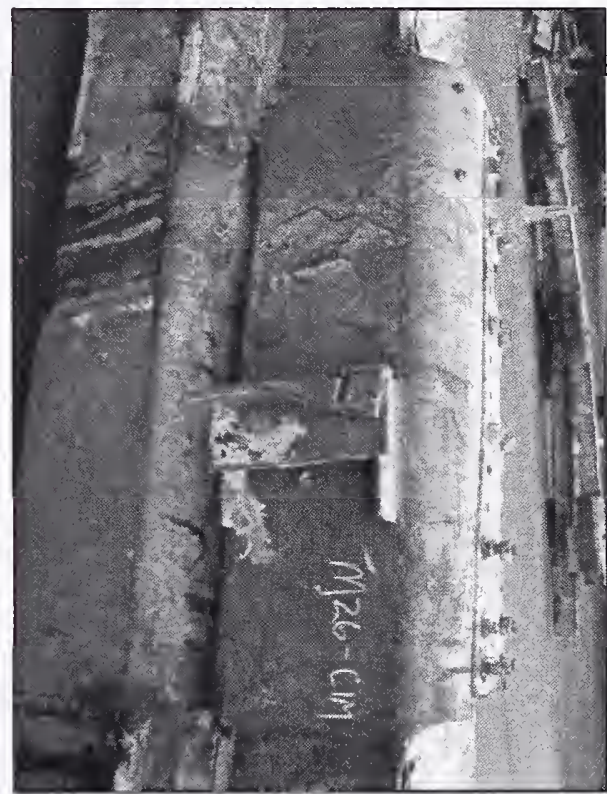

M26-C1B (WTC 1, Col.131, Fl 91)

Type A

Seat Detail: 1411

Gusset plate ripped off at weld;

Seat intact, bent downwards, 1 bolt hole remains intact, other ripped out;

Gusset for damper unit ripped off at weld.

Figure B-24. Floor truss connectors found on recovered panel M-26 (A130: 90-93) from WTC 1. Shown are connectors found on column 131 at different floor levels. 
d)

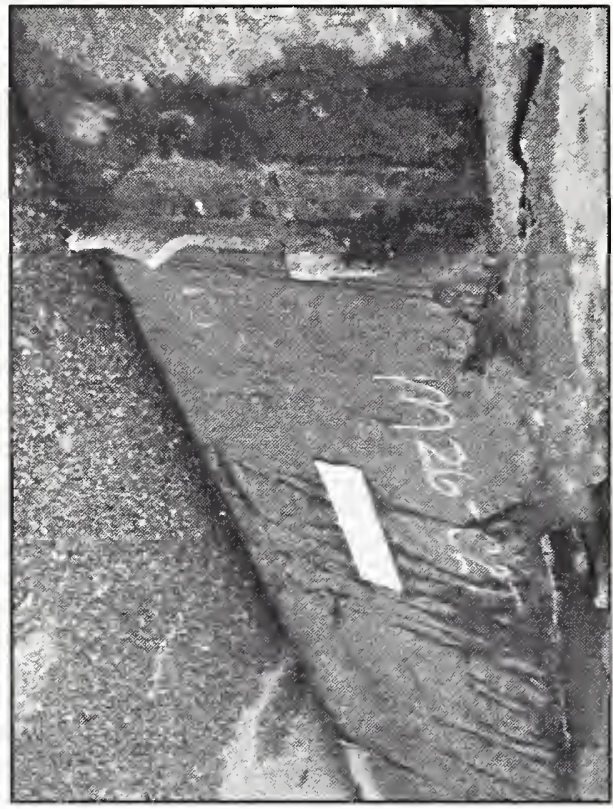

M26-C2T (WTC 1, Col.130, Fl 93)

Type C

Seat Detail: 5210

Portion of gusset plate remains, ripped in base.

M26-C2M (WTC 1, Col.130, Fl 92)

Type C

Seat Detail: 5210

Gusset plate ripped at weld.

e)

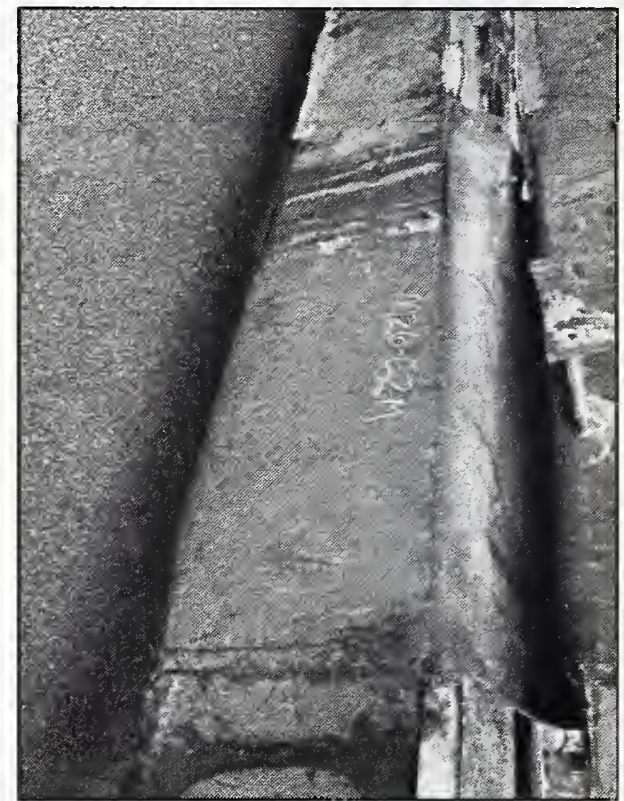

f)

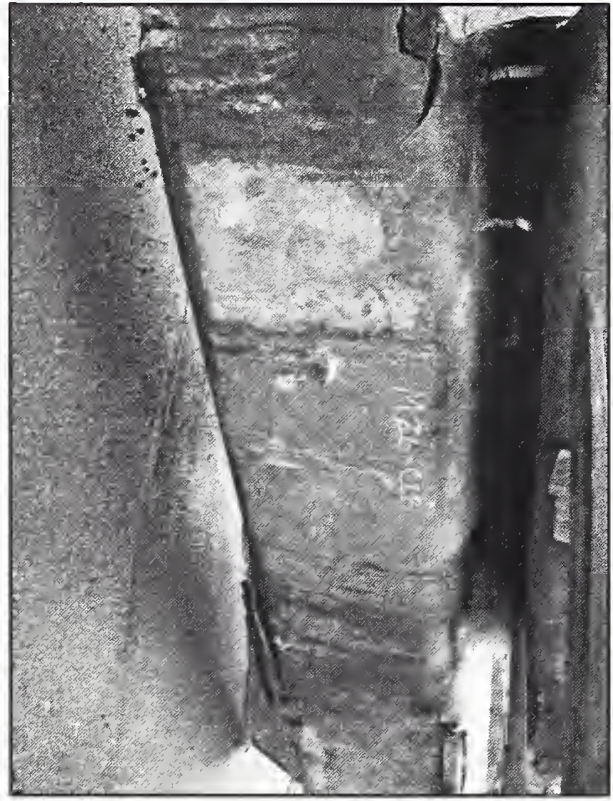

M26-C2B (WTC 1, Col.130, Fl 91)

Type C

Seat Detail: 5210

Gusset plate ripped at weld.

Figure B-24. Floor truss connectors found on recovered panel M-26 (A130: 90-93) from WTC 1. Shown are connectors found on column 130 at different floor levels (cont.). 
g)

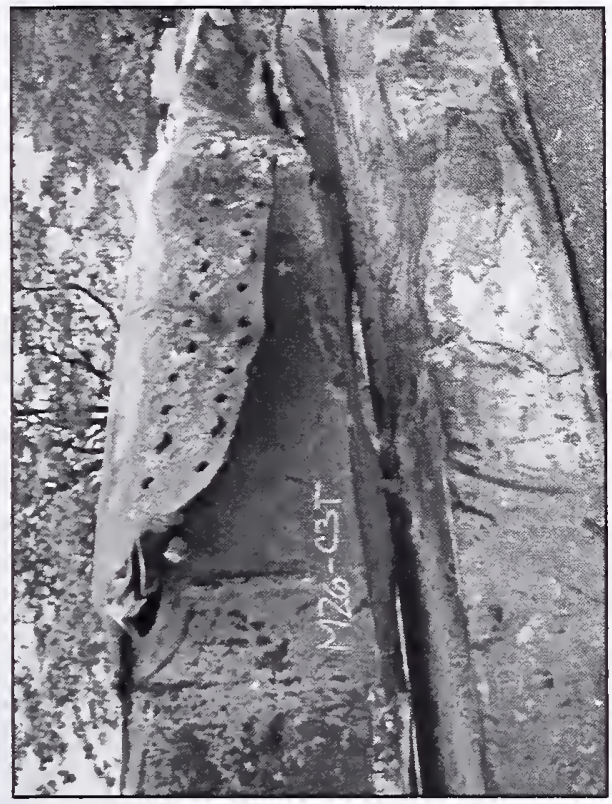

M26-C3M (WTC 1, Col.129, Fl 92)

Type A

Seat Detail: 2410

Gusset plate remains;

Only 1 standoff plate remains, seat and other standoff plate ripped off at welds;

No evidence of gusset plate for damper unit.
M26-C3T (WTC 1, Col.129, Fl 93)

Type A

Seat Detail: 1411

Gusset plate remains;

Only standoff plates remain, seat ripped off at welds;

Gusset for damper unit ripped off at weld. h)

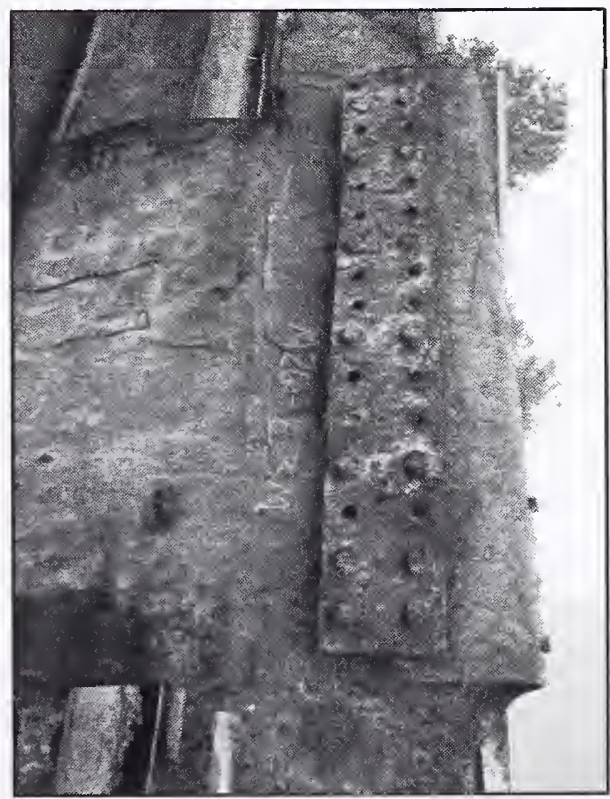

i)

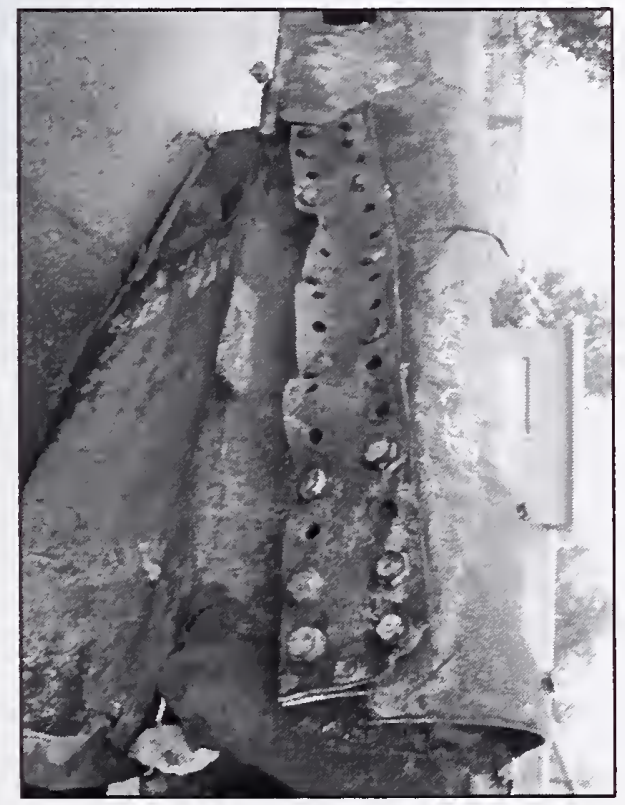

M26-C3B (WTC 1, Col.129, Fl 91)

Type A

Seat Detail: 1411

Gusset plate ripped off at weld;

Only standoff plates remain, seat ripped off at welds;

Gusset for damper unit ripped off at weld.

Figure B-24. Floor truss connectors found on recovered panel M-26 (A130: 90-93) from WTC 1. Shown are connectors found on column 129 at different floor levels (cont.). 
a)

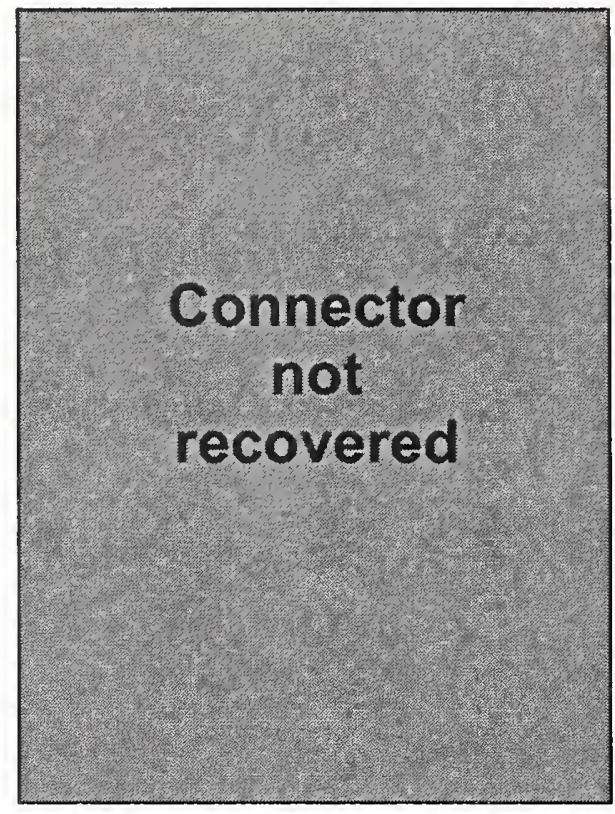

M27-C1M (WTC 1, Col.131, FI 95)

Type A

Seat Detail: 1411

Small portion of gusset plate remains;

Seat intact but deformed, bent in towards column, both bolt holes ripped out;

Gusset for damper unit ripped off at welds. c)

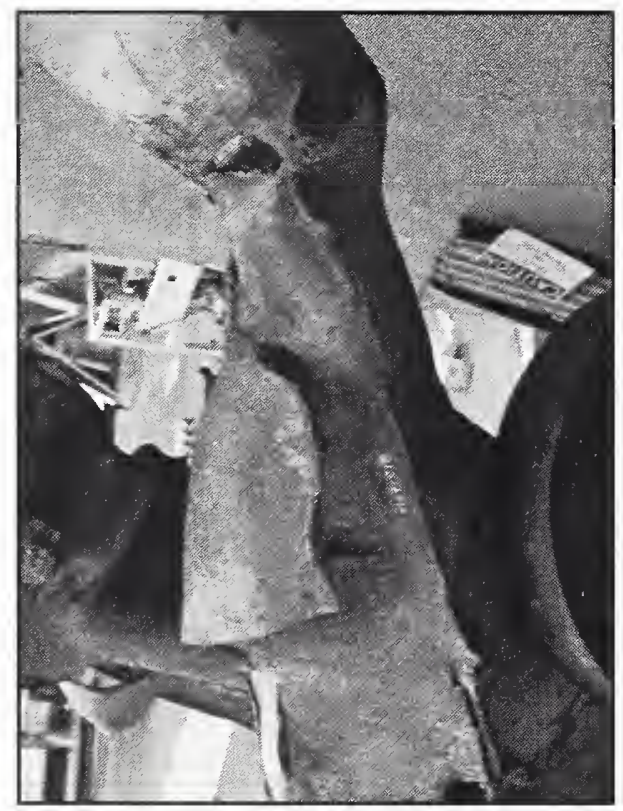

M27-C1T

NA b)

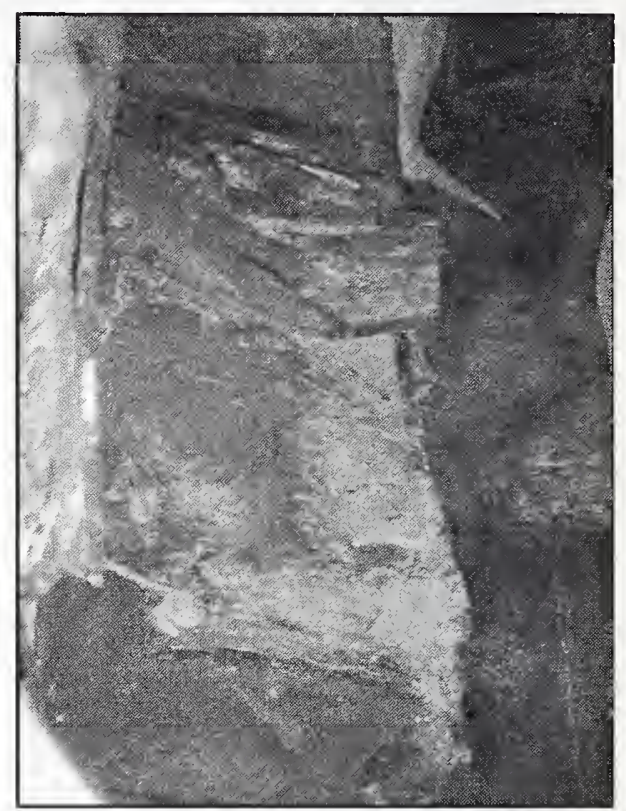

M27-C1B (WTC 1, Col.131, FI 94)

Type A

Seat Detail: 1411

Small portion of gusset plate remains;

Standoff plates ripped off at welds;

Gusset plate for damper unit ripped off at weld.

Figure B-25. Floor truss connectors found on recovered panel M-27 (A130: 93-96) from WTC 1. Shown are connectors found on column 131 at different floor levels. 
d)

Connector

M27-C2T

not

recovered

M27-C2M (WTC 1, Col.130, F1 95)

Type C

Seat Detail: 5210

Gusset plate remains, half bent upwards and half downwards, diagonal bracing strap attached.

e)

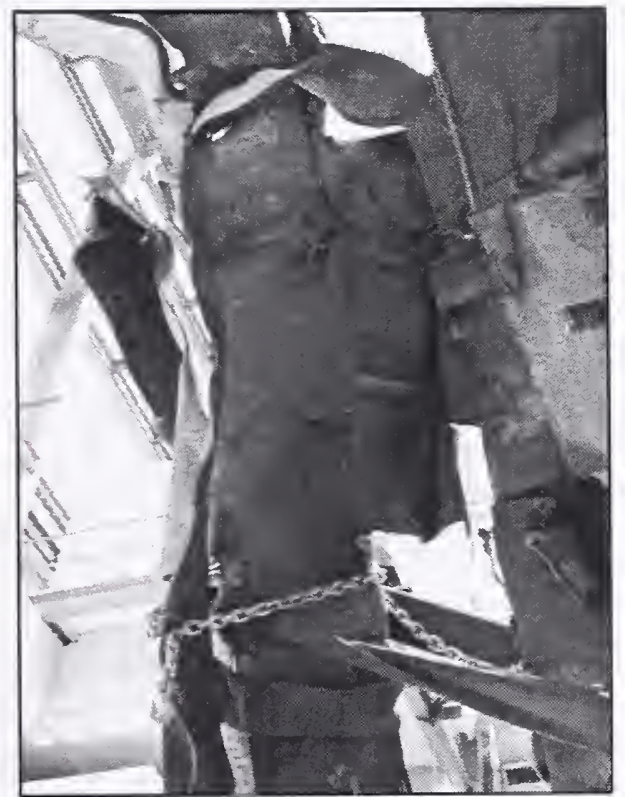

f)

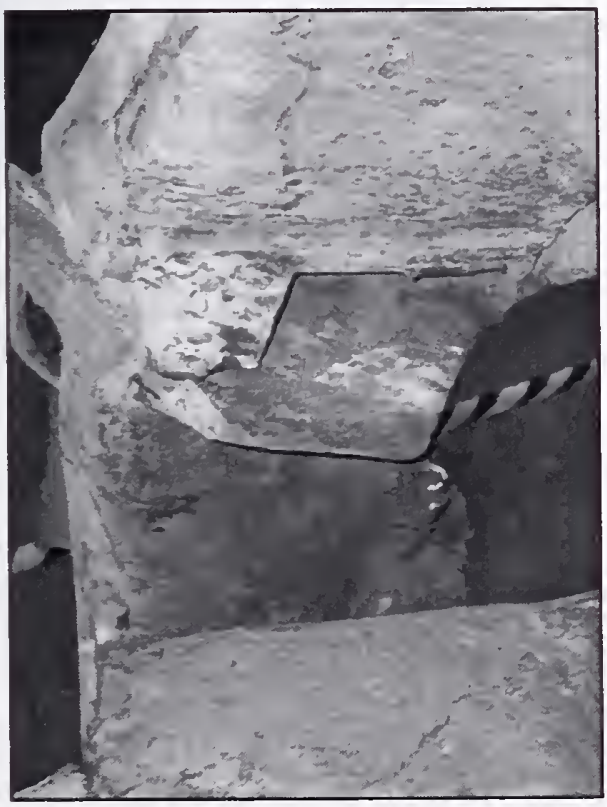

M27-C2B (WTC 1, Col.130, F1 94)

Type $\mathrm{C}$

Seat Detail: 5210

Portion of gusset plate remains, bent downwards, with diagonal bracing strap attached.

Figure B-25. Floor truss connectors found on recovered panel M-27 (A130: 93-96) from WTC 1. Shown are connectors found on column 130 at different floor levels (cont.). 
g)

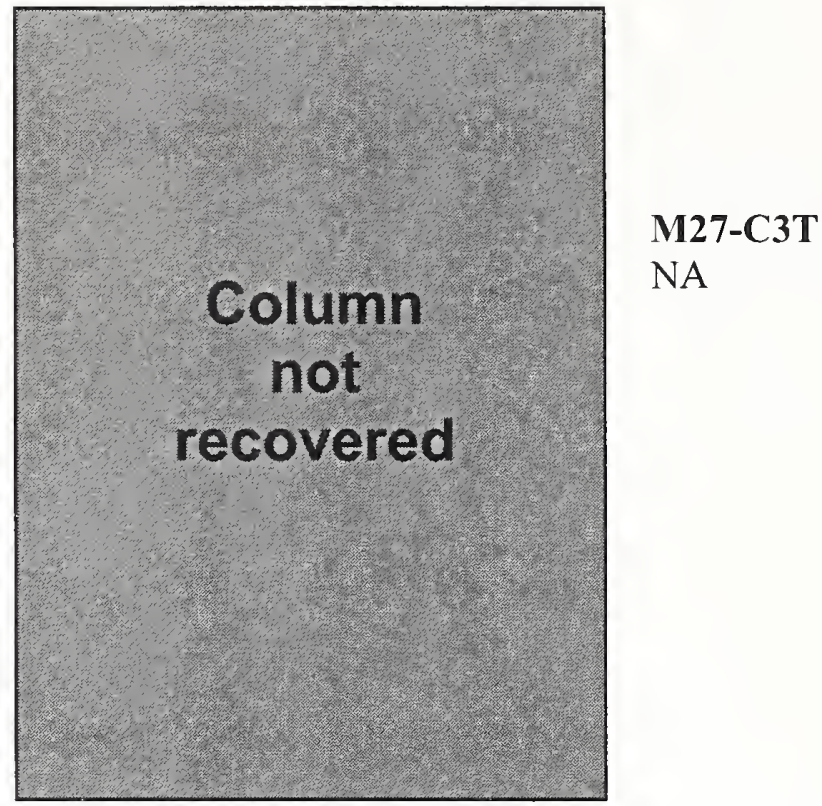

M27-C3M

NA

i)

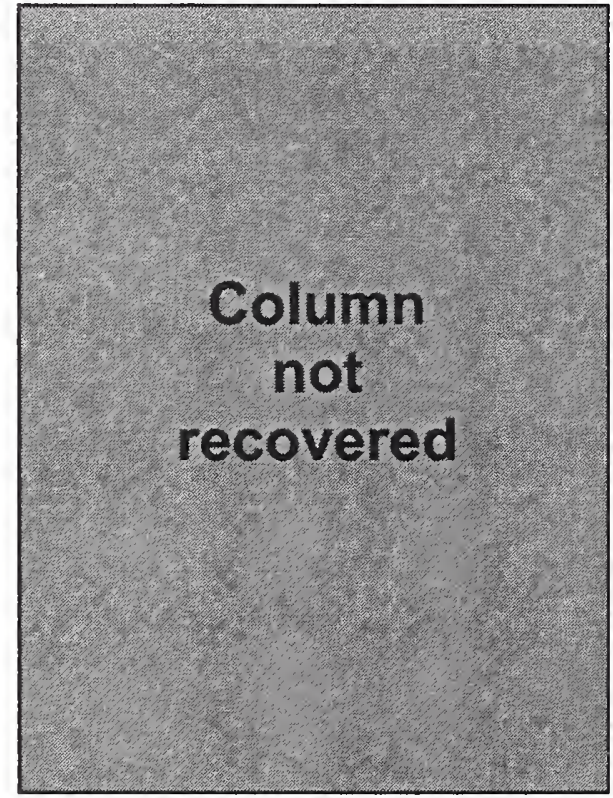

h)

\section{Column not recovered}

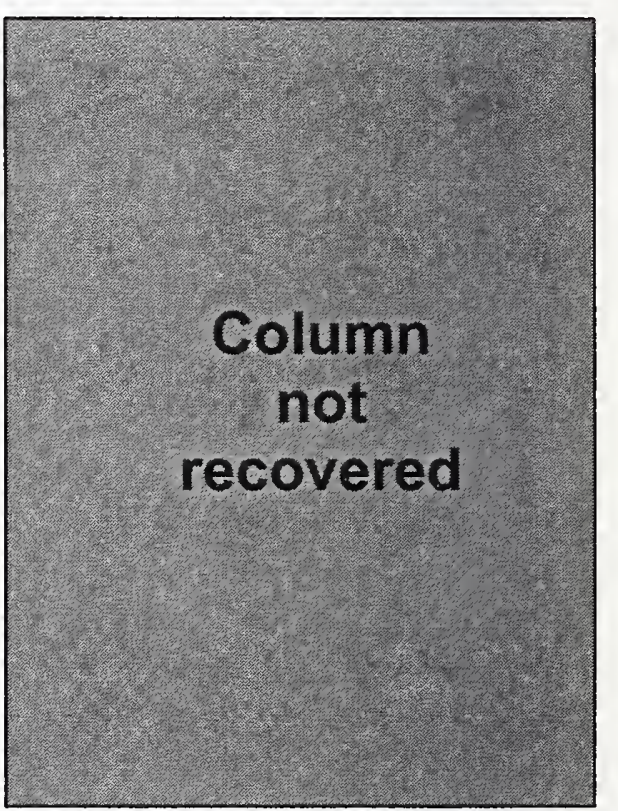

M27-C3B

NA

Figure B-25. Floor truss connectors found on recovered panel M-27 (A130: 93-96) from WTC 1. Shown are connectors found on column 129 at different floor levels (cont.). 
a)

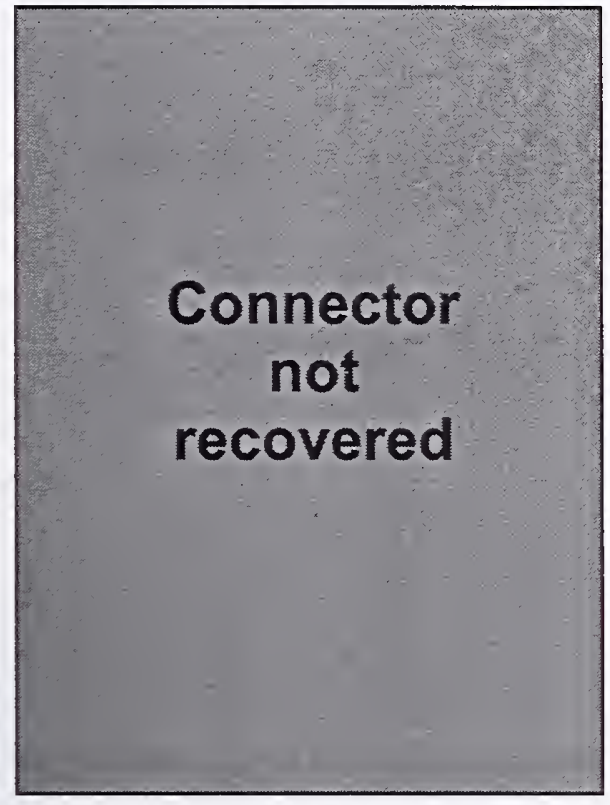

M28-C1M

NA

\section{M28-C1T}

NA b)

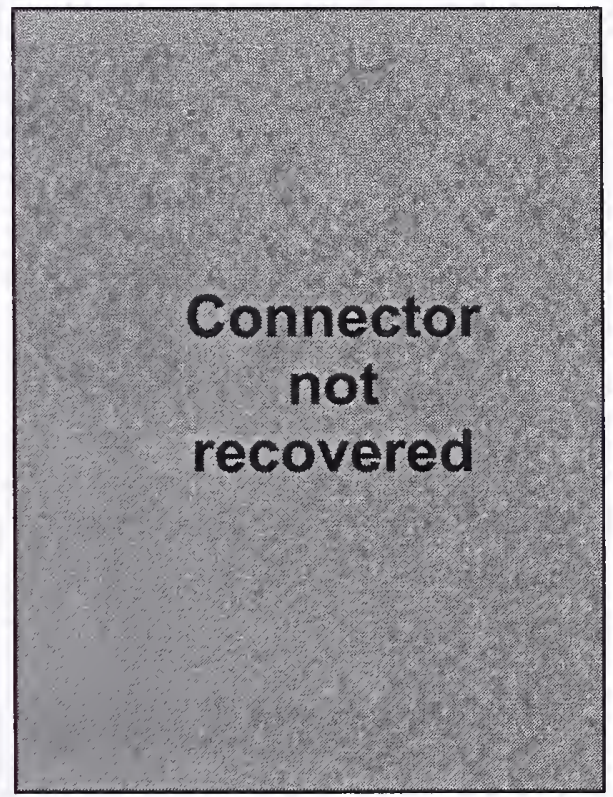

c)

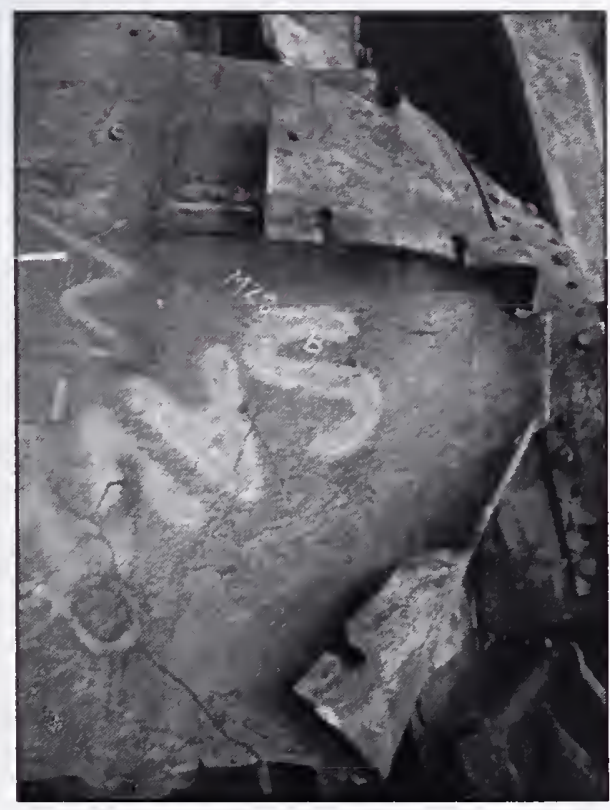

M28-C1B (WTC 2, Col.346, F1 99)

Type C

Seat Detail: 5110

Gusset plate intact with portions of diagonal bracing straps attached.

Figure B-26. Floor truss connectors found on recovered panel M-28 (B345: 98-101) from WTC 2. Shown are connectors found on column 346 at different floor levels. 
d)

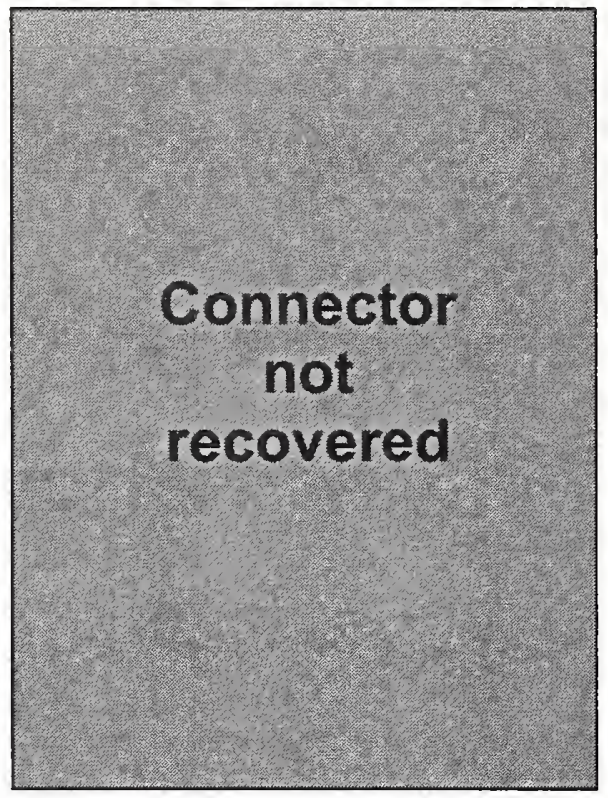

M28-C2M

NA
M28-C2T

NA f)

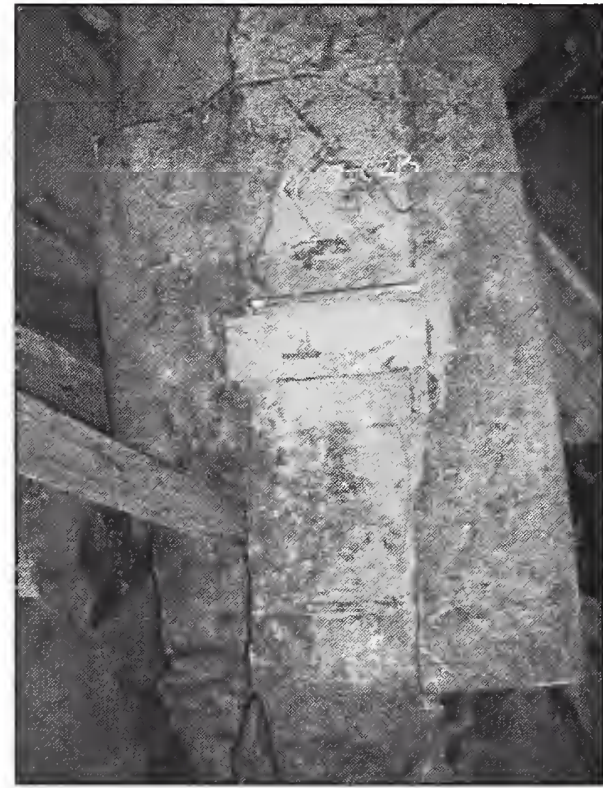

e)

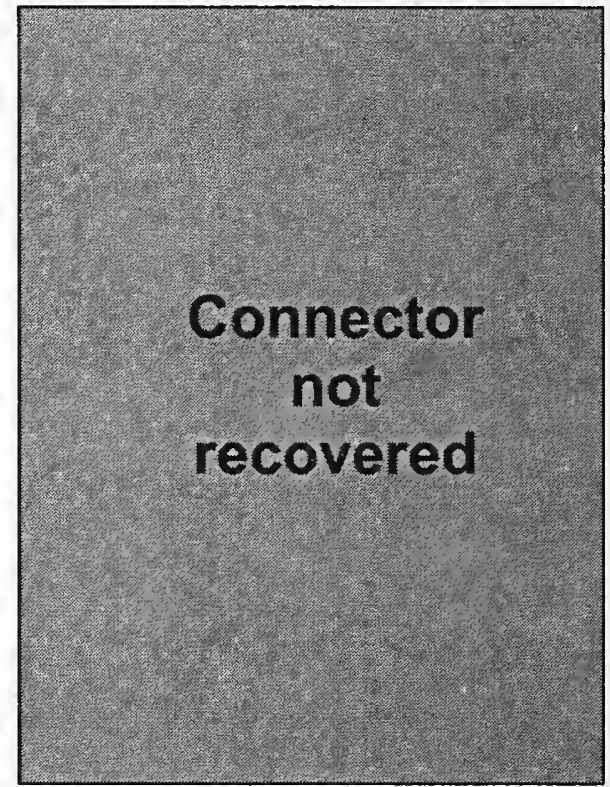

M28-C2B (WTC 2, Col.345, Fl 99)

Type A

Seat Detail: 1411

Gusset plate remains;

Seat intact, 1 bolt hole intact, other ripped through;

Gusset plate for damper unit ripped off at weld.

Figure B-26. Floor truss connectors found on recovered panel M-28 (B345: 98-101) from WTC 2. Shown are connectors found on column 345 at different floor levels (cont.). 
g)

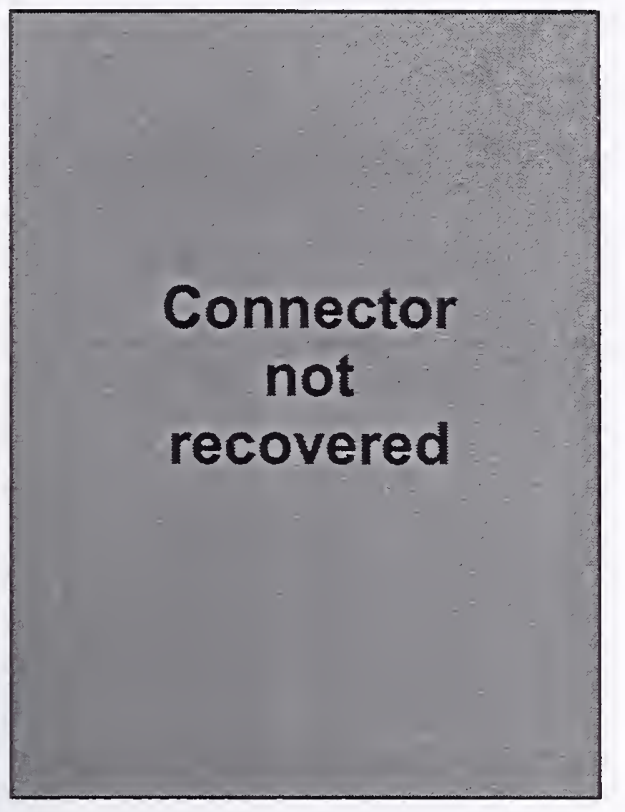

M28-C3M

NA
M28-C3T

NA h)

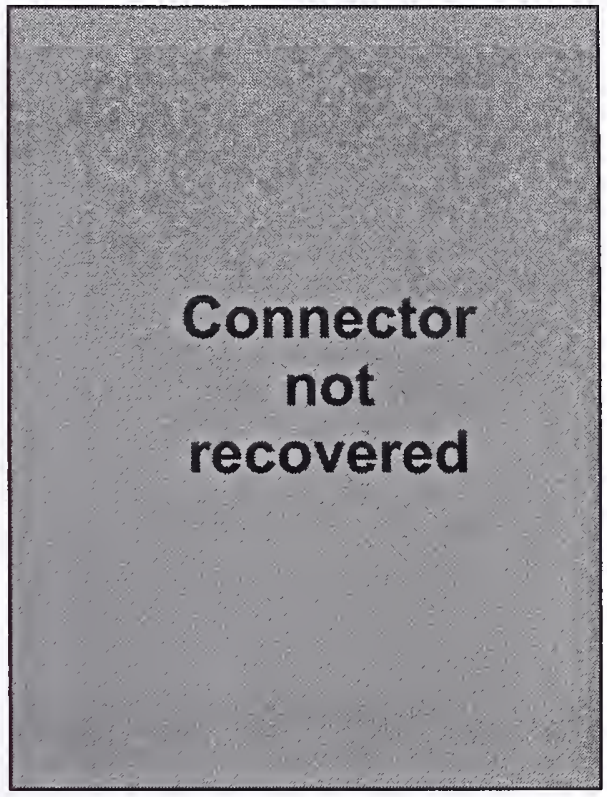

M28-C3B (WTC 2, Col.344, Fl 99)

Type C

Seat Detail: 5110

Gusset plate ripped off at weld.

Figure B-26. Floor truss connectors found on recovered panel M-28 (B345: 98-101) from WTC 2. Shown are connectors found on column 344 at different floor levels (cont.). 
a)

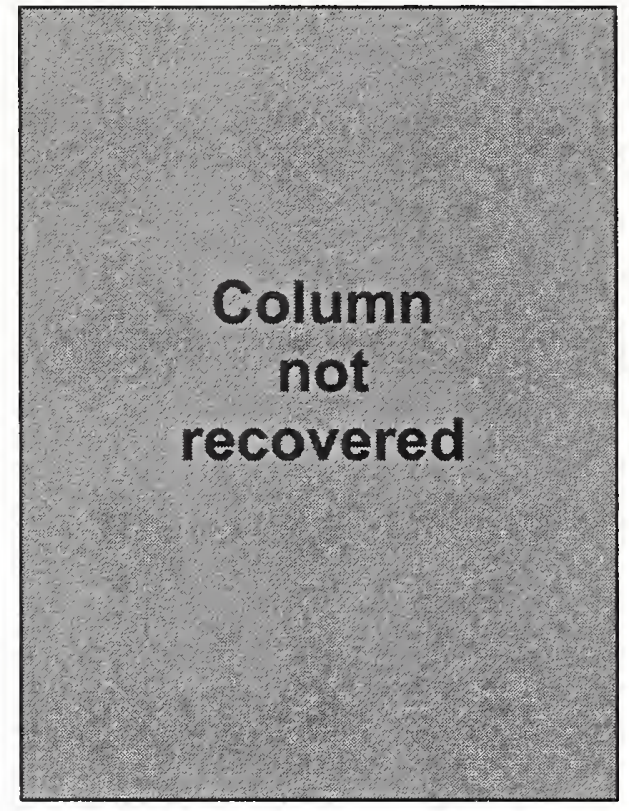

M30-C1M

NA
M30-C1T

NA b)

\section{Column not recovered}

c)

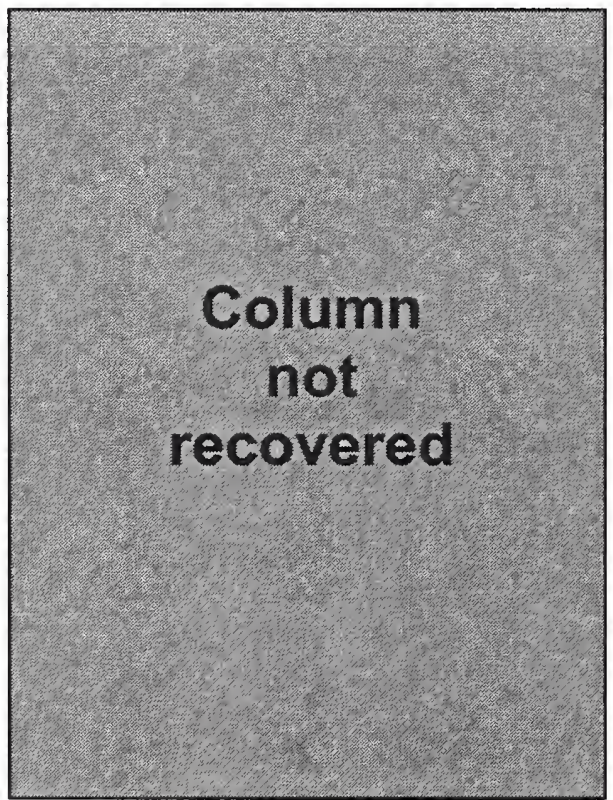

Figure B-27. Floor truss connectors found on recovered panel M-30 (A133: 94-97) from WTC 1. Column 134 was not recovered. 
d)

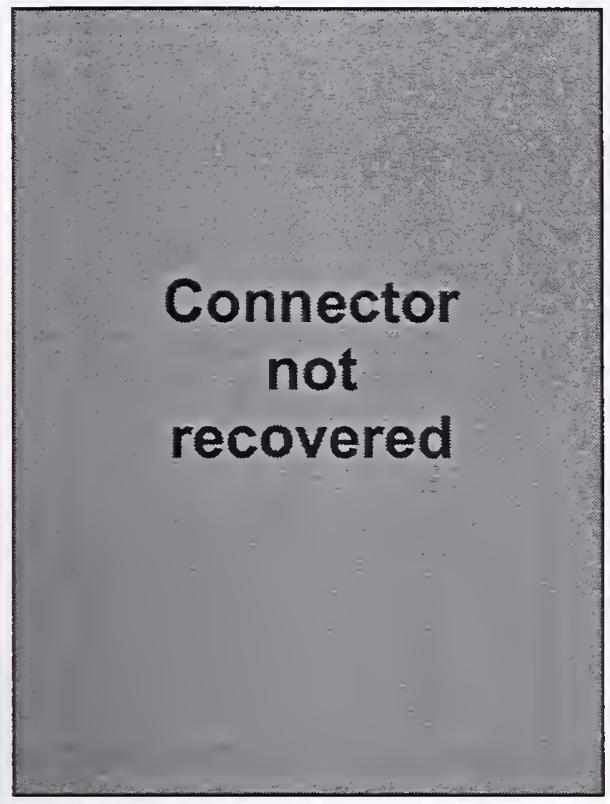

M30-C2M

NA

\section{M30-C2T \\ NA}

e)

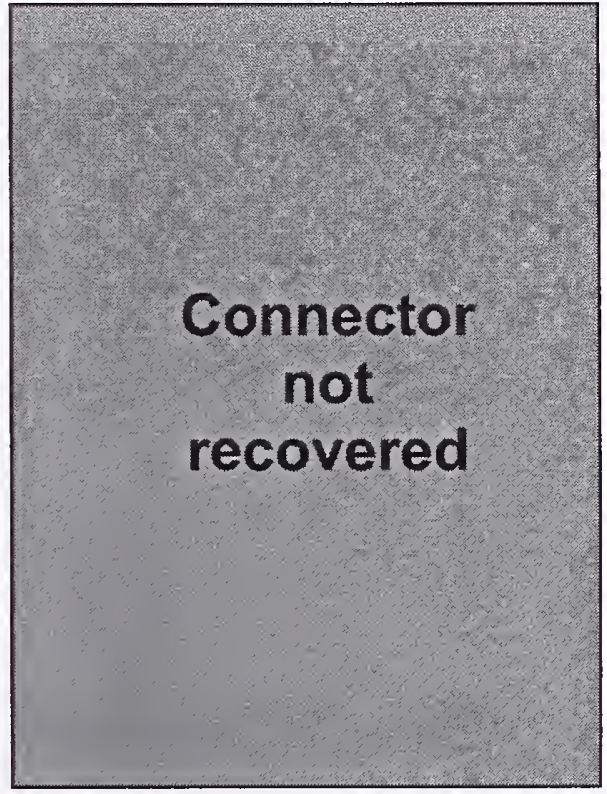

M30-C2B (WTC 1, Col.133, Fl 95)

Type A

Seat Detail: 1411

Gusset plate remains;

Seat intact, bent downwards, both bolt holes intact with one still having bolt and nut;

Gusset for damper unit ripped off at weld.

Figure B-27. Floor truss connectors found on recovered panel M-30 (A133: 94-97) from WTC 1. Shown are connectors found on column 133 at different floor levels (cont.). 
g)

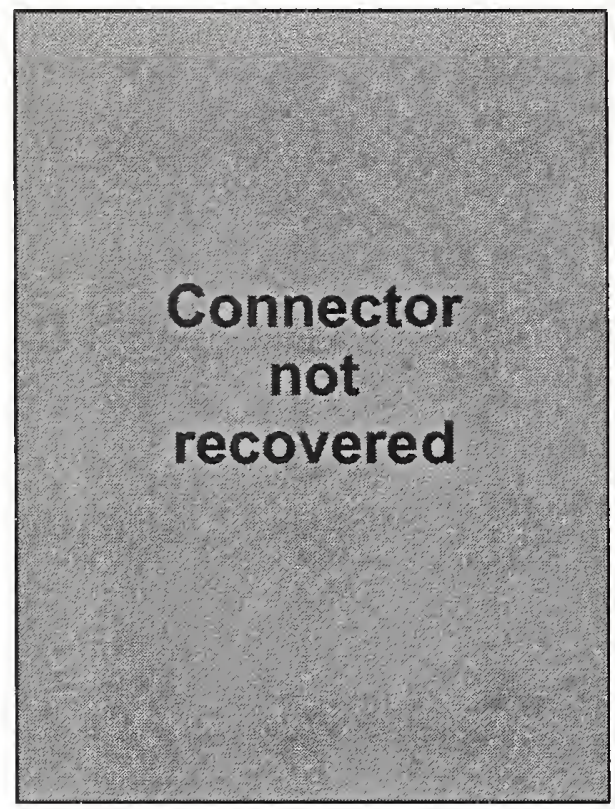

M30-C3T

NA

M30-C3M

NA

i)

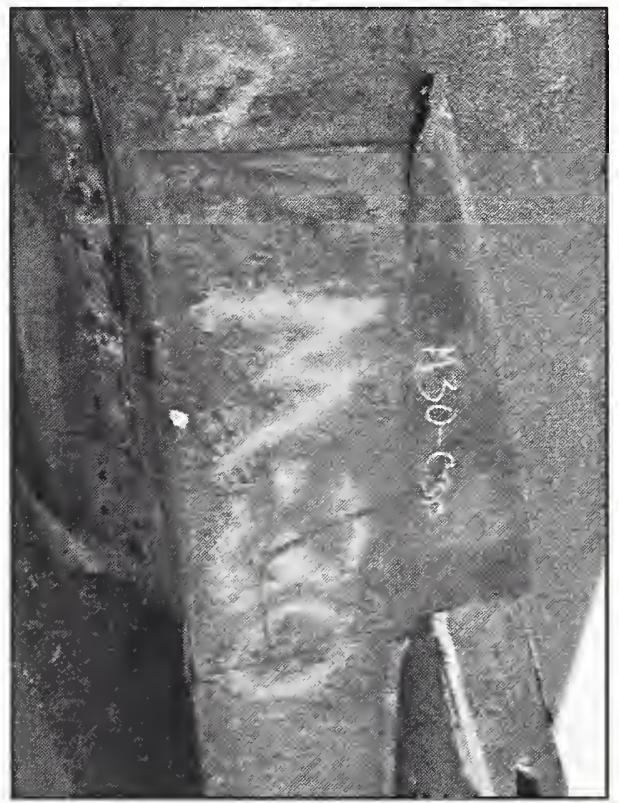

h)

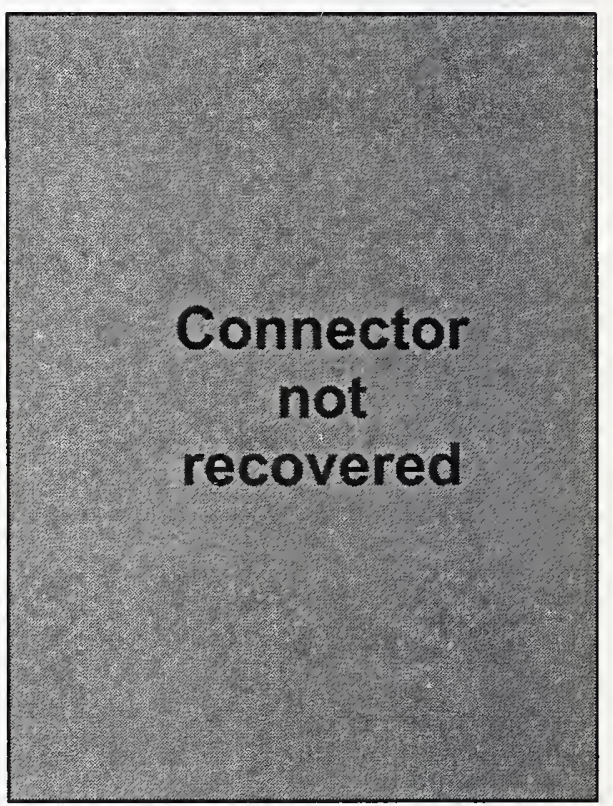

M30-C3B (WTC 1, Col.132, Fl 95)

Type $\mathrm{C}$

Seat Detail: 5210

Gusset plate ripped off at weld.

Figure B-27. Floor truss connectors found on recovered panel M-30 (A133: 94-97) from WTC 1. Shown are connectors found on column 132 at different floor levels (cont.). 
a)

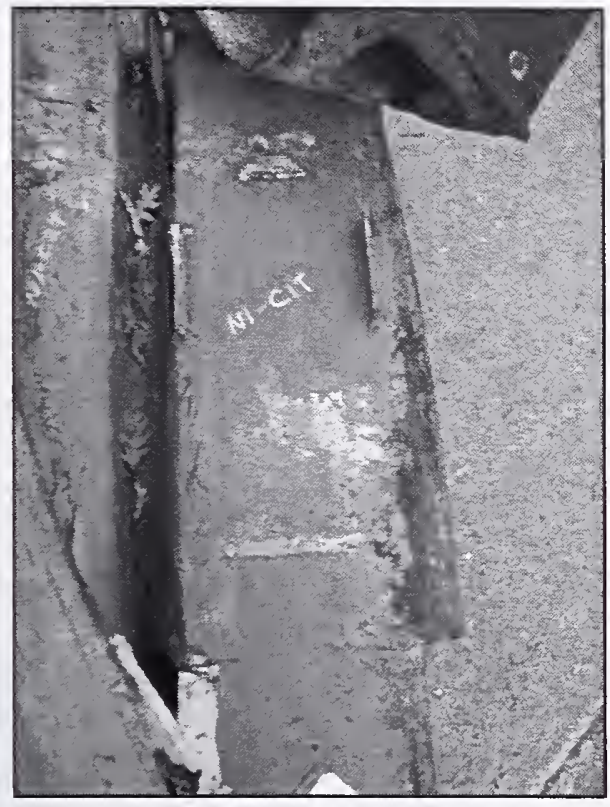

N1-C1M (WTC 1, Col.219, Fl 84)

Type A

Seat Detail: 1111

Gusset plate remains;

Seat intact, bent downwards, both bolt holes intact; Gusset plate with damper unit intact, bent downwards.

c)

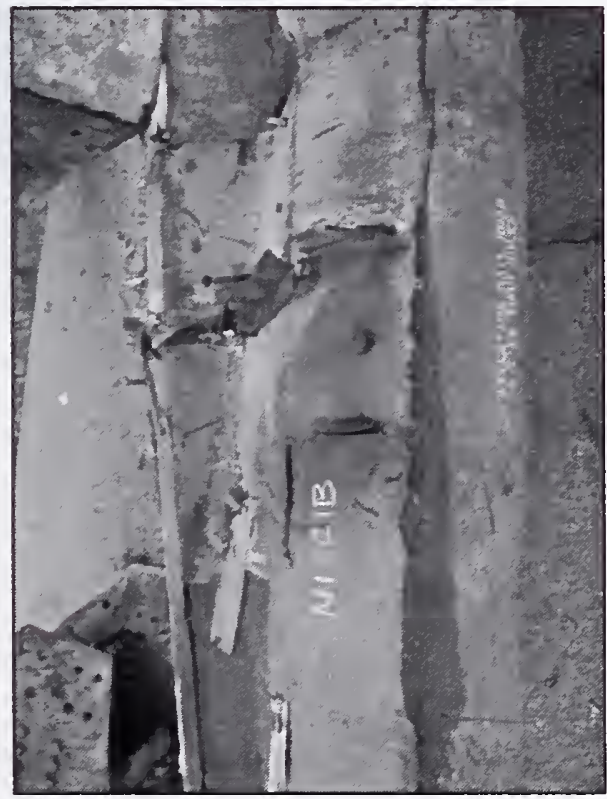

N1-C1T (WTC 1, Col.219, Fl 85)

Type A

Seat Detail: 1111

Gusset plate intact;

Only standoff plates remain;

Gusset plate for damper unit ripped off at weld. b)

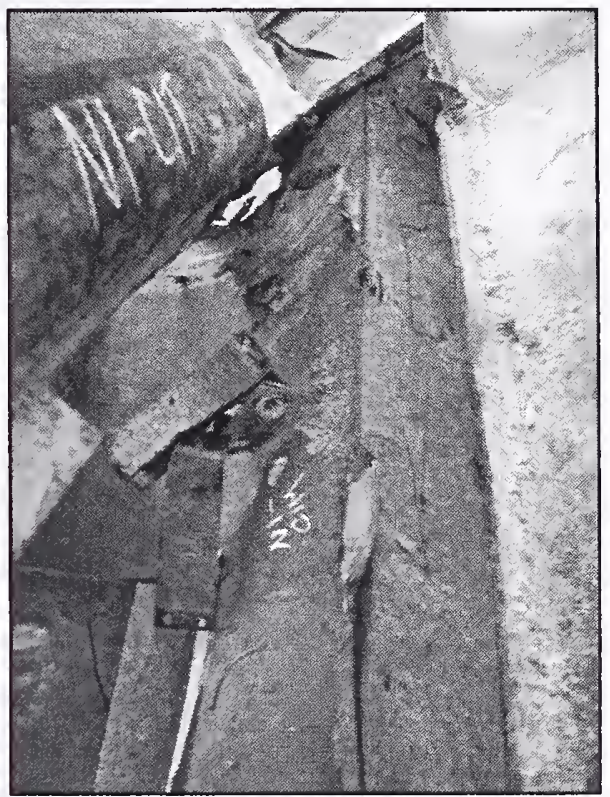

N1-C1B (WTC 1, Col.219, Fl 83)

Type A

Seat Detail: 4120

No evidence of gusset plate;

Seat welded directly to spandrel, no standoff plates, both bolt holes ripped out;

No evidence of typical gusset plate for damper unit.

Figure B-28. Floor truss connectors found on recovered panel N-1 (A218: 82-85) from WTC 1. Shown are connectors found on column 219 at different floor levels. 
d)

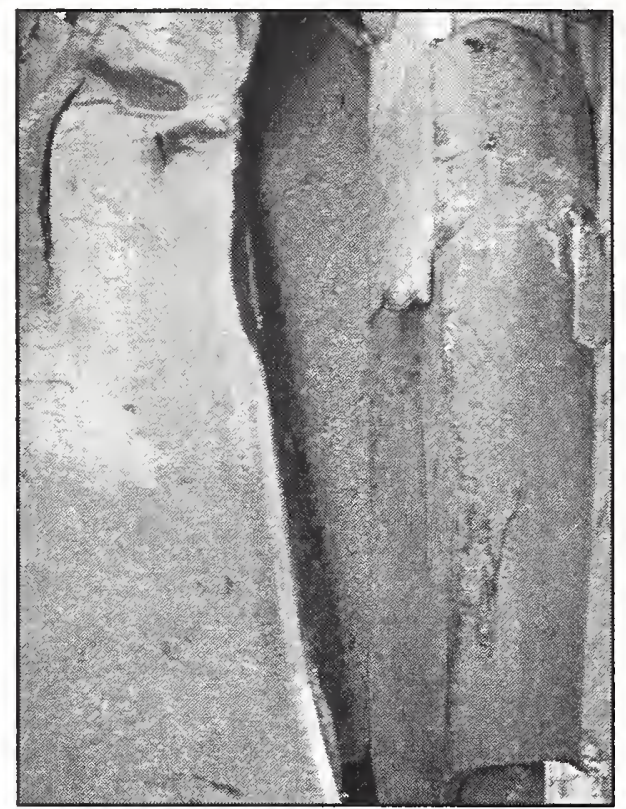

N1-C2M (WTC 1, Col.218, FI 84)

Type C

Seat Detail: 5210

Gusset plate remains, but half ripped away from spandrel at weld.

f)

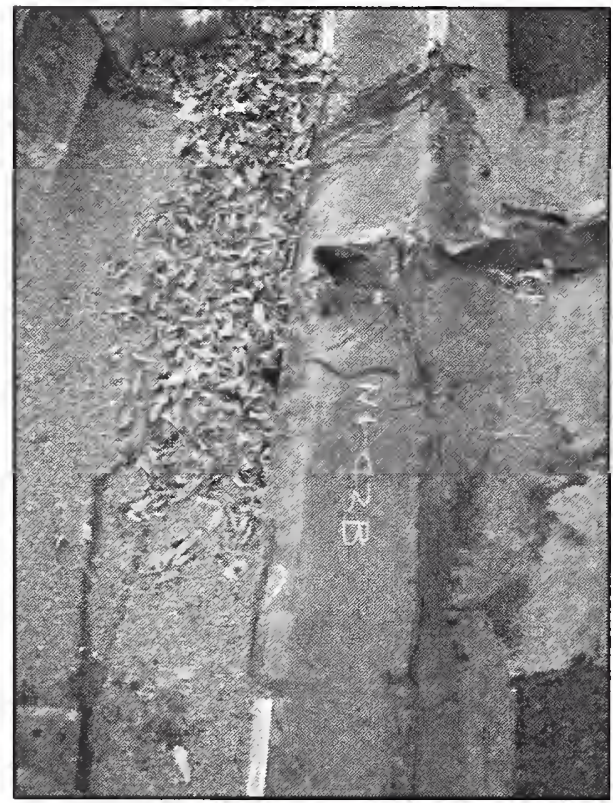

N1-C2T (WTC 1, Col.218, Fl 85)

Type C

Seat Detail: 5210

Portion of gusset plate remains, bent downwards. e)

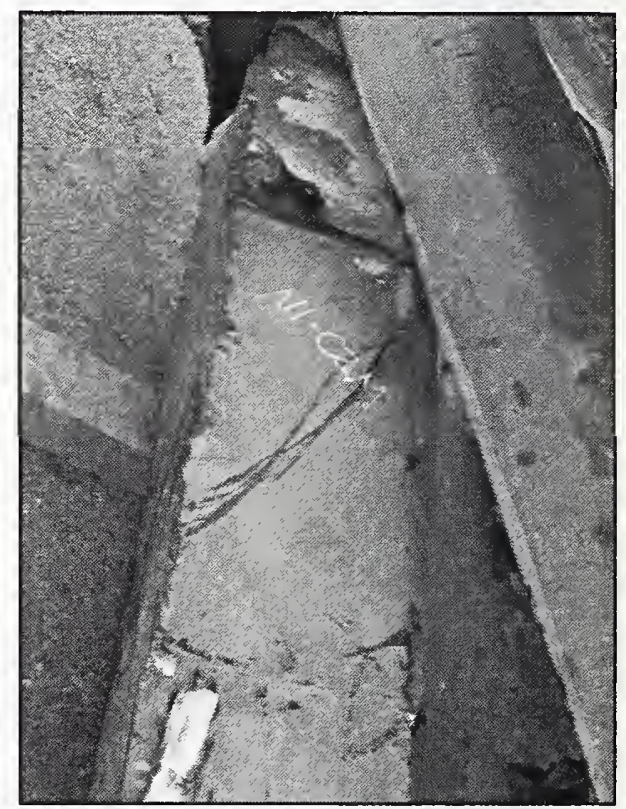

N1-C2B (WTC 1, Col.218, Fl 83)

Type C

Seat Detail: 5210

Gusset plate remains, bent downwards, with diagonal bracing strap attached.

Figure B-28. Floor truss connectors found on recovered panel N-1 (A218: 82-85) from WTC 1. Shown are connectors found on column 218 at different floor levels (cont.). 
g)

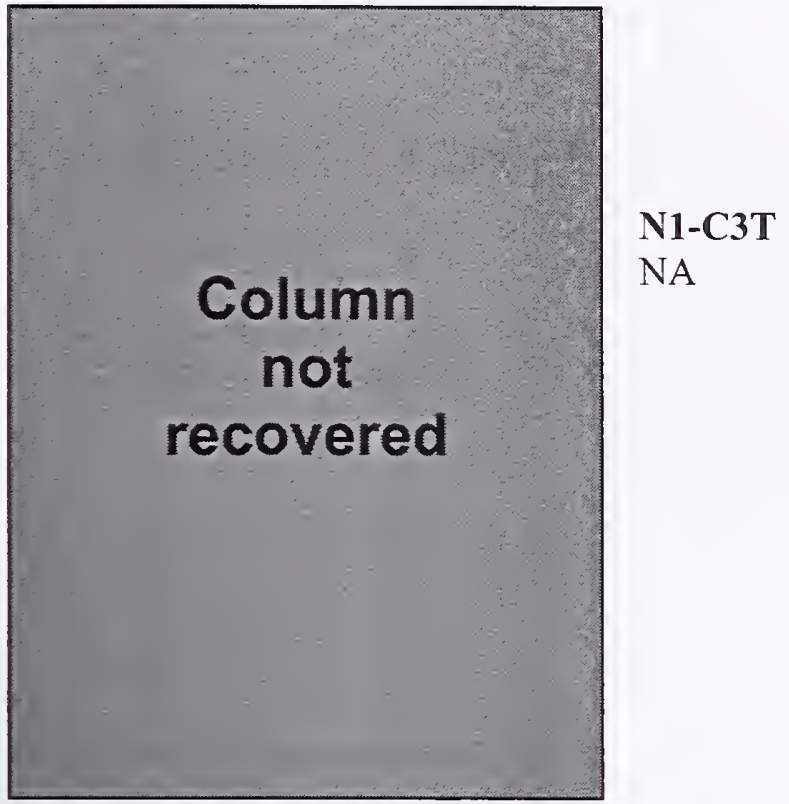

h)

N1-C3M

NA

i)

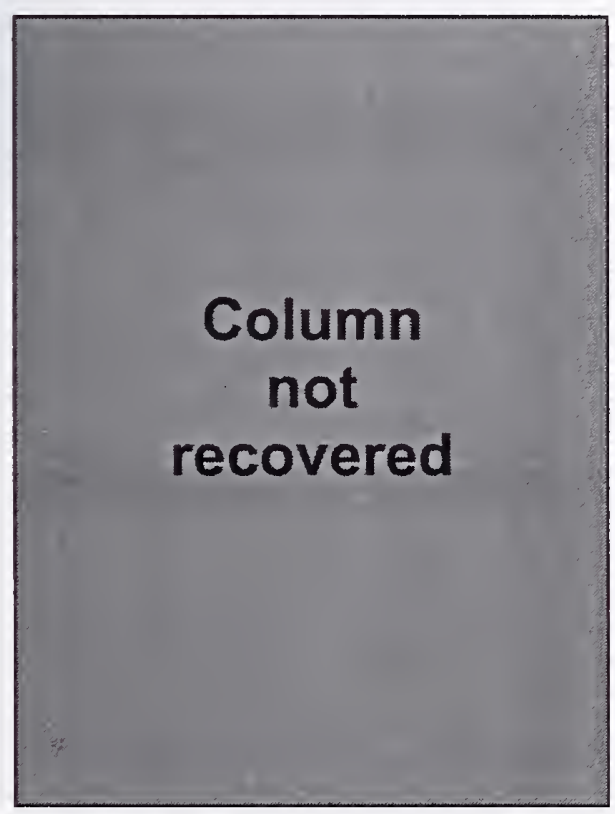

Column not

recovered

N1-C3B

NA

Figure B-28. Floor truss connectors found on recovered panel N-1 (A218: 82-85) from WTC 1. Column 217 was not recovered (cont.). 
a)

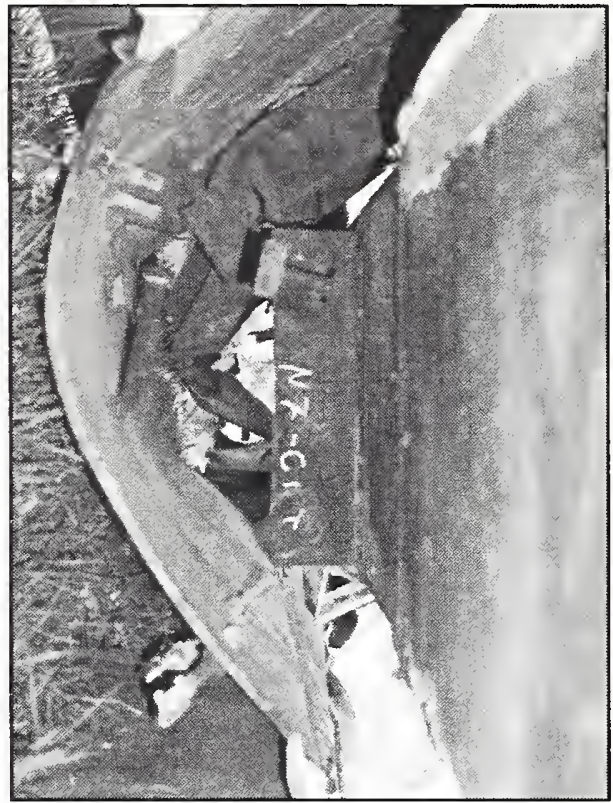

N7-C1M (WTC 1, Col.128, F1 99)

Type C

Seat Detail: 5210

Portion of gusset plate remains, bent downward.

c)

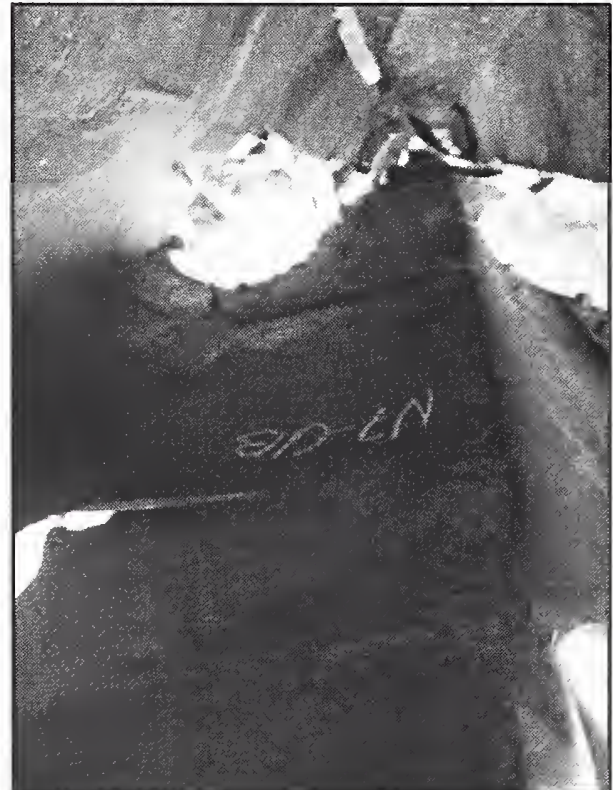

N7-C1T (WTC 1, Col.128, Fl 100)

Type C

Seat Detail: 5210

Gusset plate intact, bent upwards, with 2 diagonal bracing straps attached

b)

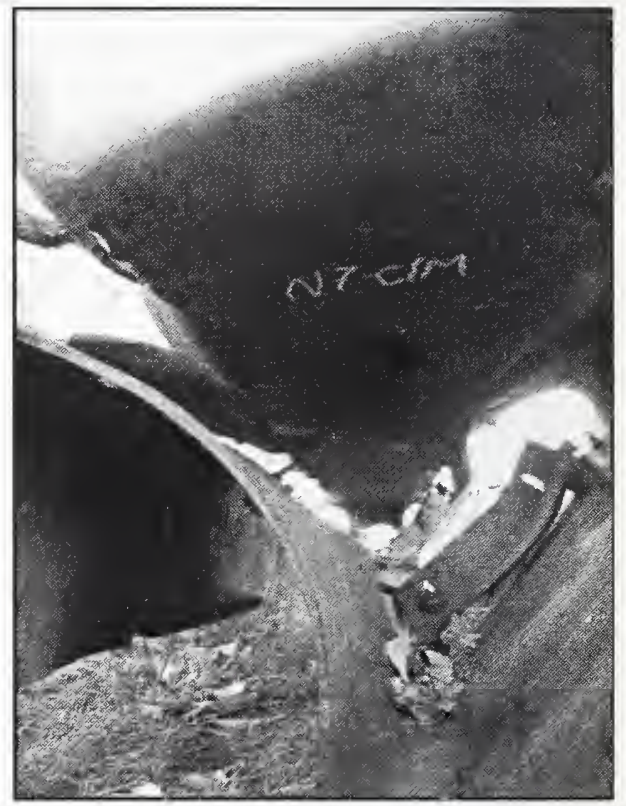

N7-C1B (WTC 1, Col.128, FI 98)

Type C

Seat Detail: 5210

Gusset plate ripped off at weld.

Figure B-29. Floor truss connectors found on recovered panel N-7 (A127: 97-100) from WTC 1. Shown are connectors found on column 128 at different floor levels. 
d)

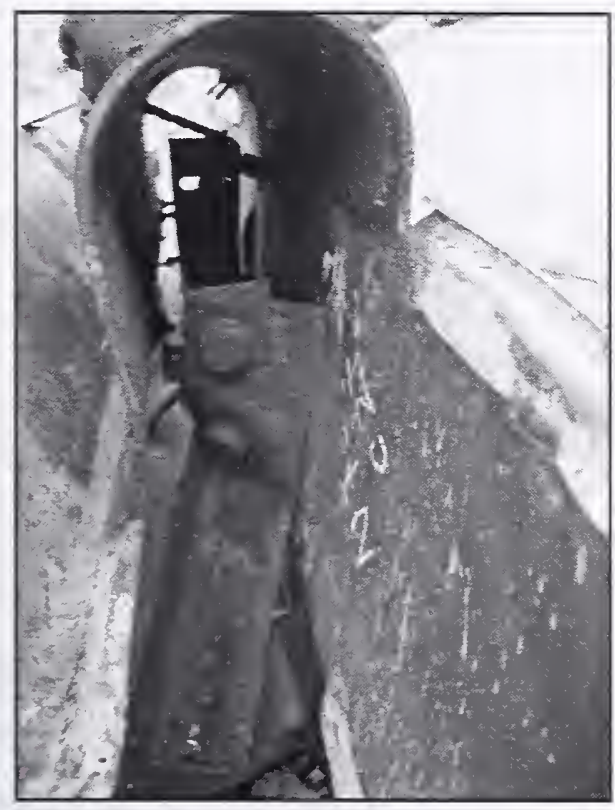

N7-C2M (WTC 1, Col.127, Fl 99)

Type A

Seat Detail: 1411

Gusset plate ripped off at weld;

Seat intact, bent in towards column, both bolt holes intact, 1 with bolt and nut;

Gusset plate for damper unit ripped off at weld.

f)

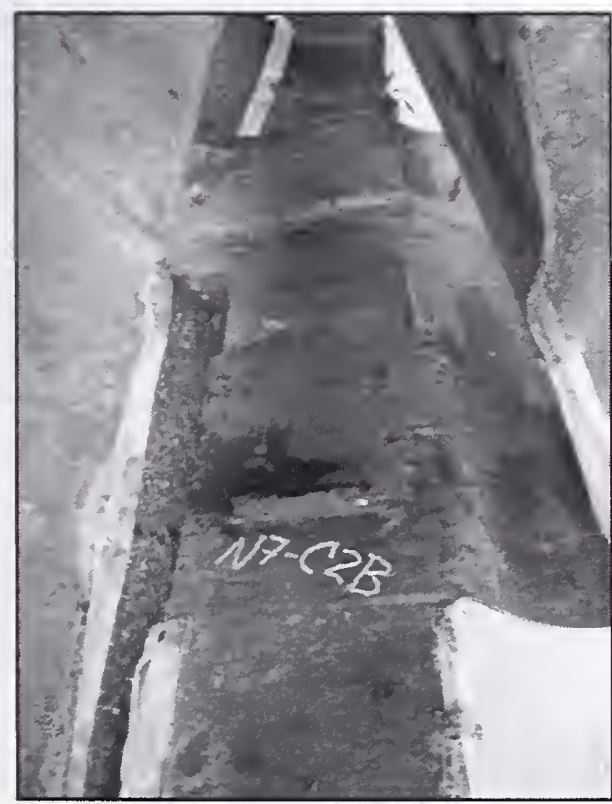

N7-C2T (WTC 1, Col.127, Fl 100)

Type A

Seat Detail: 1411

Gusset plate completely ripped out of spandrel; Seat intact, partially bent towards column, 1 bolt hole intact, other ripped out, both bolts and nuts remain;

Gusset plate with damper unit intact, bent upwards, 1 damper bent up, other bent down.

e)

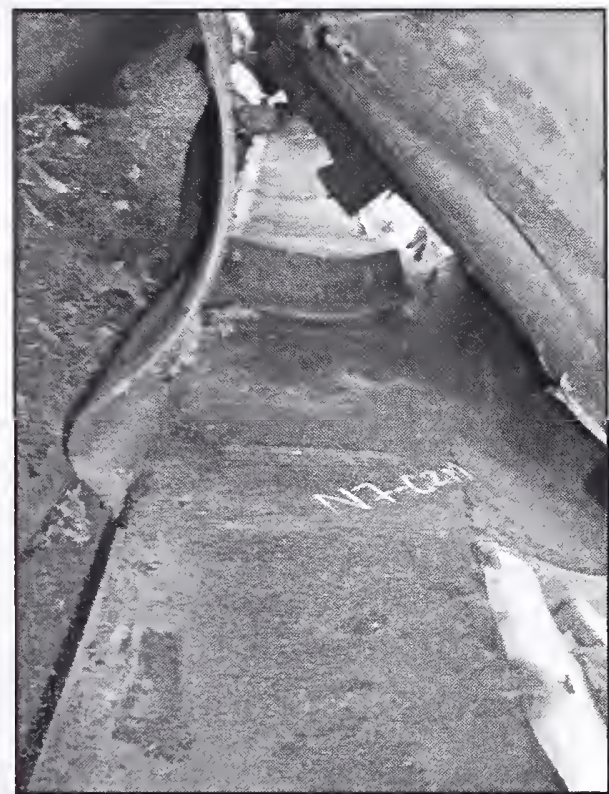

N7-C2B (WTC 1, Col.127, Fl 98)

Type A

Seat Detail: 1411

Gusset plate ripped off at weld;

Standoff plates ripped off at welds;

Gusset plate for damper unit ripped off at weld.

Figure B-29. Floor truss connectors found on recovered panel N-7 (A127: 97-100) from WTC 1. Shown are connectors found on column 127 at different floor levels (cont.). 
g)

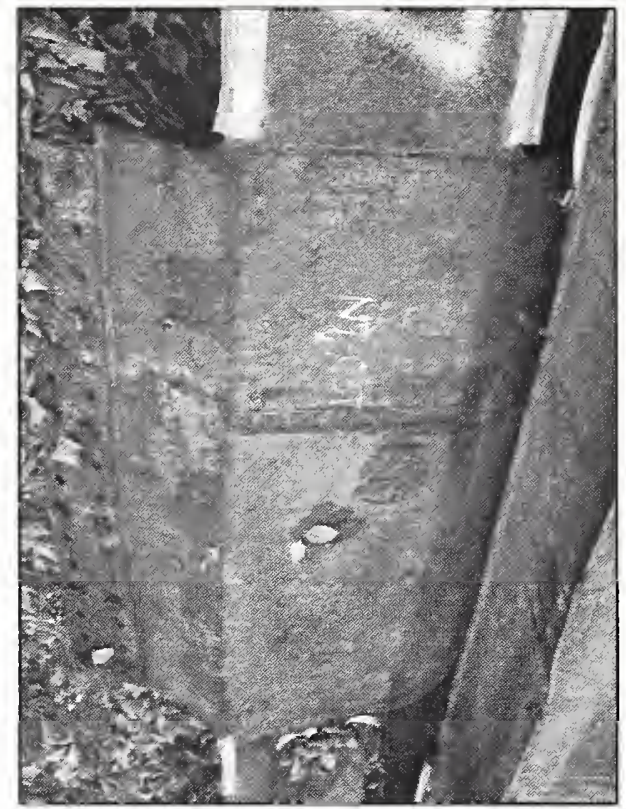

N7-C3T (WTC 1, Col.126, Fl 100)

Type C

Seat Detail: 5210

Gusset plate ripped off at welds.

N7-C3M (WTC 1, Col.126, FI 99)

Type C

Seat Detail: 5210

Gusset plate ripped off at welds.

i)

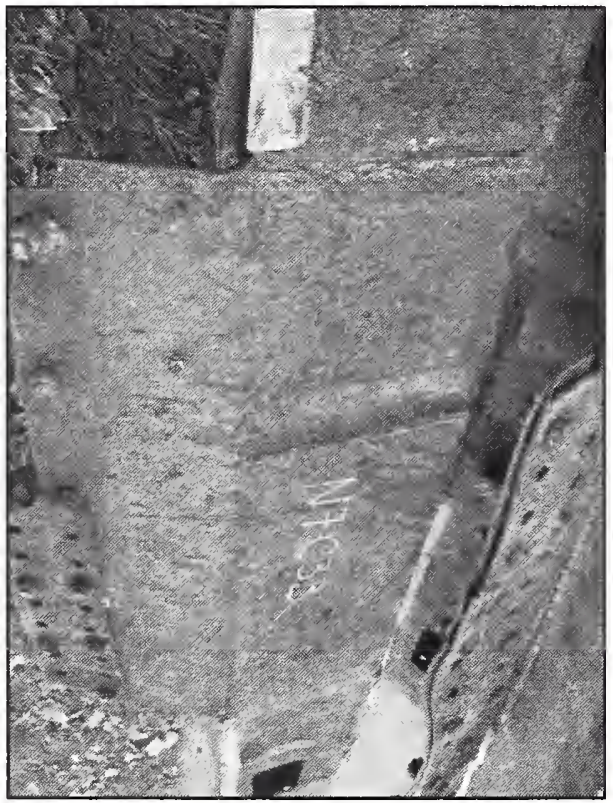

h)

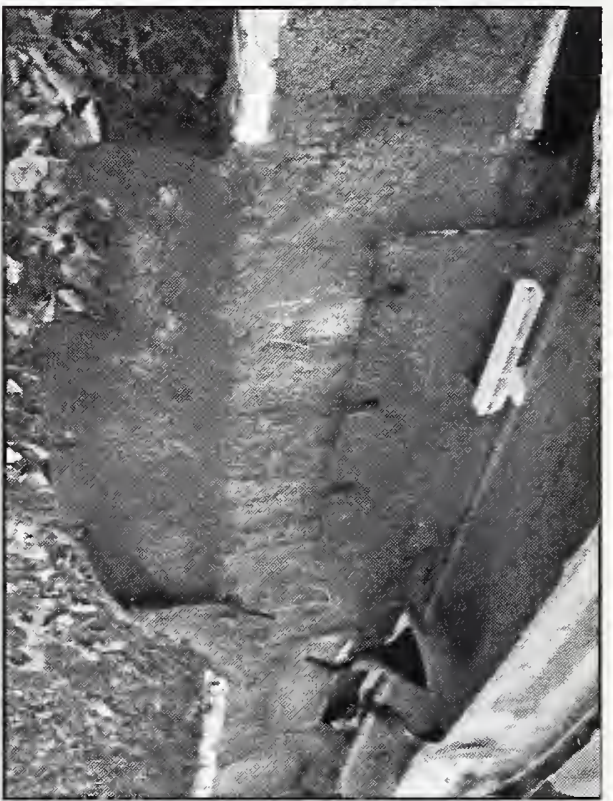

N7-C3B (WTC 1, Col.126, F1 98)

Type C

Seat Detail: 5210

Gusset plate ripped off at welds.

Figure B-29. Floor truss connectors found on recovered panel N-7 (A127: 97-100) from WTC 1. Shown are connectors found on column 126 at different floor levels (cont.). 
a)

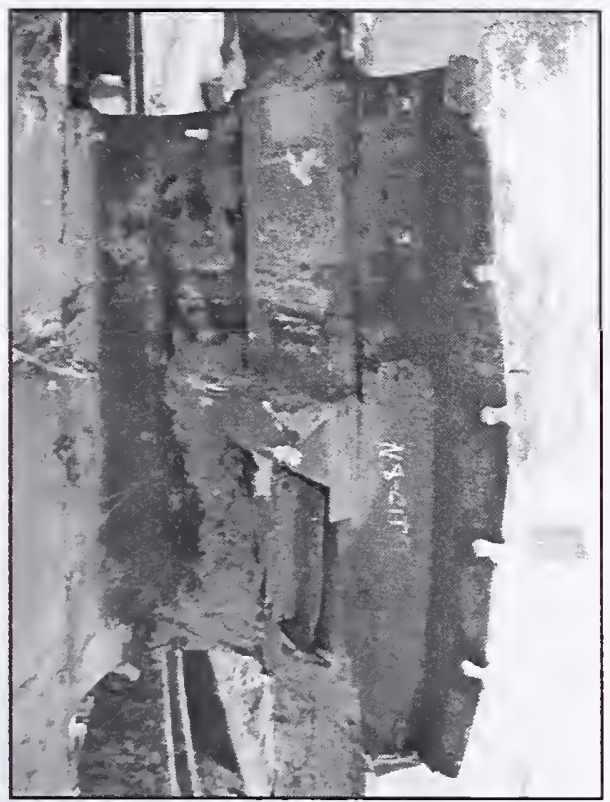

N8-C1M (WTC 1, Col.143, Fl 99)

Type A

Seat Detail: 1411

Gusset plate intact;

Seat intact, partially bent downwards, both bolt holes intact with bolts and nuts;

Gusset for damper unit ripped off at weld.

c)

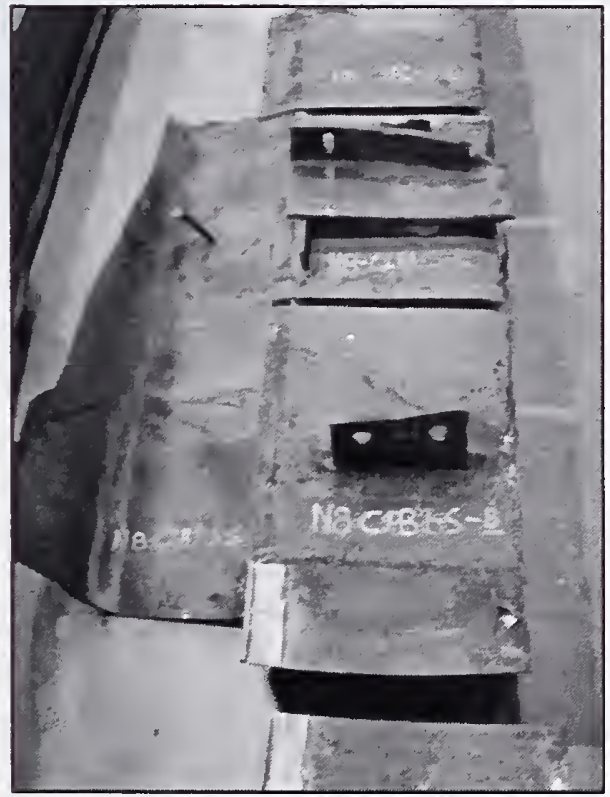

N8-C1T (WTC 1, Col.143, FI 100)

Type A

Seat Detail: 1411

Gusset plate intact;

Seat intact, partially bent downwards, both bolt holes intact, 1 with bolt and nut;

Gusset with damper unit intact, bent upwards. b)

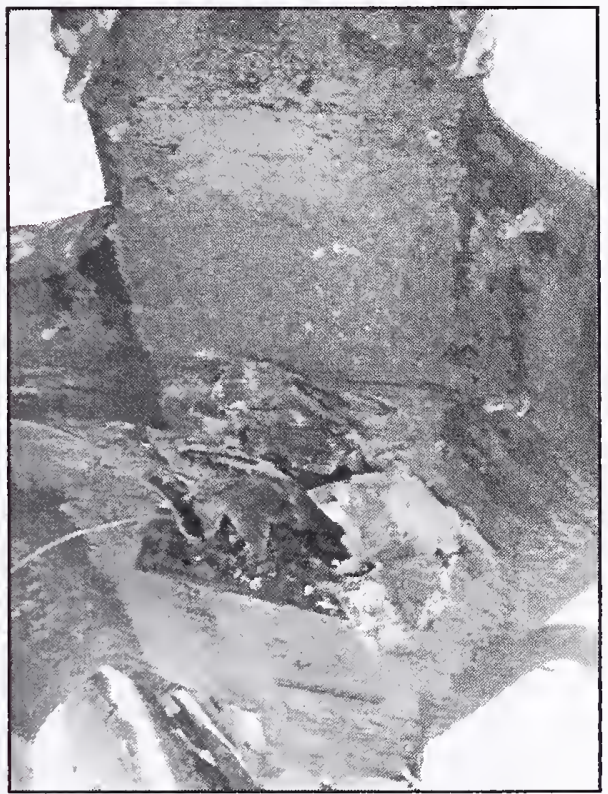

N8-C1B (WTC 1, Col.143, Fl 98)

Type A

Seat Detail: 1411

Portion of gusset plate remains;

Seat intact, partially bent downwards, one bolt hole intact, other ripped out;

Gusset plate for damper unit intact, bolt holes intact, damper unit missing

Figure B-30. Floor truss connectors found on recovered panel N-8 (A142: 97-100) from WTC 1. Shown are connectors found on column 143 at different floor levels. 
d)

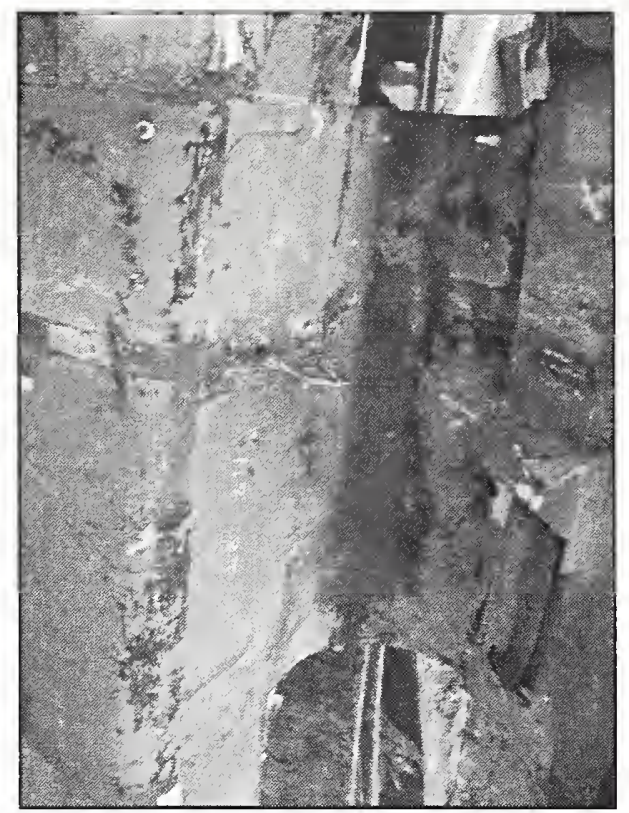

N8-C2M (WTC 1, Col.142, F1 99)

Type C

Seat Detail: 5210

Gusset plate intact with portion of diagonal bracing strap attached, bent downwards. f)

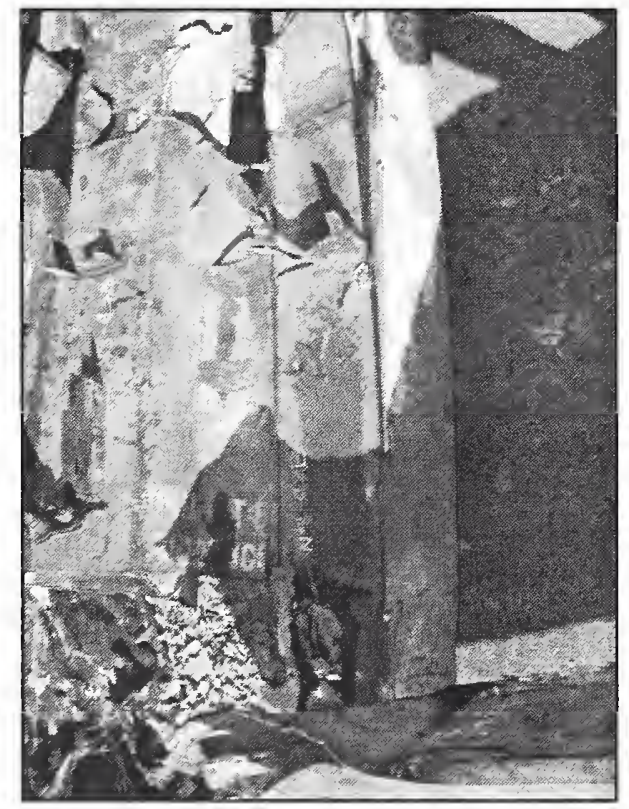

N8-C2T (WTC 1, Col.142, Fl 100)

Type C

Seat Detail: 5210

Portion of gusset plate remains, other part ripped off at weld, half bent upwards, half bent downwards, portion of diagonal bracing strap remains. e)

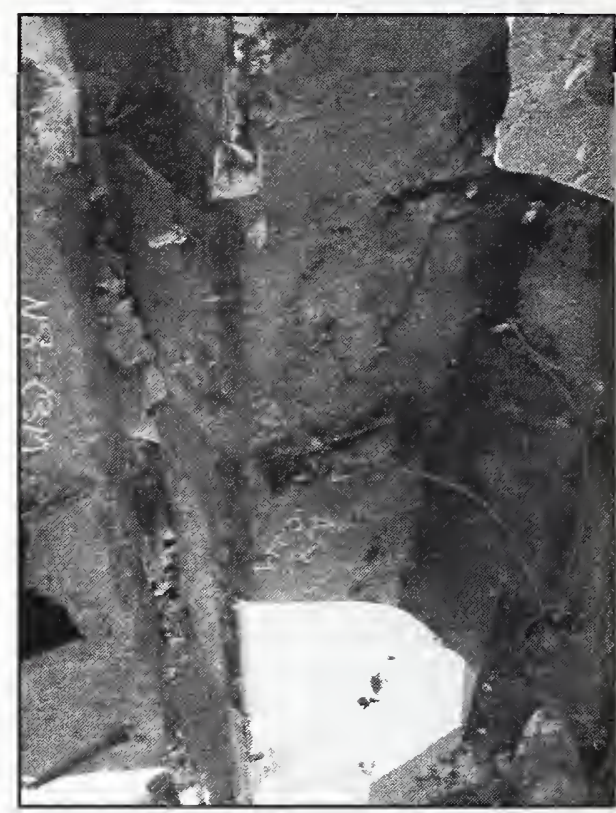

N8-C2B (WTC 1, Col.142, Fl 98)

Type C

Seat Detail: 5210

Gusset plate intact with portion of diagonal bracing strap attached, half bent upwards, other half bent downwards.

Figure B-30. Floor truss connectors found on recovered panel N-8 (A142: 97-100) from WTC 1. Shown are connectors found on column 142 at different floor levels (cont.). 
g)

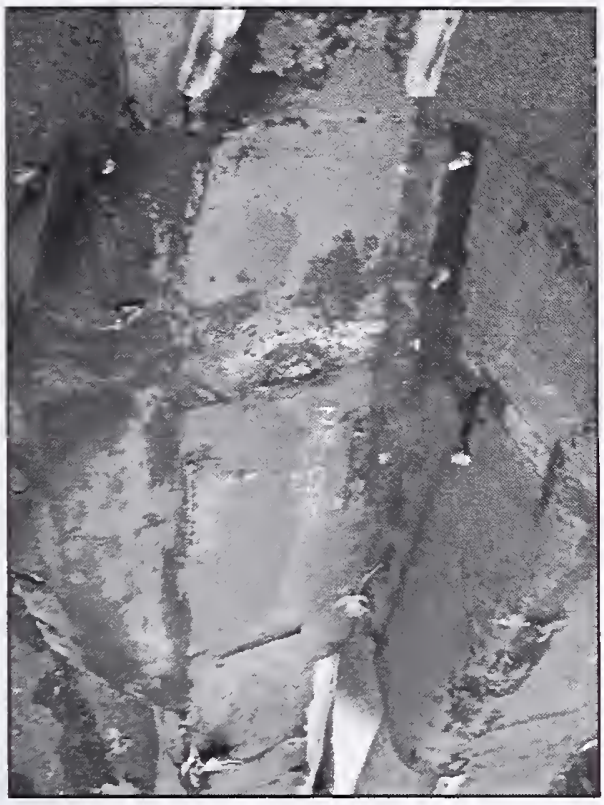

N8-C3M (WTC 1, Col.141, Fl 99)

Type A

Seat Detail: 1411

Portion of gusset plate intact;

Seat intact, both bolt holes intact with bolts and nuts;

Gusset plate for damper unit ripped off at weld.

i)

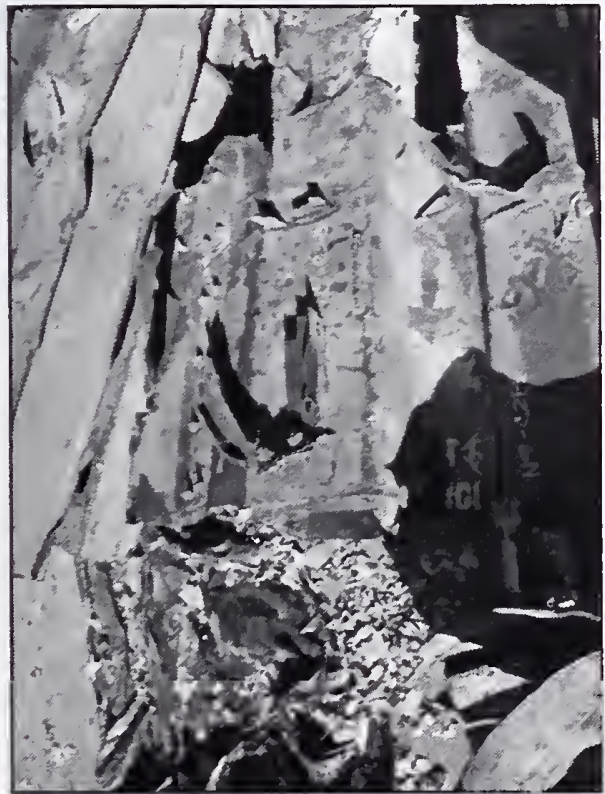

\section{N8-C3T (WTC 1, Col.141, FI 100)}

Type A

Seat Detail: 1411

Gusset plate ripped completely out of spandrel; Seat intact, 1 bolt hole intact with bolt and nut, other ripped out;

Gusset plate for damper unit ripped out at weld.

h)

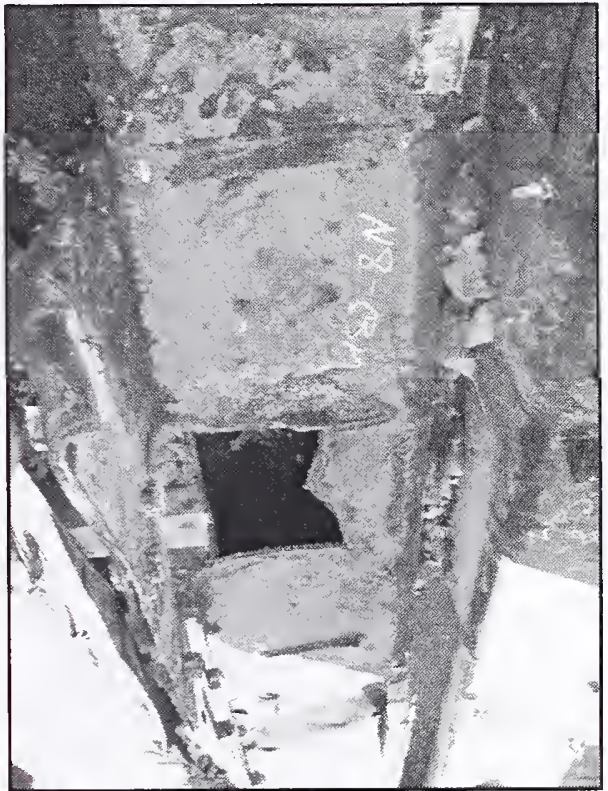

N8-C3B (WTC 1, Col.141, Fl 98)

Type A

Seat Detail: 1411

Portion of gusset plate intact;

Seat intact, 1 bolt hole intact with bolt and nut, other ripped out;

Gusset plate with damper unit bent downwards.

Figure B-30. Floor truss connectors found on recovered panel N-8 (A142: 97-100) from WTC 1. Shown are connectors found on column 141 at different floor levels. Seats on this column were removed and sent to NIST, Boulder, $\mathrm{CO}$, for mechanical property testing. See next page for removed sample images (cont.). 
j)

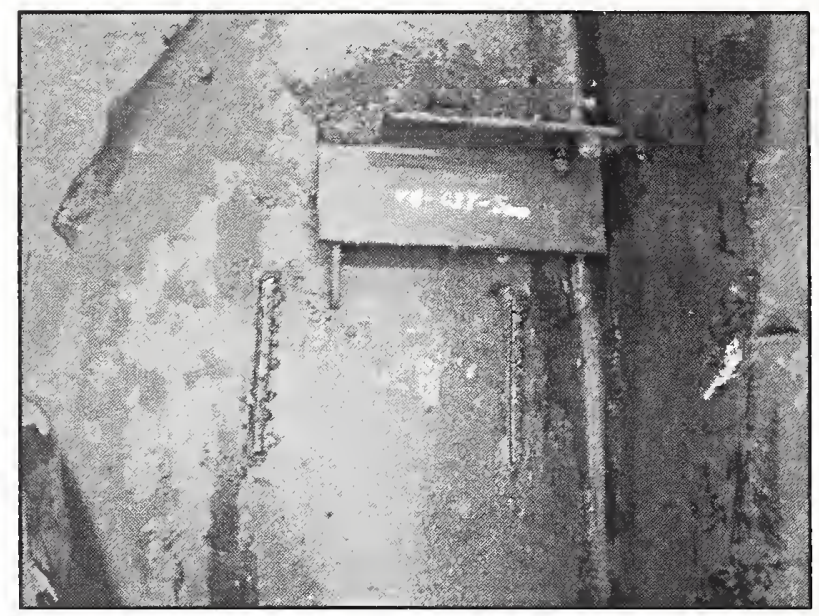

N8-C3T (WTC 1, Col.141, Fl 100)

Type A

Seat Detail: 1411

Cut for Boulder on 10/02

N8-C3M (WTC 1, Col.141, Fl 99)

Type A

Seat Detail: 1411

Cut for Boulder on 10/02

k)

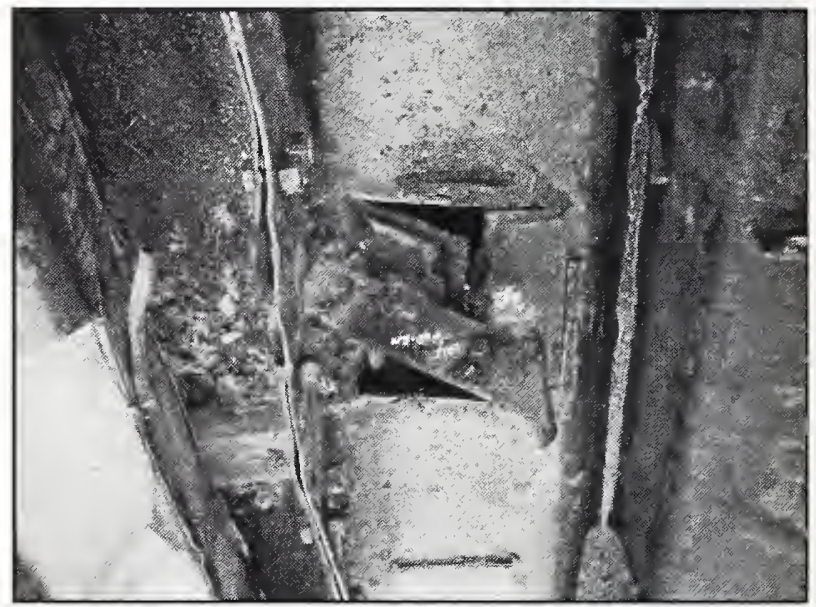

1)

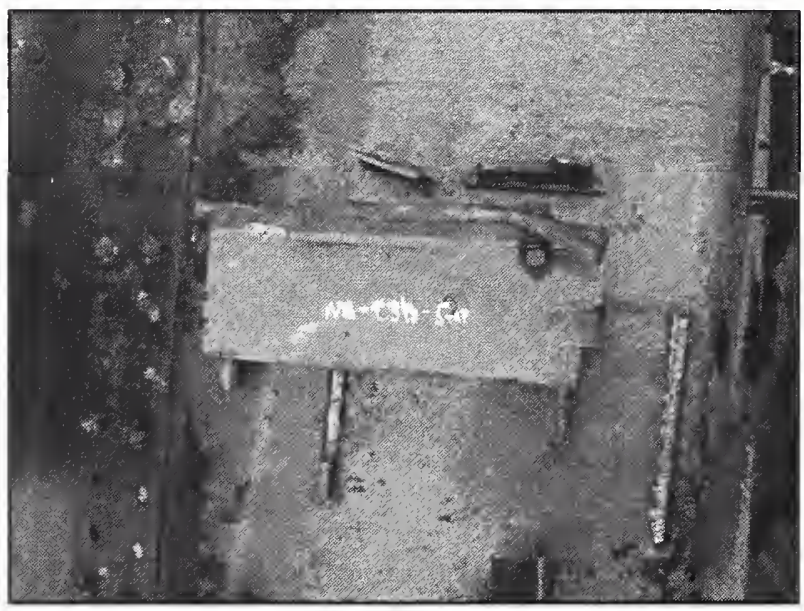

N8-C3B (WTC 1, Col.141, Fl 98)

Type A

Seat Detail: 1411

Cut for Boulder on 10/02

Figure B-30. Floor truss connectors found on recovered panel N-8 (A142: 97-100) from WTC 1. Shown are connectors found on column 141 at different floor levels subsequent to their removal for mechanical property testing (cont.). 
a)

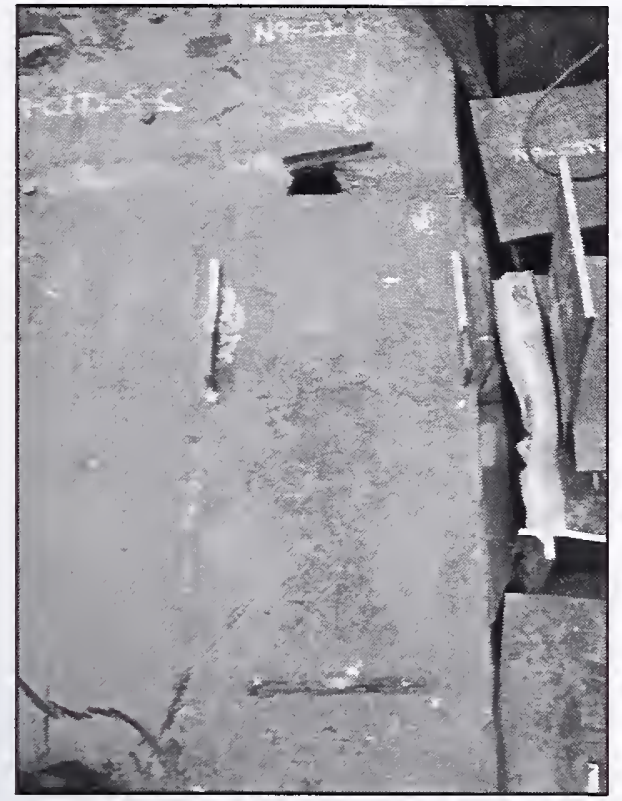

N9-C1M (WTC 1, Col.155, Fl 103)

Type A

Seat Detail: 1311

Gusset plate ripped completely out of spandrel;

Only part of 1 standoff plate remains, rest ripped off at welds;

c)

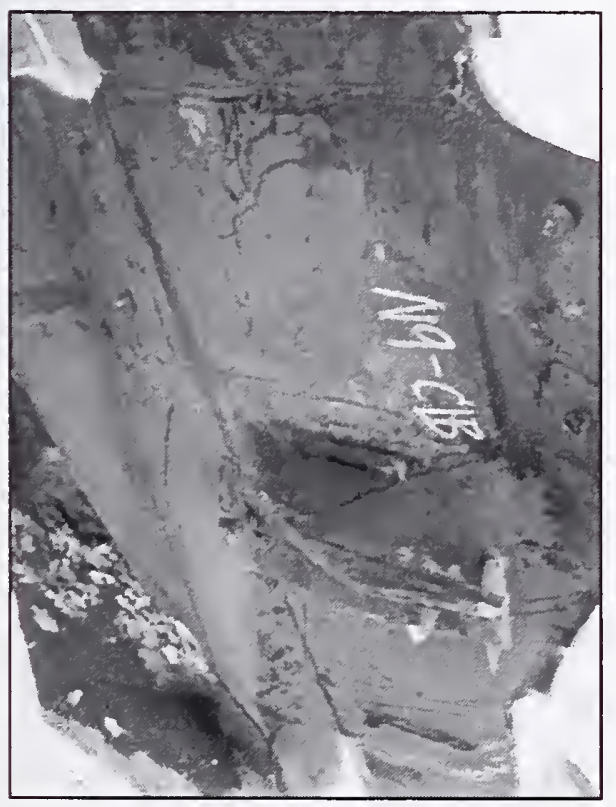

\section{N9-C1T (W'TC 1, Col.155, Fl 104)}

Type A

Seat Detail: 1311

Portion of gusset plate remains, ripped out of spandrel; Seat intact (removed by Boulder prior to picture), both bolt holes intact with bolts and nuts; Gusset for damper unit ripped off at weld. b)

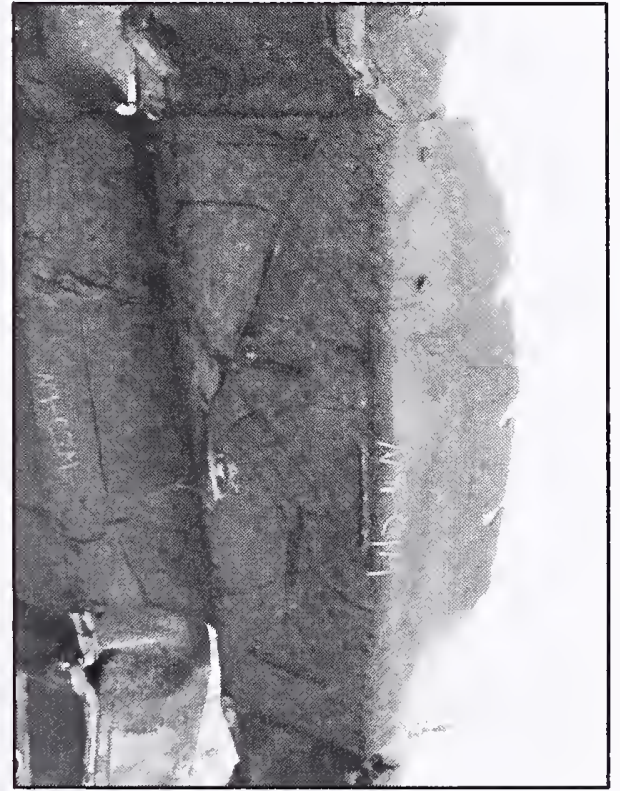

N9-C1B (WTC 1, Col.155, FI 102)

Type A

Seat Detail: 1311

Gusset plate ripped off at weld;

Seat intact, bent upwards, both bolt holes intact with bolts and nuts;

Gusset for damper unit ripped off at weld.

Figure B-31. Floor truss connectors found on recovered panel N-9 (A154: 101-104) from WTC 1. Shown are connectors found on column 155 at different floor levels. 
d)

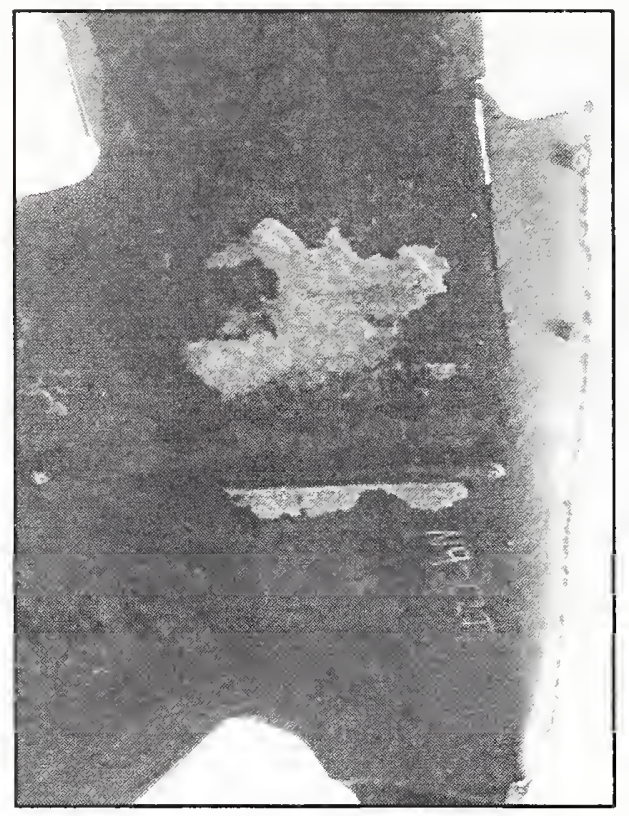

N9-C2M (WTC 1, Col.154, Fl 103)

Type C

Seat Detail: 5110

Gusset plate ripped off at weld.

f)

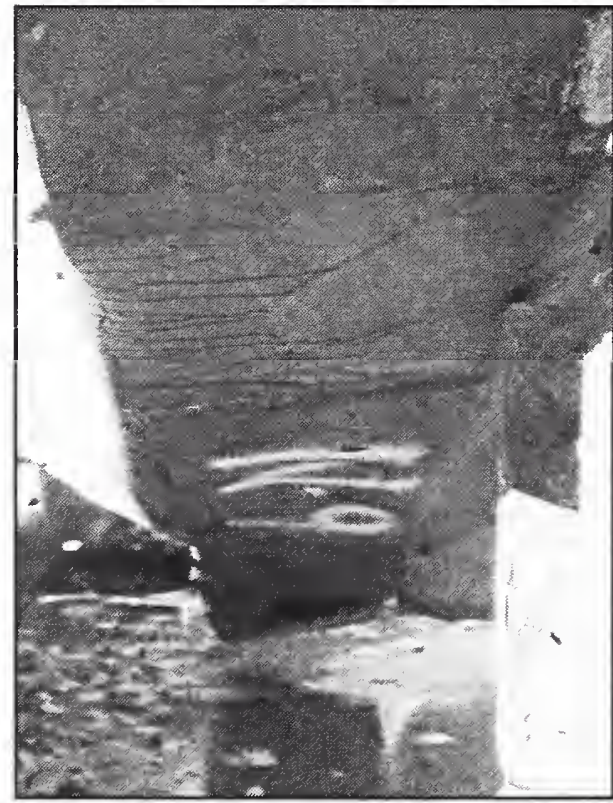

N9-C2T (WTC 1, Col.154, FI 104)

Type C

Seat Detail: 5110

Gusset plate ripped off at weld. Remnants of cement and fire proofing. e)

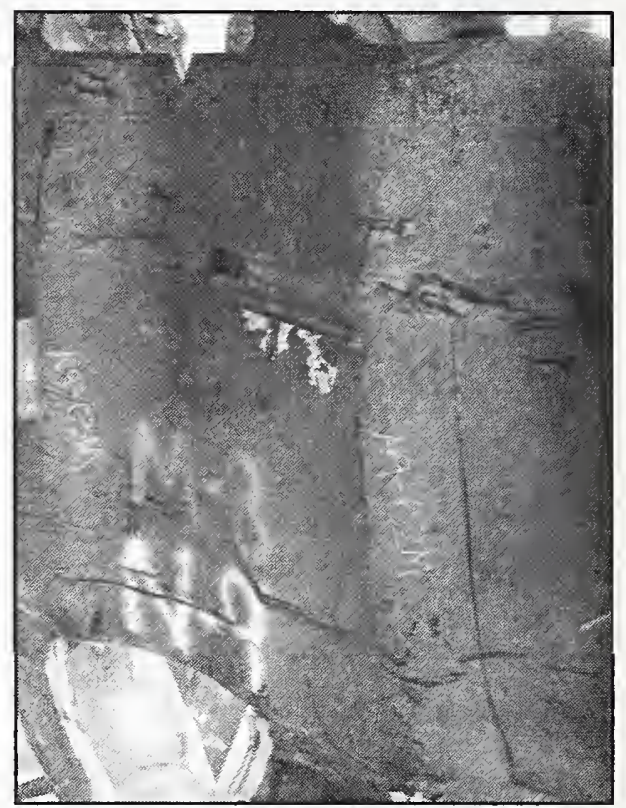

N9-C2B (WTC 1, Col.154, Fl 102)

Type C

Seat Detail: 5110

Gusset plate ripped off at weld.

Figure B-31. Floor truss connectors found on recovered panel N-9 (A154: 101-104) from WTC 1. Shown are connectors found on column 154 at different floor levels (cont.). 
g)

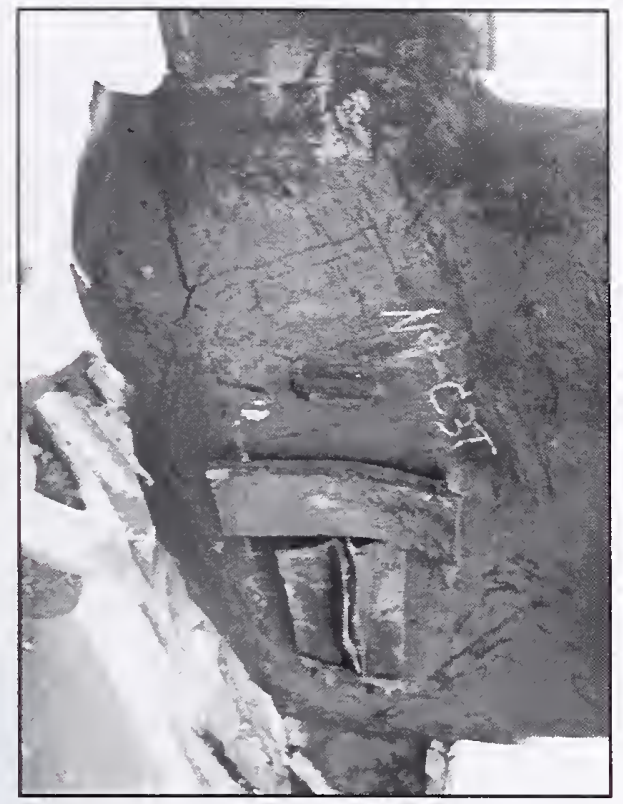

N9-C3M (WTC 1, Col.153, Fl 103)

Type A

Seat Detail: 1411

Gusset plate ripped completely out of spandrel;

Seat intact, both bolt holes intact with bolts and nuts; Gusset for damper unit ripped off at weld.

i)

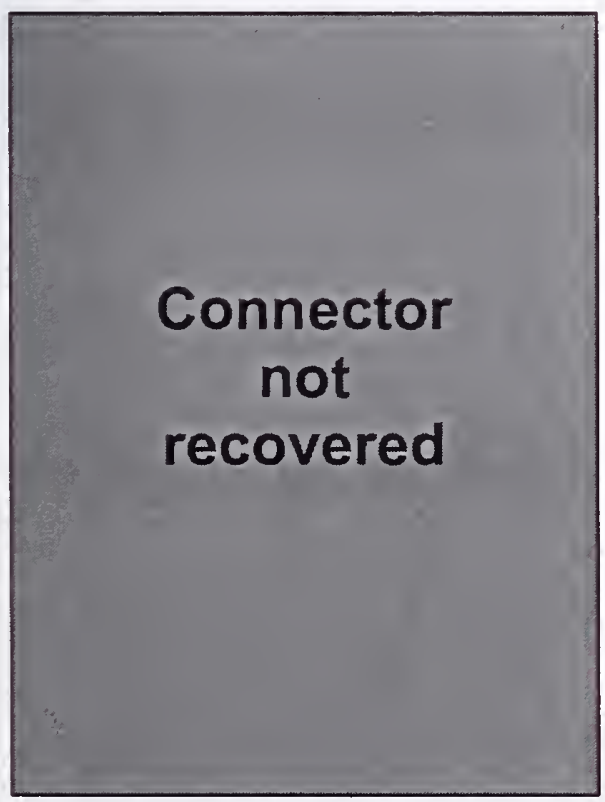

N9-C3T (WTC 1, Col.153, Fl 104)

Type A

Seat Detail: 1411

Portion of gusset plate remains;

Portion of seat is left (vertical part attached to shear plates);

Gusset with damper unit intact, bent upwards.

h)

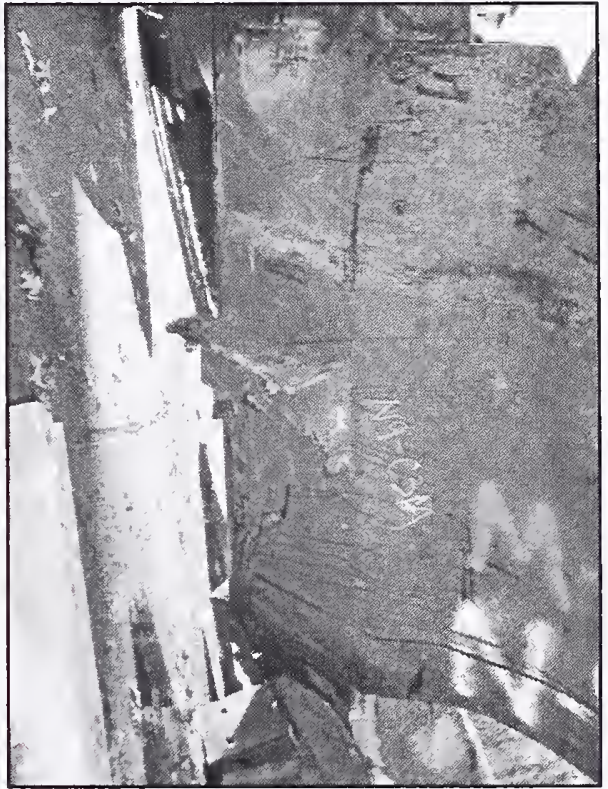

N9-C3B

NA

Figure B-31. Floor truss connectors found on recovered panel N-9 (A154: 101-104) from WTC 1. Shown are connectors found on column 153 at different floor levels (cont.). 
a)

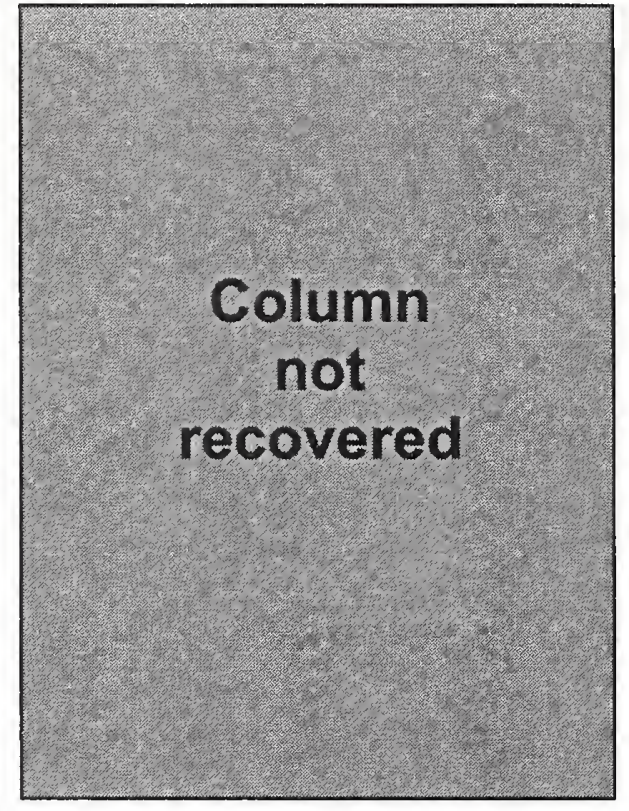

N10-C1M

NA
N10-C1T

NA c)

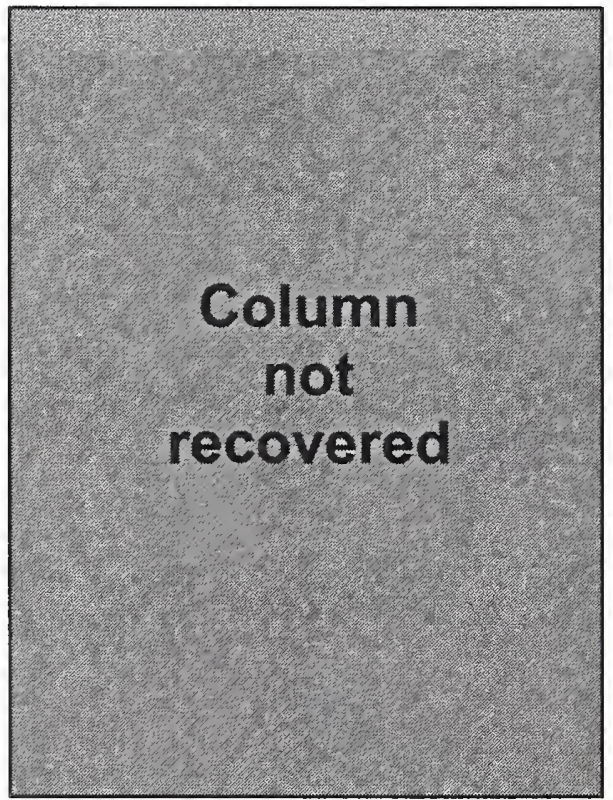

\section{Column not recovered}

b)

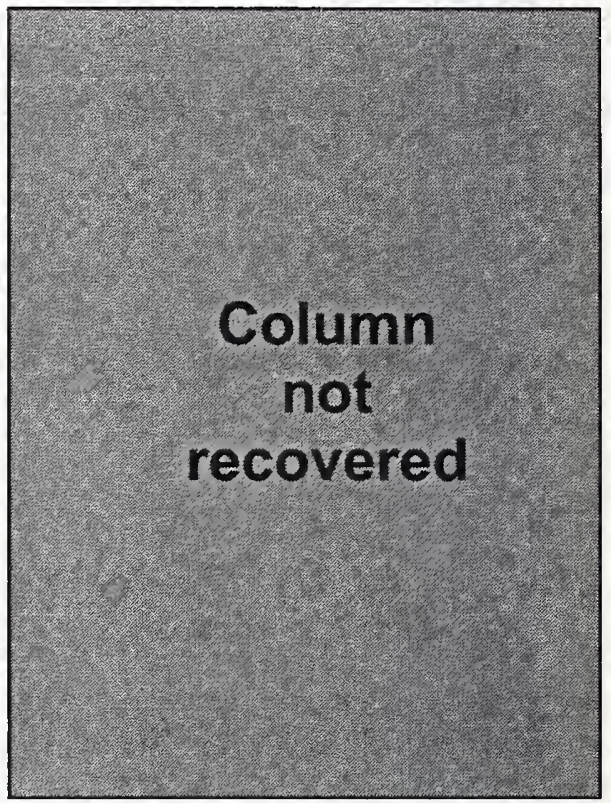

N10-C1B

NA

Figure B-32. Floor truss connectors found on recovered panel N-10 (A115: 89-92) from WTC 1. Column 116 was not recovered. 
d)

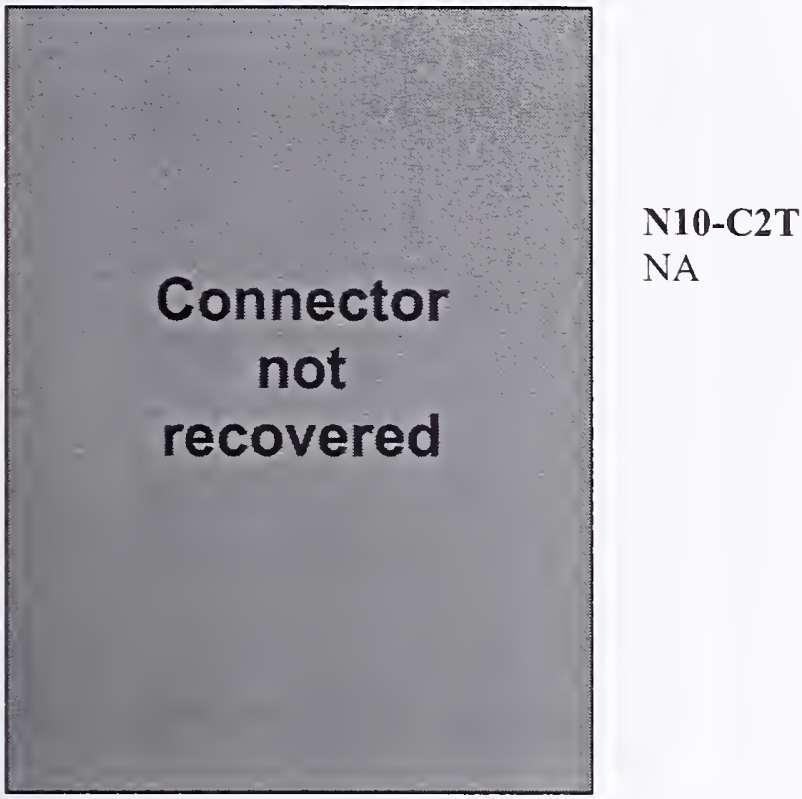

N10-C2M (WTC 1, Col.115, Fl 91)

NA

Seat Detail: 1411

Gusset plate ripped off at weld;

Seat remains, bent downward, both bolt holes intact;

Gusset with dampers intact, bent downwards.

f)

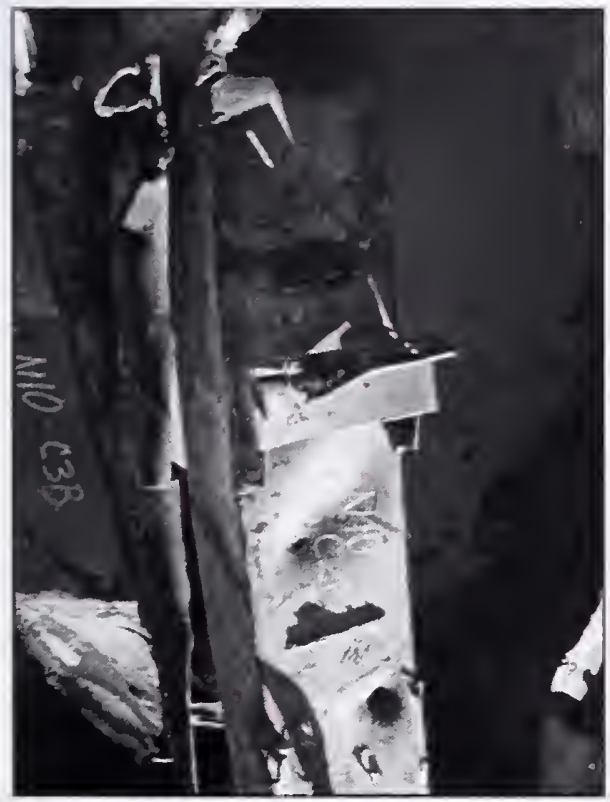

e)

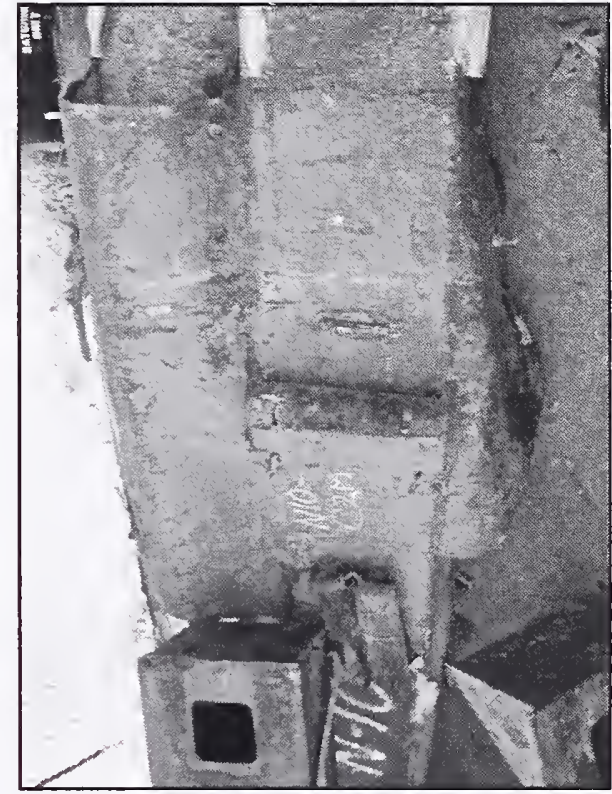

N10-C2B (WTC 1, Col.115, Fl 90)

Type A

Seat Detail: 1411

Gusset plate ripped completely out of spandrel;

Seat intact, bent downward, 1 bolt hole remains intact, other ripped out;

Gusset plate for damper unit ripped off at weld.

Figure B-32. Floor truss connectors found on recovered panel N-10 (A115: 89-92) from WTC 1. Shown are connectors found on column 115 at different floor levels (cont.). 
g)

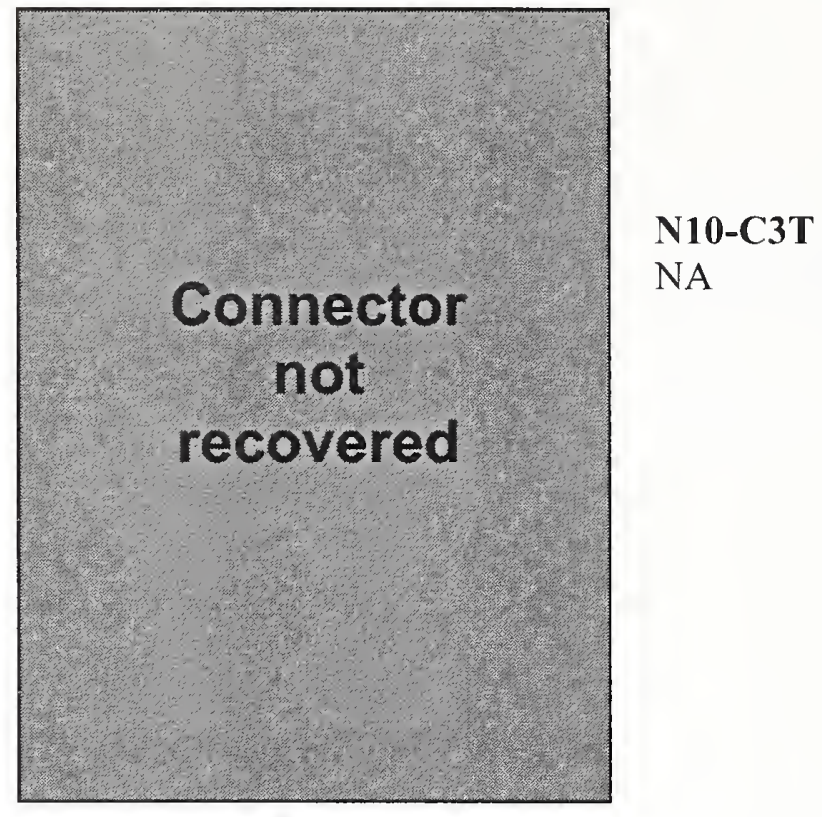

N10-C3M (WTC 1, Col.114, F1 91)

Type C

Seat Detail: 5110

Gusset plate remains, bent downwards, with portion of diagonal bracing strap still attached.

h)

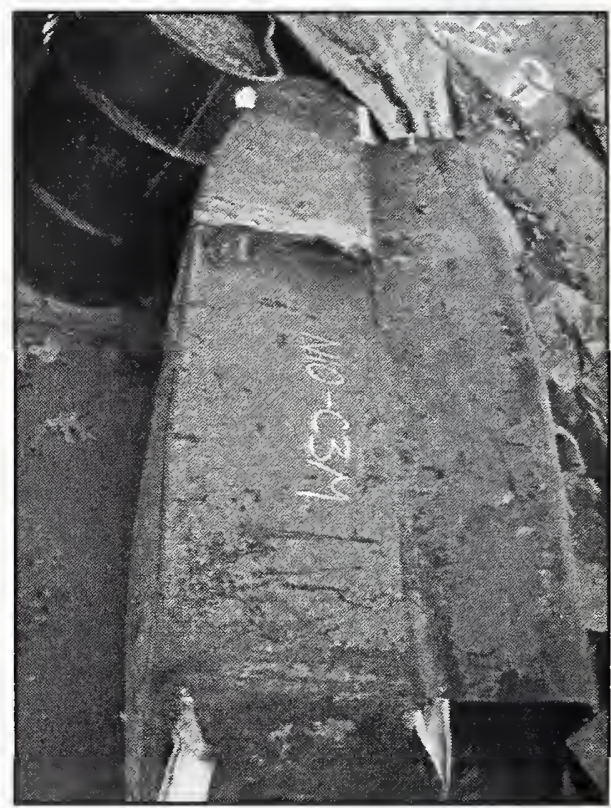

i)

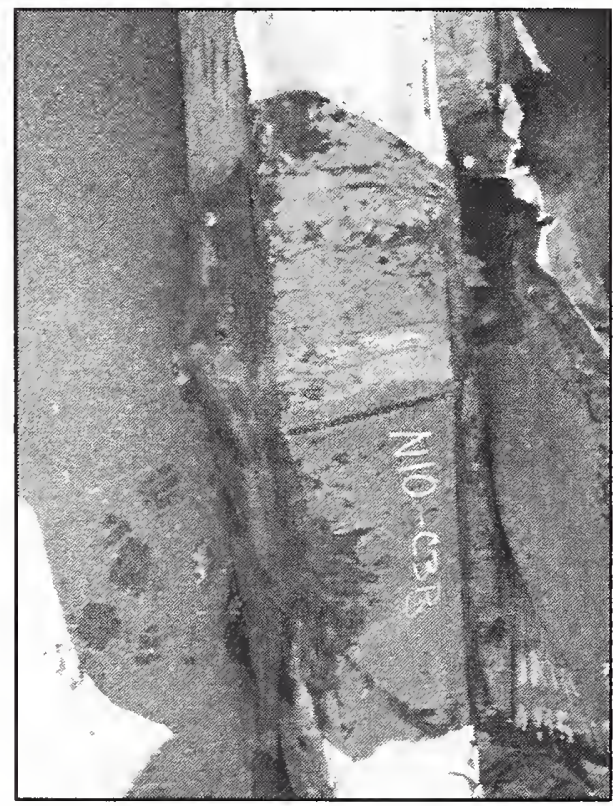

N10-C3B (WTC 1, Col.114, Fl 90)

Type C

Seat Detail: 5110

Gusset plate ripped off at weld.

Figure B-32. Floor truss connectors found on recovered panel N-10 (A115: 89-92) from WTC 1. Shown are connectors found on column 114 at different floor levels (cont.). 
a)

Column not recovered
N12-C1T

NA
N12-C1M

NA

c)

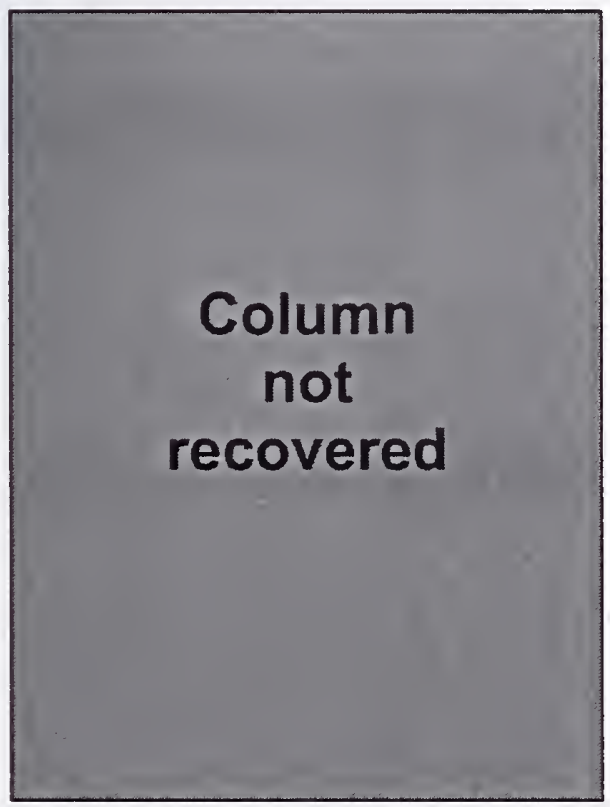

b)

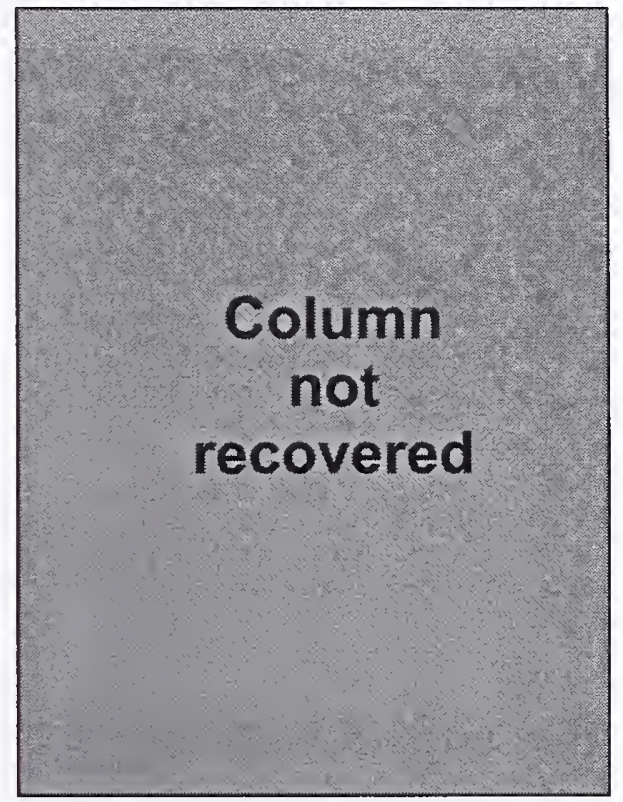

N12-C1B

NA

Figure B-33. Floor truss connectors found on recovered panel N-12 (A206: 92-95) from WTC 1. Column 207 was not recovered. 
d)

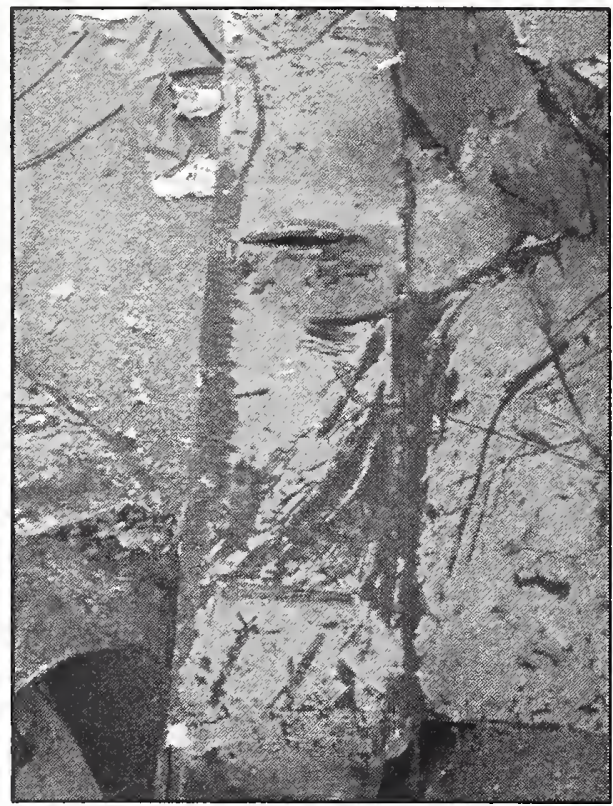

N12-C2M (WTC 1, Col.206, Fl 94)

Type A

Seat Detail: 1212

Gusset plate intact, bent downwards;

Seat intact, bent downwards and in towards column, one bolt hole intact, other ripped out; Gusset plate for damper unit ripped off at weld.

f)

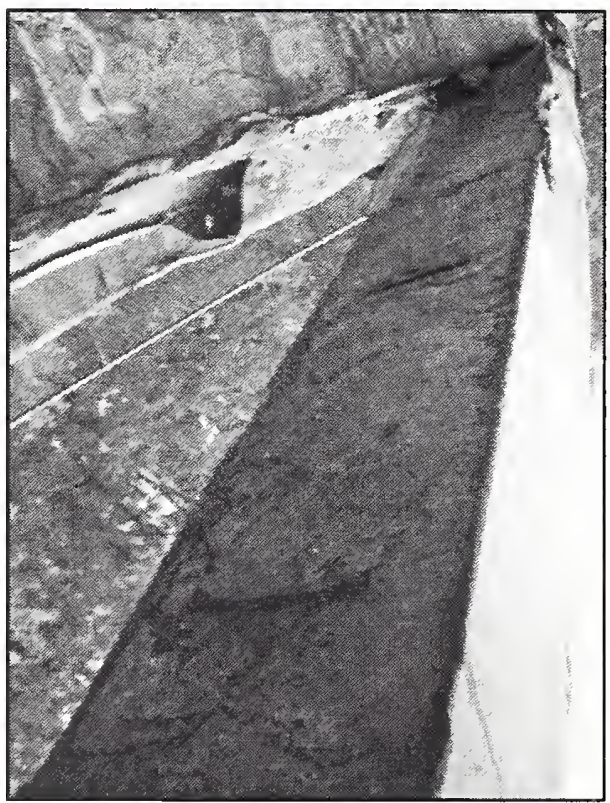

N12-C2T (WTC 1, Col.206, Fl 95)

Type A

Seat Detail: 1212

Gusset plate ripped completely out of spandrel; Only evidence of 1 standoff plate being welded to spandrel, ripped off at weld;

Gusset for damper unit ripped off at weld. e)

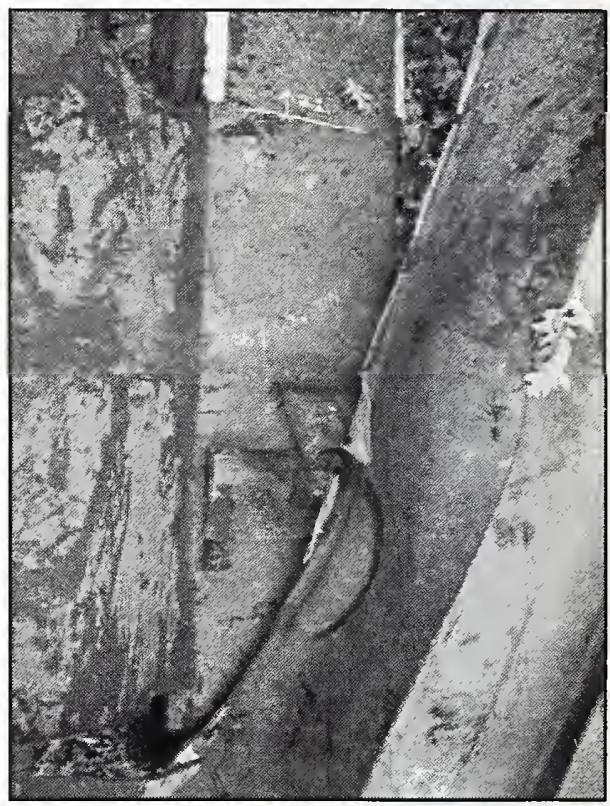

N12-C2B (WTC 1, Col.206, Fl 93)

Type A

Seat Detail: 1212

Gusset plate ripped completely out of spandrel;

No evidence of standoff plates being welded onto spandrel;

Gusset plate for damper unit ripped off at weld.

Figure B-33. Floor truss connectors found on recovered panel N-12 (A206: 92-95) from WTC 1. Shown are connectors found on column 206 at different floor levels (cont.). 
g)

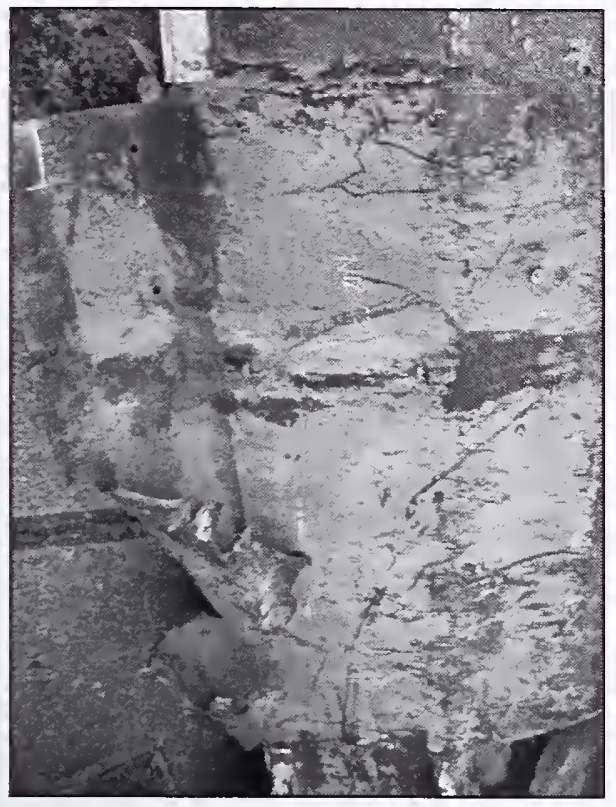

N12-C3T (WTC 1, Col.205, Fl 95)

Type D

Seat Detail: 5110

21 -in portions of diagonal bracing straps remaining

N12-C3M (WTC 1, Col.205, Fl 94)

Type D

Seat Detail: 5110

21 -in portion of diagonal bracing straps remaining.

h)

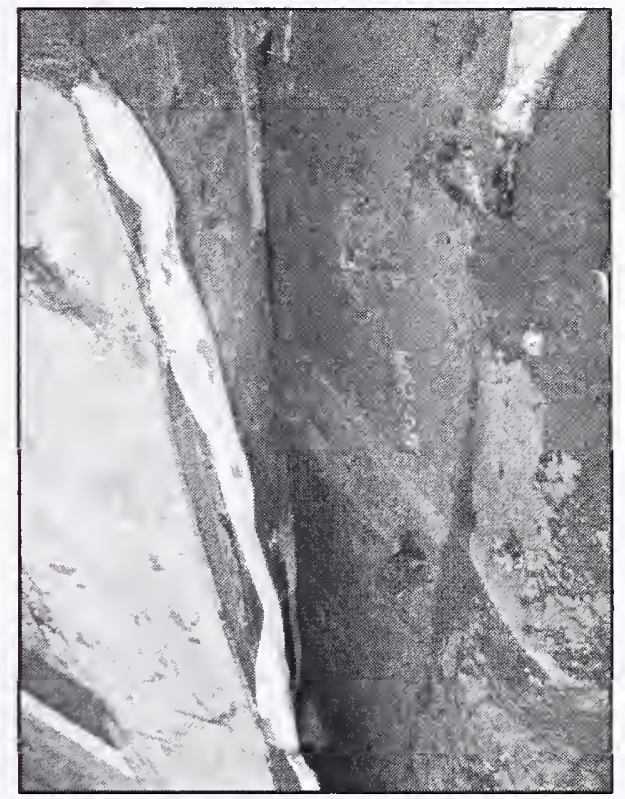

i)

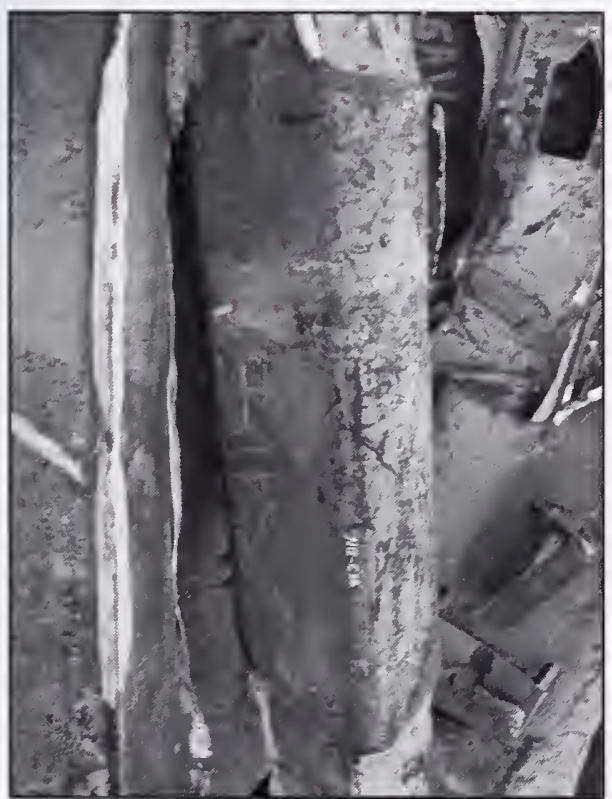

N12-C3B (WTC 1, Col.205, FI 93)

Type D

Seat Detail: 5110

21 -in portions of diagonal bracing straps remaining

Figure B-33. Floor truss connectors found on recovered panel N-12 (A206: 92-95) from WTC 1. Shown are connectors found on column 205 at different floor levels (cont.). 
a)

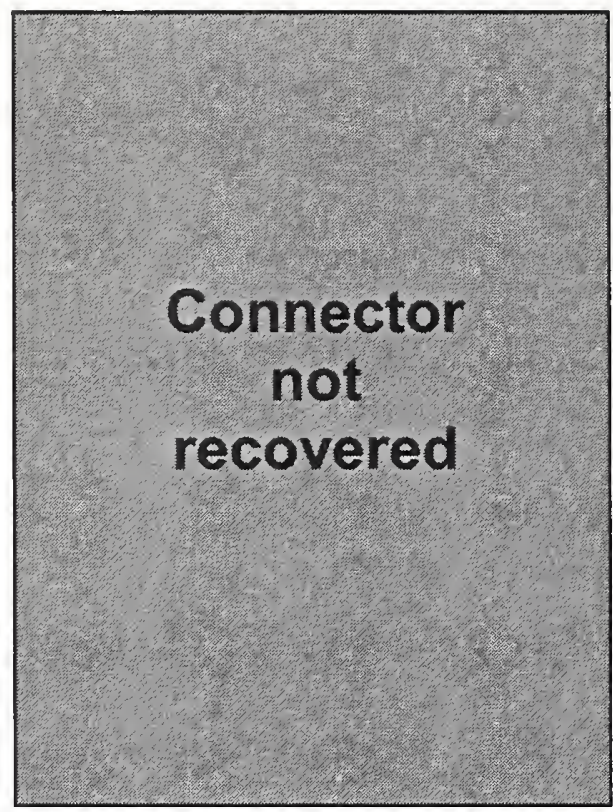

N13-C1M

NA
N13-C1T

NA b)

\section{Connector not} recovered c)

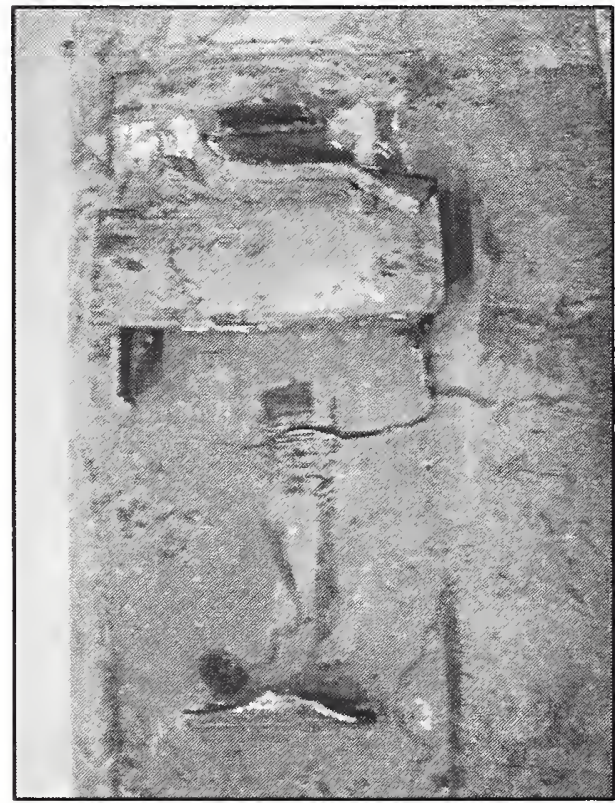

N13-C1B (WTC 1, Col.131, Fl 100)

Type A

Seat Detail: 1411

Gusset plate remains;

Seat intact, bent upwards, 1 bolt hole ripped but still has bolt and nut, other completely ripped out; Portion of gusset plate remains, bent upwards.

Figure B-34. Floor truss connectors found on recovered panel N-13 (A130: 99-102) from WTC 1. Shown are connectors found on column 131 at different floor levels. 
d)

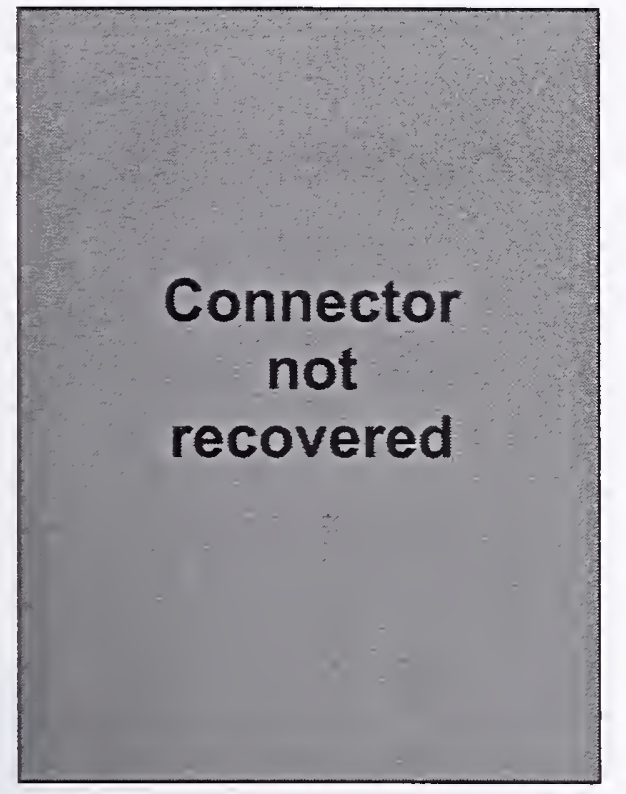

N13-C2M

NA

f)

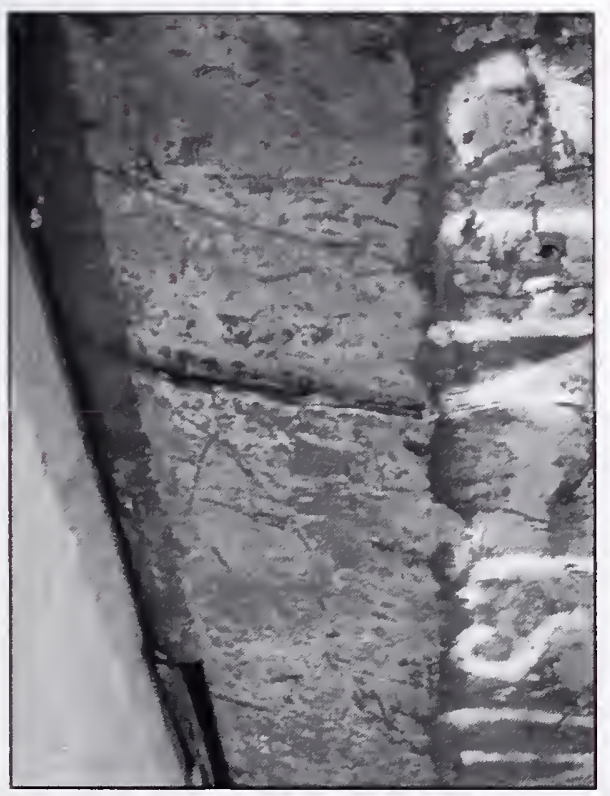

N13-C2T

NA

e)

Connector not recovered

N13-C2B (WTC 1, Col.130, Fl 100)

Type $\mathrm{C}$

Seat Detail: 5210

Gusset plate ripped off at weld.

Figure B-34. Floor truss connectors found on recovered panel N-13 (A130: 99-102) from WTC 1. Shown are connectors found on column 130 at different floor levels (cont.). 
g)

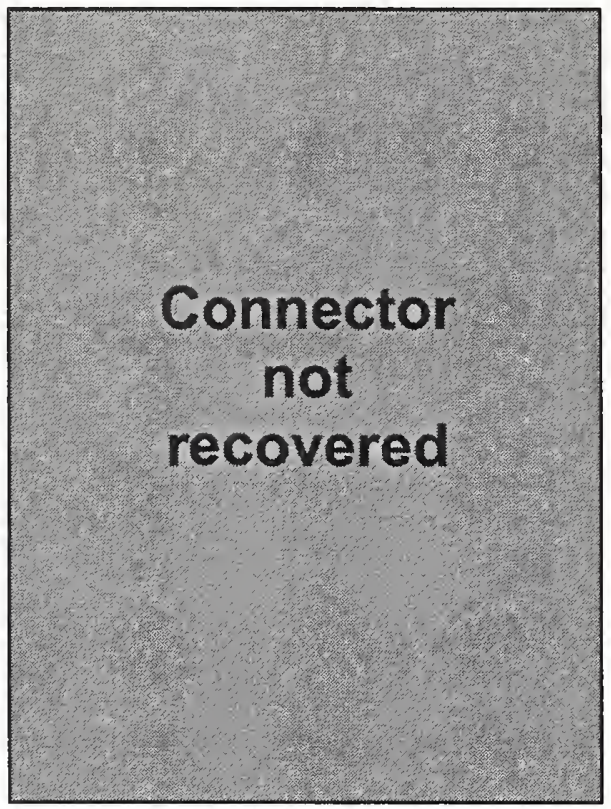

N13-C3M

NA
N13-C3T

NA h)

Connector not recovered i)

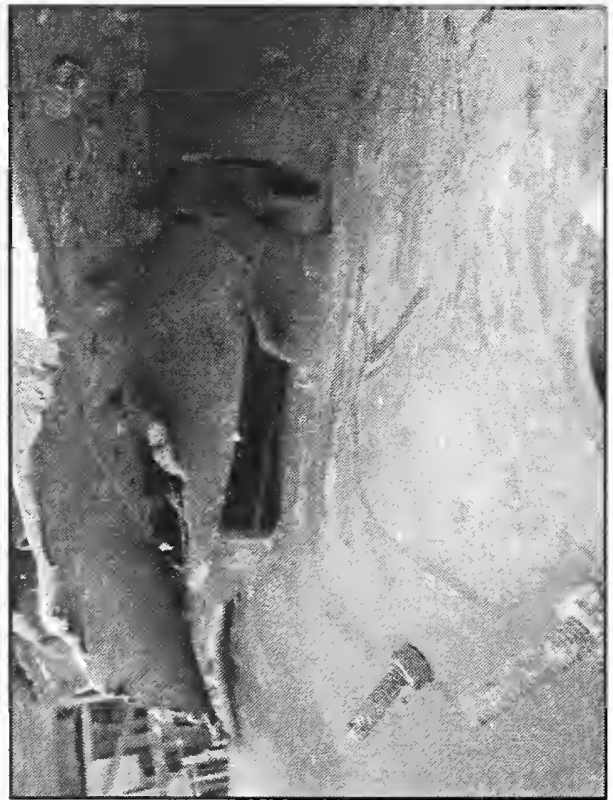

N13-C3B (WTC 1, Col.129, FI 100)

Type A

Seat Detail: 1411

Gusset plate remains;

Seat intact, bent upwards, 1 bolt hole intact with bolt and nut, other ripped out;

Gusset with damper unit intact, bent upwards.

Figure B-34. Floor truss connectors found on recovered panel N-13 (A130: 99-102) from WTC 1. Shown are connectors found on column 129 at different floor levels (cont.). 
a)

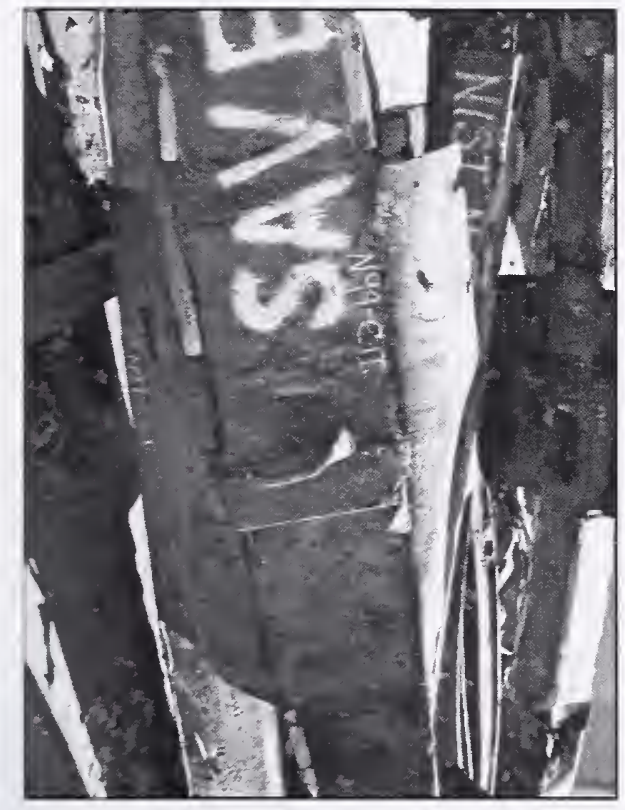

N99-C1M (WTC 1, Col.149, F1 101)

Type A

Seat Detail: 1511

Gusset plate ripped off at weld;

Seat intact, partially bent downwards, 1 bolt hole intact, other ripped through but bolt remains;

Gusset for damper unit ripped off at weld.

c)

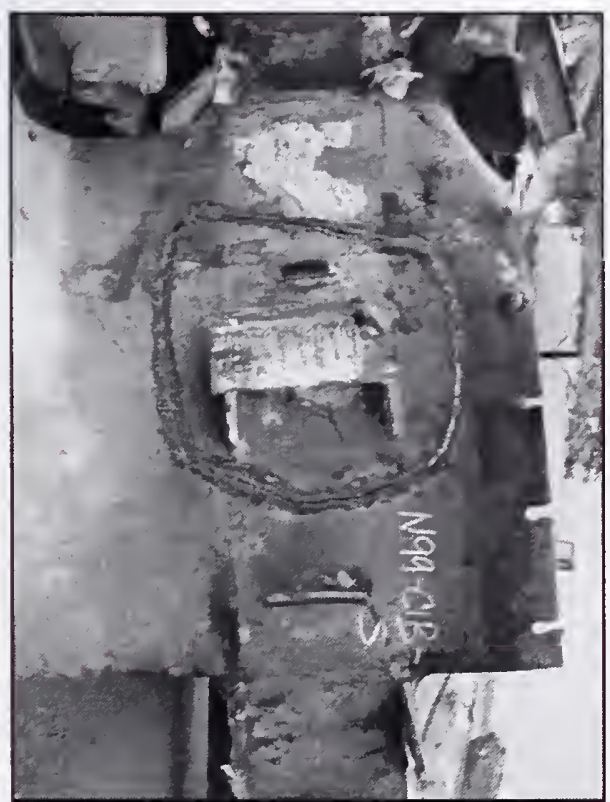

N99-C1T (WTC 1, Col.149, Fl 102)

Type A

Seat Detail: 1511

Gusset plate intact;

Seat intact, bent upwards, both bolt holes ripped out;

Gusset for damper unit ripped off at weld. b)

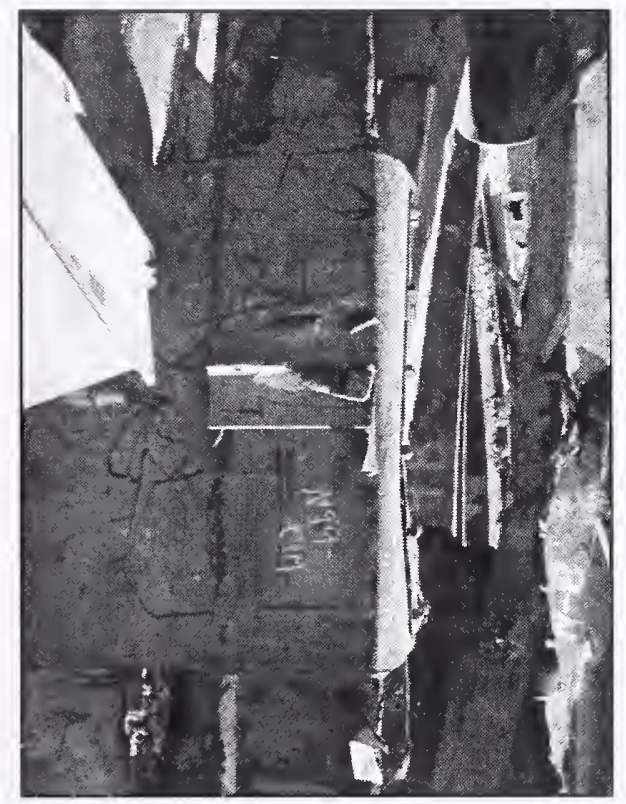

N99-C1B* (WTC 1, Col.149, Fl 100)

Type A

Seat Detail: 1511

Gusset plate intact;

Seat intact, both bolt holes intact, 1 bolt with nut remains;

Gusset with damper unit intact, bent upwards

* Boulder started cutting before picture taken, damper unit removed already in picture.

Figure B-35. Floor truss connectors found on recovered panel N-99 (A148: 99-102) from WTC 1. Shown are connectors found on column 149 at different floor levels. 
d)

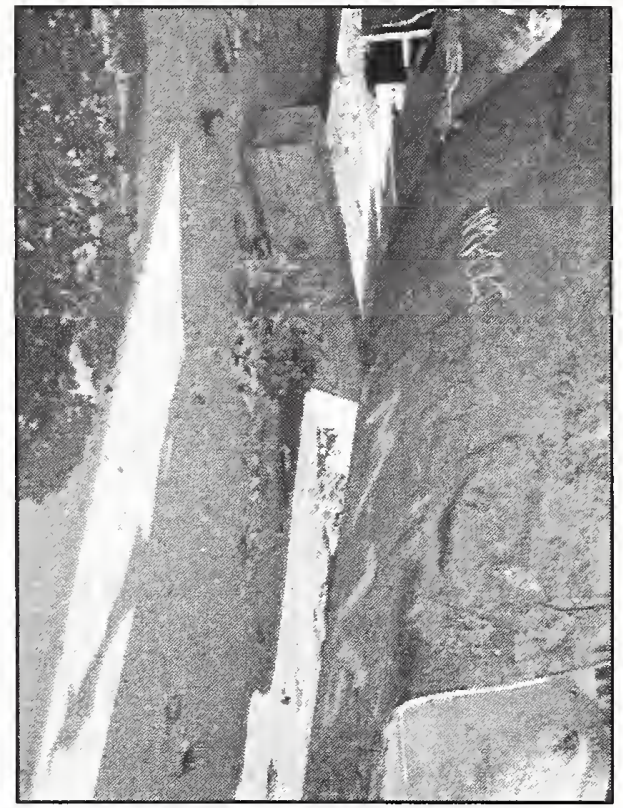

N99-C2T (WTC 1, Col.148, Fl 102)

Type C

Seat Detail: 5210

Gusset plate ripped off at weld.

N99-C2M (WTC 1, Col.148, Fl 101)

Type C

Seat Detail: 5210

Gusset plate ripped off at weld.

f)

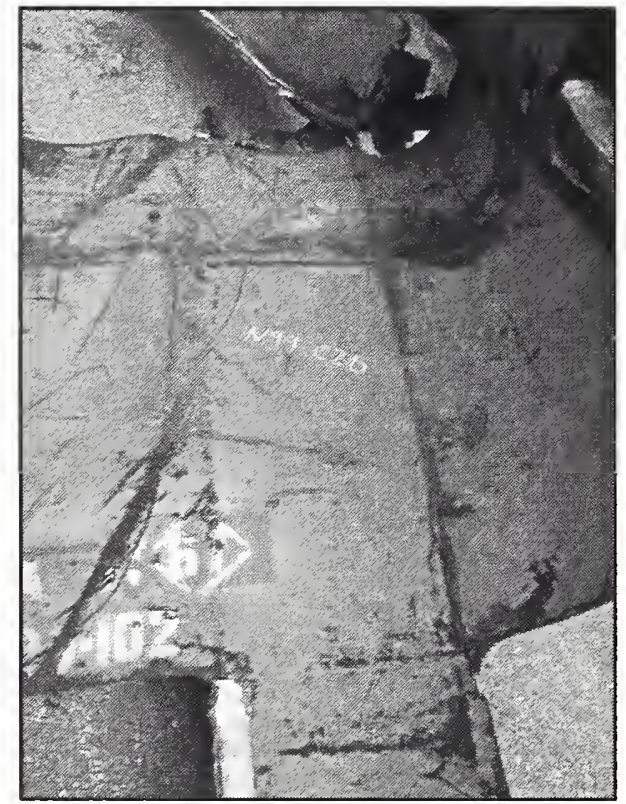

e)

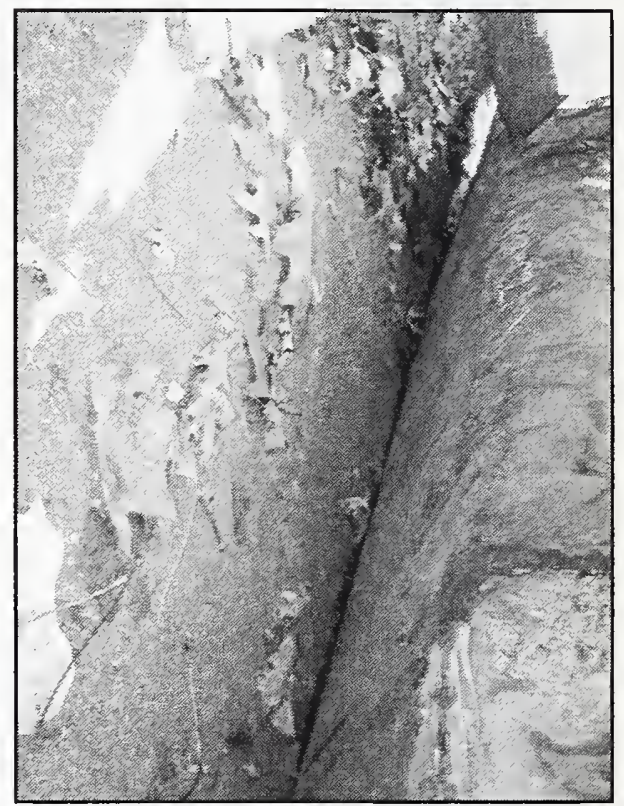

N99-C2B (WTC 1, Col.148, Fl 100)

Type C

Seat Detail: 5210

Gusset plate ripped off at weld.

Figure B-35. Floor truss connectors found on recovered panel N-99 (A148: 99-102) from WTC 1. Shown are connectors found on column 148 at different floor levels (cont.). 
g)

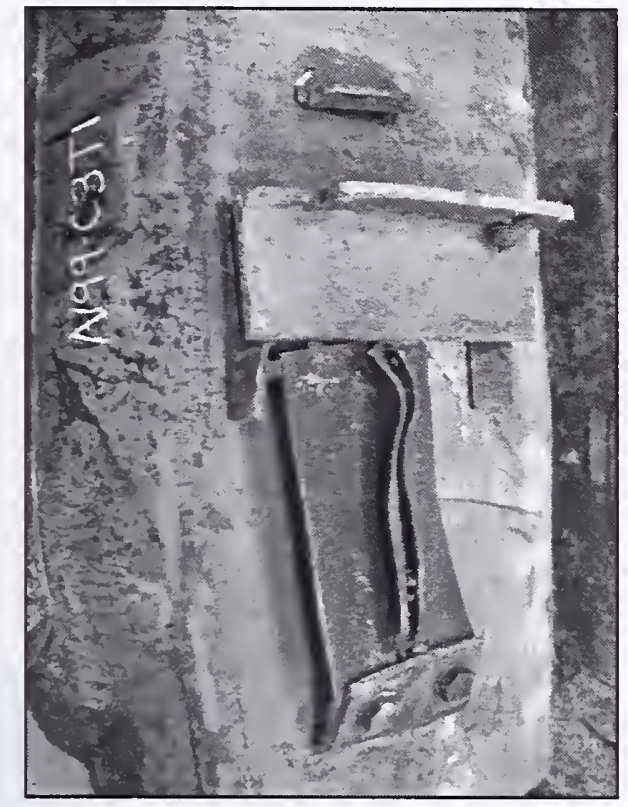

N99-C3M (WTC 1, Col.147, F1 101)

Type A

Seat Detail: 1411

Gusset plate intact;

Seat intact, 1 bolt hole intact with bolt and nut, other ripped out;

Gusset for damper unit ripped off at weld.

i)

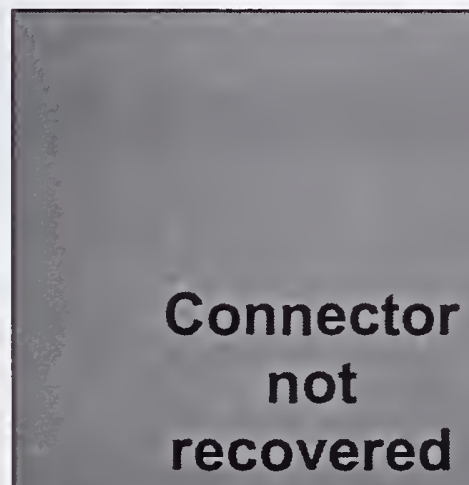

N99-C3B

NA
N99-C3T (WTC 1, Col.147, Fl 102)

Type A

Seat Detail: 1411

Portion of gusset plate remains;

Seat intact, 1 bolt hole intact with bolt and nut, other ripped out;

Gusset with damper unit intact, bent upwards. h)

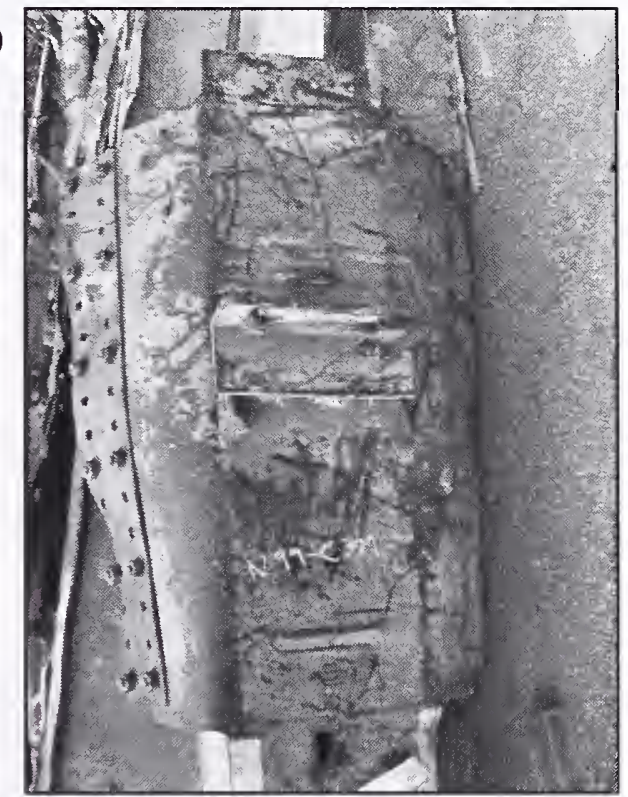

Figure B-35. Floor truss connectors found on recovered panel N-99 (A148: 99-102) from WTC 1. Shown are connectors found on column 147 at different floor levels (cont.). 
a)

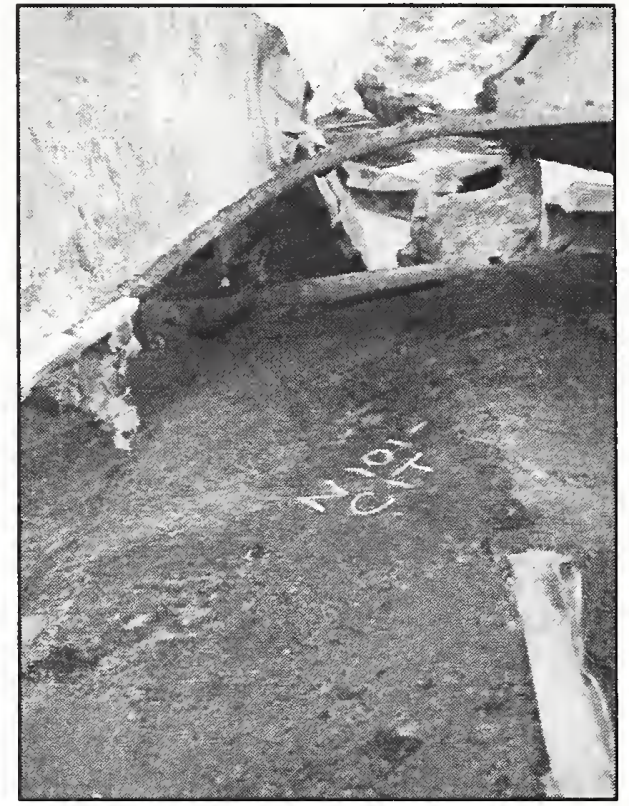

N101-C1M (WTC 1, Col.134, Fl 102)

Type C

Seat Detail: 5110

Gusset plate intact with diagonal bracing strap attached, bent upwards.

c)

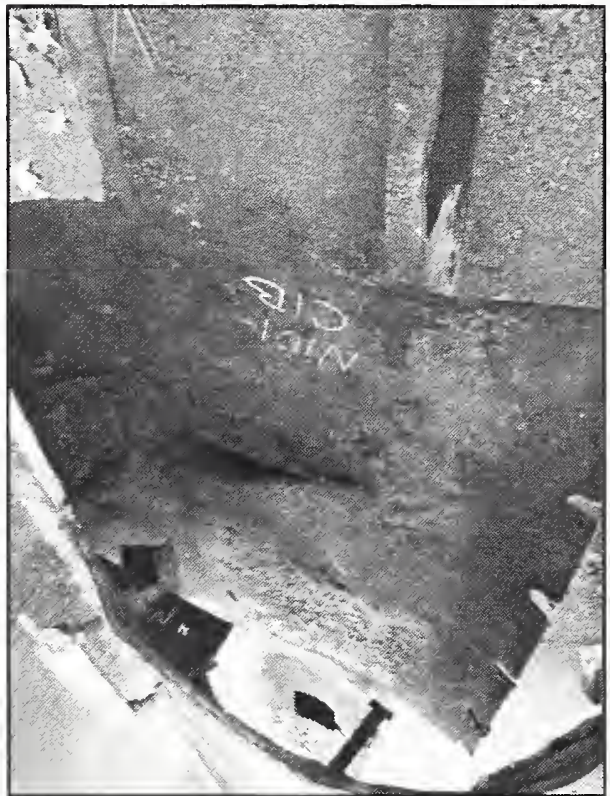

Figure B-36. Floor truss connectors found on recovered panel N-101 (A133: 100-103) from WTC 1. Shown are connectors found on column 134 at different floor levels.
N101-C1T (WTC 1, Col.134, Fl 103)

Type C

Seat Detail: 5110

Gusset plate ripped off at weld.

b)

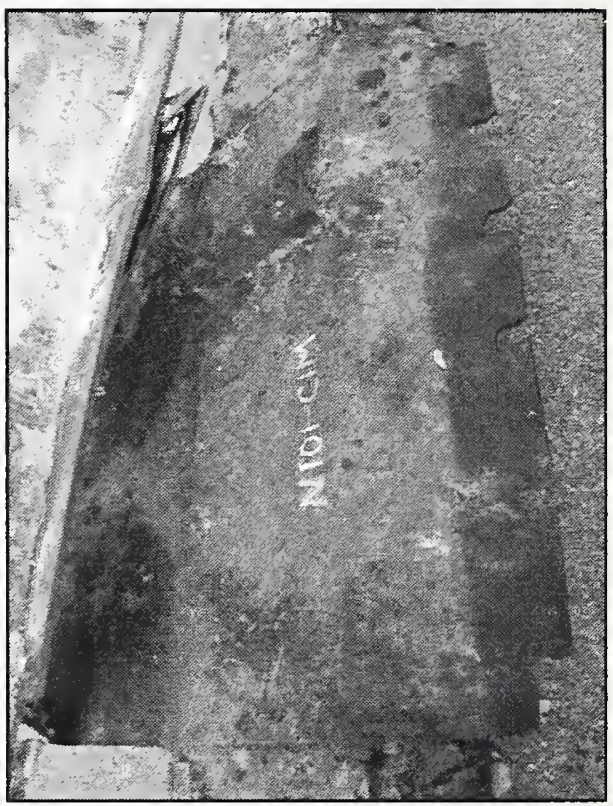

N101-C1B (WTC 1, Col.134, Fl 101)

Type C

Seat Detail: 5110

Gusset plate intact with diagonal bracing strap attached, bent upwards. 
d)

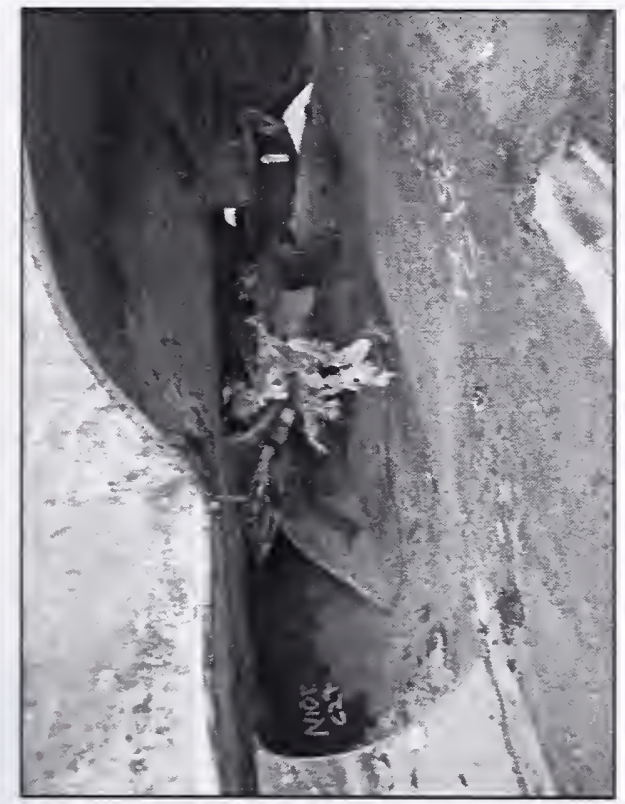

N101-C2M (WTC 1, Col.133, Fl 102)

Type A

Seat Detail: 1411

Gusset plate intact, bent upwards;

Seat intact, both bolt holes intact with bolts and nuts; Gusset with damper unit intact, bent upwards.

f)

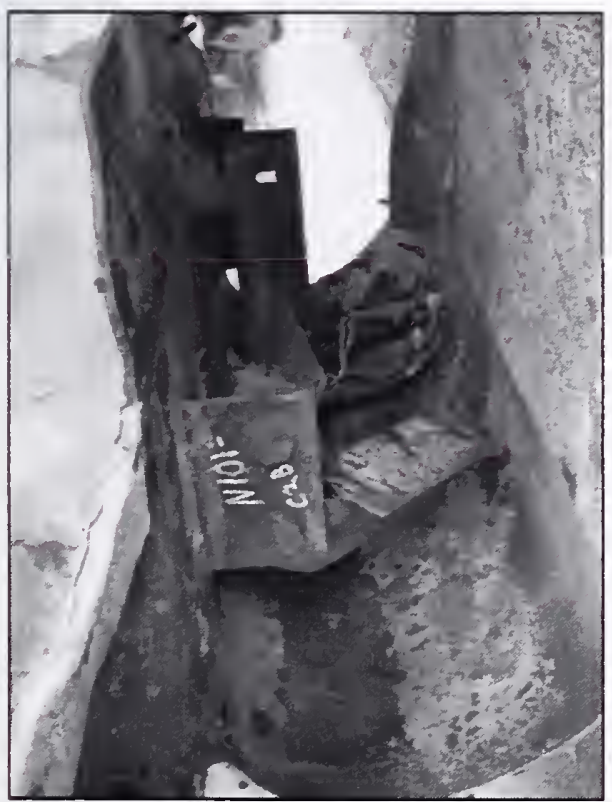

Type A

Seat Detail: 1411
N101-C2T (WTC 1, Col.133, Fl 103)

Type A

Seat Detail: 1411

Gusset plate ripped off at weld;

Seat intact, bent upwards, 1 bolt hole intact with bolt, other ripped through;

Gusset with damper unit intact, bent upwards. e)

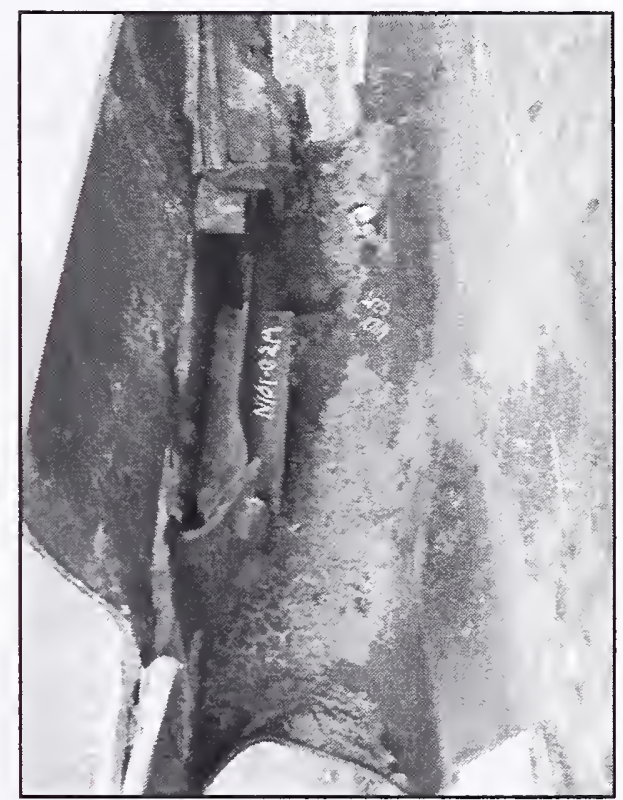

N101-C2B (WTC 1, Col.133, Fl 101)

Gusset plate intact, bent upwards;

Seat intact, both bolt holes intact with bolts and nuts; Gusset with damper unit intact, bent upwards.

Figure B-36. Floor truss connectors found on recovered panel N-101 (A133: 100-103) from WTC 1. Shown are connectors found on column 133 at different floor levels (cont.). 
g)

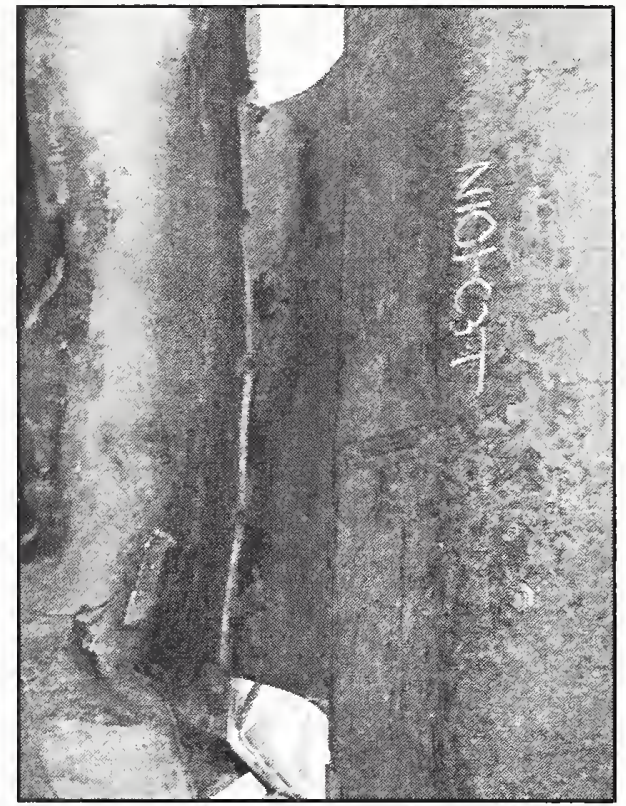

N101-C3T (WTC 1, Col.132, Fl 103)

Type C

Seat Detail: 5210

Gusset plate ripped out at weld.

N101-C3M (WTC 1, Col.132, Fl 102)

Type C

Seat Detail: 5210

Gusset plate ripped out at weld.

i)

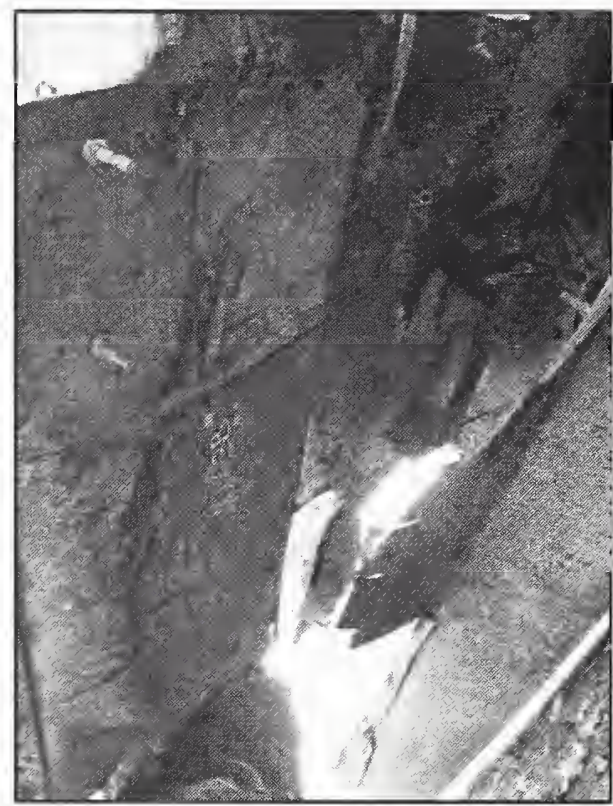

h)

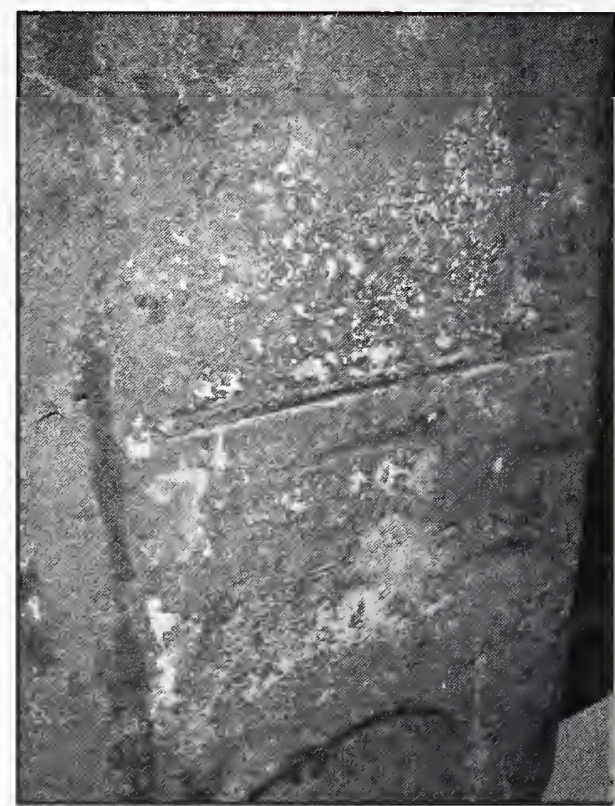

\section{N101-C3B (WTC 1, Col.132, Fl 101)}

Type C

Seat Detail: 5210

Gusset plate ripped out at weld.

Figure B-36. Floor truss connectors found on recovered panel N-101 (A133: 100-103) from WTC 1. Shown are connectors found on column 132 at different floor levels (cont.). 
a)

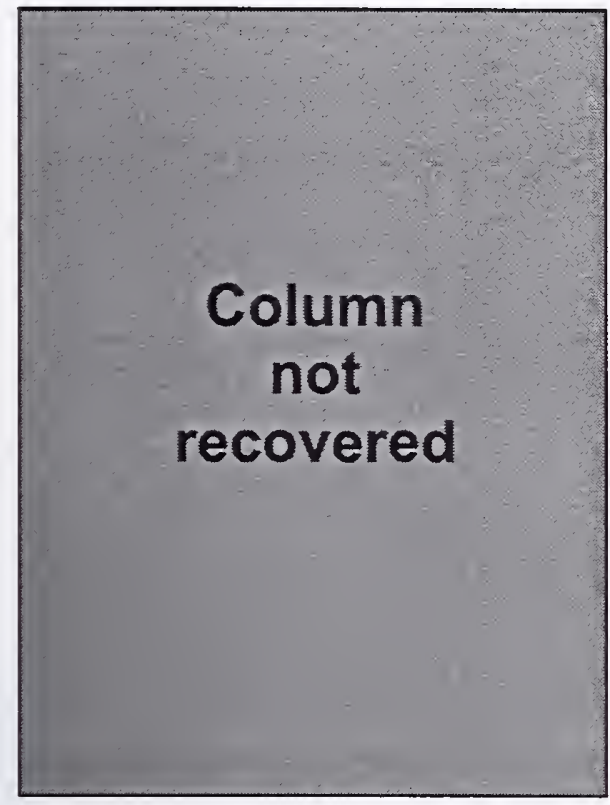

S1-C1T

NA

S1-C1M

NA

c)

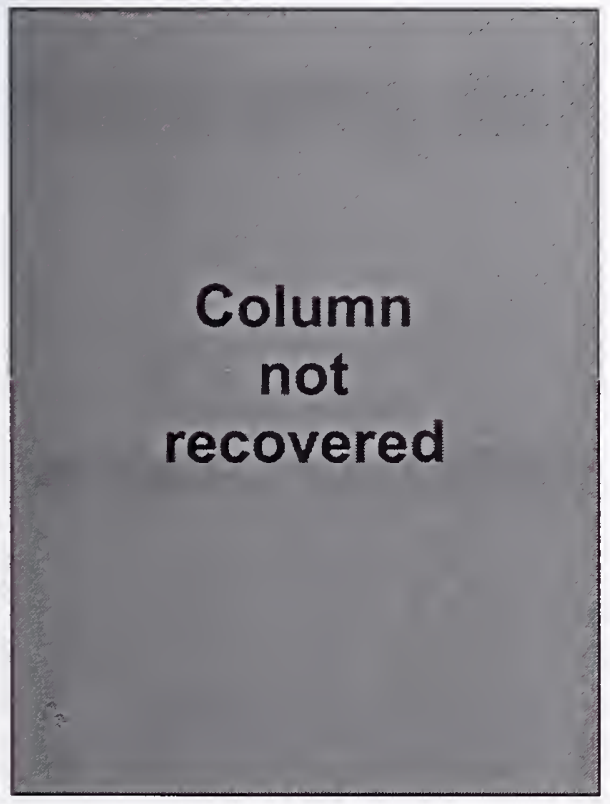

b)

\section{Column not} recovered

S1-C1B

NA

Figure B-37. Floor truss connectors found on recovered panel S-1 (A433: 79-82) from WTC 1. Column 434 was not recovered. 
d)

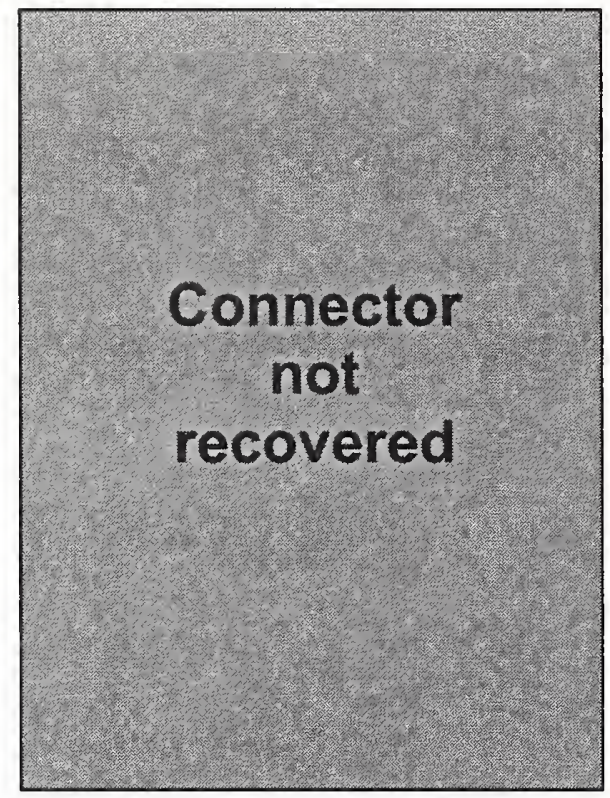

S1-C2M

NA
S1-C2T

NA e)

Connector not recovered f)

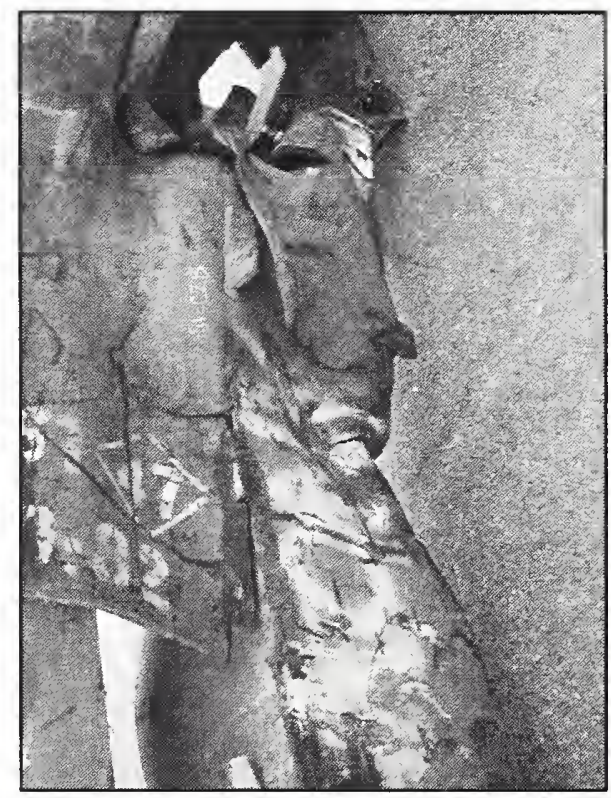

S1-C2B (WTC 1, Col.433, F1 80)

Type A

Seat Detail: 1111

Gusset plate ripped off at weld;

Only 1 standoff plate remains, rest ripped off at welds; Gusset for damper unit ripped off at weld.

Figure B-37. Floor truss connectors found on recovered panel S-1 (A433: 79-82) from WTC 1. Shown are connectors found on column 433 at different floor levels (cont.). 
g)

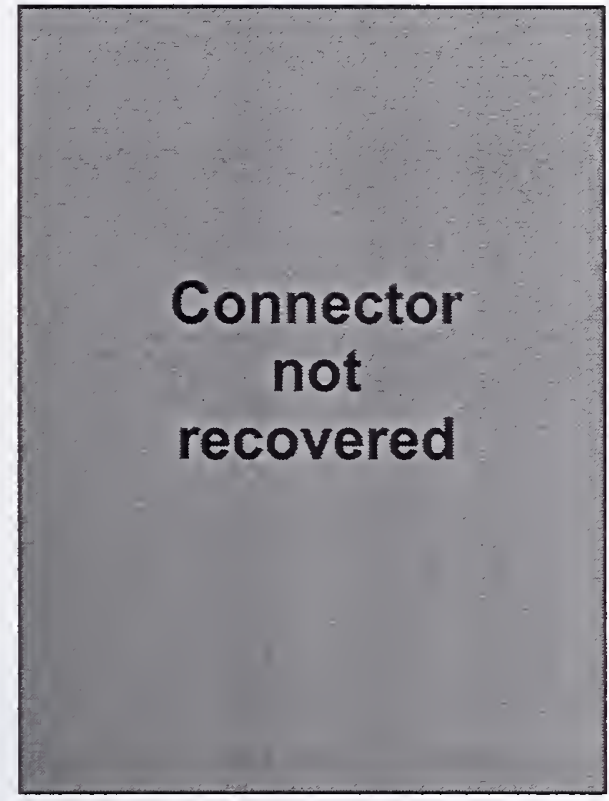

S1-C3M

NA
S1-C3T

NA h)

\section{Connector not recovered}

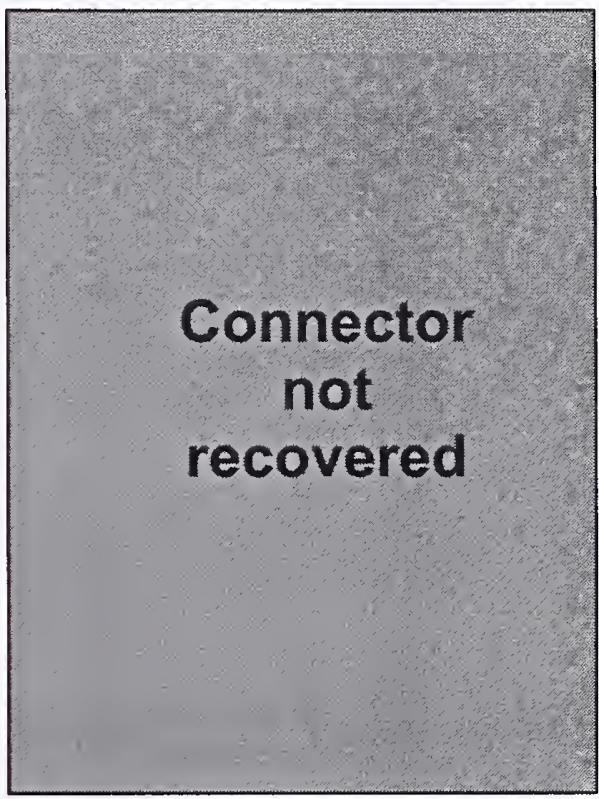

S1-C3B (WTC 1, Col.432, FI 80)

Type C

Seat Detail: 5210

Gusset plate intact, bent downwards, with 2 diagonal bracing straps attached.

Figure B-37. Floor truss connectors found on recovered panel S-1 (A433: 79-82) from WTC 1. Shown are connectors found on column 432 at different floor levels (cont.). 
a)

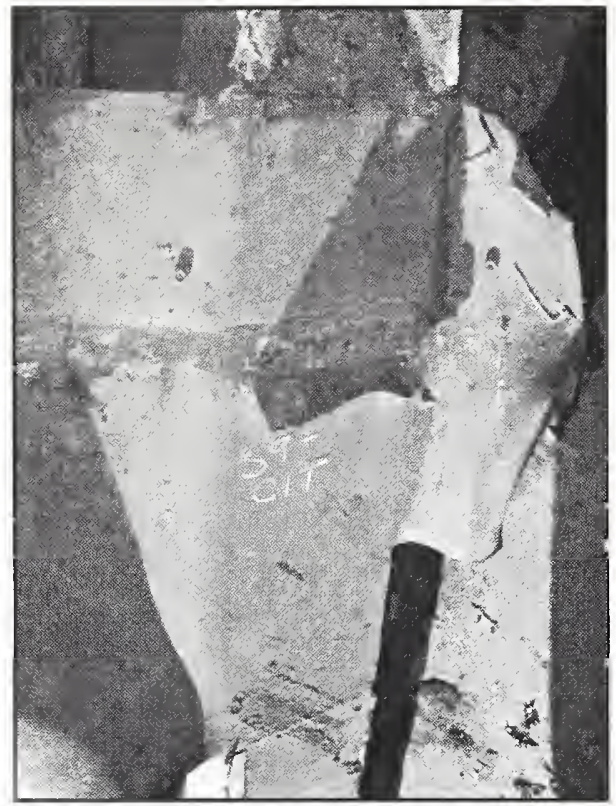

S9-C1M (WTC 1, Col.134, Fl 99)

Type $\mathrm{C}$

Seat Detail: 5110

Gusset plate ripped off at weld.

c)

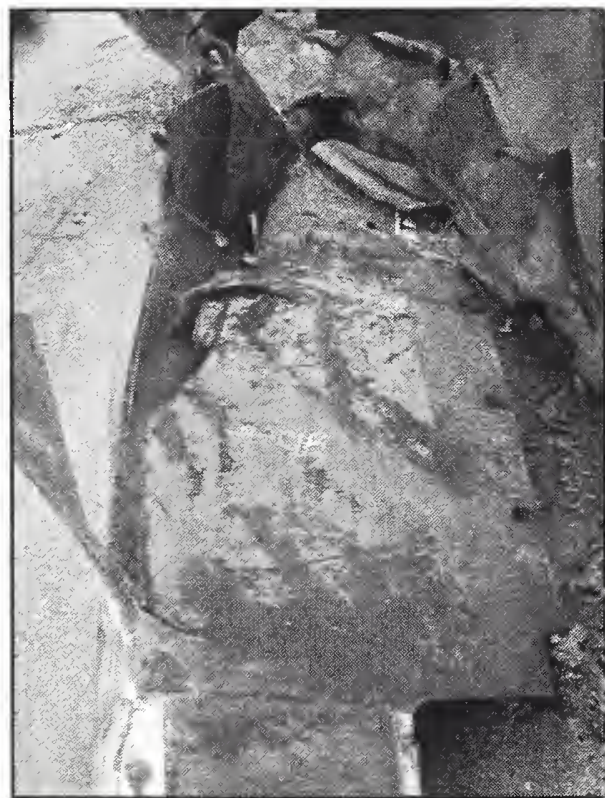

S9-C1T (WTC 1, Col.134, Fl 100)

Type C

Seat Detail: 5110

Gusset plate intact, bent downwards, with diagonal bracing strap attached. b)

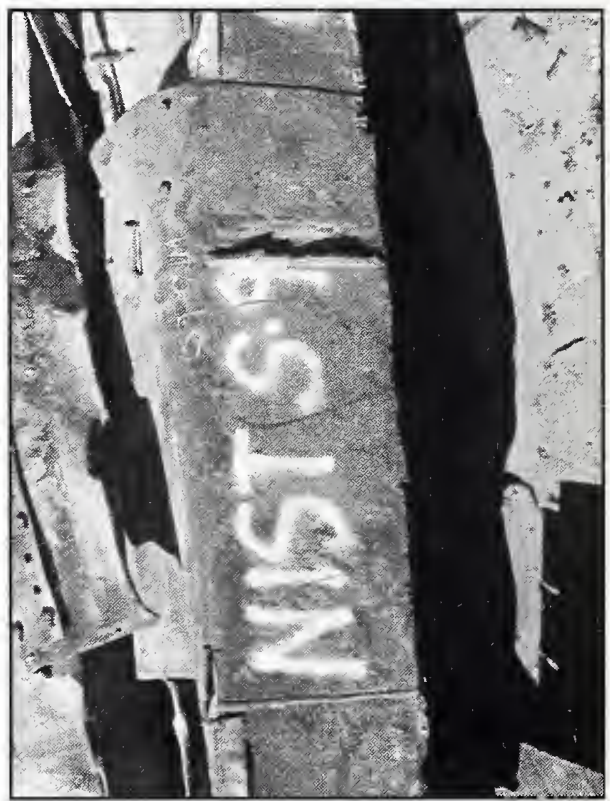

S9-C1B (WTC 1, Col.134, Fl 98)

Type C

Seat Detail: 5110

Gusset plate ripped off at weld.

Figure B-38. Floor truss connectors found on recovered panel S-9 (A133: 97-100) from WTC 1. Shown are connectors found on column 134 at different floor levels. 
d)

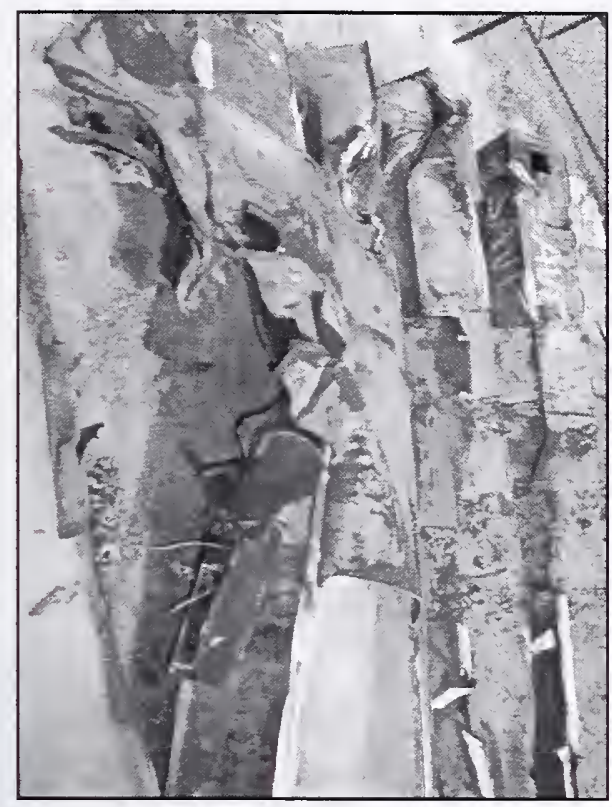

S9-C2M (WTC 1, Col.133, Fl 99)

Type A

Seat Detail: 1411

Gusset plate intact;

Seat intact, bent in towards column, 1 bolt hole intact with bolt and nut;

Gusset plate for damper unit ripped off at weld.

)

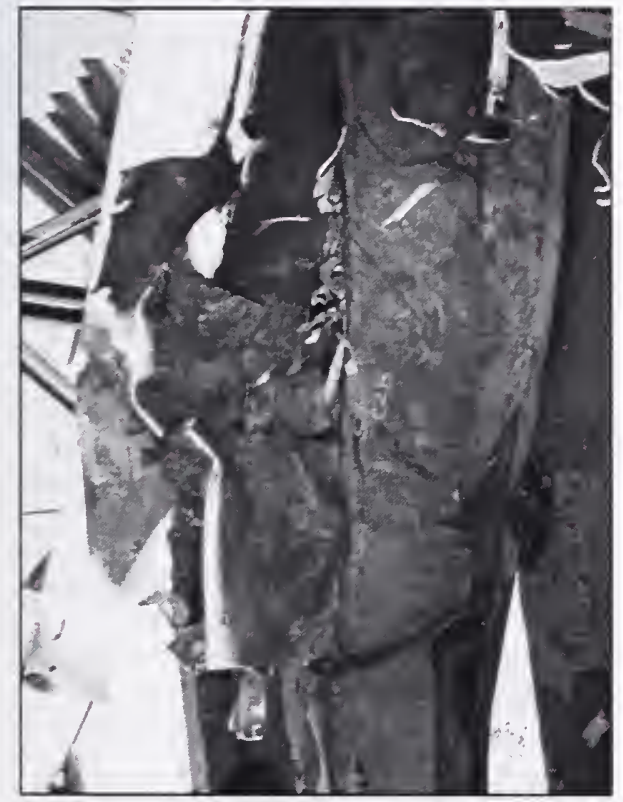

S9-C2T (WTC 1, Col.133, Fl 100)

Type A

Seat Detail: 1411

Gusset plate ripped completely out of spandrel; Standoff plates intact, seat ripped off at welds; Gusset with damper unit intact, bent downwards.

\section{S9-C2B (WTC 1, Col.133, FI 98)}

Type A

Seat Detail: 1411

Portion of gusset plate remains;

Seat intact, has extra stiffener attached, both bolt holes intact;

Gusset with damper unit intact, bent upwards.

Figure B-38. Floor truss connectors found on recovered panel S-9 (A133: $97-100)$ from WTC 1. Shown are connectors found on column 133 at different floor levels (cont.). 
g)

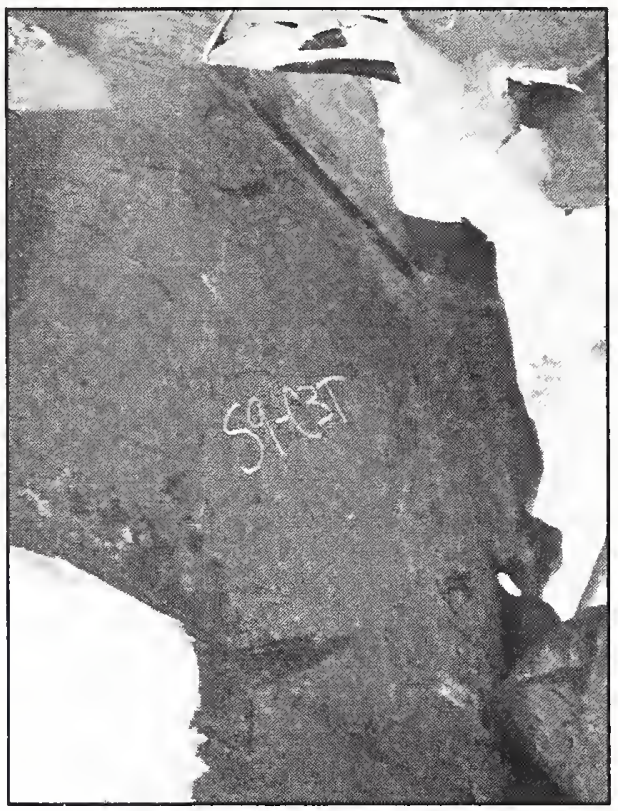

S9-C3T (WTC 1, Col.132, Fl 100)

Type C

Seat Detail: 5210

Gusset plate ripped out at weld.

S9-C3M (WTC 1, Col.132, Fl 99)

Type C

Seat Detail: 5210

Gusset plate intact with portion of diagonal bracing strap attached.

i)

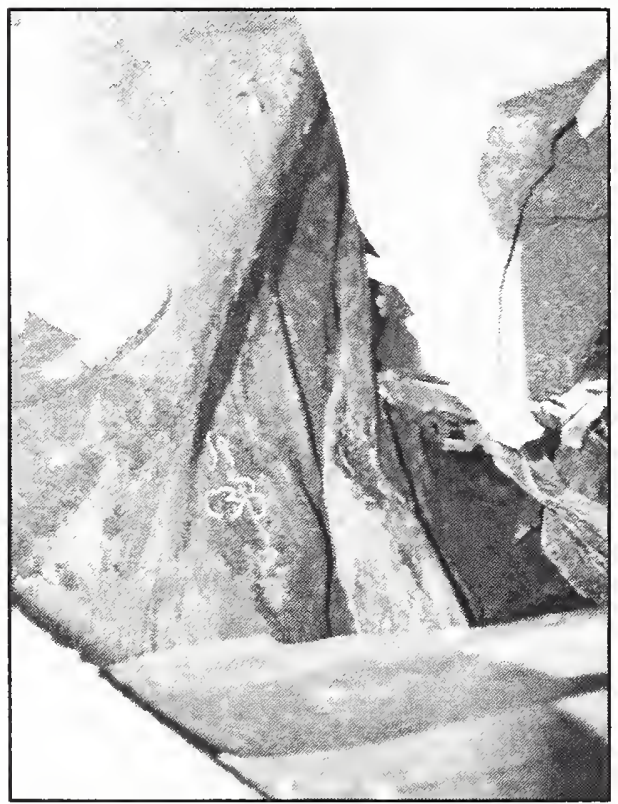

h)

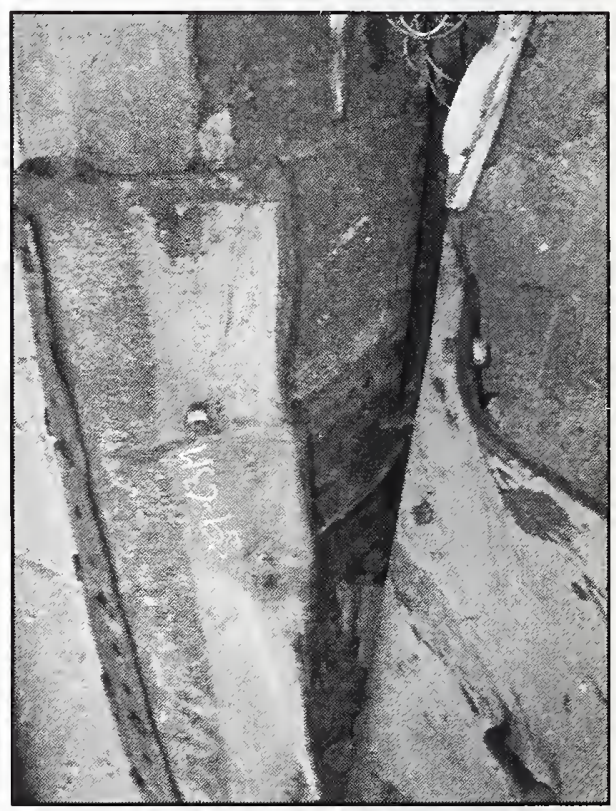

S9-C3B (WTC 1, Col.132, Fl 98)

Type C

Seat Detail: 5210

Gusset plate ripped out at weld.

Figure B-38. Floor truss connectors found on recovered panel S-9 (A133: 97-100) from WTC 1. Shown are connectors found on column 132 at different floor levels (cont.). 
a)

S10-C1M (WTC 1, Col.225, FI 94)

Type A

Seat Detail: 1111

Gusset plate ripped out of spandrel;

Shear plates ripped off at welds;

Gusset plate for damper unit ripped off at weld.

c)

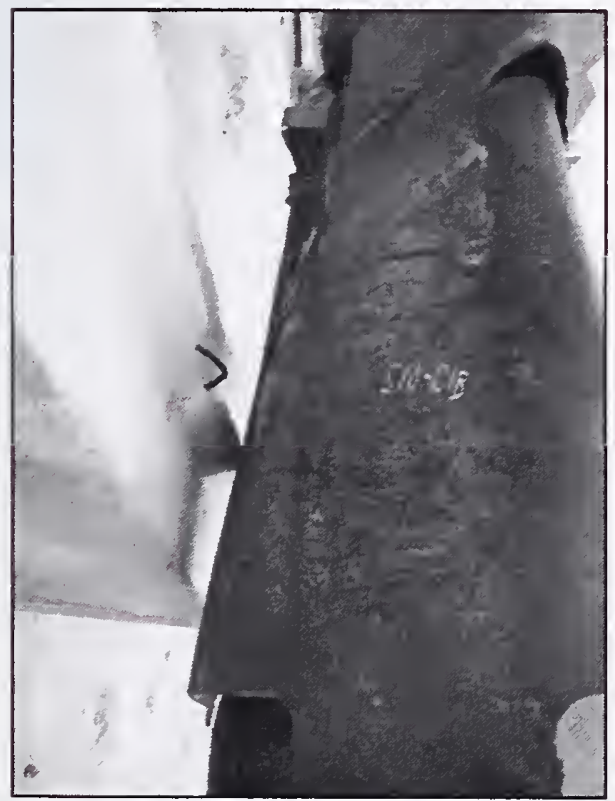

\section{S10-C1T}

NA
Type A

Seat Detail: 1111 b)

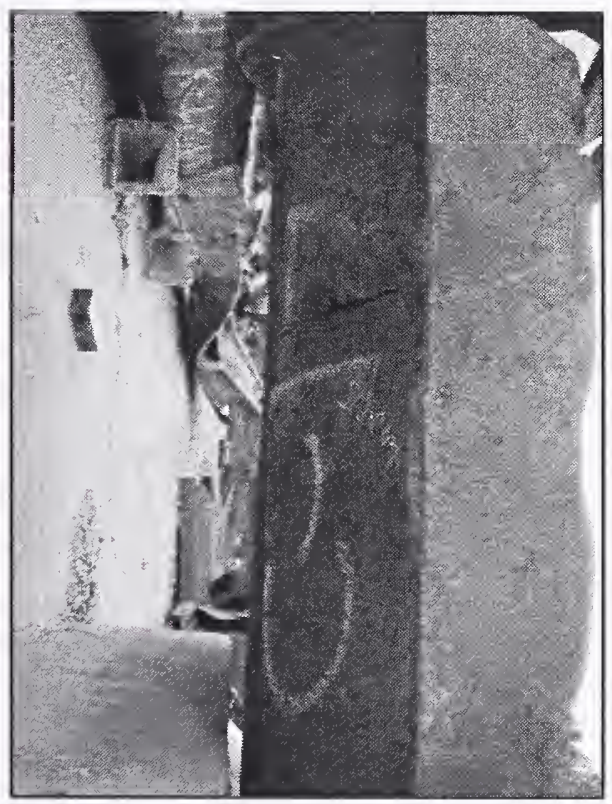

S10-C1B (WTC 1, Col.225, Fl 93)

Portion of gusset plate remains;

Standoff plates ripped off at welds;

Gusset plate for damper unit ripped off at weld.

Figure B-39. Floor truss connectors found on recovered panel S-10 (A224: 92-95) from WTC 1. Shown are connectors found on column 225 at different floor levels. 
d)

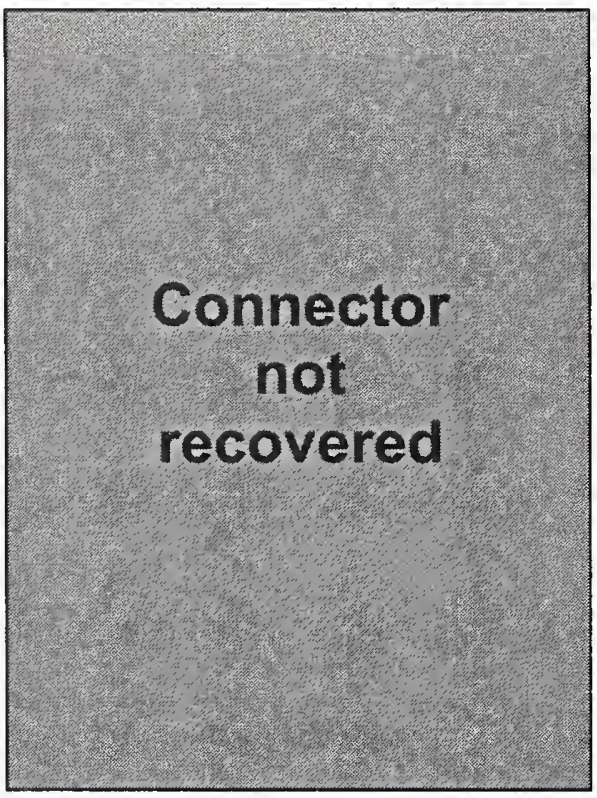

S10-C2T

NA

S10-C2M (WTC 1, Col.224, FI 94)

Type C

Seat Detail: 5110

Portion of large gusset plate remains.

f)

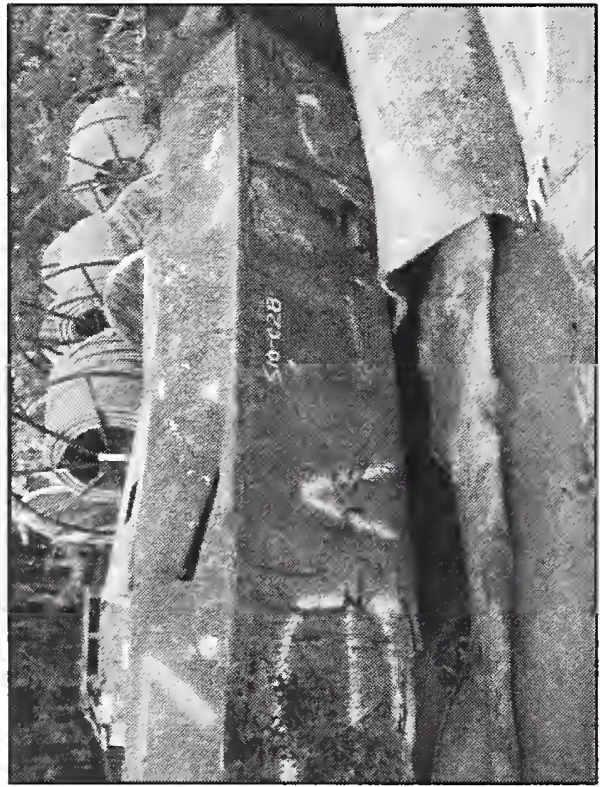

e)

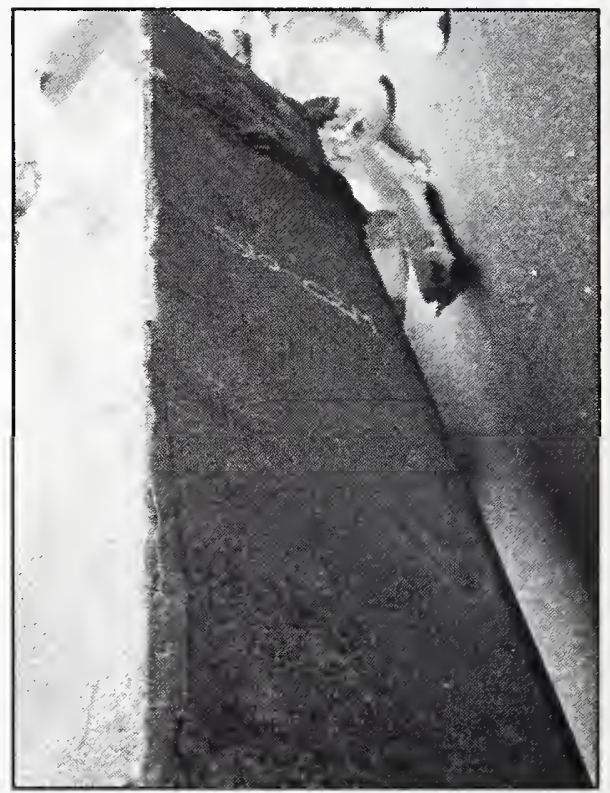

S10-C2B (WTC 1, Col.224, Fl 93)

Type C

Seat Detail: 5110

Portion of large gusset plate remains, bent downwards.

Figure B-39. Floor truss connectors found on recovered panel S-10 (A224: 92-95) from WTC 1. Shown are connectors found on column 224 at different floor levels (cont.). 
g)

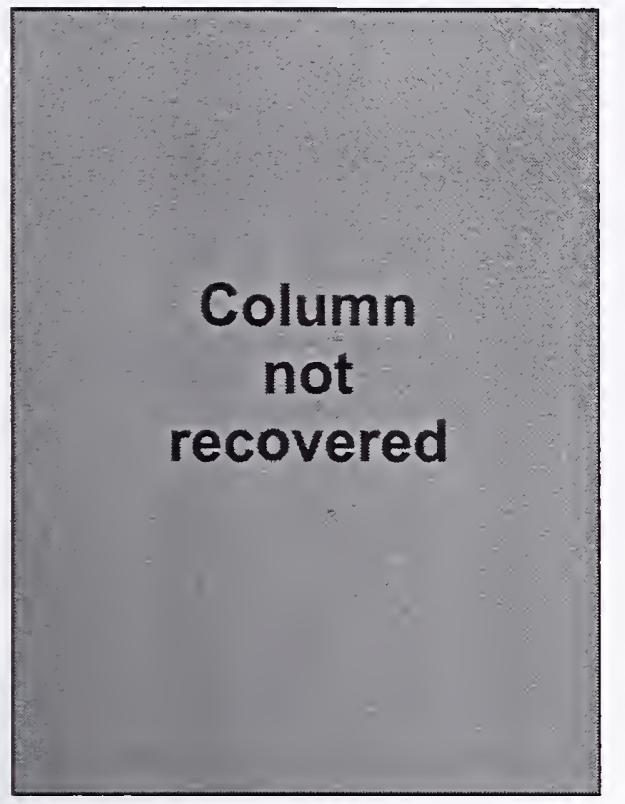

S10-C3T

NA

S10-C3M

NA

i)

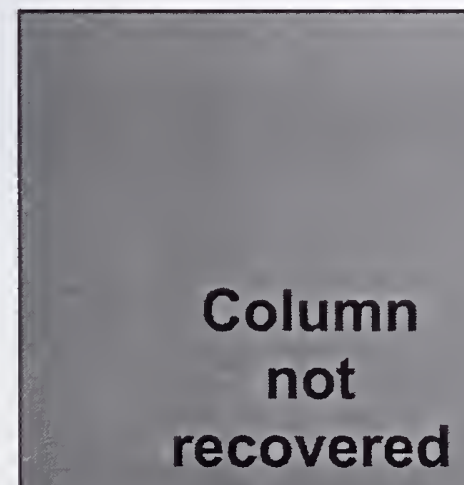

S10-C3B

NA

h)

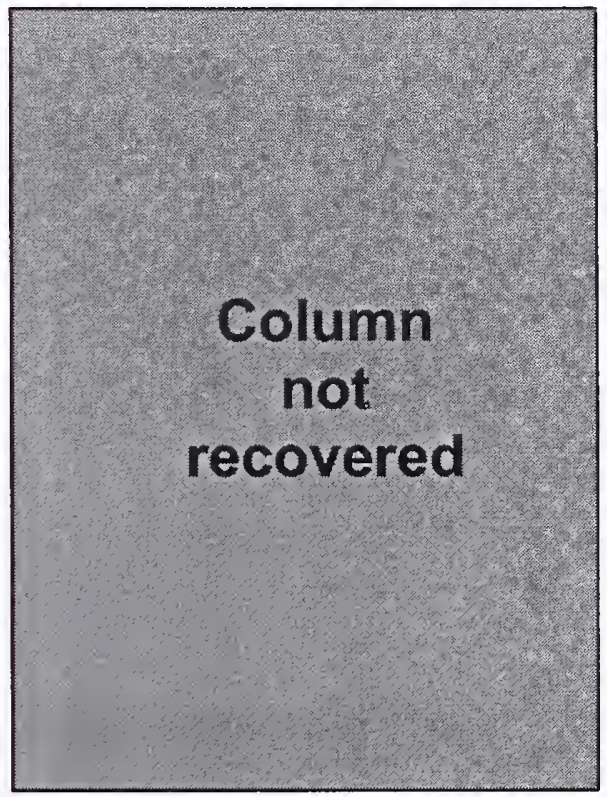

Figure B-39. Floor truss connectors found on recovered panel S-10 (A224: 92-95) from WTC 1. Shown are connectors found on column 223 at different floor levels (cont.). 
a)

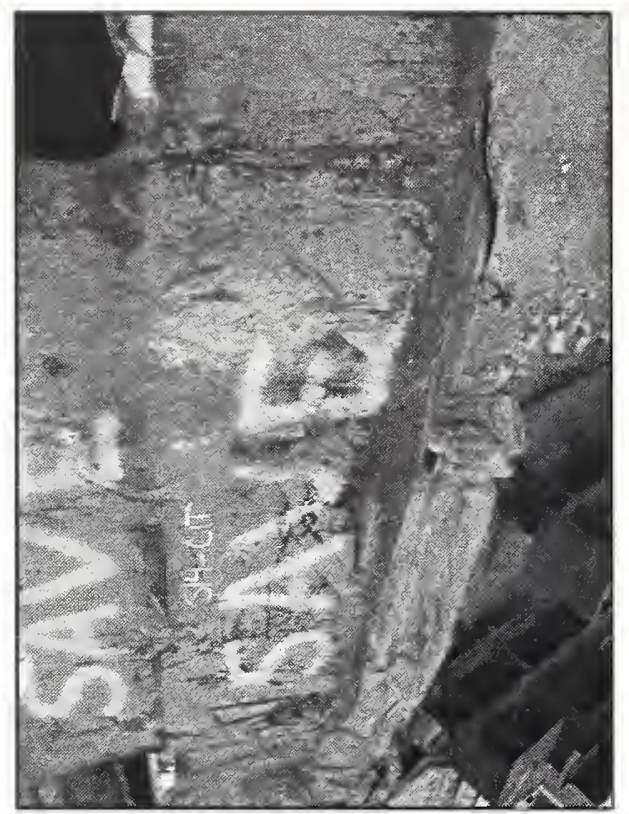

S14-C1M (WTC 2, Col.219, FI 93)

Type A

Seat Detail: 1111

Gusset plate ripped completely out of spandrel; Seat intact but badly deformed, half bent upwards and half bent downwards, both bolt holes ripped through though bolts and nuts remain;

Gusset plate with damper unit intact, bent upwards.

c)

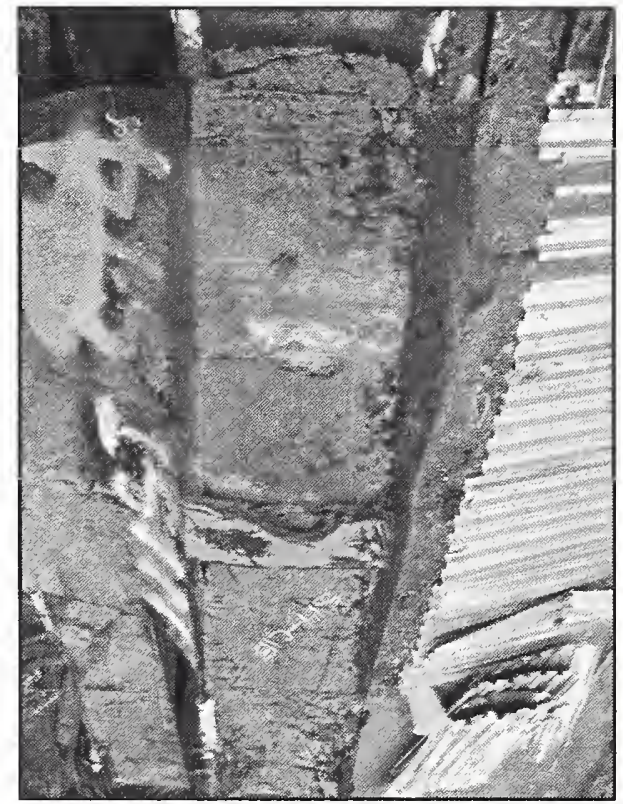

Type A

Seat Detail: 2110 out;
S14-C1T (WTC 2, Col.219, Fl 94)

Type A

Seat Detail: 1111

Gusset plate remains;

Only 1 standoff plate remains, rest is ripped off at weld;

Gusset plate for damper unit was half ripped completely out of spandrel, other half at weld. b)

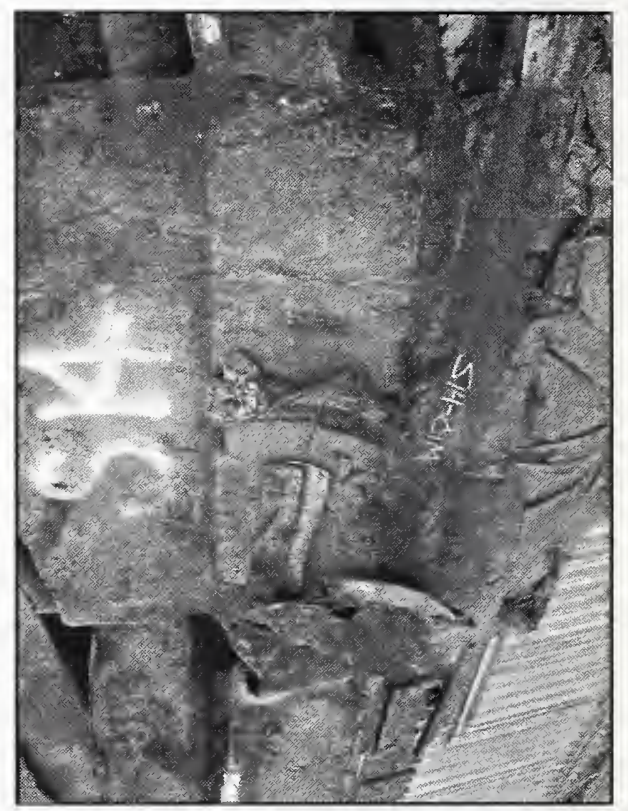

S14-C1B (WTC 2, Col.219, Fl 92)

Portion of gusset plate remains;

Seat intact, bent upwards, both bolt holes are ripped

No evidence of gusset plate for damper unit.

Figure B-40. Floor truss connectors found on recovered panel S-14 (B218: 91-94) from WTC 2. Shown are connectors found on column 219 at different floor levels. 
d)

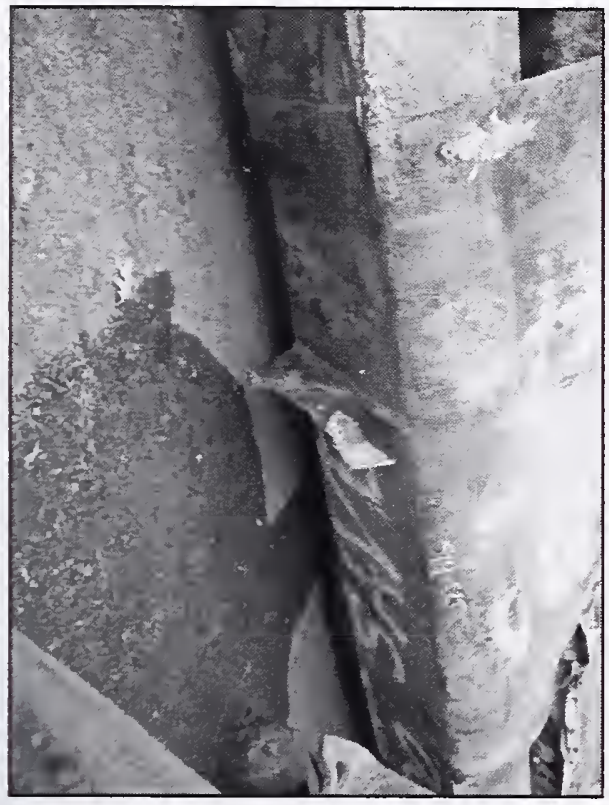

S14-C2T (WTC 2, Col.218, F1 94)

Type C

Seat Detail: 5210

Gusset plate intact, partially ripped at weld, with 2 diagonal bracing straps attached

S14-C2M (WTC 2, Col.218, Fl 93)

Type C

Seat Detail: 5210

Large gusset plate ripped off at weld.

f)

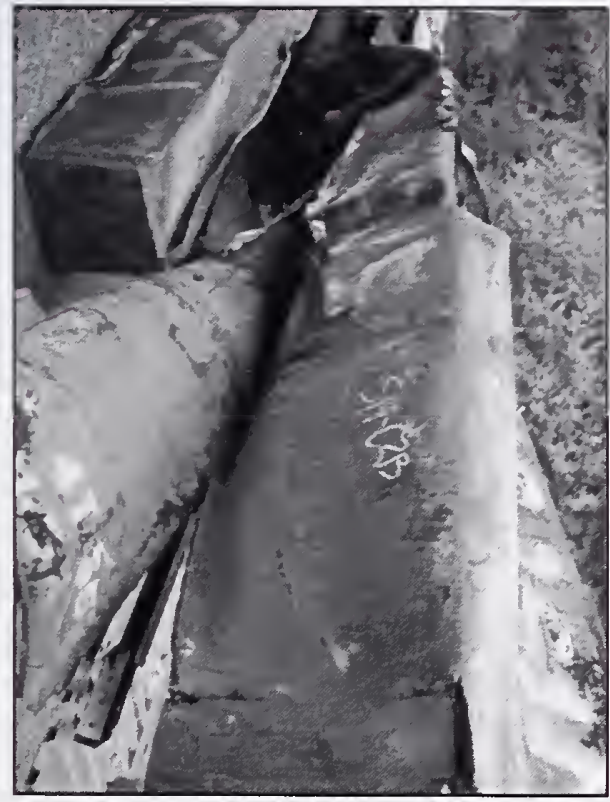

e)

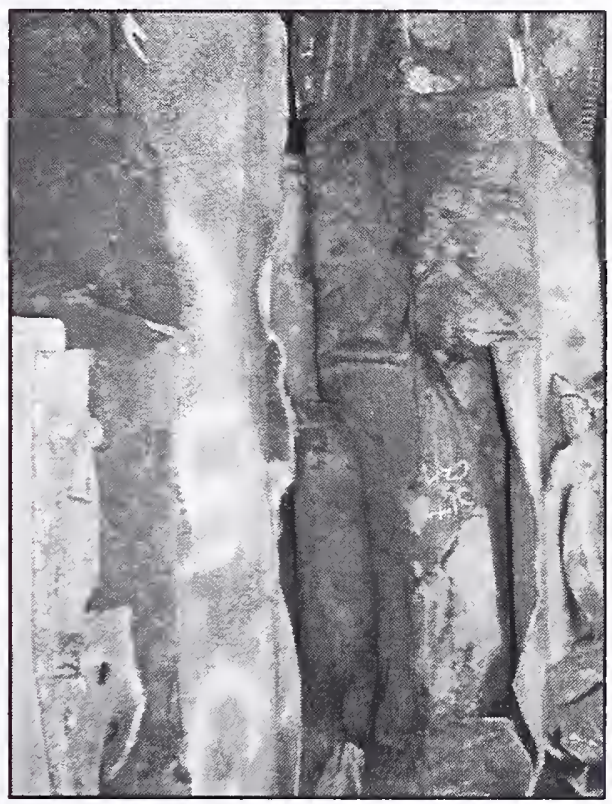

S14-C2B (WTC 2, Col.218, Fl 92)

Type C

Seat Detail: 5210

Portion of gusset plate remains with diagonal bracing strap attached, bent upwards.

Figure B-40. Floor truss connectors found on recovered panel S-14 (B218: 91-94) from WTC 2. Shown are connectors found on column 218 at different floor levels (cont.). 
g)

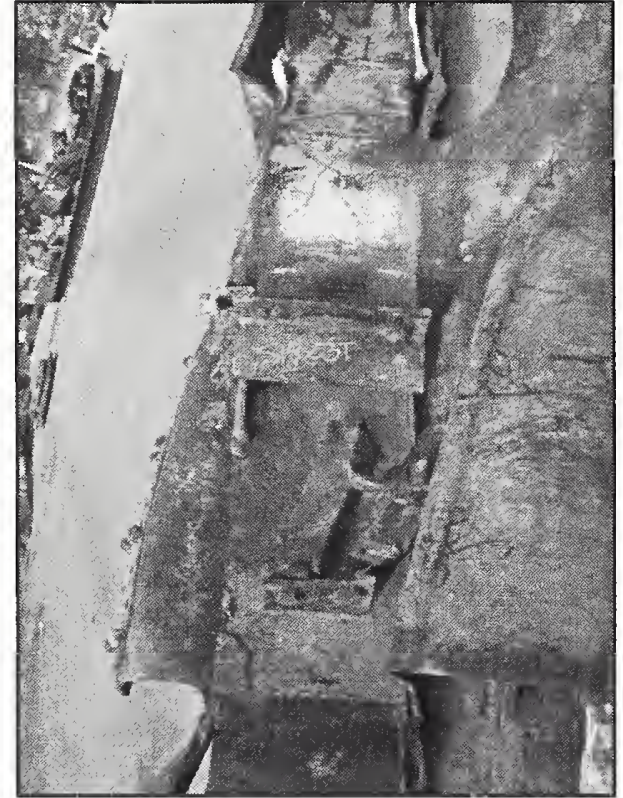

\section{S14-C3T (WTC 2, Col.217, Fl 94)}

Type A

Seat Detail: 1611

Gusset plate intact;

Seat intact, both bolt holes intact, only one has bolt and nut;

Gusset plate with damper unit intact, bent upwards.

S14-C3M (WTC 2, Col.217, Fl 93)

Type A

Seat Detail: 1611

Gusset plate ripped out of spandrel;

Only 1 standoff plate remains, rest ripped off at welds;

Gusset plate for damper unit ripped off at weld.

i)

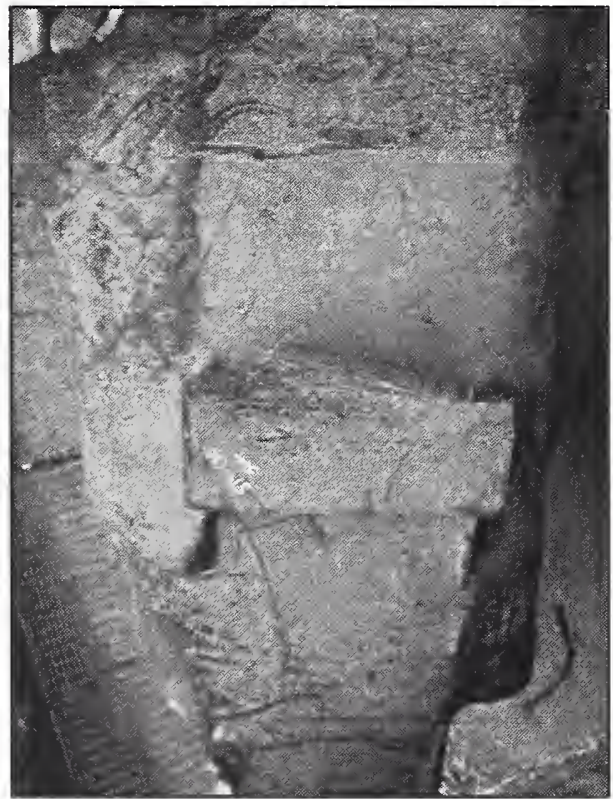

h)

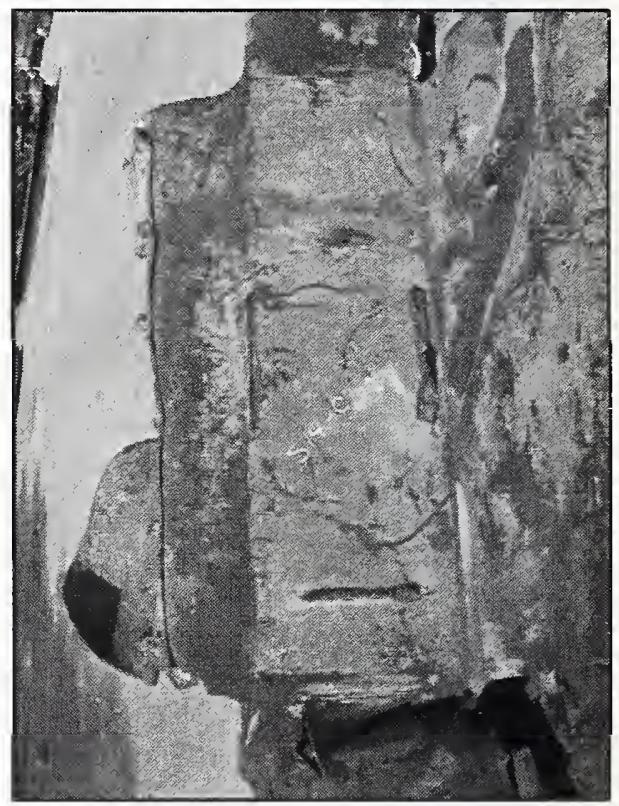

S14-C3B (WTC 2, Col.217, Fl 92)

Type A

Seat Detail: 2160

Gusset plate ripped off at weld;

Seat intact, both bolt holes ripped out;

Gusset plate for damper unit ripped off at weld.

Figure B-40. Floor truss connectors found on recovered panel S-14 (B218: 91-94) from WTC 2. Shown are connectors found on column 217 at different floor levels (cont.). 


\section{Appendix C \\ ReCOVERED CORE FLOOR TRUSS CONNECTORS}

The floor trusses were attached to the core columns via an assembly composed of core truss seats welded to sections of channels. These channels were attached directly to the core columns. The following appendix displays images, taken by National Institute of Standards and Technology (NIST), of the recovered core truss seats found on the recovered channel sections. Channels with NIST identification labels of C-99 and C-124 were not observed to have seats or the remnants of them. 
a)

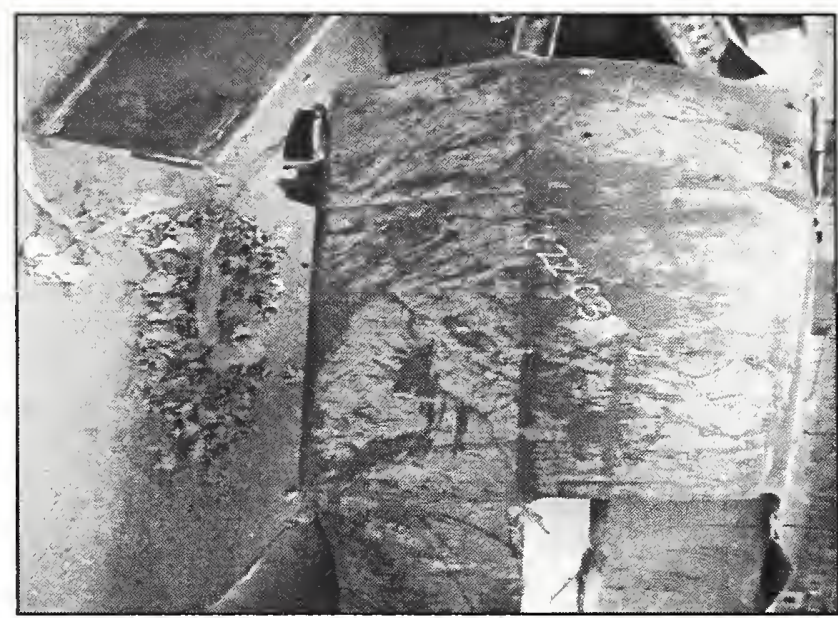

Seat 1: Both holes intact, one has bolt remaining, seat was bent

Seat 2: Both holes intact, both have bolts remaining, seat was bent

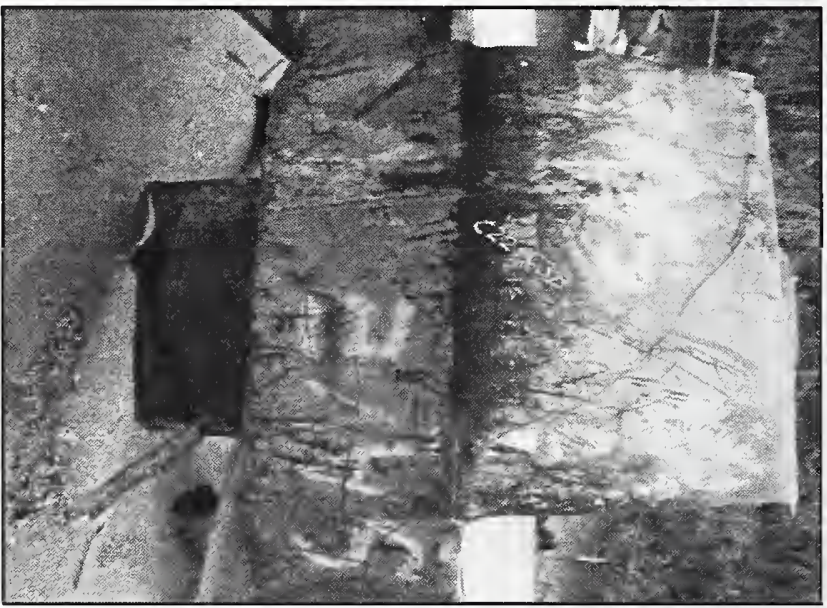

Figure C-1. Sample C-91, channel with two seats. 


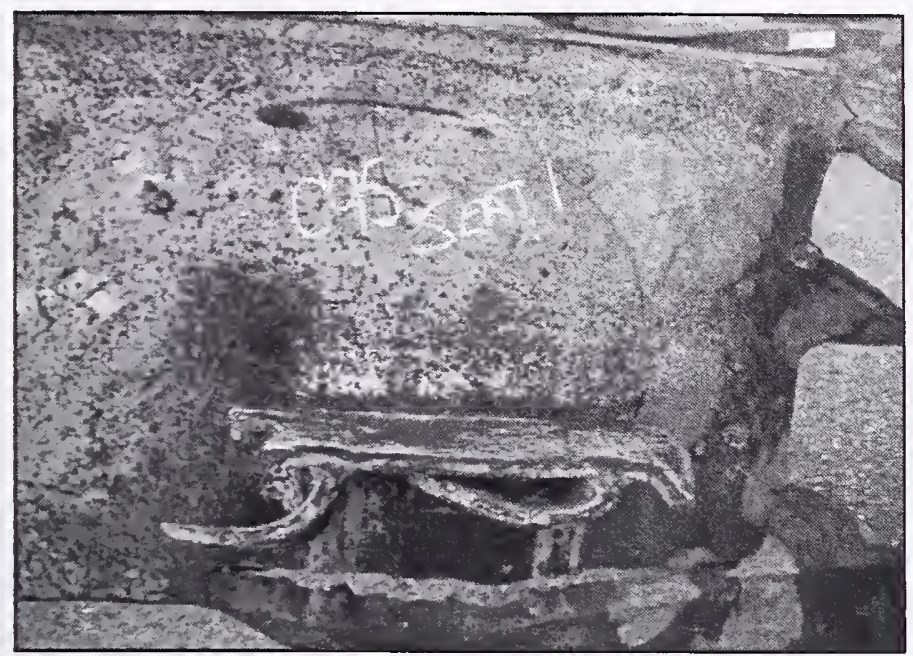

One seat with one hole intact, other ripped out, seat was bent

Figure C-2. Sample C-95, channel with one seat.

a)

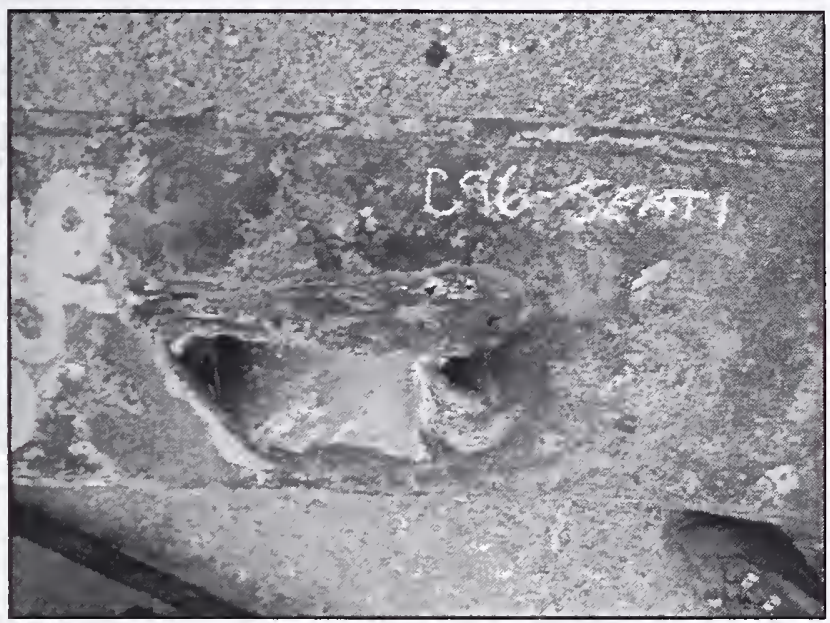

Seat 1: Both holes ripped out, seat appears corroded

Seat 2: Seat ripped off at welds

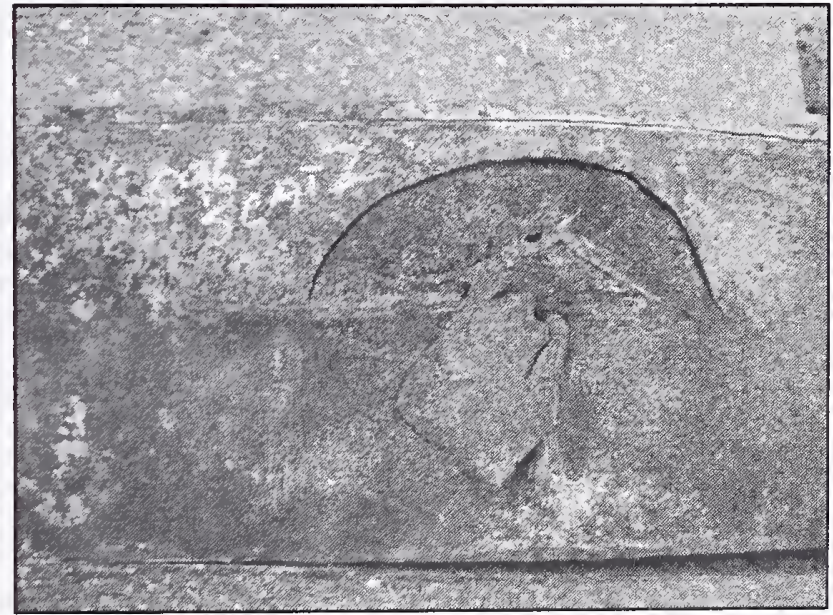

Figure C-3. Sample C-96, channel with two seats. 
a)

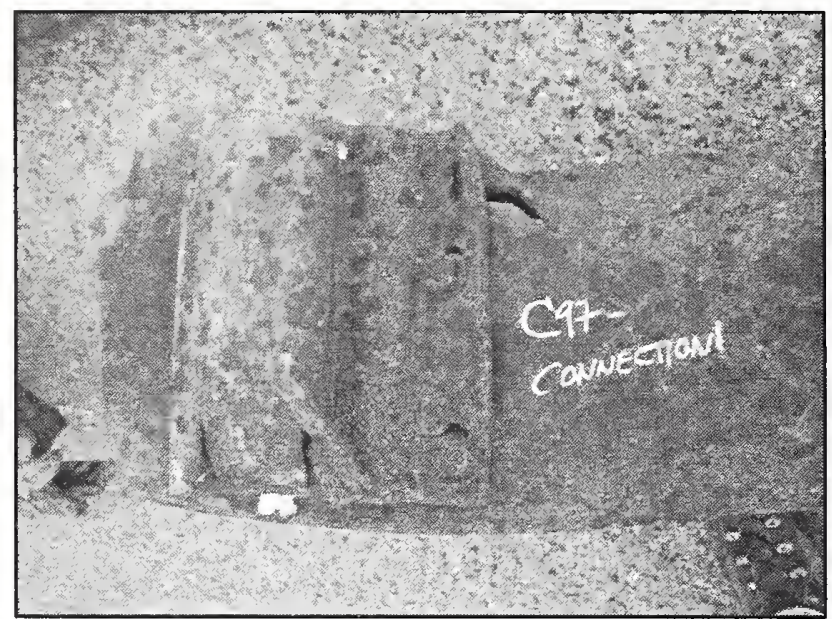

Connector 1

b)

Connector 2

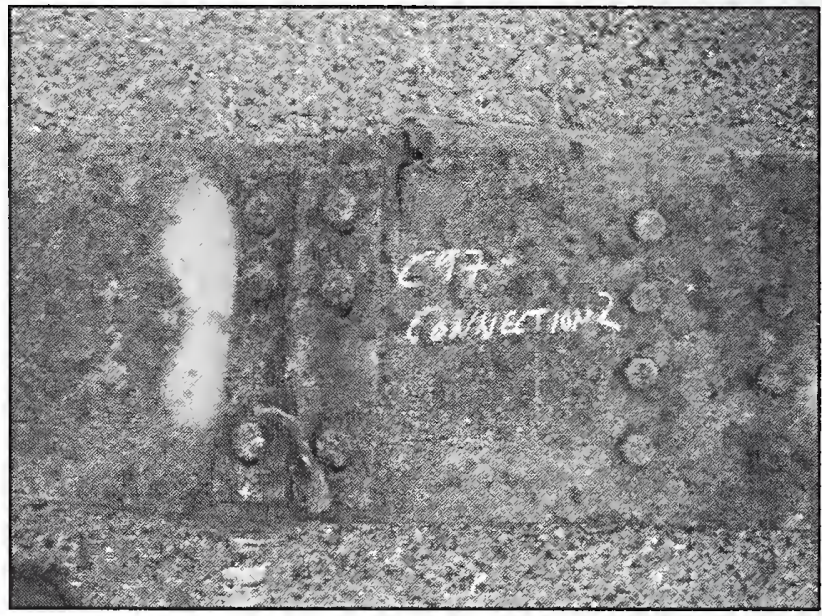

c)

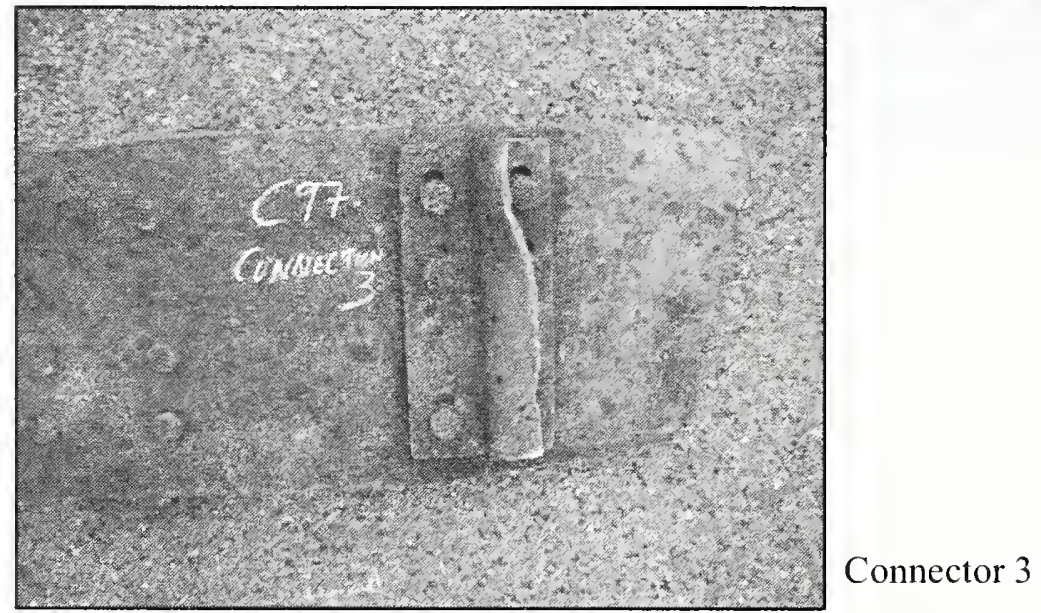

Figure C-4. Sample C-97, channel with three connectors. 


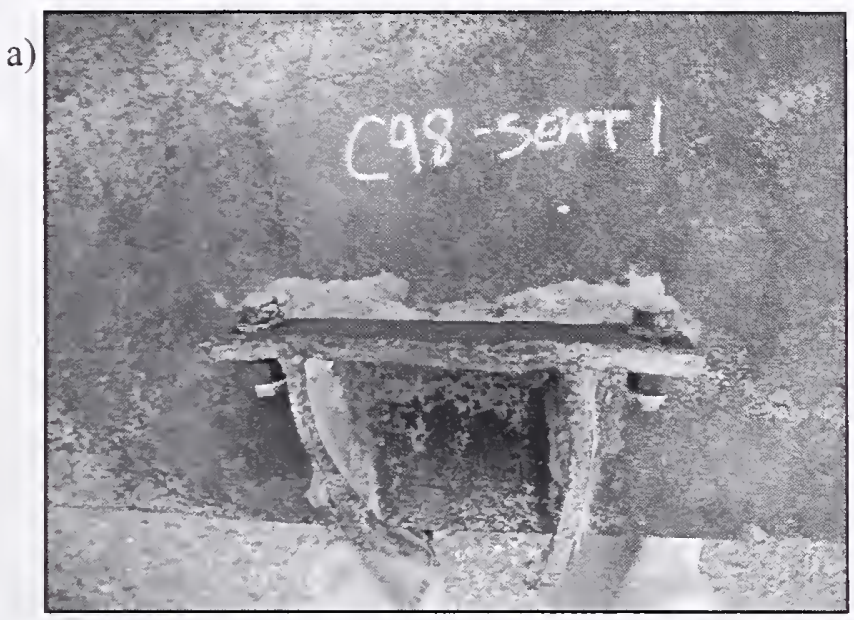

Seat 1: Both holes intact, both have bolts remaining, seat was bent

b)

Seat 2: Both holes ripped through, one has bolt remaining, seat was bent

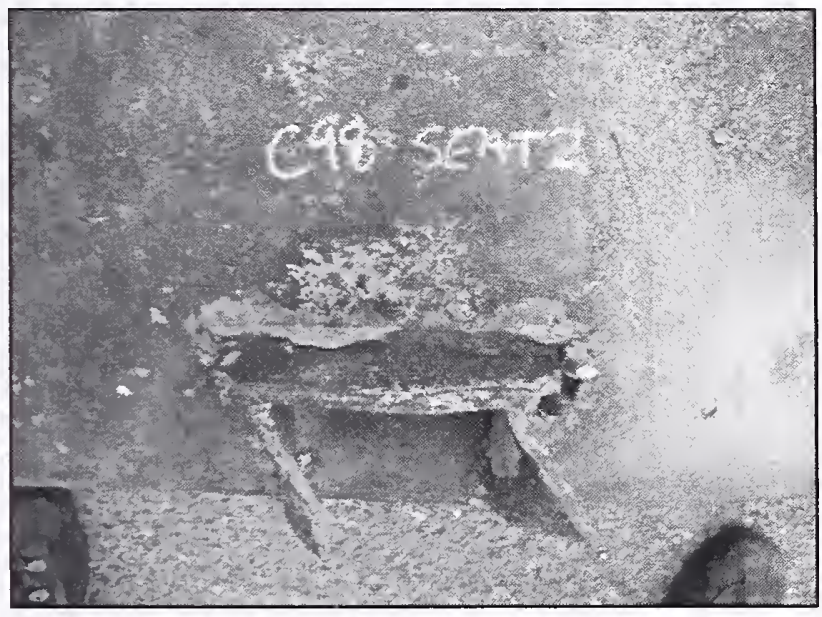

Figure C-5. Sample C-98, channel with two seats. 
a)

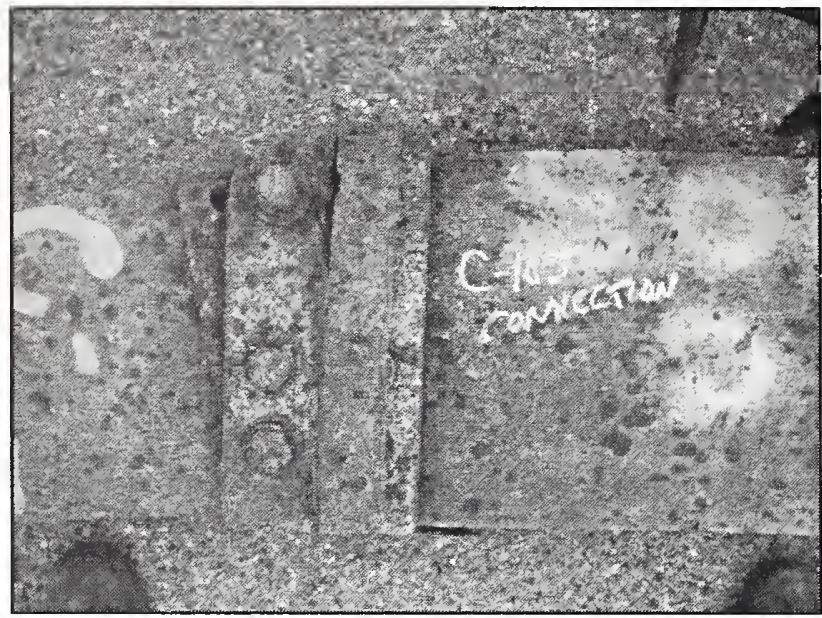

b)

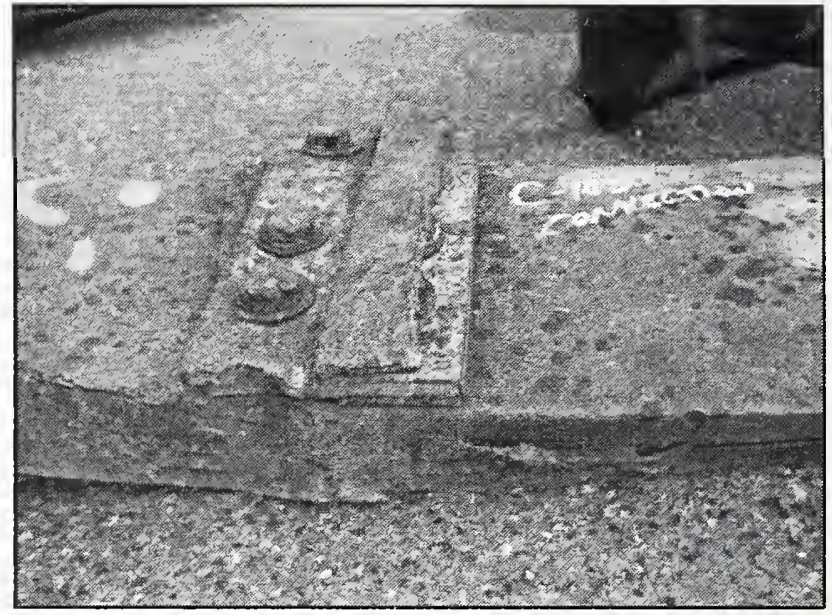

Figure C-6. Sample C-105, channel with one connector. 


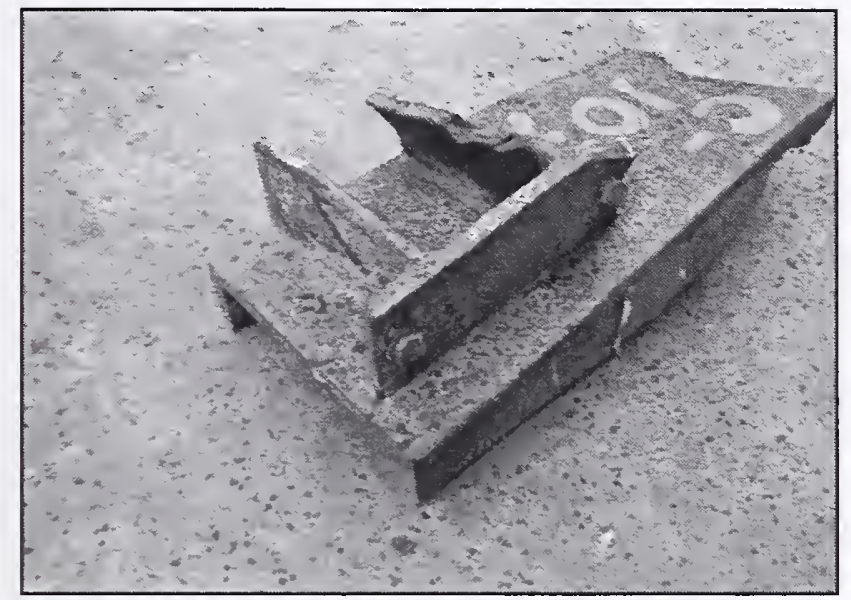

One seat with both holes intact, both holes have bolts remaining

Figure C-7. Sample C-107, channel with one seat. 


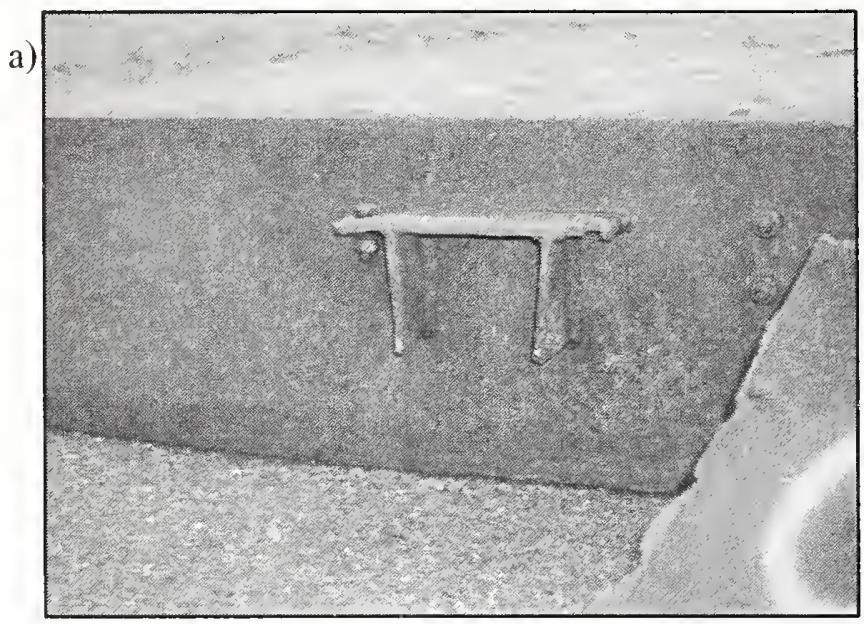

Seat 1: One hole intact with bolt, other ripped out

Seat 2: Both holes intact, seat was slightly bent

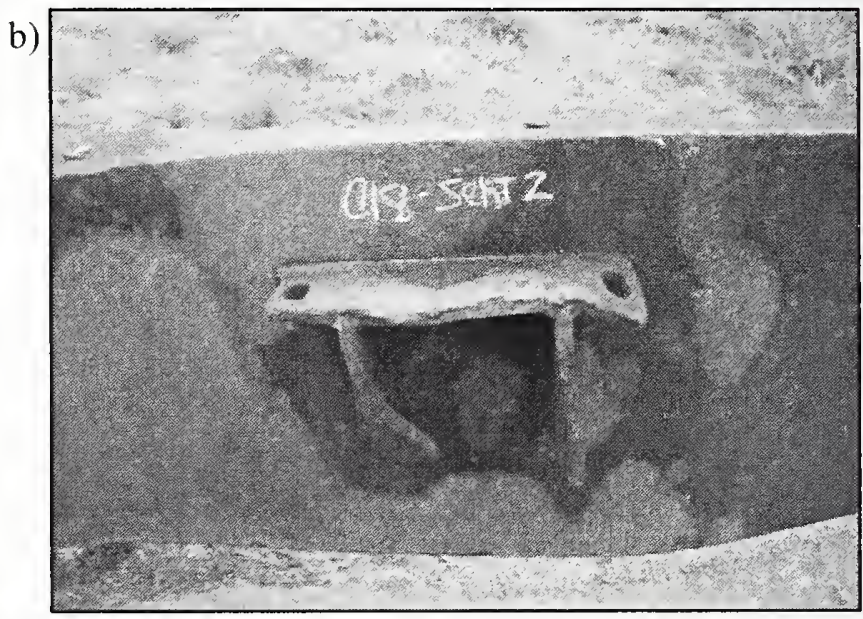

c)

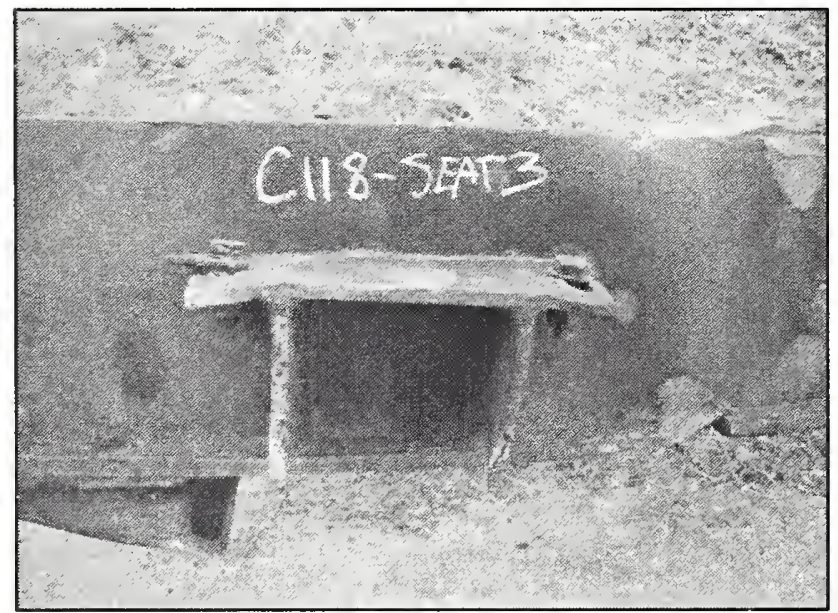

Seat 3: Both holes intact with bolts remaining

Figure C-8. Sample C-118, channel with three seats. 


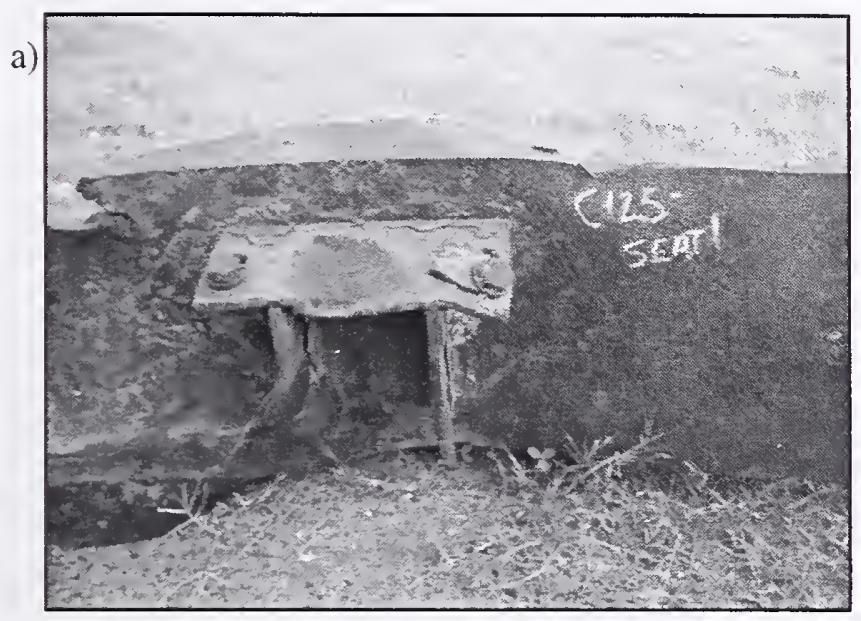

Seat 1: Both holes intact with bolts remaining, one has piece of angle attached, seat was bent

Seat 2: Both holes ripped out, seat was bent

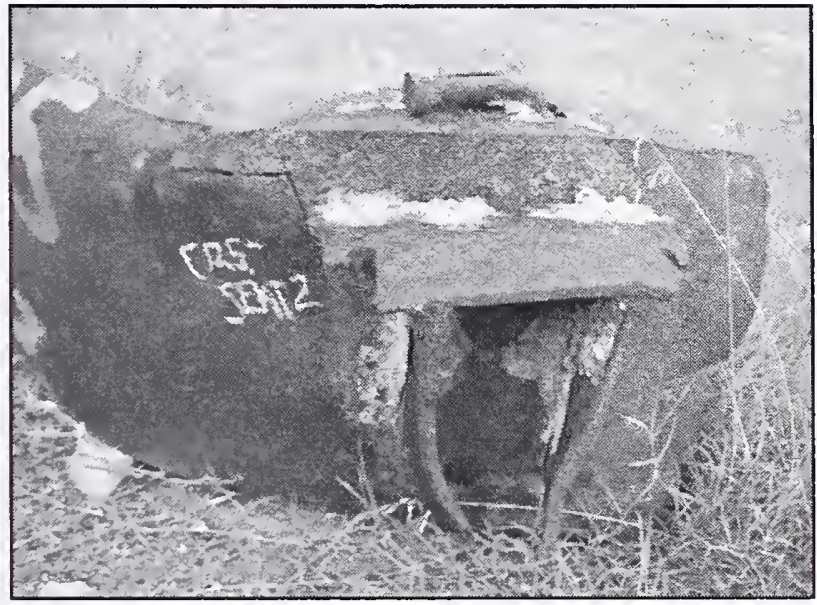

Figure C-9. Sample C-125, channel with two seats.

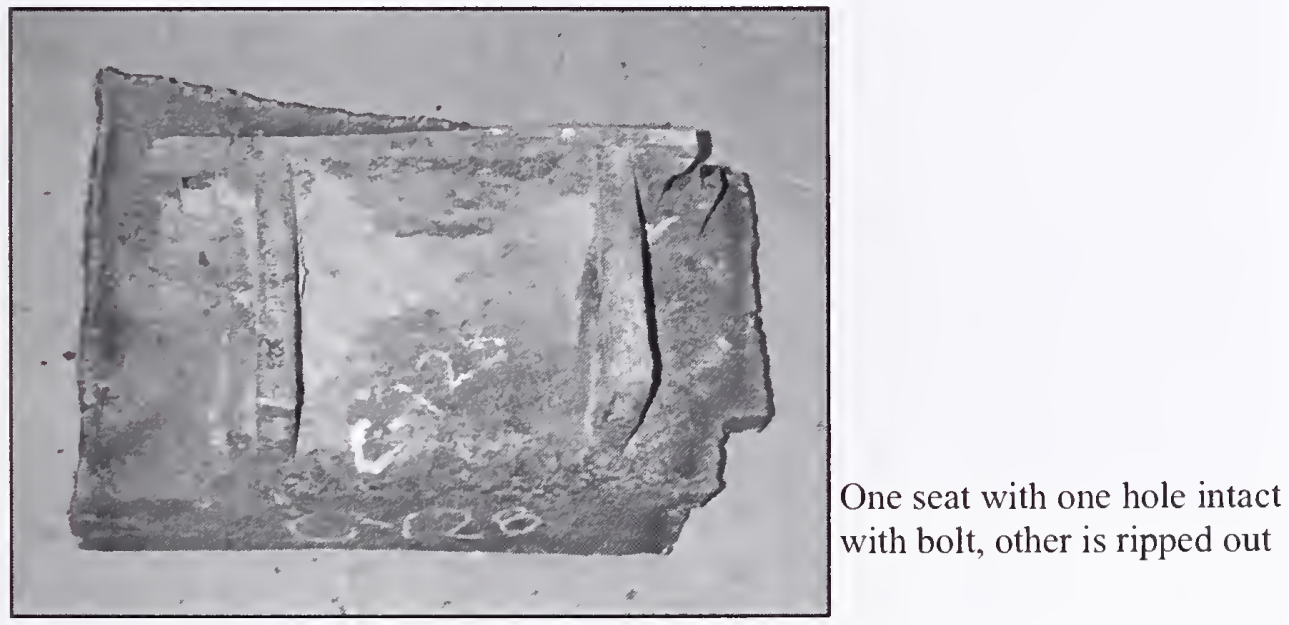

Figure C-10. Sarnple C-128, channel with one seat. 
a)

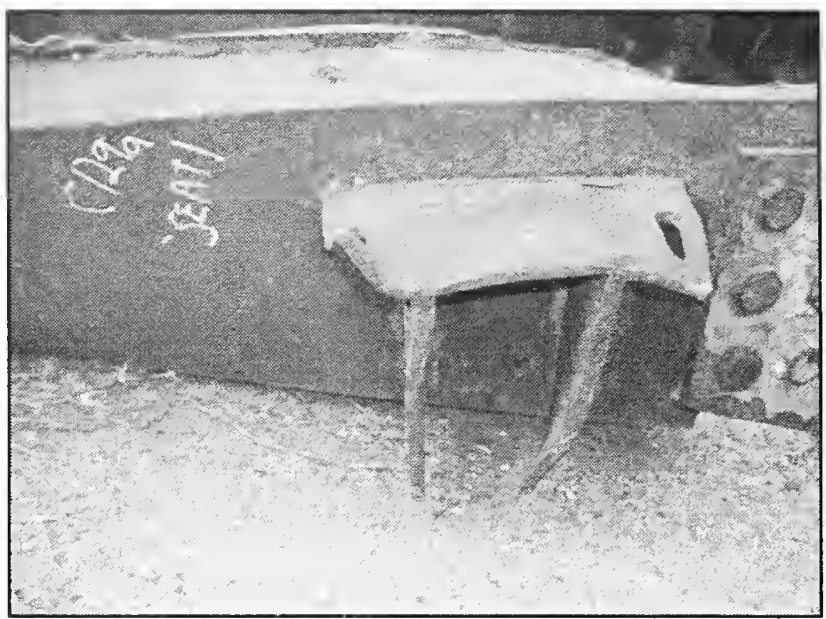

Seat 1a: One hole intact, other ripped out, seat was bent

Seat 1b: One hole intact with bolt, other is ripped out, seat was bent

b)

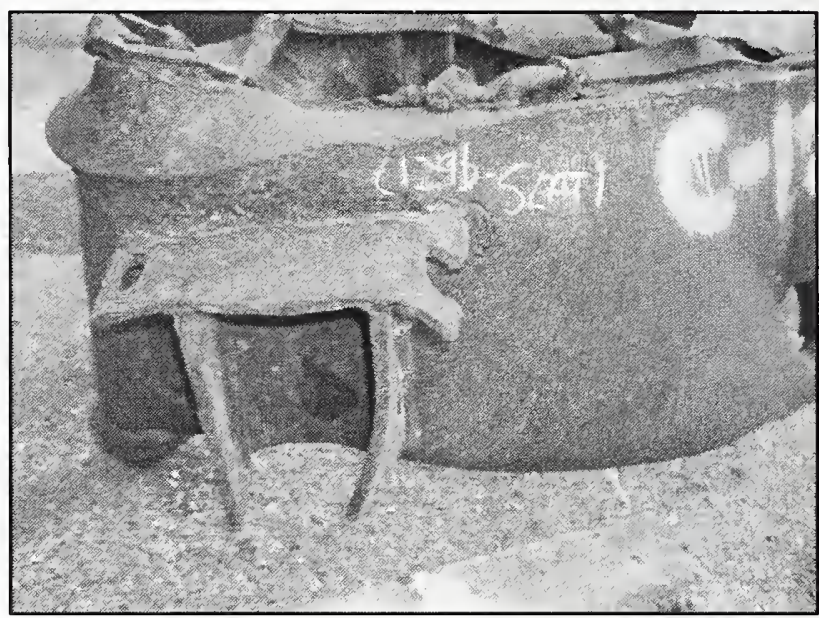

c)

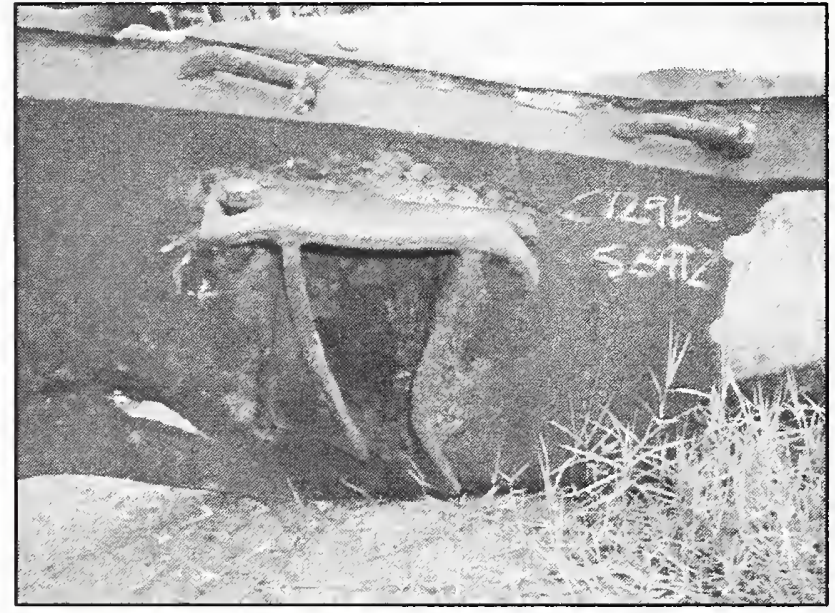

Seat 2b: Both holes intact, seat was bent

Figure C-11. Sample C-129, two connected channels with three seats. 


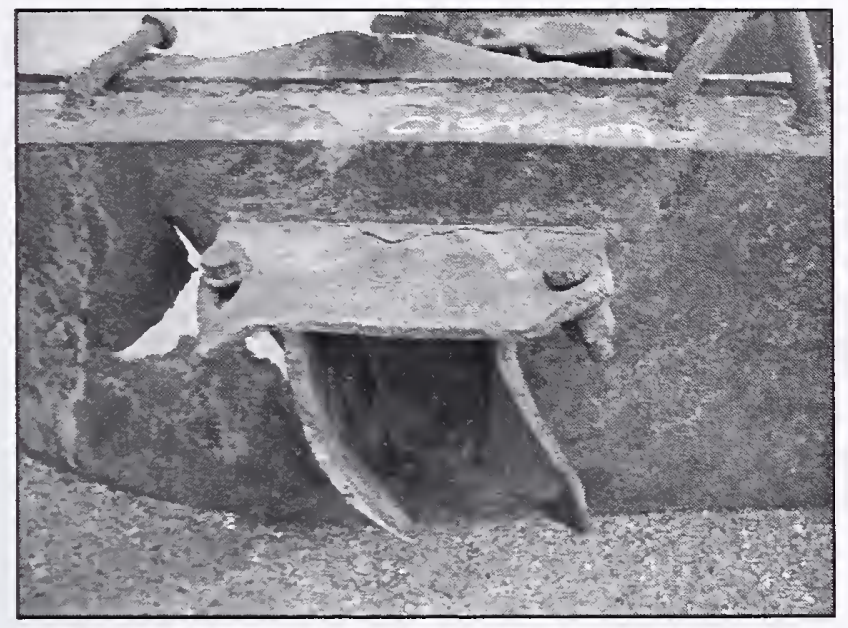

One seat with both holes intact, both holes have bolts remaining, seat was bent

Figure C-12. Sample C-134, channel with one seat.

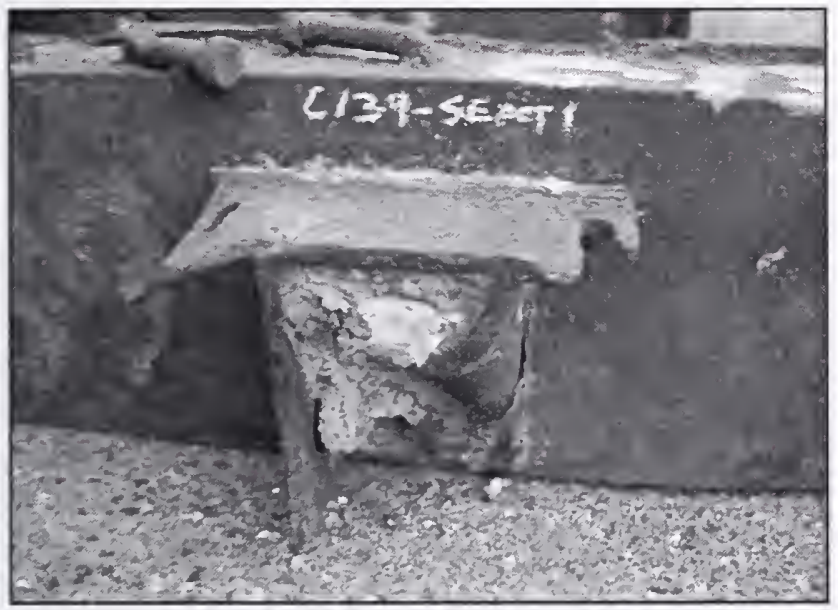

One seat with one hole intact, other ripped out, seat was damaged

Figure C-13. Sample C-139, channel with one seat. 


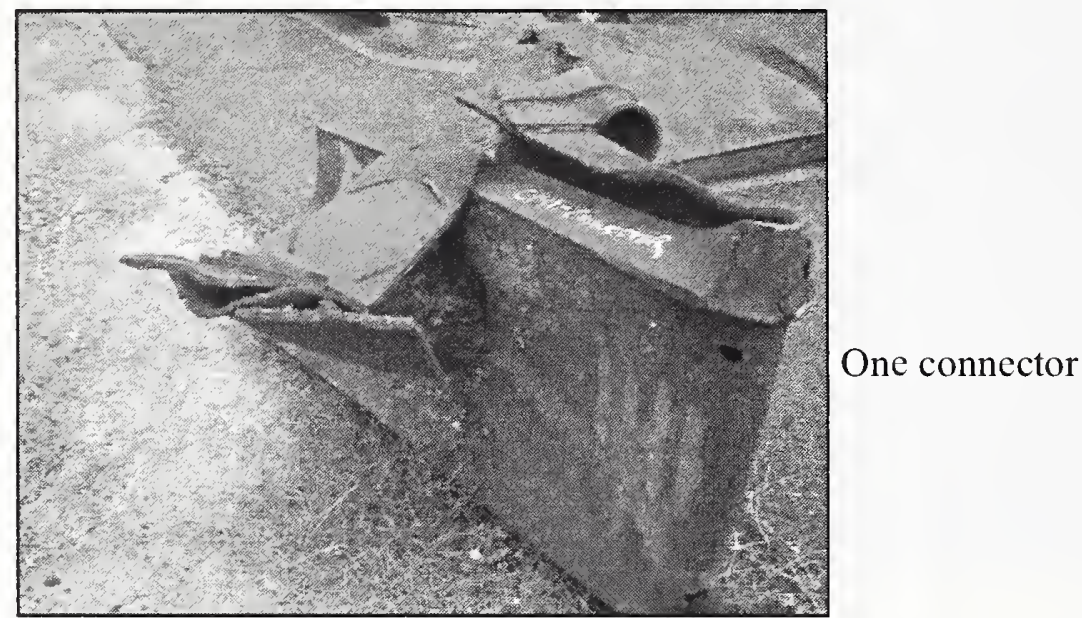

Figure C-14. Sample C-141, channel with one connector.

a)

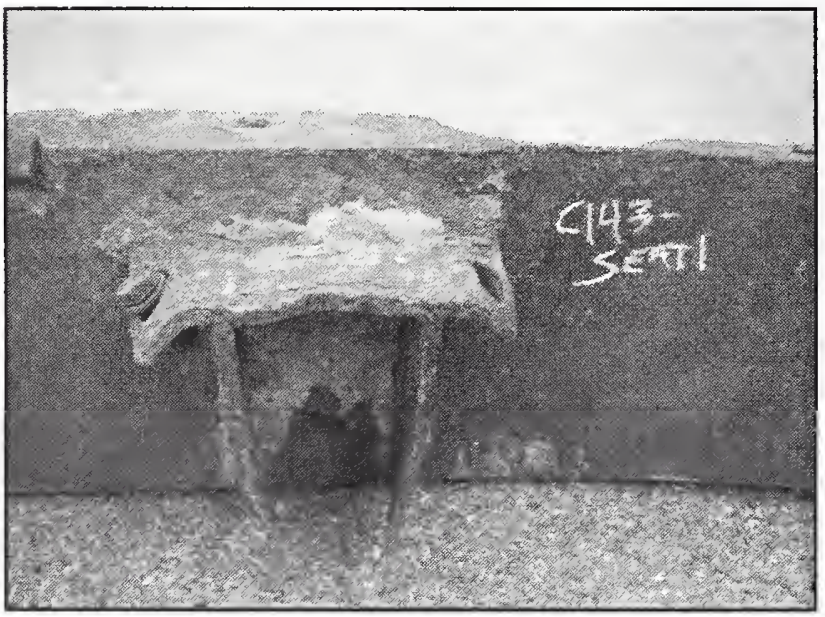

Seat 1: Both holes intact, one has bolt remaining, seat was bent

b)

Seat 2: Ripped off at the weld

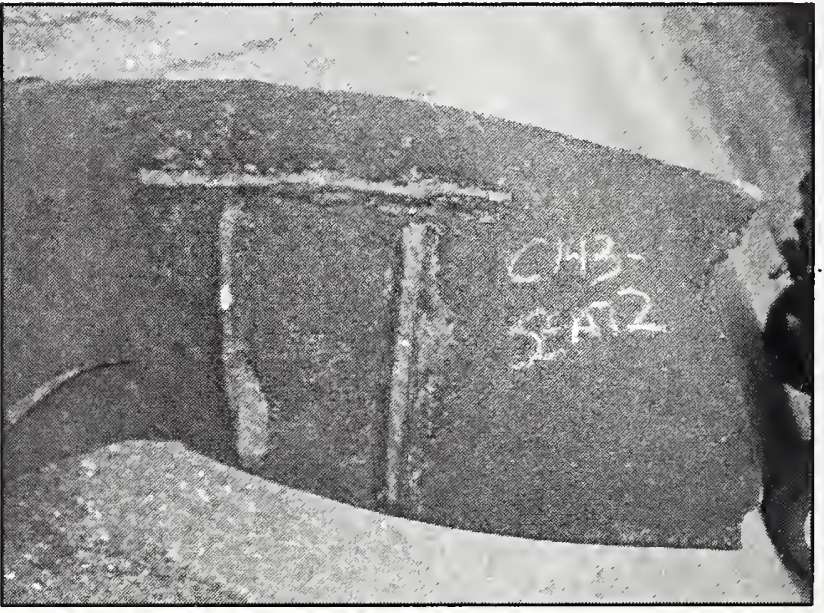

Figure C-15. Sample C-143, channel with two seats. 


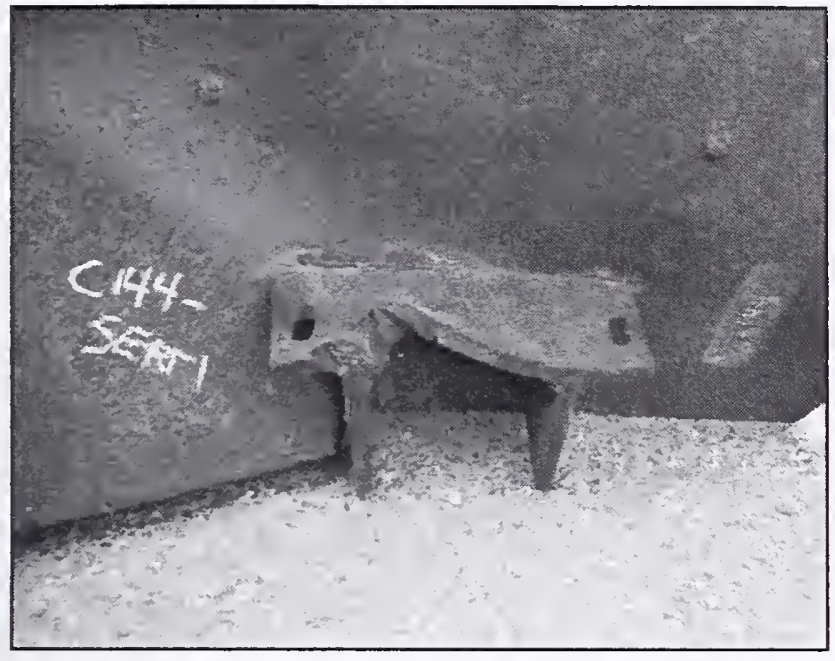

One seat with both holes intact, seat was damaged

Figure C-16. Sample C-144, channel with one seat.

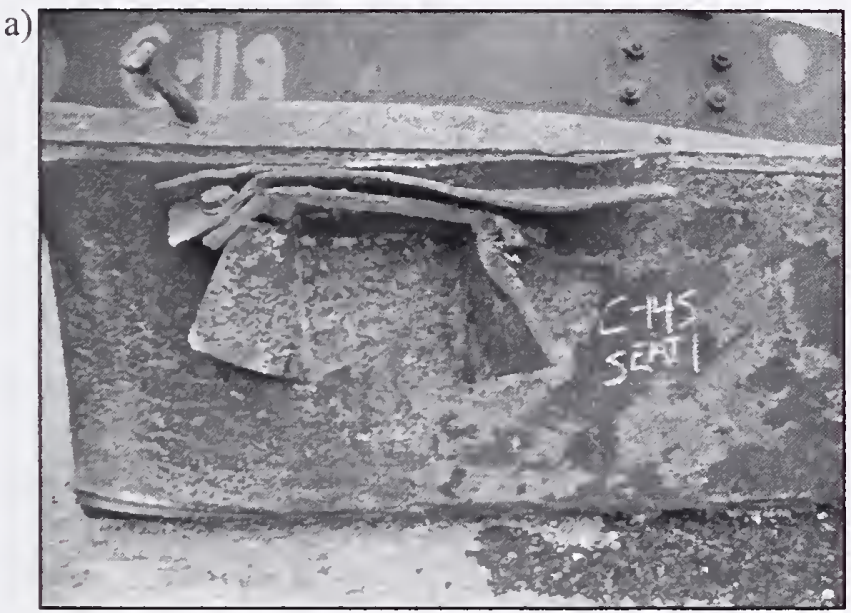

Seat 1: One hole intact with bolt and angle attached, other ripped, seat was damaged

b)

Seat 2: Both holes intact, seat was bent

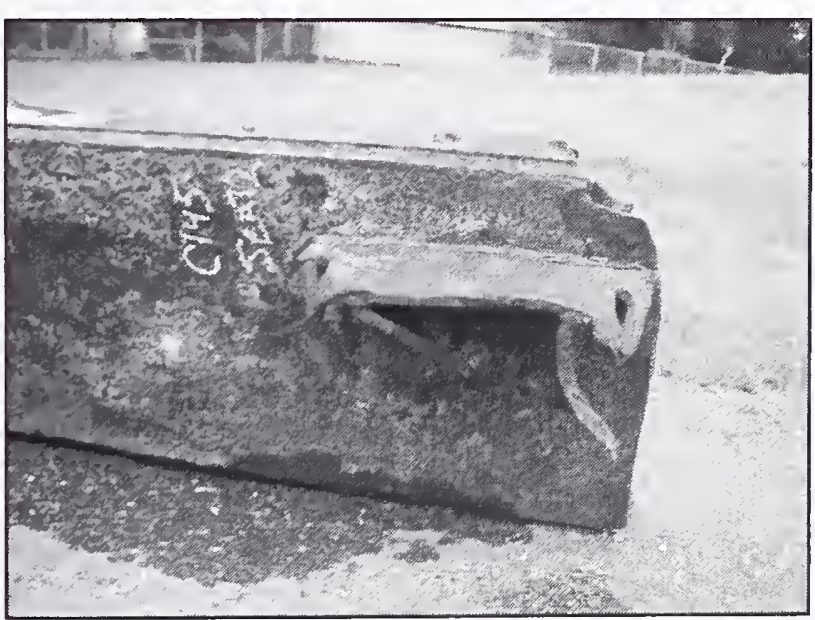

Figure C-17. Sample C-145, channel with two seats. 


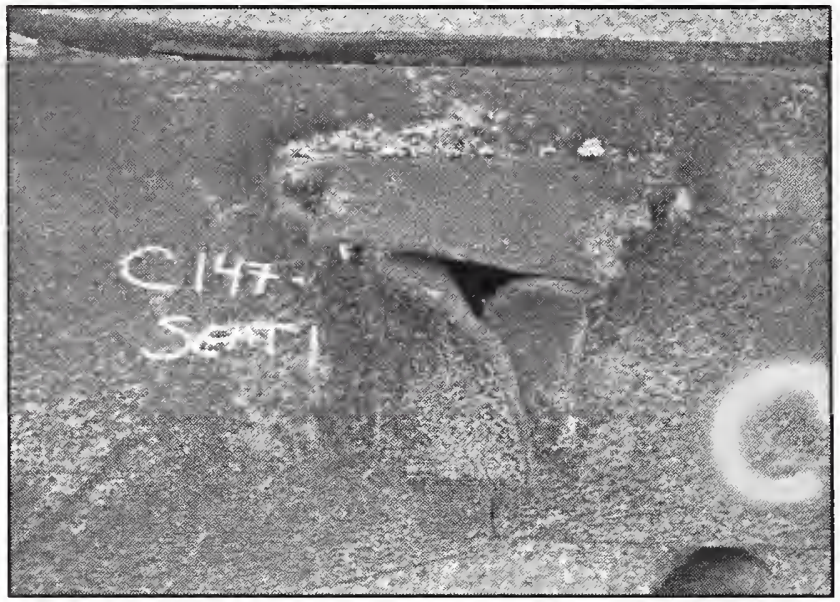

One seat with both holes ripped out, seat was bent

Figure C-18. Sample C-147, channel with one seat.

a)

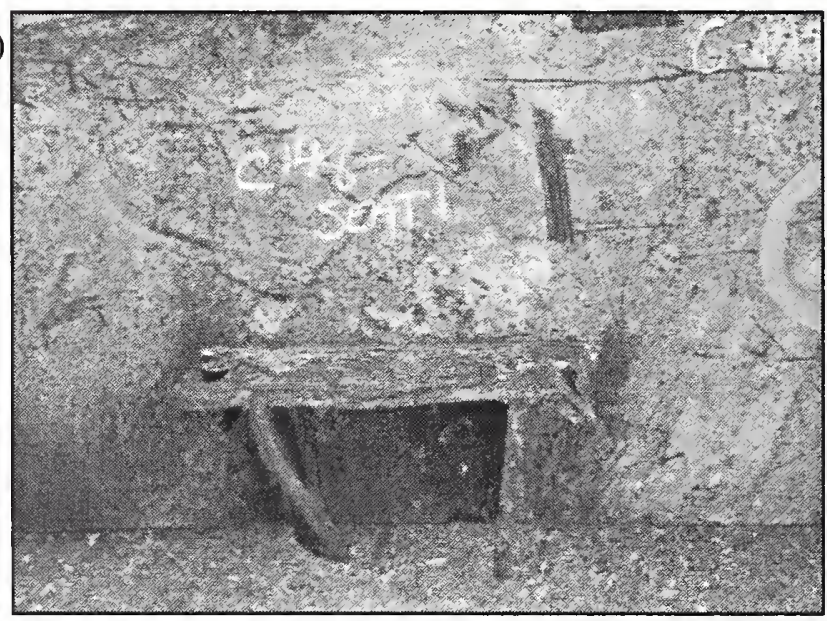

Seat 1: Both holes intact, one has bolt

Seat 2: Both holes ripped out, seat crushed

b)

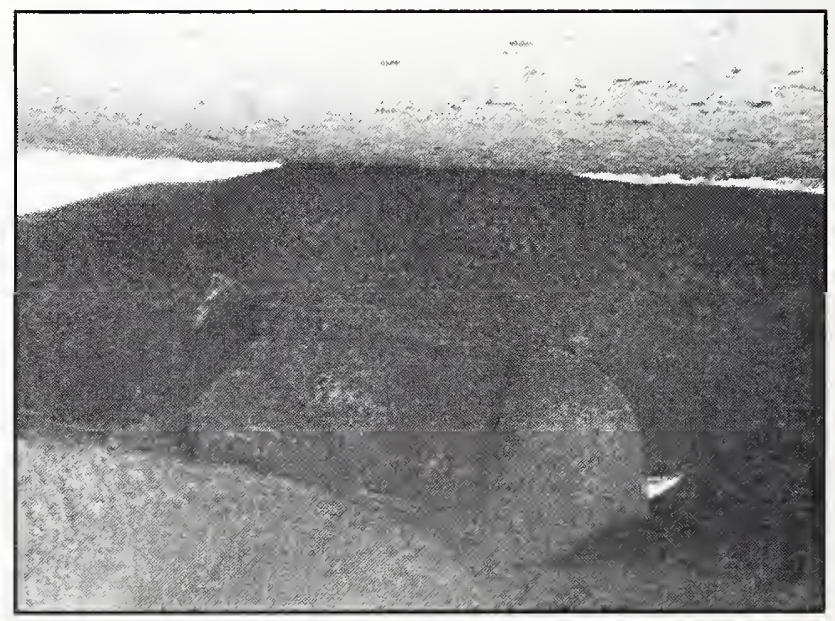

Figure C-19. Sample C-148, channel with two seats. 


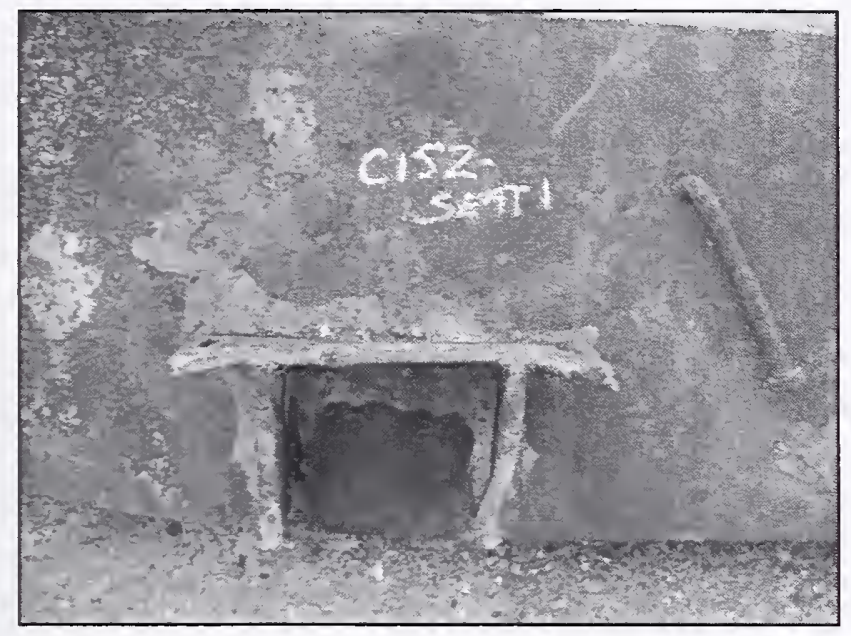

One seat with both holes intact

Figure C-20. Sample C-152, channel with one seat.

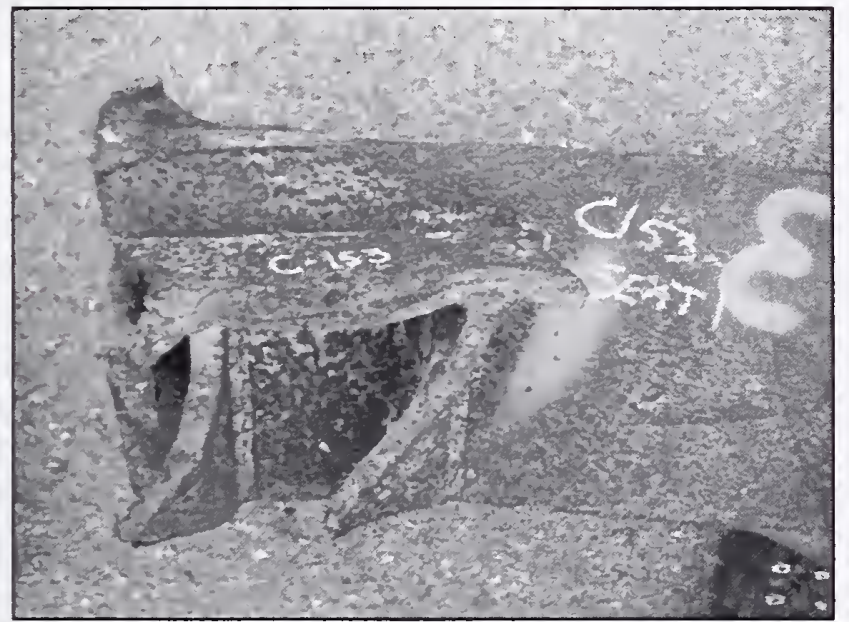

One seat with both holes ripped out, seat was bent

Figure C-21. Sample C-153, channel with one seat. 


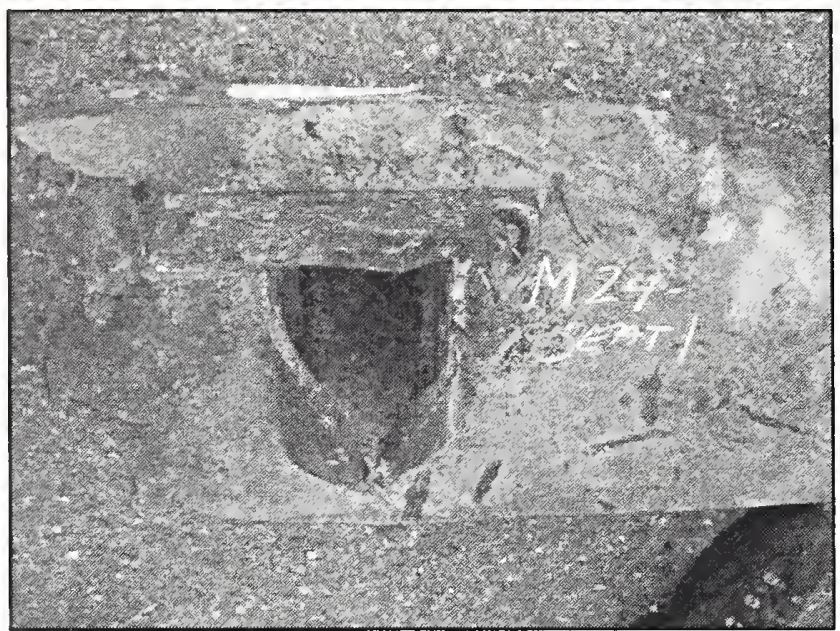

One seat with both holes intact, seat was bent

Figure C-22. Sample M-24, channel with one seat.

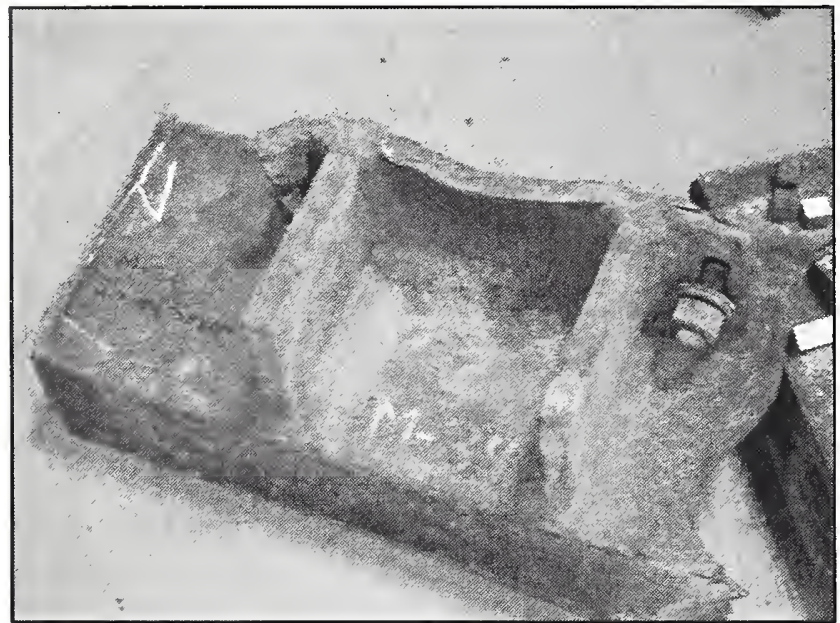

One seat with one hole intact with bolt remaining, other was ripped out

Figure C-23. Sample M-34, channel with one seat. 


\section{Appendix D \\ FORENSIC THERMOMETRY TECHNIQUE DEVELOPMENT}

\section{D.1 INTRODUCTION}

Determination of temperatures reached by the structural steel in the fires of World Trade Center (WTC) 1 and WTC 2 can provide important clues as to the intensity of the fires as well as data for input or validation of the modeling of the building response to the fires. A number of methods were examined which would allow for the determination of the maximum temperature experienced by a given steel component in the building. This appendix reports on a number of potential tests that could meet this need.

\section{D.2 CANDIDATE FORENSIC TESTS}

\section{D.2.1 Condition of Paint}

Perhaps the most obvious physical indicator of a component's exposure to high temperatures is the condition of the paint. Most paints contain organic materials in the form of binders or agents that allow them to be spread or sprayed. Upon drying, depending on the particular paint formulation, some of these organic compounds are left in the coating and burn upon exposure to high temperatures.

The structural steel components for the WTC were painted with a coating consisting of several oxide pigments suspended in a volatile liquid (Sramek 1967). The constituents of the paint are found in Table D-1. The paint was applied by the fabricators at the factory and given a low temperature (approx. $120^{\circ} \mathrm{C}$ ) bake to cure the paint and evaporate the suspending liquid. The paint was essentially a ceramic coating, consisting of predominantly of iron oxides with small additions of other oxides that provide color, and silica sand. The purpose of the sand in the mixture was not made clear in available documents, but in a traditional column and girder building, it was added for good foot traction for the steelworkers.

Under the assumption that this was a typical paint with an organic binder, initial burn tests were conducted at relatively low temperatures, looking for the temperature at which the paint will combust. The paint was unaffected at exposure temperatures below approximately $250{ }^{\circ} \mathrm{C}$ (Fig. D-la). Beginning with exposure temperatures of $250^{\circ} \mathrm{C}$, the samples began to exhibit noticeable "mud crack" patterns (Fig. D-1b). These were formed when the paint is put into biaxial tension under the influence of the thermal expansion mismatch between the paint and the base steel. This temperature where the first mud crack patterns appear did not vary between samples by more than $25^{\circ} \mathrm{C}$ and was not found to be a function of exposure time beyond this range. Additional burn tests at temperatures up to $650{ }^{\circ} \mathrm{C}$ produced mud-crack patterns (Fig. D-1c) that were indistinguishable in size or character from those produced at $250{ }^{\circ} \mathrm{C}$. Figure D-2 shows the time dependence of the mud cracking appearance at $250{ }^{\circ} \mathrm{C}$. These results would indicate that the paint has a characteristic size of the un-cracked islands of the paint that is not subdivided by heating to higher temperatures. 
Beyond approximately $650{ }^{\circ} \mathrm{C}$ (plus or minus approximately $50{ }^{\circ} \mathrm{C}$ for the samples tested), a black scale formed between the steel and the paint, Fig. D-3. This scale layer had very poor adherence to the steel, and the paint was seen to flake off with slight pressure. Above approximately $800^{\circ} \mathrm{C}$, the kinetics of the scale formation were very fast, and after short exposures to this temperature a thick scale formed and spalled off of the steel, carrying away the paint. This left a very dark blue-black colored surface to the steel.

The paint samples tested were taken from exterior columns. Burn tests were proposed for core columns to establish the same calibration, but sufficient material with intact paint on the core columns could not be isolated. The Tnemic series brand of paints was in common use in high-rise construction steel at the time of the erection of the WTC, and it is assumed that the paint on the core columns that was imaged in this study (a very limited sample) was also of this type and exhibited the same degradation mechanism and kinetics.

There are many uncertainties associated with using the condition of the paint as an indicator of temperature exposure. The formation of the scale exhibited both time and temperature dependence. In addition, the mud-crack pattern seen on the burn specimens in the lab could also be produced by plastic deformation of the underlying steel, or through the corrosion of the steel. Cracking due to plastic deformation generally had a more directional pattern to the cracks, lying perpendicular to the axis of tension and bending, and could often be separated from thermal expansion cracking patterns. At best, since there were several ways to produce mud-cracks, their absence can predict the absence of high temperature exposure, plastic deformation, or generalized corrosion for that component, but their presence cannot a priori be taken as an indicator of high temperature exposure.

\section{D.2.2 Hardness of Washers}

The washers used on the vertical and spandrel bolted connections of the panels of the exterior wall were punched from high strength steel sheet and were subjected to a post-manufacturing anneal to decrease the possibility of warping or cracking. These washers have a high hardness which relies on metastable microstructures and residual stresses that may change with high temperature exposure. The hardness of the washers of the vertical and spandrel panel connections was examined to see if they could give an indication of the maximum temperature of exposure for a given associated component.

A series of heat treatments was performed on washers taken randomly from recovered steel components. In each case, several washers were taken from the same location, so that multiple samples could be tested to establish statistics. The washers were heated in a laboratory furnace to a given temperature and time combination, air cooled, and hardness readings were made. In all cases, the washers were put directly into a furnace where the exposure temperature had been established and subsequently removed and placed in the same location to cool down, so the only difference between the heating cycles was the maximum temperature and exposure time. For the first set of hardness testing, the samples were metallographically mounted and polished to a 1 micrometer finish before indentation using Vickers microhardness ( $1 \mathrm{~kg}$ load). For those washers measured using Rockwell testing ( $\mathrm{C}$ scale), the samples were ground using silicon carbide papers to 800 grit, in order to remove any scale and oxide that formed during the heating, but were not polished. 
Results typical of these experiments are given in the plot of Vickers microhardness versus exposure temperature in Fig. D-4. As seen, the scatter within the individual measurements is larger than any effect on the hardness of heating the samples. To test whether this was an artifact of spatial variation of hardness in the washers with a size scale on the order of the Vickers indent size, Rockwell C hardness measurements, with a much larger indentation size, were also performed. The Rockwell measurements showed less scatter, but also did not show a clear trend of hardness with annealing temperature. Thus, this technique was rejected for further consideration in the investigation.

\section{D.2.3 Metastable Phases in Weld Metal}

During the welding process, the feed metal from the welder is melted along with some base metal from the two components being joined as the welding arc passes. This pool of molten metal then rapidly cools, forming the weld. As the metal rapidly cools, metastable phases are formed within the weld metal region. These phases transform to other more stable phases upon heating to some temperature which is retained upon subsequent cooling. The presence of metastable phases can be detected during a thermal scan in a differential thermal analyzer (DTA), which measures the heat absorption and emission from a sample as the temperature is changed. The transformation of metastable phases are detectable as an exothermic spike.

Samples of weld and base metal were cut from pieces retrieved from the WTC. These were subjected to DTA scans over a temperature range of $0{ }^{\circ} \mathrm{C}$ to $800{ }^{\circ} \mathrm{C}$. DTA tests were performed on samples from three locations: weld metal, inner web, and flange plate from a sample with $F_{y}=100 \mathrm{ksi}$ (panel C-10). The initial microstructure of the inner web and flange plate was quenched and tempered. Cylinders, $3 \mathrm{~mm}$ diameter and $1 \mathrm{~cm}$ long, were cut by Electrical Discharge Machining (EDM). The cylinder from the weld metal was cut with its axis parallel to and centered in the weld zone. Other samples were cut from several locations from the flange and inner web away far from the weld zone. DTA scans were performed in gettered Ar on $\sim 100 \mathrm{mg}$ slices from the EDM cylinders.

Figure D-5 show's two superimposed DTA scans. The initial heating scan at $20 \mathrm{~K} / \mathrm{min}$ exhibits a broad exothermic peak centered at $400^{\circ} \mathrm{C}$. The cooling scan at $2.5 \mathrm{~K} / \mathrm{min}$ shows no significant transformations. The second heating scan at $20 \mathrm{~K} / \mathrm{min}$ shows that the broad exothermic peak is absent. In general, DTA scans exhibit endothermic peaks on heating for normal 'equilibrium' transformations. On the other hand, exothermic peaks on heating usually indicate the return of a non-equilibrium state formed during prior processing to an equilibrium state. The interpretation of the present experiment was that the broad exothermic peak during first heating resulted from the annealing (exothermic heat release) of the weld microstructure. The second heating shows no such exothermic peak. Thus, heating into the range of $400{ }^{\circ} \mathrm{C}$ is required to remove the cast microstructure and presumably the associated residual stresses. Two more samples were tested by DTA: flange and inner web material far from the weld. The broad exothermic peak at $400{ }^{\circ} \mathrm{C}$ seen in the weld metal is absent in the DTA scans of the non-weld materials.

DTA scans of weld metal would, thus, in the case of similar welds, predict exposure of the base metal to temperatures in excess of $400^{\circ} \mathrm{C}$, and would be a "litmus" test for that temperature. Difficulties arise when trying to broadly apply this technique to the rest of the materials recovered by National Institute of Standards and Technology (NIST). Sample acquisition and preparation was time consuming, as was the actual experiment. Also, given the 14 different grades of steel and the many different welding techniques and materials used in the fabrication of the various components in the WTC, different chemistries, 
welding temperatures, and cooling rates will produce different metastable phases, each with a characteristic transformation temperature. Each weld would need to be examined in detail and calibrated with tests of identical material that was known not to have burned. These operational requirements make this technique very difficult to apply, but it was still available if a detailed examination of a critical element was necessary.

\section{D.2.4 Relaxation of Residual Stresses in Welds}

In addition to metastable microstructural phases, residual stresses develop during weld cooling. These form when the yield stress of the cooling metal increases sufficiently to resist deformation against the thermal expansion (contraction) stresses within the weld metal. If the weld metal cools fast enough, the stresses trapped within the structure are large. Annealing at a given temperature will allow the stresses to relax by lowering the flow stress of the metal and allowing plastic flow to occur. Measurement of the residual stresses in welds was surveyed as a possible indicator of the temperature that might have been reached in a fire.

Figure D-6 shows the residual stress profiles measured in prototype welds on non-WTC steel using neutron diffraction. The technique is able to resolve the stresses sufficiently to be able to determine temperature exposures between $200{ }^{\circ} \mathrm{C}$ and $500{ }^{\circ} \mathrm{C}$ with an estimated resolution of $50{ }^{\circ} \mathrm{C}$. However, this technique suffers from the same difficulties as the DTA technique described above. The sample preparation was time-consuming and critical. Since the flow stress depends on chemical composition as much as the presence or absence of metastable phases, the technique would need to be calibrated on identical welds that were known not to have burned. Nevertheless, this technique remains available if needed to apply to critical temperature determinations on a specific component.

\section{D.2.5 Microstructural Changes in Steel}

The as-fabricated microstructure of structural steel may change with exposure to high temperatures. As seen in Sec. 5.4 of NIST NCSTAR 1-3E, furnace exposure of the flange plates, spandrel plates, and floor truss seats to $625^{\circ} \mathrm{C}$ for as little as $0.25 \mathrm{~h}$ produced noticeable changes in the microstructure. For the hot rolled steels, the plates of cementite were observed to spheroidize. For the quenched-and-tempered microstructures, the lath boundaries of the martensitic structure became less distinguishable, while the cementite precipitates became more discernable as exposure time increased. The results from this study were used to determine if microstructural changes occurred in perimeter columns exposed to pre-collapse fires.

\section{D.2.6 Combustion of Architectural Aluminum with Structural Steel}

Some photographic evidence showed that architectural aluminum façade, applied to the outer surface of the exterior columns, appeared to burn while erect on the building (NIST NCSTAR 1-5A). It was postulated that determining the temperature at which the aluminum façade combusted would give an indication of the temperature of the steel components in their vicinity.

This idea was rejected and not investigated for the following reasons. The temperature of the aluminum façade while installed and experiencing flames from the building would have little relation to the temperatures experienced by the steel members. The combustion of the aluminum occurred quite rapidly, 
and the difference in thermal mass between the façade and column would make it extremely difficult to estimate the temperature of the steel from the fact that the aluminum burned. In addition, the façade was attached to the column at specific points by means of standoff bolts, and was not in direct contact being heated conductively, but rather by the flames being fed by the air rushing through the building.

\section{D.2.7 Corrosion Scale Formation Kinetics}

A final technique that was considered and rejected was the measurement of the thickness of the scale that formed between the paint and steel upon exposure to high temperature. By correlating temperature and exposure time to characteristic features of the scale, it may be possible to estimate the maximum exposure temperature of a given sample. A series of controlled burn tests were conducted in the laboratory, and it became quickly apparent that this technique would have limited utility. The scale formation was found to be a complex function of annealing temperature, annealing time, and atmosphere. Additionally, delamination of the scale and paint occurred. As many variables were found to influence the development and adherence of the reaction product scales between the steel and the paint, this analysis method was abandoned.

\section{D.3 SUMMARY}

A number of analytical techniques that might give indications as to what temperature a particular piece of erected structural steel reached were studied for this investigation. The only one that was found to be rapid and easy to perform in the field was the microscopic analysis of the condition of the paint on the columns. This paint, essentially a ceramic coating, was found to crack in a particular pattern when the base steel was heated to approximately $250^{\circ} \mathrm{C}$, and was found to spall off entirely when the steel was heated to in excess of $650{ }^{\circ} \mathrm{C}$. The test was a "negative" test, in that the cracking pattern can also be created by deforming or corroding the steel, so the absence of cracking indicated the absence of a temperature excursion above $250^{\circ} \mathrm{C}$. Other techniques, such as weld stresses and metastable phases, were found to be potentially useful but exceedingly difficult to implement. However, if needed and with proper calibration, these tests would likely yield more information.

\section{D.4 REFERENCES}

Sramek, Thomas F. 1967. Correspondence between Pittsburgh-Des Moines Steel Company and R.M. Monti, PONYA. giving clarification and attaching a product sheet for Tnemec 69 and 99 column paints. November 22. 
Table D-1. Composition of primer paint.

\begin{tabular}{|l|l|r|}
\hline \multirow{5}{*}{ Pigment } & Iron oxide & $35.9 \%$ \\
\cline { 2 - 3 } & Zinc yellow & $20.3 \%$ \\
\cline { 2 - 3 } & $\begin{array}{l}\text { Tnemec pigment (proprietary } \\
\text { composition) }\end{array}$ & $33.7 \%$ \\
\cline { 2 - 3 } & Diatomaceous silica & $10.1 \%$ \\
\hline \multirow{5}{*}{ Vehicle } & Soya alkyd resin solids & $16.5 \%$ \\
\cline { 2 - 3 } & Hard resin & $2.8 \%$ \\
\cline { 2 - 3 } & Raw linseed oil & $35.1 \%$ \\
\cline { 2 - 3 } & Bodied linseed oil & $6.4 \%$ \\
\cline { 2 - 3 } & Suspension agents & $2.2 \%$ \\
\cline { 2 - 3 } & Driers and anti-skin & $32.3 \%$ \\
\cline { 2 - 3 } & Thinners & \\
\hline
\end{tabular}

Source: Sramek 1967. 

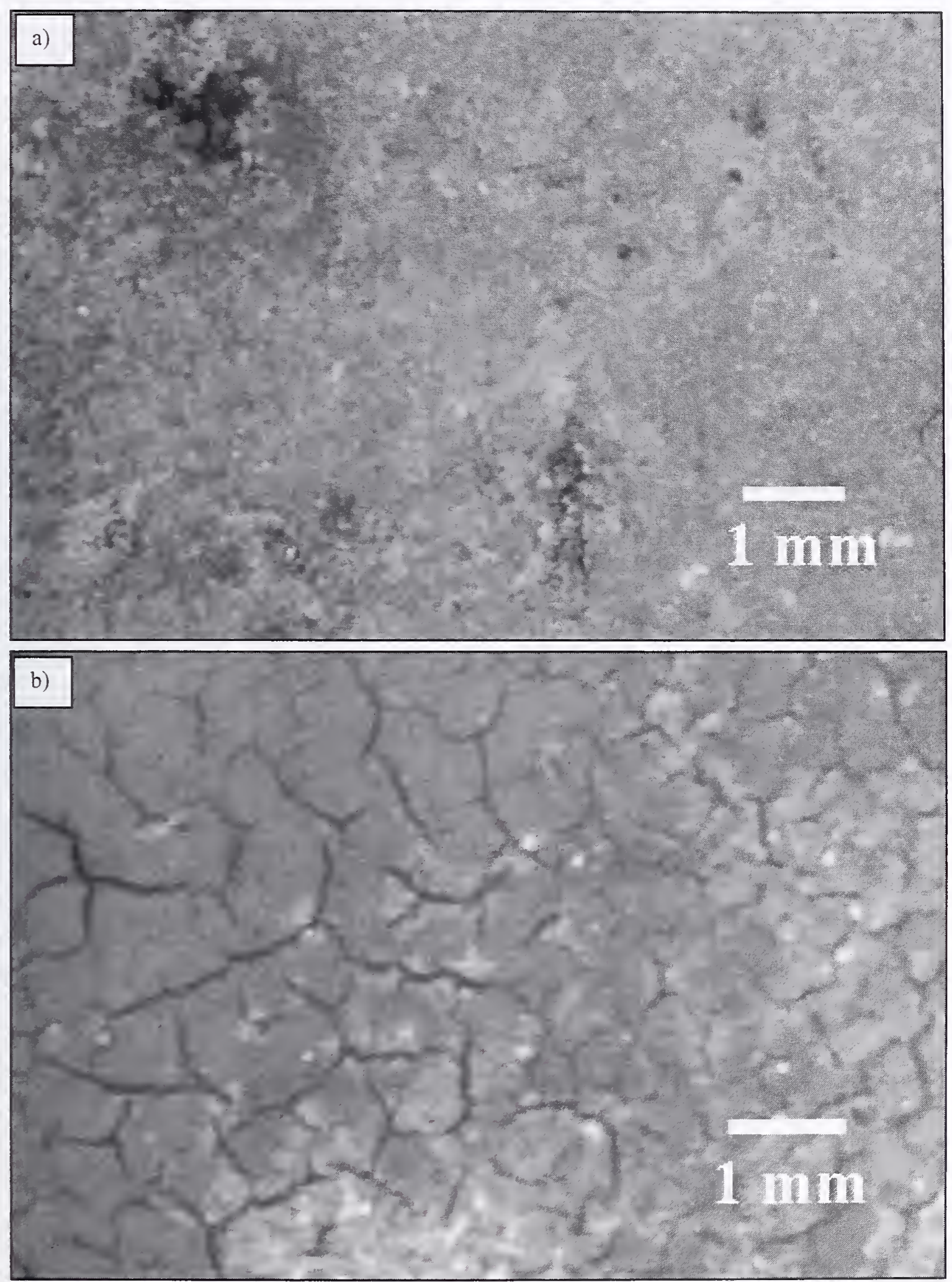

Source: NIST.

Figure D-1. Photographic images of the primer paint. a) paint in its original condition and b) mud cracking of paint after exposure at $250^{\circ} \mathrm{C}$ for $1 \mathrm{~h}$. 


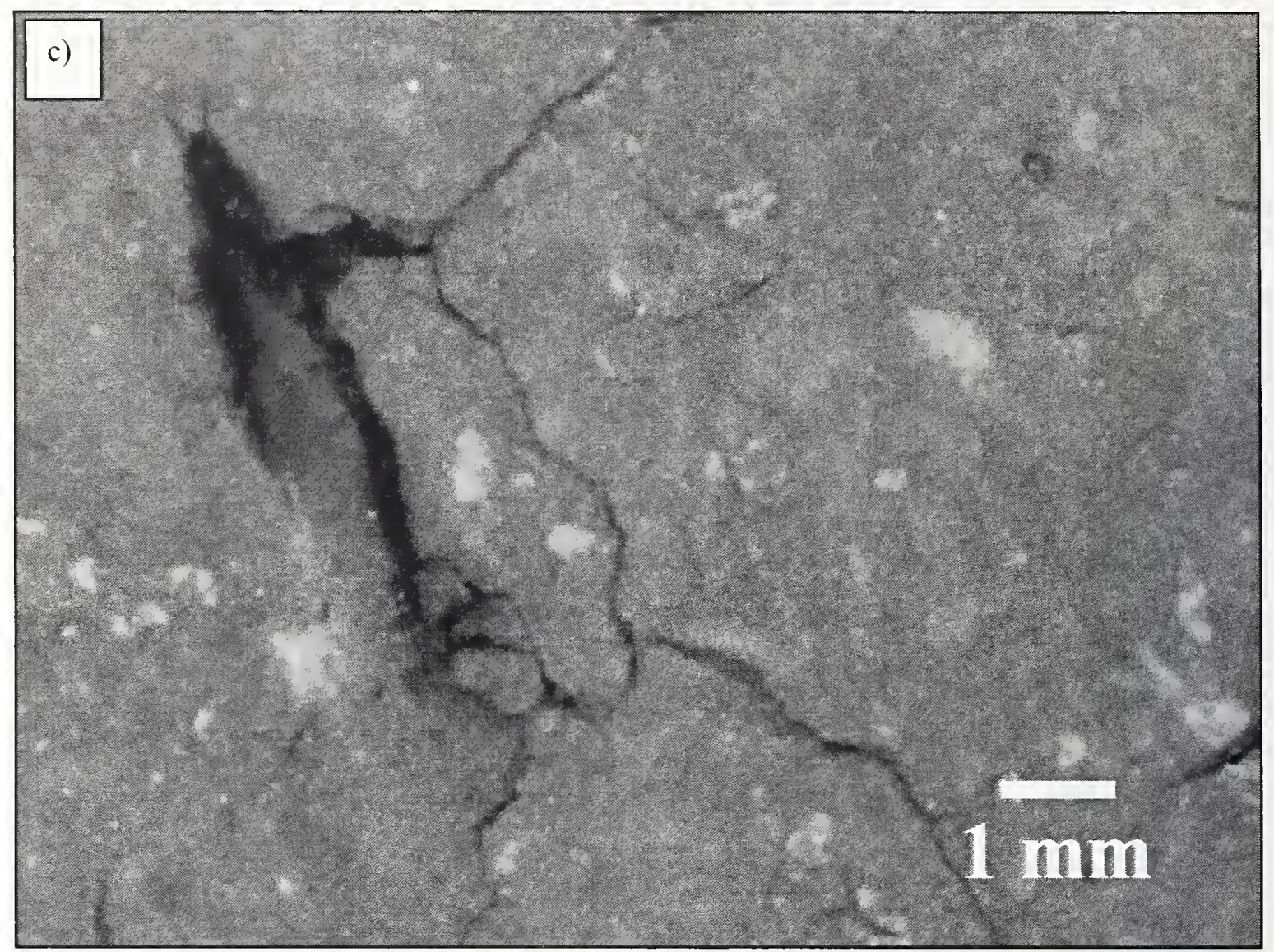

Source: NIST.

Figure D-2. Photographic images of the primer paint. c) mud cracking of paint after exposure at $650^{\circ} \mathrm{C}$ for $1 \mathrm{~h}$. 

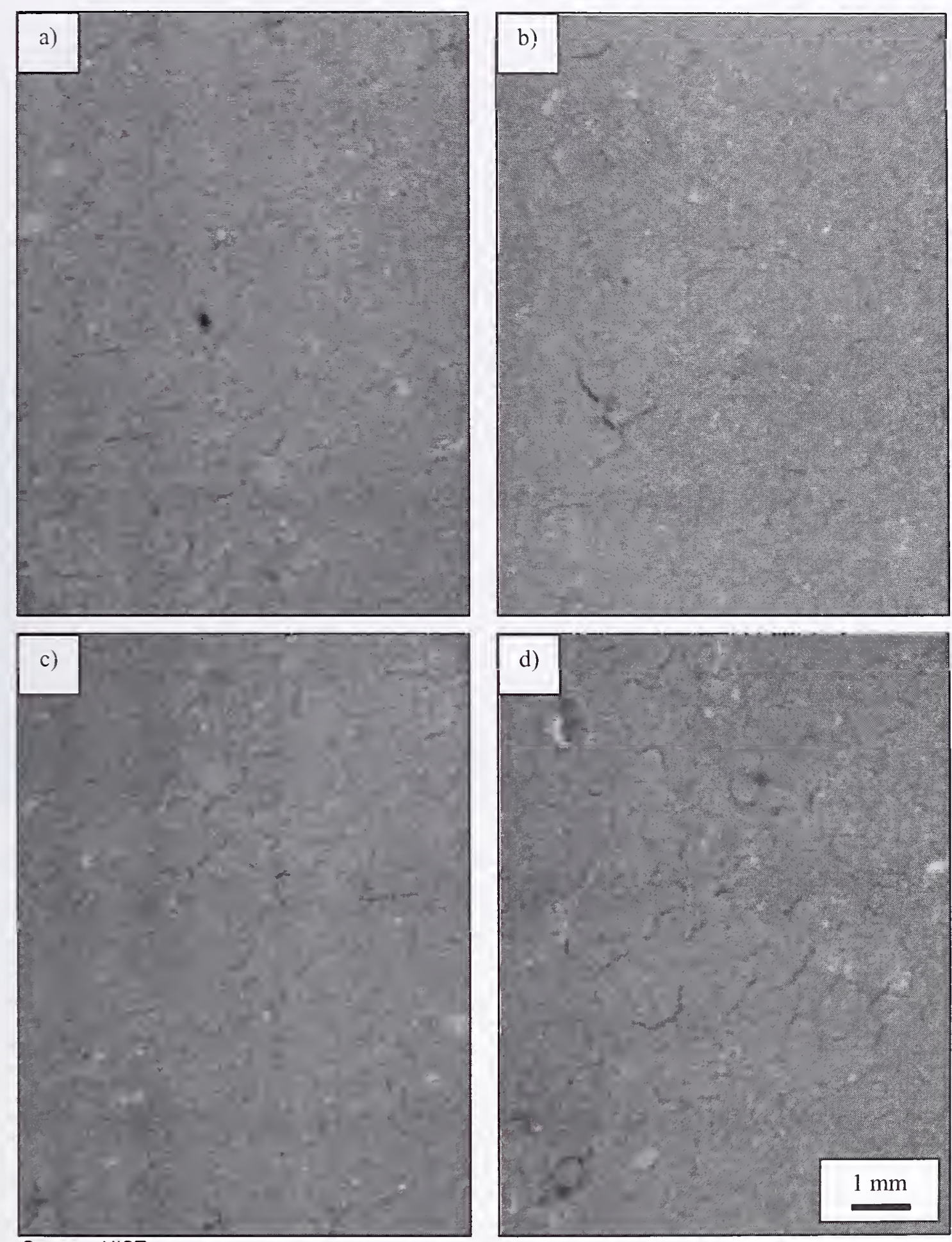

Source: NIST

Figure D-3. Mud cracking appearance as a function of time. Exposure temperature was $250^{\circ} \mathrm{C}$. a) $0.25 \mathrm{~h}$, b) $\left.0.5 \mathrm{~h}, \mathrm{c}\right) 1 \mathrm{~h}$, and d) $2 \mathrm{~h}$. Note that these images are taken under different magnification and illumination conditions than Figure D-1(b). 


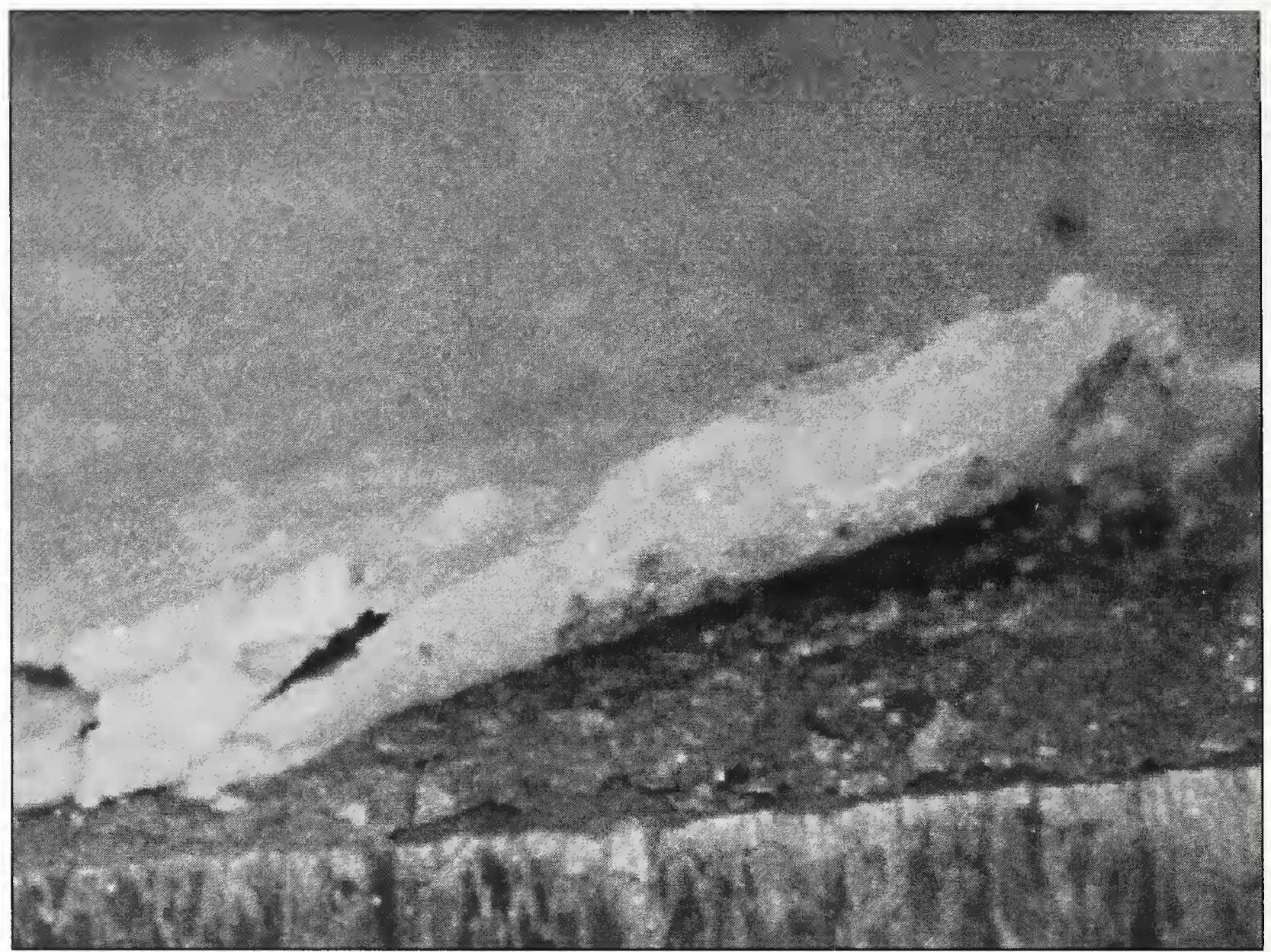

Source: NIST.

Figure D-4. Formation of a black scale between paint and steel after exposure greater than $650^{\circ} \mathrm{C}$. Paint readily spalled. 


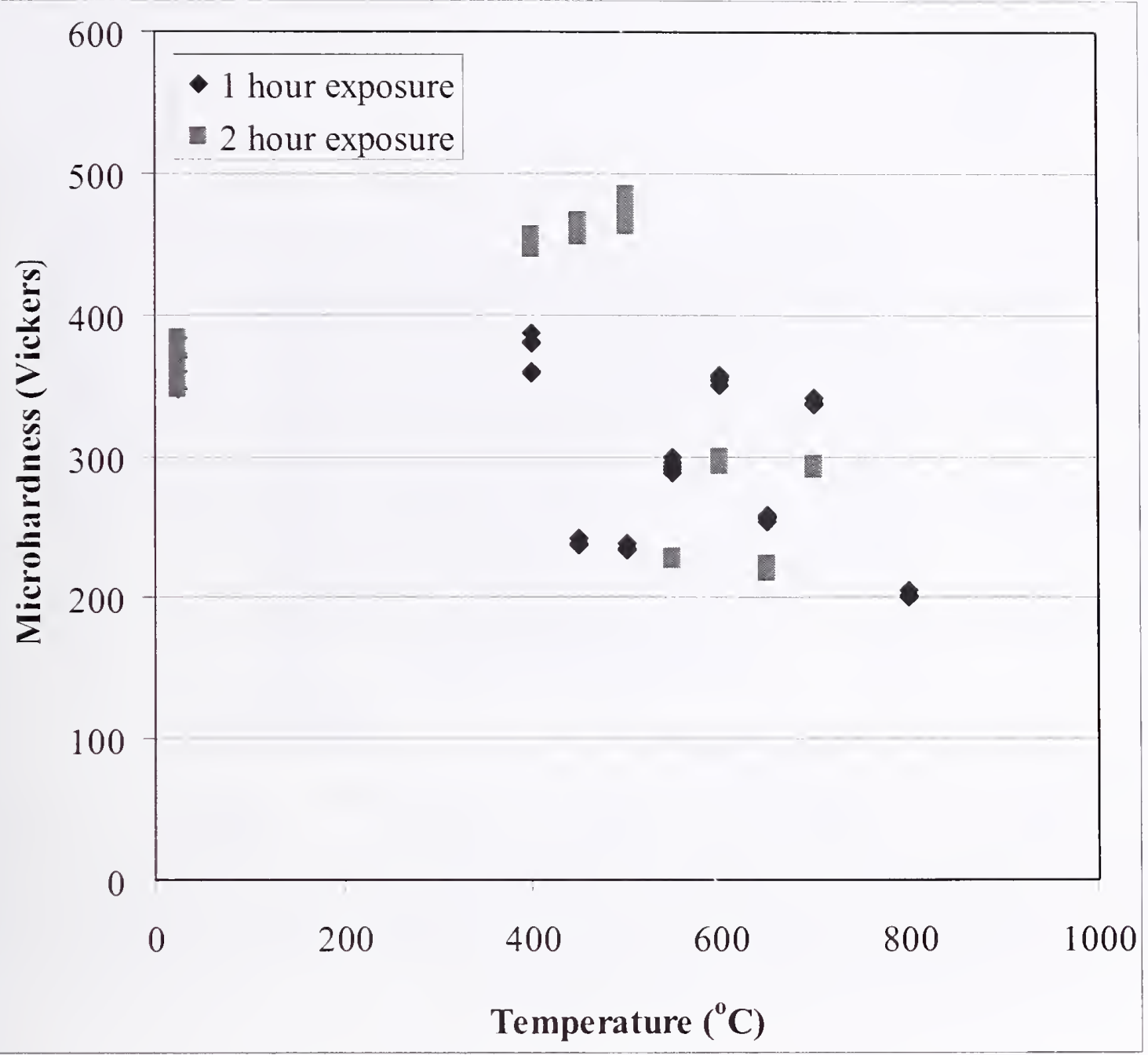

Figure D-5. Plot of Vickers microhardness versus exposure temperature for washers recovered from WTC structural steel. 


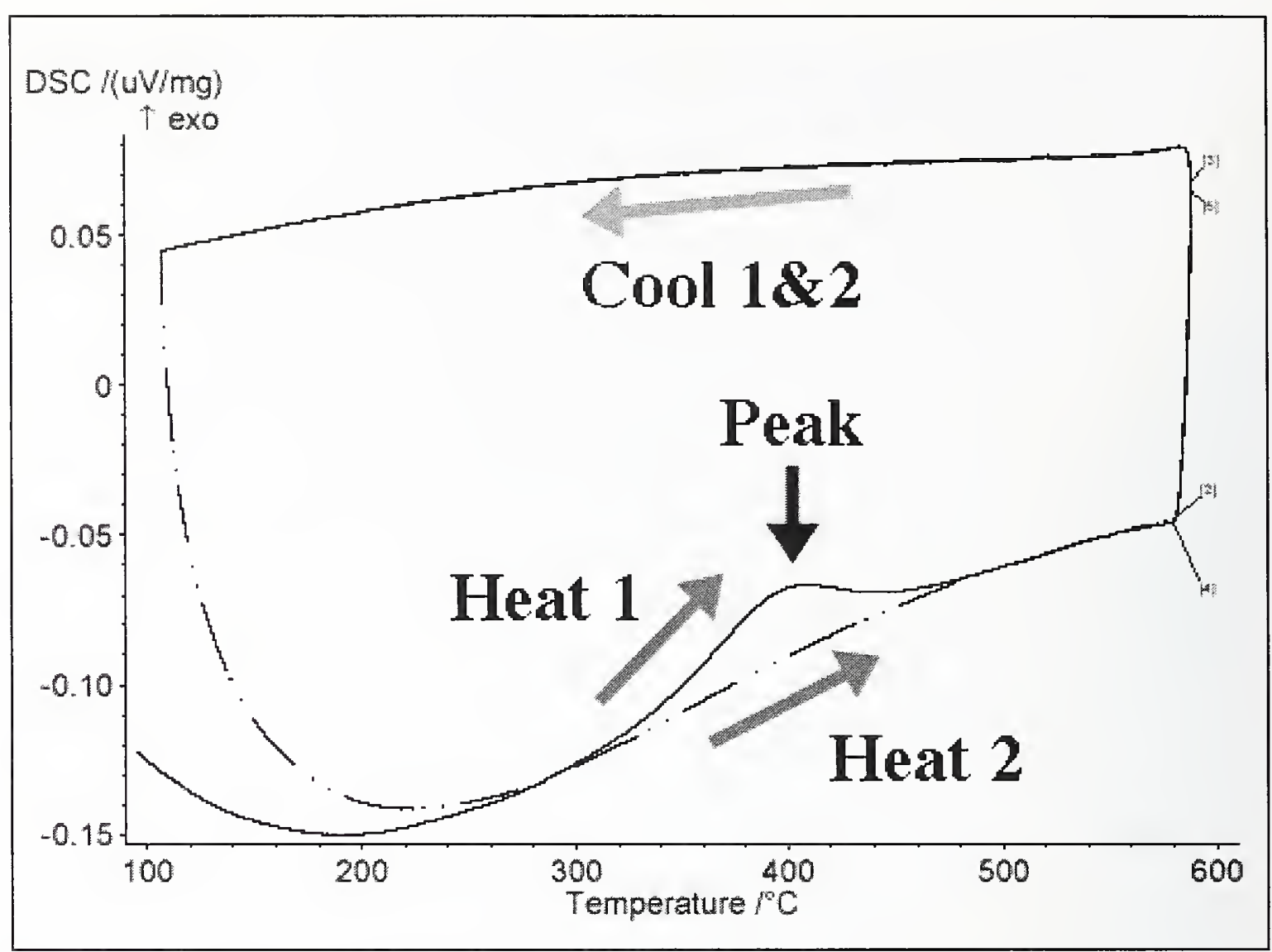

Figure D-6. DTA scans of weld metal taken from panel C-10 (A451: 85-88). First scan shows exothermic peak from non-equilibrium structure. Peak is missing in subsequent scan. 


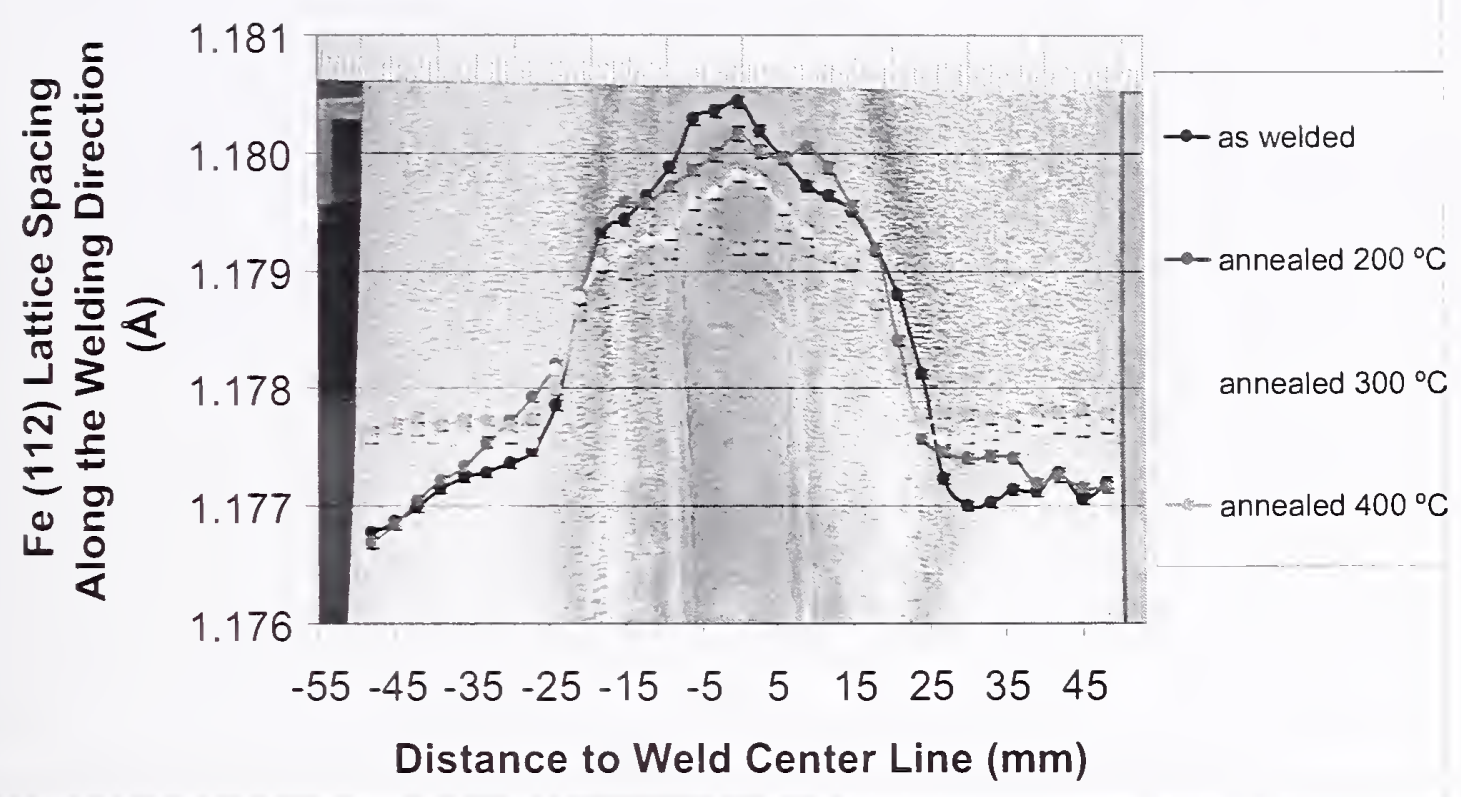

Figure D-7. Residual stress profiles measured in prototype welds on non-WTC steel using neutron diffraction. 
This page intentionally left blank. 


\section{Appendix E \\ PAINT MAPPING STUdY OF WORLD TRADE CENTER STRUCTURAL ELEMENTS}

An analysis was conducted on the condition of the primer paint of the World Trade Center structural elements. As shown in Appendix D, it was found that mud cracking of the paint occurred on samples that were exposed to temperatures in excess of $250{ }^{\circ} \mathrm{C}$. The following table lists the individual locations that were evaluated during the study, observed pre-collapse exposure of the element, and the results of the paint mapping technique. 


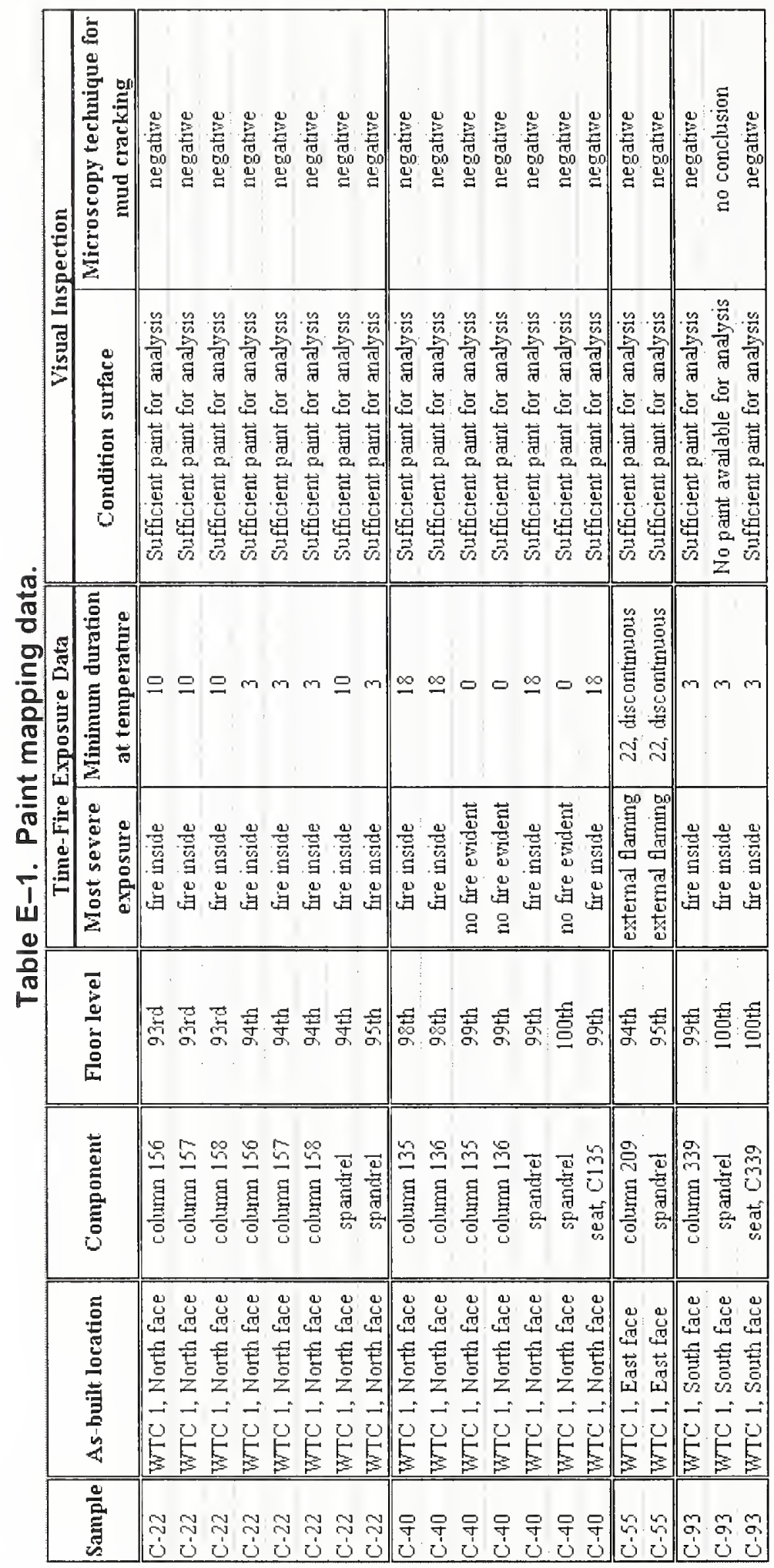




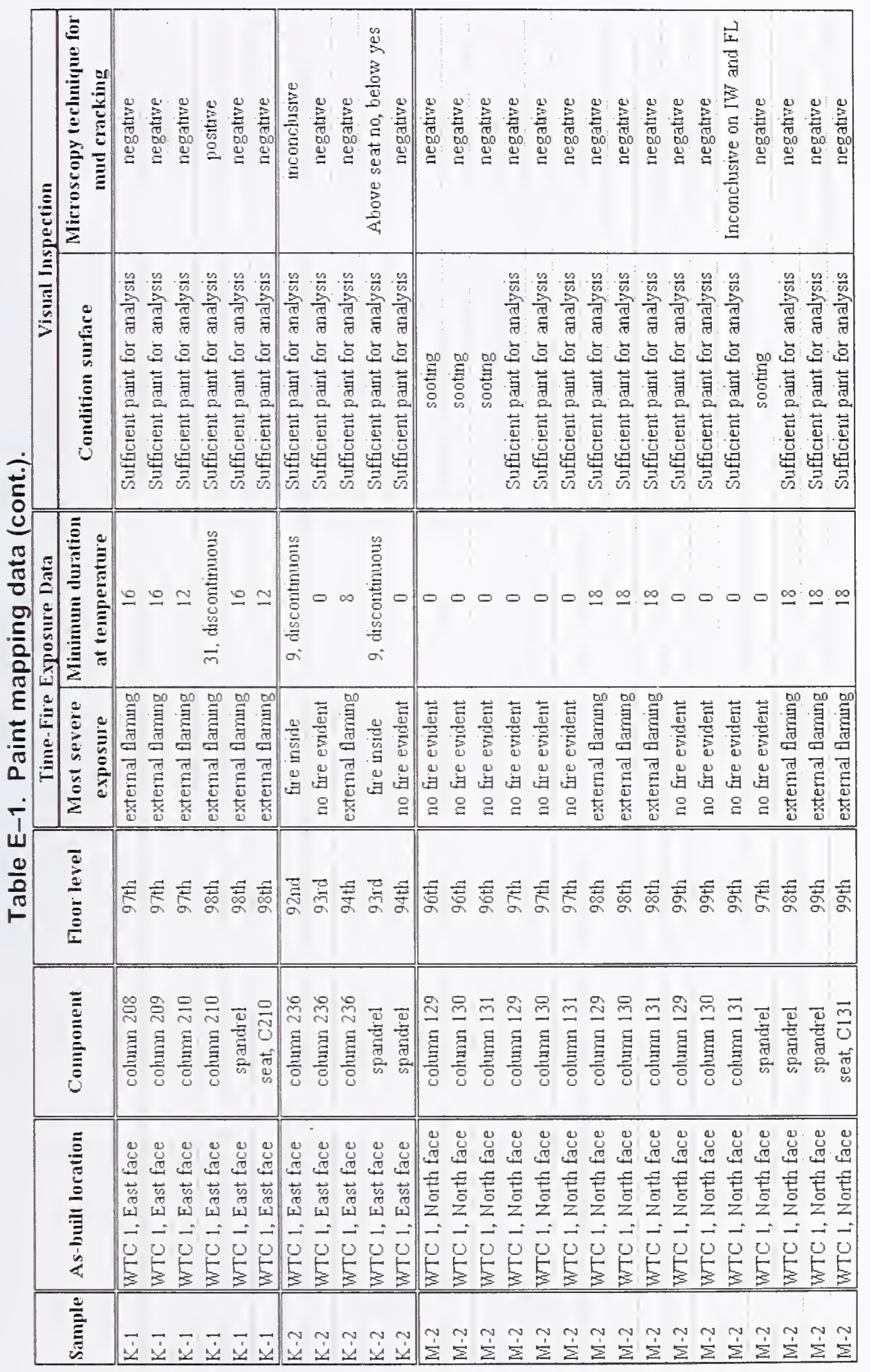




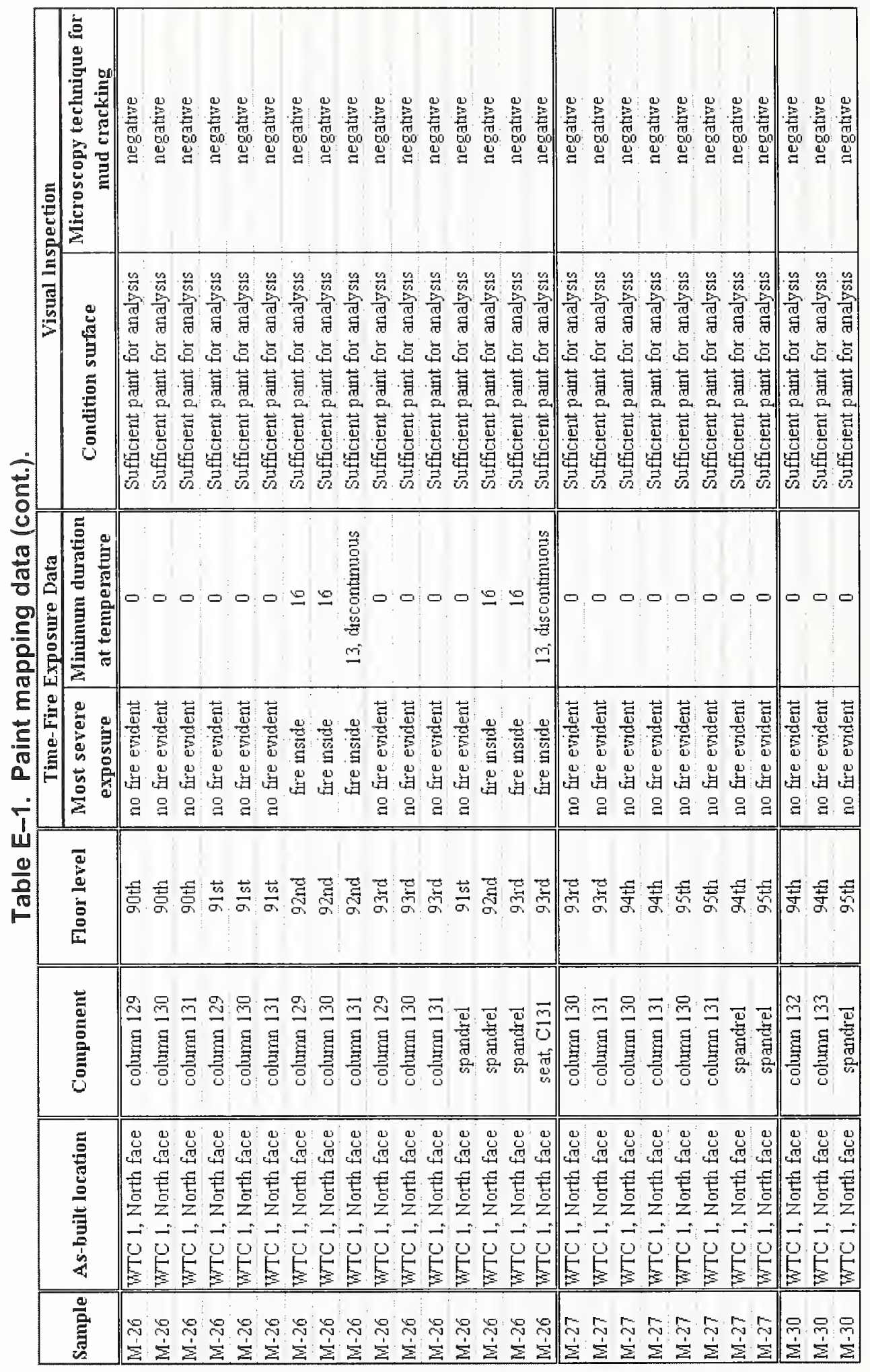




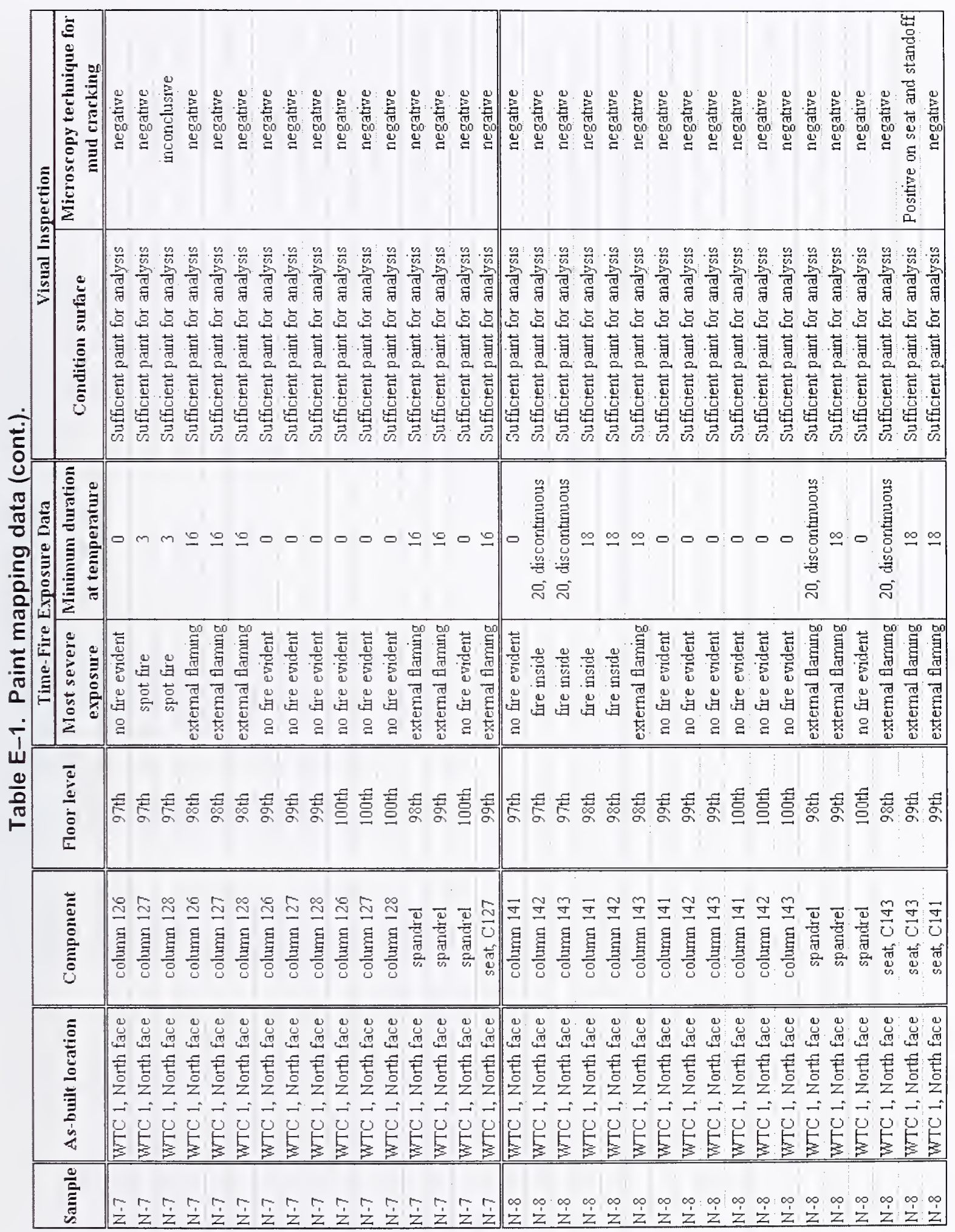




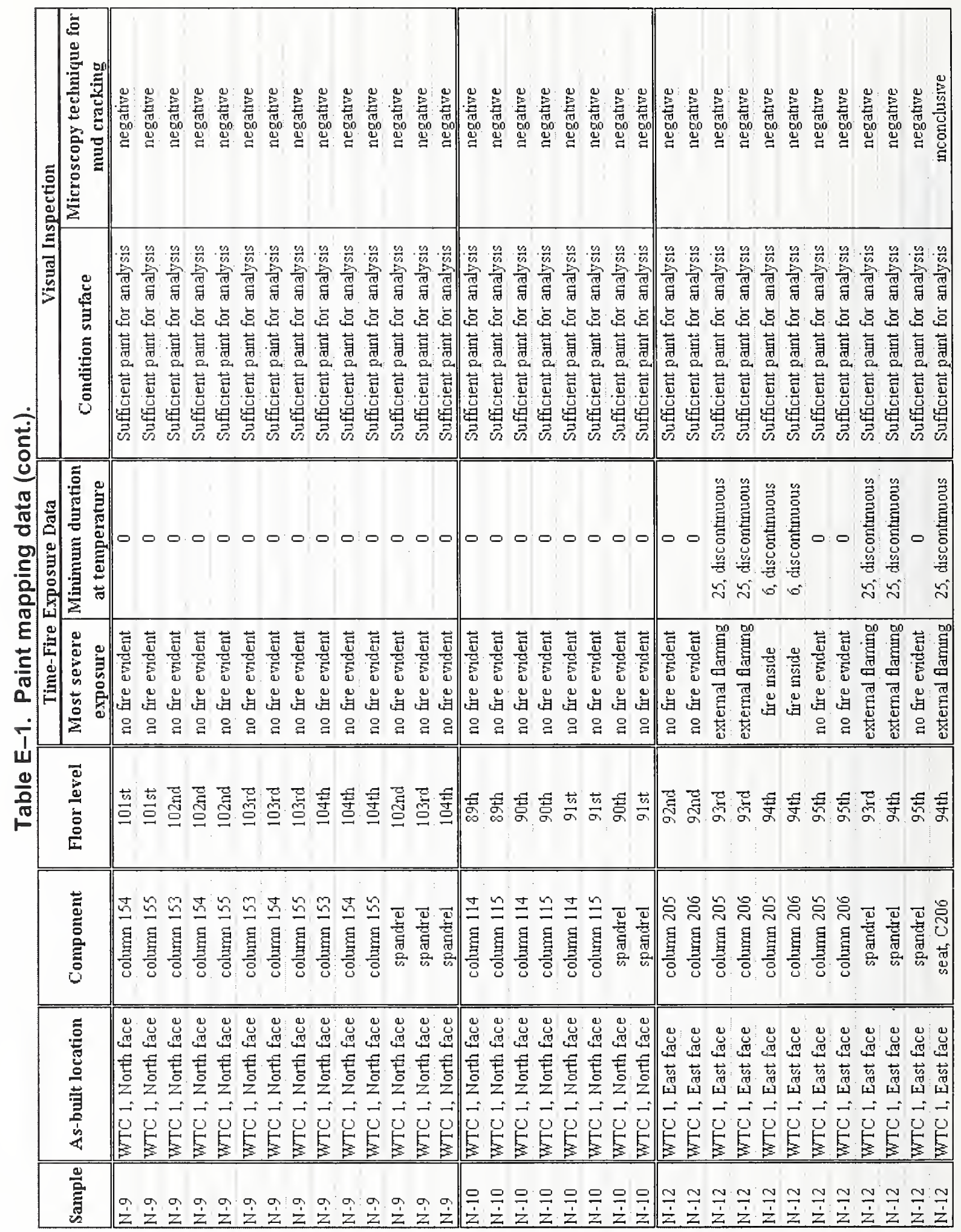




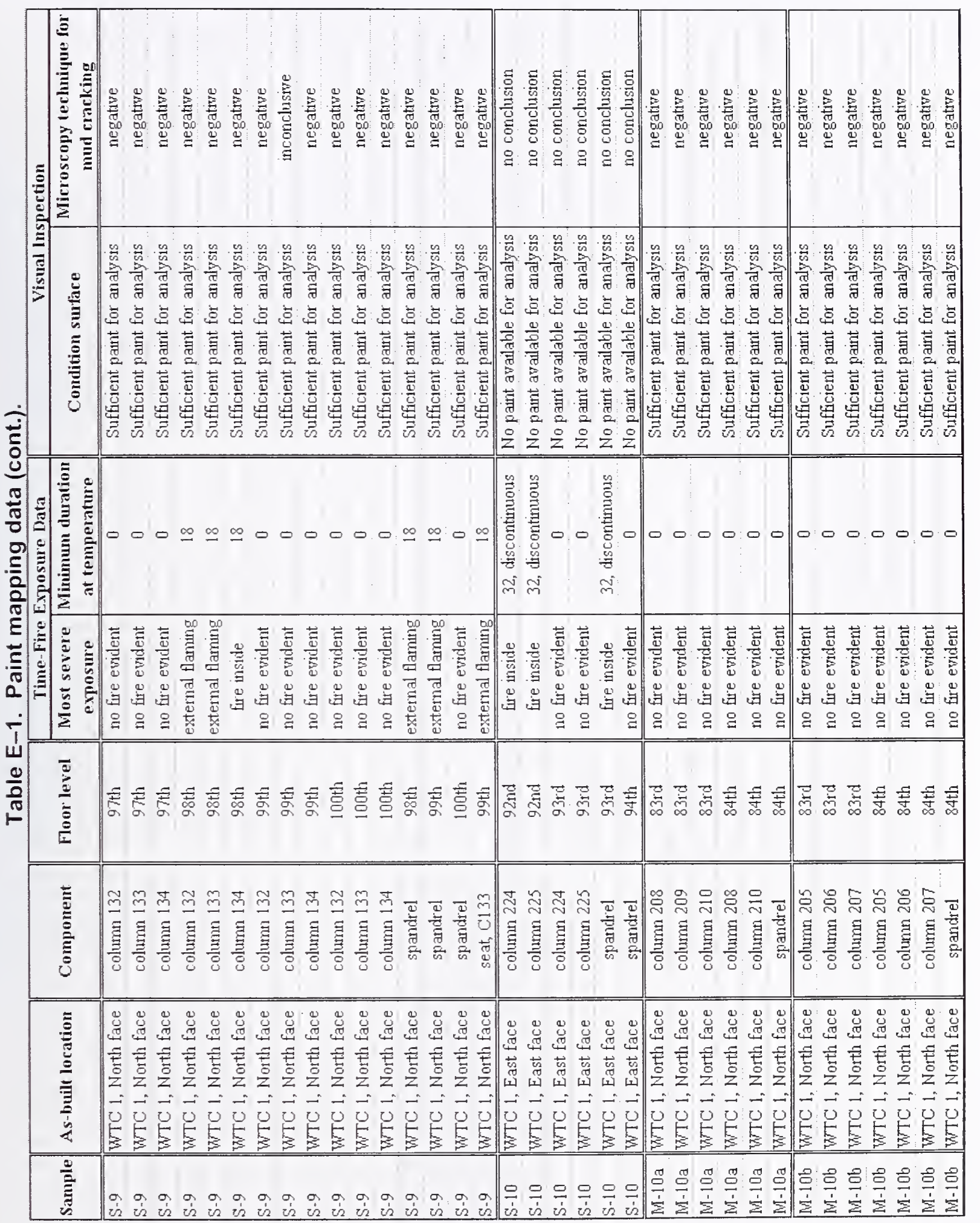




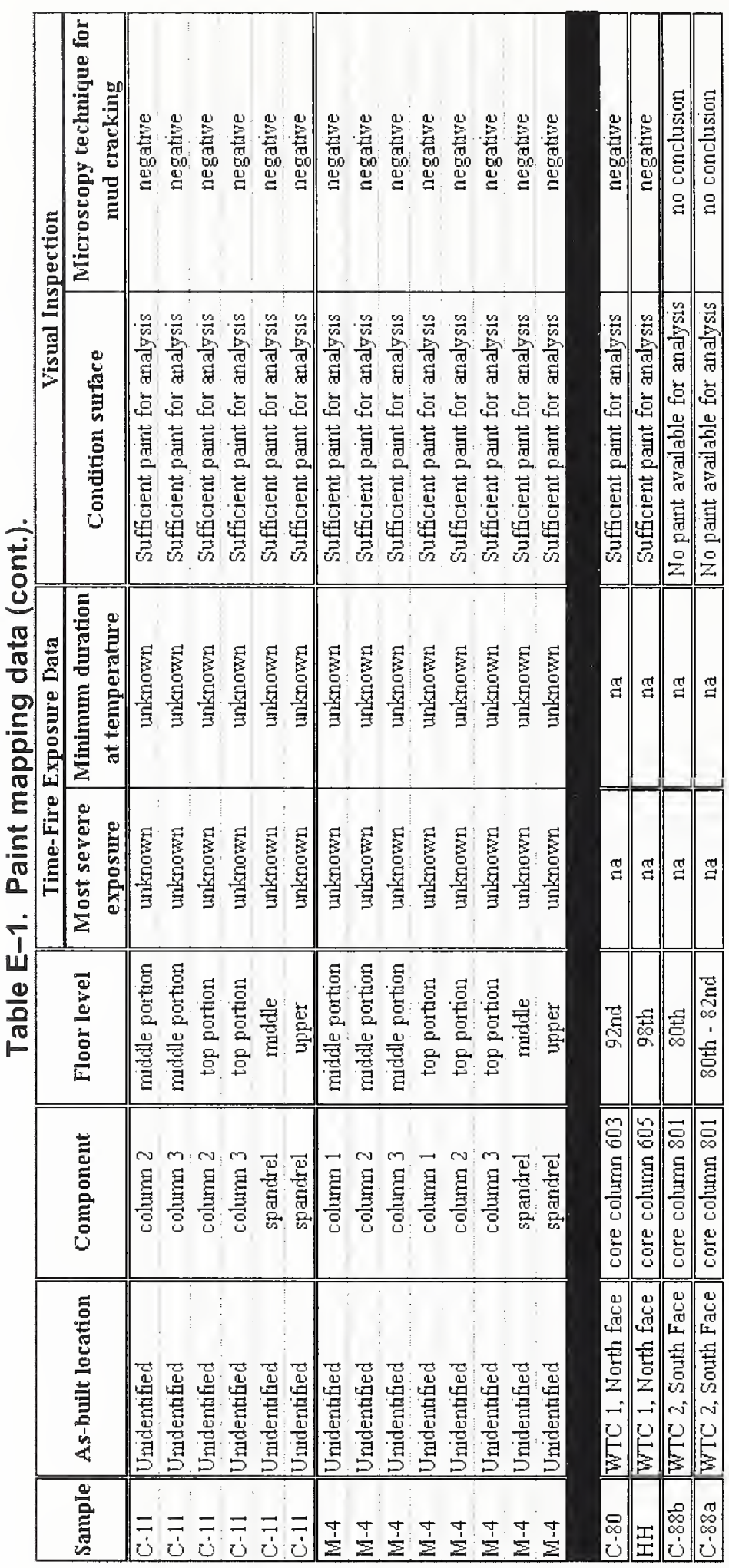




\section{Appendix F \\ VISUAL OBSERVATIONS OF THE STEEL RECOVERED FROM THE WORLD TRADE CENTER Site REPORTEd By WISS, JANNEY, ELSTNER ASSOCIATES, INC.}

In support of Task 2 of Project 3 in the National Institute of Standards and Technology World Trade Center Investigation, Wiss, Janney, Elstner Associates. Inc. (WJE) was retained to perform two tasks: (1) provide independent identification of recovered structural steel of particular interest to the furtherance of other tasks under Project 3; and (2) through unaided visual examination, identify and describe the various failure mechanisms and damage types observed on the recovered structural steel members. Thus, WJE team members surveyed and documented possible local failure mechanisms from a structural engineering perspective on exterior panels, core columns, floor trusses, and structural connections (spandrel splice plates, floor truss seat connectors). The report is given in this appendix. 
This page intentionally left blank. 
IN SUPPORT OF TASK 2 UNDER PROJECT 3 OF THE NIST WORLD TRADE CENTER INVESTIGATION Visual Observations of the Steel Recovered from the World Trade Center Site

NIST Order No. SB1341-03-Q-0155

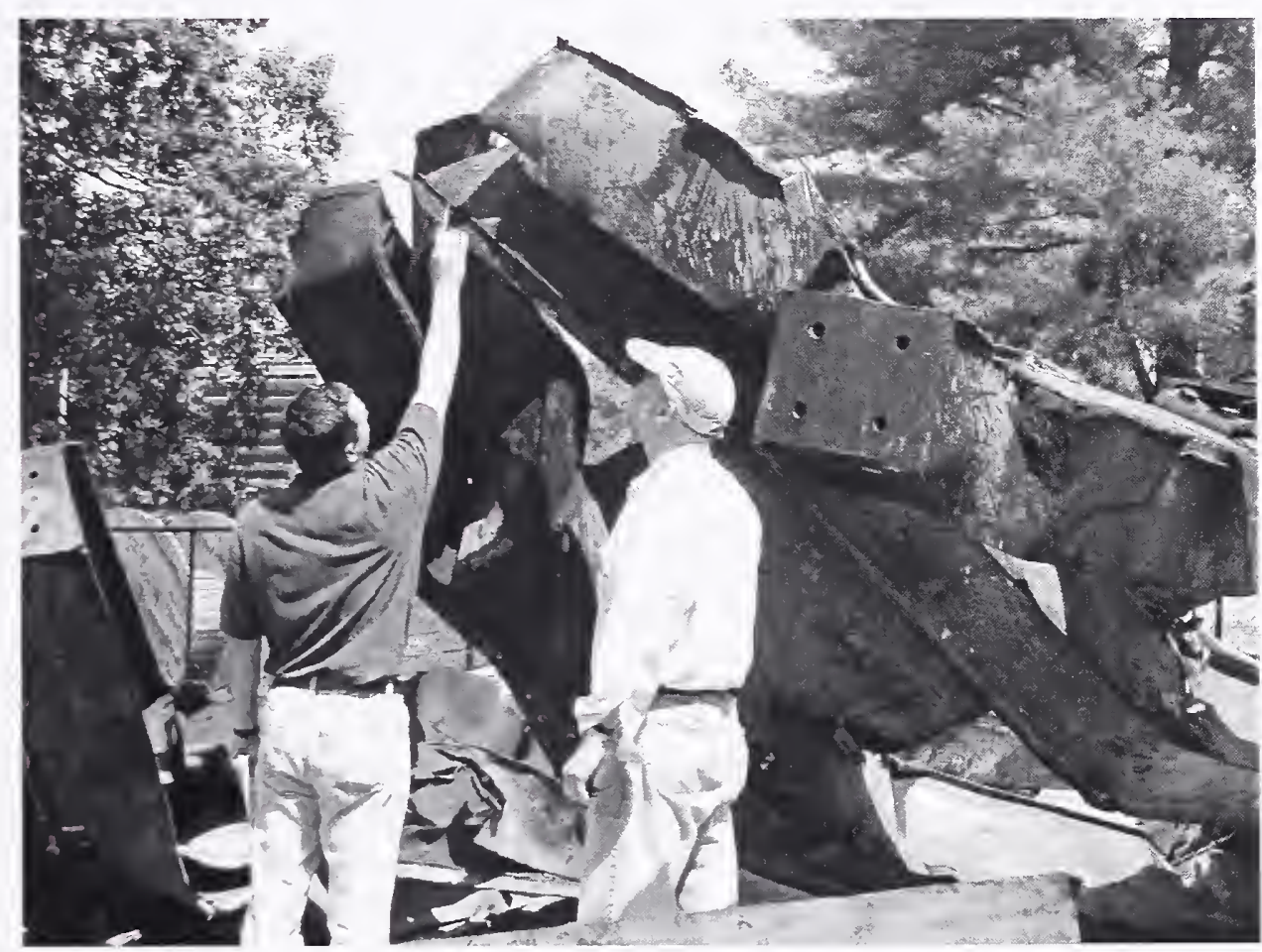

Final Report

26 November 2003

WJE No. 2003.0323.0

Prepared for:

National Institute of Standards and Technology

Prepared by:

Wiss, Janney, Elstner Associates, Inc. 
IN SUPPORT OF TASK 2 UNDER PROJECT 3 OF THE NIST WORLD TRADE CENTER INVESTIGATION Visual Observations of the Steel Recovered from the World Trade Center Site

NIST Order No. SB1341-03-Q-0155

Conrad Paulson

Principal Investigator

Raymond H.R. Tide

Co-Principal Investigator

James J. Hauck

Investigator

Final Report

26 November 2003

WJE No. 2003.0323.0

Prepared for:

National Institute of Standards and Technology

Gaithersburg, MD 20899

Prepared by:

Wiss, Janney, Elstner Associates, Inc.

120 N. LaSalle Street, Suite 2000

Chicago, Illinois 60602

312.372.0555 tel | 312.372.0873 fax 


\section{TABLE OF CONTENTS}

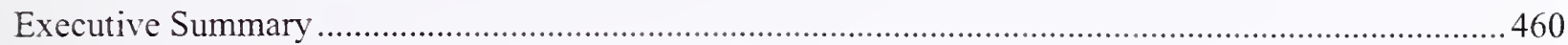

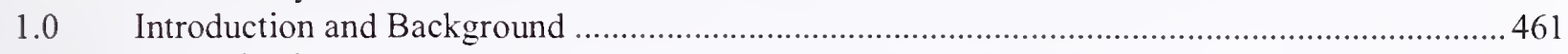

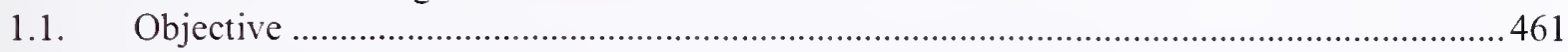

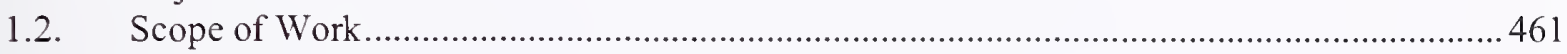

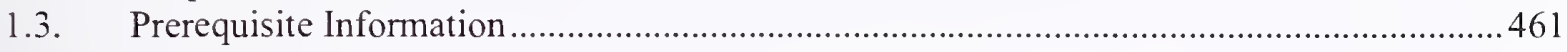

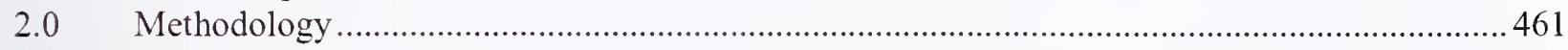

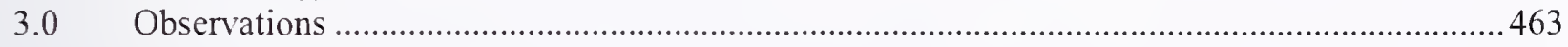

3.1. Observed Performance of Welds on Columns from WTC 1 and WTC 2 ...................... 463

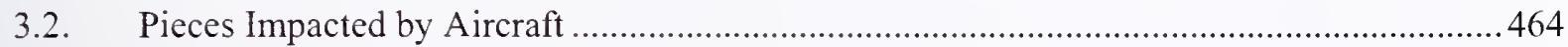

3.3. Pieces Possibly Affected by Fire Following Aircraft Impact ........................................ 465

3.4. Limited Survey of Structural Connections ................................................................... 466

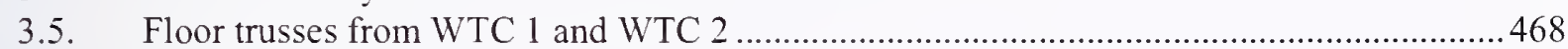

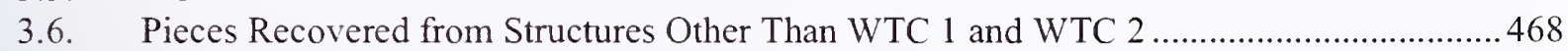

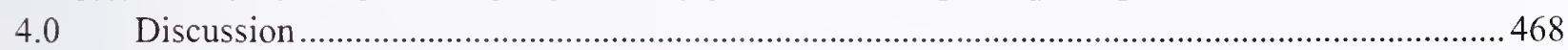

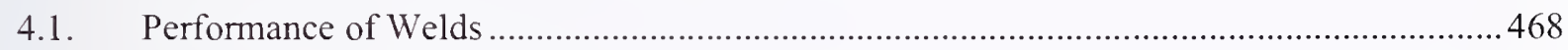

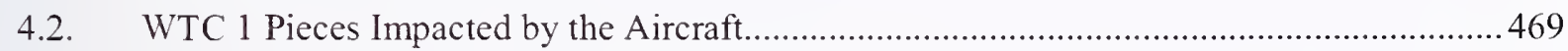

4.3. WTC 1 and WTC 2 Pieces Possibly Affected by Fire .....................................................469

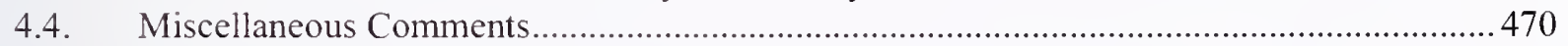

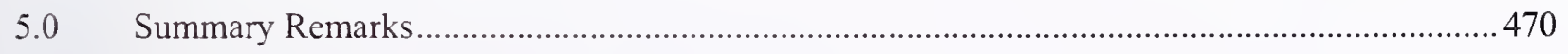

Attachment A - Identified Pieces from Floors 90 to 103 of WTC 1 and Floors 74 to 87 of WTC 2

Attachment B - Other Pieces from WTC 1 and WTC 2

Attachment C - Pieces Recovered from Structures Other Than WTC 1 and WTC 2

Attachment D - Limited Survey of Structural Connections on Selected Pieces from WTC 1 and WTC 2 


\title{
IN SUPPORT OF TASK 2 UNDER PROJECT 3 OF THE NIST WTCI Visual Observations of the Steel Recovered from the World Trade Center Site
}

\author{
NIST Order No. SB1341-03-Q-0155
}

\section{EXECUTIVE SUMMARY}

The objective of this work is to visually examine the steel pieces recovered from the WTC disaster and to document possible local failure mechanisms from a structural engineering perspective. The recovered steel is presently located at the NIST campus in Gaithersburg, Maryland. Approximately 236 pieces of steel have been recovered, a substantial number of which are large built up components such as prefabricated perimeter column-and-spandrel panel elements.

Certain extreme loading events were considered in this examination, as follows: loading related to the impact of the aircraft; loading and changes in properties related to the fire following the aircraft impact and before the collapse of the structures; and the collapse of the structures. The recovered pieces were also potentially exposed to extreme events after collapse of the towers, such as fire in the post-collapse debris pile and physical handling during recovery and salvage operations. Each of these extreme events can affect the structural elements, structural connections, and the steel material itself in a different way. By making careful visual observation of a piece, it is sometimes possible to meaningfully characterize what extreme events affected a particular piece, including identification of failure modes associated with the extreme event.

Fillet welds were examined, both in general and close-up, on numerous pieces from WTC 1 and WTC 2 Observations showed that the fillet welds joining the plates that make up the columns failed almost exclusively by rupture through the parent material at the HAZ of the weld. Extremely few fractures were observed through the weld material itself. This indicates that the welded joints performed as intended.

Three significant pieces from WTC 1 directly impacted by the aircraft were examined. Certain observations on these pieces suggest that certain columns in these panels may have resisted the aircraft impact in part by membrane tension behavior, in a fashion roughly analogous to sideways stretching of a rubber band held between two fingers. Likewise, other observations on these pieces suggest that other columns in each of these panels resisted the impact of the aircraft through shear behavior.

Numerous pieces from WTC 1 and WTC 2 were visually examined for evidence of possible effects of fire following impacts of aircrafts and before collapse of the structures. These pieces were distinguished from those exposed to fire following collapse of the towers by particular patterns of fire-related distress that would be unique to a fire occurring in the towers before their collapse.

Observations showed that the fillet welded joints at the four corners of the box column sections were ruptured on numerous sections apparently exposed to one or more extreme loading events. These ruptures were observed on pieces that were believed to have been exposed to fire as well as pieces that were not, and on pieces impacted directly by the plane as well as pieces that were away from impact. It is possible that the weld ruptures occurred during the collapse of the towers or during post-collapse recovery and salvage operations. For pieces possibly exposed to fire, there is the additional possibility that the welds may have ruptured under a particular combination of axial load in the column, pre-fire strength properties of the weld metal and column steel, and heating of the column due to fire. 


\subsection{INTRODUCTION AND BACKGROUND}

In accordance with National Institute of Standards and Technology (NIST) Purchase Order No. SB134103-Q-0155, Wiss, Janney, Elstner Associates, Inc. (WJE) has carried out unaided visual observation on selected steel pieces recovered from the World Trade Center disaster site. WJE performed this work at the request of NIST. The work by WJE is in partial support of Task 2 under Project 3 of the NIST World Trade Center Investigation (WTCI). The work reported herein accomplishes this by identifying and documenting the failure mechanisms and damage that can be visually observed on the steel recovered from the WTC structures.

\subsection{Objective}

The objective of this work, as established by NIST, is to support Task 2 "Document failure mechanisms and damage based on visual observations of recovered steel" of Project 3 in the NIST investigation of the Word Trade Center (WTC) disaster. The effort of this work focuses on documentation and evaluation of the steel recovered from the WTC towers. The recovered steel is presently located at the NIST campus in Gaithersburg, Maryland. Approximately 236 pieces of steel have been recovered, a substantial number of which are large built up components such as prefabricated perimeter column-and-spandrel panel elements. The objective of this work is to document possible local failure mechanisms from a structural engineering perspective, which will contribute to the understanding of the building performance.

\subsection{Scope of Work}

NIST established the following scope of work for this project:

- Survey of WTC structural steel at NIST, including columns, connectors and floor trusses, to identify those pieces which may provide useful information supporting the objective stated above.

- Failure analysis and other evaluation of those selected pieces from a structural engineering perspective.

- Photographic documentation of those pieces identified in the first task above.

- Providing documentation of WJE's contributions.

In addition to a report covering the above tasks, WJE shall provide non-binding technical review of the NIST Draft Project 3 Report.

\subsection{Prerequisite Information}

Throughout this report. it is assumed that the reader is familiar with the structural design, details of the structural elements, and the general as-built construction of the WTC 1 and WTC 2 towers. The unfamiliar reader can obtain the needed background information from Chapter 2 of the FEMA 403 report. The unfamiliar reader is encouraged to read and understand the background information prior to reading what is reported herein. This report does not repeat herein any background information that can be found in FEMA 403.

\subsection{METHODOLOGY}

This work is an important screening of the recovered steel to: 1) provide independent identification of those pieces of recovered steel which are of particular interest to the furtherance of other tasks under NIST WTCI Project 3; and 2) identify and describe the various failure mechanisms and damage types observed on the recovered steel. Taken together, this meant visually screening the recovered steel for indications of effects caused by the extreme events of the WTC disaster. 
The extreme loading events considered in this examination were: loading related to the impact of the aircraft; loading and changes in properties related to the fire following the aircraft impact and before the collapse of the structures; and the collapse of the structures. The pieces were also exposed to potential extreme events after collapse of the towers, such as fire in the post-collapse debris pile and physical handling during recovery and salvage operations. Each of these extreme events can affect the structural elements, structural connections, and the steel material itself in a different way. By making careful visual observation of a piece, it is sometimes possible to meaningfully characterize what extreme events affected a particular piece, including identification of failure modes associated with the extreme event.

Pieces were organized and laid out in lots at NIST, as shown in Figures 1, 2 and 3. Several pieces of significance were located indoors. Many pieces had been identified by NIST as to exact original location within WTC 1 and WTC 2. In this report, an "identified piece" is a piece for which NIST has conclusively determined the original as-built location of the piece within one of the WTC towers. If a piece is categorized as "unidentified", it means that the precise as-built location of the piece has not been conclusively determined. NIST documentation (NIST NCSTAR 1-3B (2005)) should be consulted for information on how identifications were made. Piece numbers as used herein are the same as those utilized by NIST. Where a piece may have two different numbers, the primary number utilized by NIST is used.

This work was carried out using unaided, general visual observation of the recovered steel pieces. Originally, it was anticipated that some simple aids, such as hand-held optical magnifiers and hand-held ultrasonic thickness measurement devices, would be needed while making observations. As the observations progressed, however, there was no occasion to use these devices.

The observations were made close-up, while standing on ground near the pieces, or while standing on the pieces themselves. One exception is Piece M-27, which was stored in an upright position and was therefore examined from the platform of a man-lift vehicle. The observations were recorded as hand written field notes. These notes were later transcribed into tabular format as found in the attachments to this report.

At times, areas of interest on a piece were identified using white marking fluid. The mark numbers, typically beginning with the letter $\mathrm{Z}$ followed by a single numeric digit between 0 and 9 , and the reasons for interest are given in the detailed observations. The method of photography was primarily digital. Some film photography was used; the prints of the film photography were subsequently digitized.

While all 236 pieces were observed in a general fashion, it was not possible to make detailed observations on all 236 pieces within the allotted on-site time. Therefore, the priority was to examine pieces identified by NIST to be from close to the aircraft impact locations on WTC 1 and WTC 2, and pieces that had obvious visual indications of the effects of fire following aircraft impact and before the collapse of the towers. A limited survey was made of connections on exterior column pieces from WTC 1 and WTC 2. WJE also included observations on a limited number of pieces believed to be recovered from structures other than WTC 1 and WTC 2.

The observations were made more than a year after the WTC disaster occurred. The steel pieces had prior exposure to the elements of weather, which can promote corrosion of the surfaces of the exposed steel and can also erode the primer paint from the steel. Fracture surfaces are degraded with time. Therefore, in making observations, reasonable professional judgment, based on prior experience, was used to recognize and account for effects that may be related to post-collapse degradation of the recovered steel. 


\subsection{OBSERVATIONS}

Observations were made on two different occasions, once during June 2003 and then again during July 2003, by contract engineers Mr. Conrad Paulson, Dr. Raymond H. R. Tide, and Mr. James J. Hauck of WJE. The sizes of the pieces examined ranged from small, hand-sized fragments, to complete exterior column panel assemblies from WTC 1 and WTC2, measuring $10 \mathrm{ft}-0 \mathrm{in}$. wide by $30 \mathrm{ft}-0 \mathrm{in}$. long by $1 \mathrm{ft}-2$ in. (approximately) deep. ${ }^{1}$ In Figure 4, contract engineers can be seen examining Piece M-2, an essentially complete exterior column panel assembly recovered from WTC 1, which is among the largest pieces examined. Piece M-2 had been directly impacted by the aircraft.

The majority of pieces examined were whole or partial exterior column panels from WTC 1 and WTC 2. Also examined were some core columns recovered from WTC 1 and WTC 2, remnants of floor trusses recovered from WTC 1 and WTC 2, and a limited number of column-and-beam assemblages believed to have been recovered from structures other than WTC 1 and WTC 2.

Welds were examined in the course of observations on the column panels, core columns and floor truss remnants from WTC 1 and WTC 2. General aspects of bolted connections were observed as well. Individual bolts were not examined.

The detailed observations can be found in Attachments A, B, and C, as follows:

- Attachment A includes identified pieces known to be recovered from Floors 90 through 103 of WTC 1 and Floors 74 through 87 of WTC 2. These floors encompass the region where the aircraft impacted WTC 1 and WTC 2, and also those floors in WTC 1 and WTC 2 most likely affected by fire following the aircraft impact and prior to collapse of the structures. Observations on 14 pieces are reported in this attachment.

- Attachment B includes all other pieces, identified and unidentified, recovered from WTC 1 and WTC 2. Observations on 13 pieces are reported in this attachment.

- Attachment $\mathrm{C}$ includes all pieces recovered from structures other than WTC 1 and WTC 2. Observations on six pieces are reported in this attachment.

Within each attachment, the observations are organized by order of the piece number used by NIST.

\subsection{Observed Performance of Welds on Columns from WTC 1 and WTC 2}

Failed fillet welds were examined, both in general and close-up, on numerous pieces from WTC 1 and WTC 2. Unaided visual observation was used in making the examination. It was found most often that the welds rupturcd through the parent material (i.e., base metal of the plates that make up the box columns), at or along the heat affected zone (HAZ) of the parts joined by the welds. Extremely few welded joints ruptured through weld materials (i.e., the weld filler metal). For this reason, there was no attempt made to quantify the relative extent of rupturc through parent material versus rupture through weld material.

For fillet welds that ruptured through the parent material, two different fracture paths were observed, with both paths involving the HAZ of the weld. These two paths are schematically illustrated in Figure 5. The first and most common rupture path occurs through the HAZ along one leg of the fillet weld, as shown in Figure 6. The other common rupture path extended transversely through the thickness of the plates,

\footnotetext{
${ }^{1}$ The policy of NIST is to use the International System of Units (metric units) in all publications. In this document, however, to preserve original references and quotes, units are presented using the inch-pound system throughout.
} 
appearing to initiate in the HAZ at the toe of the weld fillet, as shown in Figure 7. On a qualitative basis, it appears to WJE that the first-described rupture path appears more often than the second-described path.

These ruptures associated with the parent material HAZ zone were observed on recovered pieces regardless of the extreme effects to which a piece was exposed. For example, these types of ruptures can be seen on pieces directly impacted by an aircraft, Figure 8, on pieces having visually evident effects of the fire following aircraft impact and before collapse, Figure 9, and on pieces having visually evident mechanical effects that appear to be related to handling during the salvage and recovery operations, Figure 10. The same ruptures were also observed on the built-up box-shaped core columns.

\subsection{Pieces Impacted by Aircraft}

Three significant pieces from WTC 1 directly impacted by the aircraft were examined: Pieces M-2, M-27, and M-30. This report section summarizes the more significant observations from these pieces. Complete observations on these pieces can be found in Attachment A.

On Pieces M-2 and M-30, the edge of the floor slab nearest the aircraft impact zone served as a fulcrum point or point of lateral restraint, whereby the impact of the aircraft bent one portion of the column panel on one side of the slab (i.e., the impact side, which could be either above or below the slab) into the building while the remaining portion of the column panel on the other side of the slab edge remained restrained by the structure. On Piece M-2, the column elements extending below the 97 th floor were bent inward about the edge of the slab, Figure 11. It also appears that these same column elements were, to some degree, stretched in tension, Figure 12. On two of the three column elements, the column base plate was evidently pulled off from the end of the column due to the aircraft impact. The separation of these base plates occurred at the welds of the plates to the ends of the column sections.

On Piece M-30, the portion of the column panel that had extended above the 95th floor slab was completely severed off just above the edge of the slab, Figure 13.

Both types of actions, bending of the column into the structure and severing the column, appear to exist on Piece M-27. In particular, one of the column elements in Piece M-27 is ribbon-like, Figure 14, and may have been stretched significantly by membrane-like tension loading due to the aircraft impact.

NIST is studying samples of steel that have been subject to high rates of loading due to aircraft impact. Certain areas have been located on these specimens where NIST could potentially extract samples for testing. Photographs and detailed description of these areas of interest may be found under the individual piece observations that are tabulated in Attachment A, and a summary of these areas is as follows:

- Probable membrane compression: Piece M-2, zones marked Z1 on the 97th floor spandrel panel.

- Probable membrane tension yielding: Piece M-2, zones marked Z2 on the outside faces of the columns opposite from the 97 th floor spandrel panel.

- Visible constriction of the plate probably related to yielding in tension: Piece M-2, zone marked Z4 at end of outside face of middle column.

- Various fractures of the welded connections between the column end plate and the plates that make up the faces of the columns: Piece M-2, zones marked Z4, Z5 and Z6 at ends of columns.

- Probable membrane tension yielding: Piece M-27, the outside face of Column 131 (not marked), which is the longest of the ribbon-like plate elements on this piece.

- Probable rupture of plate in transverse shear: Piece M-30, the inside and outside faces of Column 132.

- Probable rupture of plate in longitudinal shear: Piece M-30, the side faces on Columns 132 and 133. 
- Probable rupture of plate in tension: Piece M-30, the inside and outside faces of Column 133.

- Rupture of welds as described in Section 4.1: Pieces M-2 and M-27.

Regarding the various ruptures on the ends of the columns of Piece M-30, these ruptures may have been influenced by the presence of diaphragm stiffener plates on the interior of these column sections. This is because the ruptures are coincident with the locations of the diaphragm stiffener plates. The ruptures may have initiated at the welds between the stiffener plates and the plates that make up the column faces.

\subsection{Pieces Possibly Affected by Fire Following Aircraft Impact}

Numerous pieces from WTC 1 and WTC 2 were visually examined for evidence of possible effects of fire following impacts of aircrafts and before collapse of the structures. Approximately nine exterior column panel pieces as noted in Appendices A and B were identified as exhibiting these effects. Certain visual indications on these pieces suggest that the observable fire effects were very likely attributable to fire that occurred following the impact of an aircraft. At the same time, the same patterns lead us to believe that the observable fire effects were probably not attributable to the fire that occurred in the on-site, postcollapse debris pile.

These judgments were based on visual observation of particular patterns of the presence or the absence of the visual effects of fire. The visual effects included: the presence and loss of primer paint; surface discoloration and intensity of rusting at the exposed steel; and localized distortion interpreted as possible local buckling caused by heat-related expansive strains. The last phenomenon is analogous to local buckling of exposed flanges of wide flange steel beams in structural steel floor framing beams exposed to fires. The primary pattern identified and relied upon was the presence of typically more than one visual indicator of possible fire effects on various surfaces of the individual column elements only at window openings, accompanied by the absence of these possible fire effects on the spandrel areas of the column panel. Figure 15 shows such a pattern on Piece S-10, where, from lower left to upper right in the figure, the first rusted area corresponds to possible fire effects at the 93rd floor window opening; the area of intact primer paint corresponds to the spandrel panel at the 94th floor; and the rusted remnant at the end of column corresponds to the bottom of the window opening at the 94th floor. Figure 16 shows a similar pattern on Piece M-4. where the inside faces of the column elements at the window opening exhibit possible effects of fire, while inside faces of the spandrel elements, and also the side faces of the column elements, do not exhibit these possible effects of fire. On some pieces, the observable pattern is much more subtle. Figure 17 shows the lower end of Piece K-2, where the possible fire effects are observed on the inside column face, concentrated at the head of window opening at the 92 nd floor. On Piece K-2, it may be possible that the presence of the ceiling protected the spandrel area from exposure to fire.

If these effects were due to the fire that occurred in the post-collapse debris pile at the disaster site, then it would be expected that the effects of debris-pile fire would be much more uniform, because the debris pile fire is likely to be more diffuse. A diffuse fire is not likely to exhibit the distinct, concentrated patterns of possible fire damage as observed on the pieces illustrated in Figures 15, 16, and 17. Rather, the effects of a diffuse fire in the debris pile fire would likely be evident about all surfaces of the column panels, including the faces of the spandrel plates and potentially all faces of the column elements at window openings.

Core columns from WTC 1 and WTC 2 were surveyed, and the possible effects of fire were observed on the core columns. It was not possible, however, to make a reasonable determination as to whether or not the fire effects observed on the core columns were due to fire following aircraft impact and before collapse of the tower, or due to fire in the debris piles following collapse of the tower. 


\subsection{Limited Survey of Structural Connections}

A limited survey of the structural connections found on the exterior column panels from WTC 1 and WTC 2. The focus of the survey was to identify and inventory the various failure modes associated with different categories of connections. The following four categories of connections were surveyed:

1. Bolted spandrel splice plate connections. These are the deep, bolted connections between spandrel plates on adjacent column panels.

2. Truss seat assembly connections. This includes both the welded connection of the seat assembly standoff plates to the inside face of the spandrel plate, and the bolted connection of the truss ends to the outstanding leg of the angle on the seat assembly.

3. Welded connection of the gusset plate found at the exterior end of truss top chord to the inside face of the spandrel plate.

4. Connection of damper assembly to the inside face of the spandrel plate.

Photographic illustrations of the failure modes and the detailed failure mode survey data may be found in Attachment D. In excess of 50 recovered exterior column panel pieces were surveyed. While a survey of all available connections on a piece was attempted, in many instances connections were not accessible because of limitations on direct physical access. Additionally, while each connection category may have numerous plausible failure modes, only failure modes explicitly observed are listed in the survey.

A summary of the survey data is given in Table 1 . The percentage values given in the table indicate how frequently a particular failure mode was observed within each connection category. The observed failure modes are briefly described in Table 1, and a photograph of each failure mode can be found in Attachment D. The detailed survey data are also listed in Attachment D.

In addition to summarizing the connection survey data in aggregate, Table 1 includes a break-down of data from three groups of identified pieces, as follows:

- Pieces from WTC 1 that were located above the 96th floor

- Pieces from WTC 1 that were located below the 96th floor

- Pieces from WTC 2 that were located above the 82 nd floor

In WTC 1, the 96th floor represents an approximate center of the aircraft impact zone. Likewise, in WTC 2, the 82nd floor represents an approximate center of the aircraft impact zone on that structure. For WTC 2 below the 82nd floor, there were too few pieces to make a meaningful data set on which a statistical summary could be made.

Noticeable differences in distribution of failure modes for connections above the aircraft impact floor compared to those below the aircraft impact floors can be seen for truss seat connections and truss top plate connections. Determining and describing the structural behavior that could lead to the observed differences, along with a detailed analysis of the significance of these differences, is beyond the scope of the work reported herein.

Column-to-column end plate splice connections were not surveyed in detail. These are the bolted connections between the end plate at the top of one column element and the end plate at the bottom of the column element above. These column splice connections were not surveyed because, on columns not directly impacted by an aircraft, these connections always failed by fracture of the bolts. On columns that were impacted by an aircraft, some of these splice connections failed by fracture of the welds between the 
TABLE 1 - Distribution of connection failure modes

\begin{tabular}{|c|c|c|c|c|c|c|}
\hline & & Failure Mode & $\begin{array}{l}\text { All } \\
\text { Survey } \\
\text { Data }\end{array}$ & $\begin{array}{l}\text { WTC1 } \\
\text { Above } \\
96 \text { th } \\
\text { Floor }\end{array}$ & $\begin{array}{l}\text { WTC1 } \\
\text { Below } \\
96 \text { th } \\
\text { Floor }\end{array}$ & $\begin{array}{l}\text { WTC2 } \\
\text { Above } \\
82 \text { nd } \\
\text { Floor }\end{array}$ \\
\hline \multirow{5}{*}{ 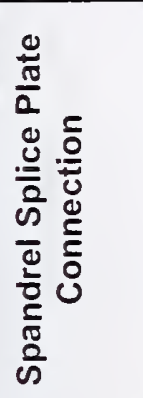 } & Type 1 & $\begin{array}{l}\text { Edge-distance bolt tear-out from } \\
\text { spandrel plate }\end{array}$ & $63 \%$ & $88 \%$ & $60 \%$ & $55 \%$ \\
\hline & Type 2 & $\begin{array}{l}\text { Edge-distance bolt tear-out from } \\
\text { connection splice plate }\end{array}$ & $14 \%$ & $0 \%$ & $0 \%$ & $0 \%$ \\
\hline & Type 3 & $\begin{array}{l}\text { Combination edge-distance tear-out: } \\
\text { tear-outs observed on both the } \\
\text { spandrel plate and the splice plate }\end{array}$ & $6 \%$ & $5 \%$ & $0 \%$ & $25 \%$ \\
\hline & Type 4 & $\begin{array}{l}\text { Fracture of the spandrel plate away } \\
\text { from the splice plate connection }\end{array}$ & $17 \%$ & $7 \%$ & $40 \%$ & $20 \%$ \\
\hline & \multicolumn{2}{|r|}{ Total number of connections surveyed } & 127 & 42 & 20 & 20 \\
\hline \multirow{5}{*}{ 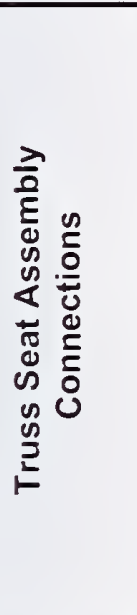 } & Type 1 & $\begin{array}{l}\text { Seat Assembly remains in place and at } \\
\text { least one erection bolt is present in the } \\
\text { seat angle }\end{array}$ & $22 \%$ & $36 \%$ & $0 \%$ & $31 \%$ \\
\hline & Type 2 & $\begin{array}{c}\text { Seat assembly remains in place and at } \\
\text { least one of the bolt holes for the in the } \\
\text { seat angle has edge distance tear-out; } \\
\text { no bolts are present }\end{array}$ & $24 \%$ & $27 \%$ & $25 \%$ & $31 \%$ \\
\hline & Type 3 & $\begin{array}{l}\text { Seat assembly remains in place with no } \\
\text { erection bolts present and no edge } \\
\text { distance tear-outs of bolt holes in the } \\
\text { seat angle }\end{array}$ & $15 \%$ & $9 \%$ & $15 \%$ & $6 \%$ \\
\hline & Type 4 & $\begin{array}{c}\text { Seat assembly is missing and appears } \\
\text { to have been sheared off where the } \\
\text { stand-off plates are welded to the face } \\
\text { of the spandrel }\end{array}$ & $38 \%$ & $27 \%$ & $60 \%$ & $31 \%$ \\
\hline & \multicolumn{2}{|r|}{ Total number of connections surveyed } & 91 & 33 & 20 & 16 \\
\hline \multirow{3}{*}{ 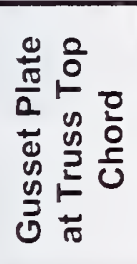 } & Type 1 & $\begin{array}{c}\text { Fracture of top plate through gross } \\
\text { section away from weld to the spandrel } \\
\text { plate }\end{array}$ & $72 \%$ & $83 \%$ & $45 \%$ & $82 \%$ \\
\hline & Type 2 & $\begin{array}{l}\text { Tear-out of top plate and weld from the } \\
\text { parent material of the spandrel plate }\end{array}$ & $28 \%$ & $17 \%$ & $55 \%$ & $18 \%$ \\
\hline & \multicolumn{2}{|r|}{ Total number of connections surveyed } & 95 & 35 & 20 & 17 \\
\hline \multirow{3}{*}{ 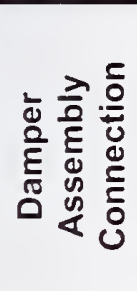 } & Type 1 & $\begin{array}{l}\text { Remnants of the damper and its } \\
\text { connection plate remain attached to the } \\
\text { column }\end{array}$ & $23 \%$ & $26 \%$ & $15 \%$ & $50 \%$ \\
\hline & Type 2 & $\begin{array}{l}\text { The damper and its connection plate } \\
\text { are missing, fracturing at the weld of } \\
\text { the connection plate to the column }\end{array}$ & $77 \%$ & $74 \%$ & $85 \%$ & $50 \%$ \\
\hline & \multicolumn{2}{|r|}{ Total number of connections surveyed } & 94 & 35 & 20 & 16 \\
\hline
\end{tabular}

end plate and plates that made up the faces of the columns. The detailed piece observations found in Attachment A should be consulted for further description of the splice plate connection fractures on pieces directly affected by aircraft impact. 


\subsection{Floor Trusses from WTC 1 and WTC 2}

The recovered remnants of floor trusses recovcred by NIST are severely distorted and the components from many individual trusses are intermingled into the same debris piles, Figures 18 and 19. It was impossible to determine whether the observed condition of the trusses was due to aircraft impact, fire following aircraft impact and before collapse of the tower, the collapse of a tower, fire in the debris pile following collapse, post-collapse recovery and salvage handling, or any combination of these extreme events. Our detailed observations may be found in Attachment B under Piece C-53.

The rolled-steel angle shapes used in making the chords of the floor trusses exhibited a shallow, round rib running longitudinally on the vertical leg of the angle. This rib is visible on truss chords seen in Figure 19. This rib is a proprietary feature of floor trusses manufactured by Laclede Steel. The web-tochord welds on the recovered truss remnants were observed to have been made by the electric resistance weld process, also typical of Laclede Steel trusses.

General observations were made of numerous web-to-chord welded connections. Despite the severely distorted condition of the trusses, many welded connections did not fail but instead remained intact. Many instances were also observed where the welds had failed, allowing the web members to become separated from the chord element. Qualitatively, the failures at the weld appeared to happen more frequently than welds remaining intact.

\subsection{Pieces Recovered from Structures Other Than WTC 1 and WTC 2}

WJE surveyed six pieces believed to have been recovered from structures other than WTC 1 and WTC 2, Figure 20. The pieces surveyed by WJE wcre prefabricated column-and-beam assemblages, typically comprised of heavy rolled wide-flange columns with pairs of rolled wide-flange beam stubs framing into the strong-axis plane of bending of the column. Typically, two floor levels of floor beam stubs were observed on each piece. In some instances, the pieces had remnants of connections for diagonal elements that also framed into the column tree. Detailed observations and photographs of pieces recovered from structures other than WTC 1 and WTC 2 can be found in Attachment C.

Over half of the surveyed pieces from structures other than WTC 1 and WTC 2 generally exhibited indications of the effects of fire. It was not possible, however, to readily distinguish whether the exposure to fire occurred before or after the collapse of the structure.

A unique connection failure mode was observed on several of these column-and-beam assemblage pieces. On these pieces, further described in Attachment $\mathrm{C}$, the beam framing connections at the free ends of the beam stubs exhibit bolt hole edge distance tear-out failures that are generally directed parallel to the longitudinal axes of the beam stubs. This suggests that the beam stubs were in axial tension at the time of tear-out. Such failures are unlikely to have been caused by ordinary gravity loads that were probably in place on the beams at the time of their collapse.

\subsection{DISCUSSION}

\subsection{Performance of Welds}

Observations of the exterior column panels from WTC 1 and WTC 2 show that the fillet welds joining the plates that make up the columns failed almost exclusively by rupture through the parent material at the $\mathrm{HAZ}$ of the weld. Extremely few fractures were observed through the weld material itself. This indicates that the welded joints performed as intended. 
As was described previously in Section 3.1 of this report, there were two types of rupture through the parent material, and both types of rupture were associated with the HAZ of the weld. One rupture progressed through the HAZ along one leg of the fillet weld, and the other fracture progressed transversely through the thickness of the parent material, appearing to initiate in the HAZ at the toe of the fillet weld. These fracture paths were illustrated previously in Figure 5. It was not possible to explicitly determine the circumstances that would cause when and how one fracture path would develop instead of the other path. Nevertheless, the following general comments can be made on this matter:

- In a weld, the fracture path is determined by the relative strengths and thicknesses of the parent pieces being joined by the fillet welds, the dimensions of the fillet welds, and the direction of stresses within the weld at the time of fracture. This assumes that weld metal strength is not a limiting factor, which is a reasonable assumption supported by the visual observations of weld failures (i.e., essentially no weld fractures were observed to have occurred through the weld metal itself). The combined assessment of these factors cannot be performed solely on the basis of visual observation.

- Based on qualitative review of the visual observations, exterior column panel elements exhibiting possible effects of significant fire exposure following aircraft impact tended to exhibit predominantly (but not exclusively) fractures that were oriented transversely through the thickness of the parent material, probably initiating in the HAZ at the toe of the weld fillet.

- Based on qualitative review of the visual observations, exterior column panel elements that had been directly affected by impact of aircraft exhibited both types of fractures in approximately equal amounts.

Regarding the electric resistance welds at the web-to-chord connections on the floor trusses, it was observed that some of these welds remained intact despite the observed severely distorted condition of the truss remnants. Qualitatively, however, failures at the weld appeared to happen more frequently than welds remaining intact.

\subsection{WTC 1 Pieces Impacted by the Aircraft}

As described previously in Section 3.2, three significant pieces from WTC 1 that were directly impacted by the aircraft were examined: M-2, M-27 and M-30. In the as-built structure, Piece M-27 was located immediately below and connected to Piece M-2, and also immediately adjacent to and connected to Piece M-30. The as-built relationship between these pieces is shown in Figure 21.

When viewed collectively, the observations on Pieces M-2 and M-27, particularly the observed apparent tensile stretching of some of the plates (as evidenced by necking down of the plates, described in Attachment A) that make up the column elements in these pieces, suggest that the columns in these panels may have resisted the aircraft impact in part by membrane tension behavior, in a fashion roughly analogous to sideways stretching of a rubber band held between two fingers. Likewise, certain observations of Pieces M-27 and M-30 can be viewed collectively; particularly the observed apparent transverse and longitudinal shear fractures in severed columns, to suggest that particular column elements in each of these panels resisted the impact of the aircraft through shear behavior. The difference between severed column elements that failed by shear, Figure 13, and column elements that failed by apparent membrane tension behavior, Figure 14, are readily distinguished on the basis of visual observation.

\subsection{WTC 1 and WTC 2 Pieces Possibly Affected by Fire}

As described in Section 3.3, numerous pieces were examined that were possibly exposed to the fire following aircraft impact and before collapse of the towers. Also as described in Section 3.3, these pieces were distinguished from those possibly exposed to fire following collapse by particular patterns of fire- 
related distress, typically a combination of observations such as loss of primer paint, surface discoloration and intensity of rusting at the exposed steel, and localized distortion interpreted as local buckling of individual plates within column elements possibly caused by heat-related expansive strains within the individual plates.

Observations showed that the welded fillet joints at the four corners of the box column sections were ruptured on numerous sections exposed to one or more different extreme loading events. The rupture of these welds causes a significant transformation in the behavior of the box column shape. After these corner welds rupture, the original, relatively stocky and closed box shape degrades into a series of independent, thin and relatively slender rectangular plates. Important sectional properties of the column, such as axial slenderness, stability and strength, are all greatly reduced as a result of this transformation. Although not a certainty, one possibility for columns exposed to fire is that the welds may have ruptured under a particular combination of axial load in the column, pre-fire strength properties of the weld metal and column steel, and sufficient heating of the column due to fire. There is also the possibility that the weld ruptures occurred during the collapse of the towers and during post-collapse recovery and salvage operations. Determining and describing the probable loading and probable sequence of fire-related events that could lead to rupture of these welds is beyond the scope of the work reported herein.

\subsection{Miscellaneous Comments}

Of note is Piece K-1, shown in Figure 22 and further described in Attachment A. The piece is unique among the recovered steel in that the upper part of one column has probably collapsed in compression in an accordion-like manner. No other similarly-collapsed piece is found among the recovered steel at NIST that was surveyed. The accordion-like collapsed part of this column remains in general concentric axial alignment with the lower portion of the same column, which is relatively undistorted even after salvage and recovery operations. It cannot be readily distinguished whether this failure took place at a high or low rate of loading; whether elevated temperatures contributed to the observed condition; whether or not this failure occurred at about the time of collapse initiation; or if the observed condition is a result of impact as the piece fell to the ground.

\subsection{SUMMARY REMARKS}

The objective of this work is to visually examine the steel pieces recovered from the WTC disaster and to document possible local failure mechanisms from a structural engineering perspective. The recovered steel is presently located at the NIST campus in Gaithersburg, Maryland. Approximately 236 pieces of steel have been recovered, a substantial number of which are large built up components such as prefabricated perimeter column-and-spandrel panel elements.

Certain extreme loading events were considered in this examination, as follows: loading related to the impact of the aircraft; loading and changes in properties related to the fire following the aircraft impact and before the collapse of the structures; and the collapse of the structures. The recovered pieces were also potentially exposed to extreme events after collapse of the towers, such as fire in the post-collapse debris pile and physical handling during recovery and salvage operations. Each of these extreme events can affect the structural elements, structural connections, and the steel material itself in a different way. By making careful visual observation of a piece, it is sometimes possible to meaningfully characterize what extreme events affected a particular piece, including identification of failure modes associated with the extreme event. 
Fillet welds were examined, both in general and close-up, on numerous pieces from WTC 1 and WTC 2 Observations showed that the fillet welds joining the plates that make up the columns failed almost exclusively by rupture through the parent material at the HAZ of the weld. Extremely few fractures were observed through the weld material itself. This indicates that the welded joints performed as intended.

Three significant pieces from WTC 1 directly impacted by the aircraft were examined. Certain observations on these pieces suggest that certain columns in these panels may have resisted the aircraft impact in part by membrane tension behavior, in a fashion roughly analogous to sideways stretching of a rubber band held between two fingers. Likewise, other observations on these pieces suggest that other columns in each of these panels resisted the impact of the aircraft through shear behavior.

Numerous pieces from WTC 1 and WTC 2 were visually examined for evidence of possible effects of fire following impacts of aircrafts and before collapse of the structures. These pieces were distinguished from those exposed to fire following collapse of the towers by particular patterns of possible fire-related distress that would be unique to a fire occurring in the towers before their collapse.

Observations showed that the welded fillet joints at the four corners of the box column sections were ruptured on numerous sections that were apparently exposed to one or more different extreme loading events. It is possible that the weld ruptures occurred during the collapse of the towers or during postcollapse recovery and salvage operations. For columns possibly exposed to fire, an additional possibility, although not a certainty, is that the welds may have ruptured under a particular combination of axial load in the column, pre-fire strength properties of the weld metal and column steel, and sufficient heating of the column due to fire. 
In Support of Task 2 under Project 3 of the NIST WTCI Visual Observations of the Steel Recovered from the World Trade Center Site 26 November 2003

Page 472

This page intentionally left blank. 
In Support of Task 2 under Project 3 of the NIST WTCI

Visual Observations of the Steel Recovered From the World Trade Center Site

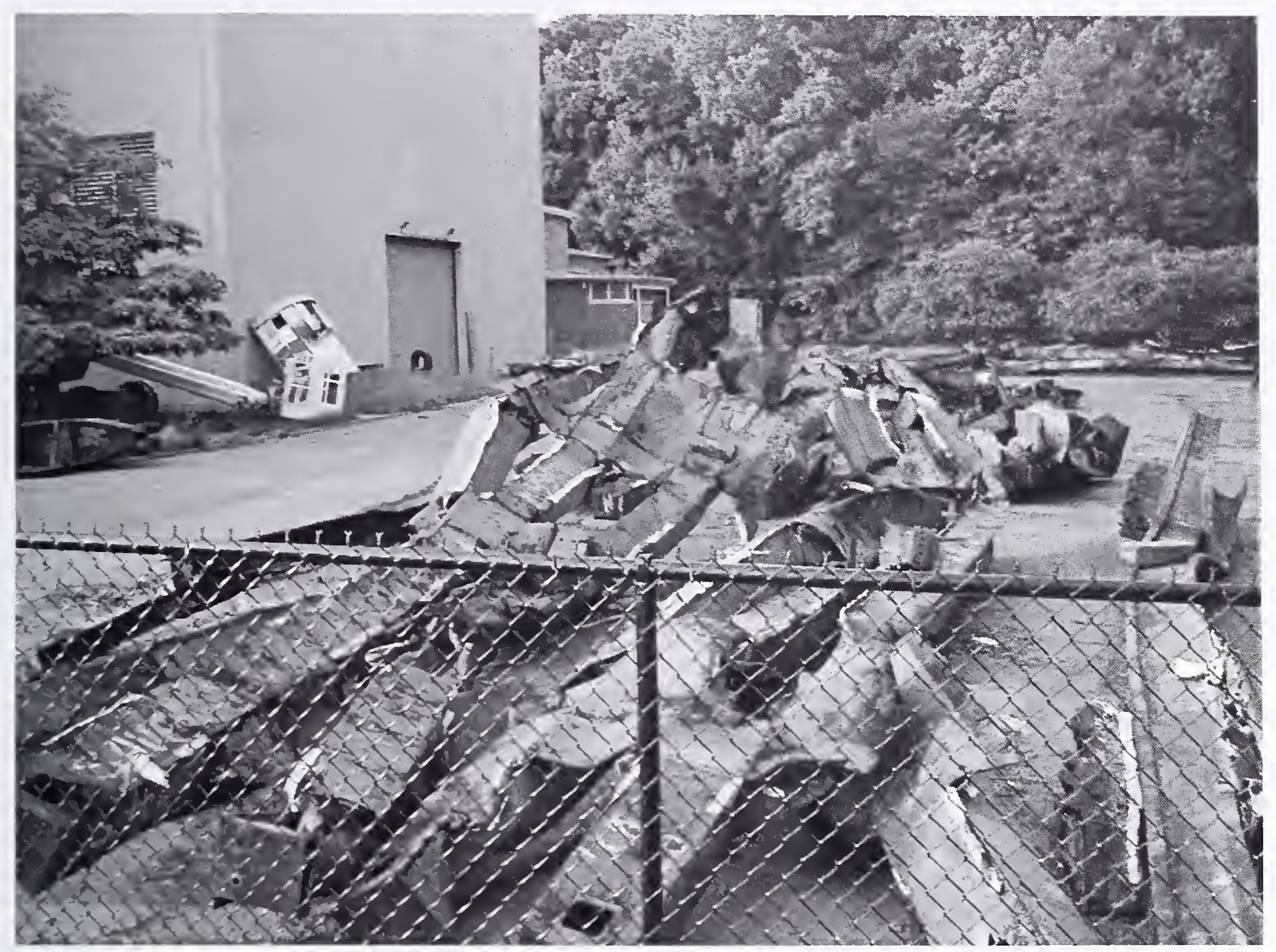

Figure 1. Debris field adjacent to Building 202 
In Support of Task 2 under Project 3 of the NIST WTCI Visual Observations of the Steel Recovered From the World Trade Center Site

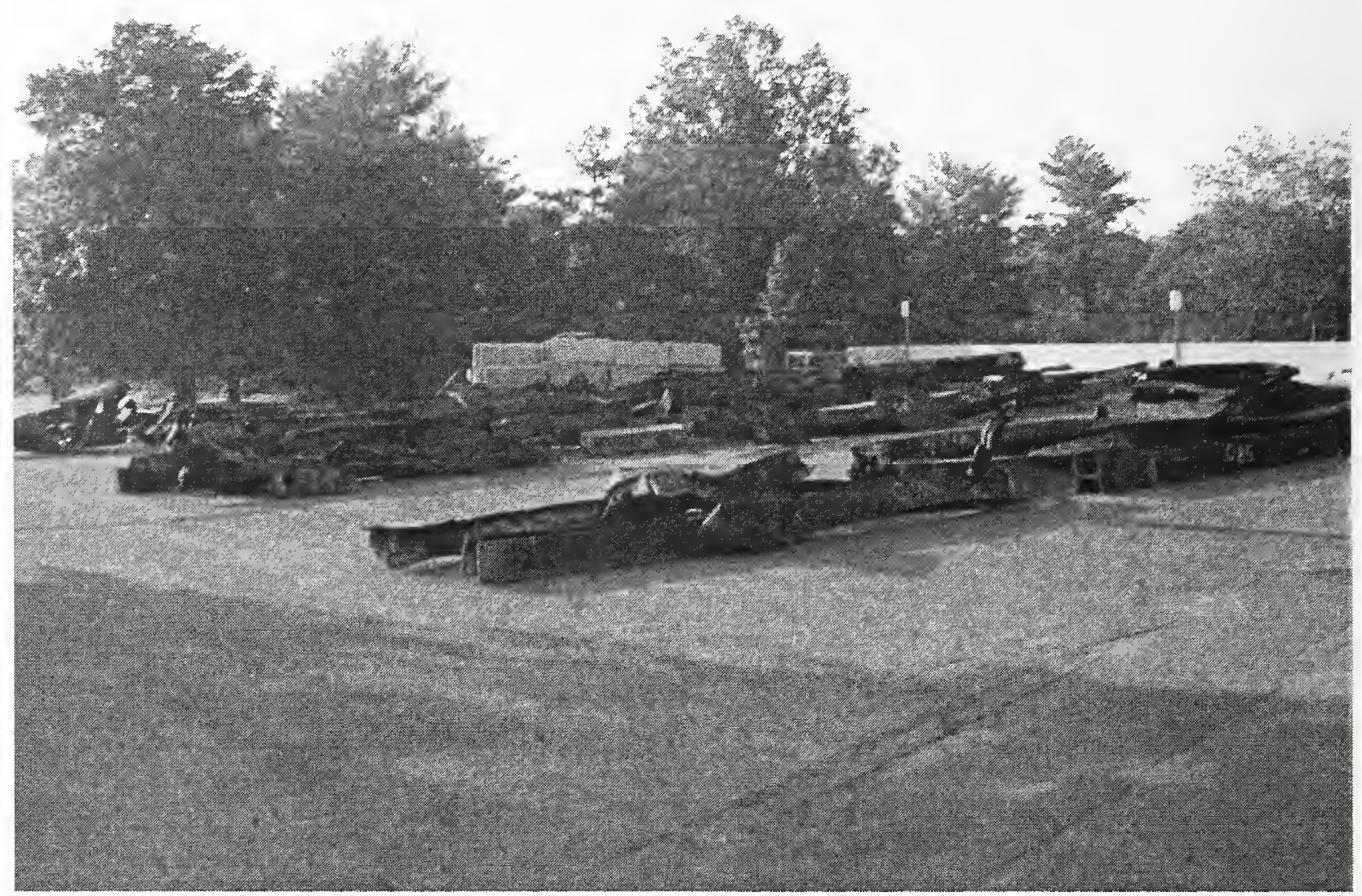

Figure 2. Debris field adjacent to Building 205 
In Support of Task 2 under Project 3 of the NIST WTCI Visual Observations of the Steel Recovered From the World Trade Center Site

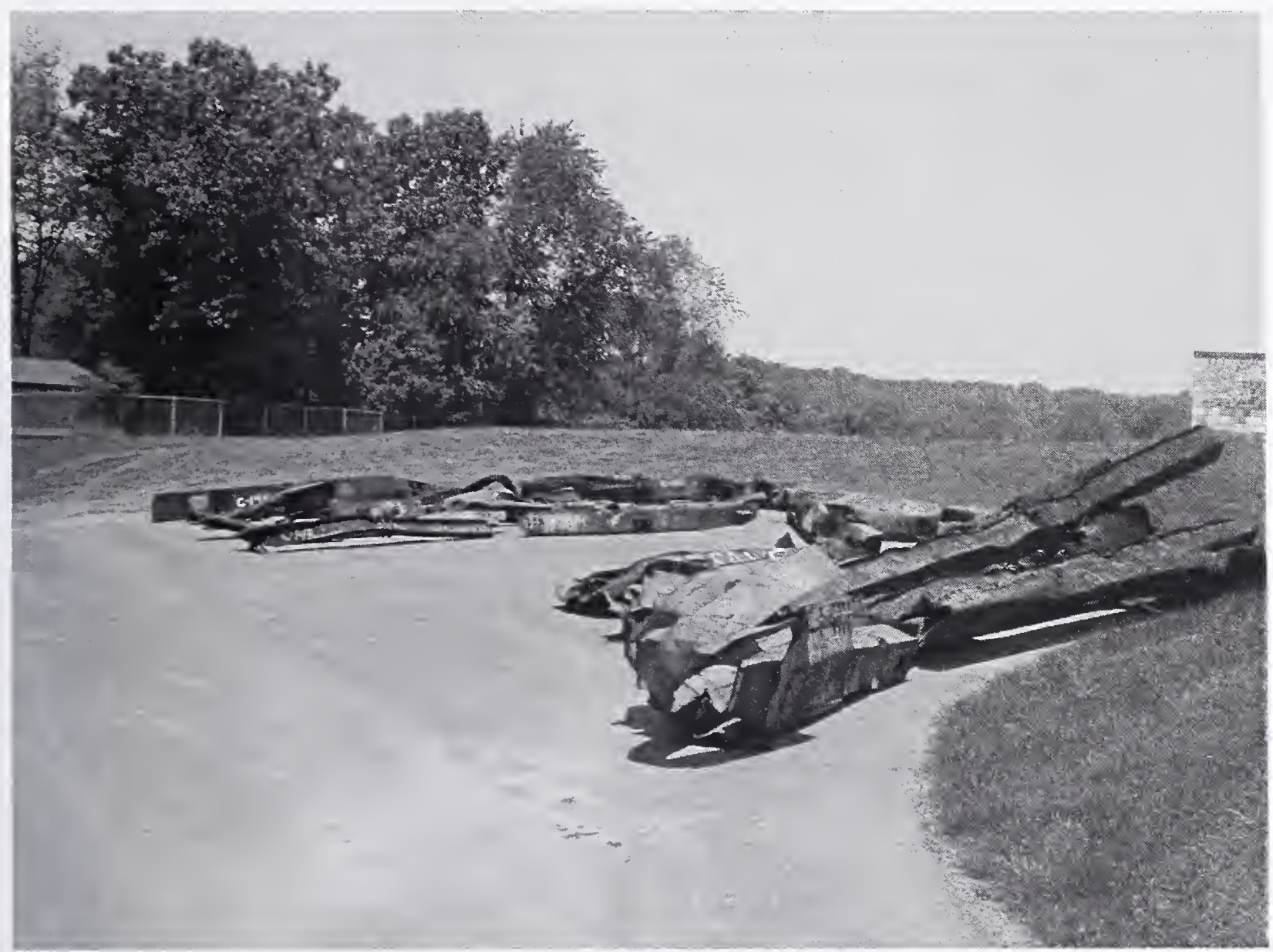

Figure 3. Debris field near Building 236 
In Support of Task 2 under Project 3 of the NIST WTCI

Visual Observations of the Steel Recovered From the World Trade Center Site

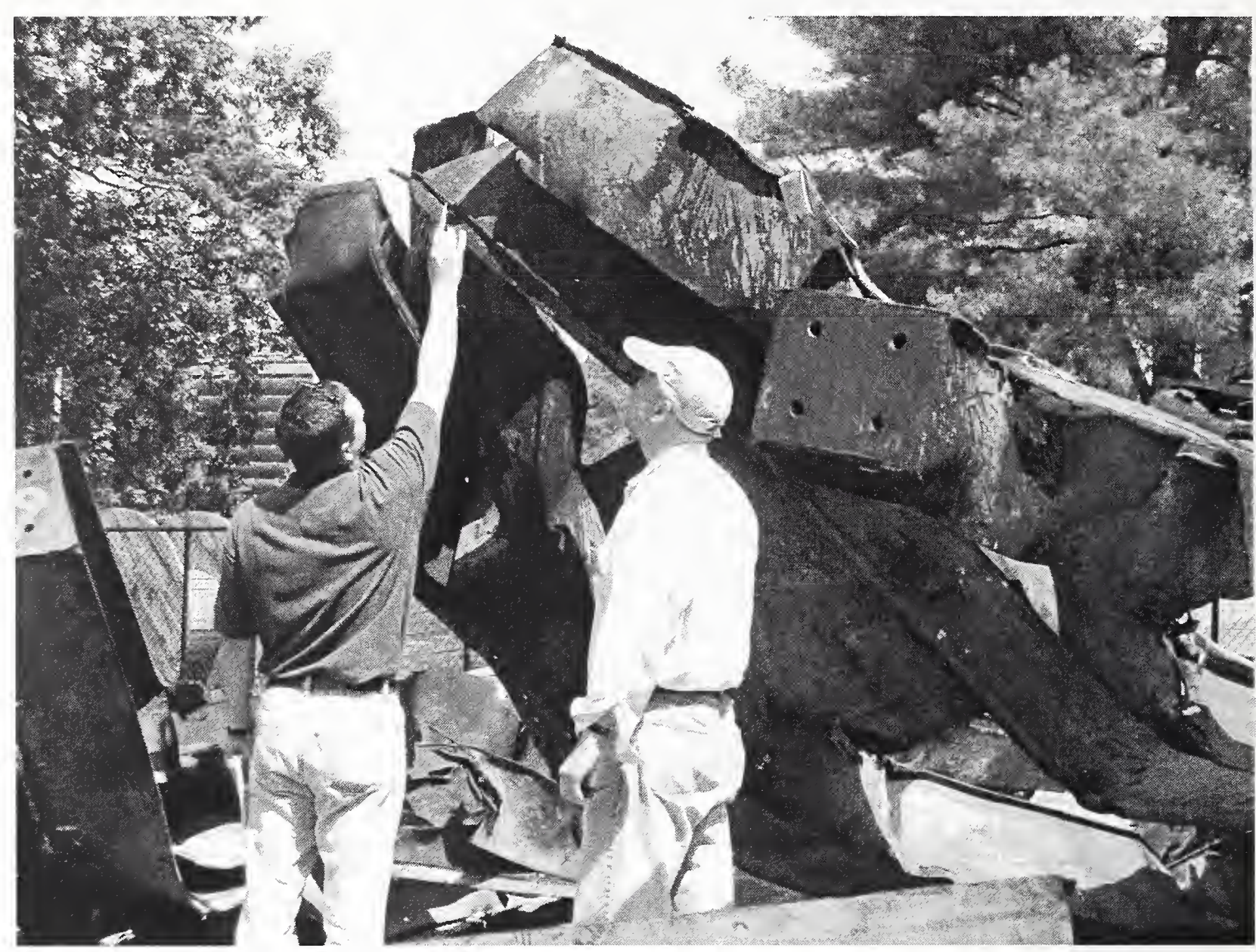

Figure 4. Contract engineers examine Piece $M-2$ 
In Support of Task 2 under Project 3 of the NIST WTCI

Visual Observations of the Steel Recovered From the World Trade Center Site
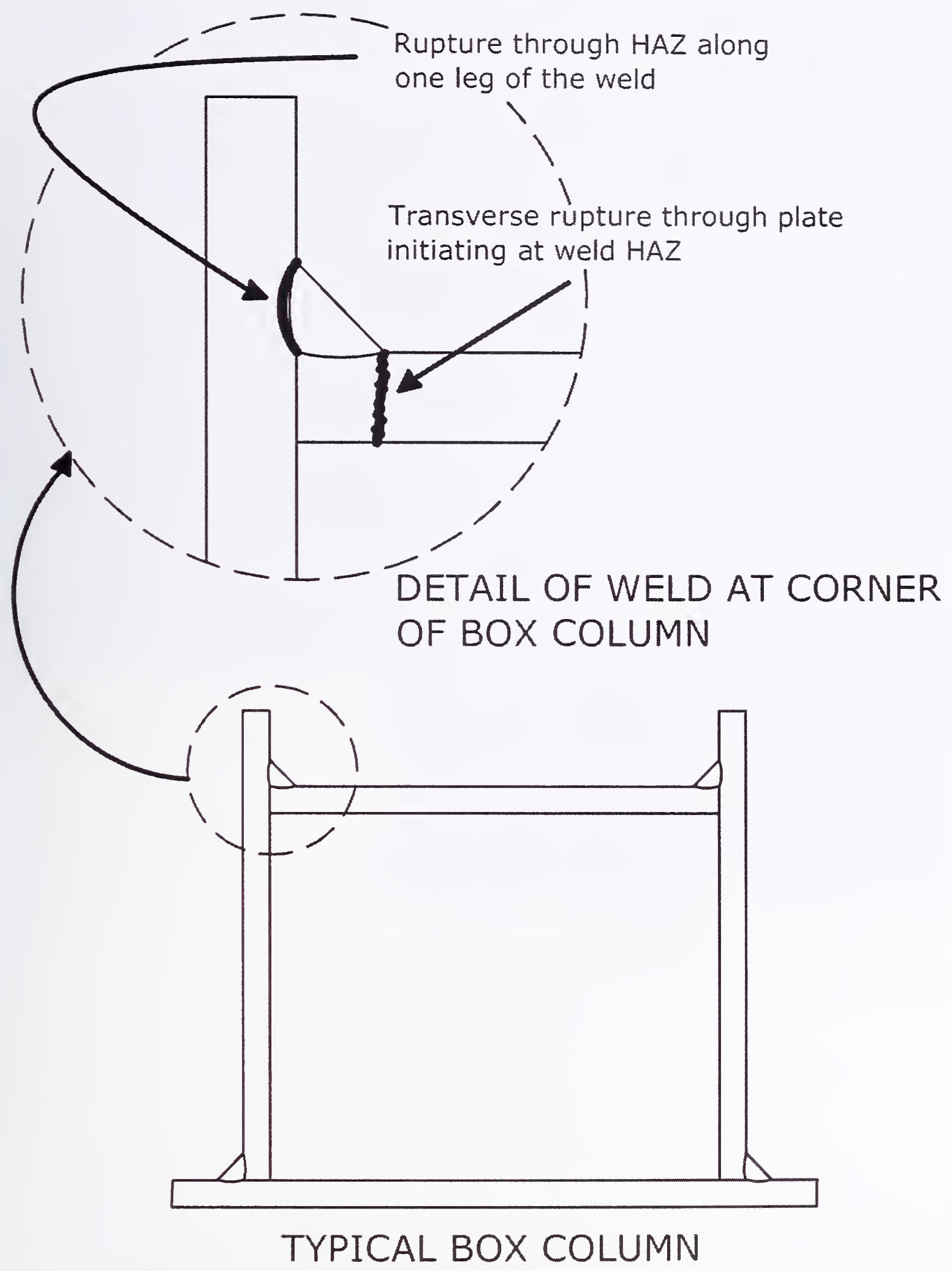

Figure 5. Schematic diagram illustrating fracture paths initiating at heat affected zone (HAZ) of weld. 
In Support of Task 2 under Project 3 of the NIST WTCI Visual Observations of the Steel Recovered From the World Trade Center Site

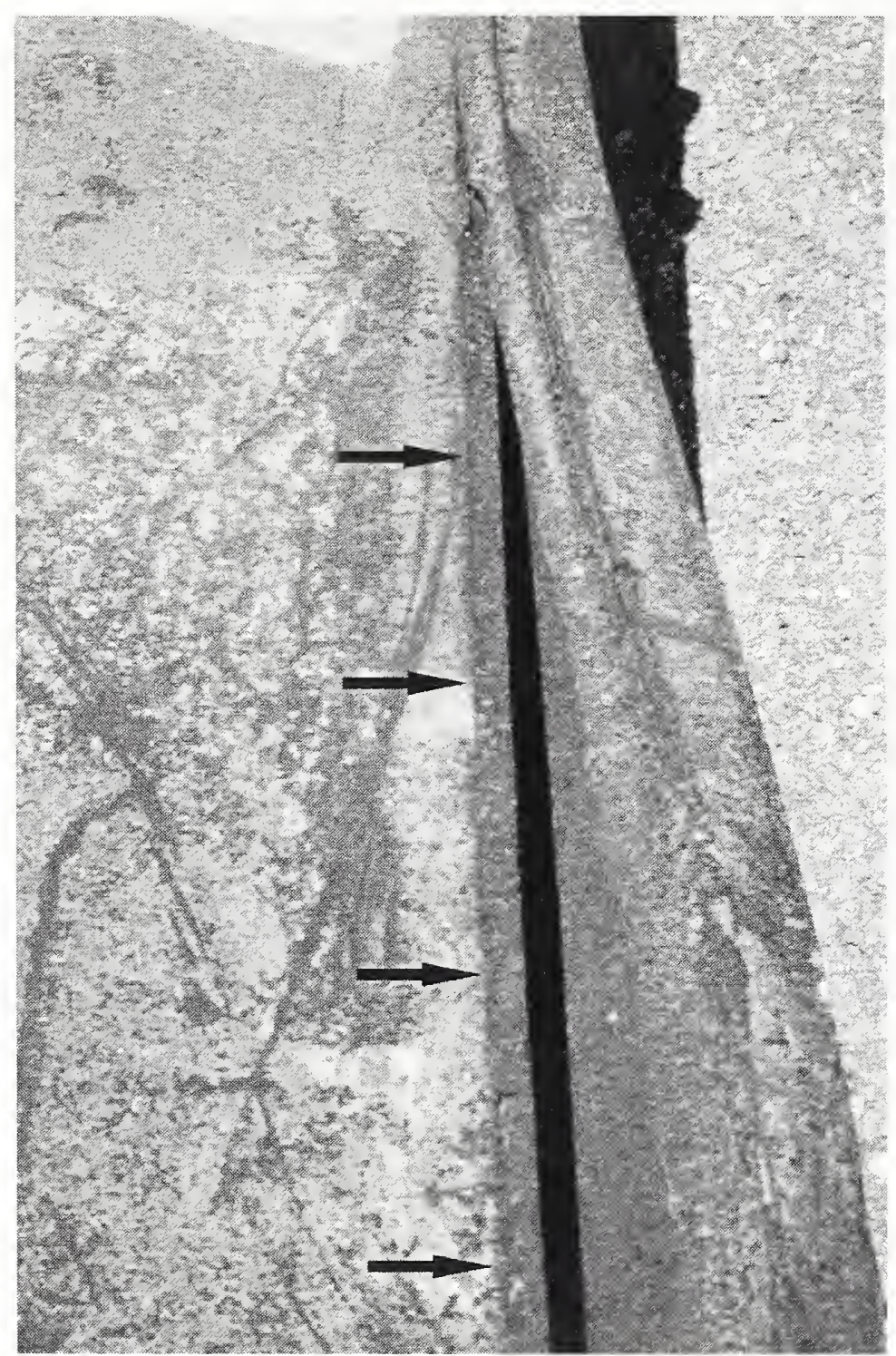

Figure 6. Close view of rupture of fillet weld through HAZ on parent material along a leg of the fillet. Arrows point to fracture surface in $H A Z$ on parent material; the mating fillet weld is at the right. 
In Support of Task 2 under Project 3 of the NIST WTCI

Visual Observations of the Steel Recovered From the World Trade Center Site

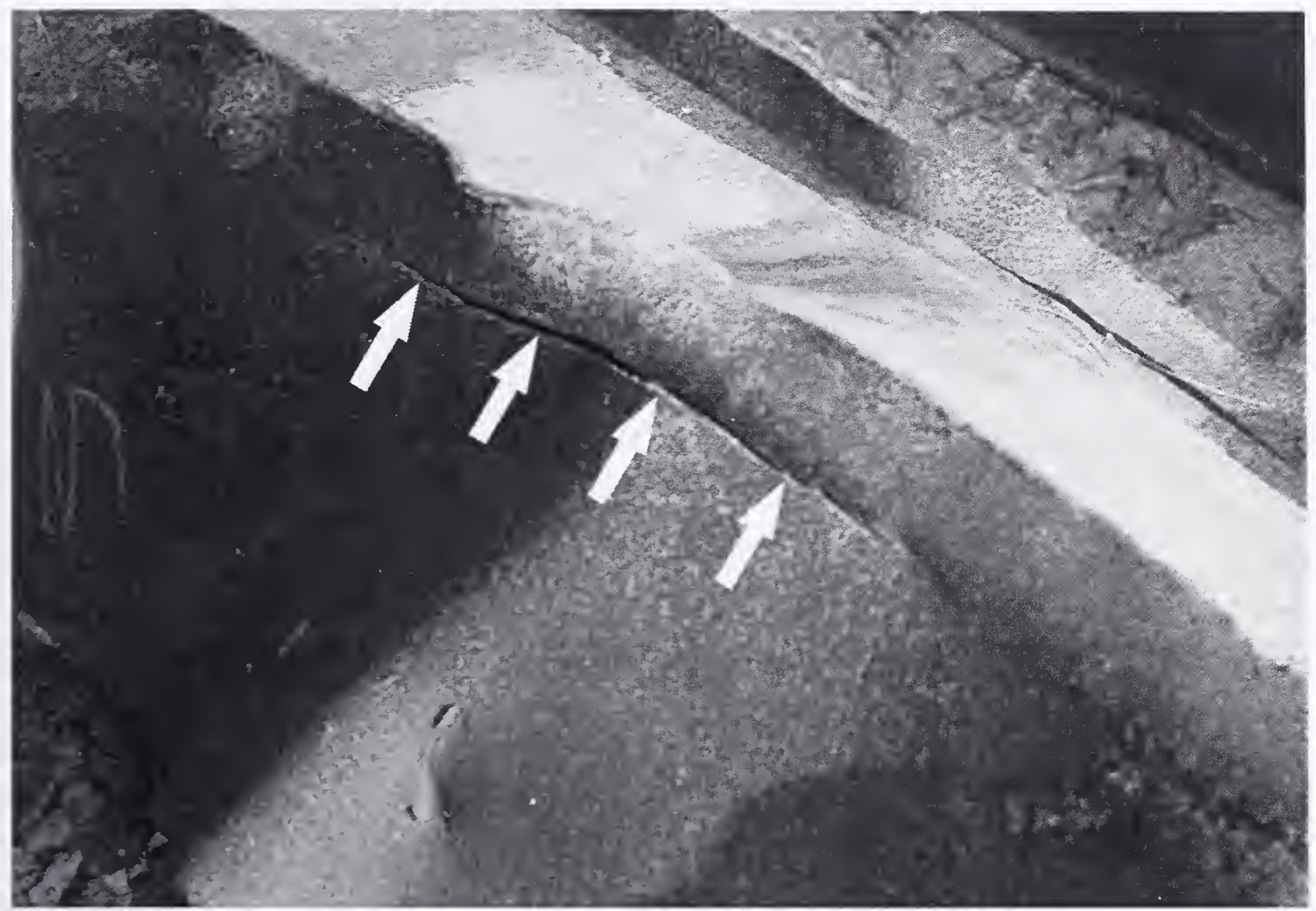

Figure 7. Transverse rupture through the thickness of the parent material, initiating in HAZ at toe of fillet welds 
In Support of Task 2 under Project 3 of the NIST WTCI Visual Observations of the Steel Recovered From the World Trade Center Site

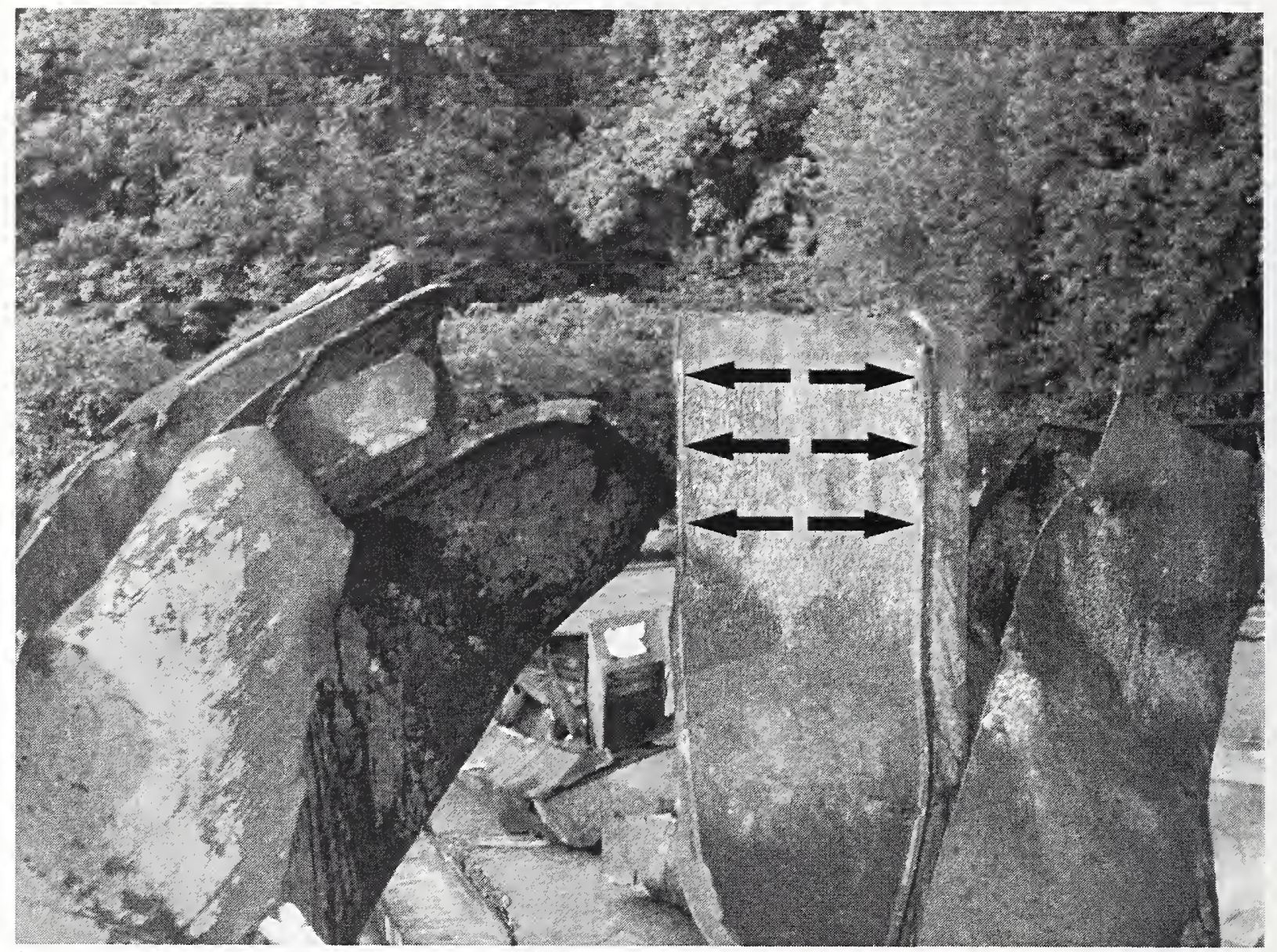

Figure 8. Ruptures of fillet welds through parent material HAZ on impact-effected region of Piece M-2 
In Support of Task 2 under Project 3 of the NIST WTCI Visual Observations of the Steel Recovered From the World Trade Center Site

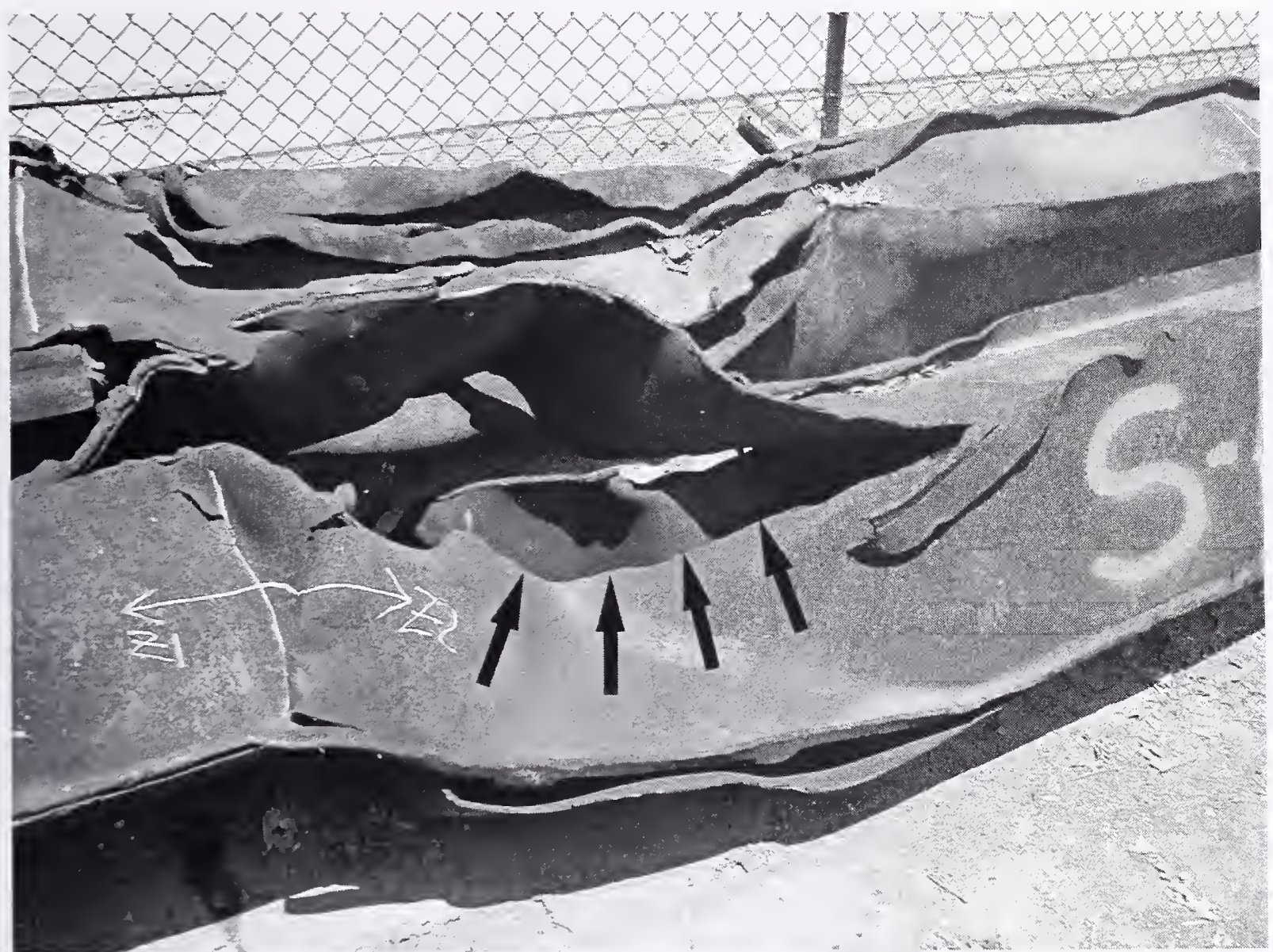

Figure 9. Ruptures of fillet welds through parent material $H A Z$ on possible fire-effected region of Piece $S-10$ 
In Support of Task 2 under Project 3 of the NIST WTCI Visual Observations of the Steel Recovered From the World Trade Center Site

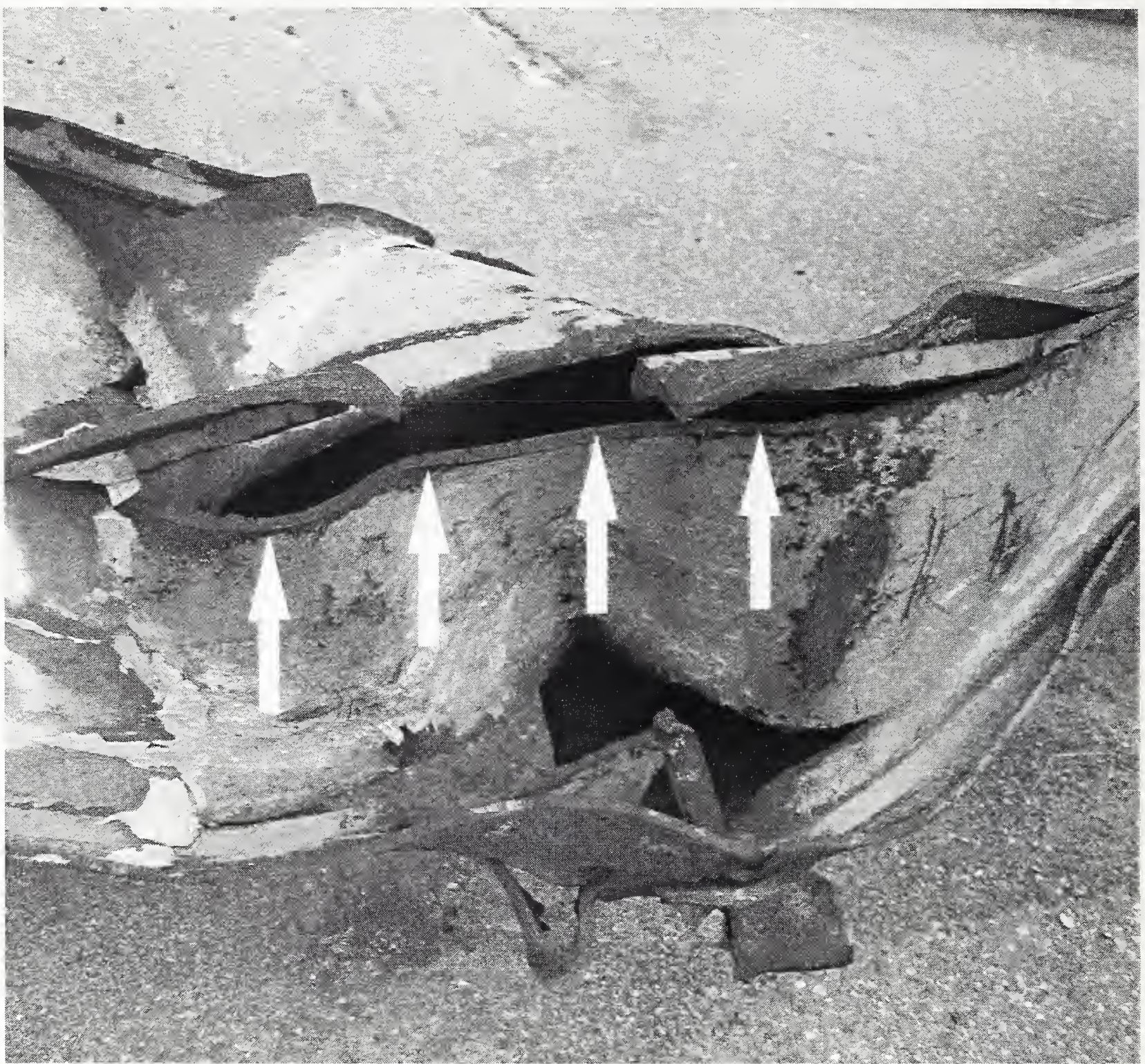

Figure 10. Ruptures of fillet welds througl parent material HAZ (at arrows) on probable mechanically pinched zone on Piece C-40 (ruptures through parent material away from welds are also observable in the photo) 
In Support of Task 2 under Project 3 of the NIST WTCI

Visual Observations of the Steel Recovered From the World Trade Center Site

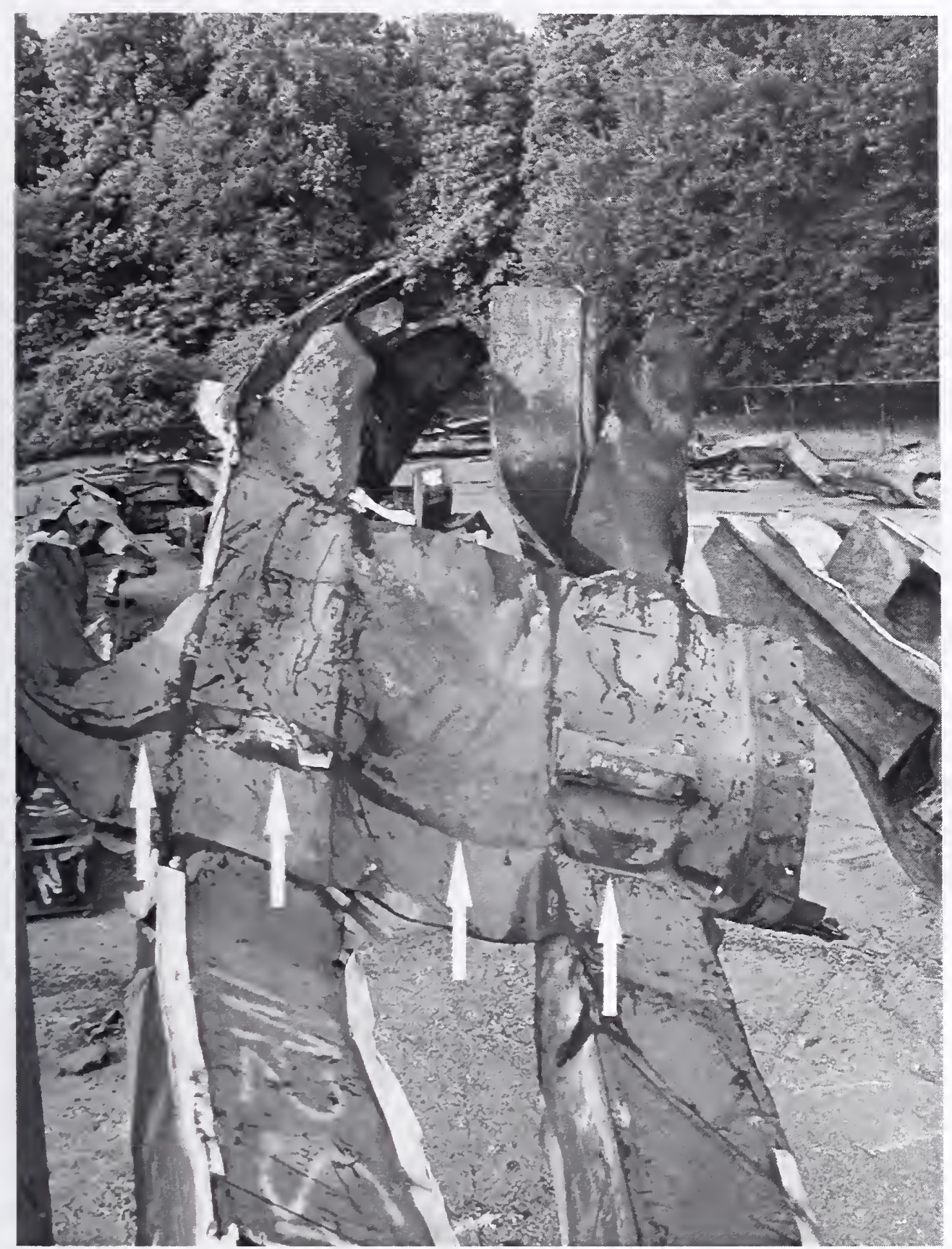

Figure 11. View of Piece M-2, showing inward bend of bottom of piece (at top of photo) about edge of the 97th floor slab(darkened line running left-10-right actoss spandrel plate, indicated by arrows) 
In Support of Task 2 under Project 3 of the NIST WTCI Visual Observations of the Steel Recovered From the World Trade Center Site

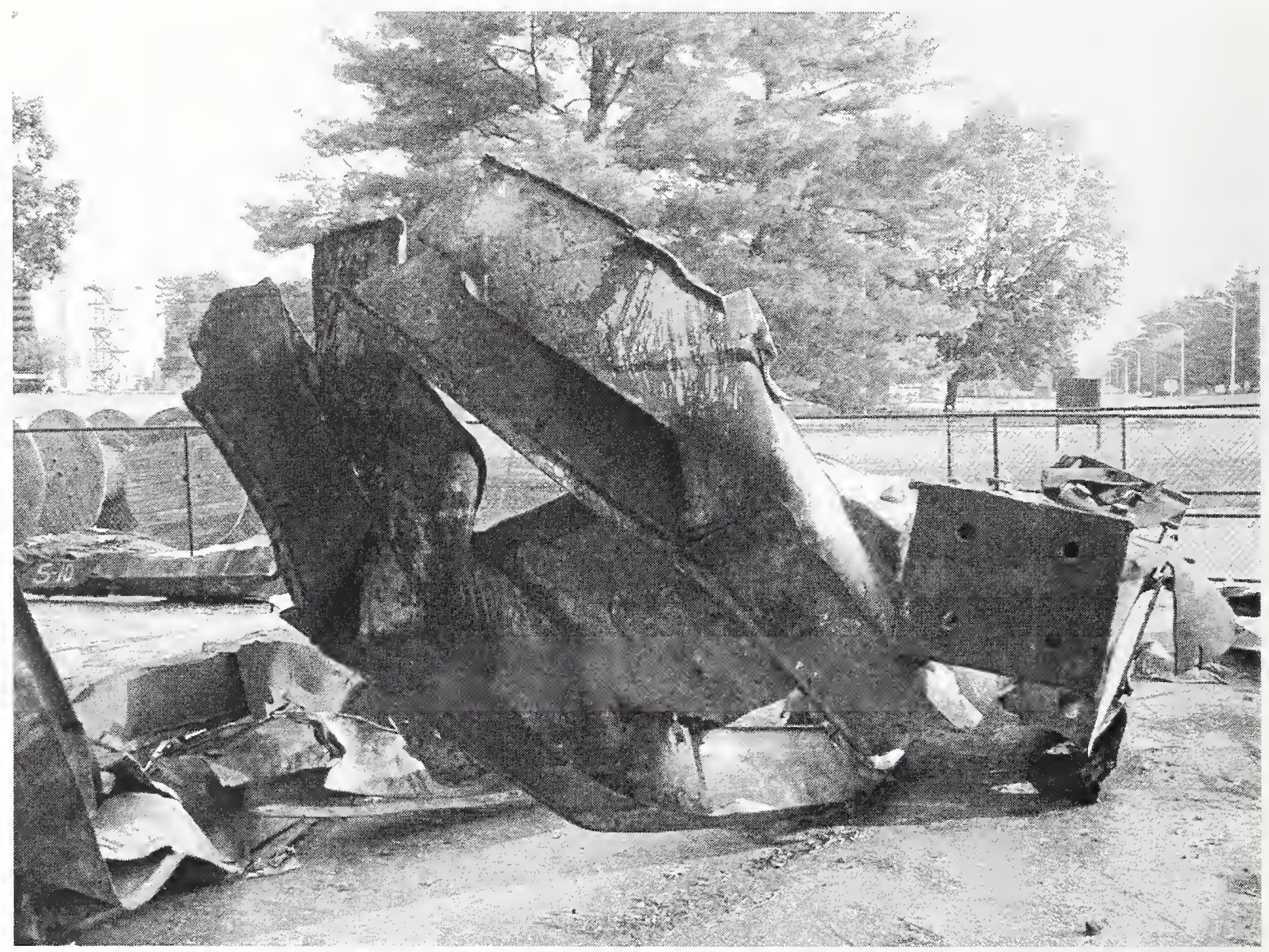

Figure 12. The extreme ends of the column elements in Piece $M-2$ 
In Support of Task 2 under Project 3 of the NIST WTCI Visual Observations of the Steel Recovered From the World Trade Center Site

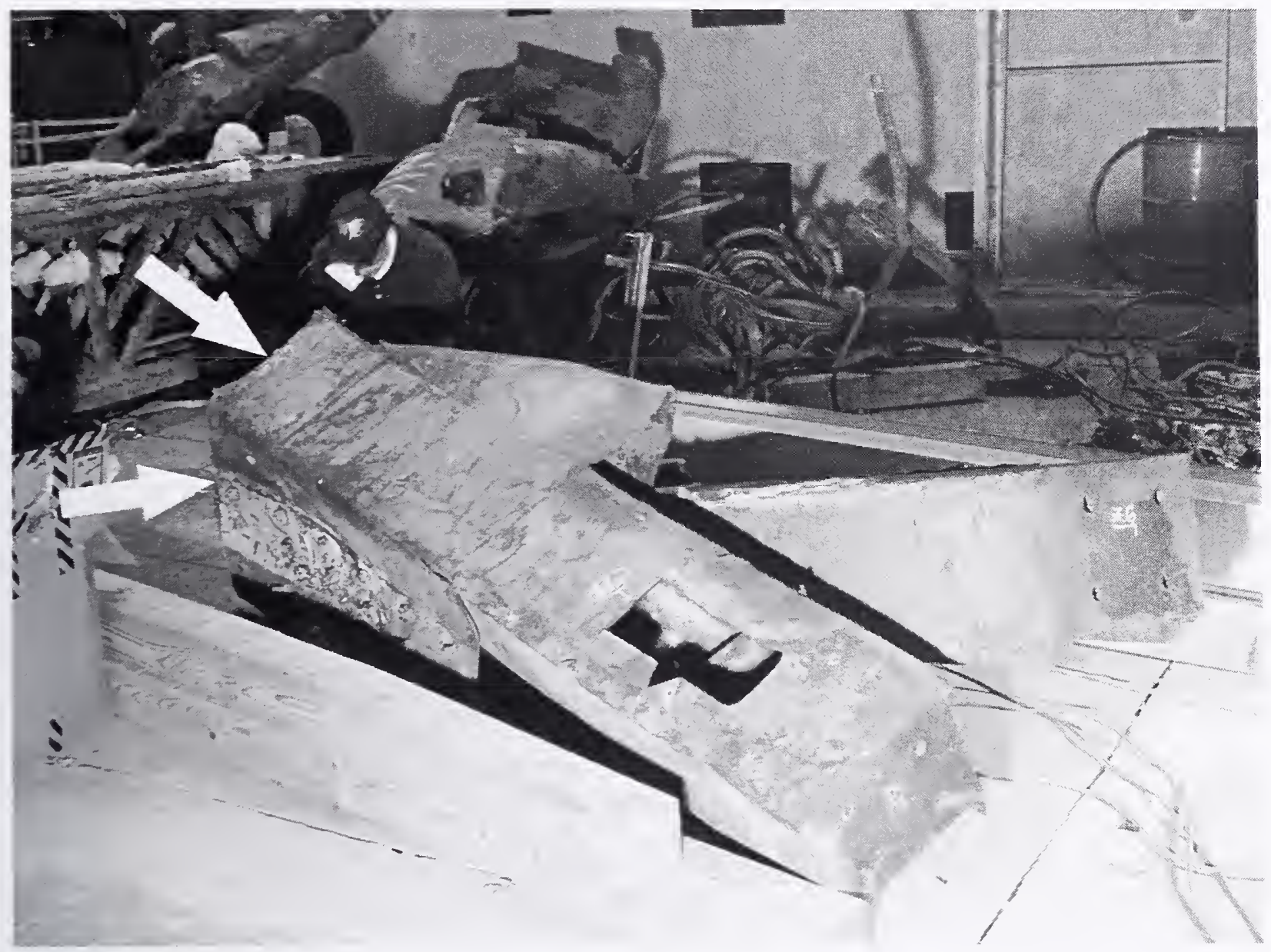

Figure 13. Severed column (at arrows) as observed on Piece M-30. 
In Support of Task 2 under Project 3 of the NIST WTCI Visual Observations of the Steel Recovered From the World Trade Center Site

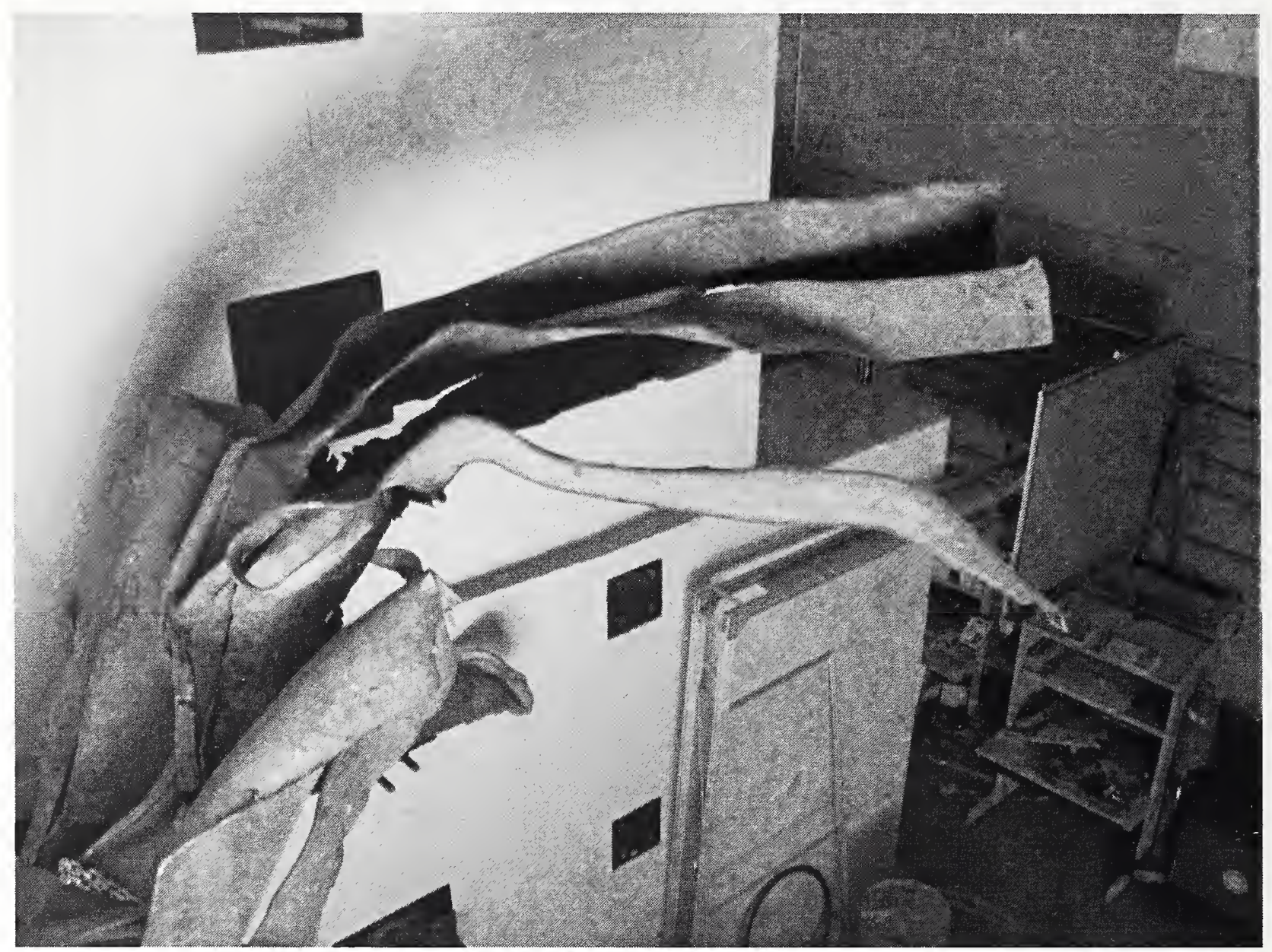

Figure 14. Column elements on Piece M-27 that have decomposed from closed box sections into ribbonlike slender plate elements as a result of the aircraft impact 
In Support of Task 2 under Project 3 of the NIST WTCI Visual Observations of the Steel Recovered From the World Trade Center Site

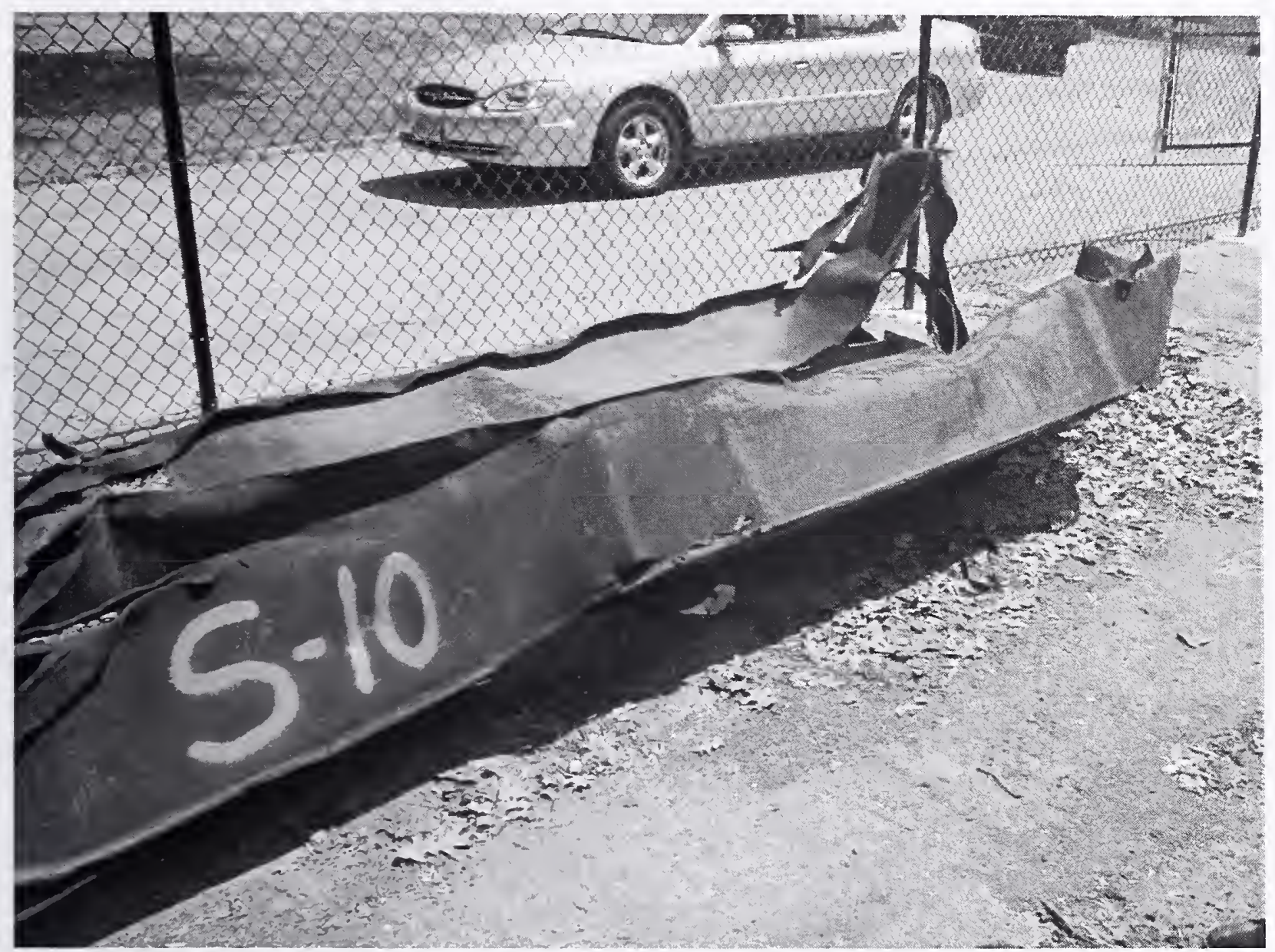

Figure 15. Pattern of possible fire effects (absence of paint at left; presence of paint at right) observable on the side face of Column 224 of Piece S-10 
In Support of Task 2 under Project 3 of the NIST WTCI

Visual Observations of the Steel Recovered From the World Trade Center Site

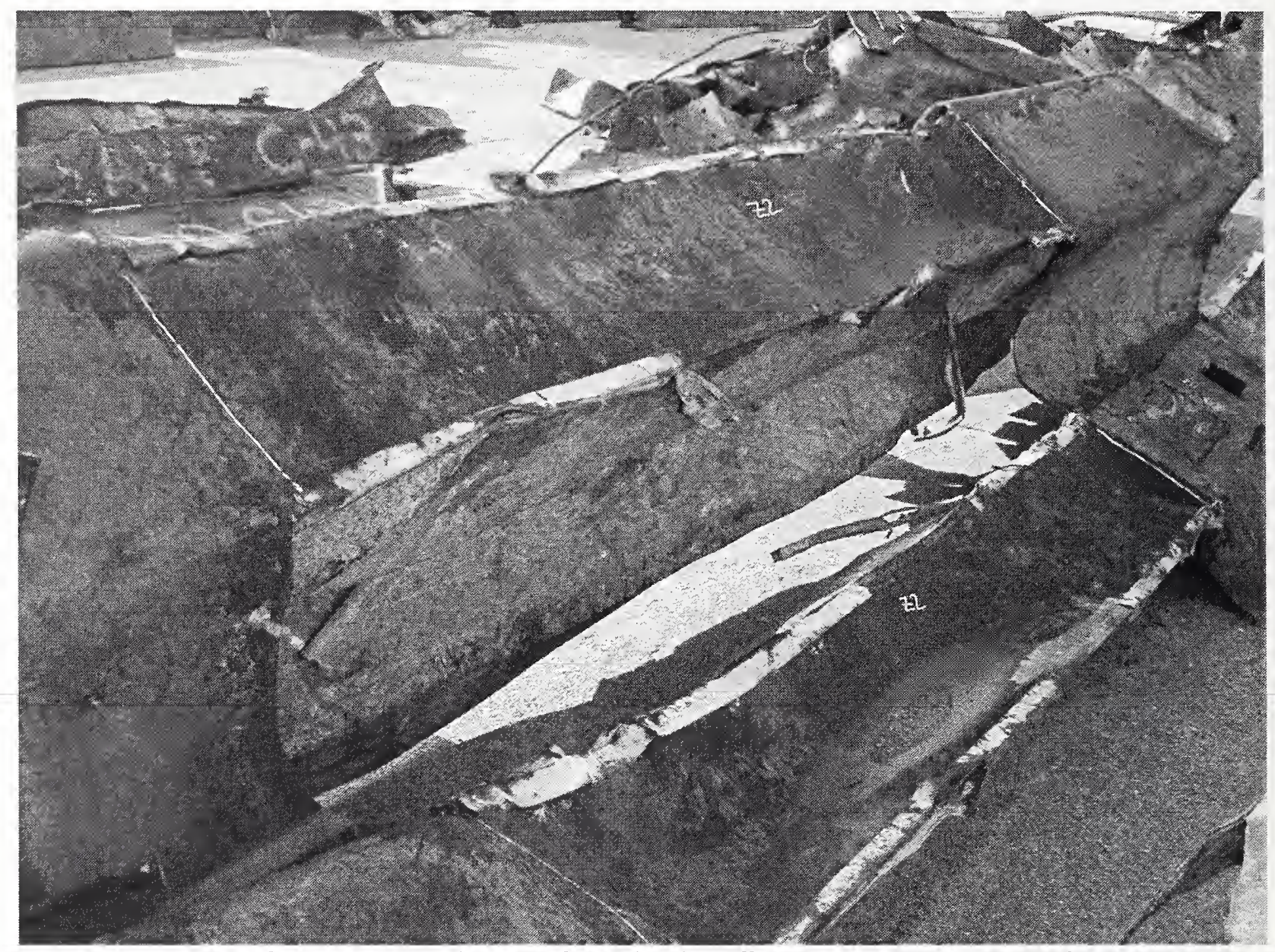

Figure 16. Pattern of presence and absence of possible fire effects on column elements of Piece M-4. Zones marked Z2 at middle are possible fire-effected areas at window openings. Spandrel plates above and below window openings appear unaffected by fire. 
In Support of Task 2 under Project 3 of the NIST WTCI

Visual Observations of the Steel Recovered From the World Trade Center Site

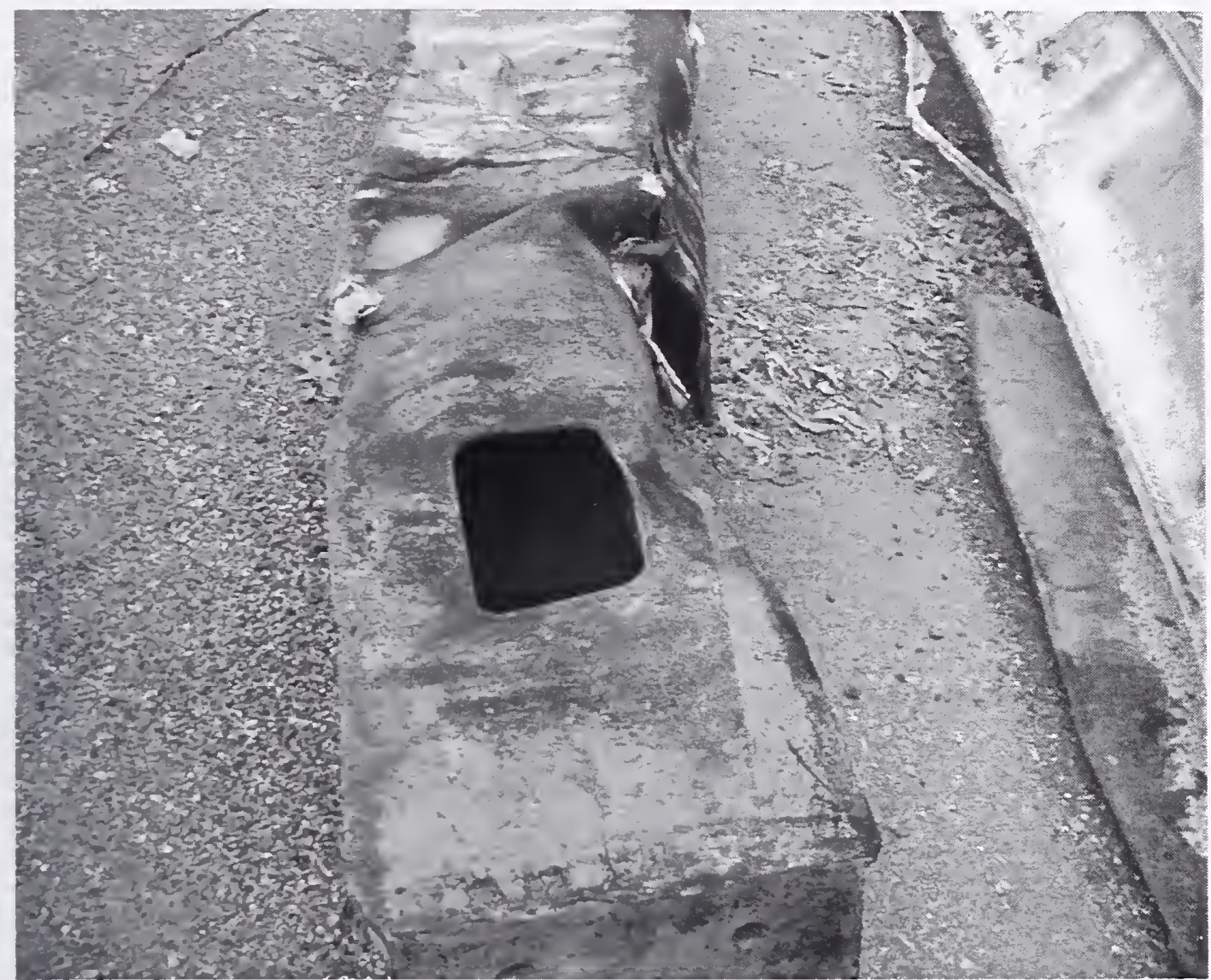

Figure 17. Possible fire effects on lower end of Piece K-2 
In Support of Task 2 under Project 3 of the NIST WTCI

Visual Observations of the Steel Recovered From the World Trade Center Site

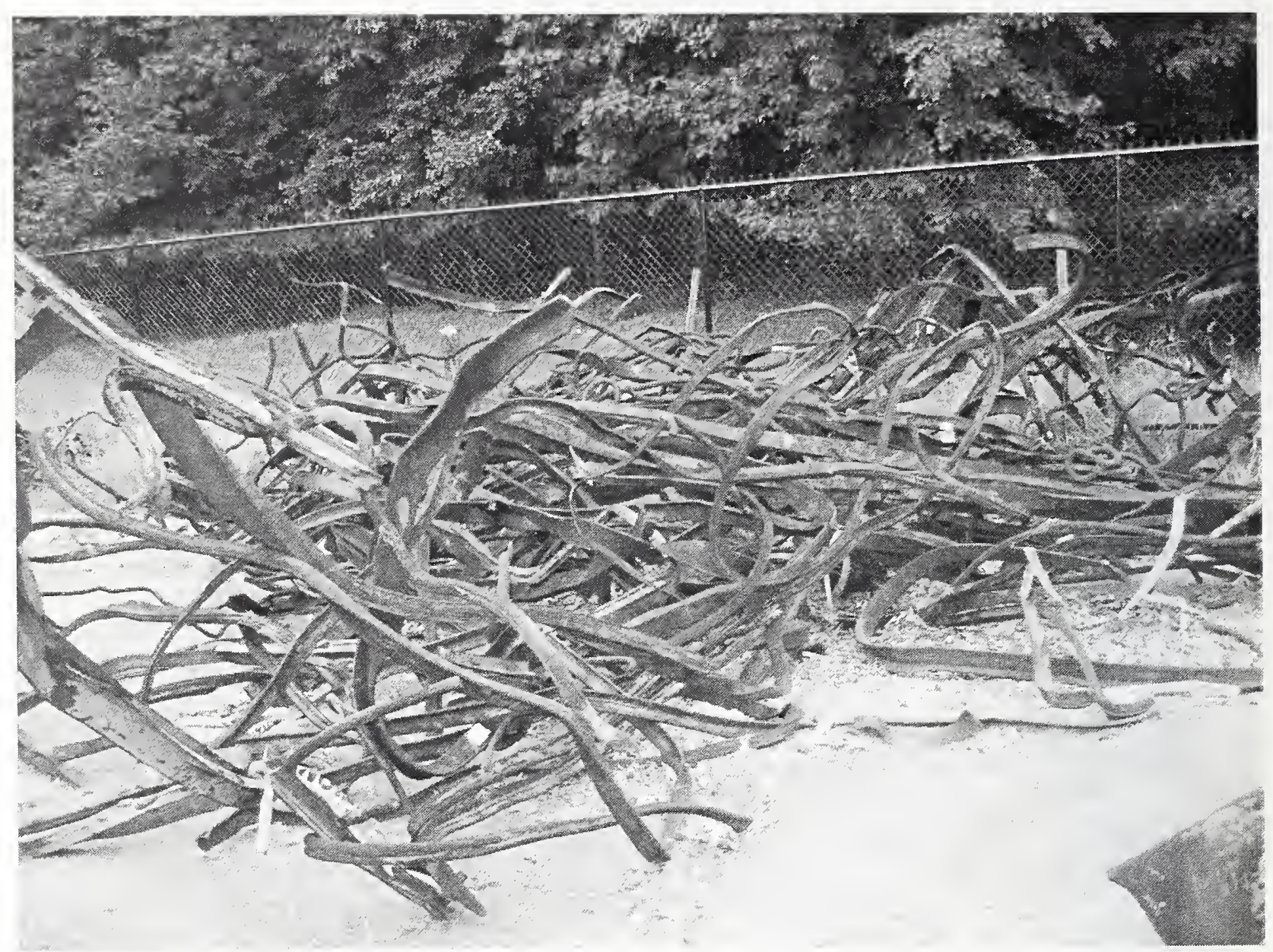

Figure 18. General view of a portion of recovered floor truss material 
In Support of Task 2 under Project 3 of the NIST WTCI

Visual Observations of the Steel Recovered From the World Trade Center Site

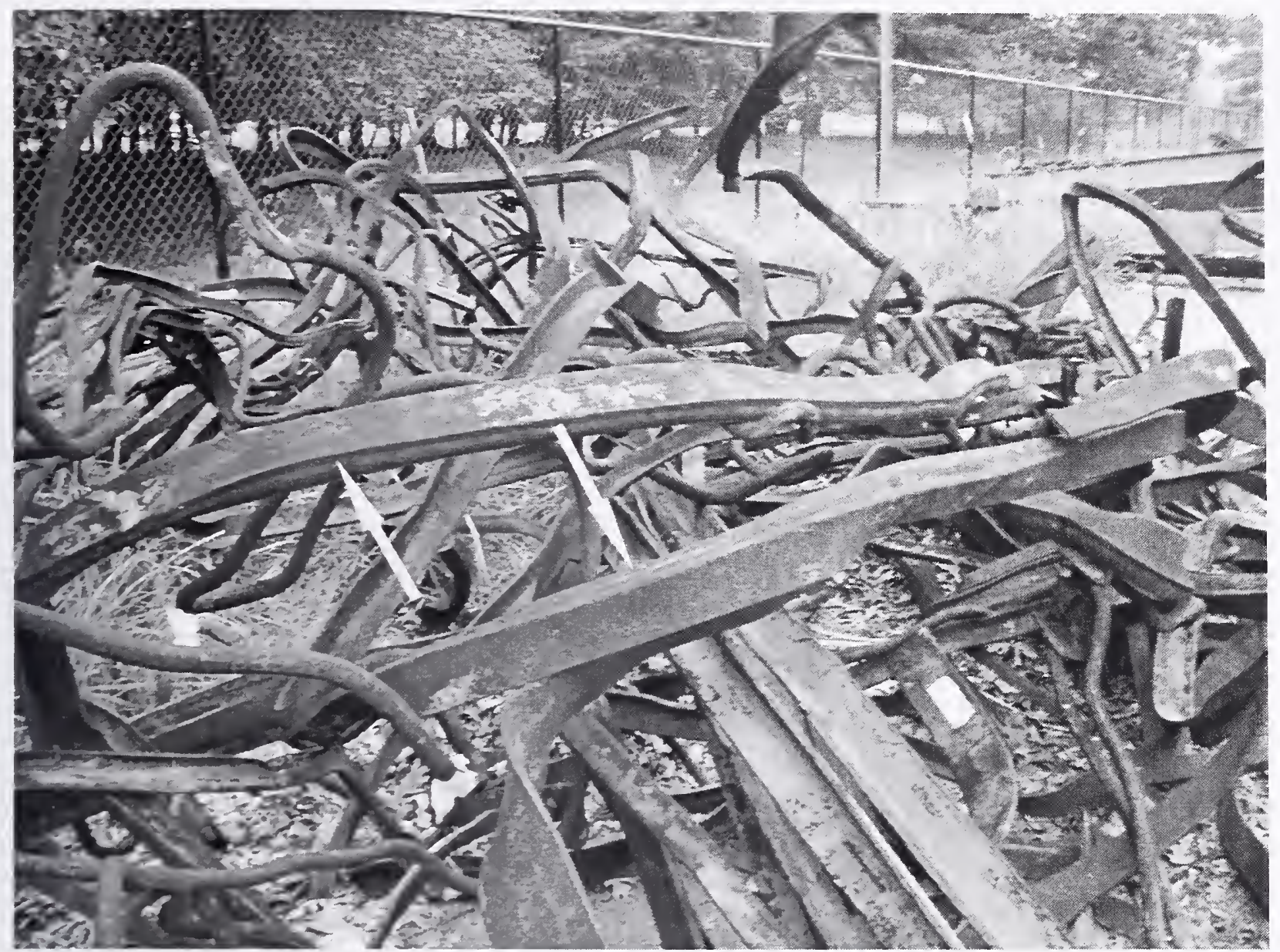

Figure 19. Close view of some recovered floor truss material. Arrows point to truss chords that exhibit longitudinal rib typical of floor trusses manufactured by Laclede Steel 
In Support of Task 2 under Project 3 of the NIST WTCI Visual Observations of the Steel Recovered From the World Trade Center Site

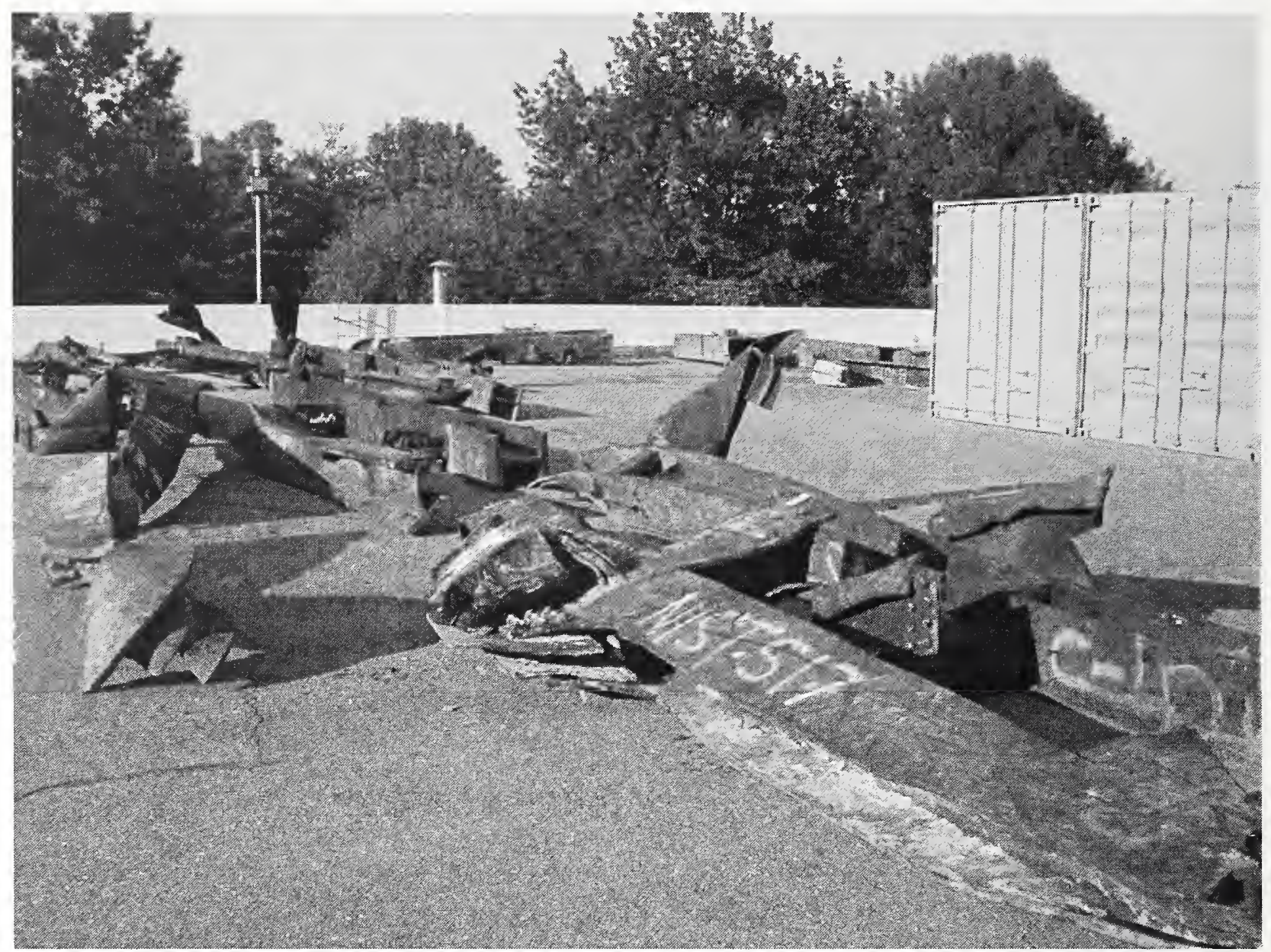

Figure 20. General view of several pieces believed to have been recovered from structures other than WTC 1 and WTC 2 
In Support of Task 2 under Project 3 of the NIST WTCI Visual Observations of the Steel Recovered From the World Trade Center Site

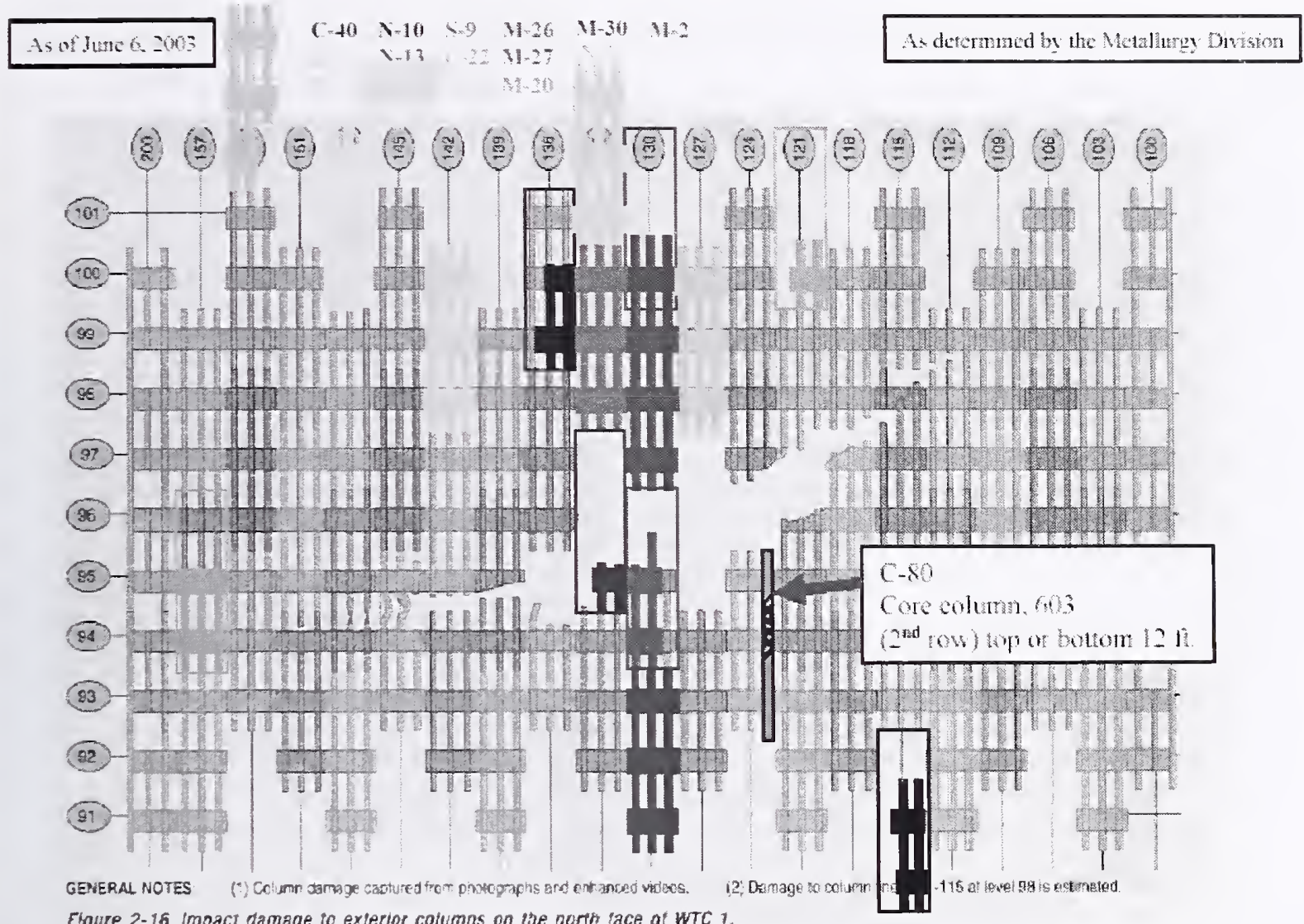

Figure 21. Original locations within WTC 1 of Pieces M-2, M-27 and M-30. 
In Support of Task 2 under Project 3 of the NIST WTCI Visual Observations of the Steel Recovered From the World Trade Center Site

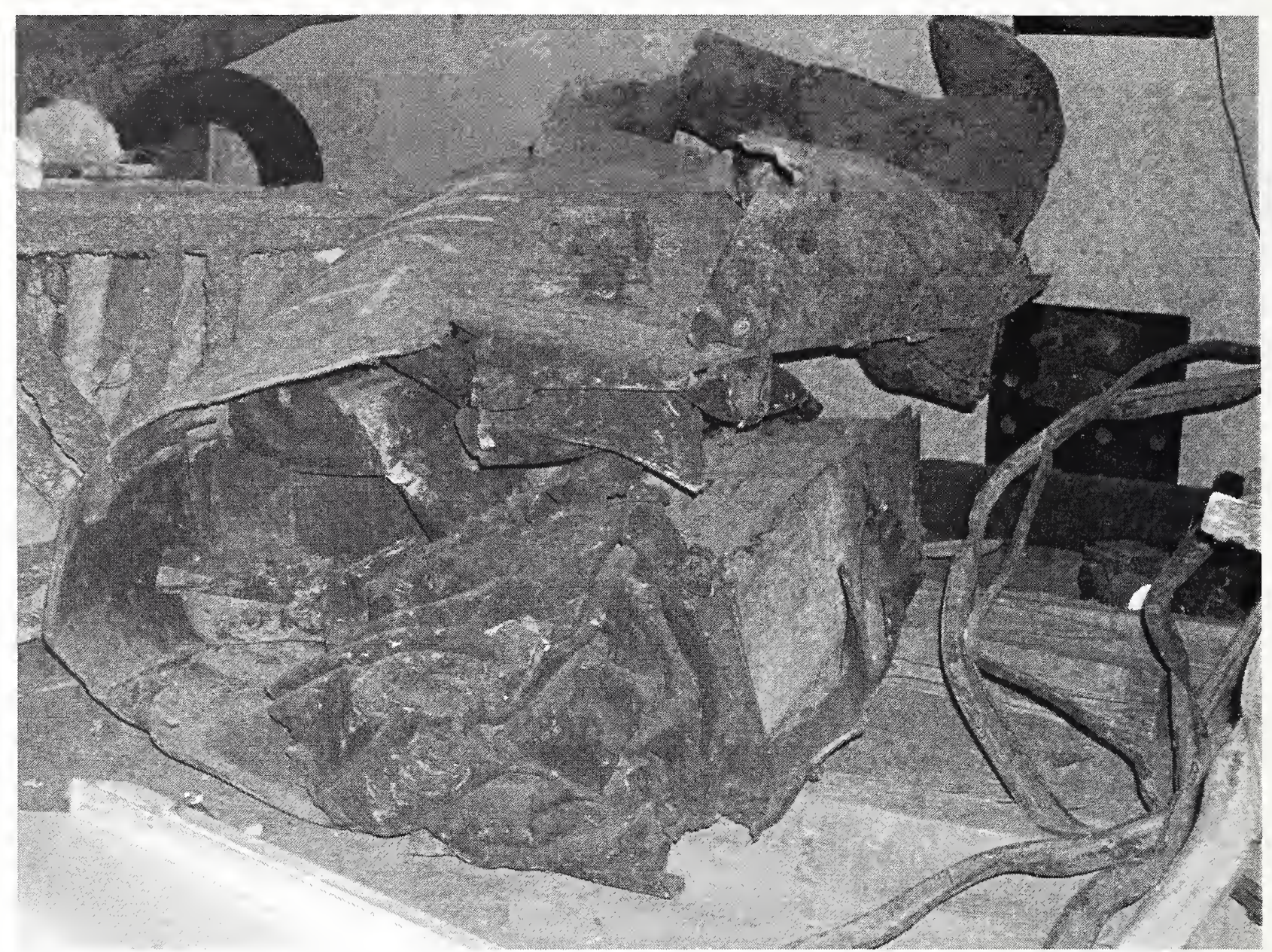

Figure 22. Piece K-1, which has collapsed in compression 

FROM FLOORS 74 TO 87 OF WTC 2 


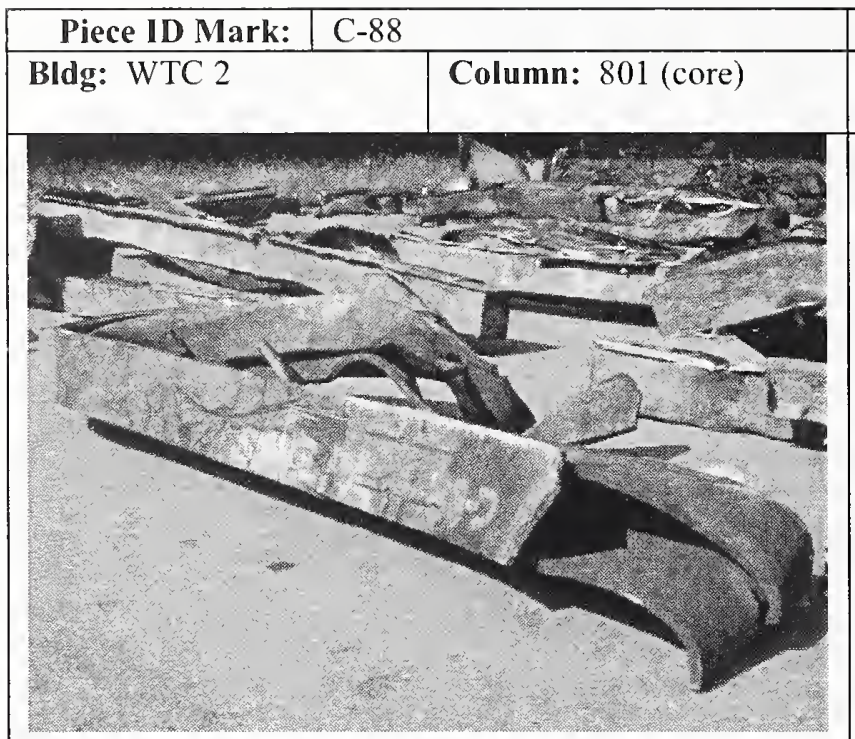

Figure F-A-1a: General view of C-88. The bottom of the column is at lower right in this view. Column splice can be observed between paint marks "SAVE" and "C-88". The side of the column with paint marks faced into a vertical shaft.

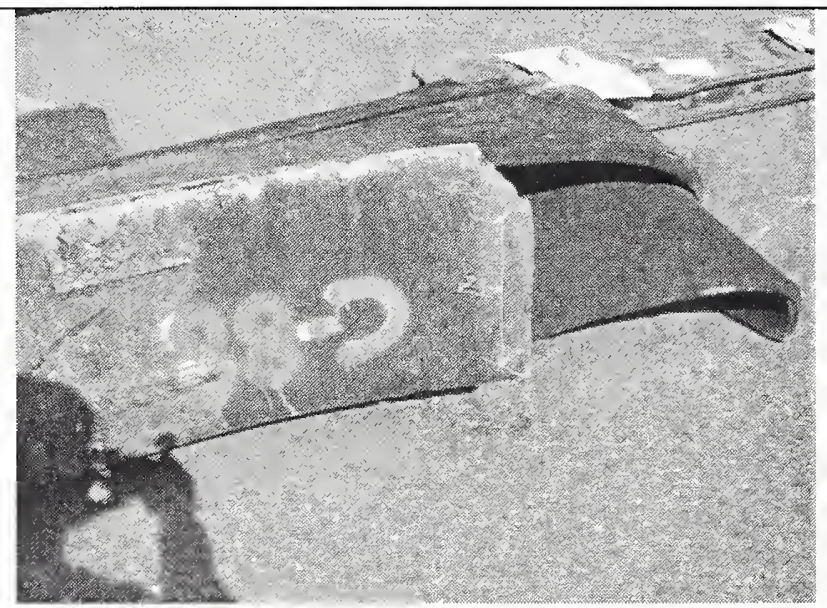

Figure F-A-lb: General view of identified areas $\mathrm{Zl}$ and $\mathrm{Z2}$.

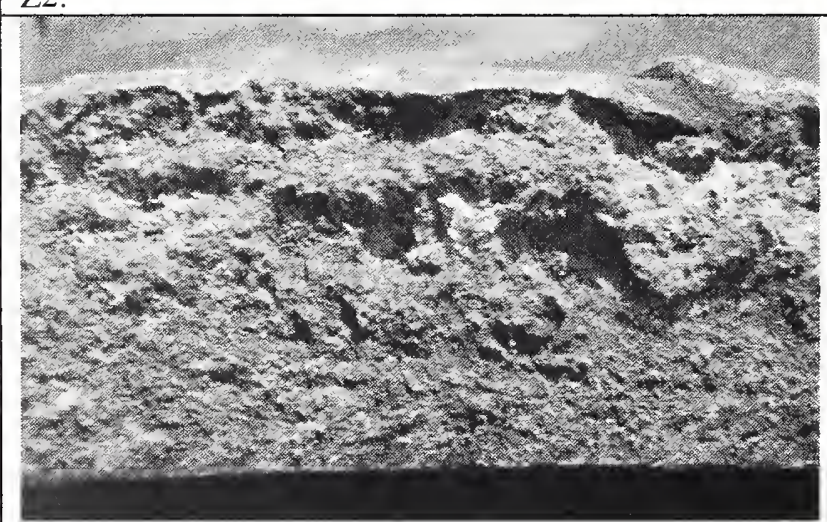

Figure F-A-1c: Close view of fracture surface at area Z1. (The upper horizontal edge of the fracture surface has been damaged due to shipping and handling of the piece.)
By \& Date: 1 CP/ JH/ RT / 24 June 2003

Floors: 79 to 82 or 83 Possible impact affected Fire effects

Box-shaped core column (Figure A-i ). Includes column splice located at floor 80 ; that portion above the splice is identified as Piece C88a, and that portion below the splice is Piece C88b. (Piece C88c is a large plate fragment cut from the upper end of C88a.) Piece C88b was at a floor level directly impacted by the aircraft.

By locating remaining fragments of connections, it was possible to determine the orientation of the piece within the structure. Floor plans showed that one face of the box column faced into an open vertical shaft.

At the lower end of the column (Piece C88b), three of the four flange plates exhibit a $\mathrm{J}$-shaped deformed shape (Figures A-1 and A-2). The direction of bend of the hook of the $\mathrm{J}$ not consistent with aircraft impact. It is likely, therefore, that the final distorted shapes of these three flanges of the box column were a result of the collapse event. The ends of these three flange plates have been flame cut.

The surface condition of these same three flanges at the immediate vicinity of the distortion is rusty (Figures A-1 and A-2), suggesting that the flanges were possibly exposed to fire. The sharpness of the bends in the distorted area, along with the lack of cracking of the steel at the sharp bends, suggests that the steel was at an elevated temperature at the time that the distortion occurred. It is possible that the fire that may have affected these pieces was the fire immediately following the aircraft impact.

The fourth flange was severed at a different vertical location and in a different fashion than the other three flanges (Figure A-2). This is the flange that faced into the open vertical shaft. The distorted shape for this flange is consistent with the direction of aircraft travel at the time of impact. This distortion could also have been caused by the collapse of the tower.

The fracture surface at the severed end was visually examined close-up. Two areas of interest along the severed end were identified as $\mathrm{Z1}$ and $\mathrm{Z2}$. Area $\mathrm{Z1}$ is a fracture of the steel that appears to have occurred in a non-ductile manner because there is no visible constriction ("neck-down") of the flange plate thickness in the immediate vicinity of the fracture. It is probable that this is a non-ductile fracture that occurred at a high rate of strain as a result of the tower collapse or aircraft impact. Representative fracture surface of $Z 1$ is shown in Figure A-3.

Identified area Z2 visually appears to be a flame cut edge. The flame cutting probably occurred sometime during the salvage, shipping and handing of the piece following collapse. 


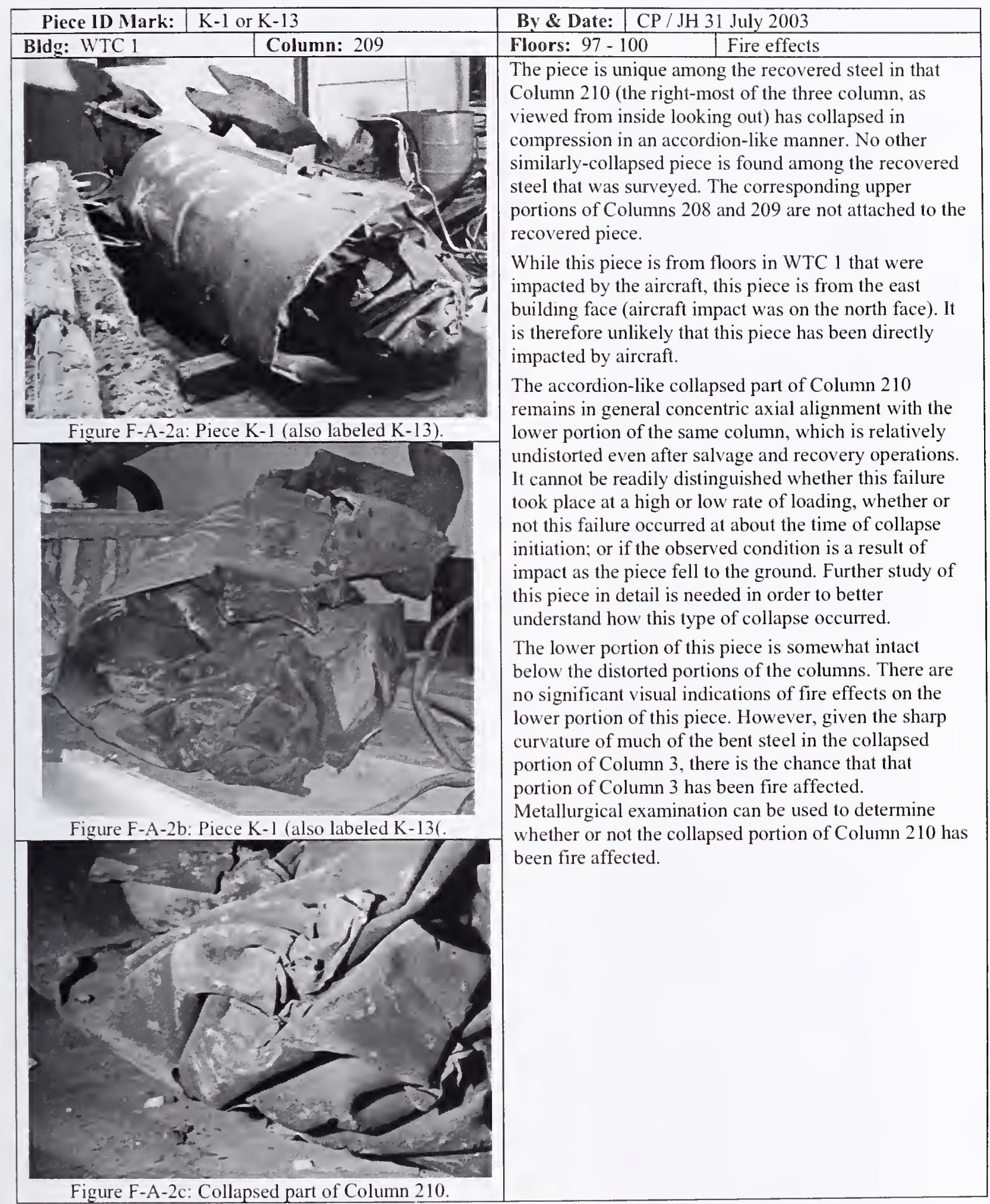




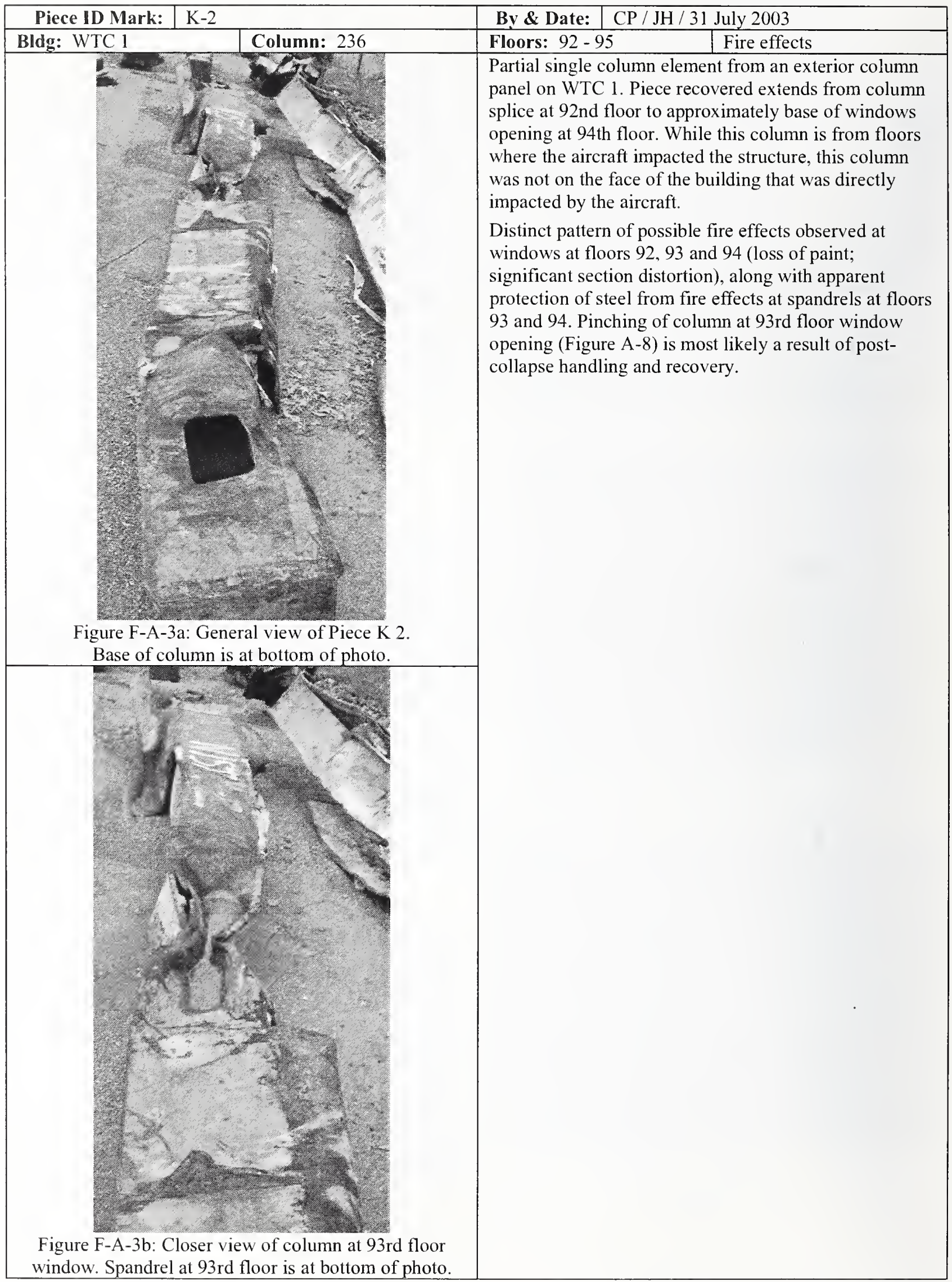




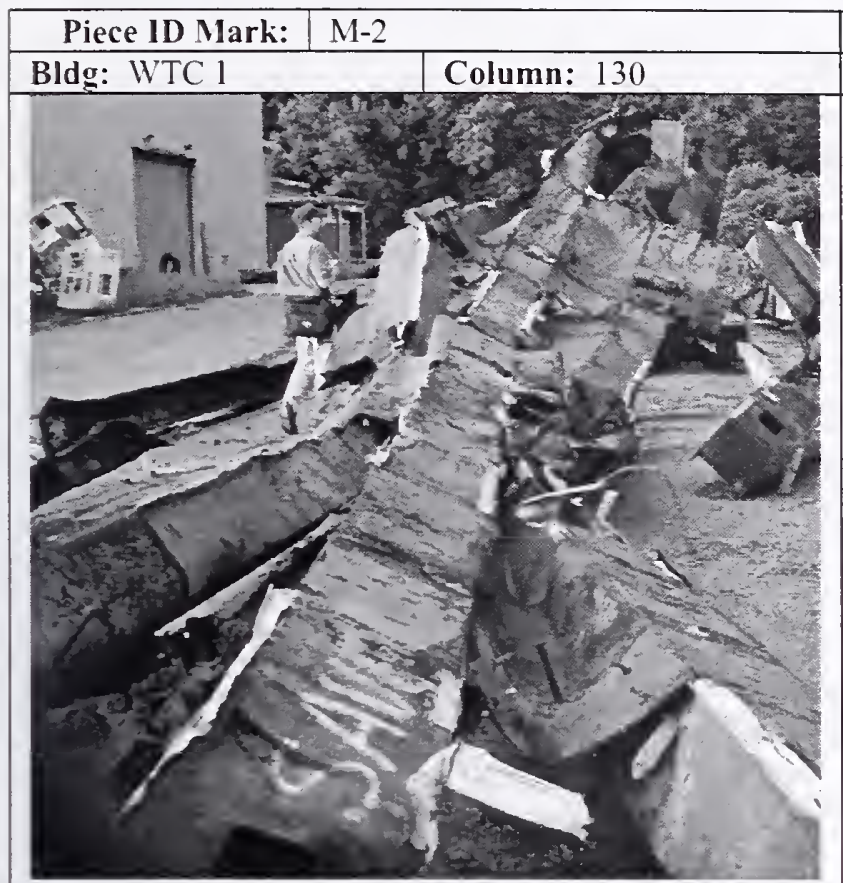

Figure F-A-4a: General view of Piece M-2; far end is bottom of panel

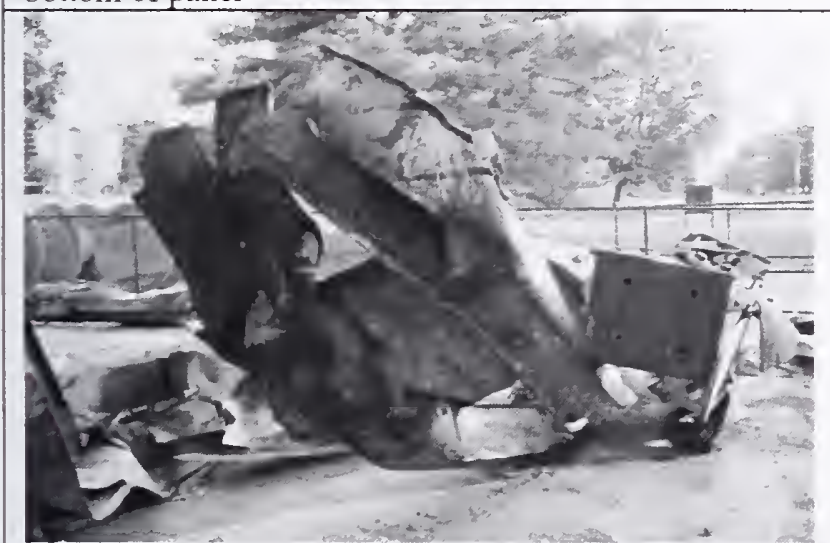

Figure $\mathrm{F}-\mathrm{A}-4 \mathrm{~b}$ : View of bottom ends of Columns 1, 2, and 3 (l to r)

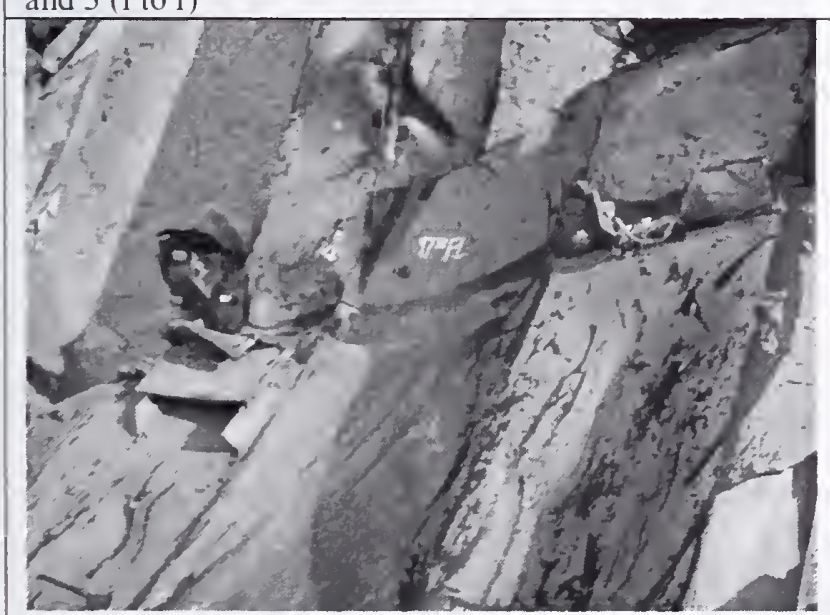

Figure F-A-4c: View of 97th floor spandrel panel. This photo shows inward bend around edge of floor slab at 97th level. \begin{tabular}{|l|l|l|}
\hline By \& Date: & $\mathrm{CP} / \mathrm{RT} / \mathrm{JH} / 24$ June 2003 \\
\hline Floors: 96 to 99 & Impact affected \\
\hline
\end{tabular} zone of WTC 1 . This piece exhibits essentially no indication of any effects of fire, as evidenced by substantially intact primer paint throughout the piece. The lowest half-story of Columns 1 and 2, which in this particular panel were located just above the column splice located at mid-height of the 96th story, were directly affected by the aircraft impact. (Columns are identified in accordance with the original structural design convention, which is from Column 1 at left through Column 3 at right, as viewed inside looking out.) These two columns exhibit a deformed shape consistent with being bent inward by the aircraft impact (Figure A-9). The column splice end plates were torn off at the welds probably by the aircraft impact, whereas the base plate for Column 3 remains attached (Figure A-10). The edge of the slab at the 97th floor probably acted as a fulcrum point for the inward bending, as evidenced by the deflected shape of the piece and by severe local distortions along the evident contact location between the slab edge and the 97 th floor spandrel plate.

Column 3 is in relatively better condition as compared to Columns 1 and 2 . Column 3 was evidently first bent inward by the aircraft impact, but then bent in the opposite direction by subsequent events (possible subsequent events are the collapse event and postcollapse handling and moving).

The following items of interest were marked onto the specimen:

Z1: In the spandrel panel, particularly at Column 1, there are inward local buckles consistent with membrane compression forces in the spandrel plate related to inward bending of the columns due to impact. The steel is cracked at some locations. It is probable that the steel in these areas was subject to high rates of compression strain related to the aircraft impact.

Z2: On Columns 1 and 2, opposite from $Z 1$ zones, are zones of evident tension yielding. It is probable that the steel in these areas was subject to high rates of tension strain related to the aircraft impact.

Z3: Local compression buckling is also evident in the column interior web plate at locations just above the spandrel plate in Column 1. Probable cause is the aircraft impact event. Similar, more pronounced local buckle in Column 2 probably initiated by aircraft impact, but amplified by collapse and subsequent events.

(comments contimue next page) 


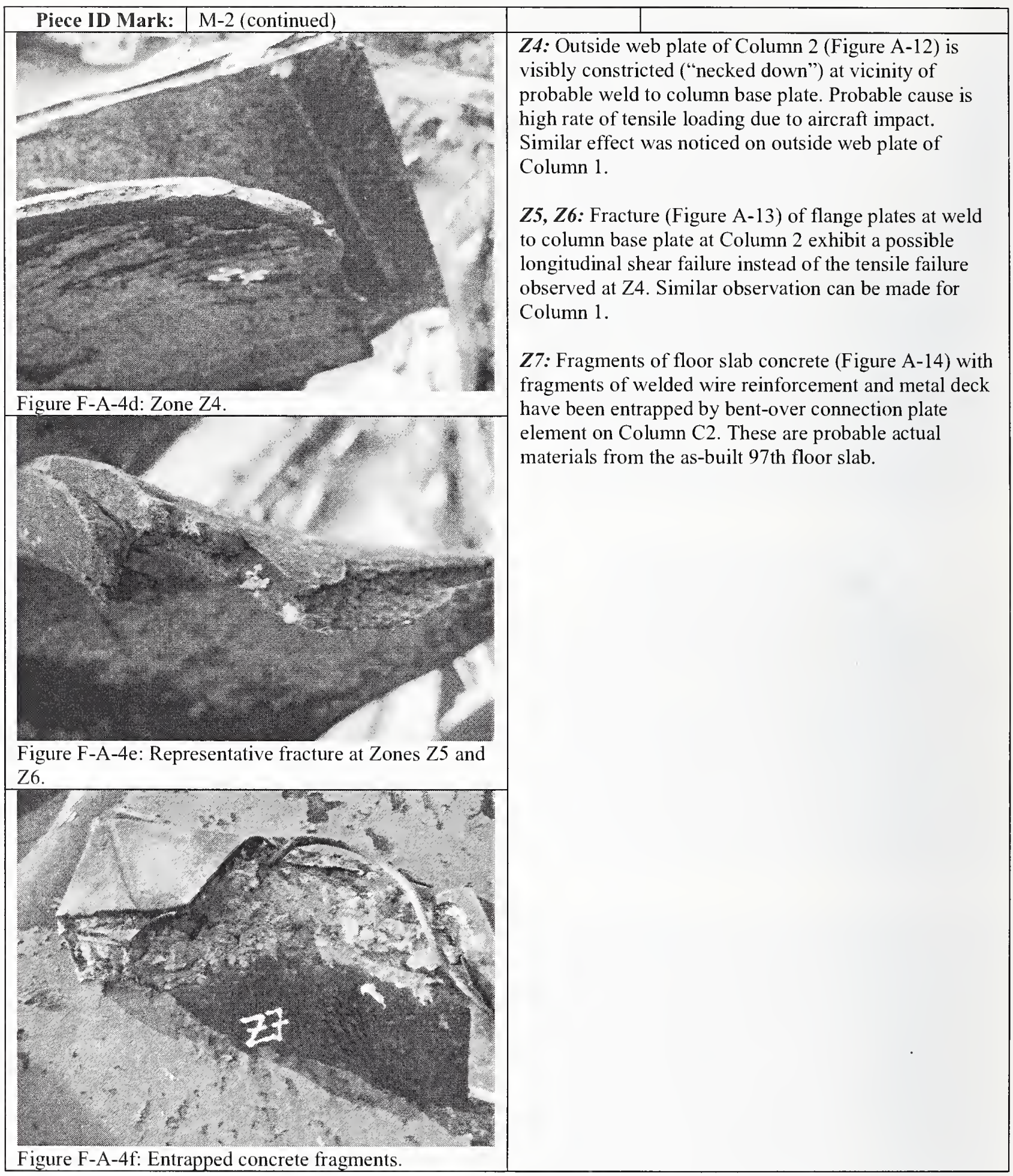

\begin{tabular}{|c|c|c|}
\hline Piece ID Mark: & M-26 & CP / 24 June 2003 \\
\hline Bldg: WTC 1 & Column: 130 & \begin{tabular}{|l|l|} 
Floors: $90-93$ & \\
\end{tabular} \\
\hline (no photo) & & $\begin{array}{l}\text { Exterior column panel. Because of location in building, } \\
\text { observed damage is not related to aircraft impact. } \\
\text { Probable cause of observed damage and distortion is } \\
\text { collapse event and subsequent handling. }\end{array}$ \\
\hline
\end{tabular}




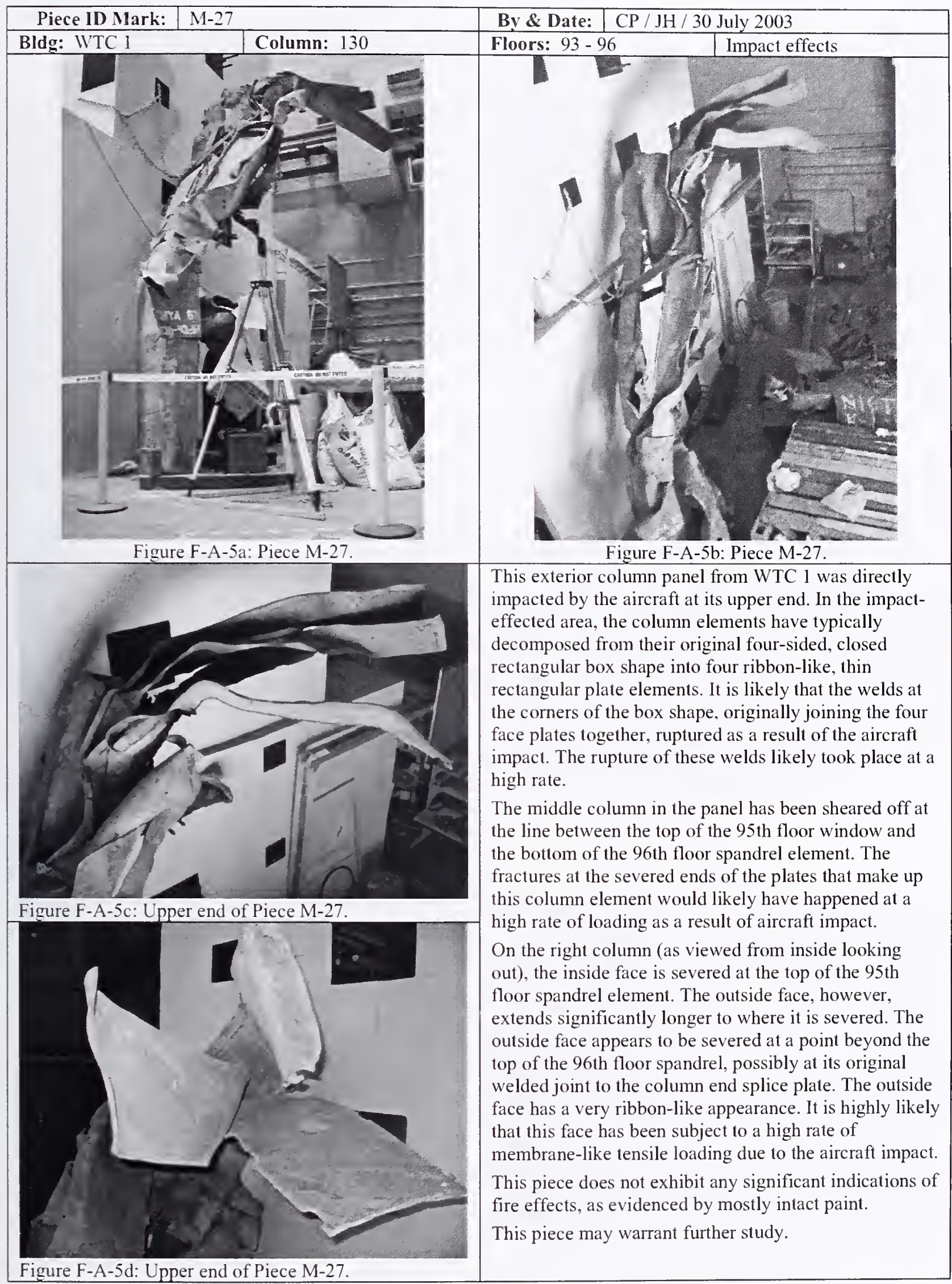




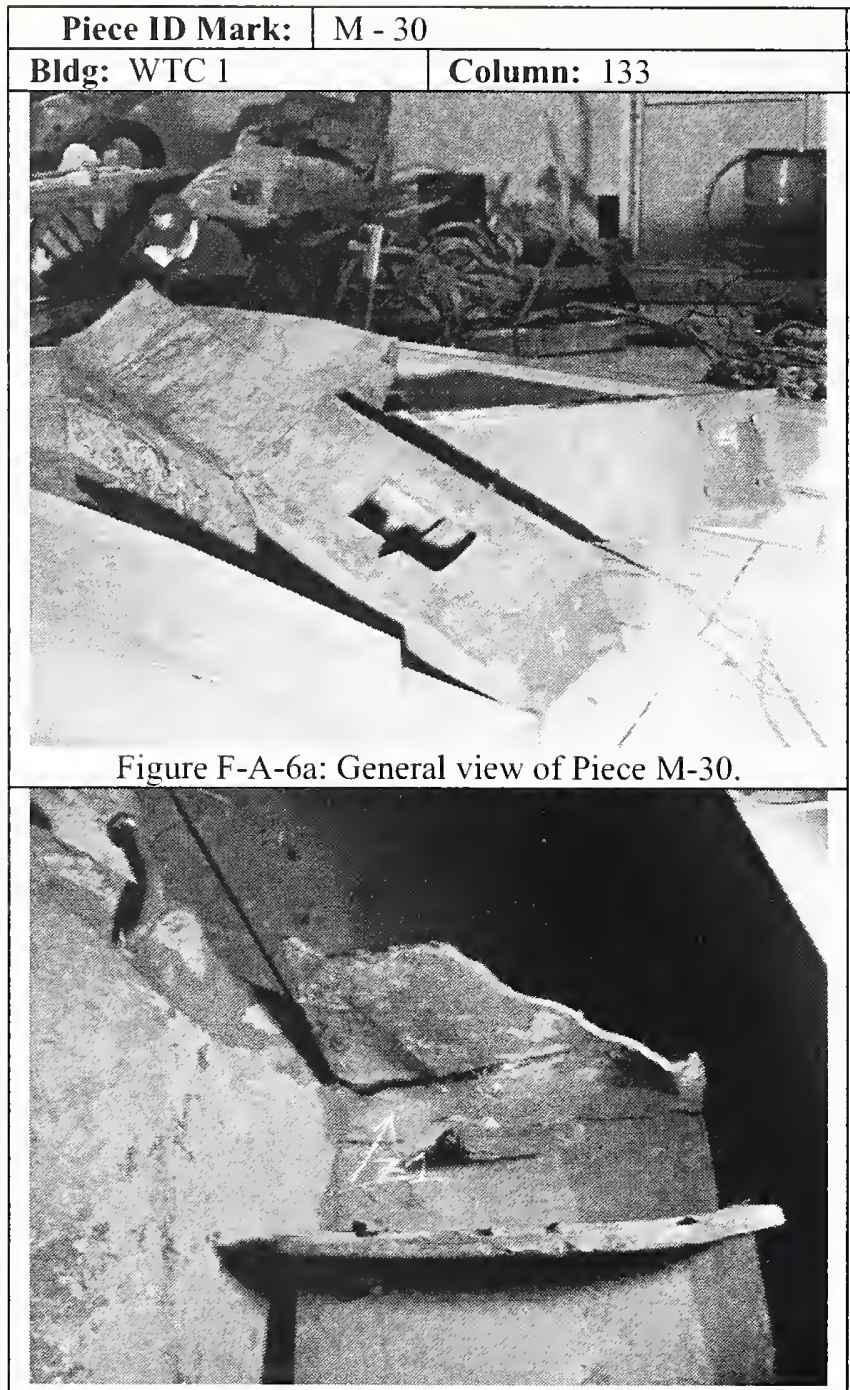

Figure F-A-6b: Close view of severed Column 133 near the top of the 95th floor spandrel. Shown here the inside face of the column, along with the 95th floor joist seat on spandrel.

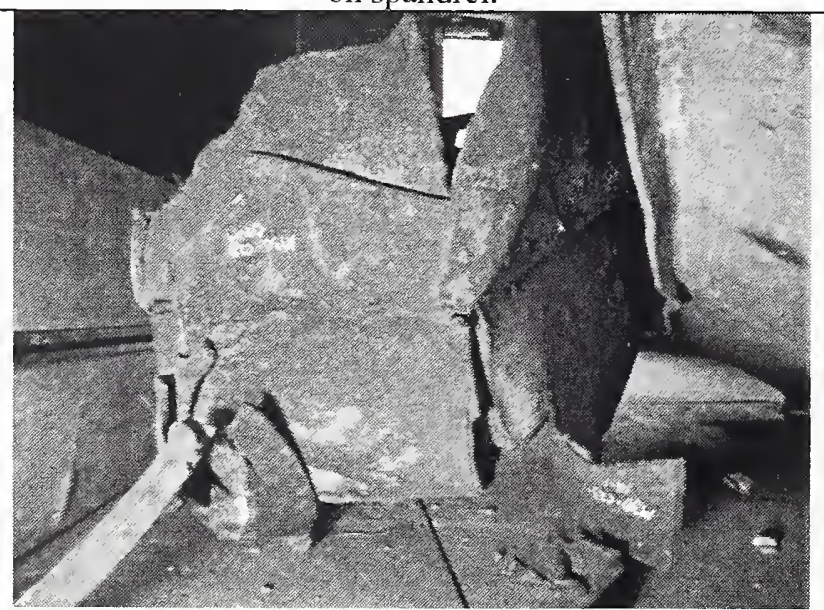

Figure F-A-6c: Close view of severed Column 133, viewed as if looking down from above. Inside face of column is at top of photo.
Column 133 was severed at approximately the 95th floor line, shown in Figures A-20 and A-21. The fractures of the column side faces occur along a line at the edge of an internal column diaphragm stiffener at this location. The side faces probably fractured in longitudinal shear along the edge of the internal stiffener. Remnants of the inside (marked $\mathrm{Zl}$ in Figure A-20) and outside faces of the column extend above the stiffener diaphragm and are bent inward along edges of the stiffener diaphragm. The inside and outside faces probably fractured in tension. It is almost certain that these fractures were caused by the impact of the aircraft and therefore occurred at a high rate of loading. 


\begin{tabular}{|c|c|}
\hline Piece ID Mark: $\mathrm{M}-30$ (continued) & \begin{tabular}{l|l} 
By \& Date: & $\mathrm{CP} / \mathrm{JH} / 31$ July 2003 \\
\end{tabular} \\
\hline 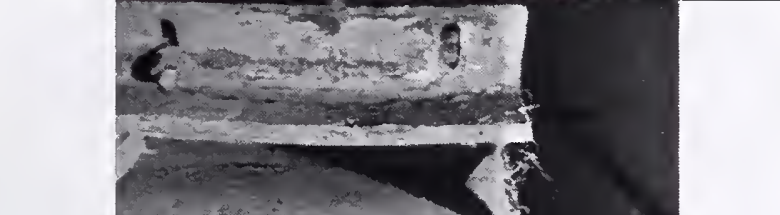 & $\begin{array}{l}\text { The 95th floor spandrel plate has probably fractured in } \\
\text { transverse shear along the right edge of Column } 133 \text {. } \\
\text { (This edge is marked Z2 in Figure A-22.) It is very } \\
\text { likely that this fracture was caused by the impact of the } \\
\text { aircraft and therefore occurred at a high rate of loading. }\end{array}$ \\
\hline $\begin{array}{l}\text { Figure F-A-6d: View of severed } 95 \text { th floor spandrel } \\
\text { plate at edge of Column 133. Column } 134 \text { would have } \\
\text { been to the right. }\end{array}$ & \\
\hline & $\begin{array}{l}\text { Column } 132 \text { was also severed at approximately the } 95 \text { th } \\
\text { floor line, shown Figures A-23 and A-24. The severed } \\
\text { edge is marked Z3 in Figure A-23. As with Column } 133 \text {, } \\
\text { the fracture of this column occurred generally at the } \\
\text { location of an internal column diaphragm stiffener. It is } \\
\text { almost certain that these fractures were caused by the } \\
\text { impact of the aircraft and therefore occurred at a high } \\
\text { rate of loading. }\end{array}$ \\
\hline $\begin{array}{l}\text { Figure F-A-6e: Close view of Column 132, showing } \\
\text { sever at the top edge of the } 95 \text { th floor spandrel. View } \\
\text { here is of the inside face of the column and spandrel. }\end{array}$ & \\
\hline
\end{tabular}




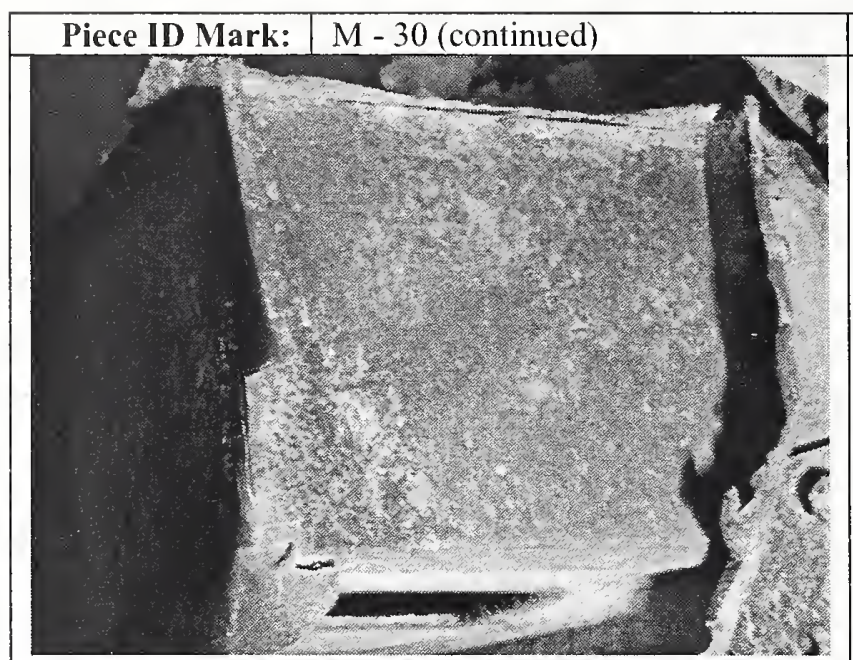

Figure F-A-6f: Close view of severed Column 132, viewed as if looking down from above. Inside face of column is at top of photo.

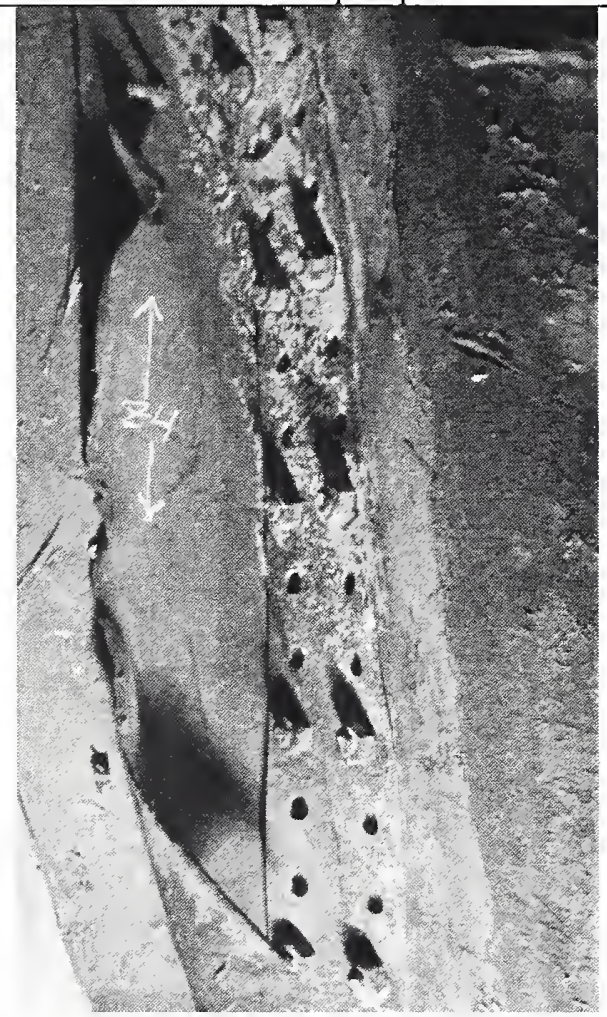

Figure F-A-6g: View of severed 95th floor spandrel plate to left of Column 132, showing spandrel connection bolts and connection plate. Column 131 would have been at left, and Column 132 is at right
By \& Date: $\mathrm{CP} / \mathrm{JH} / 31$ July 2003

The 95th floor spandrel splice at the left of Column 132 was recovered with this piece. The recovered connection materials include bolts and connection plate. A fragment of the spandrel plate that extended from the splice to Column 131 was also recovered with this piece. The opposite edge of this additional piece, marked Z4 in Figure A-25, has a fracture surface that is inclined at 45 degrees. It is not certain that this fracture was caused by impact of the aircraft, because photographic evidence (FEMA 403, Figure 2-15) suggests that this segment of the 95th floor spandrel, although bent inward, may still be intact. Column 131 and the associated spandrel panel at the 95th floor were recovered as part of Piece M-27. There is a chance that the fracture surface that would mate to the fractured edge marked Z4 may exist on piece M-27. 


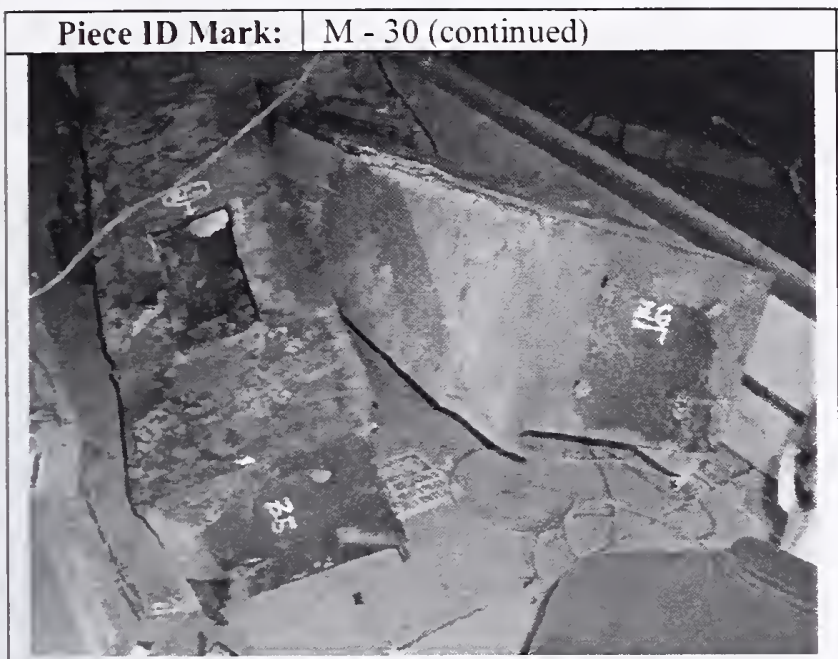

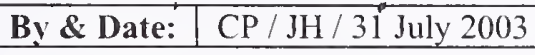

The bolt holes on the base plates for Columns 132 and 133, marked Z5 and Z6, respectively, show in Figures A-26, A-27 and A-28, were examined for hole elongation that might be related to the failure of the column splice bolts.

On the base plate of Column 132, the two bolt holes nearest the outside face of the column exhibited elongation in a direction perpendicular to the outside face. The two bolt holes closest to the interior face exhibited insignificant elongation.

On the base plate of Column 133, the direction of elongation of the bolt holes is consistent with the column twisting as the bolts were failing.

Figure F-A-6h: View of column end plates on Piece M30. Base of Column 132 is marked Z5 and base of Column 133 is marked Z6.

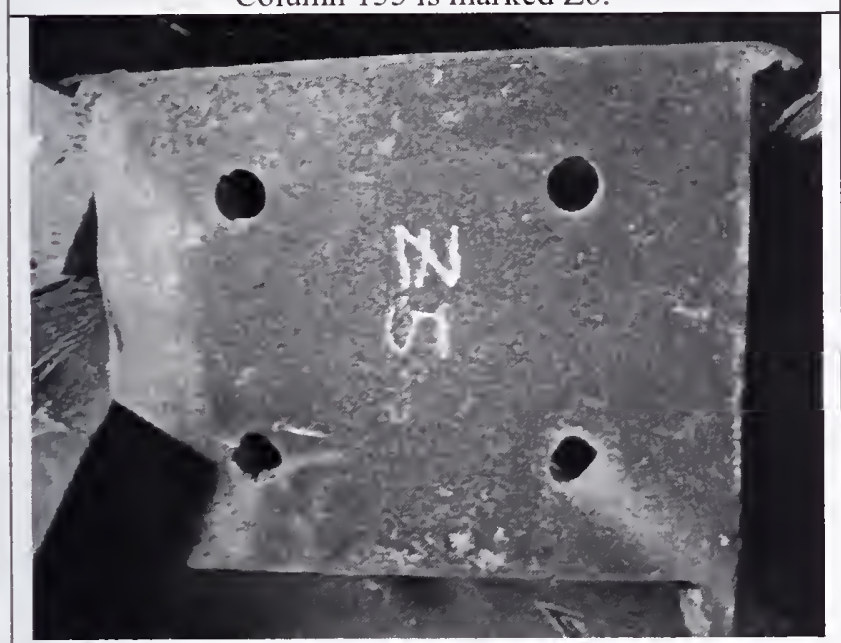

Figure F-A-6i: Base of Column 132. Outside face is down.

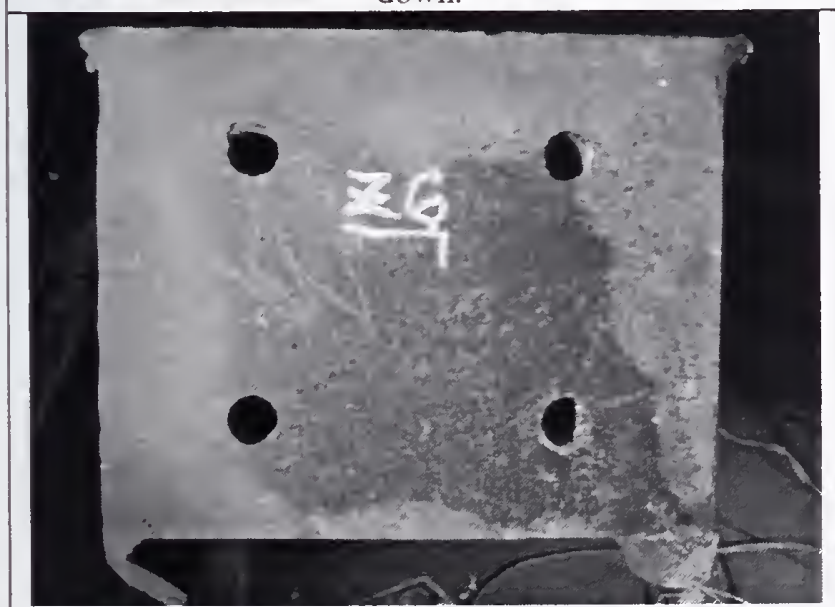

Figure F-A-6j: Base of Column 133. Outside face is down. 


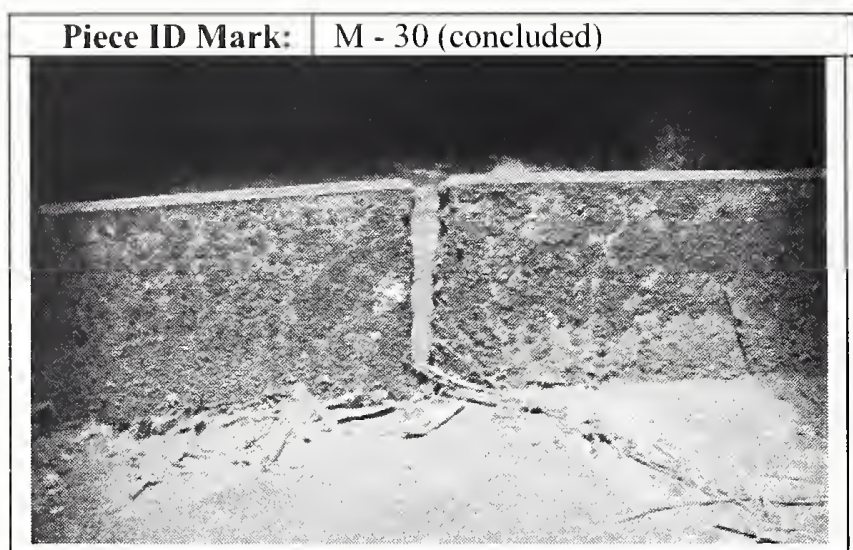

Figure F-A-6k: Separation of rectangular bar found inside access hole at base of Column 132. The inside surface of the outside column face plate is at the bottom of the photo.
By \& Date: CP / JH / 31 July 2003

A unique separation, Figure A-29, was observed in a small rectangular bar found inside the rectangular access hole at the base of Column 132. The location was marked $\mathrm{Z7}$ on the inside face of the column piece. The rectangular bar is believed to be an aid for fabrication used to support the column outside face plate at the proper position for welding. The bar appears to have ruptured, perhaps in tension, or has been flame cut.. It is not know if this condition is related to the impact of the aircraft into this piece, or is related to original fabrication.

\begin{tabular}{|c|c|c|}
\hline Piece ID Mark: & $\mathrm{N}-7$ & \begin{tabular}{l|l} 
By \& Date: & CP / 24 June 2003 \\
\end{tabular} \\
\hline Bldg: WTC 1 & Column: 127 & \begin{tabular}{l|l} 
Floors: $97-100$ & \\
\end{tabular} \\
\hline (no photo) & & $\begin{array}{l}\text { A generally complete exterior column panel from just } \\
\text { above the aircraft impact zone on WTC } 1 \text {. Column box } \\
\text { shapes generally intact. Few observed tears at welds. } \\
\text { Probable cause of the observed damage and distortion is } \\
\text { the collapse event and subsequent handling. }\end{array}$ \\
\hline
\end{tabular}




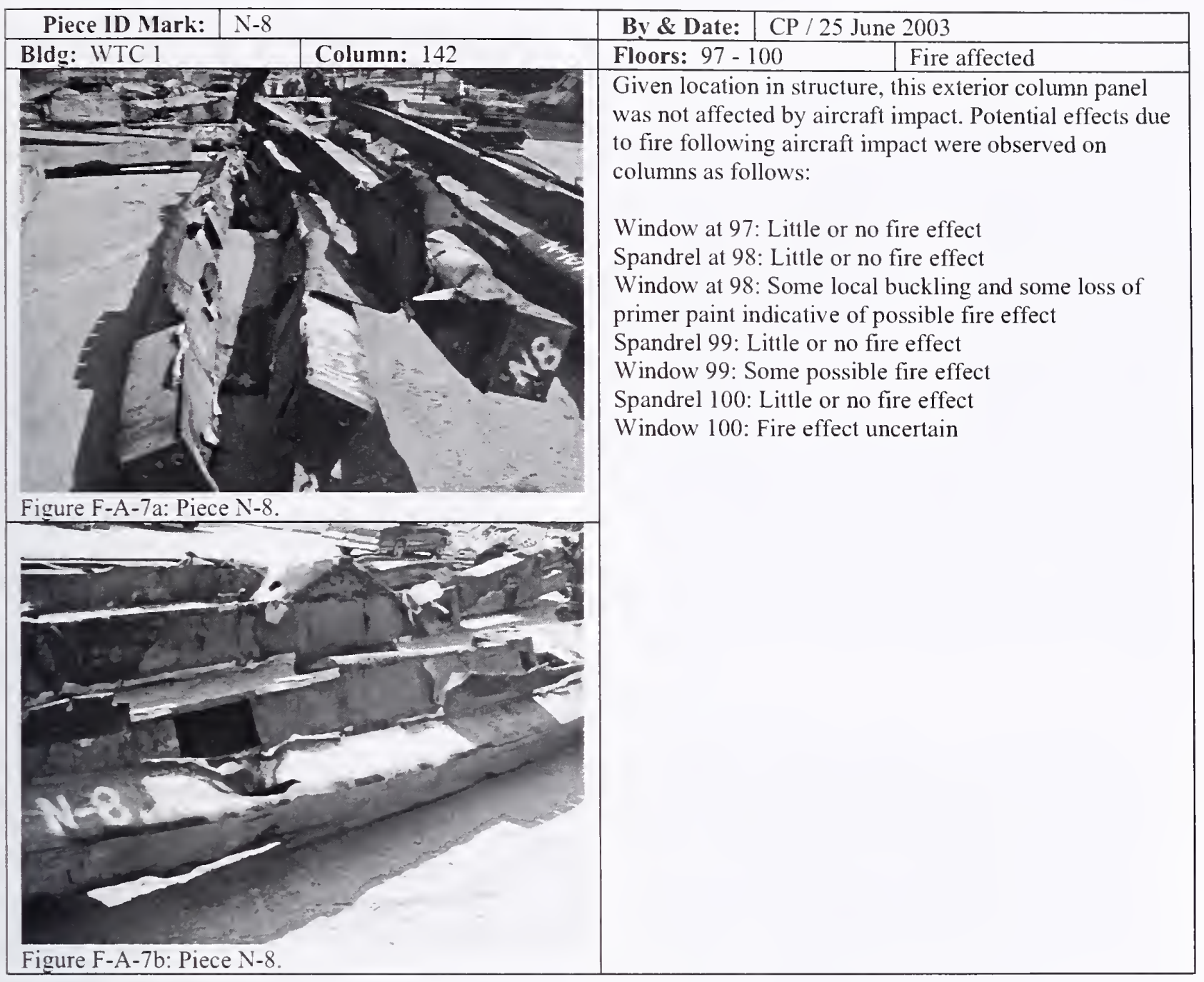

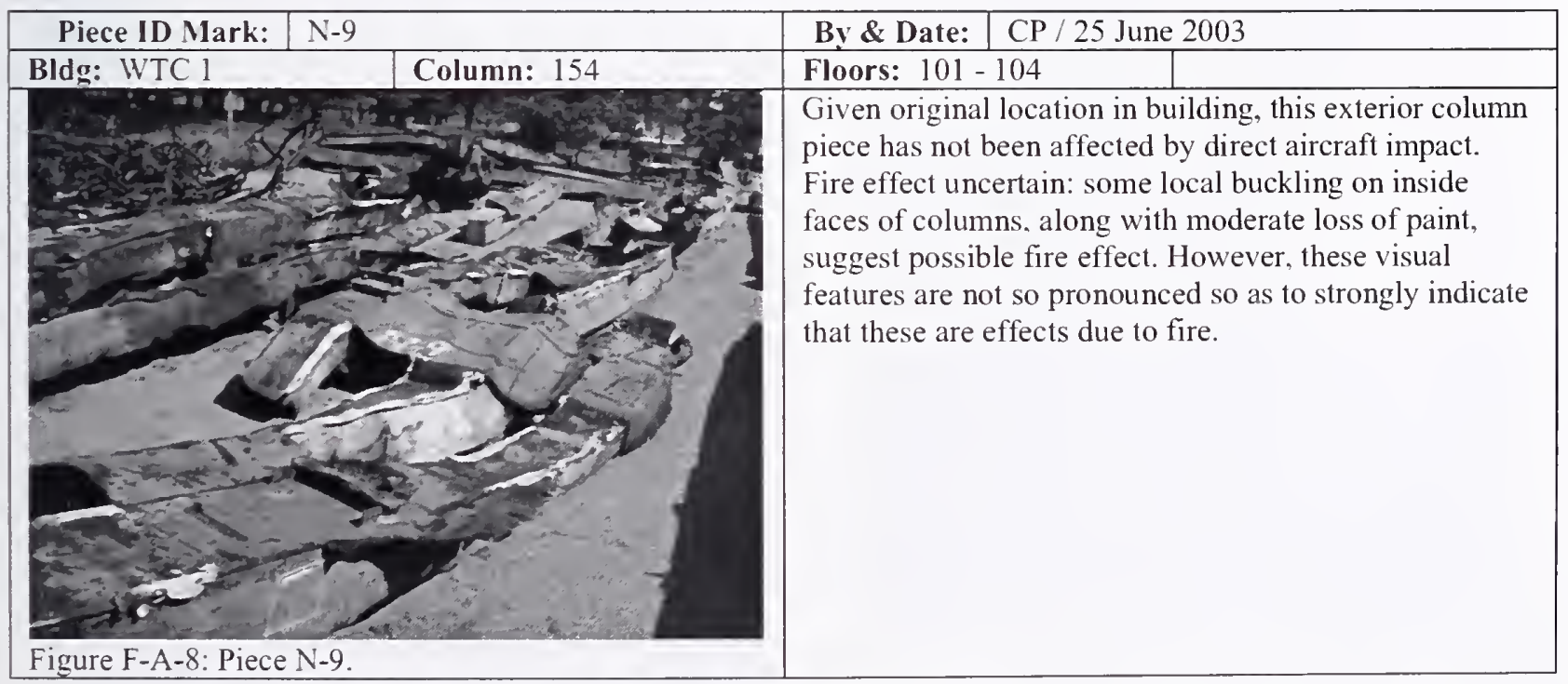




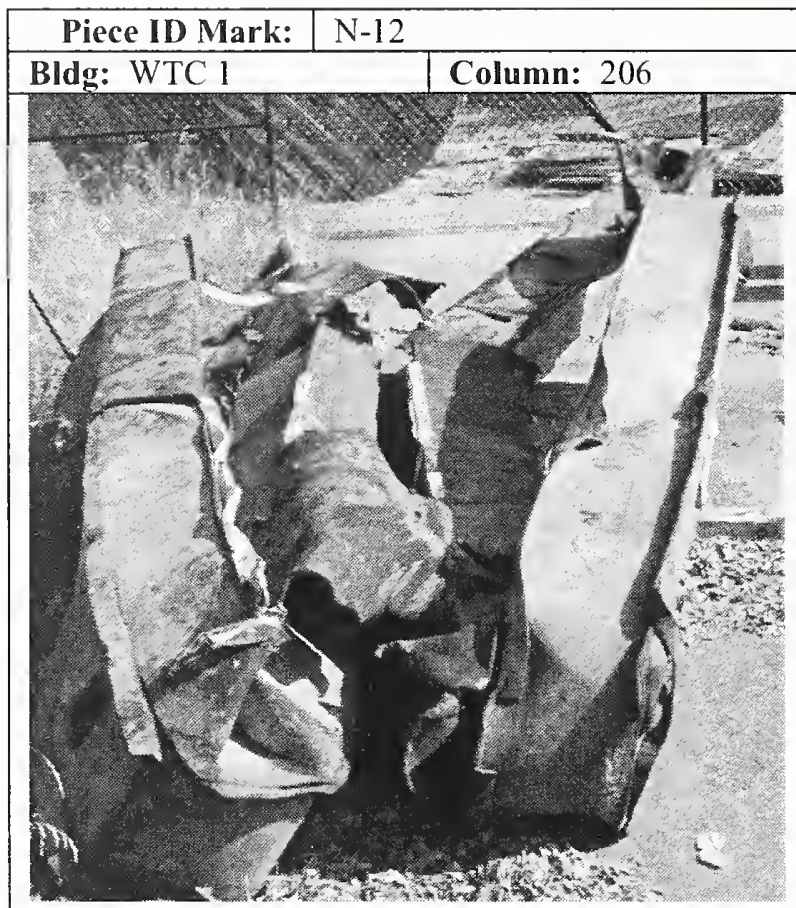

Figure F-A-9a: General view of Piece N-12, showing fold in columns.

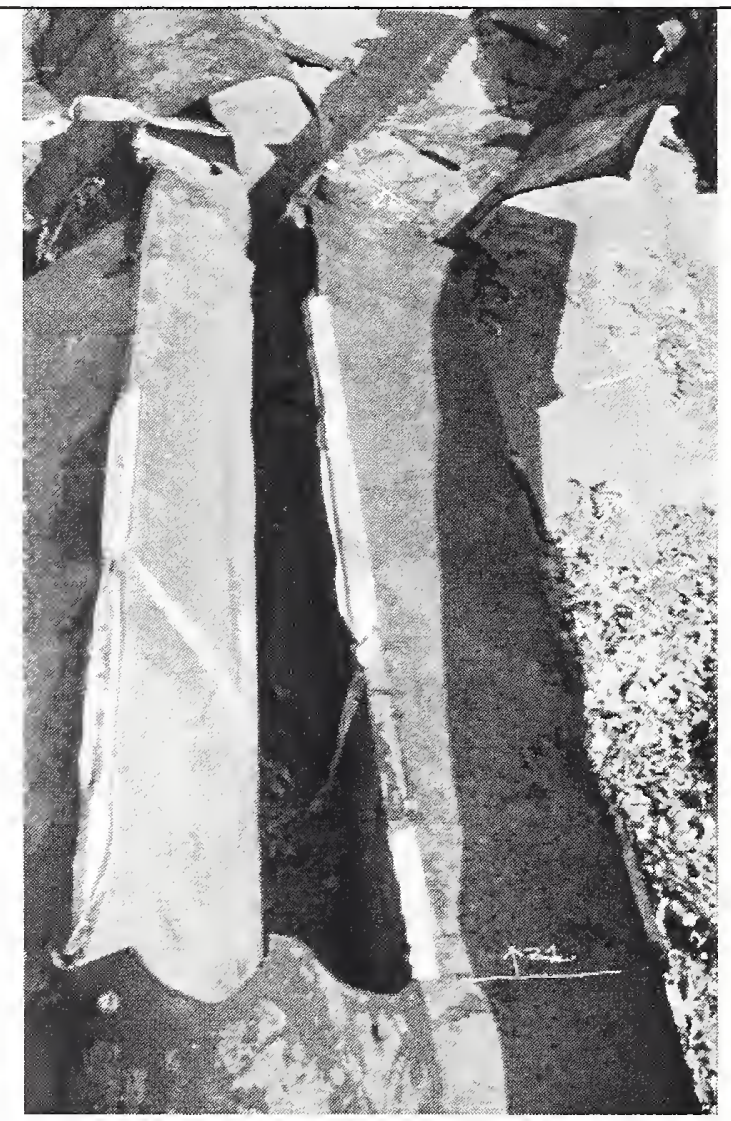

Figure F-A-9c: Column elements at 94th floor window opening. Top edge of 94 th floor spandrel is at bottom of photo.
By \& Date: $\quad$ CP / 30 July 2003

Floors: $92-95 \quad$ Fire effects

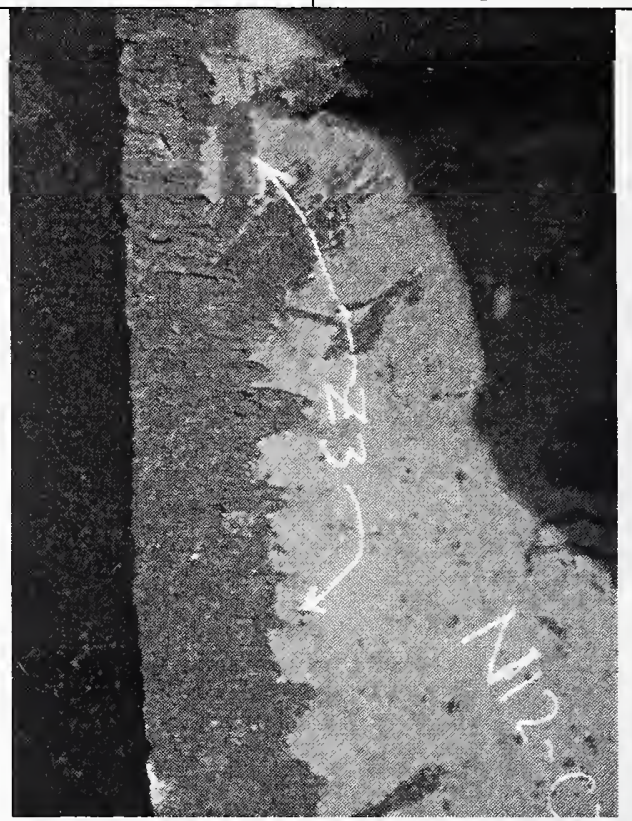

Figure F-A-9b: Facture at column edge in 95th floor spandrel plate.

While this piece is from the floors in WTC 1 that were impacted by the aircraft, this piece is from the building face to the left side (orientation of aircraft travel direction) of the impact face. It is unlikely that this piece has been directly affected by aircraft impact.

The paint is missing from approximately the upper twothirds of the inside faces of the columns adjacent to the 94th floor window openings. (The 94th floor window openings have been marked as Zone Z1.) This loss of paint is consistent with flames of an interior fire exiting through the window openings. Given proximity of this piece to the aircraft impact, it is likely that the observed loss of paint is due to fire following impact.

Near the top of the window openings, the columns are severely distorted (marked as Z2 on the piece), suggesting a local buckle or local failure in the column at this location. The corner welds that join the column face plates together have typically ruptured at this distorted region.

Similar patterns of loss of paint at window openings and probable local buckles in column elements at the top of window openings were also observed adjacent to the 93rd floor window openings. (The piece has become folded in half at the top of the 93rd floor window openings.) The 92nd floor, however, does not exhibit these patterns.

The 95th floor spandrel plate was fractured vertically along the edge of the middle column. Fracture is through the parent material but has likely initiated at the heat affected zone of the weld. Fracture probably occurred during collapse. 


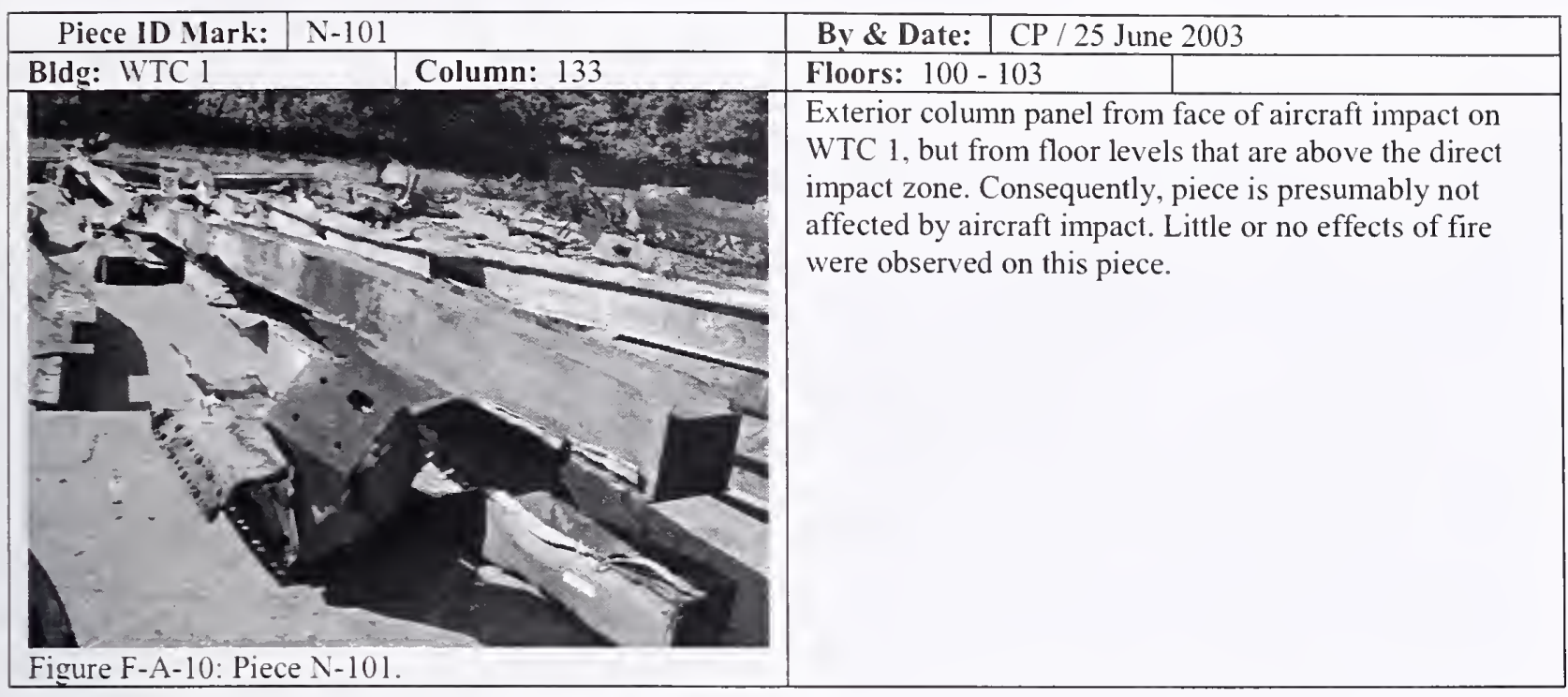

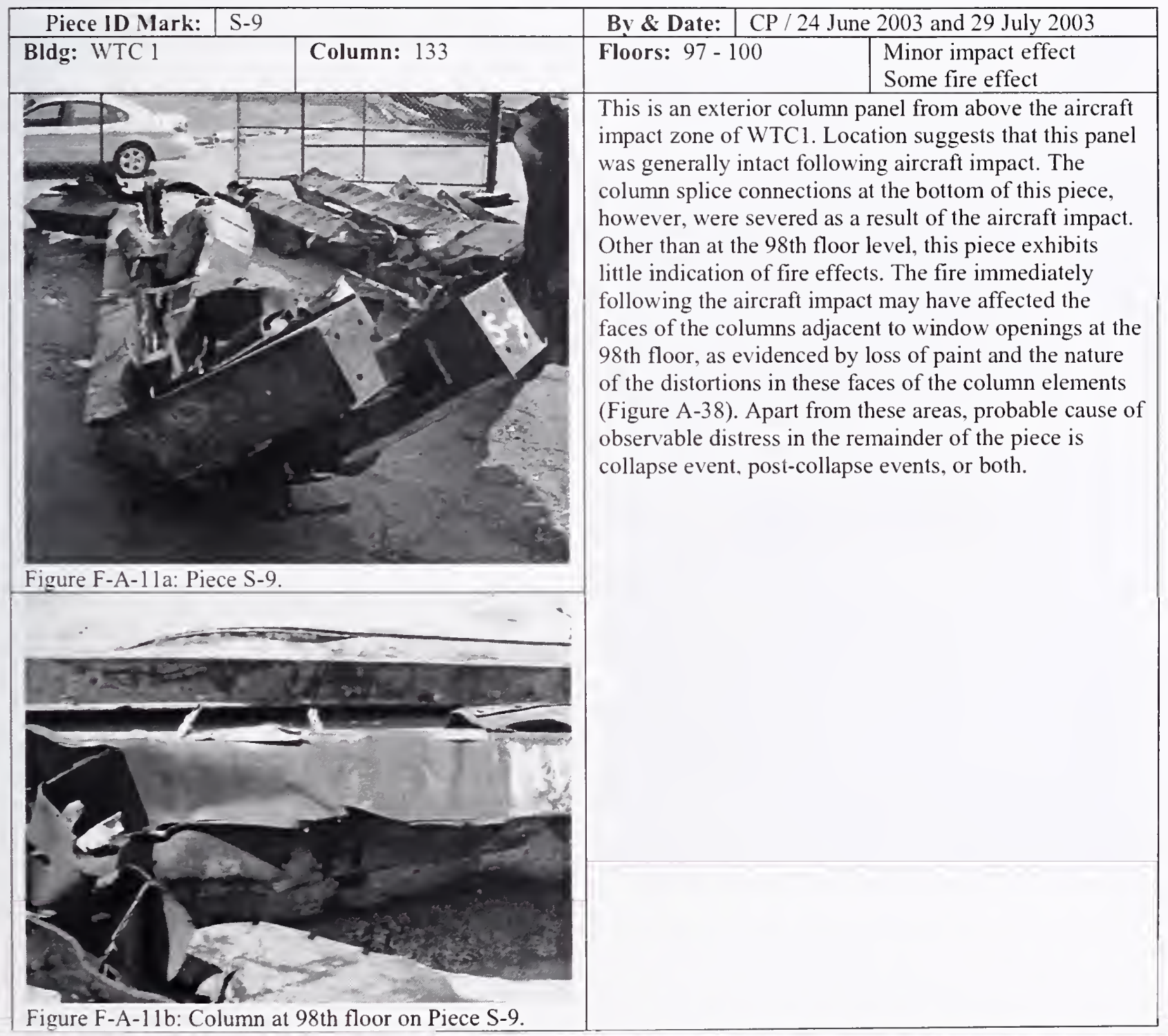




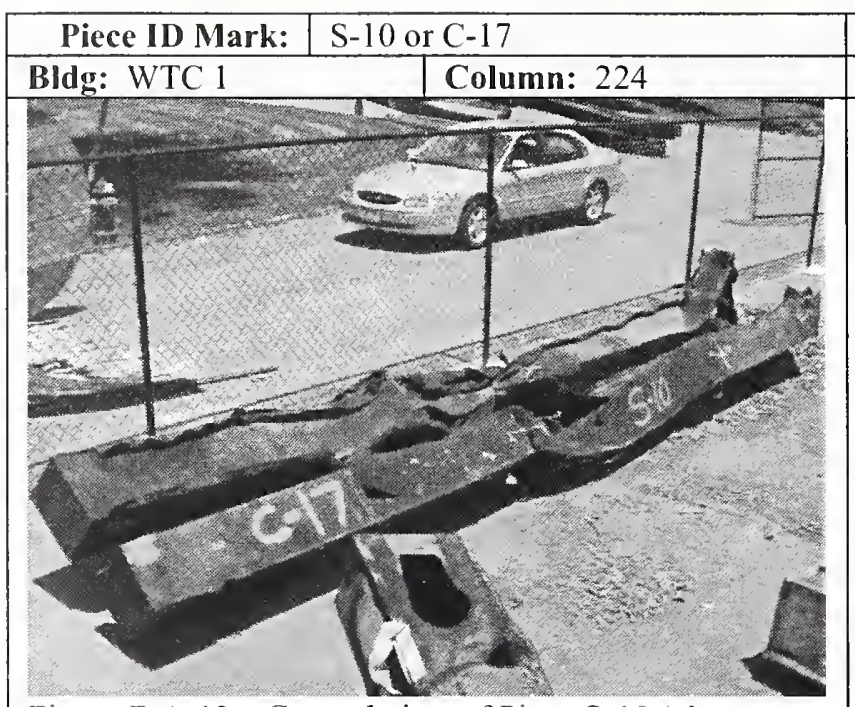

Figure F-A-12a: General view of Piece S-10 (also labeled C-17).

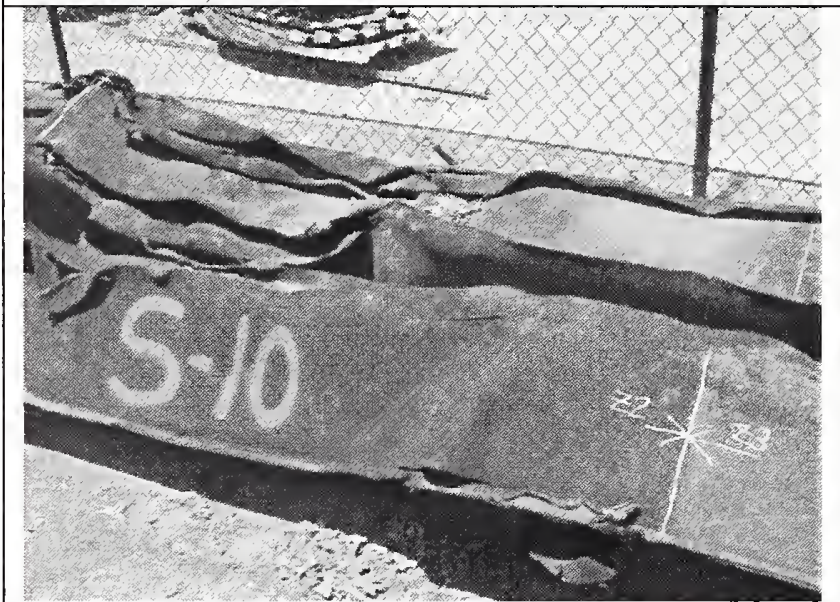

Figure F-A-12b: View of identified Zones Z2 and Z3.

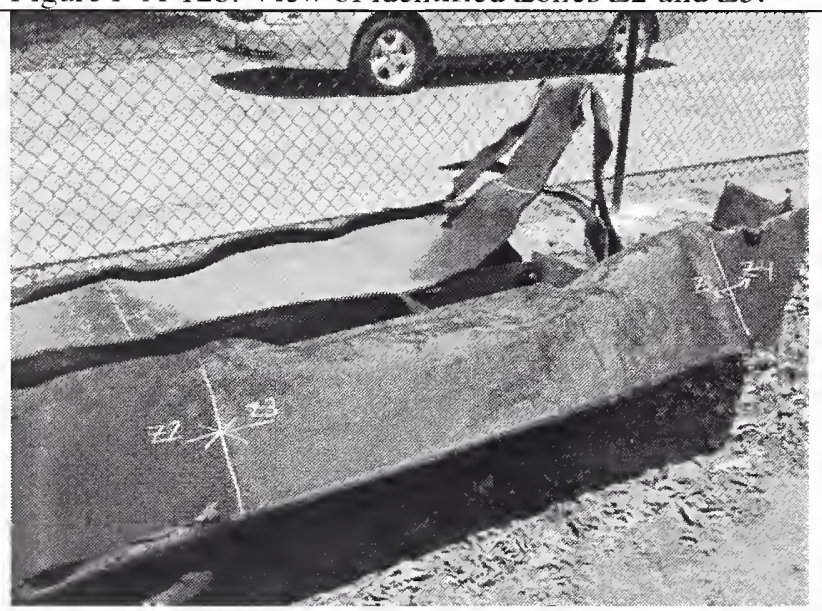

Figure F-A-12c: View of identified Zones Z2, Z3 and Z4.

\begin{tabular}{c|c|} 
By \& Date: & CP $6 / 24 / 2003$ \\
\hline Floors: $92-95$ & Fire effects
\end{tabular}

This is a partial exterior column panel, consisting of approximately the lowest two-thirds (from column splice at the window opening at the 92nd floor through part of the column elements at the window opening at the 94th floor) of the second and third column elements (left to right, inside looking out) and associated fragments of spandrel panels at the 93rd and 94th floor lines. While this column panel is from floors where the aircraft impacted the structure, this panel was not on the face of the building that was directly impacted by the aircraft.

The column panel exhibits distinct pattern of fire effects at windows openings. Zones of interest were identified on a side face of the middle column element as follows:

Z0: Window opening at 92 nd floor. Low to moderate paint remaining indicative of low degree of fire effects.

Z1: $\quad$ Spandrel at 93rd floor line. Loss of paint and moderate distortions indicative of some fire effect. Z2: Window at the 93rd floor. Lack of paint and presence of pronounced distortions are indicative of significant fire effect.

Z3: $\quad$ Spandrel at 94th floor line. Paint remains and distortions are relatively low, indicative of little fire effect.

Z4: Window at 94th floor. Lack of paint and presence of pronounced distortions are indicative of significant fire effect. The column fragments end within this zone. 


\section{ATTACHMENT B - OTHER PIECES FROM WTC 1 AND WTC 2}




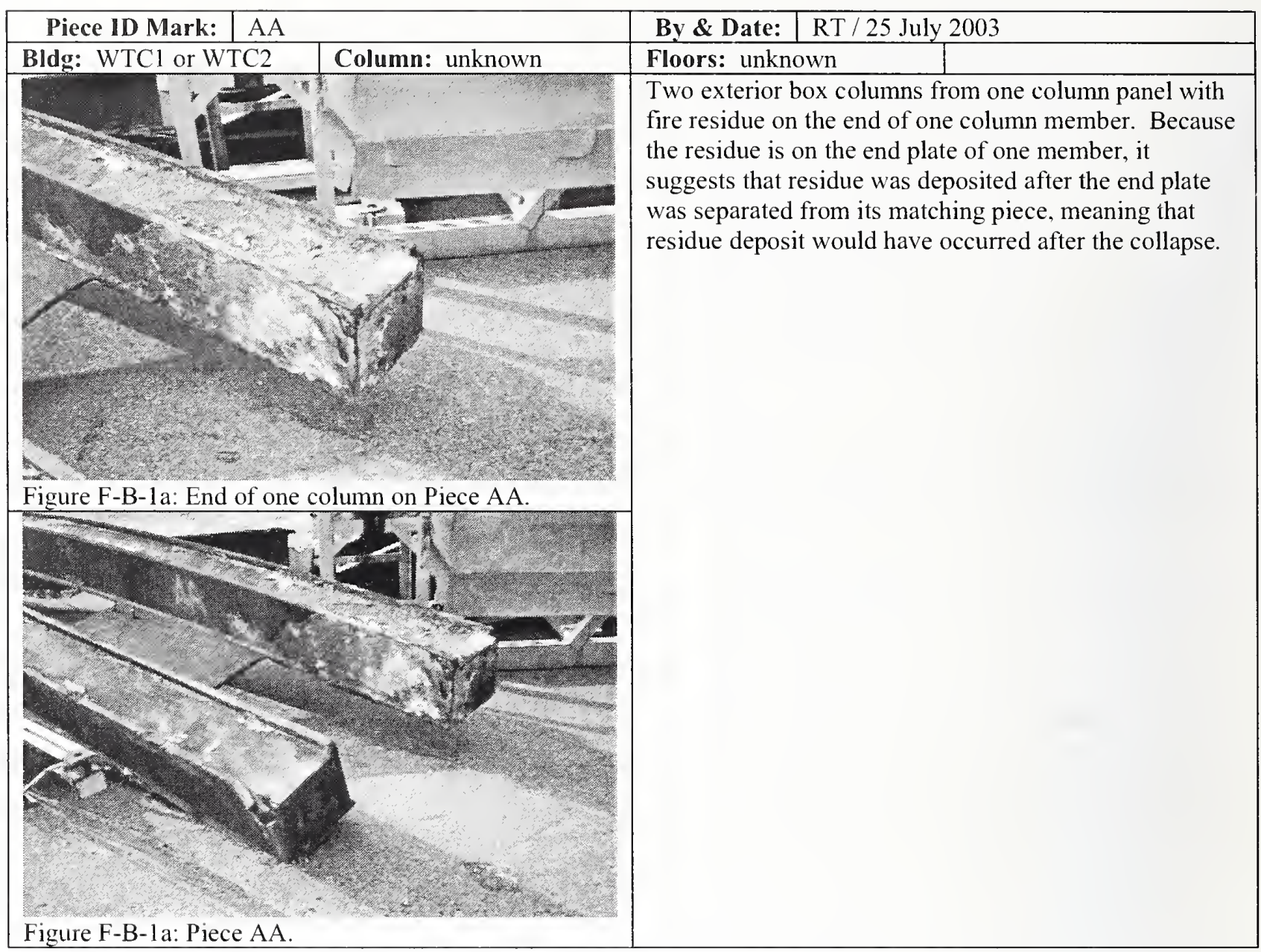




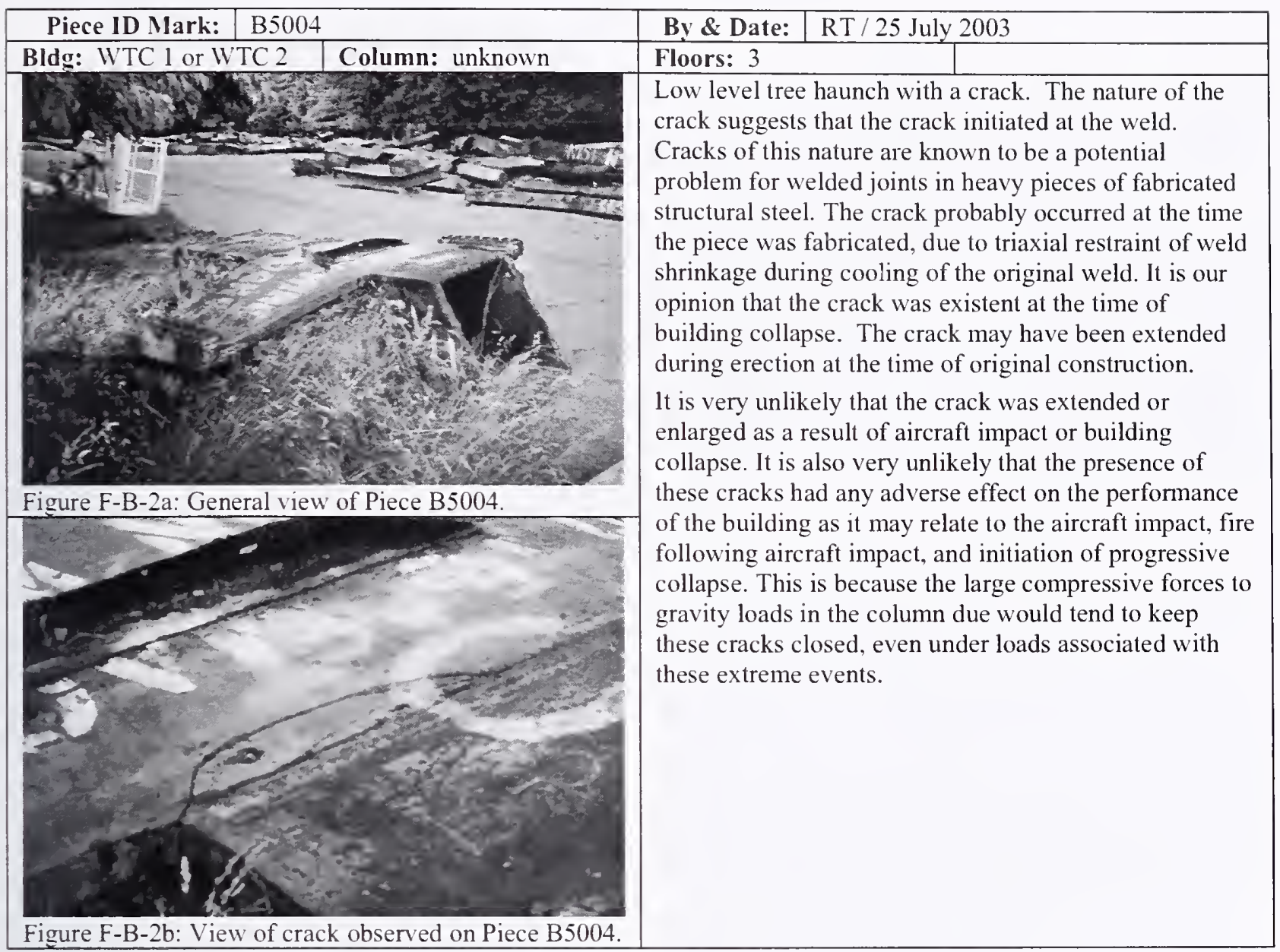




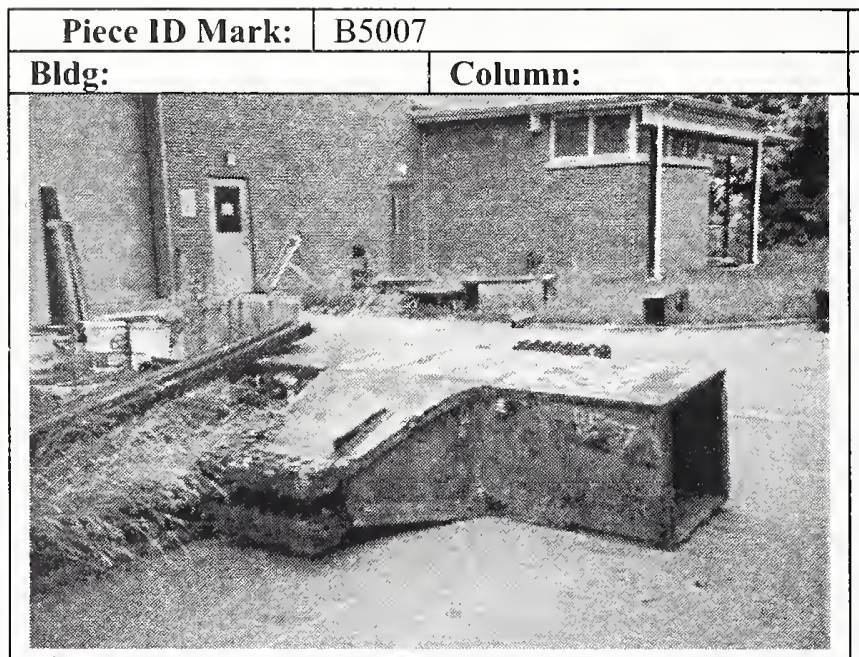

Figure F-B-3a: General view of Piece B5007.

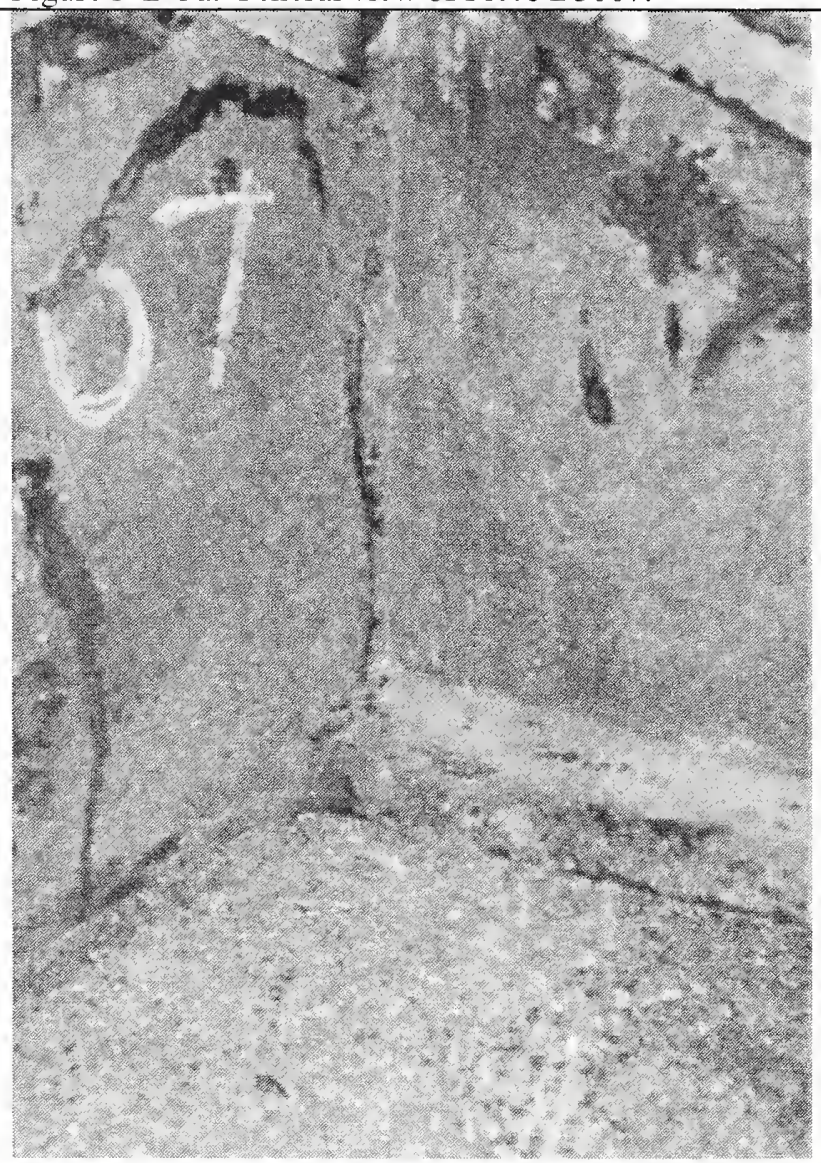

Figure F-B-3b: View of crack observed on piece.
By \& Date: 1 RT / 25 July 2003

Floors:

Low level tree haunch with a crack. The nature of the crack suggests that the crack initiated at the weld. Cracks of this nature are known to be a potential problem for welded joints in heavy pieces of fabricated structural steel. The crack probably occurred at the time the piece was fabricated, due to triaxial restraint of weld shrinkage during cooling of the original weld. It is our opinion that the crack was existent at the time of building collapse. The crack may have been extended during erection at the time of original construction.

It is very unlikely that the crack was extended or enlarged as a result of aircraft impact or building collapse. It is also very unlikely that the presence of these cracks had any adverse effect on the performance of the building as it may relate to the aircraft impact, fire following aircraft impact, and initiation of progressive collapse. This is because the large compressive forces to gravity loads in the column due would tend to keep these cracks closed, even under loads associated with these extreme events. 


\begin{tabular}{|l|l|l|l|}
\hline Piece ID Nark: & C-10 & By \& Date: & CP/25 June 2003 \\
\hline Bldg: WTC 1 & Column: 451 & $\begin{array}{l}\text { Floors: 85-88 } \\
\text { Given the location in the building, this piece was not } \\
\text { affected by aircraft impact. Visual indications of fire } \\
\text { effect were not readily observed. Observed damage and } \\
\text { distortions are probably due to collapse event and } \\
\text { subsequent handling. }\end{array}$ \\
\hline Figure F-B-4: Piece C-10. &
\end{tabular}

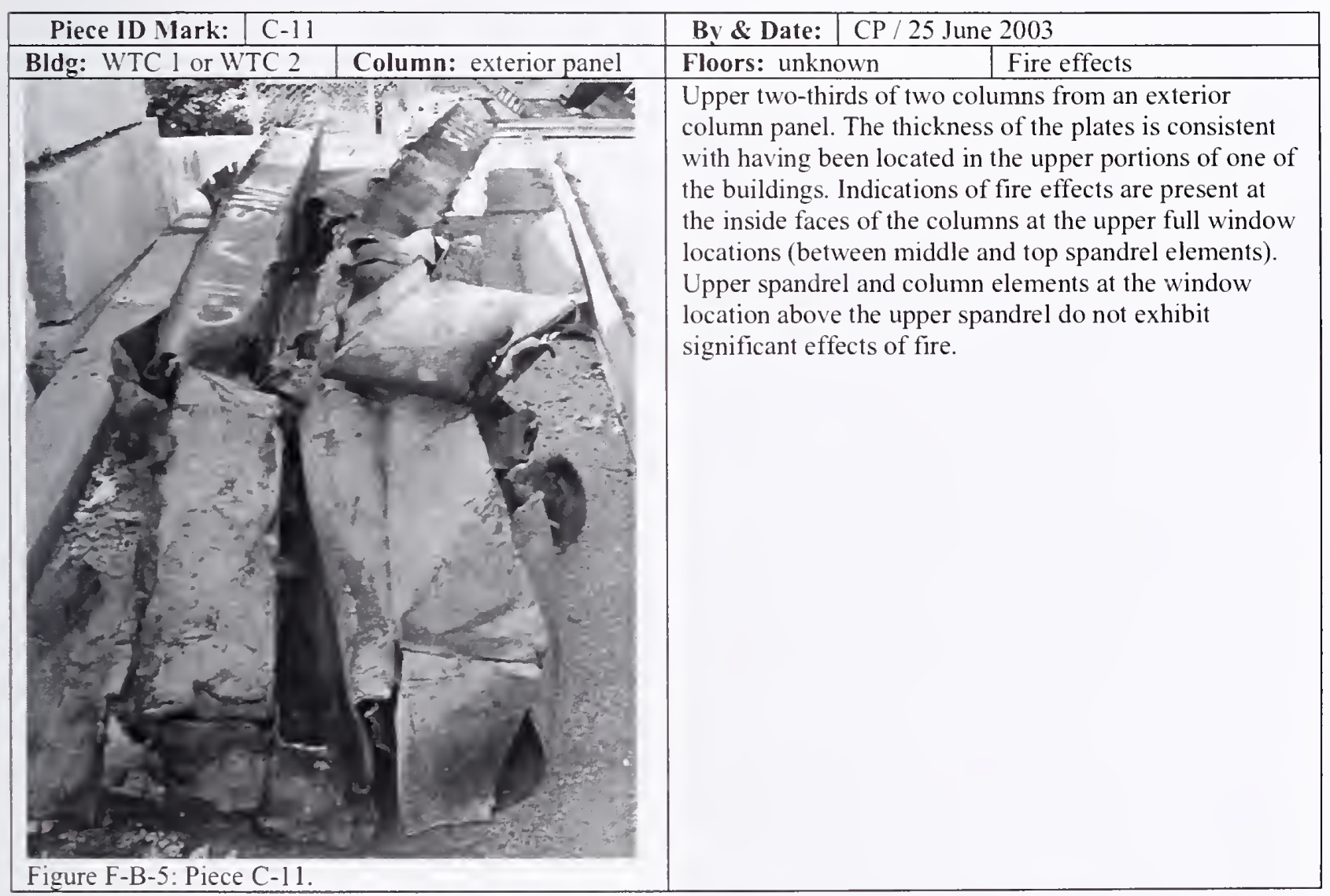




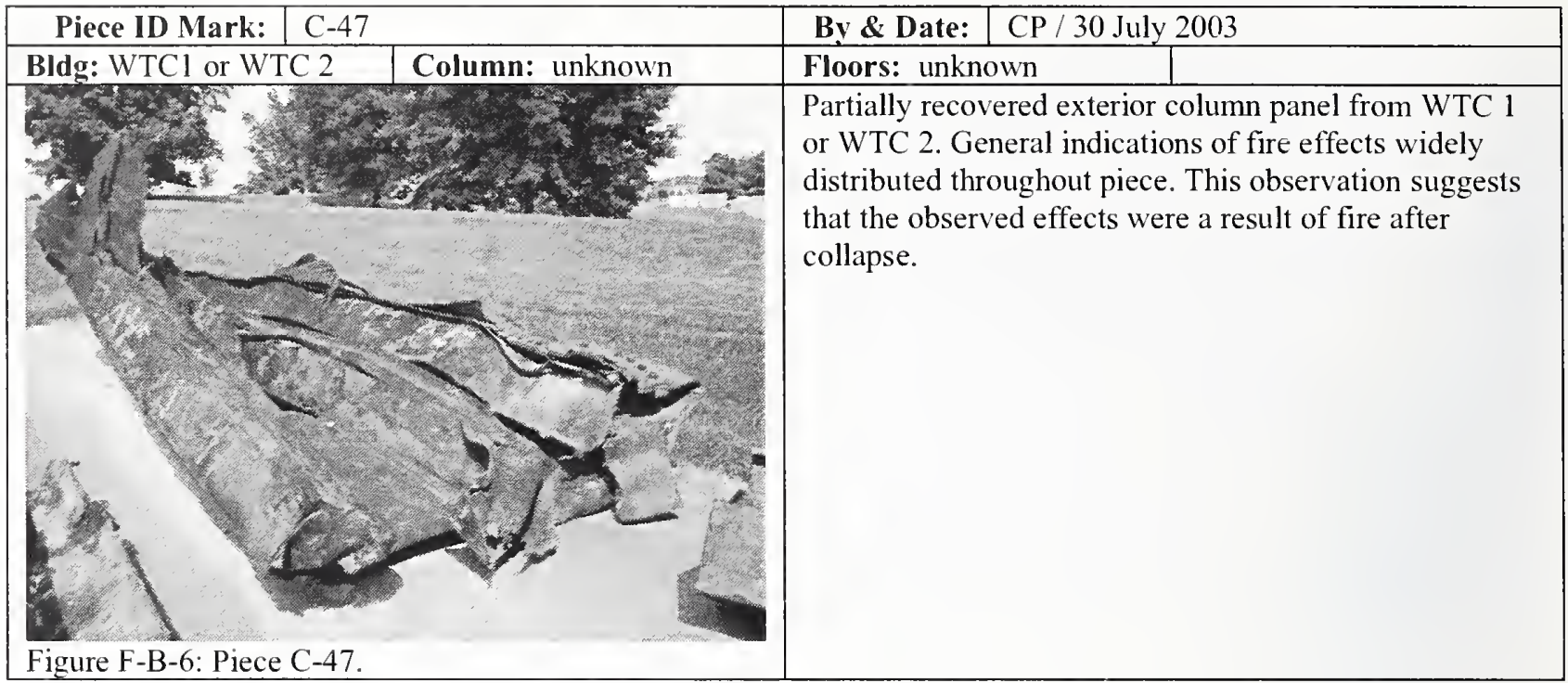

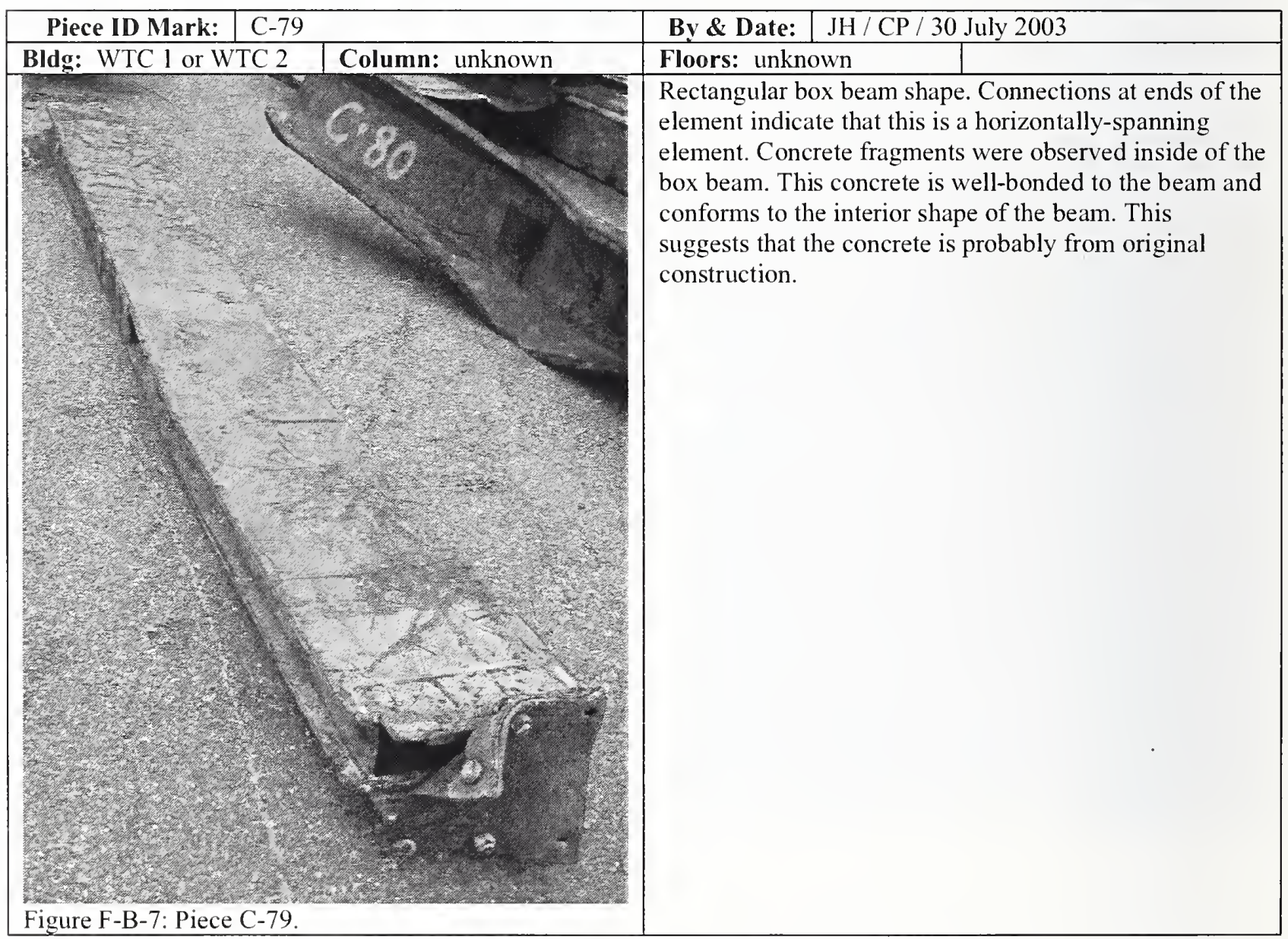




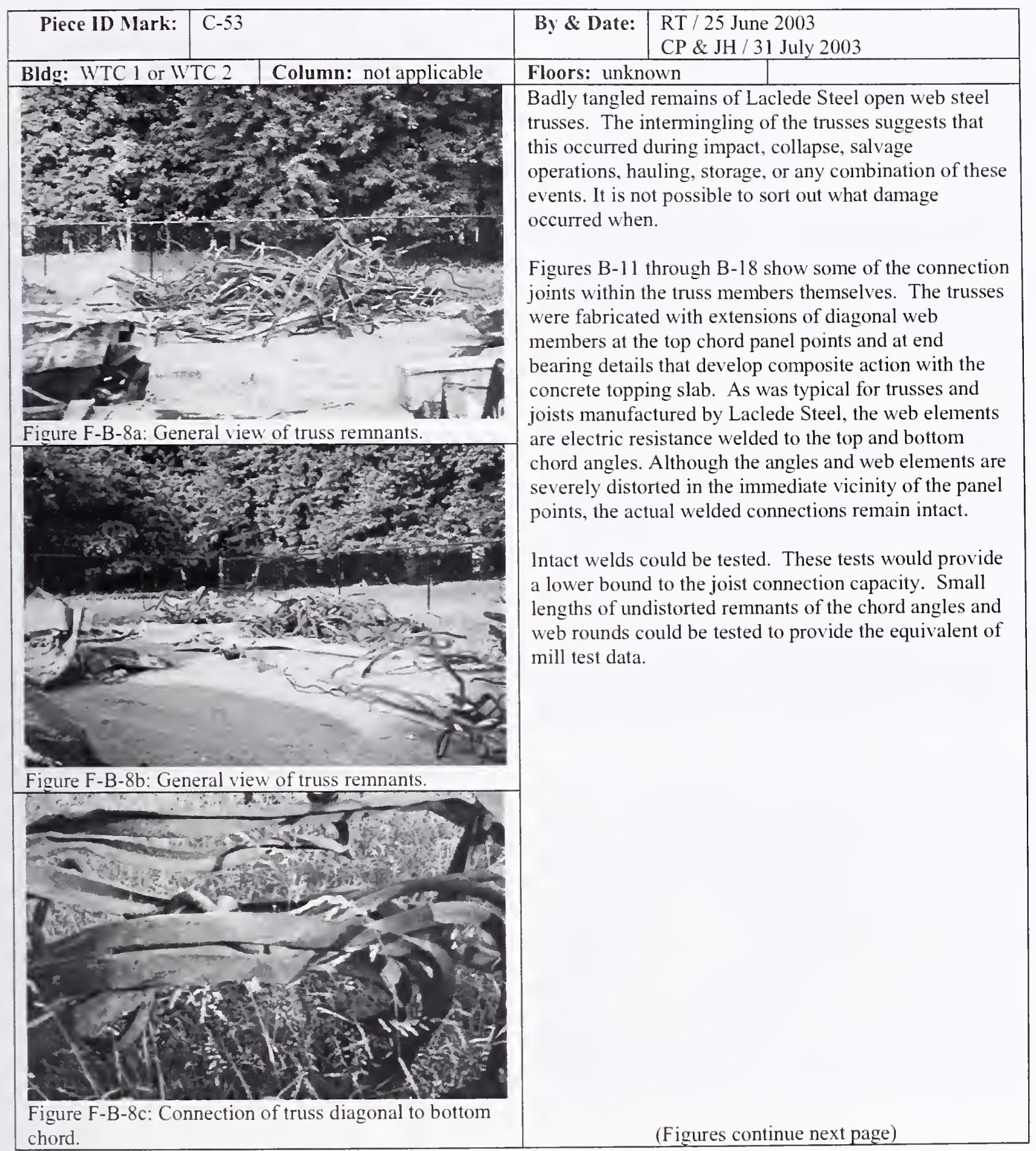




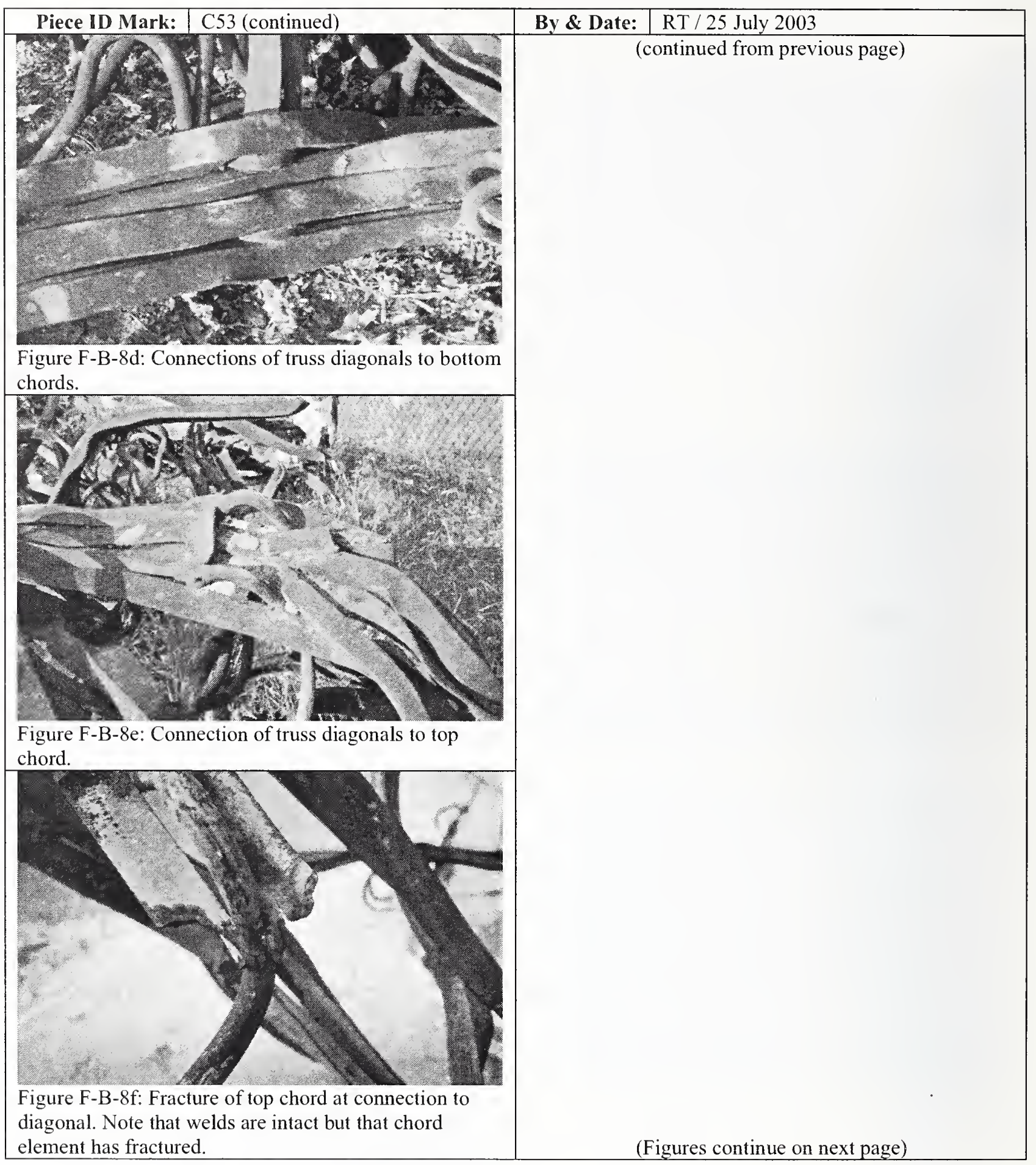


In Support of Task 2 under Project 3 of the NIST WTCI Visual Observations of the Steel Recovered From the World Trade Center Site

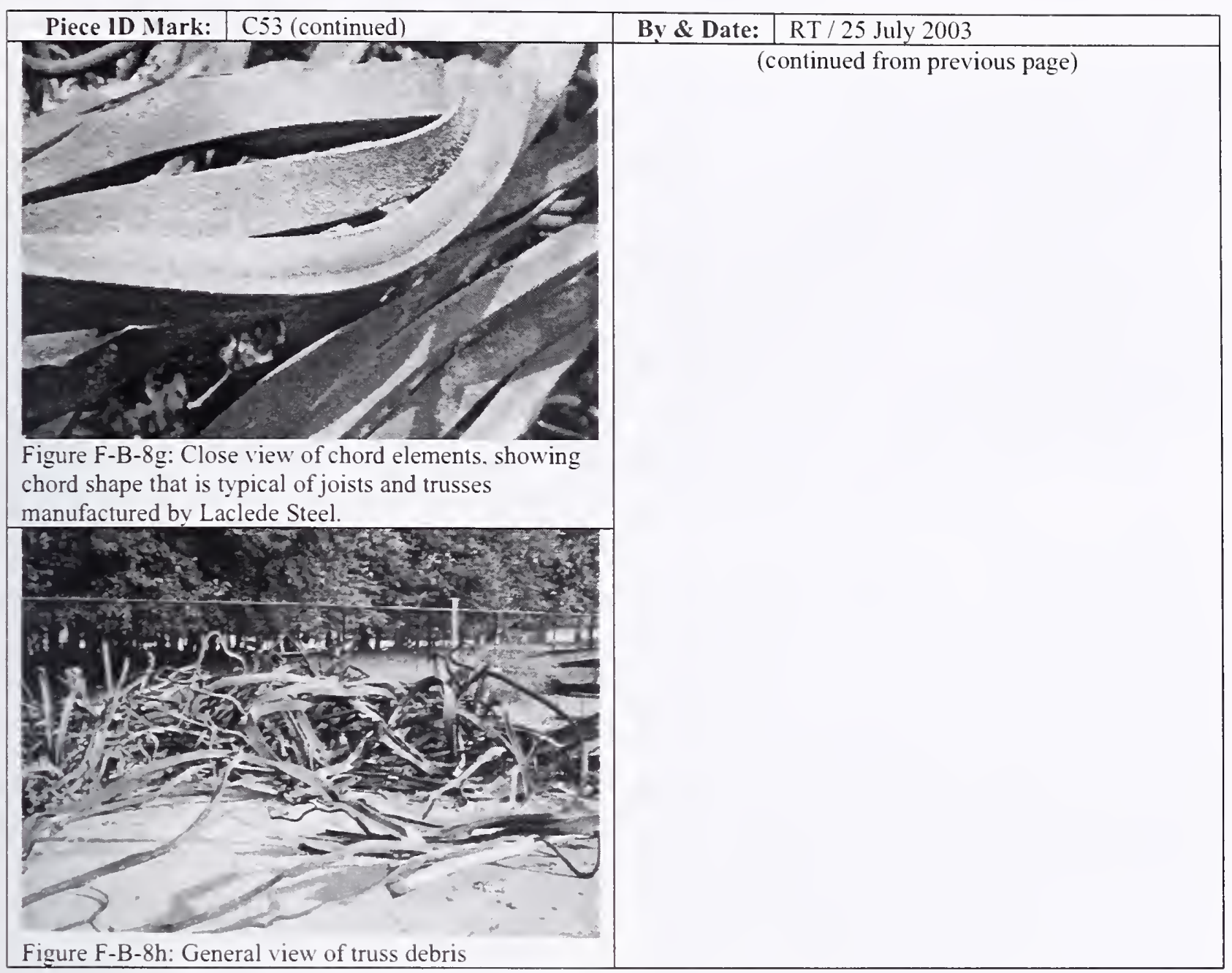




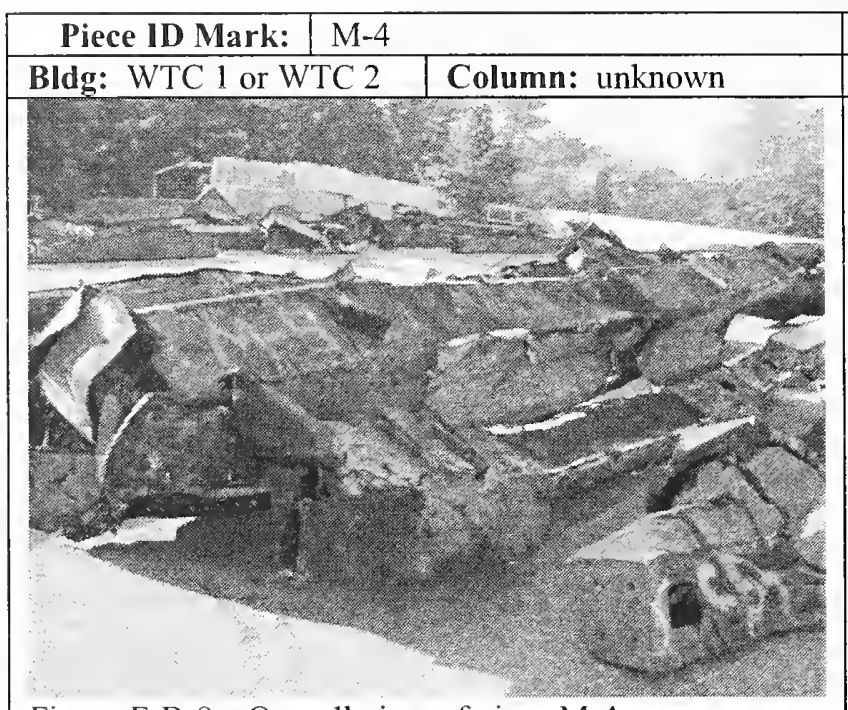

Figure F-B-9a: Overall view of piece M-4.

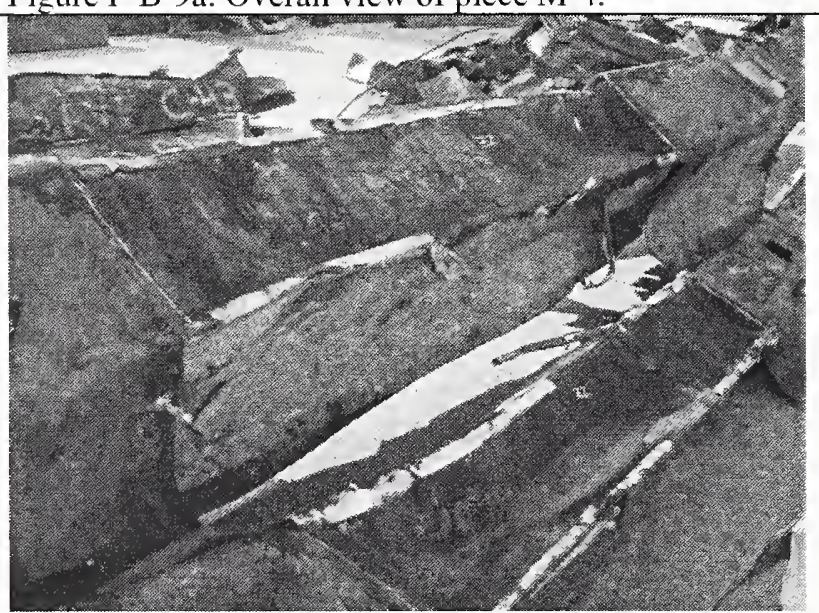

Z4: At window location just below the middle spandrel element. Loss of paint and severe distortions suggest possible fire effects.

Z3: At spandrel section at middle of panel. Little or no visible indication of fire effects.

Z2: At window location between middle and upper spandrel elements. Inside face of columns exhibit loss of paint and inward local buckling or dishing, both indicative of possible fire effects.

Z1: At upper spandrel section. Little or no visible indication of fire effects.

Z0: At window location above upper spandrel element. Little or no indication of fire effects.

Figure F-B-9b: Identified Zone Z2. Possible fire effects on inside faces of column elements at window location.

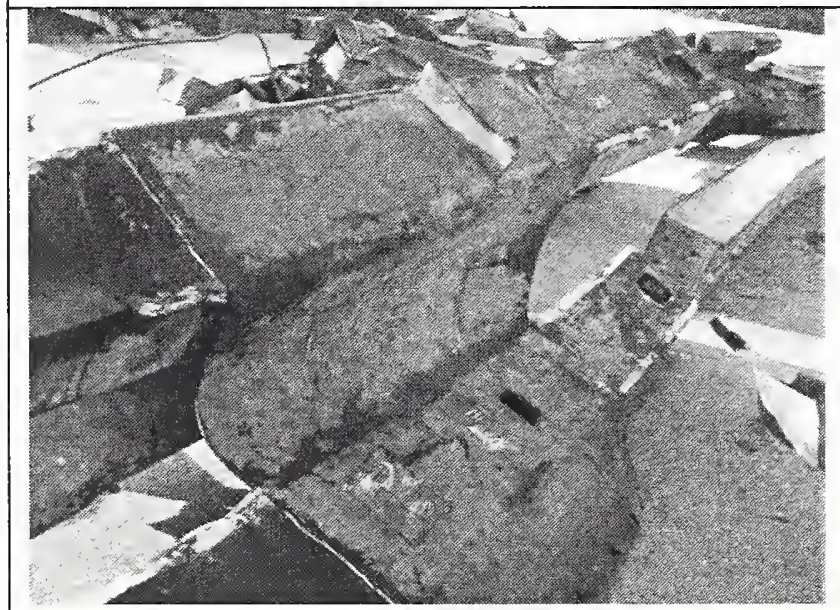

Figure F-B-9c: Identified Zones Z1 and Z0. 


\begin{tabular}{|l|l|l|}
\hline Piece ID Mark: $\mid \mathrm{N}-1$ & \multicolumn{1}{|c|}{ By \& Date: } & CP / 24 June 2003 \\
\hline Bldg: WTC 1 & Floors: $82-85$ & $\begin{array}{l}\text { This column is from well below the aircraft impact zone. } \\
\text { Panel is bent in half at about mid-length. No indication } \\
\text { of fire effect. Given location in building, probable cause } \\
\text { of observed damage and distortion is the collapse event } \\
\text { and subsequent handling. }\end{array}$ \\
&
\end{tabular}

\begin{tabular}{|l|l|l|}
\hline Piece ID Mark: & S-14 or C-20 & By \& Date: \\
\hline Bldg: WTC 2 & Column: 218 & $\begin{array}{l}\text { Full exterior column panel from high floors in WTC 2, } \\
\text { well above the aircraft impact zone for WTC 2. Possible } \\
\text { fire effects observable at some locations on the piece. } \\
\text { Given location of this piece in WTC 2, it is most } \\
\text { probable that the observed fire effects are related to } \\
\text { post-collapse fire rather than fire immediately after } \\
\text { aircraft impact. }\end{array}$ \\
\hline
\end{tabular}




\section{ATTACHMENT C - PIECES FROM STRUCTURES OTHER THAN WTC 1 AND WTC 2}




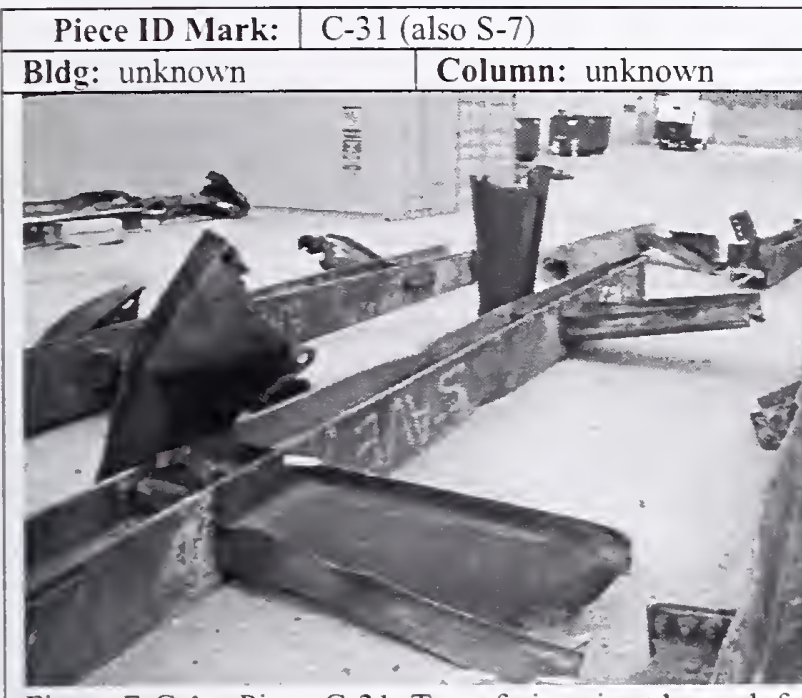

Figure F-C-1 a: Piece C-31. Top of piece is at lower left; bottom of piece is at upper right.

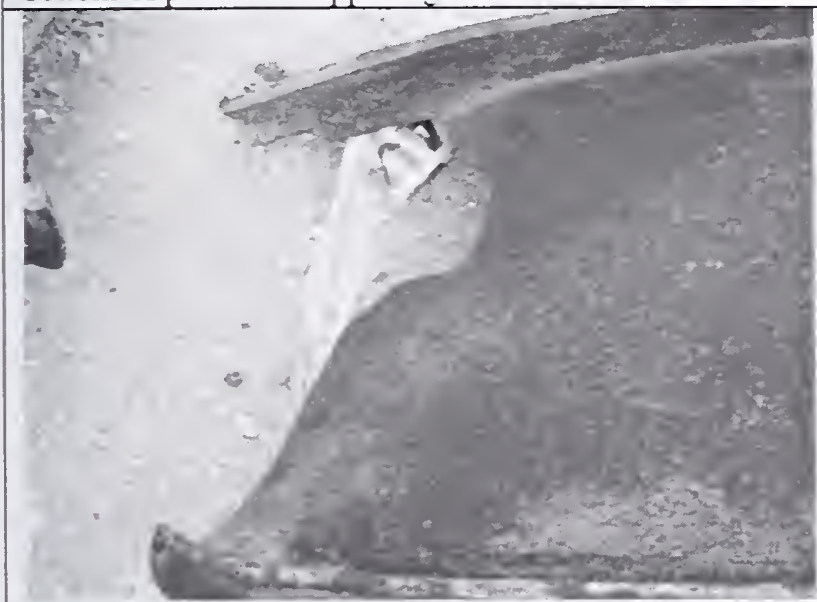

Figure F-C-1b: Piece C-31. Fracture at end of beam stub that remains in-plane

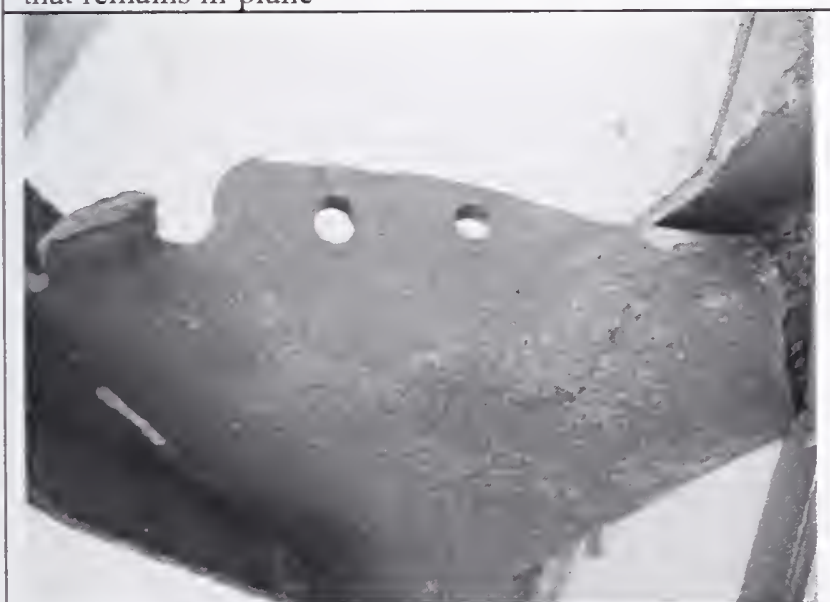

Figure F-C-1c: Piece C-31. Fracture at end of a beam stub that is bent out-of-plane
By \& Date: 1 CP \& JH / 31 July 2003

Floors: unknown $\quad$ Fire effects

Two-story wide flange column piece probably from a structure other than WTC 1 or WTC 2 . The column piece itself is generally straight.

The beam stubs framing into the flange on one side of the column (i.e., the beams are in the plane of strong axis column bending) are generally undeflected and remain in the plane of strong axis column and beam bending. The fractures at the free ends of these beam stubs suggest that these beam stubs have probably failed in tension generally directed along the longitudinal axis of the beam stub.

The beam stubs framing into the opposite flange have been bent out-of-plane, extending in a direction generally perpendicular to the plane of strong axis beam and column bending. Examination of the column piece, including examination of column space at base of column piece, suggest that these beam stubs were bent out-of-plane during collapse. Examination of bolt hole distortions at the connection at the free ends of these beam stubs suggests that these connections also fractured in tension generally directed along the longitudinal axis of the beam stub.

The outstand beam stubs appear to be fire effected. 


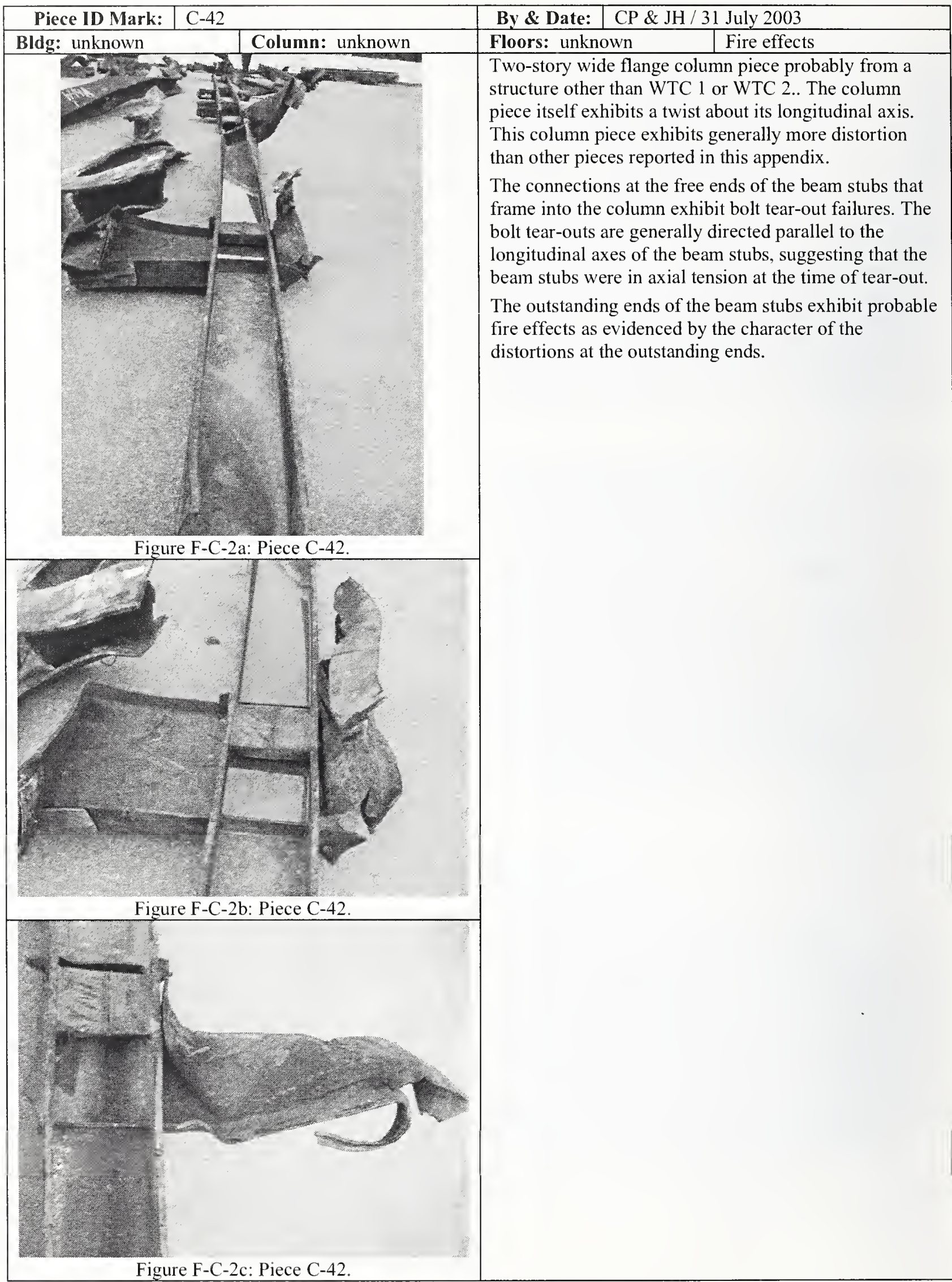




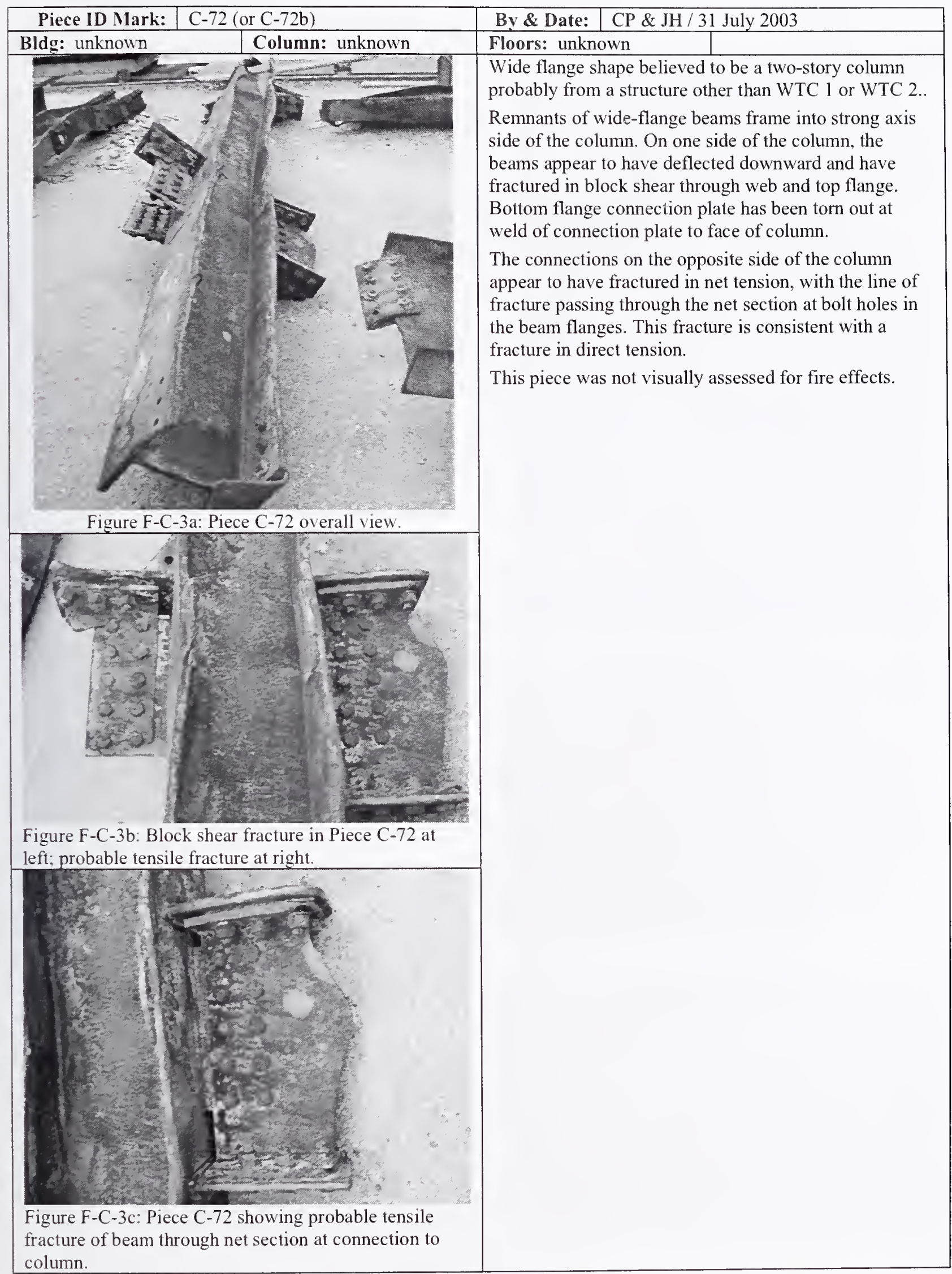




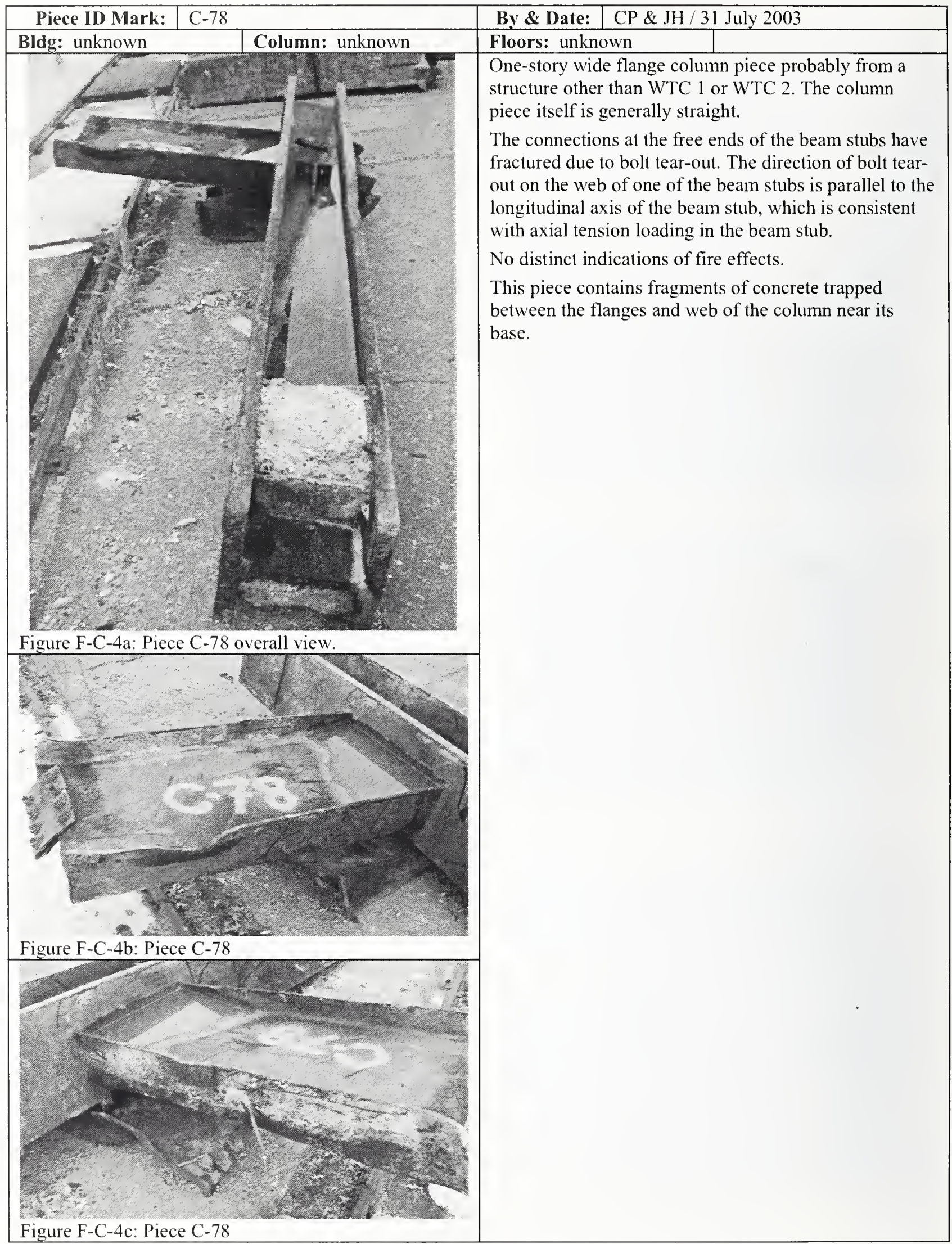




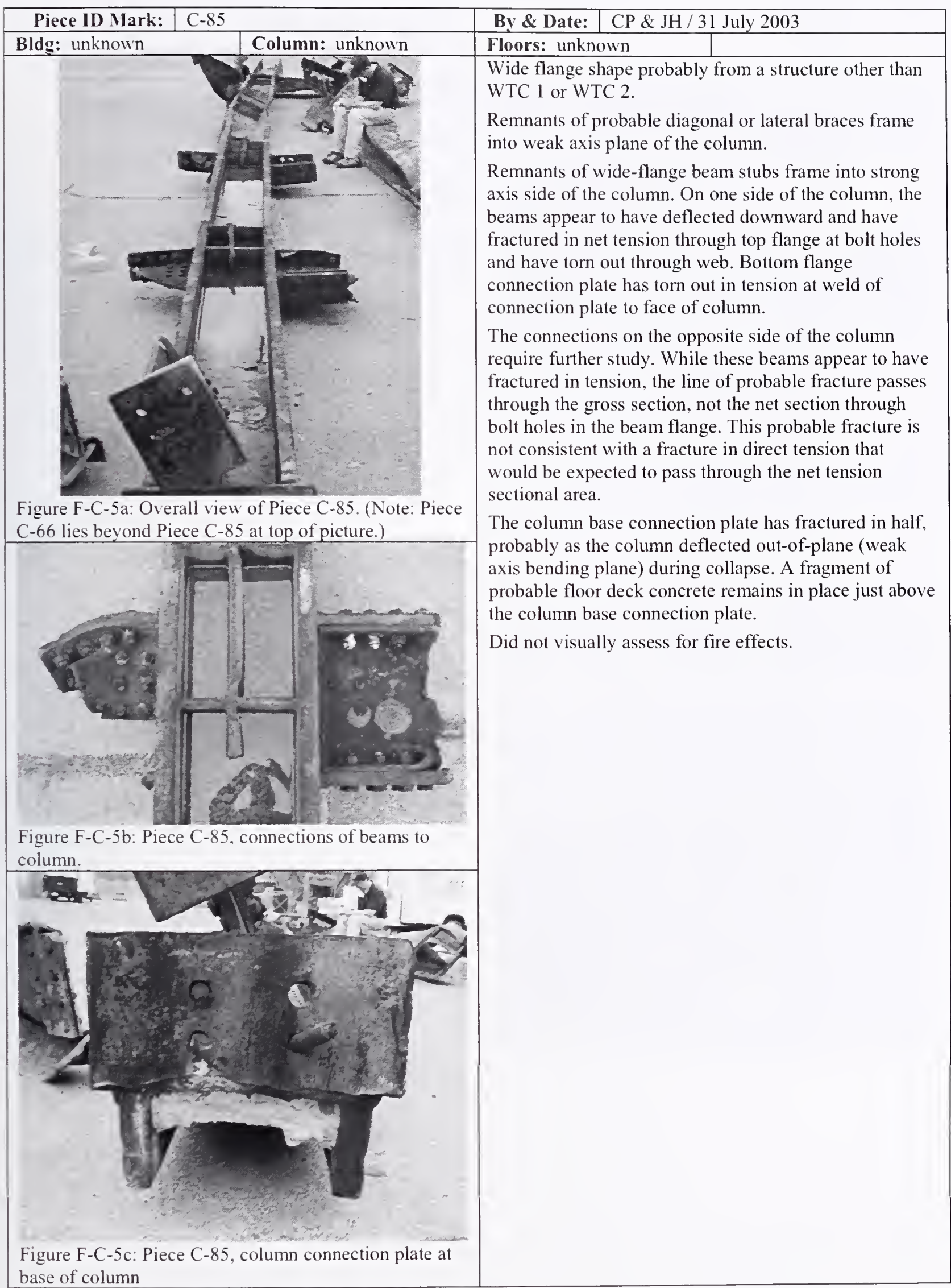




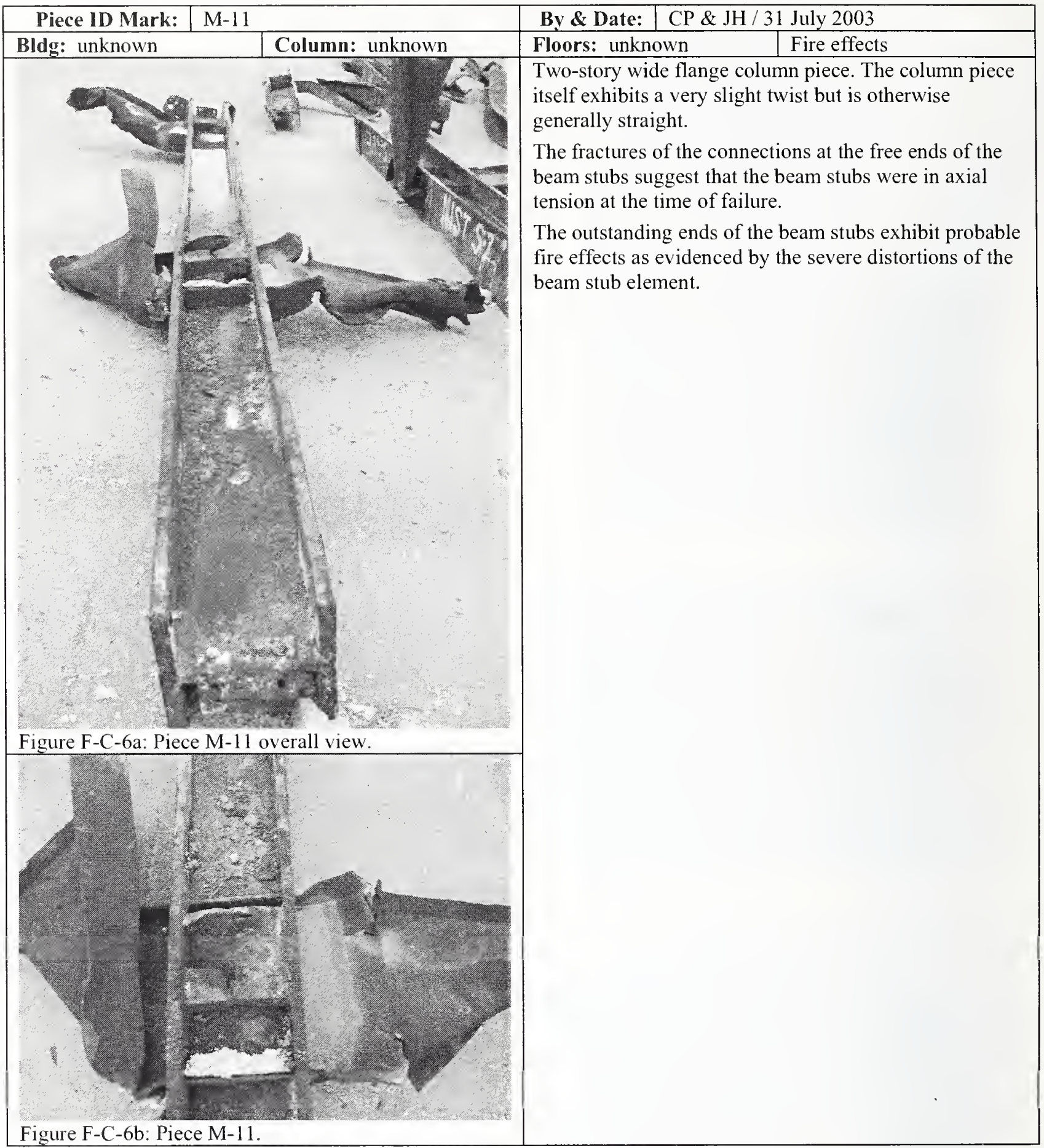



SELECTED PIECES FROM WTC 1 AND WTC 2 


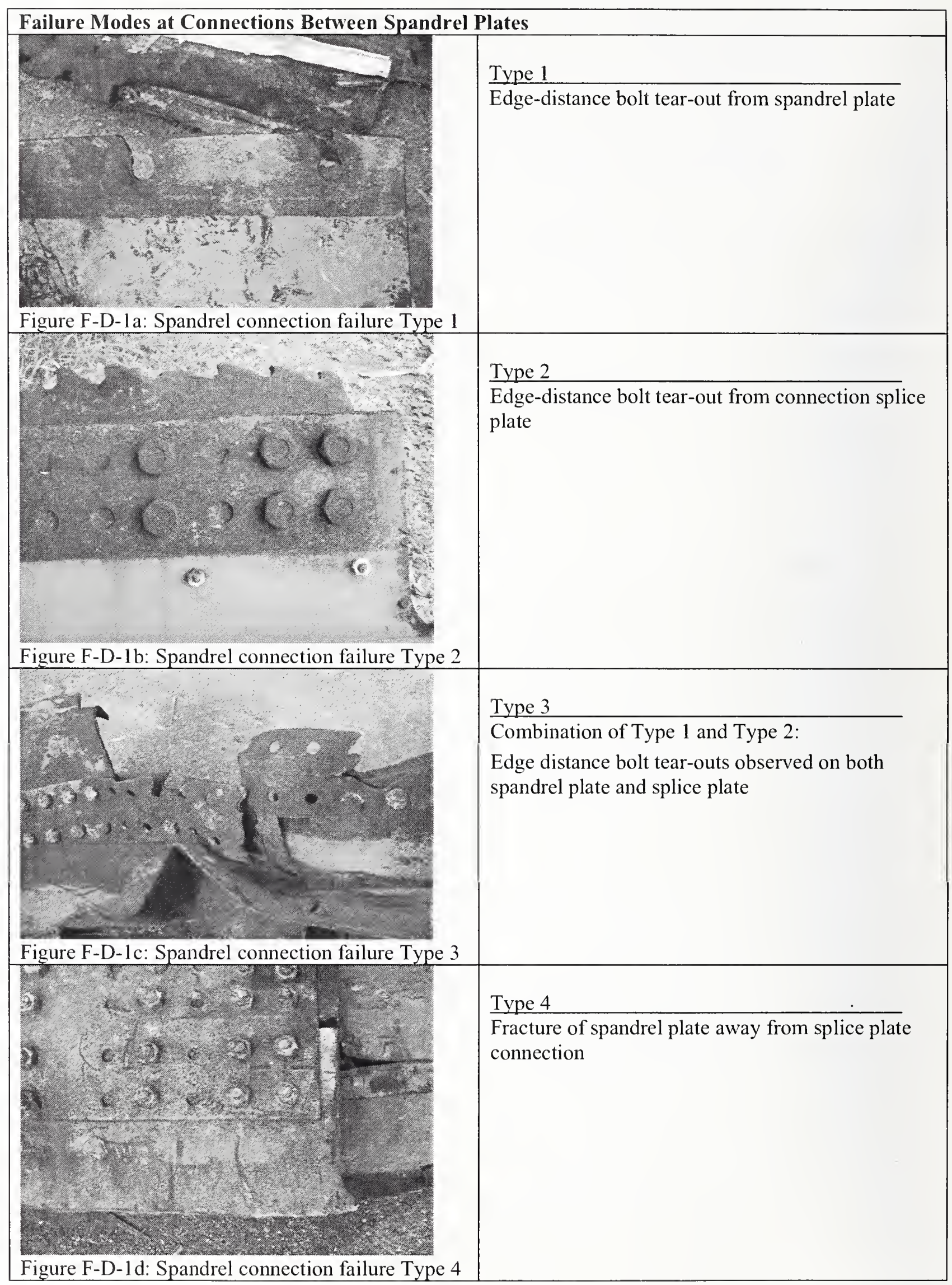




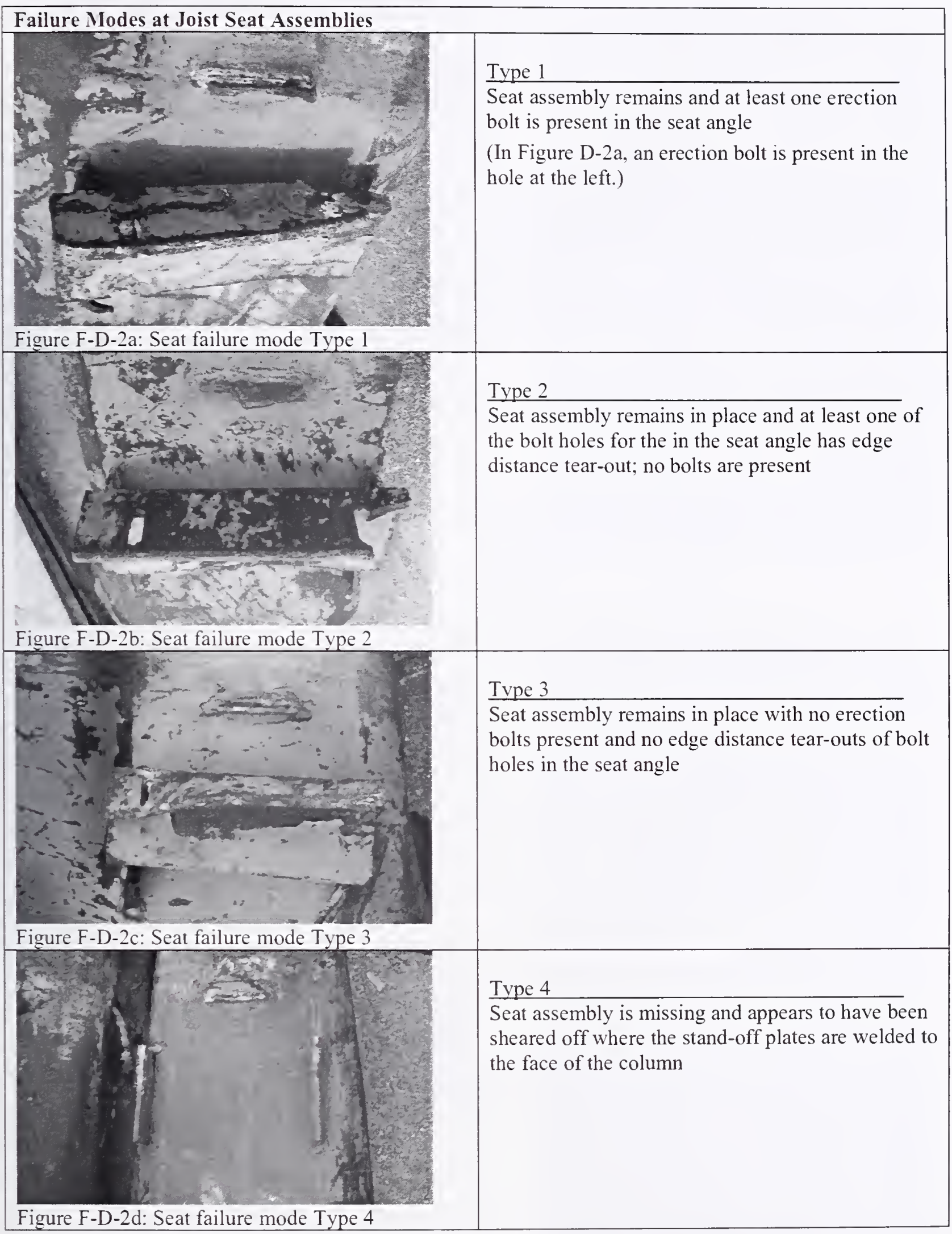




\begin{tabular}{|c|c|}
\hline \multicolumn{2}{|c|}{ Failure Modes at Joist Top Chord End Gusset Plate Connection } \\
\hline 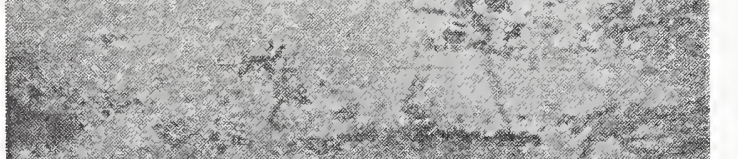 & $\begin{array}{l}\text { Type } 1 \\
\text { Fracture of top plate through gross section away } \\
\text { from weld to face of column }\end{array}$ \\
\hline Figure F-D-3a: End gusset failure mode Type 1 & \\
\hline & $\begin{array}{l}\text { Type } 2 \\
\text { Tear-out of top plate and weld from the parent } \\
\text { material of the column face }\end{array}$ \\
\hline Figure F-D-3b: End gusset failure mode Type 2 & \\
\hline
\end{tabular}

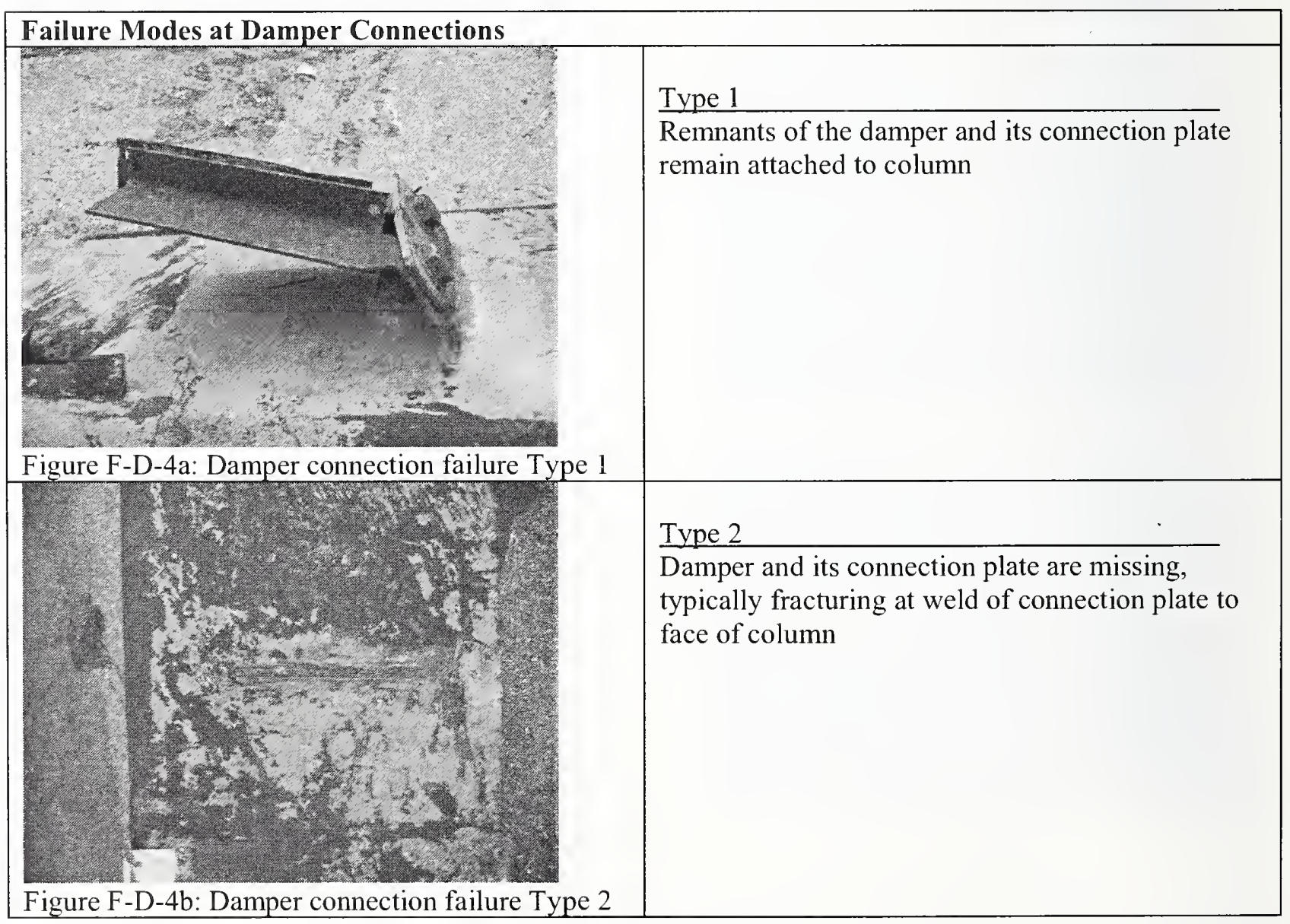


Table D-1: Quantities of Failure Mode Types as Observed on Recovered Pieces

\begin{tabular}{|c|c|c|c|c|c|c|c|c|c|c|c|c|c|c|}
\hline \multirow{2}{*}{$\begin{array}{c}\text { Piece } \\
\text { No. }\end{array}$} & \multirow[b]{2}{*}{ Bldg. } & \multirow[b]{2}{*}{ Floors } & \multicolumn{4}{|c|}{$\begin{array}{c}\text { Spandrel } \\
\text { Failure Type }\end{array}$} & \multicolumn{4}{|c|}{$\begin{array}{l}\text { Truss Seat } \\
\text { Failure Type }\end{array}$} & \multicolumn{2}{|c|}{$\begin{array}{c}\text { Top Plate } \\
\text { Failure Type }\end{array}$} & \multicolumn{2}{|c|}{$\begin{array}{c}\text { Damper } \\
\text { Failure Type }\end{array}$} \\
\hline & & & 1 & 2 & 3 & 4 & 1 & 2 & 3 & 4 & 1 & 2 & 1 & 2 \\
\hline $\mathrm{N}-9$ & WTC1 & $101-104$ & 4 & & & & 2 & 1 & & 1 & 2 & 2 & 1 & 3 \\
\hline $\mathrm{N}-101$ & WTC1 & $100-103$ & 5 & & & & 3 & & & & 3 & & 2 & 1 \\
\hline C-93 & WTC1 & $099-102$ & & & & & & 1 & & & 1 & & & 1 \\
\hline$M-20$ & WTC1 & $099-102$ & & & 1 & & 1 & & & & & 1 & & 1 \\
\hline$M-26$ & WTC1 & 099-102 & 6 & & & & & 1 & 1 & 4 & 6 & & & 6 \\
\hline$N-99$ & WTC1 & 099-102 & 4 & & & & 1 & 2 & & & 4 & & & 4 \\
\hline$C-40$ & WTC1 & 098-101 & 2 & & & & 1 & 1 & & & 2 & & & 2 \\
\hline $\mathrm{N}-7$ & WTC1 & $097-100$ & 4 & & 1 & & 1 & & 1 & 1 & 2 & 1 & 1 & 2 \\
\hline $\mathrm{N}-8$ & WTC1 & $097-100$ & 4 & & & 1 & 1 & & & & 2 & & & 2 \\
\hline S-9 & WTC1 & $097-100$ & 4 & & & 1 & 1 & & 1 & 1 & 2 & 1 & 2 & 1 \\
\hline$M-2$ & WTC1 & $096-99$ & 4 & & & 1 & 1 & 3 & & 2 & 5 & 1 & 3 & 3 \\
\hline $\mathrm{C}-22$ & WTC1 & 093-96 & 1 & & & 2 & & & & 2 & & 1 & & 1 \\
\hline $\mathrm{N}-12$ & WTC1 & $092-95$ & 2 & & & 1 & & 1 & & 2 & 1 & 2 & & 3 \\
\hline S-10 & WTC1 & $092-95$ & 1 & & & & & & & 2 & 1 & 1 & & 2 \\
\hline$C-25$ & WTC1 & $089-92$ & & & & 2 & & & & & & & & \\
\hline $\mathrm{N}-10$ & WTC1 & $089-92$ & 1 & & & & & 1 & 1 & & 1 & 1 & & 2 \\
\hline C-10 & WTC1 & $085-88$ & 2 & & & 3 & & 3 & 1 & 1 & & 6 & 2 & 4 \\
\hline $\mathrm{N}-1$ & WTC1 & $082-85$ & 2 & & & & & & 1 & 2 & 3 & & 1 & 2 \\
\hline S-1 & WTC1 & $079-82$ & 1 & & & & & & & & & & & \\
\hline $\mathrm{CC}$ & WTC1 & $070-73$ & 2 & & & & & & & 3 & 3 & & & 3 \\
\hline$M-28$ & WTC2 & 098-101 & 2 & & & & & 1 & & & 1 & & & 1 \\
\hline C-18 & WTC2 & 093-96 & 1 & & & 1 & 2 & 1 & & & 4 & & 3 & 1 \\
\hline C-92 & WTC2 & $093-96$ & 2 & & & & 1 & 1 & & & 2 & & 2 & \\
\hline S-14 & WTC2 & $091-94$ & 4 & & & 2 & 2 & 1 & & 2 & 3 & 2 & 2 & 2 \\
\hline$C-48$ & WTC2 & $091-91$ & 2 & & 1 & & & & 1 & 1 & 2 & & & 2 \\
\hline $\mathrm{C}-13$ & WTC2 & $090-92$ & & & & 1 & & & & 2 & 1 & 1 & & 2 \\
\hline$M-10 b$ & WTC2 & $083-86$ & & & 2 & & & 1 & & & 1 & & 1 & \\
\hline$M-10 a$ & WTC2 & $082-85$ & & & 2 & & & & & & & & & \\
\hline$C-46$ & WTC2 & $068-71$ & & 3 & & 1 & & & & 1 & 1 & & & 1 \\
\hline C-89 & WTC2 & 012-15 & & 3 & & & & & & & & & & \\
\hline ASCE-2 & & & & 2 & & & & & & & & & & \\
\hline ASCE-3 & & & & 1 & & & & 1 & & & 1 & & 1 & \\
\hline $\mathrm{BB}$ & & & 2 & 1 & & & & & & 3 & 3 & & & 3 \\
\hline C-102 & & & & 1 & & & & & 1 & & 1 & & & 1 \\
\hline $\mathrm{C}-11$ & & & 2 & & & & & & & & & & & \\
\hline $\mathrm{C}-117$ & & & 2 & & & & 1 & 1 & & & 2 & & 1 & 1 \\
\hline $\mathrm{C}-16$ & & & 1 & & & & & & 1 & & 1 & & & 1 \\
\hline C-24 & & & & 2 & & & & & 1 & 1 & 1 & 1 & & 2 \\
\hline C-28 & & & & & & 1 & 1 & & & & & 1 & & 1 \\
\hline$C-41$ & & & 2 & & & & & & & & & & & \\
\hline$C-43$ & & & 1 & & & & & & & & & & & \\
\hline C-52 & & & & & & 1 & 1 & & & & 1 & & & 1 \\
\hline C-64 & & & & & & 1 & & & & & & & & \\
\hline C-68 & & & 1 & & & & & & & 1 & 1 & & & 1 \\
\hline C-77 & & & & & & 2 & & & 1 & & 1 & & & 1 \\
\hline C-84 & & & & 3 & & & & & & & & & & \\
\hline$F F$ & & & & 2 & & & & & & & & & & \\
\hline$M-19$ & & & 1 & & & & & 1 & & & & 1 & & 1 \\
\hline$M-4$ & & & 4 & & & & & & 1 & 2 & 1 & 3 & & 4 \\
\hline$N-11$ & & & 3 & & & 1 & & & 2 & & 1 & 1 & & 2 \\
\hline $\mathrm{N}-\mathrm{N}$ & & & 1 & & & & & & & 1 & 1 & & & 1 \\
\hline SubTotal & & & 80 & 18 & 7 & 22 & 20 & 22 & 14 & 35 & 68 & 27 & 22 & 72 \\
\hline Total & & & & 12 & & & & & & & & & & \\
\hline$\%$ & & & $63 \%$ & $14 \%$ & $6 \%$ & $17 \%$ & $22 \%$ & $24 \%$ & $15 \%$ & $38 \%$ & $72 \%$ & $28 \%$ & $23 \%$ & $77 \%$ \\
\hline
\end{tabular}


This page intentionally left blank. 


\section{Appendix G \\ SUMMARY OF NIST REVIEW OF APPENDIX F, WJE REPORT}

\section{G.1 INTRODUCTION}

In support of Task 2 of Project 3 in the National Institute of Standards and Technology (NIST) World Trade Center (WTC) Investigation, Wiss. Janney, Elstner Associates, Inc. (WJE) was retained to perform two tasks: (1) provide independent identification of recovered structural steel of particular interest to the furtherance of other tasks under Project 3; and (2) through unaided visual examination, identify and describe the various failure mechanisms and damage types observed on the recovered structural steel members. Thus. WJE team members surveyed and documented possible local failure mechanisms from a structural engineering perspective on exterior panels, core columns, floor trusses, and structural connections (spandrel splice plates, floor truss seat connectors).

Through careful visual examination of a piece, WJE attempted to meaningfully characterize what extreme events affected a particular piece, including identification of failure modes associated with the extreme event. The extreme loading events considered in this examination, as defined by WJE, were as follows: (1) loading related to the impact of the aircraft, (2) loading and changes in properties related to the fire following the aircraft impact and before the collapse of the structures, (3) the collapse of the structures, (4) exposure to fire in the post-collapse debris pile, and (5) physical handling during recovery and salvage operations. Each of these extreme events can affect the structural components, structural connections. and the steel material itself in a different way. Observations were made with respect to physical damage sustained to the component as well as possible, pre-collapse indications of fire damage.

WJE began the examination more than a year after the collapse of the towers. The steel pieces had prior exposure to the elements of weather, which can promote corrosion of the surfaces of the exposed steel and can also erode the primer paint from the steel. Fracture surfaces also degrade with time. Therefore, in making observations, reasonable professional judgment, based on WJE's prior experience, was used to recognize and account for effects that may be related to post-collapse degradation of the recovered steel.

The complete WJE report can be found in Appendix F, with a summary of the main observations given below.

\section{G.2 SUMMARY OF MAIN OBSERVATIONS IN CONTRACTOR REPORT}

From the report prepared by WJE (found in Appendix F), the following main observations were made for recovered structural steel components from WTC 1 and WTC 2:

\section{G.2.1 Observations of Exterior Panels}

1. Observations showed that the fillet welds joining the plates that make up the columns of the exterior panels failed almost exclusively by fracture through the parent material in the heat affected zone (HAZ) of the weld. Extremely few fractures were observed through the weld 
material itself. These fractures were observed on recovered columns regardless of the extreme effects to which a piece was exposed. This indicates that the welded joints performed as intended.

2. Three significant pieces directly impacted by the aircraft from WTC 1 were examined and discussed within the text of the report. These panels were M-2, M-27, and M-30.

Observations on these pieces suggest that certain columns in these panels may have resisted the aircraft impact in part by membrane tension behavior (outer web plates), in a fashion roughly analogous to sideways stretching of a rubber band held between two fingers.

Likewise, other observations on these pieces suggest that other columns in each of these panels resisted the impact of the aircraft through shear behavior (transverse shear associated with outer and inner web plates; longitudinal shear associated with flange plates). Note: The fourth panel identified by NIST as being directly hit by the airplane was panel S-9 from WTC 1. While not addressed in the text, discussion was given within Appendix A of the WJE report that the piece was in fact hit and did sustain impact damage.

3. Visual cues were used to identify areas on exterior panels where there was evidence of possible effects of pre-collapse fires. These pieces were distinguished from those exposed to fire following collapse of the towers by particular patterns of fire-related distress that would be unique to a fire occurring in the towers before their collapse. The visual effects included: (1) the presence and loss of primer paint, (2) surface discoloration and intensity of rusting at the exposed steel, and (3) localized distortion interpreted as local buckling possibly caused by heat-related expansive strains. The primary pattern identified and relied upon was the presence of typically more than one possible fire effects on various surfaces of the individual column elements only at window openings, accompanied by the absence of these possible fire effects on the spandrel areas of the column panel. WJE observed eight panels with possible evidence of pre-collapse fires; six identified panels (K-2, N-8, N-9, N-12, S-9, S-10) and two unidentified panels (C-11 and M-4).

4. Observations showed that fracture of the plates composing the perimeter columns near the fillet welded joints occurred on numerous sections experiencing different extreme loading events. Fractures were observed on sections that were both believed to have been exposed to fire as well as those that were not and pieces impacted directly by the plane and those that were outside of the impact zone. It is possible that the fracture near the welds occurred during the collapse of the towers or during post-collapse recovery and salvage operations. For pieces possibly exposed to fire, there is the additional possibility that fracture near the welds may have come about as a result of a particular combination of axial load in the column, pre-fire strength properties of the weld metal and column steel, and heating of the column due to fire.

\section{G.2.2 Observations on Core Columns}

1. Fracturing of the plates near the welds, similar to that found on the columns of the exterior panels (Observation \#4), were observed on the built-up box shaped core columns.

2. Damage to core column C-88 indicated that fracture of one of the flanges may have occurred as a result of the plane impact as the distorted shape was consistent with the direction of 
aircraft travel. The distortion to the three other plates was believed to be a result of the collapse events, possibly at elevated temperatures.

3. Possible effects of fire were observed on the core columns examined. It was not possible, however, to make a reasonable determination as to whether or not the fire effects observcd on the core columns were due to fire following aircraft impact and before collapse of the tower, or due to fire in the debris piles following collapse of the tower.

\section{G.2.3 Floor Truss Material}

1. The electric resistance welds at many web-to-chord connections on the floor trusses did not fail but instead remained intact. Many instances were also observed, however, where the welds had failed, allowing the web members to become separated from the chord element. Qualitatively, these failures appeared to happen more frequently than the case of wclds remaining intact.

\section{G.2.4 Structural Connections}

1. Noticeable differences in distribution of failure modes for truss seat connections above the aircraft impact floor compared to those below the aircraft impact floors was seen for joist seat connections and joist top plate connections.

2. Column-to-column end plate splice connections on columns not directly impacted by the aircraft always failed by fracture of the bolts. On columns that were impacted by an aircraft, some of these splice connections failed by fracture of the welds between the end plate and plates that made up the faces of the columns.

\section{G.3 NIST REVIEW OF CONTRACTOR REPORT}

The WJE team was limited by time and analytical resources when thcir work was conducted. Additionally, the state of the NIST supplied knowledge regarding pre-collapse fire exposure (Sec. 2.3) and paint condition analysis (Sec. 6.2) had not been fully developed at the time that WJE made their observations. Thus, a complete review of the WJE observations was performed by NIST in light of the more complete information now available.

- Observation 1: NIST's findings agree with the observations of WJE in that fracture associated with weld joints on perimeter panels typically occurred in the heat affected zone of the basc material next to the weld toe. This mechanism was almost exclusively observed on pieces not directly impacted by the airplane (those hit by the plane experienced numerous failure mechanisms), and in particular, was seen on panels from all as-built locations within the towers, regardless of pre-collapse conditions.

- Observation 2: In general, NIST's findings agree with the observations of WJE concerning the failure modes of panels that were directly impacted by the airplane. Note: WJE discussed in the text the damage characteristics of only three WTC 1 panels that were impacted by the airplane and recovered (M-2, M-27, and M-30). Discussion of the damage to 
S-9 was not located in the text of the report, however, it was described in Appendix A as a piece that sustained impact damage.

- Observation 3: WJE identifies possible exposure to pre-collapse fire through the use of visual evidence of particular patterns of fire-related distress that would be unique to a fire occurring in the towers before their collapse (i.e., sooting, scorching, and/or loss of primer paint; localized distortion/buckling of column plates). Although not stated as such by WJE, this suggests the possibility that the temperature of the column steel had become elevated due to fire exposure. At the time of their investigation, WJE team members did not have access to the fire exposure-time sequence maps (Sec. 2.3) or the paint condition evaluations (Sec. 6.2) as the methods were not fully developed. From these two analysis techniques, NIST researchers did not find a correlation between the presence of sooting or other surface discoloration of the paint and the base metal reaching elevated temperatures. In addition, the loss of the primer paint can also be attributed to other external effects such as plastic straining of the painted plate, abrasion, or ambient corrosion processes. Further, WJE interpreted local buckling of individual plates within column elements as possibly caused by heat-related expansive strains. NIST identified numerous exterior panels that had similar localized plate buckling of columns that could not have experienced exposure to pre-collapse fires due to their as-built location. Thus, while the fire exposure-time sequence analysis corroborates many of WJE's visual observations (see discussion immediately below), an inferred correlation between observed failure modes and pre-collapse temperature excursions was not supported.

The WJE report indicated nine exterior panels and one core column with possible visual evidence of precollapse fire exposure. These are listed individually below as well as NIST comments made with regard to the findings of the NIST analysis in Secs. 2.3 (pre-collapse photographic evidence) and 6.2 (analysis of the paint for mud-cracking). As both of the visual observations were made directly on the primer paint, comments concerning the condition of the sprayed fire-resistive material (SFRM) immediately after the impact was also included.

- Panel K-1 (A209: 97-100)

- Recovered portion of panel: Portions of 97 th and 98 th floors.

- Condition of SFRM immediately after impact: SFRM believed to be intact as the aluminum façade was intact.

- WJE observations: Reported damage characteristics of the 98th floor (sharp curvature of the bent steel) may be a result of possible fire effects from pre-collapse fire exposure.

- NIST observations: Some loss of primer paint, but enough paint was present to conduct an analysis of the recovered portion of the panel. Mud cracking was found on the flanges and inner web of the 98 th floor.

- Pre-collapse photographic evidence: Pre-collapse fires were observed on the 97th and 98th floors, but not on the 99th and 100th floors. 


\section{- Panel K-2 (A236: 92-95)}

- Recovered portion of panel: Portions of 92nd, 93rd, and 94th floors.

- Condition of SFRM immediately after impact: Unknown as pre-collapse photographs of sufficient resolution for analysis were not available.

- WJE observations: Reported distinct pattern of possible fire effects from pre-collapse fire exposure observed at windows of the $92 \mathrm{nd}, 93 \mathrm{rd}$, and 94 th floors.

- NIST observations: Some loss of primer paint, but enough paint was present to conduct an analysis of the recovered portion of the panel. Mud cracking was only found on the lower portion of the 93 rd spandrel, indicating fire on the 92 nd floor.

- Pre-collapse photographic evidence: Pre-collapse fires were observed on the 92nd and 94th floors, but not on the 93rd and 95th floors.

- Panel N-8 (A142: 97-100)

- Recovered portion of panel: Full panel.

- Condition of SFRM immediately after impact: SFRM believed to be intact as the aluminum façade was intact.

- WJE observations: Reported local buckling and loss of primer paint at the 98th floor window as indications of possible pre-collapse fire exposure.

- NIST observations: Some loss of primer paint, but enough paint was present to conduct an analysis of the entire panel. Mud cracking was only found on the truss seat from the 99th floor indicating fires on the 98th floor.

- Pre-collapse photographic evidence: Pre-collapse fires were observed to be on the 97th and 98 th floors, but not on the 99th or 100th floors.

- Panel N-9 (A154: 101-104)

- Recovered portion of panel: Nearly full panel with portions of all floors.

- Condition of SFRM immediately after impact: SFRM believed to be intact as the aluminum façade was intact.

- WJE observations: Reported local buckling and loss of primer paint on the inside face of a column at an unidentified location as indications of possible pre-collapse fire exposure.

- NIST observations: Some loss of primer paint, but enough paint was present to conduct an analysis of the recovered portion of the panel. Mud cracking was not found on the panel. 
- Pre-collapse photographic evidence: Pre-collapse fires were not observed on any floors of the panel.

- Panel N-12 (A206: 92-95)

- Recovered portion of panel: Portions of all floors.

- Condition of SFRM immediately after impact: Unknown as pre-collapse photographs of sufficient resolution for analysis were not available.

- WJE observations: Reported local buckling and loss of primer paint at the 93rd and 94th floor windows as indications of possible pre-collapse fire exposure.

- NIST observations: Some loss of primer paint on the panel, but enough paint was present to conduct an analysis of the entire panel. Mud cracking was not found on the panel.

- Pre-collapse photographic evidence: Pre-collapse fires were observed on the 93rd and 94th floors, but not on the 92nd or 95th floors.

- Panel S-9 (A133: 97-100)

- $\quad$ Recovered portion of panel: Nearly full panel with portions of all floors.

- Condition of SFRM immediately after impact: Unknown as smoke obscured visual observation in all pre-collapse photographs.

- WJE observations: Reported loss of primer paint at the $98^{\text {th }}$ floor windows as indications of possible pre-collapse fire exposure.

- NIST observations: Some loss of primer paint on the panel, but enough paint was present to conduct an analysis of the entire panel. Mud cracking was not found on the panel.

- Pre-collapse photographic evidence: Pre-collapse fires were observed on the 98th floor, but not on the 97 th, 99 th, or 100 th floors.

- Panel S-10 (A224: 92-95)

- Recovered portion of panel: Portions of 92nd, 93rd, and 94th floors.

- Condition of SFRM immediately after impact: Unknown as pre-collapse photographs of sufficient resolution for analysis were not available.

- WJE observations: Reported loss of primer paint and local buckling on the entire portion of the recovered panel as indications of possible pre-collapse fire exposure.

- NIST observations: As no paint was available for inspection, no conclusion could be made concerning NIST's evaluation of the panel. 
- Pre-collapse photographic evidence: Pre-collapse fires were observed on the 92nd floor of the panel, but not on the 93rd, 94th. or 95th floors.

- Panel C-11 (unidentified)

- Recovered portion of panel: Upper two stories of panel.

- Condition of SFRM immediately after impact: Unknown as panel was unidentified.

- WJE observations: Reported that middle portion of recovered panel had indications of fire effects.

- NIST observations: Some loss of primer paint on the panel, but enough paint was present to conduct an analysis of the entire panel. Mud cracking was not found on the panel.

- Pre-collapse photographic evidence: No information was available on the location of precollapse fires.

- Panel M-4 (unidentified)

- Recovered portion of panel: Upper two stories of panel.

- Condition of SFRM immediately after impact: Unknown as panel was unidentified.

- WJE observations: Reported the loss of primer paint and local buckling on the entire panel as indications of possible pre-collapse fire exposure.

- NIST observations: Some loss of primer paint on the panel, but enough paint was present to conduct an analysis of the entire panel. Mud cracking was not found on the panel.

- Pre-collapse photographic evidence: No information was available on the location of precollapse fires.

- Core Column C-88b (B801: 77-80)

- Recovered portion of column: Portion of 80th floor.

- Condition of SFRM immediately after impact: Unknown as pre-collapse photographs of the core not available.

- WJE observations: Reported damage characteristics of the plates composing the column in the $80^{\text {th }}$ floor region (sharp curvature of the bent steel) may be a result of possible fire effects from pre-collapse fire exposure.

- NIST observations: As no paint was available for inspection, no conclusion could be made concerning NIST's evaluation of the column. 
- Pre-collapsc photographic evidence: While the column was located within the fire floors, no direct information was available on the exposure of pre-collapse fires.

Comparing the results from the two analyses, some discrepancies exist where the visual observations made by WJE were in opposition to that of the results of the paint mapping technique utilized by NIST. As most of the observations of sooting and paint damage made by WJE were corroborated by the fire exposure-time sequencc maps, it may be that a degradation mechanism exists where the primer paint exposed to the pre-collapse fires was damaged (resulting in the visual patterns) without increasing the local temperature of the steel above $250^{\circ} \mathrm{C}$. Additional to the discrepancy between the visual observations of WJE and the paint mapping technique of NIST, there were seven panels where precollapse fires were observable, yet neither analysis technique indicated the exposure on the recovered panel. One example of this was from piece M-2 whcre images show fires/external flaming for over 16 continuous minutes on the 98 th floor (Sec. 2.3.1).

- Observation 4: While stating that fracture near welds did occur regardless of extreme effect that the piece may have seen, WJE also mentions a possible link between the fractures and elevated temperatures near the welds on many of the pieces possibly exposed to fire. This could lead to the conclusion that perimeter columns exposed to pre-collapse fire conditions were structurally weakened due to heat-related expansive strains. However, the case for such fracture cannot bc made from the evidence now available. It is unlikely that the actual temperature rise was sufficient to induce the large heat-related expansive strains to cause a fracture near the weld. Additionally, the constraint of the columns was not likely to be great enough to allow for these strains to occur. It was possible to conjecture that fracture near one weld could occur if these strains led to buckling of the thinner inner web plate, but the amount of damage observcd by WJE (areas fracturing near all four corner welds) was unlikely as the stresses would decrease upon commencement of the single, localized distortion. In carefully reviewing panels listed as "fire affected," numerous examples were observed where fracturc ncar welds occurred both with and without localized buckling. Further, localized buckling possibly related to fire exposure was observed on columns that did not have fractures near welds. Based on this evidence, it was believed that the majority of fractures associated with welds were not correlated with heat-related expansive strains. WJE does comment in their report that the observed fractures could have been caused by other sources besides strains associated with elevated temperatures.

- Observations 5, 6, and 7: WJE's discussion of the physical and fire damage sustained by the core columns was similar to that reported by NIST. Built-up box columns, particularly those with light gauges, were observed to have fractures in the HAZ of the base plate near.the toe of the weld. Additionally, it was concluded, from the damage characteristics sustained on the column and the as-built location, that core column C-88b (801B: 77-80) may have been damaged by the impact of the airplane. It was not possible to make a reasonable determination as to whether or not pre-collapse fires had any effect on this column or any of the other threc located in the fire floors.

- Observation 8: WJE's discussion of the truss remnants is similar to that of NIST.

- Observations 9 and 10: In general, similar distributions of failure modes for the structural connections were found between the WJE and NIST analysis. Most notable was the 
distribution of failure modes for truss seat connections above the aircraft impact floor compared to those below the aircraft impact floors. This was related to the difference in collapse mechanism for each portion of the building (e.g., below impact region, the building experienced "pan caking" of the floors resulting in a majority of the truss seat either torn off or bent downward). 
This page intentionally left blank. 



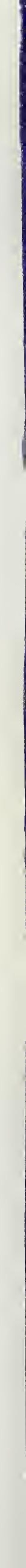

